Martin Koers

\title{
Steuerung von Markenportfolios
}




\section{Martin Koers}

\section{Steuerung von Markenportfolios}

Angesichts der Individualisierung des Konsumentenverhaltens und der damit verbundenen Fragmentierung der Märkte nimmt die Mehrmarkenstrategie in vielen Branchen eine bedeutende Stellung ein. Neben vielfältigen Chancen offenbart eine parallele Führung mehrerer selbständiger Marken im gleichen Produktbereich jedoch hohe Risiken. Werden etwa die charakteristischen Unterschiede zwischen den Marken von den Nachfragern nicht mehr differenziert wahrgenommen, steigt die Gefahr einer Kannibalisierung der Marken durch gegenseitige Marktanteilssubstitution. Die Mehrmarkenstrategie setzt somit stets eine genaue Abwägung der mit weiteren Marken erzielbaren Mehrerlöse einerseits und der zusätzlichen Kosten andererseits voraus. Vor diesem Hintergrund werden in der Arbeit Ansatzpunkte eines Mehrmarkencontrolling zur wirksamen Umsetzung und systematischen Steuerung eines Markenportfolios aufgezeigt. Dabei erweist sich die Balanced Scorecard als ein wertvolles Steuerungsinstrument, um bisher isolierte Partialansätze des Markencontrolling schlüssig in ein Gesamtkonzept zu integrieren.

Martin Koers wurde 1971 in Steinfurt geboren. Er studierte Betriebswirtschaftslehre an der Universität Münster und der Napier University of Edinburgh. Nach seinem Diplom 1996 arbeitete er zunächst als wissenschaftlicher Mitarbeiter am Lehrstuhl für Organisationstheorie und Personalmanagement der Universität Münster und war Dozent für Betriebswirtschaftslehre an der Verwaltungsakademie der Industrie- und Handelskammer Münster. Seit 1997 ist er wissenschaftlicher Mitarbeiter am Institut für Marketing bei Prof. Dr. Dr. h.c. mult. Heribert Meffert. Promotion zum Dr. rer. pol. im Juli 2000. 
Steuerung von Markenportfolios

Martin Koers - 978-3-631-75091-9

Downloaded from PubFactory at 01/11/2019 08:29:37AM

via free access 


\section{SCHRIFTEN ZU MARKETING UND MANAGEMENT}

Herausgegeben von Prof. Dr. Dr. h.c. mult. Heribert Meffert

Band 39

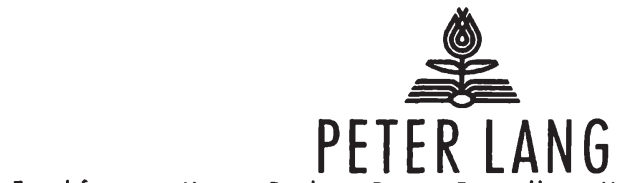

Frankfurt am Main - Berlin. Bern - Bruxelles - New York - Oxford. Wien Martin Koers - 978-3-631-75091-9 


\section{Martin Koers}

\section{Steverung von Markenportfolios}

Ein Beitrag zum Mehrmarkencontrolling am Beispiel der Automobilwirtschaft

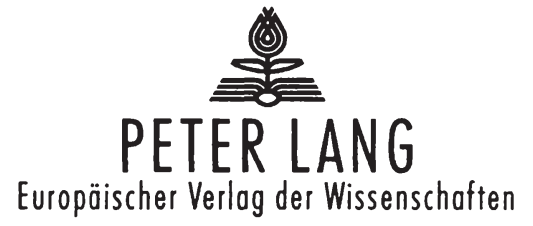

Martin Koers - 978-3-631-75091-9

Downloaded from PubFactory at 01/11/2019 08:29:37AM

via free access 
Die Deutsche Bibliothek - CIP-Einheitsaufnahme

Koers, Martin:

Steuerung von Markenportfolios : ein Beitrag zum

Mehrmarkencontrolling am Beispiel der Automobilwirtschaft /

Martin Koers. - Frankfurt am Main ; Berlin ; Bern ; Bruxelles ;

New York ; Oxford ; Wien : Lang, 2001

(Schriften zu Marketing und Management ; Bd. 39)

Zugl.: Münster (Westfalen), Univ., Diss., 2000

ISBN 3-631-37892-0

Open Access: The online version of this publication is published on www.peterlang.com and www.econstor.eu under the international Creative Commons License CC-BY 4.0. Learn more on how you can use and share this work: http://creativecommons. org/licenses/by/4.0.

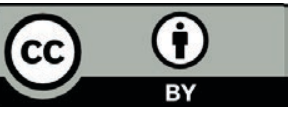

This book is available Open Access thanks to the kind support of ZBW - Leibniz-Informationszentrum Wirtschaft.

Gedruckt auf alterungsbeständigem, säurefreiem Papier.

\author{
D6 \\ ISSN 0176-2729 \\ ISBN3-631-37892-0 \\ ISBN 978-3-631-75091-9 (eBook) \\ (C) Peter Lang GmbH \\ Europäischer Verlag der Wissenschaften \\ Frankfurt am Main 2001 \\ Alle Rechte vorbehalten.
}

Das Werk einschließlich aller seiner Teile ist urheberrechtlich geschützt. Jede Verwertung außerhalb der engen Grenzen des

Urheberrechtsgesetzes ist ohne Zustimmung des Verlages unzulässig und strafbar. Das gilt insbesondere für Vervielfältigungen, Übersetzungen, Mikroverfilmungen und die Einspeicherung und Verarbeitung in elektronischen Systemen.

Printed in Germany 124567

www.peterlang.de

Martin Koers - 978-3-631-75091-9 


\section{Meinen Eltern}

und

\section{Astrid}


Martin Koers - 978-3-631-75091-9

Downloaded from PubFactory at 01/11/2019 08:29:37AM

via free access 


\section{Vorwort des Herausgebers}

Angesichts dynamischer Umfeldveränderungen haben sich die Bedingungen für die erfolgreiche Führung von Marken in vielen Branchen verschärft. Im horizontalen Wettbewerb besteht die Herausforderung, der Individualisierung des Konsumentenverhaltens und der damit verbundenen Fragmentierung der Märkte durch eine bedarfsgerechte Ausweitung des Angebotsspektrums gerecht zu werden und gleichzeitig die Wirtschaftlichkeit der Marktbearbeitung sicherzustellen. In diesem Spannungsfeld zwischen Effektivität und Effizienz der Markenführung steht nicht selten die Frage nach der Trag- bzw. Ausweitungsfähigkeit von Marken im Mittelpunkt der Betrachtung. Mit Hilfe der vielfach praktizierten Markenausdehnung wird versucht, das Leistungsspektrum etablierter Marken zu ergänzen und so in neue Marktsegmente vorzustoßen. Oftmals führt eine derartige Vorgehensweise jedoch zu einer Verwässerung ursprünglich klar profilierter Marken. Vor diesem Hintergrund gewinnt die Marktbearbeitung mit mehreren, parallel auf den Absatzmarkt ausgerichteten Marken zunehmend an Bedeutung.

Diese Beobachtung aufgreifend verfolgt die vorliegende Arbeit das Ziel, einen theoretisch begründeten und empirisch gestützten Beitrag für die Gestaltung eines Controllingsystems zur Steuerung von Markenportfolios zu leisten. Ausgangspunkt der Untersuchung bildet dabei die Überlegung, dass eine koordinierte Führung eines Markenportfolios durch eine gegenseitige Abstimmung der Marken einen höheren Gesamtgewinn erwarten lässt als eine unkoordinierte. Interdependenzen zwischen den Marken desselben Produktbereichs implizieren eine hohe Komplexität des Markenportfolios und führen letztlich dazu, dass die Freiheitsgrade der einzelnen Marken geringer werden und deren Ergebnisse nicht unabhängig voneinander sind. Die unternehmensinterne und -externe Abstimmung markenbezogener Aktivitäten wird somit zu einer zentralen Aufgabe des Mehrmarkencontrolling.

Im situativen Kontext der Automobilindustrie werden zunächst die entscheidungsfeldabhängigen planerischen und organisatorischen Aspekte eines Markenportfolios als Objekt des Controlling analysiert. Als theoretische Grundlage dient der identitätsorientierte Ansatz, welcher dem zunehmend geforderten Wechselspiel zwischen Inside-Out- und Outside-In-Perspektive der Markenführung Rechnung trägt. Auf dieser Basis analysiert der Autor den Controllingbedarf in Mehrmarkenstrategien, deren Ursache er vor allem in der Aufspaltung des Markenführungssystems in partielle Entscheidungsfelder mit der Folge von Interdependenzen und 
möglichen Zielkonflikten sieht. Zur Sicherstellung eines abgestimmten Verhaltens der Marken im Markenportfolio wird auf das Konzept der Balanced Scorecard als integrativem Controllingansatz zurückgegriffen. Um auch im Controlling dem postulierten Wechselspiel zwischen Indside-Out- und Outside-In-Orientierung zu genügen, sind mittels einer internen Perspektive Informationen im Rahmen des Aussagenkonzepts der Markenführung zu erfassen, mit der Markt-Perspektive notwendige Kennzahlen des Akzeptanzkonzepts zu erarbeiten und mit der Ergebnisperspektive die erzielte Ergebniswirkung der Markenstrategie zu bestimmen.

Aufbauend auf der Ableitung einer grundsätzlichen Controllingkonzeption zeigt der Autor verschiedene Instrumente auf strategischer und operativer Ebene zur Generierung von Kennzahlen für eine Balanced Scorecard auf. Dabei stellt er zunächst Instrumente zur Analyse der Vorteilhaftigkeit von Mehrmarkenstrategien in den Vordergrund und räumt der Wanderungsanalyse und damit Partizipations- und Substitutionseffekten einen zentralen Stellenwert im Rahmen des Controlling ein. In diesem Zusammenhang bezeichnet der Autor nur solche Wanderbewegungen als Kannibalisierung, bei denen Nachfrager von einer Marke des Portfolios zur anderen wechseln, als Alternativmarke jedoch die vorher besessene Marke des Portfolios und nicht eine Wettbewerbsmarke in Betracht gezogen haben. Markenwechsler innerhalb des Portfolios ohne Präferenz für die vorher besessene Portfoliomarke werden den Loyalitätsgewinnen des Portfolios zugerechnet, so dass zwischen einem "Bruttoeffekt" und einem "Nettoeffekt" der Mehrmarkenstrategie unterschieden werden kann.

Das im Rahmen der Arbeit verwendete GAP-Modell eröffnet dem Autor die Möglichkeit, dem Grundgedanken der Markenidentität folgend etwaige Positionierungsmängel als Ursache von Wanderungsbewegungen nicht nur nachfrager- und damit fremdbildorientiert, sondern auch angebots- und damit selbstbildorientiert zu analysieren. Im Rahmen einer ausschließlichen Fremdbildbetrachtung wird zunächst die von den Nachfragern wahrgenommene Ähnlichkeit der unternehmenseigenen Marken überprüft. In einem weiteren Schritt werden die Selbstbilder der Portfoliomarken in die Analyse integriert, wobei die bestehenden Abweichungen zwischen Selbst- und Fremdbildern als GAPs der Markenidentität interpretiert werden. Die identifizierten Gesamtabweichungen lassen sich sodann im Rahmen einer Abweichungsaufspaltung hinsichtlich möglicher Umsetzungs-, Kommunikations- und Wahrnehmungsgaps näher spezifizieren. Überdies analysiert der Autor zentrale Einflussfaktoren auf die Markenpositionierung, um einerseits zusätzliche Erkenntnisse zur Erklärung der ermittelten Realpositionen der Marken zu generieren und andererseits eine Grundlage für die Ermitt|kng einers_sityationsgeregchten 
Soll-Positionierung der Marken zu erarbeiten. Während der Autor im Rahmen des strategischen Mehrmarkencontrolling insbesondere Instrumente und Kennzahlen für den Informationsbedarf des strategischen Portfoliomanagement betrachtet, analysiert er im Rahmen des operativen Mehrmarkencontrolling zentrale Controllingaspekte bei der Ausgestaltung der Instrumentalstrategien.

Indem das Portfolio schließlich aus verschiedenen Scorecard-Perspektiven betrachtet wird, zeigt der Autor, wie sich eine adressatenorientierte Unterstützung der Markenführung mittels einer Balanced Scorecard gewährleisten lässt. Hierzu bedarf es der Integration der ermittelten Kennzahlen in ein Gesamtkonzept, wobei der Autor eine Orientierung an externe und interne Bezugsgruppen der Markenführung gemäß der Scorecard-Perspektiven propagiert. Mittels einer Hierarchisierung von Marken-Scorecards in ein umfassendes Scorecard-System fordert er letztlich eine bedarfsspezifische Informationsversorgung aller Markeneinheiten, wobei die markenspezifischen Scorecards aus der vorgelagerten PortfolioScorecard abzuleiten sind.

Insgesamt stellt die vorliegende Untersuchung nicht zuletzt aufgrund ihres integrativen Charakters eine Bereicherung der wissenschaftlichen Arbeiten auf dem Gebiet der Markenführung dar. Im Sinne eines Brückenschlags zwischen Marketing und Controlling gelingt es dem Verfasser, zahlreiche, bislang isolierte Partialansätze des Markencontrolling schlüssig in ein Gesamtkonzept zu integrieren und ihre spezifische Relevanz für die Steuerung von Markenportfolios aufzuzeigen. Richtungsweisend ist dabei die Verbindung zwischen dem identitätsorientierten Ansatz der Markenführung und dem Konzept der Balanced Scorecard. Die notwendige Fokussierung der Analyse auf die Automobilbranche erweist sich als gelungene Eingrenzung des Untersuchungsgegenstandes und liefert wertvolle Hinweise zur Überführung des Controllingansatzes auf andere Untersuchungsbereiche. Es bleibt zu wünschen, dass die Arbeit auf eine entsprechende Resonanz in Wissenschaft und Praxis stößt. 
Martin Koers - 978-3-631-75091-9

Downloaded from PubFactory at 01/11/2019 08:29:37AM

via free access 


\section{Vorwort des Verfassers}

Aufgrund ihrer hohen Relevanz für das Kauf- und Auswahlverhalten von Nachfragern stellt die Marke seit jeher einen zentralen Forschungsgegenstand des Marketing dar. In jüngster Zeit wird auch in der Praxis die herausragende Rolle der Markenführung wiederentdeckt. Die "richtige" Markenstrategie erweist sich dabei auf vielen Märkten als zentraler Erfolgsfaktor. Insbesondere die Mehrmarkenstrategie und damit die parallele Führung mehrerer selbständiger Marken im gleichen Produktbereich nimmt heute in zahlreichen Konsumgüter- und Dienstleistungsbranchen, wie etwa der Automobilindustrie, dem Versicherungsmarkt oder im Tourismus, eine dominierende Stellung ein. So wurde der Volkswagen-Konzern im Jahr 1999 für seine "konsequente Mehrmarkenstrategie" mit dem Deutschen Marketing-Preis ausgezeichnet.

Neben vielfältigen Chancen, die mit einer solchen Strategie verbunden sind, gehen mit der Mehrmarkenstrategie allerdings zentrale Risiken einher. Denn im Zielkonflikt zwischen Kostenreduktion und Produktdifferenzierung gewinnt die Nutzbarmachung von "Economies of Scale" in einem Markenportfolio häufig derart an Bedeutung, dass den einzelnen Marken durch die Verwendung von Gleichteilen, gemeinsamen Markenplattformen bis hin zum Angebot baugleicher Produkte die Differenzierungsbasis entzogen wird. Im externen Marktauftritt stellt somit die Kannibalisierung der Marken durch gegenseitige Marktanteilssubstitution ein zentrales Risiko dar. Vor diesem Hintergrund setzt die wirksame Umsetzung einer Mehrmarkenstrategie eine systematische Koordination und Steuerung des Marktauftritts der einzelnen Portfoliomarken voraus.

Einer derartigen Optimierung des Marktauftritts sowie des internen Zusammenspiels der verschiedenen Marken dient ein Mehrmarkencontrolling. Zwar wird sowohl in der wissenschaftlichen Literatur als auch in der Unternehmenspraxis immer wieder auf die Notwendigkeit von Controllingsystemen im Rahmen der Markenführung hingewiesen. Bisherige Ansätze beschränken sich jedoch auf das Controlling von Einzelmarken, wobei Einzelaspekte wie etwa Konzepte zur Messung des Markenwertes, Verfahren der Markenwahrnehmungsanalyse oder branchenspezifische Aspekte des Markencontrolling in den Fokus der Betrachtung gestellt werden. Dem Controlling komplexer Markenportfolios sowie der Analyse der vielfältigen Interdependenzbeziehungen zwischen den Marken in einem Portfolio wird demgegenüber nur unzureichend Aufmerksamkeit geschenkt. 
An diesem Defizit in der wissenschaftlichen Forschung ansetzend verfolgt die vorliegende Arbeit das Ziel, einen Beitrag zur Entwicklung und Nutzung eines Controllingsystems im Rahmen von Mehrmarkenstrategien zu leisten. Der im Rahmen dieser Arbeit vorgestellte Controllingansatz orientiert sich dabei an den differenzierten Informationsansprüchen des Portfoliomanagement. Während das strategische Mehrmarkencontrolling die einzelnen Phasen der strategischen Rahmenplanung von Mehrmarkenstrategien analysiert, fokussiert das operative Mehrmarkencontrolling die Ausgestaltung der Instrumentalstrategien im Portfolio. Um dabei der Gefahr von "Informationsinseln“ bzw. einer Suboptimierung des Mehrmarkencontrolling auf der Ebene einzelner Instrumente und Kennzahlen zu entgehen, erfolgt schließlich eine Integration der generierten Steuerungsgrößen in ein Konzept der Balanced Scorecard.

Die vorliegende Arbeit wurde im Juli 2000 von der Wirtschaftswissenschaftlichen Fakultät der Westfälischen Wilhelms-Universität Münster als Dissertationsschrift angenommen. Bei der Abfassung der Arbeit erfuhr ich umfassende Unterstützung durch verschiedene Personen. Mein besonderer Dank geht zunächst an meinen akademischen Lehrer, Herrn Prof. Dr. Dr. h.c. mult. Heribert Meffert. Nach meinem Wechsel vom Lehrstuhl für Organisationstheorie und Personalmanagement von Prof. Dr. Helmut Wagner $(\dagger)$ zum Institut für Marketing förderte er mich umfassend und stand mir bei der Erstellung der Arbeit in allen Phasen stets zur Seite. Schon zu Beginn meiner wissenschaftlichen Arbeit auf dem Gebiet des Marketing regte er die Themenstellung an und gab ihr die entscheidenden Impulse. Herrn Prof. Dr. Klaus Backhaus gebührt ebenfalls mein ausdrücklicher Dank für die Übernahme des Zweitgutachtens.

Bedanken möchte ich mich auch bei allen aktuellen und ehemaligen Kolleginnen und Kollegen am Institut für Marketing, die mich während der Abfassung der Arbeit in vielfältiger Weise unterstützt und von anderweitigen Aufgaben weitestgehend entlastet haben. Namentlich hervorheben möchte ich Herrn Dr. Dr. Helmut Schneider, der mir nicht nur in der konzeptionellen Phase wertvolle Anregungen gab, sondern auch stets aufmunternder und diskussionsbereiter Ansprechpartner war. Ein ganz besonderer Dank gilt Herrn Dipl.-Kfm. Ingo Lasslop, der durch seine mannigfaltige Hilfe und seinen moralischen Beistand unter "Markenkollegen" maßgeblich zur Fertigstellung dieser Arbeit beitrug. Aufgrund seines hohen Engagements und seiner fachlichen Kompetenz verdanke ich überdies Herrn Dr. Christoph Burmann wertvolle Hinweise bei der Durchsicht des Manuskripts. 
Darüber hinaus möchte ich meinen Eltern zutiefst danken, die einen weitaus gröBeren Anteil am Entstehen und Gelingen dieser Arbeit tragen, als sie selbst vielleicht vermuten. Denn durch das von ihnen geschaffene familiäre Umfeld haben sie die Grundlage für die Erstellung meiner Arbeit gelegt und sowohl mir als auch meinen fünf Brüdern in allen Phasen unserer Ausbildung die Freiheit eigener Entscheidungen gelassen. Ihr steter Rückhalt verbunden mit Stolz auf unsere Vorhaben gaben mir gesunde Gelassenheit und den nötigen Ansporn zugleich. Schließlich und nicht zuletzt möchte ich meiner Freundin Astrid danken, die mir nicht nur mit ihrer Ausgeglichenheit und Herzlichkeit tatkräftig zur Seite stand, sondern mir auch in vielen hitzigen Diskussionen den Stellenwert mancher Wissenschaft unermüdlich und kritisch verdeutlichte. Ihr und meinen Eltern widme ich diese Arbeit. 
Martin Koers - 978-3-631-75091-9

Downloaded from PubFactory at 01/11/2019 08:29:37AM

via free access 


\section{Inhaltsverzeichnis}

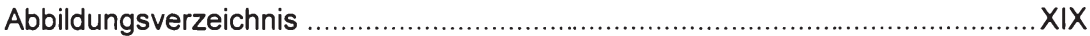

Tabellenverzeichnis ...................................................................

Abkürzungsverzeichnis ..................................................................

A. Das Controlling von Mehrmarkenstrategien als Herausforderung an die Markenführung .......................................................................1

1. Stellenwert und Gegenstand der Mehrmarkenstrategie ..........................

2. Notwendigkeit eines Mehrmarkencontrolling .................................

3. Mehrmarkencontrolling als Aufgabenbereich an der Schnittstelle zwischen Marketing und Controlling

4. Zielsetzung und Gang der Untersuchung .......................................23

B. Ausgestaltung von Markenportfolios als Objekt des Controlling

1. Markenportfolios in der Automobilindustrie als situativer Kontext eines Mehrmarkencontrolling

2. Ziele von Mehrmarkenstrategien

3. Konzeptionelle Grundlagen zur Führung von Markenportfolios.

3.1 Paradigmen der Unternehmensführung als Basis der Markenführung.

3.11 Der market-based view.

3.12 Der resource-based view.

3.2 Integration der markt- und ressourcenorientierten Sichtweise im Konzept der identitätsorientierten Markenführung. 
4. Entscheidungsfeld von Markenportfolios im Rahmen der identitätsorientierten Markenführung .56

4.1 Ausgestaltung der strategischen Rahmenplanung .58

4.11 Portfoliostruktur und Festlegung markenspezifischer Rollen ...58

4.12 Innengerichtete Ressourcenverteilung und Markenleitbilder ...60

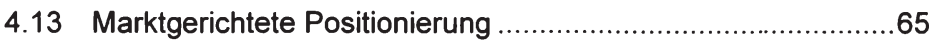

4.2 Ausgestaltung der Instrumentalstrategien .....................................69

4.3 Organisatorische Verankerung von Mehrmarkenstrategien ...............71

C. Identifikation des Controllingbedarfs und Ableitung einer Controllingkonzeption in Markenportfolios

1 Controllingbedarf in Mehrmarkenstrategien

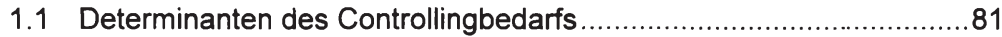

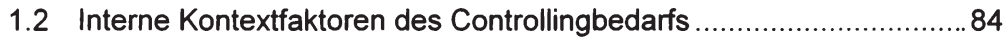

1.21 Komplexität von Markenportfolios...........................................84

1.22 Mangelnde Willensdurchsetzung und Führungsstil ................87

1.23 Opportunistisches Verhalten im Markenportfolio .....................90

1.24 Rückgriff auf gemeinsame Ressourcen .................................91

1.3 Externe Kontextfaktoren des Controllingbedarfs ...............................93

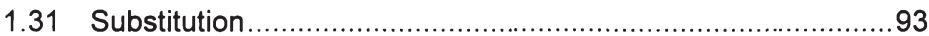

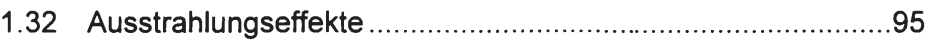

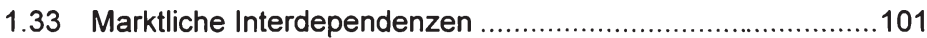

1.4 Interdependenzen zwischen den Kontextfaktoren des Controllingbedarfs

2. Controllingkonzeption zur Deckung und Reduktion des Controllingbedarfs

2.1 Controllingprozess und Anforderungen an das Controlling von Mehrmarkenstrategien

2.2 Zielsystem des Mehrmarkencontrolling.

2.3 Das Balanced Scorecard Konzept

2.4 Ableitung eines integrativen Controllingansatzes 
D Mehrmarkencontrolling zur Deckung des Controllingbedarfs am Beispiel der Automobilwirtschaft

1. Informationsgrundlagen der Analyse

2. Strategisches Mehrmarkencontrolling:

Deckung des Informationsbedarfs bei der Ausgestaltung

der strategischen Rahmenplanung.

2.1 Analyse der Vorteilhaftigkeit von Mehrmarkenstrategien ................129

2.11 Qualitative Vorteilhaftigkeitsanalyse ................................130

2.12 Quantitative Vorteilhaftigkeitsanalyse ................................133

2.2 Substitutions- und Partizipationsanalysen als Elemente der

Wanderungsanalyse

2.21 Konzeptioneller Bezugsrahmen der Wanderungsanalyse ..... 142

2.22 Substitutions- versus Kannibalisierungseffekt ..................... 148

2.23 Partizipationseffekt ................................................ 155

2.24 Wanderungsbilanzen .......................................... 157

2.3 Analyse des markenspezifischen Absatzpotenzials als Indikator der Wettbewerbsstärke.

2.4 Markenwahrnehmungsanalysen zur Aufdeckung von

Profilierungs- und Differenzierungsdefiziten im Markenportfolio

2.41 Das GAP-Modell der Markenidentität als konzeptioneller Bezugsrahmen der Markenwahrnehmungsanalyse

2.42 Analyse der marktseitigen Markenwahrnehmung: Outside-In-Betrachtung

2.421 Analyse auf Basis von Einzelurteilen

2.422 Analyse auf Basis von Globalurteilen

2.43 Analyse der angestrebten Markenwahrnehmung: Inside-Out-Betrachtung

2.431 Analyse auf Basis von Einzelurteilen .....................187

2.432 Ermittlung der Gesamtabweichung ........................190

2.433 Abweichungsaufspaltung .......................................196

2.44 Analyse ausgewählter Einflussgrößen auf die

Markenpositionierung

2.441 Markenbekanntheit und -wissen .............................202

2.442 Produkt- und Händlerzufriedenheit ...........................204

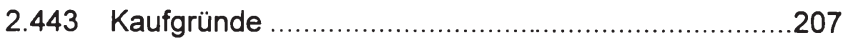

2.444 Käuferstruktur und -einstellungen ..........................209 
3. Operatives Mehrmarkencontrolling:

Zentrale Aspekte zur Deckung des Informationsbedarfs bei der

Ausgestaltung der Instrumentalstrategien 215

3.1 Leistungsvereinheitlichungs- und -differenzierungsanalyse ...........215

3.2 Preispositionierung und Ableitung von Preiskorridoren ...................221

3.3 Kommunikative Positionierung und Aufteilung des

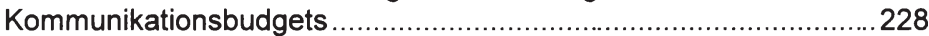

3.4 Markenidentifikation des Handels ...........................................232

4. Integration mehrmarkenspezifischer Steuerungsgrößen in ein Konzept der Balanced Scorecard

4.1 Erfassung von Leistungstreibern und Ergebnisgrößen im Rahmen von Scorecards

4.2 Erfassung von Wechselwirkungen zwischen den Marken durch Scorecard-Hierarchien

E. Schlussbetrachtung und Ausblick 251

1. Zusammenfassende Würdigung der Untersuchungsergebnisse 251

2. Implikationen für weiterführende Forschungsarbeiten 255

Anhang 261

Literaturverzeichnis .271 


\section{Abbildungsverzeichnis}

Abb. 1: Mehrmarkenstrategien in der Automobilindustrie (Stand: Februar 2000)

Abb. 2: Bestimmung der optimalen Koordinationsintensität ........................13

Abb. 3: Fragmentierung des Automobilmarktes....................................... 32

Abb. 4: Markenhierarchie am Beispiel des Volkswagen-Konzerns .................37

Abb. 5: Grundoptionen der Markenpositionierung und Marktstruktur .............41

Abb. 6: Komponenten der Markenidentität ................................................53

Abb. 7: Planungsprozess von Mehrmarkenstrategien .................................57

Abb. 8: Markenleitbilder in der Automobilindustrie ...................................63

Abb. 9: Markenspezifische und markenübergreifende Integration der Marketing-Instrumente im Rahmen von Mehrmarkenstrategien.

Abb. 10: Ausprägungen alternativer Zentralisationsgrade und Eingriffstiefen im Markenportfolio .................................................................... 75

Abb. 11: Aufgabenverteilung im Markenportfolio ....................................... 79

Abb. 12: Controllingbedarf in Mehrmarkenstrategien ...................................... 81

Abb. 13: Relative Komplexität der Portfolioführung ..................................... 85

Abb. 14: Auswirkungen der Produktionskonzepte auf Kosten und Differenzierungsposition.

Abb. 15: Substitution im Volkswagen-Konzern (nach Anzahl der Kaufverträge)

Abb. 16: Ausstrahlungseffekte zwischen Produkt- und Dachmarken ................95

Abb. 17: Dynamische und markenübergreifende Ausstrahlungseffekte ............98

Abb. 18: Spannungsfelder der Markenidentitäten................................. 100

Abb. 19: Breite, Höhe und Tiefe des Marktauftritts der Portfoliomarken........... 102

Abb. 20: Einfluss der Kontextfaktoren auf den Controllingbedarf in Mehrmarkenstrategien

Abb. 21: Mehrmarkencontrolling im Prozess der Mehrmarkenführung............107

Abb. 22: Anforderungen an das Mehrmarkencontrolling ............................ 108

Abb. 23: Zielgrößen des Mehrmarkencontrolling ....................................... 112

Abb. 24: Perspektiven und Managementprozess der Balanced Scorecard ...... 119

Abb. 25: Struktur eines Mehrmarkencontrolling

Abb. 26: Ermittlung der Bedeutungsgewichte und Erfüllungsgrad im Hinblick auf Anforderungen an eine Ein- und Mehrmarkenstrategie .. 131

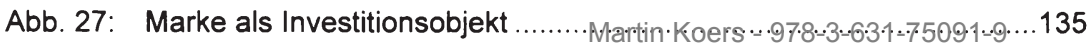


Abb. 28: Optimierungsmodell zur Bestimmung der Markenanzahl .................138

Abb. 29: Grundmodell der Substitution und Partizipation ................................. 143

Abb. 30: Grundschema der Wanderungsanalyse ........................................ 147

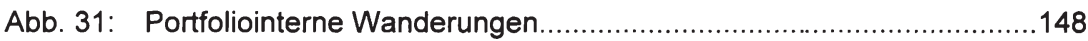

Abb. 32: Substitutionssalden der Marke $C$ in dynamischer Betrachtung ..........154

Abb. 33: Partizipationseffekt der Mehrmarkenstrategie ..................................156

Abb. 34: Konzeptioneller Rahmen der Absatzanalyse .....................................160

Abb. 35: Absatzstrukturanalyse in dynamischer Betrachtung ..........................163

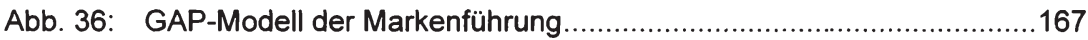

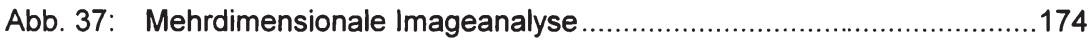

Abb. 38: Zwei- und dreidimensionaler Wahrnehmungsraum auf Basis von

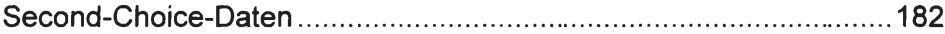

Abb. 39: Interpretation der zweidimensionalen Lösung ................................ 183

Abb. 40: Erklärung von Wanderungseffekten aufgrund der Positionierung

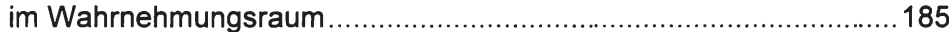

Abb. 41: Polaritätenprofil Soll-Selbstbild und Ist-Fremdbild Marke A ...............189

Abb. 42: Soll-Selbstbild und Ist-Fremdbild im zweidimensionalen Wahrnehmungsraum.

Abb. 43: Marken und Eigenschaften im Wahrnehmungsraum .......................193

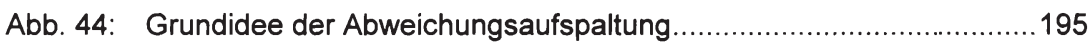

Abb. 45: Ermittlung des Zielkanals durch Abgleichung der Markenprofile ........197

Abb. 46: Markenbekanntheit, - vertrautheit und -sympathie ..........................203

Abb. 47: Produkt- und Händlerzufriedenheit..................................................206

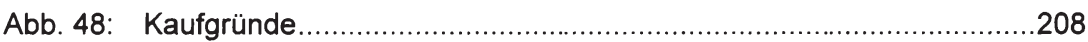

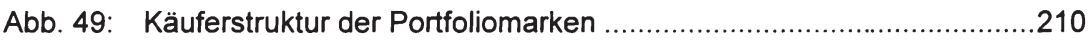

Abb. 50: Die Marken in den sozialen Milieus in Deutschland .........................211

Abb. 51: Positionierungsportfolio aufgrund der Einstellungen der

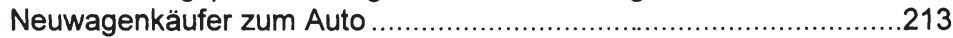

Abb. 52: Nachfragerwahrnehmung der Eigenständigkeit von Automobilen ......218

Abb. 53: Differenzierungs- und Vereinheitlichungsplan ................................220

Abb. 54: Preissegmente und Zusammenhang zwischen akzeptiertem Preis und nicht preislicher Distanz

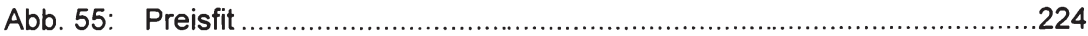

Abb. 56: Lineare Wechselneigung in Abhängigkeit von der Preisdifferenz (rein preisliche Substitution).....

Abb. 57: Bestimmung des Preiskorridors in Abhängigkeit von der Wechselneigung. 
Abb. 58: Beziehung Share of Voice - Marktanteil .................................. 232

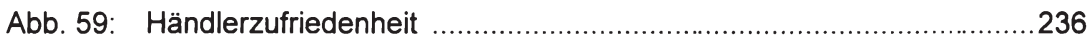

Abb. 60: Partnerschaft und Bindung zur Marke ......................................237

Abb. 61: Bezugsgruppen der Marke innerhalb der Perspektiven der BSC ......242

Abb. 63: Scorecard-Hierarchie im Markenportfolio ...................................246

Abb. 64: Beispielhafte Darstellung einer Scorecard-Hierarchie ......................249 


\section{Tabellenverzeichnis}

Tab. 1: Entwicklung der Markentreue im deutschen Pkw-Markt......................33

Tab. 2: Mergers \& Aquisitions in der Automobilindustrie .................................38

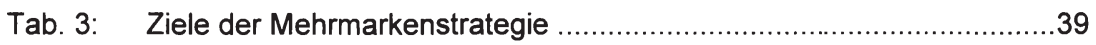

Tab. 4: Beispielhafte strategische Rollen in einem Markenportfolio eines

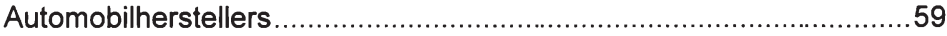

Tab. 5: Markenausdehnung ausgewählter deutscher Automobilhersteller .....103

Tab. 6: Struktur des betrachteten Markenportfolios......................................128

Tab. 7: Preisdifferenzierung strategischer Wettbewerbsmodelle im

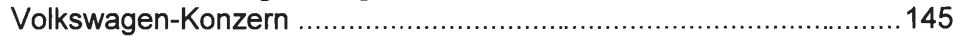

Tab. 8: Fiktives Beispiel des wertmäßigen Substitutionseffektes .................150

Tab. 9: Kannibalisierungseffekt der Mehrmarkenstrategie ..........................151

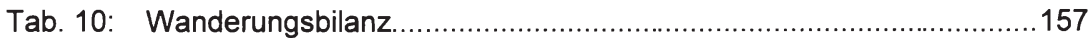

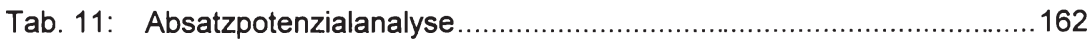

Tab. 12: Zentrale Ex-Post-Kennzahlen des Mehrmarkencontrolling ................164

Tab. 13: Ausschnitt aus der Erwägermatrix...............................................178

Tab. 14: Auswirkungen der Fremdherstellung auf Markenidentität und

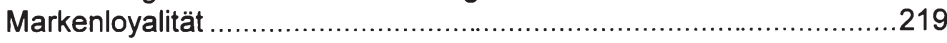




\section{Abkürzungsverzeichnis}

a.M.

a.a.O.

$A B S$

AG

Abb.

Anm. d. Verf.

Aufl.

Asw

$\mathrm{Bd}$.

BSC

bspw.

bzw.

ca.

CFO

c.p.

d.h.

DB

DBW

Diss.

DM

DSP

ED.

ECS

EMS

ER

ERW

ESK

e.V.

et al.

etc.

FAZ

f., ff.

FN

ggf.

GVO

HB

HBM

HBR

Hrsg. am Main

am angegebenen Ort

Anti-Blockier-System

Aktiengesellschaft

Abbildung

Anmerkung des Verfassers

Auflage

Absatzwirtschaft

Band

Balanced Scorecard

beispielsweise

beziehungsweise

circa

Chief Financial Officer

ceteris paribus

das heißt

Deckungsbeitrag

Die Betriebswirtschaft

Dissertation

Deutsche Mark

Deckungsspanne

Edition

European Customer Satisfaction Study

Einzelmarkenstrategie

Eroberungsrate

Erwägerrate

Ersatzkäufer

eingetragener Verein

et alii, et alia, et alteri

et cetera

Frankfurter Allgemeine Zeitung

folgende, fortfolgende

Fußnote

Gegebenenfalls

Gruppenfreistellungsverordnung

Handelsblatt

Harvard Business Manager

Harvard Business Review

Herausgeber 


\begin{tabular}{ll} 
i.d.R. & in der Regel \\
i.S. & im Sinne \\
IT & Informationstechnologie \\
insb. & insbesondere \\
JdAV & Jahrbuch der Absatz- und Verbrauchsforschung \\
Jg. & Jahrgang \\
JoAR & Journal of Advertising Research \\
JoBR & Journal of Business Research \\
JoM & Journal of Marketing \\
JoMR & Journal of Marketing Research \\
Kap. & Kapitel \\
KKV & Komparativer Konkurrenzvorteil \\
LR & Loyalitätsrate \\
MBV & market-based view \\
Mio. & Millionen \\
MDS & Multidimensionale Skalierung \\
MMC & Mehrmarkencontrolling \\
MMS & Mehrmarkenstrategie \\
Mrd. & Milliarden \\
MS & Marketing Science \\
NCBS & New Customer Buying Survey (Neuwagenkäufer-Studie) \\
No. & Number \\
Nr. & Nummer \\
NY & New York \\
O.V. & ohne Verfasser \\
PAF & Preis-Absatz-Funktion \\
PI & Profilierungsindex \\
RBV & resource-based view \\
RCP & Resource-Conduct-Performance \\
S. & Seite \\
SCP & Structure-Conduct-Performance \\
sog. & sogenannte (n, $r$ s) \\
Sp. & Spalte \\
Tab. & Tabelle \\
U.a. & und andere, unter anderem \\
UPE & Unverbindliche-Preis-Empfehlung \\
Univ. & Universität \\
\hline
\end{tabular}

Vgl. Vergleiche 


$\begin{array}{ll}\begin{array}{ll}\text { Vol. } \\ \text { vs. }\end{array} & \begin{array}{l}\text { Volume } \\ \text { Versus } \\ \text { WiSt } \\ \text { WISU }\end{array} \\ \begin{array}{l}\text { Wirtschaftswissenschaftliches Studium } \\ \text { Das Wirtschaftsstudium }\end{array} \\ \text { Z.B. } & \text { Zum Beispiel } \\ \text { ZfB } & \text { Zeitschrift für Betriebswirtschaft } \\ \text { ZfbF } & \text { Zeitschrift für betriebswirtschaftliche Forschung } \\ \text { ZFP } & \text { Zeitschrift für Forschung und Praxis } \\ \text { ZK } & \text { Zusatzkäufer }\end{array}$


Martin Koers - 978-3-631-75091-9

Downloaded from PubFactory at 01/11/2019 08:29:37AM

via free access 


\section{A. Das Controlling von Mehrmarkenstrategien als Herausfor- derung an die Markenführung}

\section{Stellenwert und Gegenstand der Mehrmarkenstrategie}

Aufgrund ihrer hohen Relevanz für das Kauf- und Auswahlverhalten von Nachfragern stellt die Marke seit jeher einen zentralen Untersuchungsgegenstand des Marketing dar. ${ }^{1}$ Heute wird die Marke vielfach als zentrale betriebswirtschaftliche Wertschöpfungsquelle interpretiert, womit ihr über das Marketing hinaus eine übergreifende Bedeutung im Rahmen der Unternehmensführung zukommt. ${ }^{2}$ Stand traditionell der Sachwert eines Unternehmens im Vordergrund, wird heute das zu erwartende Ertragspotenzial zur entscheidenden betriebswirtschaftlichen Größe, welches maßgeblich vom Ansehen und Vertrauen der Verbraucher in das Unternehmen und seine Marken bestimmt wird ${ }^{3}$. Markenführung avanciert mithin zum Kernthema des obersten Management und die Wahl der "richtigen" Markenstrategie wird zum zentralen Erfolgsfaktor vieler Unternehmen. ${ }^{4}$

Die „Grundgesetze der natürlichen Markenbildung" hat DOMIZLAFF bereits 1937 verfaßt. Vgl. Domizlaff, H., Die Gewinnung des öffentlichen Vertrauens. Ein Lehrbuch der Markentechnik, Hamburg 1939; Mellerowicz, K., Markenartikel - Die ökonomischen Gesetze ihrer Preisbildung und Preisbindung, 2. Aufl., München u.a. 1963, S. 39ff.

In nahezu sämtlichen Geschäftsberichten finden sich auf den ersten Seiten Informationen über Marken oder Markenwerte der betreffenden Unternehmen. Diese exponierte Position verdeutlicht die zunehmende Bedeutung der Marke als immateriellem Vermögensgegenstand. Esch, F.-R., Wicke, A., Herausforderungen und Aufgaben des Markenmanagements, in: Moderne Markenführung: Grundlagen - innovative Ansätze - praktische Umsetzung, Esch, F.-R. (Hrsg.), Wiesbaden 1999, S. 3. Zur volkswirtschaftlichen Bedeutung der Marke vgl. auch Güldenberg, H. G., Der volkswirtschaftliche Wert der Marke, in: Markenartikel, Heft 3, 1999, S. 8.

So besteht bei börsennotierten Unternehmen ein empirisch nachgewiesener Zusammenhang zwischen der Marke als "Kapital des Unternehmens" und der Aktienkaufbereitschaft dieser Unternehmen. Vgl. Brüne, G., IMAGERY 2 - strategische Markenführung in gehobenen Zielgruppen, in: Geo Extra, Anzeigen-Ticker, G+J (Hrsg.), Heft 7, 1998, S. 6. Vgl. auch BekmeierFeuerhahn, S., Marktorientierte Markenbewertung: eine konsumenten- und unternehmensbezogene Betrachtung, Wiesbaden 1998, S. $216 \mathrm{ff}$. 
Dabei hat sich das Aufgabenumfeld der Markenführung in den letzten Jahren entscheidend geändert. So lässt sich heute vor dem Hintergrund kosteninduzierter Standardisierungsbemühungen sowie der raschen Verbreitung von technologischem Know-how in vielen Märkten eine objektive Angleichung von Produkten und Dienstleistungen in physikalisch-funktionaler Sicht konstatieren. Die voranschreitende Homogenität objektiver Leistungsmerkmale führt dabei zu einem empirisch nachweisbaren Anstieg der vom Konsumenten wahrgenommenen Austauschbarkeit dieser Leistungen. ${ }^{5}$ Die Markenführung bietet in diesem Zusammenhang die Möglichkeit, die eigenen Leistungen von denjenigen der Wettbewerber zu differenzieren und mit einem eigenständigen Profil zu versehen.

Gleichzeitig tragen neue Informations- und Kommunikationssysteme neben einer deutlichen Erhöhung der Markttransparenz zu einer steigenden Informationsüberlastung des Nachfragers bei. ${ }^{6}$ Das Konsumentenverhalten ist dabei durch eine zunehmende Inkonsistenz geprägt, welche in vielen Branchen bereits durch eine Entwicklung vom hybriden zum multioptionalen bzw. paradoxen Konsumenten charakterisiert wird. ${ }^{7}$ Die wachsende Polarisierung und Individualisierung des Konsumverhaltens führt zu einer weiteren Fragmentierung der Märkte, so dass sich Unternehmen immer kleineren Zielgruppen mit sich schnell verändernden Bedürfnissen ausgesetzt sehen. Als Folge dieser Entwicklungstendenzen kann eine kontinuierliche Steigerung der Preissensibilität und Markenwechselbereit-

Laut einer Studie der BBDO erleben $72 \%$ der Konsumenten Leistungen in unterschiedlichen Produktgruppen als austauschbar. Vgl. BBDO, Auswege aus der kommunikativen Katastrophe, Düsseldorf 1993. BIEL weist in diesem Zusammenhang treffend darauf hin, dass rein technische Markenfähigkeiten mit zunehmender Entwicklungsgeschwindigkeit der Technologien an Diskriminationsfähigkeit verlieren. Technische Fähigkeiten werden mithin zur notwendigen Bedingung, sie reichen aber nicht zur Differenzierung aus. Vgl. Biel, A. L., Grundlagen zum Markenwertaufbau, in: Moderne Markenführung: Grundlagen - innovative Ansätze - praktische Umsetzung, Esch, F.-R. (Hrsg.), Wiesbaden 1999, S. 75.

6 Vgl. Kroeber-Riel, W., Informationsüberlastung durch Massenmedien und Werbung in Deutschland, in: DBW, 47. Jg., Heft 3, 1987, S. 257ff; Decker, R., Klein, T., Wartenberg, F., Marketing und Internet - Markenkommunikation im Umbruch?, in: Markenartikel, Heft 10 1995, S. 472.

Zur Inkonsistenz des Konsumentenverhaltens vgl. Schüppenhauer, A., Multioptionales Konsumentenverhalten: Erklärungen und Empfehlungen auf Basis der Autopoiesetheorie, Wiesbaden 1998, S. 5 ff. Im Markt für Körperpflegemittel erhöhten sich nach Angaben der Gesellschaft für Konsumforschung (GfK) der Anteil des Hochpreissegments zwischen 1982 und 1994 von 33 auf $40 \%$, der des Niedrigpreissegments von 18 auf $24 \%$. Vgl. Schüppenhauer, A., Wo die Märkte aufreißen, in: asw, 39. Jg., Nr. 6, 1996, S. 33. 
schaft der Konsumenten konstatiert werden. ${ }^{8}$ Die mit dieser Entwicklung einhergehende sinkende Markentreue kennzeichnet ein zentrales Problem von Markenartikelherstellern. ${ }^{9}$ Vor diesem Hintergrund stehen Anbieter von Markenartikeln derzeit vor der Herausforderung, den vielfältig fragmentierten Märkten mit abnehmender Markentreue durch individualisierte Leistungen gerecht zu werden und gleichzeitig wettbewerbsinduziert die Wirtschaftlichkeit der Marktbearbeitung sicherzustellen.

Die in diesem Zusammenhang vielfach praktizierte Markenausdehnung stellt dabei eine vergleichsweise einfache und kostengünstige Möglichkeit der Angebotsausweitung dar. ${ }^{10}$ Hier wird das Leistungsspektrum etablierter Marken ergänzt, um so in neue Marktsegmente vorzustoßen, wodurch das Potenzial eingeführter Marken umfassend verwertet werden kann. Allerdings erscheinen die mit Brand Ex-

8 Neben einem wettbewerbsinduzierten Markenwechsel durch den Einsatz des MarketingInstrumentariums ist der Wechsel dabei häufig Konsequenz von Unzufriedenheit, der mangelnden Verfügbarkeit von Leistungen am Point of Sale oder Resultat des sogenannten "Variety Seeking Behavior", d. h. dem Verlangen der Konsumenten nach Abwechslung und interessanten Erlebnissen. Zu den Konsequenzen von Unzufriedenheit vgl. Simon, H., Homburg, C., Kundenzufriedenheit als strategischer Erfolgsfaktor - Einführende Überlegungen, in: Kundenzufriedenheit: Konzepte-Methoden-Erfahrungen, Simon, H., Homburg, C. (Hrsg.), Wiesbaden 1995, S. 15ff. Zum „variety-seeking-behavior" vgl. ter Haseborg, F., MäBen, A., Das Phänomen Variety-Seeking-Behavior: Modellierung, empirische Befunde und marketingpolitische Implikationen, in: JdAV, 43. Jg. 1997, S. 164ff; McAlister, L., A Dynamic Attribute Satiation Model of Variety Seeking Behavior, in: Journal of Consumer Research, Vol. 9, Heft 2, 1982, S. $141 \mathrm{ff}$. Neben dem variety seeking lassen sich weitere Gründe des individuellen Markenwechsels anführen, vgl. hierzu Herrmann, A., Gutsche, J., Ein Modell zur Erfassung der individuellen Markenwechselneigung, in: ZfbF, 46. Jg., Heft 1, 1994, S. 63ff.

9 So lässt sich eine rückläufige Markentreue in vielen Warengruppen empirisch belegen. Vgl. $z$ B. o.V., Nicht nur Junge werden untreu. Markenwechsel ist keine Frage des Alters, in: Lebensmittelzeitung, Heft 24, 1995, S. 60. Vgl. auch die Studie von o.V., Markenwechsel ist in. Eine Sonderstudie zum Thema Markenwechsel/Markenbewusstsein, durchgeführt von Infratest Kommunikationsforschung im Auftrag der Bayerischen Rundfunkwerbung, München 1994, S. 8, nach der über die Hälfte der Bevölkerung in der Regel zum Markenwechsel bereit ist, "wenn man sie überzeugen kann". Vor dem Hintergrund der steigenden Markenwechselbereitschaft sind gar vereinzelte Zweifel an der grundsätzlichen Kaufverhaltensrelevanz von Marken aufgekommen, vgl. Michael, B. M., Die Marke ist tot. Es lebe die Marke!, in: Markenartikel, Heft 1, 1994, S. 22 f.; Otte, Th., Die Selbstähnlicheit der Marke, in: Jahrbuch Markentechnik, Brandmeyer, K., Deichsel, A., Otte, Th. (Hrsg.), Frankfurt a. M. 1995, S. 43 f.

Bei der Nutzung vorhandener Marken handelt es sich um eine Markendehnung durch Produktlinienerweiterungen (sog. Line Extensions) oder um Dehnung der Marke in neue Produktkategorien (Markentransfer). Vgl. zur Markenausdehnung Aaker, D. A., Brand Extensions: The Good, the Bad, and the Ugly, in: Sloan Management Review, Summer 1990, S. 47ff; Sheinin, D. A., Schmitt, B. H., Extending Brands with New Product Concepts: The Role of Category Attribute Congruity, Brand Affect, and Brand Breadth, in: JoBR, 1994, S. 1ff; Friederes, G., Priemer, V., Dachmarke versus Einzelmarke, in: der markt, Heft 2, 1998, S. 47. 
tensions verbundenen Deprofilierungsrisiken aufgrund einer möglichen Unterschreitung des notwendigen Homogenitätsgrades zwischen den unter dem Dach einer Marke angebotenen Leistungen vielfach zu hoch, so dass der kurzfristigen ökonomischen Verwertung des Markenpotenzials langfristig mit der Gefahr einer Markenüberdehnung ein zentrales Risiko gegenübersteht. In diesem Zusammenhang wird in der Literatur intensiv die Frage nach den Grenzen der Markenausdehnung diskutiert. ${ }^{11}$

Zur Vermeidung der mit einer Markenausdehnung im Rahmen einer Dach- oder Familienmarkenstrategie verbundenen Gefahr einer Deprofilierung ursprünglich konturierter Marken gewinnt eine Marktbearbeitung mit mehreren, parallel auf den Absatzmarkt ausgerichteten Marken im Rahmen einer Mehrmarkenstrategie zunehmend an Bedeutung. ${ }^{12}$ In dem Bewußtsein, dass eine klar konturierte Marke die unabdingbare Voraussetzung für das Vertrauen der Nachfrager in die Marke bildet, können auf diese Weise den individualisierten Ansprüchen der Konsumenten durch eine Erhöhung der Flexibilität im Markenauftritt begegnet und die Gefahr markenverwässender Fehlentscheidungen gesenkt werden. Im Unterschied zum Management eines Produktportfolios unter einer Marke beinhaltet die Mehrmar-

11 Vgl. Park, C. W., Milberg, S., Lawson, R., Evaluation of Brand Extensions: The Role of Product Feature Similarity and Brand Concept Consistency, in: Journal of Consumer Research, Vol. 18, Sept. 1991, S. 185-193; Aaker, D. A., Should you take your Brand where the Action is?, in: Harvard Business Review, September-October 1997, S. 136. Quelch, J. A., Kenny, D., Extend Profits, Not Product Lines, in: Harvard Business Review, Sept.-Oct. 1994, S. 153ff. So verdeutlichen auch ANDRESEN/NICKEL anschaulich: „Dem Markentechniker, der langfristig orientiert ist, läuft bei dem Begriff "Dachmarke" ein Schauer über den Rücken. Für ihn ist eine Dachmarkenstrategie lediglich eine kurzfristige Gewinnoptimierung", vgl. Andresen, Th., Nikkel, O., Führung von Dachmarken, in: Moderne Markenführung: Grundlagen - innovative Ansätze - praktische Umsetzung, Esch, F.-R. (Hrsg.), Wiesbaden 1999, S. 577.

Vgl. Meffert, H., Perrey, J., Mehrmarkenstrategie - Ein Beitrag zum Management von Markenportfolios, in: Moderne Markenführung: Grundlagen - innovative Ansätze - praktische Umsetzung, Esch, F.-R. (Hrsg.), Wiesbaden 1999, S. 625. Dabei existieren in der Literatur terminologische Unterschiede zur Beschreibung dieser markenstrategischen Option. So verwendet KAPFERER den Begriff "Multimarkenstrategie", KOTLER/BLIEMEL "Parallelmarkenstrategie", BECKER "Mehrmarken-Konzept" bzw. "A-, B-, und C-Marken-Strategie" bzw. "MehrschichtenKonzept". Vgl. Kapferer, J.-N., Die Marke - Kapital des Unternehmens, Landsberg/Lech 1992, S. 211ff; Kotler, Ph., Bliemel, F., Marketing-Management: Analyse, Planung, Umsetzung und Steuerung, 9. Aufl., Stuttgart 1999, S. 710f; Becker, J., Marketing-Konzeption: Grundlagen des strategischen und operativen Marketing-Management, 6. Aufl., München 1998, S. 230 sowie 234. In der angelsächsischen Literatur wird insbesondere der Begriff "multi-branding" oder "multibrand approach" verwendet. Vgl. stellvertretend Doyle, P., MarketingManagement and Strategy, New York 1994, S. 174, Barwise, P., Robertson, Th., Brand Portfolio, in: European Management Journal, Vol. 10, Nr. 3, September 1992, S. 281. 
kenstrategie als Management eines Markenportfolios ${ }^{13}$ eine gleichzeitige Führung mehrerer selbstständiger Marken, die auf denselben Produktbereich ausgerichtet sind. ${ }^{14}$ Um unterschiedliche Marktsegmente anzusprechen, sind die offerierten Leistungen der verschiedenen Marken dabei anhand zentraler Merkmale bzw. der voneinander getrennten Ausgestaltung der Marketinginstrumente zu unterscheiden, wobei die nachfragerseitige Wahrnehmung letztlich als Maßstab der angestrebten Differenzierung fungiert. Innerhalb der Gesamtunternehmung stellen die separaten Marken darüber hinaus zumeist organisatorisch abgegrenzte und mit der Markenführung betraute Einheiten dar. ${ }^{15}$

Heute nimmt die aus der Fusion bzw. Akquisition fremder Marken oder aus internen Wachstumsüberlegungen ${ }^{16}$ hervorgehende Führung von Markenportfolios

Unter dem Begriff des Markenportfolio-Management wird in der Literatur häufig auch die Markenausdehnung diskutiert, vgl. stellvertretend Laforet, S., Saunders, J. Managing Brand Portfolios: How The Leaders Do It, in: JoAR, Nr. 5, 1994, S. 64-76. Letztlich handelt es sich hier jedoch lediglich um Produktportfolios unter einer Marke im Sinne einer Dach- oder Familienmarkenstrategie. MEFFERT/BURMANN unterscheiden von daher zwischen einer Mikroebene eines quasi unechten Markenportfolios und einer Makroebene des Markenportfoliomanagement, vgl. Meffert, H., Burmann, Ch., Identitätsorientierte Markenführung - Grundlagen für das Management von Markenportfolios, Arbeitspapier Nr. 100 der Wissenschaftlichen Gesellschaft für Marketing und Unternehmensführung e.V., Meffert, H., Wagner, H., Backhaus, K. (Hrsg.), Münster 1996, S. 20. Hier wird das Markenportfolio als Makro-Markenportfolio verstanden.

Die von DOYLE vertretene Auffassung, dass auch die parallele Führung einer Hersteller- und Handelsmarke ein "horizontal multi-branding" darstellt, wird nicht geteilt. Vgl. Doyle, P., Marketing-Management and Strategy, a.a.O., S. $174 f$.

Zwar besteht in der Literatur Einigkeit darüber, dass die Mehrmarkenstrategie die parallele Führung mindestens zweier Marken beinhaltet, jedoch divergiert die Literatur, auf welchen relevanten Markt sie ausgerichtet ist. So bezieht sich KELLER diesbezüglich auf eine "WarenbzW. Produktkategorie", KOTLER/BLIEMEL auf eine "Produktlinie“, MEFFERT auf einen "Produktbereich", BECKER sowie KAPFERER auf die "jeweiligen Gesamtmärkte" und DOYLE auf "the same broad market". Hier soll in Anlehnung an MEFFERT von Produktbereich gesprochen werden und die subjektiv empfundene Substituierbarkeit von Produkten nach DICHTLAANDRITZKYI SCHOBERT als Kriterium der Abgrenzung verwendet werden. Vgl. hierzu Dichtl, E., Andritzky, K., Schobert, R., Ein Verfahren zur Abgrenzung des "relevanten Marktes" auf der Basis von Produktperzeptionen und Präferenzurteilen, in: WiSt, 6. Jg., Heft 6, 1977, S. $290 \mathrm{ff}$. Vgl. zu den verschiedenen Definitionen Keller, K. L., Strategic Brand Management: Building, Measuring, and Managing Brand Equity, London et al. 1998, S. 404; Kotler, Ph., Bliemel, F., MarketingManagement: Analyse, Planung, Umsetzung und Steuerung, a.a.O., S. 710f; Meffert, H., Strategien zur Profilierung von Marken, a.a.O., S. 139; Becker, J., Typen von Markenstrategien, in: Handwörterbuch Markenartikel, Bruhn, M. (Hrsg.), Band 1, Stuttgart 1994, S. 486; Kapferer, J.-N., Die Marke - Kapital des Unternehmens, a.a.O., S. 212f.; Doyle, P., MarketingManagement and Strategy, a.a.O., S. 174.

Aufgrund der hohen Kosten für die Einführung einer neuen Marke und der in vielen Märkten hohen Flopwahrscheinlichkeit entsteht hierbei ein Aufwand, der häufig über den Kosten für den Erwerb einer bereits existierenden Marke liegt. Überdies ist dieser Aufwand nur bedingt 
insbesondere in schwach wachsenden und gesättigten Märkten des Konsumgüterund Dienstleistungsbereichs bereits eine dominierende Stellung ein. ${ }^{17}$ Besondere Bedeutung hat die Mehrmarkenstrategie in der Automobilindustrie gewonnen. Aufgrund der Fusions- und Akquisitionswelle der letzten Jahre existieren in dieser Branche weltweit nur noch 13 eigenständige Hersteller, die durch ein Geflecht von Minder- und Mehrheitsbeteiligungen miteinander verbunden sind und sämtlichst mit mehreren Marken im Wettbewerb agieren (vgl. Abb. 1). ${ }^{18}$

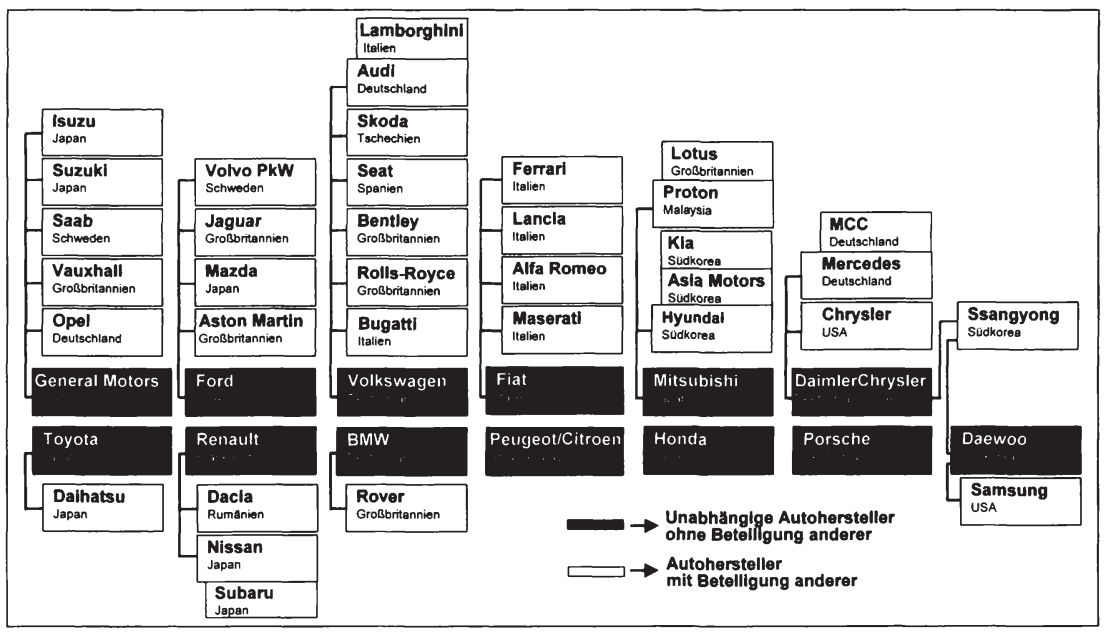

Abb. 1: Mehrmarkenstrategien in der Automobilindustrie (Stand: Februar 2000)

aktivierbar und beeinträchtigt somit unmittelbar das Ergebnis. Vgl. auch Andresen, Th., Nikkel, O., Führung von Dachmarken, a.a.O., S. 577.

17 Als Pionier dieses Konzeptes der Markenführung wird PROCTER \& GAMBLE angeführt, vgl. Solomon, J., Hymowitz, C., Procter and Gamble Makes Changes in the Way It Develops and Sells Its Products, in: Wall Street Journal, 11.8.1987, S. 1 und 12; Schiller, Z., The Marketing Revolution at Procter and Gamble, in: Business Week, 25.7.1988, S. 72-76; Mason, Ch. H., Milne, G. R., An Approach for Identifying Cannibalization within Product Line Extensions and Multi-Brand Strategies, in: JoBR, 1994, S. 163. Als weitere Beispiele im Kosumgüterbereich vertreibt etwa NESTLÉ im Produktbereich der Riegel \& Stückartikel KITKAT, LION, NUTS, ROLO, CARAMAC und BUTTERFINGER oder offeriert THOMSON im Bereich der Unterhaltungselektronik seine Produkte über die Marken DUAL, SABA, TELEFUNKEN und NORDMENDE. Im Dienstleistungssektor offeriert die TUI AG ihre touristischen Leistungen neben der Stammarke TUI über Marken wie AIRTOURS, SEETOURS, DR. TIGgeS, 1-2-FLY, WOLTERS und ROBINSON CLUB. Im Flugdienstleistungsbereich tritt BRITISH AIRWAYS mit der zusätzlichen Discount-Marke Go an.

18 Vgl. o.V., autofahren in deutschland 1999, Motor-Presse Stuttgart (Hrsg.), Stuttgart 1999, S. 73. 
Die konsequente Verfolgung einer Mehrmarkenstrategie wird vor dem dargestellten Hintergrund in jüngster Zeit vielfach als Erfolgsrezept sine qua non gesehen. ${ }^{19}$ Den Vorteilen einer solchen Strategie, wie einer breiten Marktabdeckung oder erhöhten Effizienz in Kundengewinnung und Kundenbindung, stehen jedoch mit den hohen Kosten der Mehrmarkenführung sowie der Gefahr einer potenziellen Substitution des eigenen Angebotes auch zentrale Nachteile gegenüber.

In der Vermeidung solch konkurrierender Leistungsangebote bei Sicherstellung der Wirtschaftlichkeit der Marktbearbeitung liegt damit eine zentrale Herausforderung an das Management von Markenportfolios. ${ }^{20}$ Zu Recht bemerkt die Literatur in diesem Zusammenhang, dass Markenführung letztlich nur so gut sein kann wie die Instrumente und Systeme, „...die vergangene Maßnahmen hinsichtlich ihrer erzielten Erfolge kontrollieren und als Grundlage für therapeutische Maßnahmen dienen ${ }^{\text {21 }}$. Vor diesem Hintergrund wird ein systematisches Mehrmarkencontrolling notwendig.

\section{Notwendigkeit eines Mehrmarkencontrolling}

Für die erfolgreiche Führung von Mehrmarkenstrategien im Sinne der Unternehmensoberziele erweist sich eine rein intuitive bzw. "spirituelle Markenführung" nach GERKEN nur selten als ausreichend. ${ }^{22}$ Zur Sicherstellung der Rationalität ${ }^{23}$

So wurde der Deutsche Marketingpreis 1999 an die Volkswagen AG für die Etablierung ihrer erfolgreichen Mehrmarkenstrategie verliehen. Vgl. Michael, B. M., Laudatio Deutscher Marketing-Preis 1999 an die Volkswagen AG, in: Kongressdokumentation vom Deutschen Marketing Verband des 27. Deutschen Marketing-Tag am 29. Oktober 1999 in Frankfurt unter dem Thema "Chancen im 3. Jahrtausend. Information managen - Zukunft gestalten", S. 47f. Menzel, St., Mehrmarkenstrategie als Erfolgsrezept, in: Handelsblatt Nr. 147 vom 3.8.1999, S. 43: Diekhof, R., Preisgekröntes Marketing, in: W\&V, Heft 29, 1999, S. $80 f$.

20 Zur Kritik vgl. etwa Linden, F.A., Volles Rohr voraus, in: manager magazin, Heft 4, 1998, S. $59 f f$.

21 Andresen, Th., Esch, F.-R., Messung der Markenstärke durch den Markeneisberg, in: Moderne Markenführung: Grundlagen - innovative Ansätze - praktische Umsetzung, Esch, F.-R. (Hrsg.), Wiesbaden 1999, S. 1032.

Vgl. hierzu Gerken, G., Die fraktale Marke, Düsseldorf 1994. Der Ansatz von GERKEN trennt sich von nahezu allen Grundprinzipien der Markenführung wie etwa Nutzenversprechen, Positionierung, Zielgruppenorientierung und Markenidentität. Während in der klassischen Markenführung beispielsweise einzelne Marken noch einen definierten Segmentbezug haben, hebt GERKEN das Zielgruppenkonzept auf (Interfusion statt Marketing). Ein trennscharfer Zielgruppenbezug ist jedoch gerade für die Führung von Markenportfolios aufgrund der sonst auftretenden unkontrollierbaren Kannibalisierungseffekte unumgänglich. Zu weiteren Kritikern der 
unternehmerischen Handelns bedarf es statt dessen verstärkt analytisch ausgerichteter Controllingsysteme im Rahmen der Markenführung, welche sowohl quantitative als auch qualitative Daten berücksichtigen. MEFFERT führt in diesem Zusammenhang an, dass nach dem Siegeszug des strategisch-konzeptionellen Marketing in den 80er Jahren die Implementierungsqualität des Marketing zum zentralen Wettbewerbsfaktor wird. Die wichtigsten Instrumente hierbei sind nach seiner Ansicht die Gestaltung der Unternehmens- und Marketingkulturen und -strukturen sowie die Schaffung effektiver Anreiz- und Controllingstrukturen. ${ }^{24}$ Auch WIEDMANN weist in diesem Zusammenhang auf die Notwendigkeit eines auf die jeweilige Unternehmenssituation zugeschnittenen Mischungsverhältnisses zwischen "harter und weicher" Markenführung hin: "Dort, wo etwa die Markenführung in praxi bereits stark mystizistische Züge trägt, muss Controlling mittels rationaler Planung, Steuerung und Kontrolle ein Stück weit zur „Entzauberung der magischen Markenwelt" beitragen. Dort, wo allein das Primat der "harten Fakten" regiert, ist für mehr Spiritualität und Emotionalität Sorge zu tragen. "25

Im Gegensatz zur rein intuitiven Führung von Markenportfolios erfordert eine reflexive Willensbildung eine Versorgung sämtlicher Entscheidungsträger mit allen notwendigen Informationen sowohl quantitativer als auch qualitativer Art. Dabei lässt sich in allen Entscheidungsbereichen von der Planung und Gestaltung eines Markenportfolios bis hin zur operativen Umsetzung der Mehrmarkenstrategie ein spezifischer Informationsbedarf identifizieren. Dieser Informationsbedarf bezieht

fraktalen Markenführung vgl. Paulus, J., Fraktale Marke: Verbrauchter Mythos, in: W\&V, Heft 10, 1995, S. $80 \mathrm{ff}$.

23 Nach MAX WEBER handelt ein Subjekt zweckrational, welches sein Handeln entsprechend nach Zweck, Mittel und Nebenfolgen orientiert und dabei sowohl die Mittel gegen die Zwecke als auch die Zwecke gegen die Nebenfolgen sowie die verschieden möglichen Zwecke gegeneinander abwägt. Das Subjekt handelt also weder affektuell bzw. emotional, noch traditional. Vgl. Weber, M., Soziologische Grundbegriffe, in: Gesammelte Aufsätze zur Wissenschaftslehre, Wincekmann, J. (Hrsg.), Tübingen 1973, S. 566. Vgl. auch Weber, J., Controlling - Entwicklungstendenzen und Zukunftsperspektiven, in: Die Unternehmung, 53. Jg., Heft 6, 1999, S. 465ff, der Controlling als Rationalitätssicherung der Führung bezeichnet.

Vgl. Meffert, H., Marketing-Management: Analyse, Strategie, Implementierung, Wiesbaden 1994, S. 479.

25 Wiedmann, K-P., Strategisches Markencontrolling, in: Handwörterbuch Markenartikel, Bruhn, M. (Hrsg.), Band 2, Stuttgart 1994, S. 1307. Bereits ALBACH spricht hinsichtlich des GUTENBERG'schen Begriffs des dispositiven Faktors von einem fruchtbaren Spannungsfeld zwischen rationalen und intuitiven (als irrational bezeichnet) Elementen. Vgl. Albach, H., Der dispositive Faktor in Theorie und Praxis, in: ZfB, Heft 5/6, 1990, S. $533 f f$. 
sich sowohl auf die Ist-Situation und mithin auf die Entwicklung des Markenportfolios in der Vergangenheit als auch auf zukünftige Entwicklungen und Veränderung in der Markenumwelt oder innerhalb des Portfolios, welche eine Anpassung der verfolgten Strategie ${ }^{26}$ bedingen können. Die Informationsanforderungen können dabei häufig nur durch umfangreiche Analysen und Rückgriff auf diverse Quellen wie z. B. Marktforschung und internes Rechnungswesen befriedigt werden. Vor diesem Hintergrund stellt die Zusammenführung und Aufbereitung von Daten zu entscheidungsrelevanten Informationen eine zentrale Herausforderung im Rahmen von Mehrmarkenstrategien dar.

Die Notwendigkeit einer solchen Informationsversorgung stellt indes noch kein mehrmarkenspezifisches Problem dar, da auch bei der Führung nur einer einzelnen Marke ein entsprechender, wenn auch weniger komplexer Informationsbedarf existiert. Eine eigenständige wissenschaftliche Auseinandersetzung mit einem "Mehrmarken"-Controlling wird erst dann notwendig, wenn es sich dabei um eine vom Markencontrolling abweichende oder diese zumindest ergänzende Problematik handelt. Andernfalls könnten die bisherigen Erkenntnisse des Markencontrolling analog auf die Mehrmarkenstrategie übertragen werden. Vor diesem Hintergrund bedarf es einer Identifikation von Grundsatzproblemen, die nicht unbedingt auch bei Einzelmarken vorzufinden sind, und deren Systematisierung und Lösung einen hohen Grad an Allgemeingültigkeit aufweisen. Entsprechend kann auch von originären Problemen der Mehrmarkenstrategie gesprochen werden.

Ein wesentliches Charakteristikum der Mehrmarkenstrategie kann in der Tatsache gesehen werden, dass die für eine Marke getroffenen Entscheidungen aufgrund der Ausrichtung auf einen Gesamtmarkt im Gesamtzusammenhang mit den Entscheidungen stehen, die für andere Marken getroffen werden, die Entscheidungen also interdependent sind. ${ }^{27}$ Ressourcen-, Markt- und innerbetriebliche Interde-

Vgl. zum Strategiewechsel Burmann, Ch., Strategiewechsel in turbulenten Märkten - Neuere theoretische Ansätze zur Unternehmensflexibilität, in: Arbeitspapier Nr. 134 der Wissenschaftlichen Gesellschaft für Marketing und Unternehmensführung e.V., Meffert, H., Backhaus K., Becker, J., (Hrsg.), Münster 2000, S. 4ff. Charakteristikum des Internationalen Marketing sieht. Vgl. Berekoven, L., Internationales Marketing, 2. Aufl., Berlin 1985, S. 21. Vgl. auch Backhaus, K., Büschken, J., Voeth, M., Internationales Marketing, 3. Aufl., Stuttgart 2000, S. $37 \mathrm{ff}$. 
pendenzen ${ }^{28}$ zwischen den Marken desselben Produktbereichs implizieren eine hohe Komplexität ${ }^{29}$ des Markenportfolios und führen letztlich dazu, dass die Freiheitsgrade und Ergebnisse einzelner Marken nicht unabhängig voneinander sind. Das Führen einer Marke beeinflusst dann die Art und Weise, wie mit anderen Marken des Portfolios agiert werden kann et vice versa. AAKER führt hier treffend an, „a brand decision cannot be made in isolation.... a brand should .....work within the context of other brands. ${ }^{30}$ Die dadurch notwendig werdende gegenseitige unternehmensinterne und -externe Abstimmung markenbezogener Aktivitäten und damit die Koordination der Marken wird zu einer zentralen Aufgabe des Mehrmarkencontrolling. Liegen keine Interdependenzen vor, ergeben sich für die Marken voneinander isolierbare Entscheidungsprobleme, womit letztlich auch kein Mehrmarkencontrolling erforderlich wird.

Die Komplexität hängt neben der Anzahl der Marken vor allem von der Struktur des Markenportfolios ab. In diesem Zusammenhang ist zu unterscheiden, ob sich die Führung von Mehrmarken auf einzelne Produktmarken, auf selbstständige Unternehmensmarken im Rahmen eines Konzerns oder auf Markenfamilien bzw. eine beliebige Kombination der Markentypen untereinander bezieht. ${ }^{31}$ Dementsprechend kann auch theoretisch zwischen einer Mehr-Produktmarken-, einer Mehr-Dachmarken- oder einer Mehrfamilienmarkenstrategie unterschieden wer-

In diesem Zusammenhang soll mit ADAM von Interdependenzen oder auch Erfolgskopplungen gesprochen werden, wenn das zielsetzungsgerechte Niveau einer Variablen eines Entscheidungsbereichs vom entsprechenden Niveau der Variablen eines anderen Entscheidungsbereichs mit abhängt, d. h., „wenn die zielsetzungsgerechte Lösung der Probleme nur bei simultaner Planung gefunden werden kann." Adam, D., Planung und Entscheidung: Modelle Ziele - Methoden; Fallstudien und Lösungen, 3. Aufl., Wiesbaden 1993, S. 155.

29 Komplexität kann als Varietät bzw. als Anzahl möglicher Zustände, die ein System annehmen kann, definiert werden. Ein System ist dabei eine gegenüber der Umwelt abgegrenzte Gesamtheit von Elementen, die durch Beziehungen miteinander verknüpft sind. Vgl. zur Komplexität Malik, F., Strategie des Managements komplexer Systeme, 5. Aufl., Bern 1996, S. 201. Zum Systembegriff vgl. Ulrich, H., Die Unternehmung als produktives soziales System, 2. Aufl., Bern 1970. Als System wird hier das Markenportfolio verstanden, als Elemente sind die Marken innerhalb des Portfolios zu sehen.

Aaker, D. A., Building Strong Brands, New York 1996, S. 200.

Die Mehr-Produktmarkenstrategie findet sich z. B. bei Philipp Morris mit den Zigarettenmarken MARLboro, Merit, Benson\&Hedges, Virginia slims, Parliament, SARATOga und Cambridge, die Mehr-Dachmarkenstrategie etwa im Continentalkonzern mit den Firmenmarken Continental, UniRoyal, Semperit, GislaVed, Viking, General TIRE, Barum und MABOR und eine Mehr-Familienmarkenstrategie bei dem Unternehmen Kraft Jacobs Suchard, welches im Produktbereich Tafelschokolade die Familienmarken SUCHARD, MILKA und COTE D'OR führt, welche auch Produkte in anderen Produktbereichen führen. 
den. ${ }^{32}$ Der höhere Komplexitätsgrad bei den zuletzt genannten Strategieoptionen resultiert dabei vor allem aus den interdependenten Beziehungsmustern bzw. den wechselseitigen Ausstrahlungs- und Transfereffekten, da hier insbesondere das Zusammenspiel zwischen der Unternehmens- bzw. Markenfamilienidentität einerseits und der produktbereichsspezifischen Markenidentität andererseits Beachtung finden muss. ${ }^{33}$ Häufig handelt es sich bei Markenportfolios realiter somit um eine mehr oder weniger tiefgliedrige Hierarchie von Marken.

Die horizontalen und vertikalen Interdependenzen zwischen den Portfoliomarken erzeugen dann Controllingbedarf, wenn die gegenseitige Abstimmung zwischen den Marken höhere Zielerreichungsgrade erwarten lässt als die unkoordinierte. ${ }^{34}$ Entscheidend ist hier eine auf das Gesamtportfolio gerichtete Perspektive. Die als optimal ermittelten Maßnahmen einzelner Marken sind häufig kurzfristig situativ ausgerichtet und können aus einer übergeordneten Perspektive suboptimal sein, wenn sie nicht langfristige Portfolioerfordernisse berücksichtigen. Vor diesem Hintergrund kann das Ergreifen von Koordinationsmaßnahmen dazu führen, dass im Sinne des Gesamtportfolios für einzelne Marken geringere Erträge erwirtschaftet werden, im Gesamtzusammenhang jedoch das Portfolioergebnis steigt. Die Vernachlässigung solch markenspezifischer Optima kann dabei als kennzeichnend für eine auf das Gesamtunternehmen gerichtete Managementperspektive gesehen werden.

Bei konsequenter Ausrichtung des Portfolios am erzielten Gewinn sind die controllinginduzierten Ergebnisauswirkungen letztlich das maßgebliche Kriterium für die Notwendigkeit eines Mehrmarkencontrolling ${ }^{35}$ Ergibt sich aus der Koordination der

LAFORET/SAUNDERS kommen in ihrer empirischen Untersuchung zu dem Ergebnis immer häufiger auftretender Mischformen. Vgl. Laforet, S., Saunders, J., Managing Brand Portfolios: How The Leaders Do It, in: JoAR, Nr. 5, 1994, S. 72.

Zum identitätsorientierten Ansatz der Markenführung vgl. ausführlich Meffert, H., Burmann, Ch., Identitätsorientierte Markenführung - Grundlagen für das Management von Markenportfolios, a.a.O., S. 13ff. sowie die Ausführungen in dieser Arbeit auf S. $51 \mathrm{ff}$.

34 Siehe hierzu auch Brockhoff, K., Hauschildt, J., Schnittstellen-Management - Koordination ohne Hierachie, in: Zeitschrift für Organisation, Heft 6, 1993, S. 396ff. Dabei lässt sich der Gewinn als zentrale Zielsetzung definieren.

Ein Mehrmarkencontrolling wird nicht um seiner selbst willen durchgeführt, sondern ist viel mehr eine "wirtschaftliche Veranstaltung" und hat nur dann Sinn, wenn der durch das Controlling gestiftete Nutzen die Kosten ihrer Durchführung übersteigt. Vgl. zur Überwachung als wirtschaftliche Veranstaltung Loitlsberger, E., Treuhand und Revisionswesen, 2. Aufl., Stutt- 
Marken kein positiver Beitrag zum Gesamtergebnis des Portfolios, sollten die dezentralen Markeneinheiten isoliert agieren. Hier würde das Unternehmen den $\mathrm{Ge}$ samtgewinn dann maximieren, wenn die einzelnen Markeneinheiten ihre Gewinne maximieren. Bestimmt werden die Ergebnisauswirkungen der Koordination vom Interdependenzgrad zwischen den Marken, welcher maßgeblich von den Konkurrenz- bzw. Komplementaritätsbeziehungen zwischen den Marken determiniert wird. Je höher der Interdependenzgrad ist, desto höher sind die Gewinnpotenziale der Koordination.

Der erzielbare Koordinationsgewinn stellt somit den Unterschied zwischen der Gewinnsituation bei isoliertem und optimal abgestimmtem Markenmanagement dar. Koordinationskosten umfassen neben Kontroll- und Anpassungskosten auch die markenspezifischen Gewinneinbußen aufgrund geringerer Gewinne einzelner Marken als Ergebnis der Koordination. Koordinationserlöse liegen demgegenüber in zusätzlichen Gewinnen des Gesamtportfolios, die durch die koordinierte Vorgehensweise realisiert werden können. Diese Zusatzerlöse gehen bei fehlender Koordination im Sinne einer hohen Autonomie der Marken jedoch wieder verloren, weshalb sie auch als Autonomiekosten bezeichnet werden können. Entsprechend ergibt sich der in Abb. 2 dargestellte Zusammenhang der entscheidungsorientierten Koordinationstheorie. ${ }^{36}$ Während die Autonomiekosten mit zunehmender Koordinationsintensität abnehmen, steigen die Koordinationskosten. Eine optimale Koordinationsintensität $\mathrm{K}_{\mathrm{o}}$ wird letztlich durch das Minimum der Gesamtkostenfunktion bestimmt.

Ein weiteres, dem Markenportfolio zwar nicht inhärentes, aber in der Praxis verbreitetes Problem stellen die simultanen Standardisierungs- und Differenzierungsbemühungen im Rahmen von Mehrmarkenstrategien dar. Die Portfoliomarken werden unternehmensintern häufig unter dem Primat einer größtmöglichen Synergienutzung, im Marktauftritt jedoch unter der Maxime einer möglichst deutlichen Differenzierung geführt. ${ }^{37}$ In Verbindung mit einer weitreichenden Verwendung von

gart 1966, S. 84; Vgl. Baetge, J., Überwachung, in: Vahlens Kompendium der Betriebswirtschaftslehre, Bitz, M. et al. (Hrsg.), Band 2, 3. Aufl., München 1993, S. $175 f f$.

36 Vgl. auch Meffert, H., Marketing: Grundlagen marktorientierter Unternehmensführung: Konzepte - Instrumente - Praxisbeispiele, 8. Aufl., Wiesbaden 1998, S. 942.

37 Vgl. auch Meffert, H., Perrey, J., Mehrmarkenstrategie - Ein Beitrag zum Management von Markenportfolios, a.a.O., S. 643; Meffert, H., Mehrmarkenstrategie - immer die beste Option? in: asw, Sondernummer Oktober 1999, S. $82 \mathrm{ff}$. 
dung von Gleichteilen und modularen Produktkonzepten soll die Mehrmarkenstrategie eine differenzierte Marktbearbeitung bei gleichzeitiger Ausschöpfung von Kostensenkungspotenzialen bewirken.

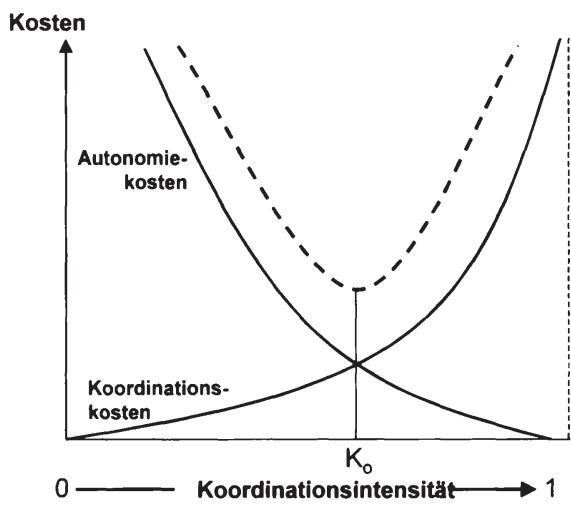

Abb. 2: Bestimmung der optimalen Koordinationsintensität

(Quelle: Benkenstein, M., F\&E und Marketing: Eine Untersuchung zur Leistungsfähigkeit von Koordinationskonzeptionen bei Innovationsentscheidungen, Wiesbaden 1987, S. 19.)

Hieraus ergibt sich die Notwendigkeit, diese beiden Sichtweisen aufeinander abzustimmen, um das zentrale Risiko einer Mehrmarkenstrategie - die Kannibalisierung durch gegenseitige Marktanteilssubstitution - einzuschränken und die übergeordneten Zielerreichungsgrade zu maximieren. Zur Optimierung des externen und internen Zusammenspiels der verschiedenen Portfoliomarken bedarf es somit eines an den unterschiedlichen Zielgrößen des Portfolios ausgerichteten Mehrmarkencontrolling, welches eine Schlüsselstellung zur Sicherstellung der Effektivität und Effizienz des Gesamtportfolios einnimmt. Betrachtet man in diesem Zusammenhang das Marketing als "Führungskonzept vom Markt" her und das Controlling als "Führungskonzept vom Ergebnis" her, so besteht die zentrale Herausforderung an ein Mehrmarkencontrolling, diese beiden Sichtweisen zu integrieren. $^{38}$

„Marketing muss heute gleichzeitig mit zwei Bällen spielen: Umsätze steigern und Kosten senken. Marketingeffizienz zielt demnach zum einen darauf ab, die Effektivität zu erhöhen [...]. Zum anderen sollen die Marketing-Kosten gesenkt werden.", vgl. Droege, W.P.J., Kricsfalussy, A., Marketingaudit: Check up the strategic fit!, in: Marketingcontrolling, Reinecke, S., Tomczak, T., Dittrich, S. (Hrsg.), St. Gallen 1998, S. 70. Gerade bei Aufkaufkonzepten beste- 
Trotz der aufgezeigten Notwendigkeit fehlt es bisher an konzeptionellen Ansätzen eines Mehrmarkencontrolling. ${ }^{39}$ Sowohl in der wissenschaftlichen Literatur als auch in der Unternehmenspraxis wird zwar immer wieder auf die Notwendigkeit von Controllingsystemen im Rahmen der Markenführung hingewiesen, bisherige Ansätze beschränken sich jedoch auf das Controlling von Einzelmarken. Dem Controlling komplexer Markenportfolios sowie der Analyse der vielfältigen Interdependenzbeziehungen zwischen den Portfoliomarken wird demgegenüber nur unzureichend Aufmerksamkeit geschenkt. ${ }^{40}$

hen besondere Schwierigkeiten, wenn das erweiterte Markenportfolio im Sinne einer trennscharfen Mehrmarkenstrategie neu geordnet werden muss. Um Konkurrenten zuvorzukommen, werden Marken häufig unter kurzfristigen Gesichtspunkten und weniger im Zeichen markenstrategischer Überlegungen als vielmehr im Fokus von Synergie- und Größenbestrebungen zur Sicherung einer Führungsposition im globalen Wettbewerb hinzugekauft. BECKER spricht in diesem Zusammenhang treffend vom "Management by Opportunities", DUDENHOFFER, DITTLER, THIES von betriebswirtschaftlich schwer nachvollziehbarem "MarkenShopping". Vgl. Becker, J., Marketing-Konzeption: Grundlagen des strategischen und operativen Marketing-Management, a.a.O., S. 234; Dudenhöffer, F., Dittler, Th., Thies, C., AutoMarkt 2005 und danach, in: Marketing Journal, Heft 4, 1998, S. 220. Im Nachhinein können erhebliche Umpositionierungs- und Eliminierungsprobleme im Markenportfolio auftreten. Der häufig eher kapitalorientierten Intention der Erhöhung des Shareholder Value durch Zukauf von Unternehmen und Marken folgt dann die Aufgabe, die so entstandenen Markenportfolios erfolgswirksam im Sinne der Unternehmensoberziele zu führen. Vgl. auch Barwise, P., Robertson, Th., Brand Portfolio, a.a.O., S. 280; Knudsen, T. R. et al., Brand Consolidation makes al lot of Economic Sense, in The McKinsey Quarterly, No. 4, 1997, S. 189ff; Doyle, P., Building Successful Brands. The Strategic Options, in: Journal of Marketing Management, No. 1, 1989, S. 77 und S. 94.

KOTLER/BLIEMEL stellen entsprechend einer Untersuchung von 75 Unternehmen unterschiedlicher Größe in verschiedenen Branchen fest, dass Unternehmen eine Steuerung durch ein funktionsfähiges Marketingcontrolling zwar benötigen, jedoch nur über unzureichende Kontrollverfahren verfügen. Bei Fehlen eines Marketingcontrolling ist das Vorliegen eines Mehrmarkencontrolling kaum vorstellbar. Vgl. hierzu Kotler, Ph., Bliemel, F., MarketingManagement: Analyse, Planung, Umsetzung und Steuerung, a.a.O., S. 1184.

Vgl. Puhlmann, M., Semlitsch, B., Wie geht das Management mit der Marke um?, in: asw, Sondernummer Oktober 1997, S. 30. Nach der Fusion DAIMLERCHRYSLER wurde eine "Markenbibel" als "Guidelines for DC Brand Management" etabliert, die zum Schutz der Markenidentität dient, vgl. Lamparter, D. H., Tenbrock, Ch., Speed, Speed, Speed, in: Die Zeit, Nr. 12 vom 18.3.1999, S. 35. Bestehende Ansätze befassen sich meist nur mit Teilaspekten wie etwa dem Controlling der Marketing-Instrumente oder der Ermittlung von Markenwert oder stärke einer Einzelmarke. Darüber hinaus sind die bestehenden Controllingansätze durch eine enge Verzahnung des Controlling mit dem Finanz- und Rechnungswesen gekennzeichnet, wobei vorökonomischen Daten der Markenführung kaum Beachtung geschenkt wird. So wurde bei einer Befragung von 100 Controllern aus der Großindustrie und mittelständischen Unternehmen in 1999 die spezifische Aufgabe, Bedürfnisse und Anforderungen der Kunden zu ermitteln, überhaupt noch nicht in den Tätigkeitsfeldern des Controller genannt. Vgl. auch Gerberich, C. W., Wohin führt der Weg des Controllers? in: FAZ, Nr. 123 vom 31.5.1999, S. 31. 


\section{Mehrmarkencontrolling als Aufgabenbereich an der Schnittstelle zwischen Marketing und Controlling}

Obwohl das Controlling mittlerweile zum festen Bestandteil der betriebswirtschaftlichen Praxis ${ }^{41}$ und damit zu einem wichtigen Erkenntnisobjekt der Betriebswirtschaftslehre geworden ist, liegt eine unumstrittene inhaltliche Festlegung des $\mathrm{Be}$ griffs und damit eine anerkannte allgemeine Definition noch nicht vor. ${ }^{42}$ So liefert das Schriftum zum Controlling eine Fülle von kontroversen Beiträgen mit im Detail heterogenen terminologischen Interpretationen und dem Führungsbegriff nahen oder gar inhärenten Aufgabenstellungen. Das Controlling wird in unterschiedlichen Zusammenhängen und mit unterschiedlichen Inhalten verwendet und vielfach finden sich sämtliche Aufgaben unter diesem Begriff, die von anderen Funktionen nicht ausreichend erfüllt werden. Betrachtet man darüber hinaus die unterschiedlichen Definitionsansätze der Marke, verursacht durch einen steten Wandel im Markenverständnis seit der Entstehung des klassischen Markenartikelkonzeptes zu Beginn dieses Jahrhunderts, ${ }^{43}$ so kann bei dem Versuch einer definitorischen

41 Die erste Controllerstelle wurde schon vor ca. 100 Jahren in den USA eingerichtet, vgl. Gnirke, K., Controlling international tätiger Unternehmen - Grundrisse eines koordinationsorientierten Bezugsrahmens unter besonderer Berücksichtigung von Netzwerkbeziehungen, in: Controlling-Konzepte im Wandel - ausgewählte Ansätze, Jöstinmeier, B. et. al (Hrsg.), Marburger Schriften zum Genossenschaftswesen 80, Göttingen 1994, S. 24.

"Der Bedeutung des englischen Begriffs „control" entsprechend geht es dabei um zweierlei, nämlich zum einen um Übenwachung und Beaufsichtigung, zum anderen um Beherrschung, Regelung und Steuerung...", Nieschlag, R., Dichtl, E. Hörschgen, H., Marketing, 18. Aufl., Berlin 1997, S. 942. Vgl. zum Begriff und zur Philosophie des Controlling auch Ahlert, D., Grundlagen des Controlling, Münsteraner Schriften zur Distributions- und Handelsforschung, Ahlert, D. (Hrsg.), 3. Aufl., Münster 1999, S, 1-42; Grob, H. L., Positionsbestimmung des Controlling, Arbeitsbericht Nr. 1 der Reihe Computergestütztes Controlling, Grob, H. L.. (Hrsg.), Münster 1996, S. 1; "Controlling lässt sich mit Fug und Recht zu den schillemdsten und umstrittensten, zugleich aber auch in der Praxis bedeutsamsten betriebswirtschaftlichen Teildisziplinen zählen.", Küpper, H.-U., Weber, J., Zünd, A., Zum Verständnis und Selbstverständnis des Controlling, in: ZfB, Heft 3, 1990, S. 281ff. Vgl. zu den Entwicklungslinien des Controlling auch Schwarzrock, K., Controlling und Führungsberichtswesen in der Praxis, in: Controlling in Theorie und Praxis, Botta, V. (Hrsg.) Aachen 1999, S. $188 \mathrm{ff}$.

Vgl. hierzu ausführlich Meffert, H., Burmann, Ch., Identitätsorientierte Markenführung Grundlagen für das Management von Markenportfolios, a.a.O., S. 3ff. Das Markenverständnis hat sich seit Anfang des Jahrhunderts gewandelt. Wurde die Marke zunächst als Eigentumszeichen bzw. Merkmalskatalog bezeichnet, ist sie sodann als Vermarktungsform bzw. als Nachfragergewinnungsinstrument verstanden worden. Im heutigen Verständnis stellt die Marke ein sozialpsychologisches Phänomen dar und wird als ein in der Psyche des Konsumenten fest verankertes, unverwechselbares Vorstellungsbild von einer Leistung beschrieben. Die zugrundliegende Leistung wird dabei einem möglichst großen Absatzraum über einen längeren Zeitraum in gleichartigem Auftritt und in gleichbleibender oder verbesserter Qualität angeboten. 
Abgrenzung eines "Mehrmarkencontrolling" von einer doppelten Begriffsheterogenität gesprochen werden.

Trotz abweichender Begriffsauffassungen besteht in der Literatur Konsens darüber, dass Controlling weit mehr als buchhaltungsorientierte ex-post Kontrolle im Rahmen rechnungswesensorientierter Ansätze bedeutet, sondern sich vielmehr als anwendungsorientierter Zweig der Betriebswirtschaftslehre zukunfts- und aktionsorientiert mit Aufgaben der Informationsversorgung, Planung und Kontrolle sowie der Koordination befasst und - um eine klare Trennung zwischen Führung und Controlling zu gewährleisten - primär als Führungsunterstützungsfunktion in eine ganzheitliche Unternehmensführung eingebunden ist. ${ }^{44}$

Die existierenden definitorischen Ansätze unterscheiden sich letztlich darin, welcher Stellenwert den einzelnen Aufgaben im Rahmen des Controlling zukommt. Einen mittlerweile weit akzeptierten Raum nimmt das in den Arbeiten von HORVÁTH ${ }^{45}$ zugrunde gelegte führungssystem- bzW. koordinationsorientierte Grundverständnis ein, das auch von KÜPPER und WEBER in unterschiedlichen Ausprägungen aufgegriffen wird. ${ }^{46}$ Bei diesem Konzept wird das gesamte Aufgabenspektrum des Controlling unter die Metaebene der Koordination des Führungssystems gestellt. Bei dem älteren, vorrangig informationsorientierten Grundverständnis, wie es insbesondere in den Arbeiten von REICHMANN zum Ausdruck kommt, wird die Koordination hingegen als gleichrangig mit der Informationsversorgung, Planung und Kontrolle angesehen. ${ }^{47}$ Hier dient das Controlling

Die Kennzeichnung des Controlling als Unterstützungsfunktion erklärt auch, warum die meisten Großunternehmen über eigenständige Controlling-Stellen verfügen (institutionales Controlling), während kleine und mittlere Unternehmungen nur relativ selten eigenständige Controllingstellen aufweisen, da hier der Eigentümer oft selber in der Lage ist, die betrieblichen Teilbereiche $\mathrm{zu}$ koordinieren und eine geeignete Informationsbereitstellung zu organisieren. Vgl. Jőstingmeier, B., Zum Verständnis der Controlling-Konzepte, a.a.O., S. 5.

Für Horváth ist Controlling ein „Subsystem der Führung, das Planung und Kontrolle sowie Informationsversorgung systembildend und systemkoppelnd koordiniert [Hervorhebung vom Verfasser] und so die Adaption und Koordination des Gesamtsystems unterstützt", vgl. Horváth, P., Controlling, 5. Aufl., München 1994, S. 144.

Vgl. Küpper, H.-U., Controlling: Konzeptionen, Aufgaben und Instrumente, Stuttgart 1995.; Weber, J. Einführung in das Controlling, 7. Aufl., Stuttgart 1998. Während HORVATH die Koordinationsnotwendigkeit insbesondere innerhalb und zwischen den Führungssubsystemen Planung, Kontrolle und Informationsversorgung sieht, zählen KüPPER und WEBER darüber hinaus das Organisations- und Personalführungssystem zu den Gestaltungsobjekten des Controlling.

Nach REICHMANN gilt Controlling als "die zielbezogene Unterstützung von Führungsaufgaben, die der systemgestützten Informationsversorgung und Informationsverarbeitung zur Planer- 
ling insbesondere der Bereitstellung führungsrelevanter Informationen. Ein weiterer Typus von frühen Definitionen stellt das Controlling auch als Teilbereich der Unternehmensführung heraus, welcher für die konsequente Zielausrichtung des Unternehmens Sorge zu tragen hat und als führungsphilosophiebezogener Ansatz interpretiert wird. ${ }^{48} \mathrm{Im}$ Zuge der Shareholder Value Orientierung finden in jüngster Zeit darüber hinaus Ansätze des Performance Measurement und Management vermehrt Eingang in die Controllingdiskussion. ${ }^{49}$ Insbesondere das mittlerweile viel diskutierte Konzept der Balanced Scorecard von KAPLAN/NORTON kann als Wegbereiter dieser Diskussion gelten. ${ }^{50}$ Letztlich handelt es sich bei diesen Konzepten jedoch um keine eigenständigen definitorischen Controllingkonzeptionen, sondern um Weiterentwicklungen von Controllinginstrumenten im Rahmen bestehender Ansätze.

Den folgenden Ausführungen wird aufgrund der aufgezeigten Problemstruktur im Markenportfolio ein primär koordinationsorientiertes Verständnis des Controlling zugrundegelegt, wobei Koordination auch den Bereich der Informationsversorgung umfassen soll, da es sich hier letztlich um eine Abstimmung zwischen Management und dem Informationsversorgungssystem handelt. ${ }^{51}$ Durch Beschaffung und

stellung, Koordination und Kontrolle dient; es ist eine [...] Systematik zur Verbesserung der Entscheidungsqualität auf allen Führungsstufen der Unternehmung.", vgl. Reichmann, Th., Controlling mit Kennzahlen und Managementberichten: Grundlagen einer systemgestützten Controlling-Konzeption, 4. Aufl., München 1995, S. 12.

Vgl. hierzu etwa Siegwart, H., Controlling-Konzepte und Controller-Funktionen in der Schweiz, in: Controlling-Konzepte im internationalen Vergleich, Mayer, E., v. Landsberg, G., Thiede, W., (Hrsg.), Freiburg i. Br., 1986, S. 109.

Vgl. stellvertretend Brunner, J., Value-Based Performance Management, Wertsteigernde Unternehmensführung: Strategien, Instrumente, Praxisbeispiele, Wiesbaden 1999.

50 Vgl. allgemein Kaplan, R. S., Norton, D. P., Balanced Scorecard: Strategien erfolgreich umsetzen, aus dem Amerikanischen übersetzt von P. Horváth, Stuttgart 1997; Weber, J., Schäffer, U., Balanced Scorecard \& Controlling: Implementierung - Nutzen für Manager und Controller - Erfahrungen in deutschen Unternehmen, 2. Aufl., Wiesbaden 2000.

GROB weisst explizit auf Planungs- und Kontrollaufgaben des Controller hin, da dieser über umfassende Kenntnisse der Instrumente und Techniken zur Unternehmenssteuerung verfügt. Würde der Controller nur koordinieren, wäre dies nach GROB ineffizient, weil das breite Fachwissen bei der Nutzung der entwickelten Systeme nicht eingesetzt würde. Obwohl sich durchaus traditionelle Grenzen zwischen Controlling und Management immer weiter aufheben, da Selbststeuerung gestützt durch Dialogsysteme wie beispielsweise SAP an Bedeutung gewinnt, kann der Auffassung von GROB hier nur eingeschränkt gefolgt werden. Würde das Controlling den kompletten Lenkungsprozess von der Planung über die Führung bis hin zur Kontrolle beinhalten, so wäre der Terminus "Controlling" überflüssig, da diese Aufgaben schon dem Managementbegriff inhärent sind. Auch die Informationsversorgung wurde schon seit jeher von spezifischen betriebswirtschaftlichen Abteilungen und Stäben wahrgenommen und 
Aufbereitung von Führungsinformationen soll das Controlling das Entscheiden und Handeln in der Unternehmung ergebnisorientiert ausrichten, wobei sich die Ergebnisorientierung auf unterschiedliche Größen wie zum Beispiel Gewinn oder Shareholder Value beziehen kann. ${ }^{52}$ Dabei hat das Controlling das Informationsversorgungs- sowie Planungs- und Kontrollsystem sowohl zu gestalten (Systembildung bzw. -gestaltung) als auch innerhalb des konzipierten Systemzusammenhangs laufend Abstimmungen vorzunehmen sowie Störungen zu beseitigen (Systemkopplung bzw. Systemnutzung). ${ }^{53}$ Vor dem Hintergrund dieser Überlegungen wird das Controlling somit als ein führungsunterstützendes Konzept zur informationellen Sicherung ergebnisorientierter Planung, Steuerung und Kontrolle verbunden mit einer übergeordneten Koordinationsfunktion verstanden, dessen Aufgabenspektrum sowohl systemgestaltende als auch systemnutzende Aktivitäten umfasst. ${ }^{54}$ Anders ausgedrückt steht Controlling somit nach WEBER für die Sicherstellung von Rationalität der Unternehmensführung. ${ }^{55}$

Die in der Praxis mit der Arbeitsteilung bzw. Spezialisierung ${ }^{56}$ einhergehenden Organisationslösungen weisen unmittelbar auf die Sinnhaftigkeit bzw. Notwendig-

kann daher nicht als Spezifikum des Controlling gesehen werden. Vgl. Grob, H. L., Positionsbestimmung des Controlling, a.a.O., S. 3. Vgl. zur Selbststeuerung auch Gerberich, C. W., Wohin führt der Weg des Controllers?, a.a.O., S. 31.

Vgl. Hahn, D., PuK. Planung und Kontrolle. Planungs- und Kontrollsysteme. Planungs- und Kontrollrechnung, Controllingkonzepte, 5. Aufl., Wiesbaden 1996, S. 175.

53 Hier werden die auf HORVATH zurückzuführenden Kriterien der Systembildung und Systemkopplung inhaltlich übernommen, vgl. Horváth, P., Controlling, in: Vahlens Großes Controllinglexikon, Horváth, P., Reichmann, Th. (Hrsg.), München 1993, S. 112. Die Kopplungsaufgabe bezeichnet den "laufenden" Betrieb bzw. die Nutzung des konzipierten Systems. HAHN, dessen Systematisierung mit der von HORVATH korrespondiert, spricht daher auch von $\mathrm{Ge}$ staltungs- und Nutzungsaufgaben des Controlling. Vgl. Hahn, D., PuK. Planung und Kontrolle. Planungs- und Kontrollsysteme. Planungs- und Kontrollrechnung. Controllingkonzepte, a.a.O., S. 188.

Vgl. hierzu auch Meffert, H., Marketing: Grundlagen marktorientierter Unternehmensführung: Konzepte - Instrumente - Praxisbeispiele, a.a.O., S. 1035 f.

Weber, J., Schäffer, U., Balanced Scorecard \& Controlling: Implementierung - Nutzen für Manager und Controller - Erfahrungen in deutschen Unternehmen, a.a.O., S. 126f.

Arbeitsteilung entsteht durch Verteilung einer Aufgabe nach Art (vertikale Arbeitsteilung bzw. Spezialisierung) und Umfang (horizontale Arbeitsteilung) auf zwei oder mehr Menschen als Aufgabenträger. Vgl. zur Arbeitsteilung Kosiol, E., Organisation der Unternehmung, 2. Aufl., Wiesbaden 1976, S. 171. 
keit unternehmensbereichsbezogener Controlling-Teilgebiete hin. ${ }^{57}$ Die damit zusammenhängende Spezialisierung des Controlling führt zu speziellen Controllingansätzen und -ausprägungen mit jeweiliger Modifikation und Anpassung der „typischen" Controlling-Instrumente. Vor dem spezifischen Hintergrund der Mehrmarkenstrategie kann das Mehrmarkencontrolling als spezielle Ausprägung des Controlling interpretiert werden, das die Informationsversorgung und Beratung aller mit der Markenführung befaßten Stellen verbunden mit einer übergeordneten Koordinationsfunktion zur Unterstützung und Ergänzung der markenspezifischen Planungs-, Steuerungs- und Kontrollprozesse im Unternehmen umfasst. Als Servicefunktion des Portfoliomanagement wirkt das Mehrmarkencontrolling subsidiär an Entscheidungen aller markenbezogenen Aktivitäten mit, ${ }^{58}$ die eigentliche Willensbildung obliegt jedoch dem Portfoliomanagement selbst. ${ }^{59}$ Sowohl die besonderen Probleme, die bei der Gestaltung eines Markenportfolios auftreten, als auch der erhöhte Informationsbedarf sowie die andauernde Koordinationsaufgabe eines bestehenden Markenportfolios sorgen dabei für eine höhere Komplexität des Mehrmarkencontrolling gegenüber einem Markencontrolling.

Analog zur Abgrenzung des Controlling von anderen betriebswirtschaftlichen Teildisziplinen besteht in Wissenschaft und Praxis kein Konsens bezüglich der direk-

57 So weisst HORVATH mit Bezug auf Cochran, E. B., What is a Controller?, Journal of Accountancy, July 1955, S. 46-53 darauf hin, dass die Aufgabe eines Gesamtcontrolling zwar weitgefächert ist, eine systemorientierte Betrachtung jedoch die Bewältigung der Komplexität ermöglicht. KLINGENBIEL spricht in diesem Zusammenhang von einer zu beobachtenden Tendenz der Dezentralisierung von Controlling-Funktionen, vgl. Klingenbiel, N., Performance Measurement: Grundlagen - Ansätze - Fallstudien, Wiesbaden 1999, S. 4.

58 Nach WEBER, SCHÄFFER sollten „Controller als Dienstleister des Managements subsidiär und engpaßbezogen dort tätig werden, wo ihn der Schuh drückt“, vgl. Weber, J., Schäffer, U., Balanced Scorecard \& Controlling: Implementierung - Nutzen für Manager und Controller Erfahrungen in deutschen Unternehmen, a.a.O., S. IV. Metaphorisch wird der Controller häufig in der Rolle als Navigator, als Informator, als Evolutionär und Innovator, als Implementator oder als Coach der Unternehmensführung gesehen. Vgl. zu den Rollen des Controllers Ahlert, D., Grundlagen des Controlling, a.a.O., S. $68 \mathrm{f}$.

Die Entscheidungsunterstützung hebt auch KELLER hervor: „A brand equity measurement system is a set of research procedures designed to provide timely, accurate, and actionable information for marketers on brands so that they can make the best possible tactical decisions in the short run and strategic decisions in the long run. [...] The ideal brand equity measurement system would provide complete, up-to-date, and relevant information on the brand and all its competitors to relevant decision makers within the organization. "Keller, K. L., Strategic Brand Management: Building, Measuring, and Managing Brand Equity, a.a.O., S. $372 f$. 
ten Zielsetzung ${ }^{60}$ des Controlling, obwohl diese letztlich die Begründung bzw. den Ausgangspunkt einer konkreten Controllingkonzeption im Unternehmen darstellt. Hier ist die Wurzel für die unzureichende theoretische Fundierung des Controlling erkennbar. Primäres Ziel des hier verwendeten Controllingbegriffs ist die Erreichung optimaler Zustände - im Allgemeinen als Gewinnmaximum definiert - für das Gesamtportfolio. Die umfassende Unterstützung des Portfoliomanagement im Hinblick auf eine Ergebnisoptimierung des Marktauftritts der Marken kann somit als Kardinalziel des Mehrmarkencontrolling interpretiert werden. Aus diesem Oberziel lassen sich weitere Subziele ableiten, wie

- Sicherstellung der Rationalität sowie Erhaltung der Reaktions- und Adaptionsfähigkeit der Mehrmarkenführung durch Versorgung mit markenrelevantem Wissen.

- Verbesserung der Wirksamkeit der Mehrmarkenstrategie durch Koordination und Ausrichtung aller markenpolitischen Aktivitäten auf die Ziele des Markenportfolios.

Aus den Zielen ergeben sich unmittelbar die Aufgaben des Mehrmarkencontrolling. Entsprechend den allgemeinen Ausführungen zum Controlling kann zwischen systemgestaltenden und systemnutzenden Aktivitäten des Mehrmarkencontrolling differenziert werden:

- Die systemgestaltende Funktion des Mehrmarkencontrolling umfasst primär die Sicherstellung einer ex-ante Koordination ${ }^{61}$ des Markenportfolios durch die Schaffung geeigneter Rahmenbedingungen, die koordinierte Entscheidungen überhaupt erst ermöglichen. Dem Mehrmarkencontrolling kommt hier die Aufgabe zu, an der Entwicklung und Implementierung von Informationssystemen,

60 Um konzeptionellen Missverständnissen vorzubeugen sind direkte und indirekte Ziele des Markencontrolling zu unterscheiden. Während die direkten Ziele Umfang und Tiefe der Mehrmarkencontrolling-Aufgaben abgrenzen, konkretisieren die indirekten Ziele die Aufgaben inhaltlich und haben die verschiedenen Ziele der Mehrmarkenstrategie zum Gegenstand. Da das Mehrmarkencontrolling nur einen mittelbaren Beitrag zu deren Erreichung leisten kann, werden sie als indirekt bezeichnet. Sie stellen den inhaltlichen Ausgangspunkt eines Mehrmarkencontrolling dar. Vgl. Schweitzer, M., Friedl, B., Beitrag zu einer umfassenden Controlling-Konzeption, in: Controlling, Spremann, K., Zur, E. (Hrsg.), Wiesbaden 1992, S. 143. Einen Überblick über die wenigen bisher in der Literatur ausführlich besprochenen Controlling-Ziele gibt Schöne, K., Controlling der Informationsinfrastruktur: Entwicklungsstand - Gestaltungskonzeptionen - Perspektiven, Wiesbaden 1997, S. 40. Vgl. auch die Übersicht über Konzeptionen zum Controlling bei Küpper, H.-U., Controlling: Konzeptionen, Aufgaben und Instrumente, a.a.O., S. 8 f.

Vgl. Kiener, J., Marketing-Controlling, Darmstadt 1980, S. 19. 
organisatorischen Richtlinien und Prozessstrukturen sowie Planungs- und Kontrollinstrumenten für das Markenportfolio mitzuwirken.

- Zu den systemnutzenden Funktionen des Mehrmarkencontrolling gehören die Unterstützung des Portfoliomanagements auf allen Ebenen der Planung, die regelmäßige Kontrolle der Umsetzung strategischer und operativer Pläne und die Sicherstellung der Informationsversorgung aller an der Markenführung Beteiligten innerhalb des durch die systemgestaltende Funktion geschaffenen Rahmens.

Während das Informationsmanagement die Infrastruktur aus IT-Sicht gestaltet, hat das Mehrmarkencontrolling eine betriebswirtschaftlich sinnvolle inhaltliche Ausprägung des Informationsbedarfs zu verantworten und im Rahmen seiner Informationsversorgungsfunktion den jeweiligen mit der Markenführung betrauten Stellen art-, zeit- und mengengerecht zur Verfügung zu stellen. Dabei ist es für das Portfoliomanagement bedeutend, mittels einiger weniger Steuerungsgrößen einen Überblick über die Lage des Portfolios zu gewinnen. Zur Gewinnung und Aufbereitung der notwendigen planungs-, entscheidungs- und kontrollrelevanten Informationen greift das Mehrmarkencontrolling auf externe und interne Daten zurück. Die Informationsversorgung entwickelt sich zur Beratung, wenn das Mehrmarkencontrolling aufgrund seines Methoden- und Fachwissens zum Gesprächspartner des Portfoliomanagement wird und Empfehlungen und Hinweise zur Entscheidungsvorbereitung gibt. ${ }^{62}$

In diesem Sinne leistet das Mehrmarkencontrolling eine Planungsunterstützung des Portfoliomanagements bei der Aufstellung des strategischen Gesamtplanes für das Markenportfolio und dessen Aufspaltung in operative, markenspezifische Teilpläne (wie Zielplan, Ressourcenplan, Terminplan). Um veränderten Anforderungen an die Mehrmarkenführung möglichst frühzeitig durch eine Anpassung der Planung Rechnung zu tragen, ist im Rahmen von Kontroll- bzw. Abweichungsanalysen ein kontinuierlicher und systematischer Vergleich von Sollvorgaben und Istzuständen hinsichtlich der vom gesamten Portfolio und den einzelnen Marken verfolgten Zielsetzungen durchzuführen. ${ }^{63}$ Dabei hat das Mehrmarkencontrolling zum einen nach dem Feed-back-Prinzip eine ex-post-Überwachung durch eine Bestandsaufnahme des Markenportfolios hinsichtlich ökonomischer, psychographischer

Vgl. Ahlert, D., Grundlagen des Controlling, a.a.O., S. 28.

63

Vgl. Meffert, H., Marketing: Grundlagen marktorientierter Unternehmensführung: Konzepte Instrumente - Praxisbeispiele, a.a.O., S. $1044 f$. 
und verhaltensbezogener Größen vorzunehmen. Zum anderen hat es als „Frühwarnsystem" feed-forward im Sinne einer ex-ante Überwachung Soll-Ist-Abweichungen zu antizipieren und damit ihr Eintreten zu verhindern. ${ }^{64}$

Die in einer übergeordneten Sichtweise zu betrachtende Koordinationsfunktion des Mehrmarkencontrolling beinhaltet die Ausrichtung aller Portfolioaktivitäten auf ein gemeinsames Ziel und mithin die markenübergreifende Abstimmung der Planung, Steuerung und Kontrolle. In diesem Sinne kann auch von einer Zielausrichtungsfunktion des Mehrmarkencontrolling gesprochen werden. ${ }^{65}$ In Rahmen der horizontalen Koordination geht es dabei um die gegenseitige Abstimmung der markenpolitischen Handlungen einzelner Marken im Hinblick auf die übergeordneten Portfolioziele. Die vertikale Koordination umfasst demgegenüber die Abstimmung über- und untergeordneter Ebenen des Portfolios. Einen zentralen Stellenwert nimmt in diesem Zusammenhang die vielfach stark verbesserungsfähige Abstimmung des strategischen und operativen Marktauftritts der einzelnen Portfoliomarken ein. ${ }^{66}$ Aktuelles Indiz für die Verbesserungsfähigkeit der auch als „bridging" bezeichneten Verbindung zwischen Strategie und operativem Management ist der Erfolg der Balanced Scorecard, welche nach der Intention von KAPLAN/NORTON unter anderem diese Verbindung schaffen will. ${ }^{67}$ Hier wird der vollständige Managementprozess auf strategischer und operativer Ebene abgebildet, indem ökonomische und vorökonomische Steuerungsgrößen bzw. Kennzahlen entsprechend ihrer Ursache-Wirkungsbeziehungen logisch miteinander verknüpft werden. ${ }^{68}$ Hierdurch lässt sich eine frühzeitige Rückkopplung auf allen

Nach BAETGE umfasst der Begriff "Überwachung" sowohl den Begriff "Kontrolle" als auch den Begriff "Prüfung". Dabei lässt sich Prüfung etymologisch ausgehend von seinem Synonym Revision als vergangenheitsbezogen (lat. revidere: wiedersehen, nachsehen), Kontrolle hingegen als gegenwartsbezogen (lat. contra-rotulus: Gegenrolle) erklären. Vgl. Baetge, J., Überwachung, a.a.O., S. 169.

Vgl. Küpper, H.-U., Controlling: Konzeptionen, Aufgaben und Instrumente, a.a.O., S. 18. Koordination beinhaltet letztlich die zielgerichtete, gegenseitige Abstimmung arbeitsteilig vollzogener Handlungen im Hinblick auf ein übergeordnetes Gesamtziel. Vgl. hierzu etwa SchulteZurhausen, M., Organisation, München 1995, S. 187.

Vgl. Köhler, R., Beiträge zum Marketing-Management. Planung, Organisation, Controlling, 3. Aufl., Stuttgart 1993, S. 166ff.

Vgl. hierfür stellvertretend Kaplan, R. S., Norton, D. P., Balanced Scorecard: Strategien erfolgreich umsetzen, a.a.O. 
Ebenen des Portfolios sicherstellen und mithin die Anpassungsfähigkeit der Marken im Marktauftritt erhöhen. Die möglichst vollständige Vernetzung des strategischen und operativen Management mit meß- und kontrollierbaren SteuerungsgröBen kann somit als zentrale Voraussetzung eines wirksamen Mehrmarkencontrolling angesehen werden. Schließlich beinhaltet die Koordination in zeitlicher Hinsicht die Abstimmung der kurz-, mittel und langfristigen Portfolioplanung. ${ }^{69}$

Art und Umfang der Controllingaufgaben ergeben sich letztlich aus dem jeweils vorliegenden Stand der Rationalität der Markenführung sowie aus der Intensität der Beziehungen zwischen den Portfoliomarken. Auch unterscheidet sich der Controllingbedarf beim Aufbau des Markenportfolios von dem beim kontinuierlichen Portfoliomanagement. So steht beim Aufbau eines Markenportfolios primär die Informationsfunktion als Rationalitätsengpass im Vordergrund. Hier ergibt sich beispielsweise ein erhöhter Informationsbedarf beim Kauf oder Aufbau einer Marke sowie deren Integration in das bestehende Markenportfolio. Noch vielschichtiger sind die Aufgaben indes in der Phase des Portfoliomanagement. Mit zunehmender Intensität der teils konfliktären Beziehungen zwischen den Marken geht eine verstärkte markenübergreifende Koordination einher. Letztlich unterliegt die konkrete Entwicklung und Ausgestaltung eines Mehrmarkencontrolling keinem standardisierten Vorgehen, sondern ist im Einzelfall vor dem Hintergrund des spezifischen situativen Kontextes, der Struktur des Markenportfolios sowie der angestrebten Ziele vorzunehmen.

\section{Zielsetzung und Gang der Untersuchung}

Vor dem Hintergrund der dargelegten Problemstellung besteht die generelle Zielsetzung dieser Arbeit darin, einen Beitrag zur Entwicklung und Nutzung eines Controllingsystems im Rahmen von Mehrmarkenstrategien zu leisten. Im Mittelpunkt der Betrachtung steht die Frage, wie ein Mehrmarkencontrolling auszugestalten ist, das sich auf die Lösung von Koordinations- und Steuerungsproblemen bei der Führung von Markenportfolios bezieht und zur Optimierung des externen und internen Zusammenspiels der verschiedenen Portfoliomarken dient. 
Um dieser generellen Zielsetzung gerecht zu werden, ist die Formulierung und Bearbeitung aufeinander aufbauender Teilziele notwendig, die auch die wesentlichen Forschungsschwerpunkte der Arbeit widerspiegeln. Als erstes Teilziel ist eine konzeptionelle Analyse des Controllingobjektes vorzunehmen. Um die Mehrmarkenstrategie als komplexes realweltliches Gebilde zu verstehen, sind die entscheidungsfeldabhängigen planerischen und organisatorischen Aspekte einer Mehrmarkenstrategie als Objekt des Controlling zu erarbeiten. Darauf aufbauend stellt eine weiteres Teilziel der Arbeit die Bestimmung des Controllingbedarfs einer Mehrmarkenstrategie dar. Hierzu sind die zentralen Spannungsfelder und Interdependenzbeziehungen bei der Führung von Markenportfolios zu identifizieren und zu präzisieren. Eine solche Analyse kann dabei nicht losgelöst vom situativen Kontext und der sich daraus für das Unternehmen ergebenden Umweltsituation erfolgen. ${ }^{70}$ Bezieht sich das Mehrmarkencontrolling etwa auf Konsumgüter, so ist entscheidend, ob das Markenportfolio quasi homogene, in der Qualitätswahrnehmung des Konsumenten kaum unterscheidbare Konsumgüter wie Benzin oder Zigaretten umfasst, oder ob eine Differenzierung im Bereich langlebiger Konsumgüter, ${ }^{71}$ wie etwa Automobile, angestrebt wird. Bewegen sich die Differenzierungsbemühungen der Mehrmarkenstrategie bei homogenen Gütern insbesondere im kommunikativen Bereich, so erfordern langlebige Konsumgüter zur Sicherstellung

Der situative Ansatz bzw. Kontingenzansatz stellt unter Rückgriff auf die Mitte der sechziger Jahre in der Organisationstheorie gewonnenen Erkenntnisse kontextbezogene, d. h. sich aus dem Umfeld der Unternehmung ergebende Anpassungsnotwendigkeiten in den Vordergrund. Solche Anpassungsnotwendigkeiten ergeben sich entsprechend auch bei der Gestaltung und Nutzung eines Mehrmarkencontrolling. Vgl. zum Kontingenzansatz insbesondere Kast, F., Rosenzweig, J., Organization and Management: A Contingency Approach, Tokio 1970. Aus der Kritik an "reinen" Kontingenzansätzen, die sich insbesondere darauf bezieht, dass das Unternehmensverhalten hier als lediglich reaktiv bezeichnet wird und dem Handlungsspielraum der Entscheidungsträger somit nicht hinreichend Rechnung getragen wird, ist die Entwicklung unternehmenspolitisch-situativer Erklärungsansätze entstanden, welche das Unternehmensverhalten sowohl in Abhängigkeit der Ziel- und Strategieentscheidung des Management als auch der situativen Einflüsse untersucht. Das Mehrmarkencontrolling ist hiernach sowohl vor dem Hintergrund situativer Einflüsse als auch vor dem Hintergrund des "Managementgebarens" zu sehen. Vgl. hierzu Lehnert, St., Die Bedeutung von Kontingenzansätzen für das strategische Management, Frankfurt a. M., Bern, New York, 1983, S. 115.

71 Während langlebige Konsumgüter als Gebrauchsgüter mehrmalig genutzt werden und in der Regel über eine längere Lebensdauer verfügen, werden Verbrauchsgüter im Laufe eines oder weniger Verwendungseinsätze konsumiert und sind demnach von kurzlebiger Natur. Vgl. zur Unterscheidung Kotler, Ph., Bliemel, F., Marketing-Management: Analyse, Planung, Umsetzung und Steuerung, a.a.O., S. 673. 
des markenspezifischen Kompetenzfeldes eine umfassendere Differenzierung auch in den übrigen Komponenten des Marketing-Mix. ${ }^{72}$

In dieser Arbeit wird die Untersuchung an den Problemstrukturen der Automobilindustrie ausgerichtet. Die Automobilindustrie als situativer Kontext eines Mehrmarkencontrolling bei langlebigen Konsumgütern bietet sich insbesondere aus folgenden Gründen an:

- In der Automobilwirtschaft kommt der Markenführung zur Differenzierung im Wettbewerb sowie zur Präferenzbildung beim Konsumenten eine herausragende Bedeutung zu, da sich Marken hier in einem Umfeld profilieren müssen, in dem nur markierte Leistungen und keine No Names angeboten werden.

- Gleichzeitig kommt der Führung von Markenportfolios im Automobilmarkt eine bedeutende Rolle zu. Wie bereits aufgezeigt, agieren heute nahezu sämtliche Automobilhersteller mit einem umfassenden Markenportfolio im Wettbewerb.

- Dabei ist die Automobilindustrie durch eine wachsende Ähnlichkeit der Produktkonzepte geprägt, deren Austauschbarkeit eine große Herausforderung an die Markenführung von Automobilherstellern stellt. Hohe Ressourcen-, Marktund innerbetriebliche Interdependenzen lassen gerade in dieser Branche ein Mehrmarkencontrolling um so notwendiger erscheinen.

Die auf der Grundlage der speziellen Marktsituation gewonnenen Erkenntnisse sollten nicht allein für die Steuerung von Markenportfolios in der Automobilindustrie von Interesse sein, sondern darüber hinaus insoweit verallgemeinerungsfähig sein, als sie auf Märkte bzw. Branchen übertragen werden, die durch eine dem Automobilmarkt vergleichbare Bedingungskonstellation gekennzeichnet sind. Hierbei ist insbesondere an diejenigen Märkte langlebiger Gebrauchsgüter zu denken, die ebenfalls durch eine dominante Rolle der Marke, eine hohe Konkurrenzverbundenheit, lange Wiederkaufszyklen und durch eine vergleichsweise hohe Markenbindung zu charakterisieren sind. ${ }^{73}$

Aufbauend auf dem identifizierten Controllingbedarf stellt die strukturelle Ausgestaltung eines den Erfordernissen des Portfoliomanagement Rechnung tragenden

Vgl. Meffert, H., Mehrmarkenstrategie - immer die beste Option?, a.a.O., S. 82

Die wissenschaftliche Forschung bewegt sich letztlich stets im Spannungsfeld zwischen dem Wunsch einer Generalisierung über den spezifischen Einzelfall hinaus (induktive Methode der Erkenntnisgewinnung) und der Notwendigkeit, in bestimmten Erklärungsansätzen allgemeine Grundsätze auf den individuellen Einzelfall übertragen zu müssen (deduktive Methode). Vgl. hierzu Knoblich, H. K., Die typologische Methode in der Betriebswirtschaftslehre, in: WiSt, Heft 4, 1972, S. 141. 
Controllingsystems ein weiteres Teilziel der Arbeit dar. Vor dem Hintergrund der situativen Gegebenheiten soll ein Ansatz entwickelt werden, der es erlaubt, aus einer Vielzahl einzelner Größen mosaiksteinartig ein aussagefähiges System zu konstruieren, welches dem Portfoliomanagement ein umfassendes Bild über das externe und interne Zusammenspiel der Portfoliomarken vermittelt. ${ }^{74}$ Dabei soll insbesondere der Frage nachgegangen werden, welchen Beitrag die Balanced Scorecard zur Willensdurchsetzung im Rahmen einer Mehrmarkenstrategie leisten kann. Neben der Entwicklung stellt schließlich die Nutzung einer solchen zusammenhängenden Controllingkonzeption ein weiteres Teilziel der Arbeit dar. Hierzu wird der Analyse ein reales Markenportfolio eines Automobilherstellers zugrunde gelegt.

Die inhaltlich-sachliche Zielsetzung der Untersuchung geht mit einem theoretisch methodischen Ziel einher. Dieses konzentriert sich mit Blick auf den geringen Erkenntnisstand auf die Erarbeitung einer Grundlagenstudie des Mehrmarkencontrolling, wobei die Forschungsstrategie aufgrund des außerordentlich komplexen Untersuchungsobjekts als eher heuristisch anzusehen ist. Grundlage der Erkenntnisgewinnung sind dabei insbesondere literaturgestützte Plausibilitätsüberlegungen, die anhand empirischer Studien der Automobilhersteller gestützt bzw. illustriert werden.

Der Gang der Untersuchung ergibt sich unmittelbar aus der Bearbeitung der Teilzielsetzungen. Kapitel B dient der Analyse des Controllingobjektes. Hierzu ist zunächst die Automobilindustrie als situativer Kontext der Mehrmarkenstrategie darzustellen. Aufbauend auf den Zielsetzungen sowie den theoretischen Grundlagen der Mehrmarkenführung wird sodann die Ausgestaltung von Markenportfolios sowohl in strategischer und operativer Hinsicht als auch in Bezug auf die organisatorische Verankerung umfassend analysiert. Anknüpfend an die im Rahmen der Analyse des Controllingobjektes gewonnen Erkenntnisse lässt sich in Kapitel C der Controllingbedarf von Mehrmarkenstrategien im Automobilbereich identifizieren. Auf dieser Basis kann die Entwicklung eines integrativen Controllingansatzes

74 Die Qualität der Entscheidungsfindung auf der Grundlage eines Mehrmarkencontrollingsystems ist umso höher einzustufen, je größer die Homomorphie zwischen dem zu erfassenden Realsystem „Marke" und dem Controllingsystem ausfällt. Trägt die Forderung nach Strukturgleichheit zwar dem Grundproblem der Optimierung eines solchen Rechnung, so sind ihrer Realisierung aufgrund des kaum zu befriedigenden Informationsbedarfs und der hohen Komplexität sehr enge Grenzen gesetzt. 
vorgenommen werden. Dabei nimmt die Diskussion und Würdigung der Balanced Scorecard im Rahmen des Mehrmarkencontrolling einen besonderen Stellenwert ein. Um die zu behandelnde Problemstellung nicht nur auf theoretischem, sondern auch auf empirischem Wege zu erhellen, setzt sich Kapitel D mit der konkreten Nutzung des in Kapitel $\mathrm{C}$ konzipierten Controllingansatzes in der Automobilindustrie auseinander. Dabei kommen unterschiedliche Bausteine und Instrumente auf strategischer und operativer Ebene eines Mehrmarkencontrolling unter Beachtung der Wechselwirkungen der Portfoliomarken zum Einsatz. Im Sinne einer konsistenten und koordinierenden Informationsversorgung erfolgt schließlich eine hierarchische Informationsintegration der relevanten Steuerungsgrößen in ein Konzept der Balanced Scorecard. Abschluss der Arbeit bildet Kapitel E, in dem die gewonnen Erkenntnisse der Mehrmarkenführung und des Mehrmarkencontrolling eine abschließende Würdigung erfahren sowie Ansatzpunkte für weiterführende Forschungsarbeiten aufgezeigt werden. 
Martin Koers - 978-3-631-75091-9

Downloaded from PubFactory at 01/11/2019 08:29:37AM

via free access 


\section{B. Ausgestaltung von Markenportfolios als Objekt des Con- trolling}

\section{Markenportfolios in der Automobilindustrie als situativer Kontext ei- nes Mehrmarkencontrolling}

Die Automobilindustrie ist durch eine starke internationale Ausrichtung und zunehmende Globalisierung gekennzeichnet. Im Automobilmarkt als einem klassischen oligopolistischen Markt produzieren die zehn größten Automobilunternehmen drei Viertel der weltweiten Pkw-Fabrikation. Dabei sind die Pkw-Märkte in den Ländern der Triade obgleich ihrer hohen Bedeutung hinsichtlich der Stückzahlen durch eine Sättigung der Nachfrage gekennzeichnet, so dass hier auch mittelfristig lediglich ein geringes Wachstum im Neuwagenmarkt zu erwarten ist und Marktanteilsgewinne primär durch Verdrängung bestehender Anbieter möglich sind. ${ }^{75}$ Die durch Globalisierung, stagnierende Nachfrage und oligopolistische Marktform hervorgerufene hohe Wettbewerbsintensität wird zusätzlich trotz weltweiter Überkapazitäten durch einen preisaggressiven Marktauftritt neuer Konkurrenten verstärkt, die insbesondere aus finanzstarken asiatischen Unternehmen hervorgehen. $^{76}$

In einem solchen Wettbewerbsumfeld sehen sich Automobilhersteller mit steigenden Investitionen bei geringeren Entwicklungszeiten ${ }^{77}$ für neue Fahrzeugpro-

75 Zu den Triadeländern gehören die Länder bzw. Regionen USA, Europa und Japan. Dabei ist der europäische Markt im Wesentlichen bestimmt durch die sogenannten EURO 5-Länder Frankreich, Großbritannien, Italien, Spanien und Deutschland, auf die rund 85 Prozent des Gesamtmarktvolumens in Europa fallen. Trotz neu aufstrebender Länder ist die Bedeutung der Triade in der Automobilindustrie sowohl als Produktionsstandort als auch als Absatzmarkt ungebrochen. Vgl. Heise, G., Internationale Marktsegmentierung im Automobilmarketing, Wiesbaden 1997, S. 2f.; auch Wolters, H., Hocke, R., Auf dem Weg zur Globalisierung Chancen und Risiken, in: Die Zukunft der Automobilindustrie, Wolters, H. et al. (Hrsg.), Wiesbaden 1999, S. 14.

76 Hierbei handelt es sich insbesondere um die koreanischen Anbieter DAEWOO oder HYUNDAl, die malayischen Anbieter PROTON und PERODUA oder die indischen Automobilhersteller MARUTTI und TATA. Zum intensiven Preiswettbewerb vgl. Lederer, M., Clement, R., Mit innovativen Marktleistungskonzepten zu globaler Wettbewerbsfähigkeit, in: Markendynamik, Weinhold-Strünzi, H., Reinecke, S., Schögel, M. (Hrsg.), St. Gallen 1997, S. 146f.

77 Nach Angaben der Hersteller kann zwischen 1990 und 1998 bei AUDI eine Verkürzung der Entwicklungszeiten um 50 Prozent, bei BMW um 35 Prozent und bei DAIMLERCHRYSLER um 30 Prozent konstatiert werden. Vgl. Diez, W., Plattorm-Strategien: Irrweg oder Königsweg in der Modellpolitik, Arbeitspapier Nr. 2 des Instituts für Automobilwirtschaft, Gelsenkirchen 1999, S. 11. 
jekte konfrontiert. Zunehmend längere Pay-Off-Perioden bei sich verkürzenden Produktlebenszyklen ${ }^{78}$ münden in ein gesteigertes Ertragsrisiko, da Automobilhersteller Gefahr laufen, die Break-Even-Menge zu verfehlen. Bei kleineren Herstellern betragen die Gesamtinvestitionen für ein neues Fahrzeug bis zu zehn Prozent des Gesamtumsatzes, so dass die Gefahr eines Misserfolgs auch unter Berück-

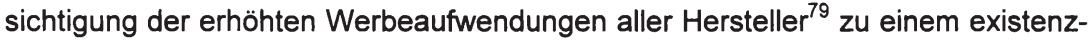
bedrohenden Risiko werden kann.

Die hohe Produktqualität nahezu sämtlicher Anbieter führt zu eingeschränkten technologischen Profilierungsmöglichkeiten gegenüber Konkurrenzprodukten, wobei auch Produktinnovationen aufgrund rascher Imitationen nur eine zeitlich befristete exponierte Marktstellung bewirken. ${ }^{80}$ Gleichzeitig wird die im Gleichschritt vollzogene Erhöhung der Produktsubstanz durch serienmäßige Ausstattungsverbesserungen ohne äquivalente Preiserhöhung an die Verbraucher weitergegeben, womit Ausstattungsmehrungen zu real sinkenden Preisen führen. ${ }^{81}$ Infolgedessen kann im Automobilmarkt in Anlehnung an D'AVENI von einem Trend zum Wertoptimum gesprochen werden. ${ }^{82}$

Lag die Lebenszyklusdauer etwa beim Golf I noch bei 9 Jahren, so verkürzte sich der Lebenszyklus beim Golf II auf 8 und beim Golf III auf 6 Jahre. Der Golf IV erschien 1997, wobei der Golf $\mathrm{V}$ bereits seit längerem in Planung ist. Vgl. auch Schaaf, A., Marktorientiertes Entwicklungsmanagement in der Automobilindustrie: Ein kundennutzenorientierter Ansatz zur Steuerung des Entwicklungsprozesses, Wiesbaden 1999, S. 4.

Allein 1998 wurden fast 3 Milliarden DM für Werbung im deutschen Automobilmarkt aufgewendet. Dabei stiegen in ganz Deutschland die Werbeaufwendungen zwischen 1989 und 1998 um 111 Prozent, die für Automobile hingegen um 139 Prozent. Gleichzeitig nahm die Anzahl der Neuzulassungen nur um 32 Prozent zu, so dass sich die durchschnittlichen Werbeaufwendungen pro neu zugelassenem Pkw von 444 DM auf 806 DM erhöhte. Vgl. o.V., autofahren in deutschland 1999, a.a.O., S. 62

Vgl. Ealey, L., Troyano-Bermúdez, L., Are Automobiles the next Commodity?, in: The MkKinsey Quarterly, No. 4, 1996, S. 65.

Beispielsweise zeigt ein Vergleich der ausstattungsbereinigten Preise ausgewählter Produkteinführungen im Oberklassesegment reale Preissenkungen. Bei Einführung des Opel Omega, Volvo 850, Audi A4, Mercedes E-Klasse und Passat mussten ausstattungsbereinigt Preissenkungen von bis zu 5,5 Prozent hingenommen werden. Der amerikanische Markt weist hier ähnliche Tendenzen auf. Vgl. Maxton, G., Wormald, J., Driving over a Cliff? Business Lessons from the World's Car Industry, Cambridge 1995, S. $47 f$.

Vgl. D'Aveni, Hypenwettbewerb: Strategien für die Dynamik der Märkte, Wiesbaden 1995, S. 85. Beispielsweise hatten 1996 in Deutschland 59 Prozent aller Neuzulassungen einen Airbag, während im Durchschnitt aller Fahrzeuge dieser Anteil bei nur 12 Prozent lag. Haben alle Anbieter ihre Preise und Qualität angeglichen, sind Preispremien, also Preise oberhalb der technischen Grundleistung, nur schwer durchsetzbar. Vgl. Proff, H., Proff, H. V., Bedeutung vorwärtsgerichteter Strategien für die Automobilindustrie, in: Strategien für die Automobilindu- 
Den real sinkenden Preisen versuchen die Automobilhersteller bei einer zurückhaltenden Preispolitik insbesondere über Produktivitätssteigerungen zu begegnen. Mit der Rezession von 1993 hat ein massiver Restrukturierungsprozess eingesetzt, um vor allem Kostensenkungen durchzusetzen. Hierdurch hat sich der Kostendruck in der gesamten Branche insgesamt weiter erhöht. ${ }^{83}$ Die Kostensituation einzelner Hersteller wird infolgedessen in hohem Maße von der Nutzbarmachung von Economies of Scale bestimmt. Gerade kleine Hersteller erweisen sich wegen grundsätzlicher Kostennachteile häufig als nicht überlebensfähig und werden vielfach von anderen Herstellern übernommen, so dass die Automobilindustrie durch eine zunehmende Konzentration gekennzeichnet ist. ${ }^{84}$

Auf der Nachfragerseite geht die Sättigung der Märkte mit einer zunehmenden Individualisierung der Kundenwünsche und einer daraus resultierenden weiteren Fragmentierung der Märkte einher. Zusätzlich zur Basisfunktion des reinen Transports dient das Auto in immer stärkerem Maße als ein Mittel zum Ausdruck des eigenen Lebensstils. Mehrdimensionaler Wertewandel, Individualisierung und die damit verbundene Pluralisierung von Lebensstilen bei gleichzeitiger Freizeitorientierung führen zu veränderten Einstellungen der Konsumenten zum Automobil, so dass das Auto zunehmend seine Rolle als klassisches Prestigeobjekt und Statussymbol verliert. Der Automobilmarkt fächert sich infolgedessen zunehmend auf, wobei neben einer Erhöhung der Varianten bestehender Baureihen früher häufig unbeachtete Marktnischen zur Befriedigung der Erlebnisorientierung des Konsumenten zunehmend an Bedeutung gewinnen. ${ }^{85}$ Dabei werden entweder neue

strie: Ansatzpunkte im strategischen Management und in der Industriepolitik, Proff, H., Proff, H. V., (Hrsg.), Wiesbaden 1998, S. 13.

83 Vgl. Baldwin, C. Y., Clark, K. B., Modularisierung: Ein Konzept wird universell, in: Harvard Business Manager, Heft 2, 1998, S. 42.

Gab es um 1964 noch weltweit rund 52 unabhängige Automobilhersteller, so sank die Zahl in 1980 auf 30 . Zum heutigen Zeitpunkt befinden sich nur noch 13 unabhängige Automobilhersteller im Markt, wobei eine weitere Reduktion erwartet wird. Vgl. zum Stand Weißenberg, P., Hochzeiten mit Hindernissen, in: W\&V, Heft 38, 1999, S. 96. Experten gehen davon aus, dass Hersteller und Marken, die weniger als fünf Prozent Marktanteil in den nationalen Märkten haben, langfristig nicht überlebensfähig sind, da hohe Marketing-Kosten, Kosten hochwertiger Händlernetze sowie breit angelegtes Markenmanagement nur bei ausreichendem Verkaufsvolumen darstellbar sind. Vgl. Dudenhöffer, F., Dittler, Th., Thies, C., Schlüssel-Trends im Automobilgeschäft Europas, in: Internationales Verkehrswesen, Heft 10, 1998, S. 444.

SCHAAF verweist beispielhaft auf die extensive Produktdifferenzierung anhand der Variantenzahl der BMW 3er Modellreihe: Aus fünf Karosserievarianten, neun Hubraumversionen, zwei Schaltungen und zwei Lenkungen ergeben sich 135 Typkombinationen. Durch 12 länderspezifische Marktanpassungspakete, 21 Außenfarben, 30 Innenausstattungskombinationen sowie mindestens 60 frei kombinierbare Sonderausstattungen ergibt sich eine Angebotsvielfalt von 
Marktsegmente geschaffen (Micro-Vans, Großraumlimousinen oder Fun-Cars) oder aber "brachliegende" Segmente wiederbelebt (z. B. Roadster). Die klassische Aufgliederung des Automobilmarktes nach Ober-, Mittel- und Unterklasse löst sich damit immer weiter auf. Statt dessen gewinnt der Automobilmarkt stärker eine horizontale Dimension, welche durch eine zunehmende individuelle Nutzungsweise und stilistische Differenzierung der Autofahrer gekennzeichnet ist. ${ }^{86}$ Laut empirischen Untersuchungen im deutschen Automobilsektor haben Konsumenten im Jahr 1987 nur zwischen neun unterschiedlichen Marktsegmenten differenziert, während sich in 2000 die Zahl der wahrgenommenen Segmente bei gleichzeitig rückläufiger Segmentgröße verdreifacht hat (Abb. 3). ${ }^{87}$

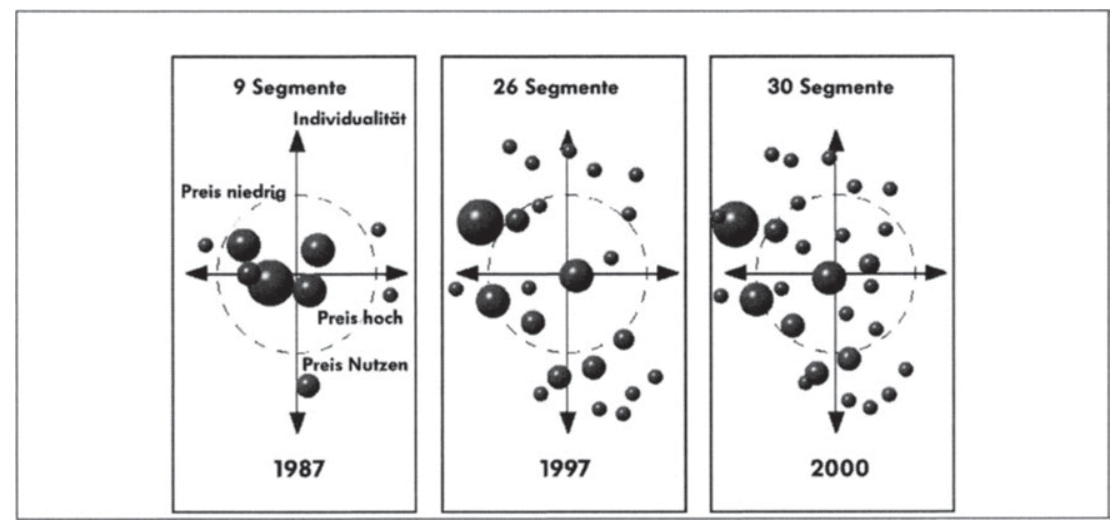

\section{Abb. 3: Fragmentierung des Automobilmarktes}

Diese Entwicklung trägt zu einer tendenziell abnehmenden Markenbindung bei, da Kunden durch anhaltend neue Angebote zum Fahrzeug- und Markenwechsel animiert werden. So ist im deutschen Pkw-Markt in den vergangenen Jahren eine

$135 * 12 * 21 * 30 * 2^{60}=\sim 1,2 * 2^{24}$. Vgl. Schaaf, A., Marktorientiertes Entwicklungsmanagement in der Automobilindustrie: Ein kundennutzenorientierter Ansatz zur Steuerung des Entwicklungsprozesses, a.a.O., S. 3.

Vgl. Heise, G., Internationale Marktsegmentierung im Automobilmarketing, a.a.O., S. 7.

Grundlage der Darstellung sind die beim Automobilkauf von über 15.000 Befragten angegebenen Alternativfahrzeuge, die statt des tatsächlich erworbenen Fahrzeugs bei der Kaufentscheidung in Betracht gezogen wurden. Auf Grundlage der modellspezifischen Substitutionsbeziehungen wurden die Zahl und die wahrgenommene Ähnlichkeit der Segmente berechnet. Vgl. Clef, U., Mehrmarkenstrategie für die Pole-Position, in asw, Sondernummer Oktober 1999, S. 72. 
sinkende Markentreue zu verzeichnen. Während im Modelljahr 1992 noch im Durchschnitt 65,9 Prozent aller Pkw-Besitzer mit deutscher Marke, die sich einen neuen Pkw erwarben, wieder die Marke ihres bisherigen Fahrzeugs kauften, sank die Anzahl markentreuer Kunden in 1998 um 4,7 Prozent auf 62,8 Prozent. Im gleichen Zeitraum verringerte sich die Markentreue bei italienischen Marken um 7,9 bei französischen um 3,5 und bei japanischen Marken gar um 9 Prozentpunkte (vgl. Tab. 1). ${ }^{88}$

\begin{tabular}{|c|c|c|c|}
\hline Marken & $1992(\%)$ & $1998(\%)$ & $\begin{array}{l}\text { Veränderung in } \\
\text { Prozent }\end{array}$ \\
\hline $\begin{array}{l}\text { Deutsche Marken } \\
\text { (WW, Audi, Ford, Opel, } \\
\text { Daimler-Benz, BMW) }\end{array}$ & 65,9 & $62,8^{*}$ & $-4,7$ \\
\hline $\begin{array}{l}\text { Itallenische Marken } \\
\text { (Fiat, Lancia, Alfa) }\end{array}$ & 53,2 & 49,0 & $-7,9$ \\
\hline $\begin{array}{l}\text { Franzosischen Marken } \\
\text { (Citroen, Peugeot, } \\
\text { Renault) }\end{array}$ & 62,9 & 60,7 & $-3,5$ \\
\hline $\begin{array}{l}\text { Japanische Marken } \\
\text { (Toyota, Nissan, Mazda, } \\
\text { Mitsubishi, Honda, } \\
\text { Suzuki, Daihatsu, Subaru) }\end{array}$ & 66,5 & 60,5 & $-9,0$ \\
\hline \multicolumn{4}{|c|}{$\begin{array}{l}\text { - Lesebeispiel: Im Modelljahr } 1998 \text { kauften im Durchschnitt } 62,8 \% \text { aller Besitzer eines Pkw } \\
\text { mit deutscher Marke, die sich einen neuen PKW kauften, wieder die Marke ihres } \\
\text { bisherigen Fahrzeugs. }\end{array}$} \\
\hline
\end{tabular}

Tab. 1: Entwicklung der Markentreue im deutschen Pkw-Markt (Quelle: Volkswagen AG)

Um dem Trend zur Individualisierung zu entsprechen bzw. die Marktchancen bei Nischenfahrzeugen zu nutzen, wird von vielen Herstellern eine Abkehr einer Fokussierung auf ausgewählte Kernbereiche hin zu einer gesamtmarktabdeckenden segmentspezifischen Differenzierung verfolgt. Hersteller, die sich bisher durch eine Fokussierung auf bestimmte Premiumsegmente auszeichneten, enweitern den Grad der bisherigen Marktabdeckung. Beispielsweise konkurrieren der BMW 3ER COMPACT, die MERCEDES A-KLASSE und der AUDI A3 mit den etablierten Modellen der Kompaktklasse wie dem WW GoLF. Umgekehrt gehen die Volumenhersteller den Weg zu neugeschaffenen Segmenten wie Großraumlimousinen oder Microgeführten Neuwagenkäufer-Studie berechnet. In dieser Gemeinschaftsstudie werden jährlich 41.000 Pkw-Käufer 4-6 Monate nach der Fahrzeugübernahme schriftlich befragt. Die Zahlen für 1997 finden sich bei Meffert, H., Burmann, Ch., Abnutzbarkeit und Nutzungsdauer von Marken, in: GfK JdAV, Heft 3, 1999, S. 258. 
Vans. Zielsetzung der meisten Hersteller ist es, sich zu Full-Line-Anbietern zu entwickeln, da ein breites Produktprogramm ein zielgruppenspezifisches Angebot erlaubt. $^{89}$

Vor dem dargestellten Hintergrund befindet sich der Automobilmarkt insgesamt in einem Konzentrationsprozess auf Herstellerseite und einem Differenzierungsprozess im Produktangebot. Die meisten Automobilhersteller stehen vor der Herausforderung, einerseits in einem zum Verdrängungswettbewerb neigenden saturierten Automobilmarkt dem wettbewerbsinduzierten Kostendruck und Preiskampf stand zu halten, andererseits der zunehmenden Individualisierung der Nachfrage und der damit einhergehenden Fragmentierung der Märkte durch Bereitstellung von immer mehr Fahrzeugvarianten bzw. Nischenfahrzeugen gerecht zu werden. Letztlich befinden sich Automobilhersteller somit in einem Spannungsfeld zwischen

- kostenorientierter Standardisierung einerseits und

- kundenorientierter Individualisierung andererseits,

so dass vor allem Volumenhersteller gehalten sind, eine Kombination der klassischen wettbewerbsstrategischen Optionen der Qualitäts- bzw. Kostenführerschaft im Sinne eines "Outpacing-Ansatzes“ anzustreben. ${ }^{90}$ Für Automobilhersteller stellt somit die Beherrschung steigender variantenabhängiger Kosten zur Schaffung eines breiten Produktprogramms ein zentrales betriebswirtschaftliches Problem dar. Zur Bewältigung variantenabhängiger Kosten greifen Automobilhersteller daher auf unterschiedliche strategische Optionen zurück: ${ }^{91}$

Nur im Luxusbereich (z. B. FERRARI oder PORSCHE) erscheinen aufgrund der vorherrschenden Snob-Effekte im Käuferverhalten Nischenstrategien weiterhin sinnvoll, da hier hohe Preise und ein begrenztes Produktangebot nicht nur aufgrund enwarteter überdurchschnittlicher Qualität, sondern als Voraussetzung für Exklusivität akzeptiert werden. Vgl. Heise, G., Internationale Marktsegmentierung im Automobilmarketing, a.a.O., S. 40.

90 Hier wird davon ausgegangen, einen hohen Produktnutzen für den Kunden anzubieten, also möglichst differenziert seinen Bedürfnissen zu entsprechen, und dabei dennoch gleichzeitig relativ geringe Kosten zu realisieren. Vgl. auch Funaki, Y., Produktentwicklung zwischen kundenorientierter Individualisierung und kostenorientierter Standardisierung, in: Markt für Mobilität, Meinig, W., Mallad, H. (Hrsg.), Darmstadt 1996, S. 254. Zum Outpacing-Ansatz vgl. Gilbert, X., Strebel, P., Strategies to Outpace the Competition, in: The Journal of Business Strategy, Heft 1, 1987, S. $28 \mathrm{ff}$.

Diese Veränderungen werden von verschiedenen Autoren so oder ähnlich immer wieder genannt. Vgl. stellvertretend Dudenhöffer, F., Produktstrategien, die Industrien umkrempeln: Befunde aus der Automobilindustrie, in: THEXIS, Heft 3, 1997, S. 24ff; Lederer, M., Clement, 
- Im Rahmen des Outsourcing erfolgt eine Verlagerung von Wertschöpfungsaktivitäten auf externe Zulieferer bzw. Kooperationspartner. Eine Reduktion der Wertschöpfungstiefe ermöglicht eine Konzentration auf Kernaktivitäten zur Realisierung von Kostenvorteilen. ${ }^{92}$ Durch ein Simultaneous Engineering wird vielfach selbst die Fahrzeugentwicklung aufgrund des Engineering Knowhows auf Systemlieferanten als „Entwicklungsabteilung für die ganze Industrie $^{\text {‘ै } 3}$ übertragen. Damit wird eine Differenzierung über Qualität und Technik erschwert, da der Systemlieferant die gleichen Teile bzw. das gleiche Knowhow an alle interessierten Hersteller liefert.

- Während im Rahmen einer Gleichteile-Strategie zumeist auf Teile-Baukästen und Module („mix-and-match"-Strategie ${ }^{94}$ ) zurückgegriffen wird, die eine technische Differenzierung zwischen Modellen und Varianten aufgrund ihres variablen Einsatzes in einem gewissen Rahmen ermöglichen, geht eine PlattformStrategie durch den Einsatz identischer Normteile mit einer Vereinheitlichung kompletter innerer Fahrzeugstrukturen einher, auf welcher sich unterschiedliche Modelle aufbauen lassen. Eine Plattform stellt die Gesamtheit derjenigen Komponenten, Schnittstellen und Funktionen dar, die über eine Produktfamilie hinweg standardisierbar und zeitlich stabil sind. ${ }^{95}$ Eine Differenzierung der Modelle und Marken erfolgt lediglich durch eine sichtbare markentypische Gestaltung der Karosserie und des interieurs. ${ }^{96}$ Die Verwendung von Plattformen ermöglicht somit in besonderem Maße eine kostengünstige Produktion von Nischenfahrzeugen und Varianten, impliziert jedoch gleichzeitig eine hohe "innere Austauschbarkeit" der Modelle. ${ }^{97}$

R., Mit innovativen Marktleistungskonzepten zu globaler Wettbewerbsfähigkeit, a.a.O., S $144 \mathrm{ff}$.

Vgl. Dyer, J. H., Specialized Supplier Networks as a Source of Competitive Advantage: Evidence from the Auto Industry, in: Strategic Management Journal, Vol. 17, 1996, S. $271 \mathrm{ff}$.

Dudenhöffer, F., Marken-Management bei Produktkonvergenz - Neue Ansätze im Automobilmarketing, in: GfK JdAV, 1997, S. 6.

Vgl. Sanchez, R., Strategic Product Creation: Managing New Interactions of Technology, Markets and Organizations, in: European Management Journal, Vol. 14, Heft 2, 1996, S. 126.

Plattform-Strategien werden heute von einer Vielzahl von Automobilherstellern verfolgt, wobei eine weitere Reduktion der Plattformen angestrebt wird: Ford 1999: 32, Ziel: 16; Volkswagen: 16/4; Renault: 5/3; Fiat: 5/3. Vgl. Diez, W., Plattform-Strategien: Irrweg oder Königsweg in der Modellpolitik, a.a.O., S. 16

96 Vgl. in diesem Zusammenhang die Analogie zum ${ }_{n}$ Haarschneideautomaten" bei Meffert, H., Mehrmarkenstrategie - immer die beste Option?, a.a.O., S. 84; Vgl. auch Kirsch, W., Roventa, W., Trux, W., Wider den Haarschneideautomaten - Ein Plädoyer für mehr Individualität bei der Strategischen Unternehmensführung, Arbeitspapier Nr. 5 der Wissenschaftlichen Gesellschaft für Marketing und Unternehmensführung e.V., Meffert, H., Wagner, H. (Hrsg.), Münster 1982.

In diesem Zusammenhang kommen Test- und Motorzeitschriften vielfach zu dem Ergebnis, dass sich technische Spezifika zwischen Modellen und Marken kaum mehr unterscheiden. So etwa die Zeitschrift Auto, Motor und Sport für den WW POLO und den SEAT CORDOBA, die beide auf der AO-Plattform des Volkswagen-Konzerns basieren. Vgl. o.V., Doppeltest WW Polo 
- Der Kauf von Motoren (Motoren-Shopping) bei anderen Herstellern spart Forschungs- und Entwicklungskosten, geht jedoch gleichzeitig mit einer Nichtdifferenzierung bei der Prestigekomponente Motor einher. Ein prominentes Beispiel des Motoren-Shopping stellt die Motorenlieferung von BMW an ROLLSROYCE dar. ${ }^{98}$ Bleibt beim Zukauf strategisch wichtiger Teile jedoch noch ein gewisser Spielraum zur Differenzierung, geht dieser Spielraum beim Fahrzeugzukauf und Badge Engineering als Vermarktung identischer Automobile unter verschiedenen Marken völlig verloren. Das Badge-Engineering kann dabei Ergebnis einer Kooperation zwischen zwei Herstellern sein (z. B. WW SHARAN und FORD GALAXY) oder sich aber innerhalb der Marken eines Herstellers abspielen (z. B. Peugeot 106 und Citroen SAXo). ${ }^{99}$

Alle diese primär kostengetriebenen Entwicklungen führen zu einer zunehmenden Austauschbarkeit der Automobile verschiedener Marken, so dass die traditionelle wettbewerbsstrategische Differenzierung durch spezifische technische Eigenschaften eines Automobils erschwert wird. In diesem Umfeld nimmt die Markenpolitik als Differenzierungs- und Profilierungsinstrument eine exponierte Stellung in der Automobilindustrie ein. Um in allen Fahrzeugsegmenten bei konsequenter Nutzbarmachung von Economies of Scale präsent zu sein, verfolgen heute nahezu sämtliche Automobilhersteller eine Mehrmarkenstrategie in Kombination mit den genannten Produktionsstrategien. In dem Bewusstsein, dass nur eine Marke aufgrund der Gefahr einer Deprofilierung nicht unbegrenzt ausgedehnt werden kann, verfügen die meisten Automobilhersteller über ein umfassendes Markenportfolio und agieren mit unterschiedlichen Hersteller- bzw. Dachmarken im Wettbewerb.

Demzufolge sind in der Automobilindustrie Mehr-Dachmarken-Strategien vorherrschend, wie die Markenhierarchie des Volkswagen-Konzerns exemplarisch verdeutlicht (vgl. Abb. 4). Dabei stehen letztlich die Dachmarken im Fokus der Strategieüberlegungen, da diese die markenprägenden Leistungen beinhalten,

Classic gegen Seat Cordoba, in: Auto, Motor und Sport, Heft 6, 1996, S. 42ff. Vgl. zur kritischen Presse auch Scholtys, F., Riskante Tour, in: managermagazin, Heft 10, 1999, S. $130 \mathrm{ff}$.

Weitere Beispiele des Motoren-Shopping sind: CITROEN-PEUGEOT, AUDI-WW-SEAT-SKODA, OPEL-SAAB, MAZDA-FORD, MERCEDES-WW. VOLVO-WW, ROVER CHRYLSLER. Vgl. hierzu Dudenhöffer, F., Marken-Management bei Produktkonvergenz - Neue Ansätze im Automobilmarketing, a.a.O., S. 10. Vgl. auch Lederer, M., Clement, R., Mit innovativen Marktleistungskonzepten zu globaler Wettbewerbsfähigkeit, a.a.O., S. 152.

99 Zum Badge-Engineering Projekt Ford Galaxy und WW Sharan vgl. die Fallstudie Meffert, H., Koers, M., Positionierung im Wettbewerb - dargestellt am Beispiel der Automobilbranche, Fallstudie des Instituts für Marketing, Münster 2000, S. $18 \mathrm{ff}$. 
welche den Ausgangspunkt der Markenwahrnehmung durch die Nachfrager bilden. ${ }^{100}$

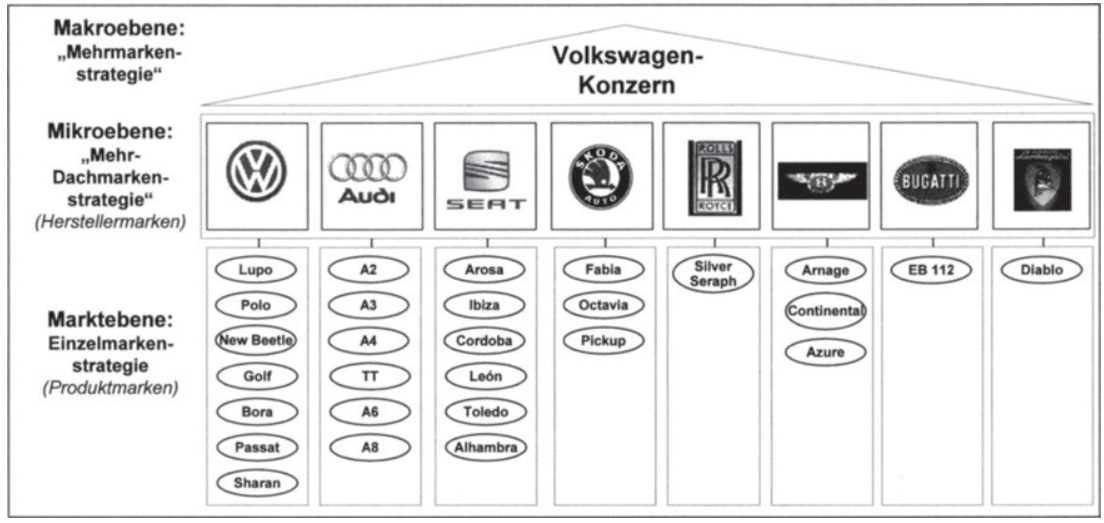

Abb. 4: Markenhierarchie am Beispiel des Volkswagen-Konzerns

Erweisen sich Marken als zu eng, um eine angestrebte akquisitorische Wirkung in einem neuen Marktsegment auszuüben oder besteht die Gefahr einer Markenüberdehnung, werden Marken entweder neu geschaffen ( $\mathbf{z}$. B. SMART) oder im Rahmen von Mergers and Aquisitions hinzugekauft. ${ }^{101}$ Tab. 2 zeigt in diesem Zusammenhang einen Überblick über die Unternehmenszusammenschlüsse der letzten Jahre. ${ }^{102}$

Vgl. zu Dachmarken der weiteren Hersteller die Abbildung auf Seite 6. Vgl. hier auch Meffert, H., Perrey, J., Mehrmarkenstrategie - Ein Beitrag zum Management von Markenportfolios, a.a.O., S. 627

101 Neben einer solch bewussten Erweiterung leiten sich Markenportfolios häufig auch aus der geschichtlichen Entwicklung eines Unternehmens ab. Vgl. auch Kapferer, J.-N., Die Marke Kapital des Unternehmens, a.a.O., S. 203.

102 Vgl. auch Hünerberg, R., Heise, G., Hoffmeister, M., Internationales Marketing für die Automobilwirtschaft - Eine Einführung, in: Internationales Automobilmarketing, Hünerberg, R., Heise, G., Hoffmeister, M., (Hrsg.), Wiesbaden 1995, S. 20. 


\begin{tabular}{|c|c|c|c|}
\hline Jahr & Käufer & Marke & $\begin{array}{c}\text { Kaufsumme } \\
\text { (in DM) }\end{array}$ \\
\hline 1986 & $\begin{array}{l}\text { GM } \\
\text { Fiat } \\
\text { WW }\end{array}$ & $\begin{array}{l}\text { Lotus } \\
\text { Alfa Romeo } \\
\text { Seat }\end{array}$ & $\begin{array}{l}75 \text { Mio. } \\
\text { 1,5 Mrd. } \\
\text { 1,3 Mrd. }\end{array}$ \\
\hline 1987 & $\begin{array}{l}\text { Chrysier } \\
\text { Ford }\end{array}$ & $\begin{array}{l}\text { Lamborghini } \\
\text { Aston Martin }\end{array}$ & $\begin{array}{l}54 \text { Mio. } \\
6 \text { Mio. }\end{array}$ \\
\hline 1989 & $\begin{array}{l}\text { Ford } \\
\text { GM }\end{array}$ & $\begin{array}{l}\text { Jaguar } \\
\text { Saab }\end{array}$ & $\begin{array}{l}\text { 4,7 Mrd. } \\
\text { 1,1 Mrd. }\end{array}$ \\
\hline 1990 & WW & Skoda & 1,4 Mrd. \\
\hline 1993 & $\begin{array}{l}\text { Megatech } \\
\text { Fiat }\end{array}$ & $\begin{array}{l}\text { Lamborghini } \\
\text { Maserati }\end{array}$ & $\begin{array}{l}35 \text { Mio. } \\
4 \text { Mio. }\end{array}$ \\
\hline 1994 & BMW & Rover & $2,1 \mathrm{Mrd}$. \\
\hline 1998 & $\begin{array}{l}\text { Daimler-Benz' } \\
\text { Toyota } \\
\text { Toyota } \\
\text { WW } \\
\text { Audi } \\
\text { WW }\end{array}$ & $\begin{array}{l}\text { Chrysler } \\
\text { Daihatsu } \\
\text { Hino } \\
\text { Rolls-Royce/Bentley } \\
\text { Lamborghini } \\
\text { Bugatti } \\
\end{array}$ & $\begin{array}{l}8,9 \text { Mrd. } \\
\text { n.b. } \\
\text { n.b. } \\
\text { 1,3 Mrd. } \\
600 \text { Mio. } \\
130 \text { Mio. }\end{array}$ \\
\hline 1999 & Ford & Volvo & 11,0 Mrd. \\
\hline 2000 & GM & Fiat & $5,1 \mathrm{Mrd}$ \\
\hline 2000 & DaimlerChrysler & Mitsubishi $^{3}$ & 2,54 Mrd. \\
\hline
\end{tabular}

Tab. 2: Mergers \& Aquisitions in der Automobilindustrie

(Quelle: In Weiterführung von Hünerberg, R., Heise, G., Hoffmeister, M., Internationales Marketing für die Automobilwirtschaft - Eine Einführung, in: Internationales Automobilmarketing, a.a.O. S. 20.)

Die Motivation zur Übernahme von Marken ist dabei weniger im Erwerb des Anlage- und Umlaufvermögens unter Einschluss des Mitarbeiterstamms zu sehen, sondern lässt sich vornehmlich mit der Übernahme der Rechte und damit des akquisitorischen Potenzials eingeführter Marken begründen. KAPFERER führt diesbezüglich an: „Wenn der Käufer ein Unternehmen sehr teuer bezahlt, erwirbt er damit einen Platz im Kopf des potenziellen Kunden. [...] Bekanntheit, Image, Vertrauen und Reputation, mühsam erreicht im Laufe der Zeit, sind die besten Garanten für zukünftige Erträge und rechtfertigen den bezahlten Preis. “103 Vor diesem Hintergrund sind die Ziele der Mehrmarkenstrategie zu erläutern.

103 Kapferer, J.-N., Die Marke - Kapital des Unternehmens, a.a.O., S. 9. Der Tobin's q Koeffizient, d.h. das Verhältnis von Marktwert zu Buchwert der Aktien eines Unternehmens, wird von BURMANN für die an der Frankfurter Börse gelisteten Automobilhersteller auf 4,46 beziffert (ungewichteter arithmetischer Mittelwert auf Basis der Kurse vom 26.10.1999). Vgl. Burmann, Ch., Strategiewechsel in turbulenten Märkten - Neuere theoretische Ansätze zur Unternehmensflexibilität, a.a.O., S. 7. 


\section{Ziele von Mehrmarkenstrategien}

Die grundsätzlichen Ziele von Mehrmarkenstrategien lassen sich aus den allgemeinen Unternehmens- und Marketingzielen ableiten und stehen in einer engen Beziehung zueinander. Eine solche Unterordnung ist insbesondere durch zu vermutende Mittel-Zweck-Zusammenhänge zu begründen. ${ }^{104}$ Zur Sicherstellung des Oberziels der Ertrags- und Renditesteigerung stellt der Markenwert die relevante ökonomische Zielgröße dar. ${ }^{105}$ Der Markenwert kennzeichnet den mit dem Markennamen bzw. Markensymbol verbundenen inkrementalen Wert einer Marke. Basierend auf der Nutzenwahrnehmung der Nachfrager bestimmt sich der Markenwert als Differenz des wahrgenommenen Nutzens eines Markenartikels gegenüber dem Nutzen einer technisch-physikalisch gleichen, aber unmarkierten Leistung. ${ }^{106}$

\begin{tabular}{|c|c|}
\hline MarktgerichteteZiele & InnengerichteteZiele \\
\hline $\begin{array}{l}\text { - breite Marktabdeckung durch } \\
\text { differenzierte Positionierung } \\
\text { - gezielte und bedarfsgerechte } \\
\text { Konsumentenansprache } \\
\text { - Absicherung der Wettbewerbsposition } \\
\text { durch Markteintrittsbarrieren } \\
\text { - Reduktion des Marktrisikos und höhere } \\
\text { Aktionsflexibilität } \\
\text { - Markterschließung mit jeweils } \\
\text { bestgeeigneten Marken } \\
\text { - Ausweitung der Kundengewinnung und } \\
\text { Kundenbindung }\end{array}$ & $\begin{array}{l}\text { - Nutzung von Synergiepotenzialen } \\
\text { entlang der Wertschöpfungskette } \\
\text { (Plattform, Gleichteile etc.) } \\
\text { - Förderung des internen Wettbewerbs }\end{array}$ \\
\hline
\end{tabular}

Tab. 3: Ziele der Mehrmarkenstrategie

In Mehrmarkenstrategien gilt es, den Markenwert des Gesamtportfolios verstanden als Summe der Werte der einzelnen Marken zu maximieren. Diesem Zweck

Vgl. Haedrich, G., Tomczak, T., Strategische Markenführung: Planung und Realisierung von Marketingstrategien für eingeführte Produkte, Bern, Stuttgart 1990, S. 76.

OTTE geht davon aus, dass nur diejenigen Unternehmen alle externen und internen Interessen befriedigen können, die einen über den marktüblichen Kundennutzen liegenden Mehrwert für ihre Kunden schaffen. Entsprechend sei der Markenwert Ursache von und folglich Voraussetzung für Shareholder Value als ökonomisches Oberziel. Vgl. Otte, Th., Sind die Marken milleniumfähig?, in asw, Sondernummer Oktober 1999, S. $191 \mathrm{f}$.

Vgl. Sander, M., Die Bestimmung und Steuerung des Wertes von Marken. Eine Analyse aus Sicht des Markeninhabers, Heidelberg 1994, S. 43ff. 
dienen markt- und innengerichtete Zielsetzungen der Mehrmarkenstrategie als Subziele (vgl. Tab. 3). ${ }^{107}$

Marktgerichtet stellen die Erhöhung des Absatzvolumens sowie die Ausweitung der Marktanteile des gesamten Markenportfolios zentrale Zielsetzungen von Mehrmarkenstrategien dar. ${ }^{108}$ Obwohl jede zusätzliche Portfoliomarke Umsatzeinbußen bei bestehenden Marken verursachen kann (sog. Substitutionseffekt), soll der Gesamtumsatz durch Eroberung von speziell angesprochenen Abnehmern (sog. Partizipationseffekt) sowie durch Halten von Markenwechslern innerhalb des eigenen Portfolios gesteigert werden. ${ }^{109}$ Durch eine differenzierte Positionierung der Marken besteht die Chance, eine breite Marktabdeckung zu erzielen und auftretende Nischenbedürfnisse mit entsprechenden Leistungen zu begegnen. Sind die verschiedenen Marken des Portfolios durch eigenständige und nachfragerseitig wahrgenommene Identitäten geprägt, läßt sich zudem eine gezielte und bedarfsgerechte Konsumentenansprache vornehmen, die zu einer zusätzlichen Verbesserung der Marktdurchdringung beiträgt.

Durch die hohe Attraktivität segmentspezifischer Markenangebote erlaubt die Mehrmarkenstrategie ein hohes Maß an Bedürfnisbefriedigung im funktionalen bzw. emotionalen Sinne und somit eine intensivere Ausschöpfung des Marktpotenzials, als dies mit einer an den Durchschittsbedürfnissen aller Nachfrager orientierten Einheitsleistung möglich wäre. ${ }^{110}$ Aufgrund des auch als "Verlust-in-derMitte-Phänomen" benannten Polarisierungseffekts reicht es in der Regel nicht aus,

107 Zur folgenden Systematisierung und Darstellung der Ziele von Mehrmarkenstrategien vgl. Meffert, H., Perrey, J., Mehrmarkenstrategie - Ein Beitrag zum Management von Markenportfolios, a.a.O., S. $627 \mathrm{ff}$.

Voraussetzung für die Vorteilhaftigkeit der Mehrmarkenstrategie (MMS) gegenüber alternativen Markenstrategien ist, dass ceteris paribus der durch die parallele Marktbearbeitung generierte Gewinn größer ist als der alternativer marktstrategischer Optionen. So muss etwa im Vergleich zur Einzelmarkenstrategie (EMS) gelten $U(M M S)-K(M M S)>U(E M S)-K(E M S)$, wobei $\mathrm{U}$ den Umsatz und $\mathrm{K}$ die Kosten kennzeichnet.

Vgl. auch Meffert, H., Entscheidungsorientierter Ansatz der Markenpolitik, in: Handwörterbuch Markenartikel, Bruhn, M. (Hrsg.), Band 1, Stuttgart 1994, S. 184. Wie erfolgreich sich die Mehrmarkenstrategie der Volkswagen AG entwickelt hat, lässt sich prägnant an einigen Unternehmenszahlen des Konzerns verdeutlichen. In Westeuropa konnte der Konzern mit seinen vier Kernmarken WW, AUDI, SEAT und SKODA seinen Marktanteil binnen 5 Jahre von $16 \%$ in 1994 auf mittlerweile knapp 19\% erhöhen. Die Umsatzerlöse des Konzerns stiegen dabei seit 1993 von 76.856 Mio. DM auf 134.243 Mio. DM in 1998. Vgl. hierzu den Geschäftsbericht der Volkswagen AG 1998.

110 Vgl. Kotler, Ph., Marketing Management, 6. Aufl., Englewood Cliffs 1988, S. 469. 
den Gesamtmarkt mit nur einer Marke abzudecken. Ein solches Vorgehen mündet vielfach in der in Abb. 5 dargestellten "Zwischen-den-Stühlen-Position" ${ }^{111}$, d.h. eine Lage, in der die Marke in der Wahrnehmung der Abnehmer weder einen entsprechenden Preis-/Leistungsvorteil noch einen imagemäßigen Nutzenvorteil aufweisen kann.

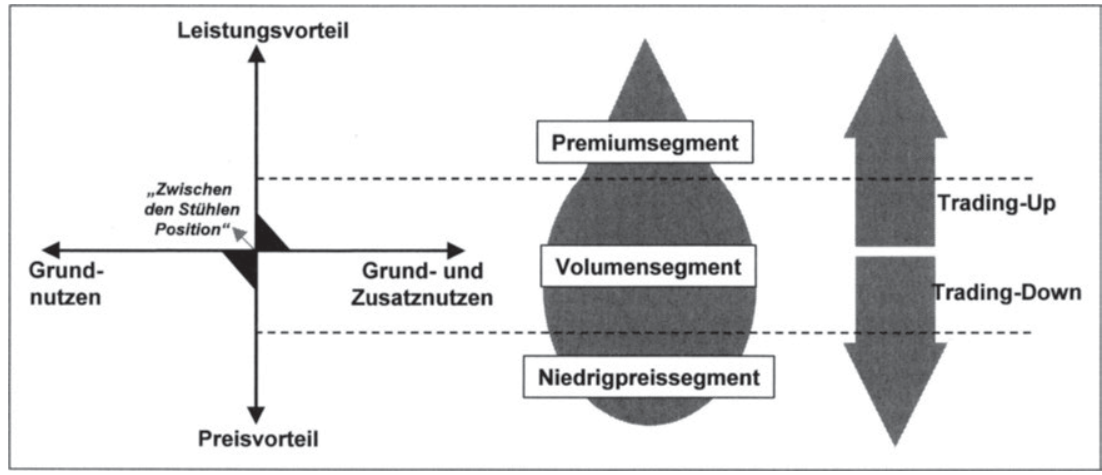

Abb. 5: Grundoptionen der Markenpositionierung und Marktstruktur ${ }^{112}$

Automobilhersteller, die sich mit nur einer Marke im Volumensegment bzw. der mittleren Preisschicht angesiedelt haben, stehen letztlich vor dem Problem, die Marke in die Segmente nach oben (Trading-up) ins Premiumsegment oder nach unten (Trading-down) ins Niedrigpreissegment ausdehnen zu müssen, um evtl. Nachfrageverluste in der Mitte ausgleichen zu können. Eine gleichzeitige Markenausdehnung nach oben und unten übersteigt jedoch in der Regel den Kompetenzrahmen einer Marke. Aufgrund der damit einhergehenden Gefahr einer Markendeprofilierung kann der Markt mit nur einer Marke zumeist nur in eine Richtung abgedeckt werden. ${ }^{113}$ Ein ausschließliches Trading-up oder Trading-down impli-

111 Vgl. Porter, M. E., Wettbewerbsvorteile: Spitzenleistungen erreichen und behaupten, 5. Aufl., Frankfurt a. M., New York 1999, S. 44. Ein Unternehmen zwischen den Stühlen verfügt über keinen Wettbewerbsvorteil. Ein solches Unternehmen wird nach PORTER nur dann Gewinne erwirtschaften, wenn die Branchenstruktur günstig ist oder wenn es Konkurrenten hat, die auch zwischen den Stühlen sitzen.

112 Eine solche Marktstruktur erscheint im Automobilmarkt plausibel. So teilte sich das Gesamtvolumen 1998 in Deutschland wie folgt auf die einzelnen Klassen auf: Mini-Klasse: 6,4 Prozent, Kleinwagen: 26,2, untere Mittelklasse: 32,2 , Mittelklasse 22,2, Ober-/Luxusklasse 5,9 sowie sonstige 7,1 Prozent.

113 Vor solchen Problemen steht etwa die Marke VOLKSWAGEN, die bisher die unteren und mittleren Marktsegmente des Automobilmarktes bediente, nun aber auch den Luxusmarkt mit ei- 
ziert jedoch ein Verzicht auf Ausschöpfung von Nachfragepotenzial im jeweiligen anderen Segment. Durch eine speziell auf das Premium- bzw. Niedrigpreissegment zugeschnittene Marke lässt sich ein solcher Verlust vermeiden. ${ }^{114}$

Die breite Marktabdeckung im Rahmen einer Mehrmarkenstrategie verhilft überdies zur Marktabsicherung durch Schaffung von Markteintrittsbarrieren sowohl gegenüber potenziellen Wettbewerbern als auch gegenüber neuen Leistungen aktueller Konkurrenten. ${ }^{115}$ Die gleichzeitige Führung einer deutlich höherwertigen Referenzmarke und einer niedrigpreisigen Kampfmarke bietet den Automobilherstellern die Möglichkeit, Wettbewerber verstärkt in die beschriebene „Zwischenden-Stühlen-Position" zu drängen. Zudem können die übrigen Marken des Portfolios durch Einführung einer Kampfmarke vor einem Preiswettbewerb geschützt werden. ${ }^{116}$ Eine solche „Schutzschild-Funktion" kommt beispielsweise der Marke SKODA für VOLKSWAGEN und AUDI zu.

In Analogie zur Risikostreuung in einem Finanzportfolio zielt die Mehrmarkenstrategie überdies darauf $a b$, durch das Agieren mit mehreren Marken einen Risikoausgleich im Markenportfolio zu erreichen. Sind etwa die Erfolgschancen eines

nem Fahrzeug unter der Marke VOLKSWAGEN (sog. D1-Konzept) glaubhaft abdecken will. In diesem Zusammenhang ist entscheidend, ob eine Marke eher von "unten kommend" obere Segmente abdecken will oder von "oben kommend" eine Abdeckung auch unterer Segmente anstrebt. Während es sich für eine von unten kommende Marke als schwierig erweist, in oberen Segmenten Marktakzeptanz zu finden, ist bei einer von oben kommenden Marke eine vergleichsweise höhere Marktakzeptanz in unteren Segmenten bei gleichzeitiger Imageverwässerung zu erwarten ist. Vor diesem Hintergrund ist die Entscheidung von DAIMLERCHRYSLER zu verstehen, das Kleinwagen-Segment nicht mit der Marke MERCEDES-BENZ, sondern der Marke SMART zu bearbeiten.

114 Ist ein Unternehmen allerdings in der Lage, seine Leistungen in der Wahrnehmung der Konsumenten nachvollziehbar zu differenzieren, kann ein und dieselbe Marke eine relativ breite Spanne von Preis-Leistungsverhältnissen glaubhaft abdecken. Häufig wird in diesem Zusammenhang die Marke BMW genannt, die mit der 3er-Reihe (zukünftig auch 2er-Reihe) bis zur 7er-Reihe von der unteren Mittelklasse bis zur Oberklasse den Pkw-Markt abdeckt. Allerdings muss auch hier die Differenzierungsfähigkeit der Marke BMW angezweifelt werdenn, wie die interne Substitution zwischen der 3er- und 5er-Reihe zeigen: nahezu im gleichen Umfang wie der 3er BMW an Kunden gewinnt (plus 30,1 Prozent im ersten Halbjahr 1999 gegenüber dem entsprechenden Vorjahreszeitraum), verliert der 5er BMW im deutschen Markt (minus 20,6 Prozent).Vgl. zu den Zahlen Diez, W., Plattform-Strategien: Irrweg oder Königsweg in der Modellpolitik, a.a.O., S. 37.

115 Im Rahmen der Marktabsicherung bietet die Mehrmarkenstrategie - zwar weniger für den Neuwagenmarkt aufgrund des markenexklusiven Vertriebs als vielmehr für den Gebrauchtwagenmarkt - überdies die Möglichkeit, ein größeren Anteil der verfügbaren räumlichen Kapazität im Distributionssystem (Werkstätten, Tankstellen etc.) zu belegen.

116 Vgl. Kapferer, J.-N., Die Marke - Kapital des Unternehmens, a.a.O., S. 214. 
neuen Modells aufgrund seines innovativen Charakters nur schwer abschätzbar, so beschränken sich mögliche Floprisiken und damit einhergehende Deprofilierungspotenziale in der Regel nur auf eine Marke des Portfolios. Da neue Märkte mit den jeweils dafür geeignetsten Marken erschlossen werden können, kann darüber hinaus das Risiko der Markterschließung reduziert werden. ${ }^{117}$ Die Mehrmarkenstrategie impliziert somit neben einer Diversifikation im Markenportfolio eine Erhöhung der Aktionsflexibilität von Automobilherstellern im Wettbewerb. ${ }^{118}$

Nachfragerseitig besteht eine zentrale Zielsetzung von Mehrmarkenstrategien darin, Markenwechsler durch "Konkurrenz im eigenen Haus" aufzufangen und folglich das Ertragspotenzial innerhalb des eigenen Unternehmens zu halten. Anstatt eine Marke der Konkurrenz zu kaufen, sollen Nachfrager zu Marken des eigenen Portfolios wechseln. Vor diesem Hintergrund wird im Rahmen von Mehrmarkenstrategien der Aufbau von Loyalität zur einzelnen Marke durch die Erhöhung der Portfolioloyalität erweitert. Die Tiefe und Breite der Marktabdeckung durch das Markenportfolio eines Automobilherstellers und die damit einhergehende hohe Attraktivität segmentspezifischer Markenangebote bietet zudem vielseitige Ansatzpunkte in der Kundengewinnung und vielfach gar die Möglichkeit einer lebenslangen Kundenbindung. ${ }^{119}$ So kann der Automobilkäufer entsprechend seines Alters und seiner Einkommensverhältnisse vom Kleinwagen zur Mittelklasse und schließlich zu einem Fahrzeug des Premiumsegments wechseln, wobei er zwar innerhalb des Markenportfolios wandert, letztlich aber der Gesamtunternehmung treu bleibt.

Vor dem Hintergrund eines wachsenden Kostendrucks treten die mit der Ausübung von Mehrmarkenstrategien verfolgten marktgerichteten Ziele häufig hinter

117 Diese Überlegungen bildeten eine wichtige Grundlage bei der Akquisition der Marken SEAT und SKODA durch den Volkswagen-Konzern. Konnten mit SEAT die durch mediterrane Bedürfnisse geprägten südeuropäischen Automobilmärkte erschlossen werden, wird mit SKODA insbesondere der wachsende osteuropäische Markt abgedeckt. Vgl. Burmann, G., Marktarealstrategien der internationalen Automobilhersteller, in: Internationales Automobilmarketing, Hünerberg, R., Heise, G., Hoffmeister, M., (Hrsg.), Wiesbaden 1995, S. $121 \mathrm{ff}$.

Zur Aktionsflexibilität vgl. auch Meffert, H., Größere Flexibilität als Unternehmungskonzept, in: ZfbF, 37. Jg., Heft 2, 1985, S. 126f. Meffert unterscheidet darüber hinaus zwischen einer Prozessflexibilität und einer Strukturflexibilität zur Erhöhung der Handlungsflexibilität.

119 Vgl. zum „Customer-Life-Cycle“-Konzept Meffert, H., Marketing: Grundlagen marktorientierter Unternehmensführung: Konzepte - Instrumente - Praxisbeispiele, a.a.O., S. 827. 
die innengerichtete Zielsetzung der Nutzung von Synergiepotenzialen zurück. Die in der Praxis zu beobachtende Akquisitions- und Fusionswelle steht in diesem Zusammenhang zuweilen weniger im Zeichen markenstrategischer Überlegungen, als vielmehr im Fokus von Größenbestrebungen zur Sicherung einer Führungsposition im globalen Wettbewerb. Größenvorteile münden dabei vielfach in Effizienzvorteile bedingt durch Economies-of-Scale- und Fixkostendegressions-Effekte. ${ }^{120}$ Das Markenportfolio eines Automobilherstellers bietet hier entlang der gesamten Wertkette $^{121}$ zahlreiche Ansatzpunkte zur Ausschöpfung von Synergien (Gleichteilen, Plattformen etc.). ${ }^{122}$ So ermöglicht die Mehrmarkenstrategie in der Fertigung eine intensive Nutzung von Synergien durch die markenübergreifende Verwendung von Gleichteilen, Plattformen etc. Auch sind Größenvorteile in der Forschung \& Entwicklung durch gemeinsame Grundlagenforschung und Konzeptentwicklungen erzielbar, wodurch sich Entwicklungskosten reduzieren lassen.

Da die Mehrmarkenstrategie im Vergleich zu anderen Markenstrategien wie der Einzel- oder Dachmarkenstrategie grundsätzlich das Problem einer suboptimalen Ressourcenverwendung in sich birgt ${ }^{123}$, kann die Identifikation und Nutzung möglicher Synergien im Markenportfolio auch als unabdingbare Voraussetzung einer effizienten Mehrmarkenstrategie angesehen werden. ${ }^{124}$ In diesem Zusammenhang

Während Economies-of-Scale die sinkenden Kosten durch zum Beispiel effizientere Maschinen in der Produktion oder Beschaffungskostenvorteile wiedergeben, beziehen sich die Fixkostendegressionen auf sinkende durchschnittliche Kosten. Vgl. zu dieser Abgrenzung Meffert, H., Marketing: Grundlagen marktorientierter Unternehmensführung: Konzepte - Instrumente Praxisbeispiele, a.a.O., S. 270.

Die Wertkette im Sinne PORTERS stellt ein Instrument zur systematischen Suche nach Kostensenkungs- und Differenzierungspotenzialen dar, welche die Quelle von Wettbewerbsvorteilen bilden. Vgl. Porter, M. E., Wettbewerbsvorteile: Spitzenleistungen erreichen und behaupten, a.a.O., S. $67 \mathrm{ff}$.

So stieg etwa das Jahresergebnis des Volkswagen-Konzerns seit konsequenter Nutzbarmachung bestehender Synergiepotenziale im Rahmen der verfolgten Mehrmarkenstrategie von 1.940 Mio. DM in 1993 auf 2.243 Mio. DM in 1998 und die Umsatzrentabilität nach Steuern im gleichen Zeitraum von -2,53 auf 1,6 Prozent. Zahlen auf Grundlage des Geschäftsberichts der Volkswagen AG 1998 sowie eigener Berechnungen.

Vgl. Quelch, J. A., Kenny, D., Extend Profits, Not Product Lines, a.a.O., S. 153ff. Neben der Anzahl der Marken bestimmt auch die Anzahl der beteiligten Hierarchieebenen die beschriebenen Kosten. So sind bei Großunternehmen in der Regel bis zu 7 Hierarchieebenen an den Marketingentscheidungen beteiligt. Vgl. Weber, D., Wenn Line Extensions gefährlich werden, in: W\&V, Heft 39, 1997, S. 112.

Entsprechend ist die Aussage von JÜRGEN SCHREMPP, Vorstandsvorsitzender der DAIMLERCHRYSLER AG zu verstehen: „Wir müssen die Quadratur des Kreises erreichen - Synergien herausholen, ohne Markenidentitäten zu verwässern." Linden, F.A., Schlacht um die Spitze, in: manager magazin, Heft 8, 1998, S. 57. 
sind die innengerichteten Zielsetzungen keine originären Zielsetzungen der Mehrmarkenstrategie, sondern als Bedingungen einer effizienten, d.h. „rechenbaren" Mehrmarkenstrategie zu interpretieren. So führt MICHAEL in der Laudatio zum Marketing-Preis-Träger Volkswagen AG 1999 treffend an: „Mit maßgeschneiderten Markenprofilen beantwortet der VW-Konzern den Trend zur Individualisierung der Käufer. [....] Verbunden mit dem nachgewiesenen wirtschaftlichen Erfolg ist dies ein erfolgreicher unternehmerischer Kraftakt, der höchsten Respekt verdient. "125

Vor allem im Marketing bzw. den betrieblichen Funktionen mit unmittelbarem Kundenkontakt erfolgt vielfach ein Duplizieren von Anstrengungen, da sich hier aufgrund der markenindividuellen Profilierungsnotwendigkeit nur wenig Ansatzpunkte zur Bündelung von Ressourcen identifizieren lassen. Da die einzelnen Marken den erforderlichen Profilierungsaufwand in der Regel alleine tragen müssen, sind vergleichsweise hohe Kosten durch die parallele Marktbearbeitung die Folge. Entsprechend kann sich die Rentabilität bei der Ausübung einer Mehrmarkenstrategie mit wachsender Anzahl an Marken trotz eines Umsatzanstiegs durchaus verschlechtern. ${ }^{126}$ Durch die koordinierte Ausnutzung produktions- und forschungsseitiger Synergie- und Verbundvorteile soll das Portfolioergebnis der Mehrmarkenstrategie letztlich höher sein als die Summe der markenspezifischen Einzelergebnisse. ${ }^{127}$ Im Fall von zwei Marken soll bei koordinierter Vorgehensweise für den Gewinn (G) gelten: ${ }^{128}$

$$
G(\text { Marke } A+\text { MarkeB })>G(\text { MarkeA })+G(\text { MarkeB }),
$$

d.h. der von zwei Marken im Rahmen eines Markenportfolios generierte Gewinn soll ceteris paribus größer als der im Zuge eines Alleingangs beider Marken sein.

Der aus der gleichzeitigen Führung mehrerer Marken resultierende Wettbewerb unter den Marken kann schließlich dazu beitragen, die Leistungsmotivation und

Michael, B. M., Laudatio Deutscher Marketing-Preis 1999 an die Volkswagen AG, a.a.O., S. $47 f$.

126 So weisst KAPFERER darauf hin, dass die Kosten für den Erhalt einer Marke häufig so hoch sind, dass es nicht möglich ist, zu viele Marken im Portfolio zu halten; vgl. Kapferer, J.-N., Die Marke - Kapital des Unternehmens, a.a.O., S. 203. Vgl. auch Doyle,P., Building Successful Brands. The Strategic Options, in: Journal of Marketing Management, No. 1, 1989, S. 79

127 In diesem Zusammenhang wird häufig vom 2+2 = 5-Effekt gesprochen. Vgl. Bühner, R., Betriebswirtschaftliche Organisationslehre, a.a.O., S. 12; Ansoff, H. I., Management-Strategie, München 1966, S. 97.

128 Vgl. Barney, J. B., Gaining and Sustaing Competitive Advantage, Mass. u.a. 1997, S. 286. 
-effizienz der Mitarbeiter der Markeneinheiten zu steigern. ${ }^{129}$ Zum einen lassen sich durch den „Wettbewerb im eigenen Haus" unternehmensinterne Benchmarks setzen, welche als Richtgrößen für die einzelnen Markeneinheiten fungieren. Zum anderen fördert die operative Eigenverantwortlichkeit der Marken marktorientiertes unternehmerisches Denken im Rahmen des sogenannten Intrapreneuring. ${ }^{130}$

Um die mit der parallelen Führung mehrerer Marken verfolgten externen und internen Zielsetzungen zu erreichen, haben die Automobilhersteller Entscheidungen im Hinblick auf die Gestaltung des Markenportfolios zu treffen, die sich insbesondere in der Ausgestaltung und Führung der Mehrmarkenstrategie niederschlagen.

\section{Konzeptionelle Grundlagen zur Führung von Markenportfolios}

Primäres Anliegen bei der Ausgestaltung von Markenportfolios ist es, mit Hilfe geeigneter Marken ein Portfolio zusammenzustellen, das zum Aufbau bzw. zur Bewahrung von Wettbewerbsvorteilen beiträgt. Als Wettbewerbsvorteil einer Marke ist eine dauerhafte Leistung bei einem für den Kunden wichtigen Merkmal zu verstehen, dem ein höherer Wert beigemessen wird als dem eines Konkurrenzunternehmens. ${ }^{131}$ Angesichts der bislang unzureichenden theoretischen Grundlage ist zunächst zu überprüfen, welche Forschungsansätze in diesem Zusammenhang einen unmittelbaren Beitrag zur Markenprofilierung und damit zur Schaffung eines Wettbewerbsvorteils zu leisten vermögen. MEFFERT folgend zeichnet „[...] langfristig erfolgreiche Unternehmen [...] neben Marktorientierung auch Ressourcenausrichtung aus. In Zukunft werden Unternehmen somit die Inside-Out- und die Outside-In-Perspektive stärker dialogisch entwickeln müssen". ${ }^{132}$ Vor diesem Hintergrund werden im Folgenden beide Forschungsansätze kurz dargestellt.

Vgl. Kotler, Ph., Bliemel, F., Marketing-Management: Analyse, Planung, Umsetzung und Steuerung, a.a.O., S. 710.

130 Zum Intrapreneuring vgl. Pinchot III, G., Intrapreneuring, New York 1985 zitiert in Bühner, R., Betriebswirtschaftliche Organisationslehre, a.a.O., S. 390.

131 Vgl. Ghemawat, P., Dauerhafte Wettbewerbsvorteile aufbauen, in: Simon, H., Wettbewerbsvorteile und Wettbewerbsfähigkeit, Stuttgart 1988, S. 18ff. Für einen Wettbewerbsvorteil müssen die Kriterien Wichtigkeit, Wahrnehmbarkeit und Dauerhaftigkeit erfüllt sein. BACKHAUS spricht vom komparativen Konkurrenzvorteil, vgl. Backhaus, K. Investitionsgütermarketing, 6. Aufl., München 1999, S. $26 \mathrm{f}$.

132 Meffert, H., Dogmen, in: Horizont Magazin, Heft 1, 1999, S. 107; Vgl. Meffert, H., Herausforderungen an die Betriebswirtschaftslehre - Die Perspektive der Wissenschaft, in: DBW, 58. Jg., Heft 6, 1998, S. 709ff. 


\subsection{Paradigmen der Unternehmensführung als Basis der Markenführung}

\subsection{Der market-based view}

Aufbauend auf der marktstrategischen Perspektive der Industrial Organization (IO)-Theorie nach BAIN ${ }^{133}$ sowie dem daraus abgeleiteten Structure-ConductPerformance (S-C-P)-Paradigma bezeichnet der market-based view (MBV), dass ein dauerhafter Unternehmenserfolg (performance) zum einen durch die Struktur (structure) des Produkt-Markt-Feldes, in dem ein Unternehmen tätig ist, und zum anderen durch das strategische Verhalten des Unternehmens in seinen Produktmärkten (conduct) determiniert wird. ${ }^{134}$ Insbesondere PORTER zeigt, wie sich die Überlegungen der 10 -Forschung für das strategische Management nutzen lassen und wie sie den unternehmensindividuellen Unternehmenserfolg erklären.

Nach PORTER ist ein Unternehmen in der Lage, durch eine geeignete Positionierung in attraktiven Produkt-Marktsegmenten eine dauerhafte, verteidigungsfähige Wettbewerbsposition zu erlangen, um so einen nachhaltigen Unternehmenserfolg bzw. eine dauerhafte strategiebedingte Rente zu sichern. ${ }^{135}$ Attraktive Branchen sind solche mit geringer Wettbewerbsintensität entsprechend dem Konzept der fünf Wettbewerbskräfte. ${ }^{136}$ In der IO-Theorie werden vor allem die Rivalität zwischen den etablierten Wettbewerbern und die potentielle Bedrohung durch neue Konkurrenten als Determinanten der Struktur des Produkt-Markt-Feldes betrachtet. Dem SCP-Paradigma folgend wird das strategische Verhalten der Unternehmen in einem Produkt-Markt-Feld nun insoweit von der Marktstruktur bestimmt, als Preispolitik, Kooperationsstrategien oder auch der Aufbau von Markteintritts- und Mobilitätsbarrieren in Abhängigkeit von der Anbieterkonzentration erfolgen und

133 Vgl. Bain, J., Industrial Organization, 2. Aufl., New York 1968.

134 Vgl. Proff, H., Mikroökonomische Wurzeln eines strategischen Managements zwischen Marktund Ressourcenorientierung, in: Strategien für die Automobilindustrie: Ansatzpunkte im strategischen Management und in der Industriepolitik, Proff, H., Proff, H. V., (Hrsg.), Wiesbaden 1998, S. 26. Eine empirische Bestätigung dieser Sichtweise wurde in der viel zitierten Studie von SCHMALENSEE gesehen. Vgl. Schmalensee, R., Do Markets Differ Much?, in: American Economic Review, Vol 75, 1985, S. $341 \mathrm{ff}$

135 Vgl. Porter, M. E., What is Strategy, in: Harvard Business Review, Heft 6, Nov.-Dez. 1996, S 61ff.; Meffert, H., Marketing-Management: Analyse, Strategie, Implementierung, a.a.O.; S. $113 \mathrm{ff}$.

136 Zu den Elementen der Branchenstrukturanalyse vgl. Porter, M. E., Wettbewerbsvorteile: Spitzenleistungen erreichen und behaupten, a.a.O., S. 32. 
den Eintritt für potenzielle neue Wettbewerber erschweren bzw. verteuern sollen. Hierauf basieren die Wettbewerbsvorteilsklassen „Kostenvorteil" oder „Differenzierungsvorteil" als essentielle Bedingung der Kostenführerschafts- oder auch Differenzierungsstrategie PORTERS, d.h. die Positionierung im Produkt-Markt-Feld durch Ausrichtung aller Wertaktivitäten im Unternehmen auf eine der Wettbewerbsvorteilsklassen. ${ }^{137}$

Allerdings bleibt die marktorientierte Sichtweise im strategischen Management nicht unkritisiert. ${ }^{138}$ So wird angeführt, dass der MBV die wechselseitige Beeinflussung von Marktstruktur, -verhalten und -erfolg ignoriert und sich das Marktverhalten auf reines Anpassen an Marktstrukturveränderungen beschränkt. Die Marktstruktur wird als gegeben interpretiert, obwohl sie von Unternehmen verändert werden kann. Letztlich vernachlässigt der MBV unternehmensinterne Prozesse als Grundlage von Wettbewerbsvorteilen, so dass das Unternehmen selbst eine black box bleibt.

\subsection{Der resource-based view}

Ursprünglich als Gegenkonzept zur marktorientierten Forschungsrichtung gibt der resource-based view (RBW) die Betrachtung von Unternehmen als black box bei der Analyse von Wettbewerbsvorteilen auf. ${ }^{139}$ Auf Basis empirischer Ergebnisse wird argumentiert, dass die klassischen branchenstrukturellen Variablen der 10 Forschung nicht ausreichen, um Unterschiede in der Profitabilität von Geschäftseinheiten zu erklären. ${ }^{140}$ Der RBV bezweifelt insbesondere die Dominanz exter-

Vgl. zu den Typen von Wettbewerbsstrategien Porter, M. E., Wettbewerbsvorteile: Spitzenleistungen erreichen und behaupten, a.a.O., S. 37ff. In neueren Arbeiten werden hybride Strategien basierend auf Wettbewerbsvorteilen der kostenminimalen Differenzierung empirisch erklärt und nachgewiesen, sog. Outpacing-Ansatz.

Vgl. zur Kritik auch Proff, H., Mikroökonomische Wurzeln eines strategischen Managements zwischen Markt- und Ressourcenorientierung, a.a.O., S. 31.

Zu einer umfassenden Darstellung bzw. einem Überblick der Vertreter des RBV vgl. Rasche, Ch., Wettbewerbsvorteile durch Kernkompetenzen - Ein ressourcenorientierter Ansatz, Wiesbaden 1994, S. 38.

Vgl. etwa die Untersuchung von Hansen, G. S., Wernerfelt, B., Determinants of Firm Performance: The Relative Importance of Economic and Organizational Factors, in: Strategic Management Journal, Vol. 10, 1989, S. 399ff. RUMELT weist überdies nach, dass in der Studie SCHMALENSEES (vgl. FN 134) durchaus ein signifikanter und beträchtlicher Teil der Profitabilitätsvarianz geschäftseinheitenspezifisch ist. Vgl. Rumelt, R. P., How much does Industry matter?, in: Strategic Management Journal, Vol. 12, 1991, S. $168 \mathrm{ff}$. 
ner Faktoren und unterstellt, dass Unternehmenserfolg insbesondere durch unternehmensspezifische und einzigartige Ressourcen bzw. Fähigkeiten determiniert wird. ${ }^{141}$ Entsprechend wird vom Resource-Conduct-Performance (R-C-P)Paradigma gesprochen. Um dauerhafte Wettbewerbsvorteile bzw. strategiebedingte Renten zu erreichen (performance), wird ausgehend von der unternehmensspezifischen Ressourcenausstattung (resources) das adäquate strategische Verhalten des Unternehmens (conduct) abgeleitet.

Prämisse des RBV ist die Annahme dauerhaft heterogener Ressourcen, welche sich in einer asymmetrischen Ressourcenallokation und damit einer unterschiedlichen Ressourcenausstattung der Unternehmen widerspiegelt. Ressourcen stellen im Folgenden mit BARNEY „all assets, capabilities, organizational processes, firm attributes, information, knowledge, etc. controlled by a firm that enable the firm to conceive of an implement strategies." ${ }^{142}$ Dauerhafter Unternehmenserfolg setzt somit bei der Nutzung der Ressourcenheterogenität an. Die sich von anderen Unternehmen unterscheidenden Fähigkeiten werden auch als Kernkompetenzen bezeichnet. ${ }^{143}$ Im Unterschied zum MBV ist die allgemeine Zielrichtung des RBV somit weniger auf eine ausgewogene Portfolioposition in der Unternehmung als vielmehr auf nachhaltiges Wachstum durch Entwicklung, Nutzung und Transfer von Kernkompetenzen ausgerichtet. ${ }^{144}$

141 Vertreter des RBV führen letztlich in Anlehung an den SWOT-Ansatz an, dass der Erfolg einer Strategie von dem Grad der Anpassung der internen Stärken und Schwächen einer Unternehmung mit den in der Umwelt liegenden Chancen und Risiken abhängt. Vgl. auch Bukhari, I., Europäisches Brand Management. Entwicklung und Umsetzung erfolgreicher Marketingkonzepte, Wiesbaden 1999, S. 131.

142 Barney, J., B., Firm Resources and Sustained Competitive Advantage, in: Journal of Management, Vol. 17, Heft 1, 1991, S. 101; zitiert auch bei Burmann, Ch., Strategiewechsel in turbulenten Märkten - Neuere theoretische Ansätze zur Unternehmensflexibilität, a.a.O., S. 24. In der Literatur wird der Ressourcenbegriff ansonsten uneinheitlich verwendet (asset, skill, capability etc.), RASCHE spricht vom "Fehlen einer vermittelnden terminologischen Basis". Rasche, Ch., Wettbewerbsvorteile durch Kernkompetenzen - Ein ressourcenorientierter Ansatz, a.a.O., S. 93 .

143 Vgl. Teece, D.J., Pisano, G., Shuen, A., Dynamic Capabilities and Strategic Management, in: Strategic Management Journal, Vol. 18, 1997, S. 509ff.; Prahalad, C. K., Hamel, G., The Core Competence of the Corporation, in: Harvard Business Review, Vol. 68, May-June 1990, S. $79 \mathrm{ff}$.

144 Werden die beiden Ansätze unter dem Aspekt des strategischen Marketing im Hinblick auf ihr Zusammenspiel und eine mögliche Priorisierung analysiert, so sind situativ unterschiedliche Zuordnungen - z. B. in Käufer- oder Verkäufermärkten bzw. unterschiedlichen Stadien des Markt- und Produktlebenszyklus - vorstellbar. Beispielsweise erscheint es plausibel, dass in jungen, schnell wachsenden Märkten und in stagnierenden bzw. schrumpfenden Märkten angesichts der Markteintritts- und -austrittsbarrieren der RBV dominiert. Vgl. Meffert, H., Marke- 
Um als Quelle eines strategischen Wettbewerbsvorteils zu fungieren, sollten die Unternehmensressourcen

- wertvoll (valuable),

- rar (rare)

- nicht vollkommen imitierbar (imperfectly imitable) und

- nicht substituierbar (no strategically equivalent substitutes)

sein. ${ }^{145}$ Sind diese Eigenschaften vorhanden, liegen die Aufgaben des Management primär darin, die Ressourcen derart miteinander zu kombinieren, dass sie den Anforderungen der Märkte gerecht werden. Dabei geht es entsprechend der Anforderungen zum einen um die Akquisition und interne Entwicklung einzigartiger, schwer imitierbarer, dauerhaft haltbarer Ressourcen, zum anderen um ihre markt- bzw. kundenorientierte Bündelung und Integration. ${ }^{146}$ Über die Relevanz von Ressourcen und Fähigkeiten entscheidet dabei immer der Markt, so dass der RBV nicht auf die Marktperspektive verzichten kann. Marktrelevant sind Kernkompetenzen letztlich nur, wenn sie einen überlegenen Kundennutzen stiften.

Als unübersehbare Schwäche und damit zentrale Kritik des RBV kann vor allem die Vernachlässigung des dynamischen Aspekts der Unternehmensentscheidungen, d. h. die Notwendigkeit des Wechsels vorhandener Ressourcen und Fähigkeiten bei sich wandelnden Marktbedingungen, interpretiert werden ${ }^{147}$ Dieser wird jedoch in jüngster Zeit in einer Weiterentwicklung des Ressourcenparadigmas, dem Dynamic Capability-Ansatz ${ }^{148}$, aufgegriffen, bei dem neben der Ressourcenausstattung eines Unternehmens die Managementprozesse und damit die Entwicklung spezifischer Fähigkeiten im Zeitablauf in die Betrachtung einbezogen

tingwissenschaft im Wandel - Anmerkungen zur Paradigmendiskussion, in: HHL-Arbeitspapier Nr. 30, Leipzig 1999, S. 27.

Vgl. ausführlich Barney, J., B., Firm Resources and Sustained Competitive Advantage, a.a.O., S. $105 f$. Vgl. auch Rasche, Ch., Wettbewerbsvorteile durch Kernkompetenzen - Ein ressourcenorientierter Ansatz, a.a.O., S. 69.

Vgl. Meffert, H., Marketingwissenschaft im Wandel - Anmerkungen zur Paradigmendiskussion, a.a.O., S. 27. Als Beispiele führt Meffert etwa Sony an, welche die Kernkompetenz der Miniaturisierung besitzt und auf dieser Basis im Markt für Hifi-Geräte zahlreiche neue Produkte herausgebracht hat. Ebenso wird IBM aufgrund langjähriger Erfahrungen eine besondere Forschungs- und Servicekompetenz zugeschrieben. $\mathrm{OBI}$ beherrscht demgegenüber mit über 300 Baumärkten die besondere Fähigkeit der Steuerung von Franchise-Netzwerken.

147 Vgl. Meffert, H., Marketingwissenschaft im Wandel - Anmerkungen zur Paradigmendiskussion, a.a.O., S. 28.

148 Vgl. Teece, D.J., Pisano, G., Shuen, A., Dynamic Capabilities and Strategic Management, a.a.O., S. 509ff. 
werden. Hier zeichnen sich Unternehmen mit dauerhaft überdurchschnittlichen Kapitalrenditen vor allem durch überlegene Lernfähigkeiten, $d$. h. die Fähigkeit zur Erneuerung und zum Wechsel der Ressourcen aus. MEFFERT und auch BURMANN sprechen in diesem Zusammenhang mit Blick auf die traditionelle betriebswirtschaftliche Begriffswelt auch von einer "Renaissance bzw. Neuinterpretation der Unternehmensflexibilität" ${ }^{149}$

Zusammenfassend sind somit die frühzeitige Identifikation neuer Kunden-NutzenPotenziale und der Aufbau aller hierfür notwendigen Ressourcen erfolgsentscheidend. Hier wird somit das Wechselspiel zwischen Inside-Out- und Outside-InPerspektive deutlich. Aufgrund der hohen Bedeutung der Identifikation neuer Kunden-Nutzen-Potenziale bereitet der Dynamic Capability-Ansatz somit einer Synthese der markt- und ressourcenorientierten Perspektive den Weg.

\subsection{Integration der markt- und ressourcenorientierten Sichtweise im Kon- zept der identitätsorientierten Markenführung}

Ähnlich dem Wandel der Unternehmensparadigmen lässt sich ein Wandel im Rahmen der Markenführung aufzeigen. Stellte die Marketingwissenschaft ihre Aussagen über Markenführung bisher dominant auf eine breite Outside-inPerspektive, wird diese Sicht in jüngster Zeit um eine Inside-out-Betrachtung ergänzt. MEFFERT zur Folge kann erst durch eine dialogorientierte Verfolgung beider Perspektiven dem Anspruch erfolgreicher Markenführung Rechnung getragen werden. ${ }^{150}$ Genau diesem Zweck dient das Konzept der identitätsorientierten Markenführung, welches ähnlich dem Dynamic Capability-Ansatz eine Synthese der markt- und ressourcenorientierten Sichtweise anstrebt.

Im Vergleich zu älteren Markenführungsansätzen erweitert der identitätsorientierte Ansatz der Markenführung die bisher vorherrschende absatzmarktbezogene Sichtweise um eine innengerichtete Perspektive und interpretiert Glaubwürdigkeit und Kompetenz einer Marke als wichtige Voraussetzung für die Gewinnung des

149 Meffert, H., Marketingwissenschaft im Wandel - Anmerkungen zur Paradigmendiskussion, a.a.O., S. 28; vgl. auch Burmann, Ch., Strategiewechsel in turbulenten Märkten - Neuere theoretische Ansätze zur Unternehmensflexibilität, a.a.O., S. 30ff.

150 Vgl. auch Errichiello, O. C., GroB, A., Pirck, P., Forschungsbericht 2000/2001, in: Brandmeyer, K., Deichsel, A. (Hrsg.), Jahrbuch Markentechnik 2000/2001: Markenwelt, Markentechnik, Markentheorie, Forschungsbericht, Horizonte, Frankfurt a. M. 1999, S. 421. 
öffentlichen Vertrauens. ${ }^{151}$ Ältere Markenführungsansätze verstehen Markenkompetenz in erster Linie als Qualität bzw. Leistungsfähigkeit der Marke, ignorieren dabei allerdings die Beziehung der Marke zu verschiedenen Anspruchsgruppen. Im Gegensatz dazu konstituiert sich Markenidentität als sozialpsychologische Interpretationsform der Marke ${ }^{152}$ über einen längeren Zeitraum infolge gegenseitiger dynamischer Wirkungsbeziehungen von aktiven, marktorientierten Handlungen eines Markenartikelherstellers sowie der Wahrnehmung dieser Handlungen durch den Konsumenten. Obwohl der Identitätsbegriff in der Literatur kontrovers diskutiert wird, setzt er sich unabhängig von der jeweiligen Definition aus vier konstitutiven Merkmalen zusammen: ${ }^{153}$ So wird die Wechselseitigkeit als "Paradigma der Identitätsforschung" bezeichnet und beschreibt, dass die Identität einer Marke nur in der Wechselwirkung zwischen Menschen und den sie umgebenden Objekten der Außenwelt entstehen kann. Gäbe es nur eine Marke ohne Konkurrenzbeziehungen zu anderen Marken, so wäre Markenidentität völlig bedeutungslos. Die Identität ist überdies durch Kontinuität geprägt, d.h durch die Beibehaltung wesentlicher Merkmale einer Marke. Die Konsistenz als drittes Identitätsmerkmal bezieht sich im Gegensatz zur Kontinuität auf einen Zeitpunkt und ist durch eine Vermeidung von Widersprüchen gekennzeichnet. Das vierte Identitätsmerkmal, die Individualität, beschreibt schließlich die Einmaligkeit eines Identitätsobjektes.

Infolge der Wechselseitigkeit wird zwischen dem Selbstbild der Markenidentität aus Sicht der internen Anspruchsgruppen wie Führungskräfte und Mitarbeiter sowie dem Fremdbild der Markenidentität aus Sicht der externen Anspruchsgruppen wie etwa Kunden und Lieferanten differenziert (vgl. Abb. 6). ${ }^{154}$

151 Identität verschafft Glaubwürdigkeit und kann als notwendige Bedingung für die Entstehung von Vertrauen verstanden werden, während Kompetenz als hinreichende Bedingung das Vertrauen zusätzlich festigt. Vgl. Meffert, H., Burmann, Ch., Identitätsorientierte Markenführung - Grundlagen für das Management von Markenportfolios, a.a.O., S. 24

Vgl. auch Meffert, H., Burmann, Ch., Markenbildung und Markenstrategien, in: Handbuch Produktmanagement, Albers, S., Herrmann, A., (Hrsg.), Wiesbaden 2000 (im Druck); Chernatony, L. de, Dall'Ollmo Riley, F., Defining A "Brand“: Beyond the Literature with Experts' Interpretations, in: Journal of Marketing Management, Heft 14, 1998, S. 427; Kapferer, J.-N., Die Marke - Kapital des Unternehmens, a.a.O., S. 17ff.; Keller, K. L., Strategic Brand Management: Building, Measuring, and Managing Brand Equity, a.a.O., S. 86

Vgl. zu einer Diskussion der Identität ausführlich Meffert, H., Burmann, Ch., Identitätsorientierte Markenführung - Grundlagen für das Management von Markenportfolios, a.a.O., S. $23 \mathrm{ff}$. Identity, in: Handwörterbuch Markenartikel, Bruhn, M. (Hrsg.), Band 2, Stuttgart 1994, S. 


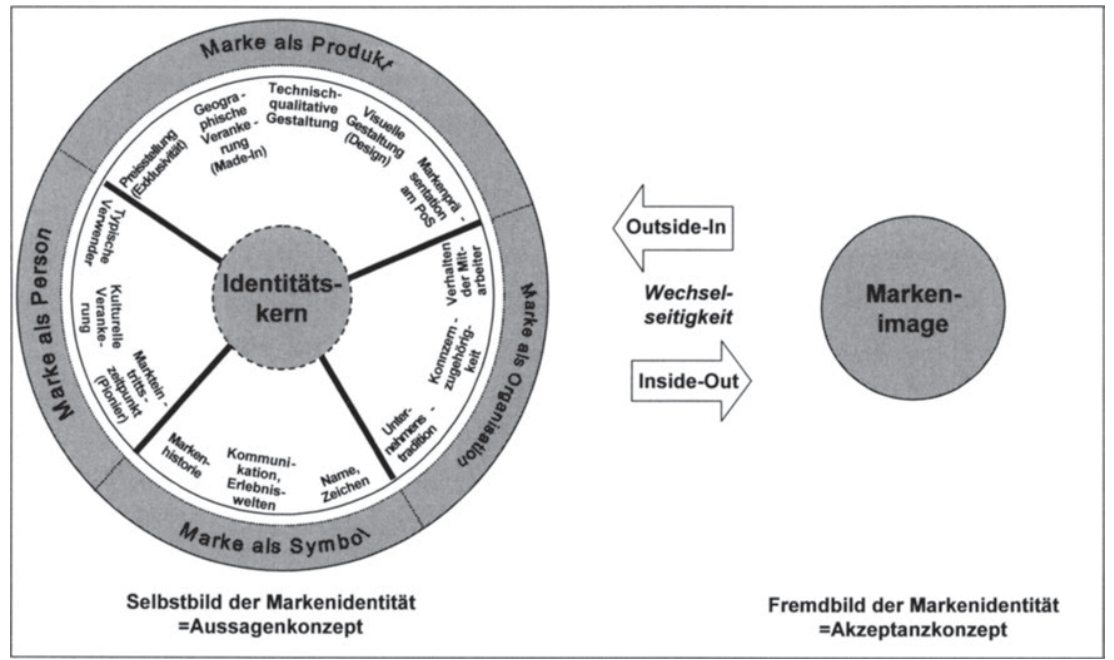

Abb. 6: Komponenten der Markenidentität

(Quelle: Meffert, H., Burmann, Ch., Identitätsorientierte Markenführung, a.a.O., S. 35.)

Während sich das Selbstbild der Markenidentität im Unternehmen aktiv konstituiert und beschreibt, wie die Marke aus unternehmensinterner Sicht gegenüber externen Gruppen auftreten soll, formt sich das Fremdbild erst langfristig bei den verschiedenen externen Anspruchsgruppen und spiegelt sich letztlich im Image der Marke wider. Das Markenimage beschreibt als mehrdimensionales Einstellungskonstrukt die Gesamtheit aller Vorstellungen eines Individuums hinsichtlich der Eignung einer Marke zur Befriedigung seiner rationalen und emotionalen Bedürfnisse. ${ }^{155}$ Das Markenimage ist somit als ein Akzeptanzkonzept der Konsumenten in ihrer Beurteilung der Marke zu sehen und stellt daher kein Managementkonzept dar, denn „um akzeptiert zu werden, muss die Marke zunächst konzipiert sein." 156

1036ff; Schmitt, B. H., Pan, Y., Managing Corporate and Brand Identities in the Asia Pacific Region, in: California Management Review, Winter 1995, S. $15 \mathrm{ff}$.

155 Vgl. zum Markenimage als Fremdbild der Markenidentität Meffert, H., Burmann, Ch., Identitätsorientierte Markenführung - Konsequenzen für die Handelsmarke, in: Handelsmarken. Entwicklungstendenzen und Zukunftsperspektiven der Handelsmarkenpolitik. Bruhn, $M$. (Hrsg.), 2. Aufl., Stuttgart 1997, S. 34ff.

Kapferer, J.-N., Die Marke - Kapital des Unternehmens, a.a.O., S. 45. 
Insofern erweitert das Konzept der Markenidentität das Akzeptanzkonzept um ein Aussagenkonzept, d.h. Inhalt, Idee und Eigendarstellung der Marke werden spezifiziert. Im Mittelpunkt des Aussagenkonzepts der Markenidentität steht die Formulierung eines Kundennutzen, den die Marke aus Sicht des Anbieters erfüllen soll, wobei vor allem die spezifische Kernkompetenz der Marke zum Ausdruck gebracht werden muss. Auf der jeweiligen Kernkompetenz der Marke basiert die Markenidentität. Sie wird vor allem durch essentielle, wesensprägende Eigenschaften einer Marke geprägt, welche mit KAPFERER auch als genetischer Code ${ }^{157}$ der Marke interpretiert werden können. Diese Eigenschaften dienen als zentrale Grundlage der Positionierung einer Marke. Im Gegensatz zur essentiellen Markenidentität umfasst die erweiterte Markenidentität akzidentielle Merkmale, die den Identitätskern anreichern und ausfüllen und im Unterschied zu den essentiellen Merkmalen bis zu einem bestimmten Grad verändert werden können. ${ }^{158}$ Dieser Teil der Identität dient mithin dazu, die Kernidentität zu vervollständigen. KAPFERER führt entsprechend an, dass sich Stil und Positionierung einer Marke mit der Entwicklung der Technologie, Produkte und Käufer im Sinne eines stilistischen Codes der Marke durchaus ändern können, wenn der Ursprung und mithin Kern der Markenidentität bewahrt wird. ${ }^{159}$ Je nach Marke können einzelne oder mehrere der

157 KAPFERER zur Folge ist die Marke eine Art genetisches Programm, UPSHAW spricht von der Marken-DNA. Vgl. Kapferer, J.-N., Die Marke - Kapital des Unternehmens, a.a.O., S. 111; Upshaw. L. B., Building Brand Identity: A Strategy for Success in an hostile Marketplace, New York u.a. 1995, S. 25; Arnold, D., Modernes Markenmanagement, Wien 1992, S. 43; Brandmeyer, K., Schmidt, M., Der "Genetische Code der Marke ${ }^{\| \otimes}$ als Management-Werkzeug, in: Brandmeyer, K., Deichsel, A. (Hrsg.), Jahrbuch Markentechnik 2000/2001: Markenwelt, Markentechnik, Markentheorie, Forschungsbericht, Horizonte, Frankfurt a. M. 1999, S. $271 \mathrm{ff}$.

Vgl. Meffert, H., Burmann, Ch., Identitätsorientierte Markenführung - Konsequenzen für die Handelsmarke, a.a.O., S. 34ff.

Das Spannungsfeld von Veränderung und Kontinuität kann am Beispiel Volkswagen verdeutlicht werden. Für die Marke Volkswagen war über lange Jahre hinweg der Name Sinnbild für die Markenphilosophie. Die Aufgabe des Herstellers war es, ein "Auto für das Volk" zu entwikkeln, wobei die Gründungsidee in der Solidarität (ein Volk, ein Wagen) lag. Später stand der Hersteller vor dem Problem, die Marke, die mit dem WW-Käfer anfangs auf nur einem Produkt basierte, an die soziologische Evolution, die technologischen Entwicklungen und die wachsenden Ansprüche der Autofahrer anzupassen. Es galt somit, die Markenidentität an die neuen Erfordernisse anzugleichen, ohne den Kern der Marke zu verändern. Entsprechend wurde verstärkt der sparsame Verbrauch, die Werthaltigkeit, Robustheit und Sicherheit des Autos betont, ohne jedoch den Identitätskern der Marke Volkswagen wirklich zu verändern. Vgl. Kapferer, J.-N., Die Marke - Kapital des Unternehmens, a.a.O., S. 110f. Zum Spannungsfeld von "Statik und Dynamik" einer Marke vgl. auch Werner, H., Vom Marken- zum Preiswettbewerb? - Strategisches Marketing in der Automobilindustrie für das Jahr 2000 und darüber hinaus!, in: Das Handbuch für das Automobilmarketing - Strategien, Konzepte, Instrumente, Diez, W. (Hrsg.), 3. Aufl., Landsberg/Lech 1997, S. 31. 
Identitätskomponenten in Abb. 6 die essentiellen oder akzidentiellen Merkmale sein.

AAKER unterscheidet vier Dimensionen, nach denen die Komponenten der Markenidentität kategorisiert werden können. ${ }^{160}$ Wie Abb. 6 zeigt, wird die Identität der Marke von der Art der Produkte und Dienstleistungen, der Markenorganisation, bestimmten Symbolen und spezifischen Persönlichkeitsmerkmalen geprägt. Die technisch-qualitative und visuelle Gestaltung der Modelle, die Preisstellung und Präsentation am Point of Sale, das Verhalten der Mitarbeiter im Verkauf und Kundendienst, das Bild vom typischen Verwender oder die geographische, kulturelle und historische Verankerung der Marke seien hier nur exemplarisch als zentrale Quellen der Identität einer Automobilmarke genannt.

Insofern kann die Markenidentität zusammenfassend auch als eine in sich widerspruchsfreie Summe von Merkmalen einer Marke interpretiert werden, die diese von anderen Marken dauerhaft unterscheidbar macht. ${ }^{161}$ Aussagen über die Relevanz der verschiedenen Identitätskomponenten für die spezifische Identität einer Marke lassen sich nur situativ unter Berücksichtigung der jeweiligen Zielgruppenstruktur, der Art des zentralen Markennutzens, der Markenidentität der Hauptwettbewerber sowie der Struktur des individuellen Markenportfolios treffen. Da Markenidentität erst durch die Wechselseitigkeit zwischen Selbstbild und Fremdbild der Marke entsteht, ist in diesem Zusammenhang entscheidend, dass die verschiedenen internen und externen Anspruchsgruppen der Marke ein einheitliches Verständnis von der Leistungskompetenz einer Marke haben. Die Stärke der Identität einer Marke hängt letztlich vom Grad der Übereinstimmung zwischen dem internen Selbstbild und dem externen Fremdbild einer Marke ab und basiert damit auf der Synthese von RBV und MBV.

Um dem Anspruch moderner Makenführung gerecht zu werden, ist es zur Schaffung konturierter Marken im Portfolio zweckmäßig, auch die Mehrmarkenführung

161 Vgl. Meffert, H., Burmann, Ch., Identitätsorientierte Markenführung - Konsequenzen für die Handelsmarke, a.a.O., S. 64. AAAKER, JOACHIMSTHALER führen in diesem Zusammenhang fünf Fragen an, die bei der Identifikation relevanter Identitätskomponenten helfen können: "Does it capture an element important to the brand and its ability to provide the customer value or support customer relationships? Does it help differentiate the brand from its cpmpetitors? Does it resonate with the customer? Does it energize employees? Is it believable?" Aaker, D. A., Joachimsthaler, E., Brand Leadership, New York u. a 2000, S. 57.
} 
nicht auf eine ausschließliche an der Kundenwahrnehmung und mithin dem Markenimage orientierte Outside-In-Perspektive zu stellen, sondern um eine in das Unternehmen gerichtete und damit ressourcenorientierte Perspektive zu ergänzen. Vor diesem Hintergrund wird das Entscheidungsfeld bei der Führung von Markenportfolios dargestellt.

\section{Entscheidungsfeld von Markenportfolios im Rahmen der identi- tätsorientierten Markenführung}

Im Rahmen der Ausgestaltung der Mehrmarkenstrategie gilt es, eigenständige Marken zu schaffen, um hierdurch sowohl eine dauerhafte Differenzierung gegenüber der Konkurrenz als auch eine dominierende Stellung in der Psyche des Konsumenten ${ }^{162}$ im Sinne eines strategischen Wettbewerbsvorteils zu erreichen. Für jede Marke ist ein eigenständiges Profil zu erarbeiten, mit dem sich eine Steigerung des Marken- und damit eine Erhöhung des übergeordneten Unternehmenswertes erzielen lässt. Die Marken des Portfolios stellen hierzu dauerhafte und selbstständige Planungseinheiten dar, so dass jede Marke als strategische $\mathrm{Ge}$ schäftseinheit interpretiert werden kann. ${ }^{163}$

Im Sinne eines Planungsansatzes beginnt die identitätsorientierte Führung des Markenportfolios auf der Unternehmensebene. ${ }^{164}$ Hier ist es Aufgabe des Management, die Soll-Identitäten der Portfoliomarken näher zu konkretisieren und die jeweiligen Markenidentitäten in diesem Sinne innen- wie aussengerichtet zu gestalten. ${ }^{165}$ Den Ausgangspunkt der Überlegungen bildet die strategische Rahmen-

DOMIZLAFF spricht von einer Monopolstellung im Kopf der Konsumenten. Vgl. Domizlaff, H., Die Gewinnung des öffentlichen Vertrauens, a.a.O., S. $67 \mathrm{ff}$.

Die Sinnhaftigkeit einer Interpretation der Marke als Strategische Geschäftseinheit bzw. als Strategisches Geschäftsfeld wird auch von HAEDRICH, TOMCZAK betont. Sie benutzen die Begriffe Marke und strategisches Geschäftsfeld synonym, vgl. Haedrich, G., Tomczak, T., Strategische Markenführung: Planung und Realisierung von Marketingstrategien für eingeführte Produkte, Bern, Stuttgart 1990, S. 29. Vgl. zum strategischen Geschäftsfeld auch Abell, D. F., Defining the Business. The Starting Point of Strategic Planning, Englewood Cliffs 1980, S. $177 \mathrm{ff}$. Zur strategischen Geschäftseinheit vgl. Hinterhuber, H., Strategische Unternehmensführung, II. Strategisches Handeln, 6. Aufl., Berlin u.a. 1997, S. $103 \mathrm{ff}$.

Vgl. Meffert, H., Burmann, Ch., Identitätsorientierte Markenführung - Konsequenzen für die Handelsmarke, a.a.O., S. 64.

165 So fodert DEMUTH, "Markenführung in jedem Unternehmen zur Chefsache zu machen ...", Demuth, A., Corporate Branding als Herausforderung an das Marketing, in: Kongressdokumentation vom Deutschen Marketing Verband des 27. Deutschen Marketing-Tag am 29. Oktober 1999 in Frankfurt unter dem Thema "Chancen im 3. Jahrtausend. Information managen 
planung von Mehrmarkenstrategien, welche den Ordnungsrahmen für den Marktauftritt der Portfoliomarken festlegt (vgl. Abb. 7).

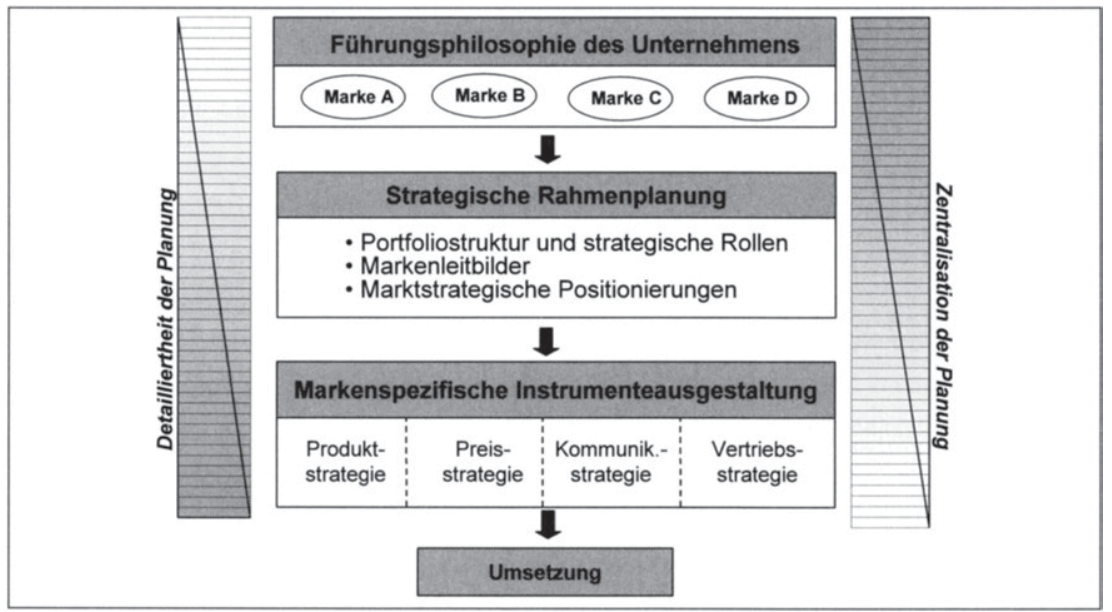

Abb. 7: Planungsprozess von Mehrmarkenstrategien

(Quelle: In Anlehnung an Meffert, H., Perrey, J., Mehrmarkenstrategie - Ein Beitrag zum Management von Markenportfolios, a.a.O., S. 634.)

Unter Berücksichtigung der Führungsphilosophie des Unternehmens sind strategische Rollen, Leitbilder und Positionierungen der Portfoliomarken zu fixieren, welche den Rahmen für den zielgerichteten und auf die relevanten Absatzmärkte abgestimmten Einsatz der Marketinginstrumente der einzelnen Portfoliomarken vorgeben. Die institutionelle Zuständigkeit für die einzelnen Teilprobleme - Definition der strategischen Rahmenplanung und instrumentelle Ausgestaltung der Mehrmarkenstrategie - verlagert sich dabei in der Regel mit zunehmender Detailliertheit auf die Ebene der einzelnen Markeneinheiten. ${ }^{166}$

- Zukunft gestalten“, S. 42. Vgl. hier auch Brabeck-Letmathe, P., Marketing ist Chefsache, mehr denn je, in: asw, Heft 1, 2000, S. $32 f$.

Strategische Entscheidungen werden wegen ihrer großen Bedeutung für den Unternehmenserfolg als echte Führungsentscheidungen bezeichnet, die von der obersten Unternehmensleitung wahrzunehmen sind und nicht delegiert werden können. Vgl. Gutenberg, E., Grundlagen der Betriebswirtschaftslehre, Erster Band, Die Produktion, 24. Aufl., Berlin, Heidelberg, New York 1983, S. 133, Adam, D., Planung und Entscheidung: Modelle - Ziele Methoden; Fallstudien und Lösungen, a.a.O., S. 270. 


\subsection{Ausgestaltung der strategischen Rahmenplanung}

\subsection{Portfoliostruktur und Festlegung markenspezifischer Rollen}

Aufbauend auf den mit der Mehrmarkenstrategie verfolgten Zielen stehen die Gestaltung und Festlegung strategischer Stoßrichtungen des Markenportfolios im Mittelpunkt der strategischen Rahmenplanung von Mehrmarkenstrategien. Hier gilt es, ein Markenportfolio zu gestalten, mit dem eine zieladäquate und in aller Regel möglichst breite Abdeckung des Gesamtmarktes sichergestellt werden kann. Lassen sich die differenzierten Bedürfnisse der Nachfrager nicht mit den bereits vorhandenen Marken befriedigen, ist zu fragen, inwieweit eine Erhöhung der Zielerreichungsgrade durch Ausweitung des Markenportfolios erzielt werden kann. ${ }^{167}$ Grundsätzlich bieten sich hierzu die Möglichkeiten eines eigenständigen Aufbaus neuer Marken, der Akquisition fremder Marken sowie eines kooperativen Vorgehens. ${ }^{168}$ In der Praxis wird zunehmend auf eine gezielte Akquisition von Fremdmarken zurückgegriffen, da mit diesen eine unmittelbare Erschließung ausgewählter Käufersegmente und damit eine Abrundung des Markenportfolios erzielt werden kann. Im Vergleich zum eigenständigen Aufbau präferenzfördernder Marken, welcher insbesondere bei langlebigen Konsumgütern zumeist viele Jahre in Anspruch nimmt, lässt sich auf diese Weise ein erheblicher Zeitvorteil generieren.

Auf Grundlage der festgelegten Portfoliostruktur sind die Ziele und Aufgaben der einzelnen Marken innerhalb des Portfolios und im Wettbewerbsumfeld zu präzisieren. Dies kann mit Hilfe der Definition von strategischen Rollen für die einzelnen Marken des Portfolios erfolgen. So fordert auch AAKER: „.... a brand should have well-defined roles and work within the context of other brands. "“69 Um eine möglichst breite Abdeckung des Gesamtmarktes sicherzustellen, sollten die strategischen Rollen der Marken im Portfolio möglichst verschiedenartig sein. Als konträre

167 Vgl. Meffert, H., Perrey, J., Mehrmarkenstrategie - Ein Beitrag zum Management von Markenportfolios, a.a.O., S. 637.

Vgl. Meffert, H., Burmann, Ch., Identitätsorientierte Markenführung - Konsequenzen für die Handelsmarke, a.a.O., S. 55.

Aaker, D. A., Building Strong Brands, a.a.O., S. 200. Vor diesem Hintergrund ist auch KRÜGER, Marketing-Leiter der Marke WW, zu verstehen: „Jede Marke vertritt eine Mission im Gesamtauftritt des Volkswagen-Konzerns, die auf den Wurzeln ihrer Herkunft aufsetzt und konsequent zu einer umfassenden Persönlichkeit mit Werten weiterentwickelt worden ist. " Krüger, B, Die Mehrmarkenstrategie des Volkswagen-Konzerns, unveröffentlichter Vortrag zum Deutschen Marketing-Preis 1999 am 30.06.1999 in Düsseldorf, S. 17. 
Rollen zweier Portfoliomarken lassen sich die Premiummarke zur Befriedigung gehobener Automobilansprüche sowie die Preismarke für funktionsnutzen- und preisorientierte Automobilkäufer nennen. Insbesondere in der angelsächsischen Literatur werden unterschiedliche Rollen der Marken im Portfolio hervorgehoben. So klassifiziert KELLER unter Bezugnahme auf die Konsumenten-, Wettbewerbsund Unternehmenssituation die in Tab. 4 (ergänzt um aktuelle Beispiele) benannten Rollen von Marken im Portfolio. ${ }^{170}$

\begin{tabular}{|c|l|l|}
\hline $\begin{array}{c}\text { Strategische } \\
\text { Rolle }\end{array}$ & \multicolumn{1}{|c|}{ Konkretisierung } & \multicolumn{1}{|c|}{ Beispiele } \\
\hline Flagship & $\begin{array}{l}\text { Flaggschiffmarken in der } \\
\text { Rolle als Marktführer }\end{array}$ & $\begin{array}{l}\text { Die Marke WW fungiert als Leitmarke im Port- } \\
\text { folio des Volkswagen-Konzerns. }\end{array}$ \\
\hline $\begin{array}{c}\text { Fighter / Flan- } \\
\text { ker }\end{array}$ & $\begin{array}{l}\text { Kampfmarken mit der Auf- } \\
\text { gabe des Flankenschutzes } \\
\text { im Wettbewerb }\end{array}$ & $\begin{array}{l}\text { Die Marke SKODA dient als Preismarke zur } \\
\text { Absicherung der Marken WW und AUDI, um } \\
\text { diese vor Preiskämpfen zu schützen. }\end{array}$ \\
\hline Cash Cow ${ }^{\mathbf{1 7 1}}$ & $\begin{array}{l}\text { Lieferant überdurchschnitt- } \\
\text { lich hoher finanzieller Über- } \\
\text { schüsse }\end{array}$ & $\begin{array}{l}\text { Mit den erzielten Gewinnen der Marke WW } \\
\text { konnte etwa die Marke AUDI als Premium- } \\
\text { marke aufgebaut werden. }\end{array}$ \\
\hline $\begin{array}{c}\text { Low-End Entry } \\
\text { bzw. High-End } \\
\text { Prestige Brands }\end{array}$ & $\begin{array}{l}\text { Erhöhung der Kaufattrakti- } \\
\text { vität für Einsteiger bzw. } \\
\text { Steigerung des Images des } \\
\text { gesamten Portfolios }\end{array}$ & $\begin{array}{l}\text { Die Marke RoLLSROYCE vervollständigt das } \\
\text { Markenportfolio als Luxusmarke in obere } \\
\text { Segmenten, die Marke SEAT dient als Trend- } \\
\text { marke insbesondere für junge Einsteiger. }\end{array}$ \\
\hline
\end{tabular}

Tab. 4: Beispielhafte strategische Rollen in einem Markenportfolio eines Automobilherstellers

Einer solchen Umschreibung der strategischen Rollen folgend sind die einzelnen Portfoliomarken unter Berücksichtigung der Führungsphilosophie des Gesamtportfolios (Was wollen wir?) innengerichtet anhand ihrer spezifischen Kompeten-

170 Vgl. Keller, K. L., Strategic Brand Management: Building, Measuring, and Managing Brand Equity, a.a.O., S. 407ff. AAKER unterscheidet Flankers (Schutz einer hochpreisigen Premiummarke), Strategic Brands, Cash Cows (zur Abschöpfung von Gewinnen), High End or LowEnd Entry Brands (Vervollständigung des Portfolios nach oben und unten), Silver Bullet (signalisieren Innovationen im Rahmen des Portfolios), Driver Brand (im Markenportfolio sollte jede Marke treibende Kraft besitzen, da jede Marke ein eigenes Kompetenzfeld zu besetzen hat). Vgl. Aaker, D. A., Building Strong Brands, a.a.O., S. 243ff. HAEDRICH/TOMCZAK differenzieren hingegen mit Bezug auf das konkurrenzorientierte Rollenkonzept von KOTLER Marken in Marktführer, Herausforderer, Mitläufer und Nischenbearbeiter. Vgl. Haedrich, G., Tomczak, T., Strategische Markenführung: Planung und Realisierung von Marketingstrategien für eingeführte Produkte, Bern, Stuttgart 1990, S. 127ff. Zum Rollenkonzept vgl. auch Meffert, H., Marketing-Management: Analyse, Strategie, Implementierung, a.a.O., S. 112.

„Reine" Cash Cow Marken existieren im Automobilbereich nicht. Denn charakteristisch für eine solche Marke ist, dass nur minimale Investitionen erfolgen. Automobilhersteller investieren aber letztlich in jede Marke, da im Automobilmarkt nur Marken und keine No Names agieren. Ein klassisches Beispiel aus der Konsumgüterindustrie ist hingegen die Marke Lux der Unilever-Gruppe, die in den letzten Jahren nahezu keine Unterstützung durch Marketingmaßnahmen erfahren hat und dennoch über 25 Mio. Dollar Umsatz p.a bei ca. 3 Prozent Marktanteil generiert. Vgl. Aaker, D. A., Building Strong Brands, a.a.O., S. 257. 
zen (Was können wir?) und aussengerichtet an den Erwartungen und Strukturen des Absatzmarktes (Was sollen wir?) zu konkretisieren. In der Spezifizierung der strategischen Rollen manifestiert sich somit prägnant die angestrebte dialogorientierte Verfolgung der ressourcenorientierten Inside-Out- und der marktorientierten Outside-In-Perspektive der Mehrmarkenführung.

\subsection{Innengerichtete Ressourcenverteilung und Markenleitbilder}

Zur Konkretisierung der strategischen Rollen sind die einzelnen Marken des Portfolios hinsichtlich inrer essentiellen Merkmale bzw. Fähigkeiten und damit ihrer Kernkompetenzen abzugrenzen. Analog zum RBV ist hierzu insbesondere auf die Merkmals- bzw. Ressourcenheterogenität der einzelnen Marken Bezug zu nehmen. Durch die inhaltliche Charakterisierung können somit die Besonderheiten und Einzigartigkeiten der verschiedenen Marken zum Ausdruck gebracht werden.

Um mit jeder Marke einen strategischen Wettbewerbsvorteil zu erzielen, haben die hinter den einzelnen Marken stehenden Ressourcen und Fähigkeiten den in Kap. 3.12 dargestellten formalen Anforderungen zu genügen. Damit die markenspezifischen Fähigkeiten eine wertvolle Ressource im Portfolio darstellen, müssen die einzelnen Marken einen aus Kundensicht wahrgenommenen Nutzen schaffen. Folglich sind die Abnehmerbedürfnisse für die verschiedenen Marken des Portfolios zu identifizieren, nach innen in das Unternehmen zu kommunizieren und in den markenspezifischen Erstellungsprozess einzubinden. Ferner sollten die markenspezifischen Fähigkeiten rar und begrenzt imitierbar sein. Die Kompetenz einer jeweiligen Marke kann nur dann Quelle eines Wettbewerbsvorteils für die Marke sein, wenn die anderen Marken des Portfolios bzw. Wettbewerber nicht über die gleichen Fähigkeiten verfügen. ${ }^{172}$ Insbesondere dann, wenn Marken größtenteils auf "weichen", intangiblen Faktoren beruhen, erscheinen die zu einer Marke gehörenden Fähigkeiten in ihrer Summe nur begrenzt imitierbar. Letztlich entsteht die Marke ausschließlich im Kopf des Konsumenten und ist insoweit stets immateri-

172 Die geringe Wahrscheinlichkeit dafür, dass produktbezogene Dimensionen einer Marke (Marke als Produkt, vgl. Abb. 6) zentrale Quellen eines markenspezifischen Wettbewerbsvorteils darstellen, liegt i.d.R. an der mangelnden Spezifität bzw. an der leichten Imitierbarkeit derartiger Fähigkeiten. Dies lässt sich insbesondere durch die bereits aufgezeigte physischtechnische Angleichung und Homogenität von Produkten verdeutlichen. Vor diesem Hintergrund sind im Markenportfolio insbesondere intangible markenspezifische Ressourcen und Fähigkeiten zur Schaffung von markenspezifischen Wettbewerbsvorteilen hervorzuheben, welche eine Transferierbarkeit bzw. Imitierbarkeit verhindern. 
ell. ${ }^{173}$ Schließlich sollten die markenspezifischen Fähigkeiten schwer substituierbar sein, d.h. der aus ihrem Einsatz resultierende Nutzen darf nicht ohne großen Aufwand auch mit anderen Ressourcen erreichbar sein.

Zur Erreichung einer möglichst breiten Gesamtmarktabdeckung durch das Portfolio sollte jede Marke des Portfolios über andere Kernkompetenzen verfügen, auf deren Basis eine glaubhafte Differenzierung begründet sein kann. Ziel sollte es sein, differenzierte, auf unterschiedliche Kundenbedürfnisse abzielende Marken zu schaffen. Hierzu sind die in der Abb. 6 dargestellten Komponenten der Markenidentität für jede Marke nunmehr im Sinne eines markenspezifischen „Kompetenzbündels" derart miteinander zu kombinieren, dass sie die Marke von anderen Marken dauerhaft unterscheidbar macht, wobei sich die einzelnen Merkmale gegenseitig verstärken und ergänzen können. So werden mit der Produktdimension produktbezogene Attribute der Marke betont, wobei die technisch-qualitative Gestaltung eine der wichtigsten Komponenten darstellt. ${ }^{174}$ Insofern kann mit der "Marke als Produkt" eine spezifische Technologie-Kompetenz der Marke besonders betont werden. ${ }^{175}$ Eine solche Kompetenz ermöglicht der Marke vor allem den Aufbau objektiv-technischer Wettbewerbsvorteile. In der "Marke als Organisation" manifestiert sich operationale Kompetenz, indem weniger auf spezifische Produkteigenschaften einer Marke, als vielmehr auf die Kompetenz und Leistungsfähigkeit des Unternehmens als Organisation abgestellt wird. Diese kann sich beispielsweise in besonderen Fähigkeiten bei der Auftragsabwicklung, im Verhalten der Mitarbeiter oder in Kundendienstprozessen ausdrücken, wobei die Kompetenz

173 Zur Markenbildung vgl. auch Meffert, H., Burmann, Ch., Markenbildung und Markenstrategien, a.a.O., S. 5f; Farquhar, P.H., Managing Brand Equity, in: Journal of Advertising Research, Aug./ Sept. 1990, S. $7 \mathrm{ff}$.

Die wichtigsten Kaufkriterien sind einer repräsentativen Befragung von Automobilfahrern in Deutschland aus dem Jahr 1998 zu Folge [Nennungen in Prozent, Mehrfachnennungen möglich]: Hohe Zuverlässigkeit [71], Geringer Spritverbrauch [67]. Airbag für den Beifahrer [63], Außerordentliche Sicherheit [59], Gute Straßenlage [57], Bequemlichkeit/Komfort [56], Serienm. Ausstattung [56], ABS [54], Geräumigkeit [50], Hohe Umweltfreundlichkeit [48], Neueste Technik [46], Seitlicher Aufprallschutz [46], Niedriger Kaufpreis [44], Styling [42], Wiederverkaufspreis [40], Service-Netz [38], Herkunftsland des Wagens [31], Hohe Motorleistung [30], Hohes Ansehen der Marke [28], Sportlichkeit/Schnelligkeit [25], Geländetauglichkeit [11]. Vgl. o.V., autofahren in deutschland 1999 , a.a.O., S. 41.

Zur Unterscheidung der Kompetenzen vgl. Burmann, Ch., Strategiewechsel in turbulenten Märkten - Neuere theoretische Ansätze zur Unternehmensflexibilität, a.a.O., S. 28 sowie die dort angegebene umfangreiche Literatur. Zur Markenkompetenz vgl. auch Schütz, K., Markenkompetenz als Ansatz der Markenführung - dargestellt an einem ausgewählten Beispiel, unveröffentlichte Diplomarbeit am Institut für Marketing der Universität Münster, Münster 2000. 
einer Marke durch ihre spezifische Zugehörigkeit zu einem Unternehmen besonders betont werden kann. In diesem Zusammenhang wurden die in der Vergangenheit schwachen Markenidentitäten von SKODA und SEAT erheblich durch die Integration in den Volkswagen-Konzern beeinflusst. In der "Marke als Person" kann sich schliesslich eine besondere Beziehungskompetenz ausdrücken. So ermöglichen eine spezifische kulturelle Verankerung der Marke (RollsRoyce mit britischer Herkunft) bzw. typische Verwender der Marke (RollsRoyce als Fahrzeug der Monarchen) dem Konsumenten verstärkt, sich mit der jeweiligen Marke zu identifizieren und ihr gegenüber ein Vertrauen aufzubauen. ${ }^{176}$ Die "Marke als Symbol" fasst letztlich die spezifischen Kompetenzen einer Marke zusammen, indem etwa mit Hilfe des Markenzeichens bzw. -namens oder durch eine besondere Kommunikation die Besonderheiten zum Ausdruck gebracht werden. Symbolische Werte einer Marke wie Kult und Mythos ${ }^{177}$ tragen insofern nur dann zu einer starken Markenidentität bei, wenn sie durch spezifische Kompetenzen der Marke begleitet werden.

Sind die spezifischen Merkmale der einzelnen Marken identifiziert und zu einem markenspezifischen Kompetenzbündel integriert, lässt sich dieses Kompetenzbündel schliesslich in Form eines Markenleitbildes anschaulich verdeutlichen. ${ }^{178}$ Das Markenleitbild bringt als "realistisches Idealbild" einer Marke die spezifische Kompetenz, die Visionen, die grundlegenden Wertvorstellungen und Ziele sowie

176 Der Aufbau von Vertrauen lässt sich in diesem Zusammenhang insbesondere mit der hohen Affinität zwischen dem Selbstbild des Konsumenten und den mit der Marke assoziierten Persönlichkeitsmerkmalen begründen. Nach FOURNIER ist die Persönlichkeit einer Marke eine wesentliche Grundlage der Beziehung Marke-Konsument. Vgl. Fournier, S. M., Consumers and Their Brands: Developing Relationship Theory, in: Journal of Consumer Research, Vol. 24., March 1998, S. 343ff.

177 Automobile haben besondere Chancen, Mythen zu zeugen, da sie dem Menschen immanente Sehnsüchte nach individueller Mobilität verkörpern. Die Mythodologie basiert dabei auf der Theorie des Animismus (Theorie der Kulturanthropologie), die durch S. FREUD (Mythos ist Reflex verdrängter individueller Wünsche) und C. G. JUNG (Mythos ist die seelische Erfahrung überindividueller Wahrheiten) psychologisch gedeutet wurde. Vgl. hierzu Kinast, K., Das Entstehen von Mythos und die Erstarrung einer Marke als Höhepunkt ihrer Entwicklung, in: der markt, Heft 2, 1995, S. 73ff. Vgl. hier auch Schweiger, G., Friederes, G., Vom Markenmythos zum Markenwert, in: Werbeforschung und Praxis, Heft 1, 1995, S. $26 \mathrm{ff}$.

Vgl. Kapferer, J.-N., Die Marke - Kapital des Unternehmens, a.a.O., S. 110f; Matje, A., Unternehmensleitbilder als Führungsinstrument: Komponenten einer erfolgreichen Unternehmensidentität, Wiesbaden 1996, S. 54. Zur Rolle des Leitbildes eines Unternehmens vgl. Roth, F., Ickstadt, C., Die geheime Quelle der Stärke, in: Horizont Magazin, Nr. 3, 1999, S. 92. Vgl. zu Leitbildern auch Carbon, M., Leitbilder, in: Effizientes Informationsmanagement in dezentralen Organisationsstrukturen, Bullinger, H.-J. (Hrsg.), Berlin u.a., S. $87 f f$. 
das Verhältnis der Marke zu den wesentlichen internen und externen Bezugsgruppen „plakativ"179 zum Ausdruck. In Abb. 8 sind beispielhaft die Leitbilder aus dem Markenportfolio des Volkswagen-Konzerns angeführt. ${ }^{180}$

The Gentleman's
Sporting Tourer

\section{Abb. 8: Markenleitbilder in der Automobilindustrie}

Dabei kommen Markenleitbildern unterschiedliche Funktionen zu:

- Entwurf einer realistischen Zukunftsvorstellung der Marke

- Festigung der Markenidentität nach innen und aussen.

- Identifikations- und Motivationsfunktion

- Orientierungs- und Stabilisierungsfunktion

- Erleichterung der Koordination

Im Markenleitbild drückt sich das artikulierte, zukunftsorientierte Selbstbild der Marke aus Sicht der Unternehmung aus, so dass seine Formulierung alle Beteiligten zum Entwurf einer derartigen Zukunftsvorstellung zwingt. ${ }^{181}$ Infolge der anschaulichen Darstellungsform entfalten Markenleitbilder eine Kommunikationswir-

So COLLINS/PORRAS: „Anders als die Unternehmensphilosophie, die als dauerhaftes Tragwerk im Hintergrund bleibt, steht das Leitbild vorn im Blickfeld und lenkt die Aufmerksamkeit aller im Unternehmen auf ein konkretes Ziel. Die Unternehmensphilosophie ist ihrem Wesen entsprechend tief und ruhig, das Leitbild hingegen kühn, aufregend und emotional fordernd." Vgl. Collins, J., Porras, J., Werkzeug Vision - Wie Unternehmensphilosophie und Leitbilder visionäre Unternehmen prägen, in: Harvard Business Manager, Heft 4, 1992, S. 113. Ähnlich für das Unternehmensleitbild Bleicher, K., Das Konzept integriertes Management, 4. Aufl., Frankfurt a. M., New York, 1996, S. 218.

180 Vgl. auch Clef, U., Mehrmarkenstrategie für die Pole-Position, a.a.O., S. 78.

181 Nach BLEICHER fordert die vielfach vorhandene Lücke zwischen tatsächlichem und für die Bewältigung der Zukunft notwendigem Verhalten aller Mitarbeiter den Entwurf eines Zukunftsfits von externen und internen Unternehmensentwicklungen, welcher über eine längere zeitliche Strecke gültig sein kann. Vgl. Bleicher, K., Leitbilder: Orientierungsrahmen für eine integrative Management-Philosophie, Stuttgart 1992, S. 21. 
kung zur innen- und aussengerichteten Festigung der Markenidentität im Sinne gelebter "shared values". Als Identifikations- und Motivationsanker dient das Markenleitbild unternehmensintern zur Bündelung der „Zentrifugalkräfte“"182 aller durch die Arbeitsteilung bedingten bereichsbezogenen Aktivitäten und fördert damit eine integrative Wirkung aller markenbezogenen Maßnahmen im Portfolio. Gleichzeitig stellt das Markenleitbild einen Fokus zur Imagebildung bei den externen Anspruchsgruppen dar, auf den sich die operative und strategische Markenführung beziehen kann und das damit als Grundlage für jegliche Markendarstellung dient.

Zur Erfüllung ihrer Funktionen müssen Markenleitbilder prägnant, glaubwürdig und authentisch sowie auf längere Sicht bestimmt sein. Dabei tragen insbesondere das Verhalten und die Wertvorstellungen der obersten Markenführungskräfte zur Glaubwürdigkeit des Markenleitbildes bei. Allerdings „gibt es kein Rezept für die Formulierung und Gestaltung von Leitbildern [...] Würden diese Grundsatzpapiere nämlich nach normierten Erkenntnissen verfaßt, entstünden uniforme [Marken, Anm. d. V.]. Nicht die Eigenständigkeit würde damit gefördert, sondern gerade das Gegenteil [...]. ${ }^{4183}$

Zusammenfassend umfasst die innengerichtete Definition von strategischen Rollen somit die Identifikation einzigartiger und dauerhaft haltbarer Merkmale und Fähigkeiten für jede Marke des Portfolios. ${ }^{184}$ Durch die Merkmalsbündelung lässt sich die spezifische Kompetenz einer jeden Portfoliomarke zum Ausdruck bringen. Mit Bezug auf den RBV kann mit BURMANN gerade in dieser kombinatorischen bzw. koordinativen Fähigkeit des Management eine wichtige Bedingung zum Aufbau von Wettbewerbsvorteilen mit den Marken im Portfolio gesehen werden. ${ }^{185}$ Die inhaltliche Charakterisierung der einzelnen Marken kann jedoch nicht losgelöst von der Marktperspektive vorgenommen werden, da über die Relevanz von

Bleicher, K., Leitbilder: Orientierungsrahmen für eine integrative Management-Philosophie, a.a.O., S. 5. Zentrifugalkräfte rekurrieren auf ein suboptimales Streben bei der Durchsetzung von individuellen Interessen.

Fankart, P., Widmer, H., Rezept - Corporate Identity. Leitbild, Erscheinungsbild, Kommunikation, Zürich, Wiesbaden 1987, S. 25. Vgl. zu den Anforderungen auch Langen, A., Leitbild und Unternehmenskultur: Die Rolle des Topmanagements, in: Herausforderung Unternehmenskultur, Simon, H., (Hrsg.), Stuttgart 1990, S. 43.

So wird Audi beispielsweise eine besondere Technologiekompetenz, VW aufgrund eines umfangreichen Händlernetzes eine besondere Servicekompetenz zugeschrieben.

Vgl. Burmann, Ch., Strategiewechsel in turbulenten Märkten - Neuere theoretische Ansätze zur Unternehmensflexibilität, a.a.O., S. $24 f$. 
Merkmalen und Fähigkeiten einer Marke letztlich immer der Markt entscheidet. Vor diesem Hintergrund soll die marktgerichtete Sichtweise näher beleuchtet werden.

\subsection{Marktgerichtete Positionierung}

Das Ziel der auch als Kernentscheidung des Marketing ${ }^{186}$ bezeichneten Positionierung besteht darin, mit jeder Marke des Portfolios sowohl eine dominierende Stellung in der Psyche der Konsumenten als auch eine hinreichende Differenzierung gegenüber den Marken der relevanten Wettbewerber und den eigenen Marken des Portfolios zu erzielen. Hierzu sind sowohl

- die mit Priorität zu bearbeitenden Zielsegmente zu bestimmen (z. B. preisbewusste Käufergruppen oder Käufergruppen mit gehobenen Ansprüchen) bzw. der Grad der durch die einzelne Marke angestrebten Marktabdeckung festzulegen ${ }^{187}$, als auch

- eine Auswahl der relevanten Wettbewerber vorzunehmen, welche vor allem unter Berücksichtigung der im Markt beobachteten Konkurrenzbeziehungen erfolgt.

Zur Sicherstellung einer eindeutigen Orientierung der Portfoliomarken im Wettbewerbsumfeld sowie zur Vermeidung eines Intramarkenwettbewerbs im Markenportfolio ist es dabei zweckmäßig, zwischen strategischen und operativen Wettbewerbern zu differenzieren. Während operative Wettbewerber solche Hersteller

Vgl. Hooley, G. H., Saunders, J., Competitive Positioning: The Key To Marketing Strategy, New York 1993. Zuweilen wird Positionierung allerdings auch als obsolet gewordenes Relikt der Marketingvergangenheit bezeichnet. Vgl. etwa Gerken, G., Abschied vom Marketing, Düsseldorf 1991, S. 1ff.

Die segmentspezifische Markenpolitik setzt grundsätzlich eine verhaltenswissenschaftliche Analyse voraus, in welcher die Konsumenten anhand ihrer Ausprägungen von Bestimmungsfaktoren des Markenwahlverhaltens in Segmente zusammengefasst werden, die in sich möglichst homogen und untereinander möglichst heterogen sind, um sodann eine gezielte Bearbeitung der Marktsegmente mit Hilfe der verschiedenen Portfoliomarken vorzunehmen. Für das Führen mehrerer Automobilmarken sind die Segmente idealtypisch so abzubilden, dass die Konsumenten innerhalb eines Segments eine entsprechend ähnliche Reaktion auf die spezifischen Merkmale einer Marke A ungleich der Reaktion auf die spezifischen Merkmale einer Marke B etc. aufweisen, d.h. im Portfolio mit zwei Marken muss gelten $X_{k}=f_{k}$ (Marke $A$ ) $\neq f_{k}$ (Marke $B$ ), wobei $X_{k}$ das Kaufverhalten des Konsumenten $k$ kennzeichnet und $f_{k}$ den funktionellen Zusammenhang zwischen den Merkmalen der Marke A bzw. B und der Reaktion des betrachteten Konsumenten. Da sich die Messung solch markenbezogener Reaktionsfunktionen als äußerst schwierig erweist, wird zumeist auf geeignete Ersatzkriterien zurückgegriffen. Diese lassen sich nach unterschiedlichen Gesichtspunkten zu Kriteriengruppen zusammenfassen, wobei eine verbreitete Systematisierung eine Unterscheidung in geographische, soziodemographische, psychographische und verhaltensorientierte Kriterien der Marktsegmentierung vornimmt. Zur Problematik der Bestimmung Marketing-Mix bezogener Reaktionskoeffizienten Freter, H., Marktsegmentierung, Stuttgart 1983, S. $45 \mathrm{ff}$. 
darstellen, die eine Homogenität von Leistungsspektrum, Marketingstrategie und arealstrategischer Präsenz aufweisen, orientiert sich die Definition strategischer Wettbewerber am langfristigen, visionären Kompetenzanspruch der Marken im Wettbewerbsumfeld. ${ }^{188}$ In der zu präzisierenden Rolle der Marke im Wettbewerb wird schließlich das Marktverhalten der Marke gegenüber der Konkurrenz festgelegt, wobei grundsätzlich innovative bzw. imitative sowie wettbewerbsstellende bzw. -vermeidende Verhaltensweisen verfolgt werden können.

In dem Bewusstsein, dass nur solche Marken auf Dauer erfolgreich sein können, die mit ihren Leistungen möglichst genau den artikulierten oder latent vorhandenen Bedürfnissen der Konsumenten in den zu bearbeitenden Segmenten entsprechen, sind spezifische Marktpositionen festzulegen, in denen die Bedürfnisse der priorisierten Zielsegmente mit "maßgeschneiderten" Angeboten in der subjektiven Wahrnehmung der Konsumenten dauerhaft besser als von anderen Anbietern erfüllt werden. ${ }^{189}$ Da die Positionierung für den Konsumenten erst durch konkrete Markeneigenschaften als Fundament der Wahrnehmung "greifbar" wird, sind die Marktpositionen aufbauend auf den innengerichtet identifizierten, wesensprägenden Merkmalen der Marken derart zu formulieren, dass für jede Marke eine positive Alleinstellung im Verhältnis zum Wettbewerb und den übrigen Marken des Portfolios geschaffen wird. ${ }^{190}$ Zur Erreichung dieser angestrebten Soll-Positionen

Beispielhaft seien hier operative und strategische Wettbewerber im WW-Konzern aufgeführt:

\begin{tabular}{|l|l|l|l|l|}
\hline & WW & SEAT & SKODA & AUDI \\
\hline Strategisch & MERCEDES-BENZ & ALFA ROMEO & VOLVO & BMW \\
\hline Operativ & OPEL & FIAT & FIAT & BMW \\
\hline
\end{tabular}

Vgl. Clef, U., Mehrmarkenstrategie für die Pole-Position, a.a.O., S. 72ff. Während der Intramarkenwettbewerb die Konkurrenzbeziehungen der Portfoliomarken untereinander beschreibt, bezieht sich der Intermarkenwettbewerb auf den Wettbewerb zwischen den Portfoliomarken und den sonstigen Anbietern auf den jeweiligen Märkten.

189 Vgl. Tomczak, T., Roosdorp, A., Positionierung - Neue Herausforderungen verlangen neue Ansätze, in: Positionierung: Kernentscheidung des Marketing, Tomczak, T., Rudolph, Th., Roosdorp, A., (Hrsg.), St. Gallen 1996, S. 26.

190 Vgl. auch Kotler, Ph., Über die Entwicklung von Wertangeboten zur Unique Selling Proposition, in: absatzwirtschaft, Heft 3, 2000, S. 46ff.; KELLER unterscheidet in diesem Zusammenhang eine Point-of-difference- Assoziation (,assoziations that are unique to the brand ") und eine Point-of-parity-Assoziation (, assoziations that are not necessarily unique to the brand but may be shared with other brands"). Vgl. Keller, K. L., Strategic Brand Management: Building, Measuring, and Managing Brand Equity, a.a.O., S. 116f. 
im Wahrnehmungsraum der Nachfrager lassen sich folgende Voraussetzungen anführen: ${ }^{191}$

- mit jeder Portfoliomarke sollte ein anderer zielgruppenspezifischer Nutzen angesprochen werden (Zielgruppenbezug).

- der Nutzen sollte für die jeweilige Zielgruppe deutlich wahrnehmbar sein (Wahrnehmbarkeit).

- der Nutzen sollte durch die Marke in ein physikalisch funktionales Angebot oder im Rahmen der werblichen Umsetzung in eine Unique Advertising Proposition (UAP) transformiert werden können (Realisierbarkeit).

- der Nutzen sollte die einzelnen Marken vom Wettbewerb und innerhalb des Portfolios abgrenzen (Inter- und Intradiskriminanz).

- der Nutzen sollte möglichst lange Gültigkeit besitzen (zeitliche Stabilität).

Die Herausforderung im Rahmen der Positionierungsentscheidungen in Mehrmarkenstrategien besteht darin, mit dem Gesamtportfolio eine bestmögliche Abdekkung des Gesamtmarktes sicherzustellen. Grenzen der Positionierungsfreiräume werden durch die zuvor identifizierten essentiellen, wesensprägenden Merkmale der Marken gesetzt. ${ }^{192}$ Der als Grundlage der Positionierung dienende und von den einzelnen Marken angesprochene Nutzen kann sowohl auf physikalisch funktionale Leistungsmerkmale rekurrieren als auch einen psychologischen Zusatzbzw. Geltungsnutzen darstellen. ${ }^{193}$

Wichtig ist, dass sich die Positionierungen nicht ausschließlich an den artikulierten Wünschen der Konsumenten im Sinne einer reaktiven Positionierung orientieren. ${ }^{194}$ Die fortschreitende Annäherung vieler Marken an das Idealbild der Konsumenten erschwert einerseits eine klare Wettbewerbsdifferenzierung, andererseits

191 Vgl. hierzu Meffert, H., Perrey, J., Mehrmarkenstrategie - Ein Beitrag zum Management von Markenportfolios, a.a.O., S. 639ff; Haedrich, G., Tomczak, T., Strategische Markenführung, in: Handwörterbuch Markenartikel, Bruhn, M. (Hrsg.), Band 2, Stuttgart 1994, S. 932 f.

192 So wird nicht jede Automarke jeden Autotyp anbieten können. Ein Mercedes Pick-Up ist ebenso wenig vorstellbar wie ein Rolls-Royce MPV.

Vgl. Nommensen, J. N., Die Prägnanz von Markenbildern: Prüfung der Kommunikationsstrategie bei Produktrepositionierung, Heidelberg 1990, S. 13 ff; Vgl. Vershofen, W., Handbuch der Verbrauchsforschung, Band 1, Berlin 1940, S. 71. Vgl. zur sachorientierten Positionierung bei hohem kognitiven Involvement sowie zur erlebnisbetonten Positionierung bei hohem emotionalen Involvement Esch, F.-R., Markenpositionierung als Grundlage der Markenführung, in: Moderne Markenführung: Grundlagen - innovative Ansätze - praktische Umsetzung, Esch, F.R. (Hrsg.), Wiesbaden 1999, S. $242 f$.

$\mathrm{Zu}$ Recht weist Trommsdorff darauf hin, dass klassische Positionierungsmodelle eine fundamentale Schwäche aufweisen. Vgl. Trommsdorf, V., Wettbewerbsorientierte ImagePositionierung, in: Markenartikel, Heft 10, 1992, S. 460. 
wird das Spektrum möglicher Innovationen auf die artikulierten Bedürfnisse beschränkt. Vor diesem Hintergrund sind auch latent vorhandene Kundenwünsche bzw. zukünftig relevante Bedürfnisse und Nutzenkombinationen zu eruieren und mit entsprechenden Marken zu bedienen. Mit RIES/TROUT liegt ein wirklicher Wettbewerbsvorteil nur dann vor, wenn zwar dem Kunden bisher unbekannte, für seine Kaufentscheidung aber wichtige Eigenschaftsdimensionen besetzt werden. ${ }^{195}$ Letztlich lassen sich nur so Innovationen am Markt realisieren.

Insgesamt setzt die wirksame Ausübung von Mehrmarkenstrategien eine fortlaufende Aktualisierung und Anpassung der Markenpositionierungen voraus, wobei im Einzelfall sowohl Umpositionierungen als auch Neupositionierungen vorzunehmen sind. ${ }^{196}$ Dabei steigt die Erfolgswirksamkeit der einzelnen Markenpositionierung allerdings mit deren Kontinuität, die zum Aufbau eines klaren Markenimages bei den Nachfragern beiträgt und damit eine wesentliche Voraussetzung zur Erzielung dauerhafter Wettbewerbsvorteile darstellt. ${ }^{197}$

Zusammenfassend stellt die Formulierung strategischer Rollen für die einzelnen Marken des Portfolios die zentrale Herausforderung bei der strategischen Ausgestaltung von Mehrmarkenstrategien dar. Die Festlegung der Markenpositionierungen setzt unmittelbar an den hierin für die einzelnen Marken definierten Ausrichtungen an, welche durch die Markenleitbilder innen- und außenwirksam gefördert werden. Die als Basis zur Entwicklung und Festigung des Vertrauens der Nachfrager in die Marke zu interpretierenden Markenidentitäten können dabei nicht im Sinne einer deterministischen Mittel-Zweck-Beziehung durch die einmalige Konzeption der strategischen Rahmenplanung abgeleitet werden, sondern entstehen über einen längeren Zeitraum in einer Wechselseitigkeit marktgerichteter Handlungen der Marken (Selbstbild der Markenidentitäten) und der Wahrnehmung dieser Handlungen (Fremdbild der Markenidentitäten) durch die Nachfrager. Die Förderung differenzierter Markenidentitäten setzt somit eine fortgesetzt zu aktualisierende Umsetzung der durch die strategische Rahmenplanung vorgegebenen Stoßrichtungen des Marktauftritts voraus.

195 196 197

Vgl. Ries, A., Trout, J., Positioning: Die neue Werbestrategie, Hamburg 1986, S. $79 \mathrm{ff}$.

Vgl. Haedrich, G., Tomczak, T., Strategische Markenführung, a.a.O., S. $934 f$.

97 Vgl. Esch, F.-R., Andresen, Th., 10 Barrieren für eine erfolgreiche Markenpositionierung und Ansätze zu deren Überwindung, in: Positionierung: Kernentscheidung des Marketing, Tomczak, T., Rudolph, Th., Roosdorp, A. (Hrsg.), St. Gallen 1996, S. 78. 


\subsection{Ausgestaltung der Instrumentalstrategien}

Die Ausgestaltung der Instrumentalstrategien in Markenportfolios zielt auf die Schaffung eigenständiger und damit innerhalb des Markenportfolios differenzierender Markenidentitäten durch eine integrierte Gestaltung und Ausrichtung aller Marketing-Instrumente ab. Das markenpolitische Entscheidungsfeld der einzelnen Marken wird in diesem Zusammenhang unmittelbar von den in der strategischen Rahmenplanung formulierten Stoßrichtungen des Marktauftritts eingegrenzt. Konkret bedarf es auf der Maßnahmenebene der Entwicklung einer strikt an der Positionierung der einzelnen Portfoliomarken ausgerichteten widerspruchsfreien Instrumentekombination. Eine widerspruchsfreie Umsetzung der vorgegebenen Leitbilder und Positionierungen durch markenspezifische Marketing-Programme fördert die Glaubwürdigkeit und Unverwechselbarkeit der Marken in der Wahrnehmung durch die Nachfrager und bildet damit die Voraussetzung zur Schaffung differenzierter Markenidentitäten. ${ }^{198}$

Im Markenportfolio bestehen sowohl zwischen den Instrumenten des MarketingMix einer Marke als auch zwischen den Instrumentekombinationen der verschiedenen Marken zahlreiche Wechselwirkungen, deren Grad der Berücksichtigung die Wirksamkeit des Marktauftritts durch das Markenportfolio determiniert (vgl. Abbildung 9).

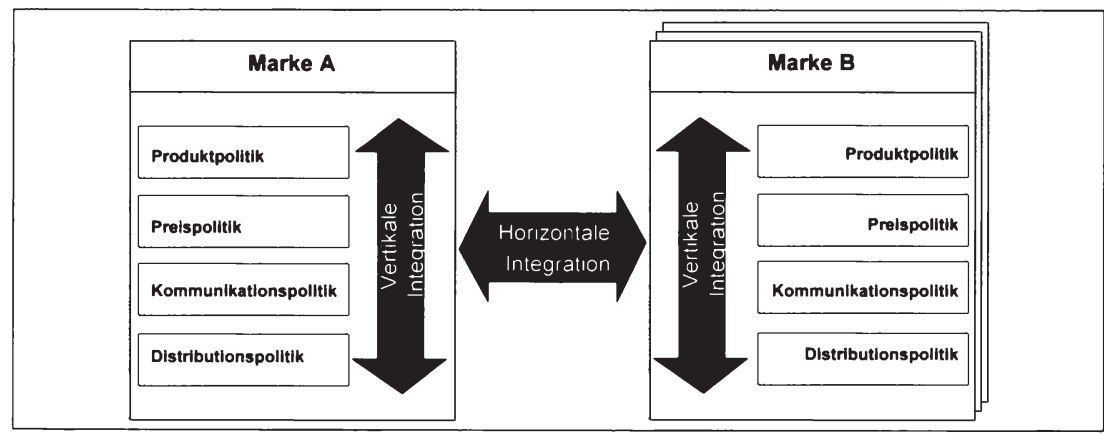

Abb. 9: Markenspezifische und markenübergreifende Integration der MarketingInstrumente im Rahmen von Mehrmarkenstrategien

198 Vgl. hierzu und im Folgenden auch Meffert, H., Perrey, J., Mehrmarkenstrategie - Ein Beitrag zum Management von Markenportfolios, a.a.O., S. $642 f$. 
Bei der Festlegung einer optimalen Instrumentekombination bedarf es somit sowohl einer markenspezifischen (vertikalen) als auch markenübergreifenden (horizontalen) Integration der Marketing-Instrumente. Die an den Oberzielen des Portfolios orientierte Ausgestaltung der Instrumentalstrategien setzt somit gleichermaßen an der Ableitung markenübergreifender Marketing-Programme - etwa im Rahmen der Nutzung vorhandener Synergien in der Leistungspolitik - wie an der Entwicklung spezifischer, auf die Positionierung der einzelnen Marken basierender Maßnahmen an.

In diesem Zusammenhang stellt die Identifikation der zur Sicherstellung einer starken Markenidentität von jeder Marke selbstständig zu produzierenden Leistungskomponenten bzw. autonom auszuführenden Geschäftsprozesse eine zentrale Aufgabe dar. Zur Erreichung der übergeordneten Zielsetzungen ergibt sich somit in Mehrmarkenstrategien das Optimierungsproblem einer bestmöglichen Synergienutzung innerhalb des Portfolios, ohne den auf die Eigenprofilierung abstellenden Marktauftritt der einzelnen Marke zu gefährden. Eine Abstimmung der Instrumentebündel erfolgt dabei aufbauend auf den strategischen Rollen der einzelnen Marken durch die

- Festlegung des markenübergreifenden Standardisierungsgrades bzw. notwendiger technischer Differenzierungskorridore im Rahmen der Produktpolitik,

- Fixierung von Preiskorridoren zwischen den Marken im Rahmen der Preispolitik,

- Definition von Kommunikationsplattformen im Rahmen der Kommunikationspolitik sowie durch die

- leitbildgerechte Ausgestaltung des Vertriebsweges ${ }^{199}$ bzw. Kundendienstes. $^{200}$

Die Gestaltung des Händlernetzes nimmt in der Automobilbranche einen besonderen Stellenwert ein, da es sich beim Automobil um ein komplexes Gebrauchsgut handelt, das sowohl einen intensiven persönlichen Verkauf erfordert als auch wartungs- und reparaturbedürftig ist, so dass neben dem Verkaufsaspekt auch dem Serviceaspekt eine hohe Relevanz beizumessen ist. Zudem kommt der Vertriebsorganisation von Automobilherstellern eine bedeutende präferenzbildende Funktion zu, da die Zufriedenheit mit der Verkaufs- und Kundendienstleistung eines Händlers als eine wesentliche Komponente in die Gesamtzufriedenheit eines Kunden während sowie nach dem Automobilkauf eingeht. Vgl. hierzu etwa Burmann, Ch., Konsumentenzufriedenheit als Determinante der Marken- und Händlerloyalität - Das Beispiel der Automobilindustrie, in: Marketing ZFP, Heft 4, 1991, S. 249ff.; Hoffmeister, M., MultiFranchise-Konzepte im Automobileinzelhandel: Entwicklungen und Auswirkungen auf die Absatzkanalpolitik der Automobilhersteller, Wiesbaden 1998, S. 1.

Da technische Differenzierungspotenziale durch den markenübergreifenden Rückgriff auf gemeinsame Produktkomponenten zunehmend ausgeschöpft werden, gewinnen zur marken- 
An zusätzlicher Komplexität gewinnt die Ausgestaltung der Instrumentalstrategien durch die Berücksichtigung länderspezifischer Besonderheiten im Marktauftritt des Portfolios. So lassen die international unterschiedlichen Markenwahrnehmungen oftmals eine modifizierte Ausgestaltung des absatzpolitischen Instrumentariums erforderlich werden, welche nicht selten mit einer Anpassung der strategischen Rahmenplanung einhergehen. Zudem werden verschiedene Absatzmärkte häufig mit unterschiedlichen Marken bearbeitet, so dass die Festlegung einer Kombination aus länderübergreifend standardisierten und länderspezifisch variierten Instrumentekombinationen ein äußerst komplexes Problem im Rahmen der Umsetzung von Mehrmarkenstrategien darstellt. ${ }^{201}$ Eine situative Anpassung der strategischen Rahmenplanung im Sinne hybrider Markenstrategien lassen sich etwa an der Marke SKODA des Volkswagen-Konzerns verdeutlichen: Stellt die Marke SKODA im osteuropäischen Raum aufgrund ihres dortigen hohen Images und ihrer vergleichsweise hohen Qualität in der Wahrnehmung der Konsumenten eine Premiummarke dar, wird SKODA in Westeuropa als Preismarke definiert. Entsprechend sind die geographisch unterschiedlich definierten Rollen in der Instrumenteausgestaltung zu berücksichtigen.

\subsection{Organisatorische Verankerung von Mehrmarkenstrategien}

Die Notwendigkeit, in Mehrmarkenstrategien eigenständige und innerhalb des Markenportfolios scharf abgegrenzte Markenidentitäten zu schaffen, lässt eine weitgehende Zusammenfassung der an der Entwicklung und Ausprägung der einzelnen Markenidentitäten mitwirkenden Personen in organisatorischen Einheiten als zweckmäßig erscheinen. ${ }^{202}$ Eine mehrmarkenadäquate Organisationsstruktur

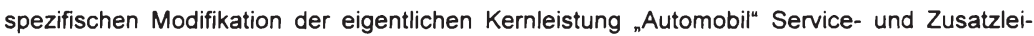
stungen an Bedeutung. Entsprechend kommt der Service- bzw. Kundendienstpolitik eine zunehmende Relevanz für die Zufriedenheit des Kunden zu. Vgl. zu Bedeutung und Zielen der Kundendienstpolitik auch Meffert, H., Kundendienstpolitik - Eine Bestandsaufnahme zu einem komplexen Marketinginstrument, in: Marketing ZFP, Heft 2, Mai 1987, S. 93ff. Zu ValueAdded Services vgl. Laakmann, K., Value-Added Services als Profilierungsinstrument im Wettbewerb. Analyse, Generierung und Bewertung, Frankfurt a.M. U.a. 1995

201 Vgl. Keller, K. L., Strategic Brand Management: Building, Measuring, and Managing Brand Equity, a.a.O., S. 550; Meffert, H., Perrey, J., Mehrmarkenstrategie - Ein Beitrag zum Management von Markenportfolios, a.a.O., S. 642. Zu den Gefahren globaler Marken vgl. auch Aaker, D. A., Joachimsthaler, E., The Lure of Global Branding, in: Harvard Business Review, No. 6, Nov.-Dec. 1999, S. $137 f f$.

202 Vgl. Meffert, H., Burmann, Ch., Identitätsorientierte Markenführung - Konsequenzen für die Handelsmarke, a.a.O., S. 56. 
kann dabei sowohl mit einer Anpassung der Primärorganisation des Unternehmens einhergehen als auch eine Hierarchieergänzung bzw. -überlagerung der bisherigen Unternehmensstruktur in Form einer Sekundärorganisation bewirken. ${ }^{203}$ In Analogie zur organisatorischen Eingliederung von strategischen Geschäftseinheiten lassen sich folgende Alternativen zur Einbindung der Markeneinheiten in die Unternehmensorganisation anführen: ${ }^{204}$

- Organisatorische Markeneinheiten in Form von interfunktionalen, hierarchieergänzenden Ausschüssen, Gremien und Komitees zur Ziel- und Strategievereinbarung sowie zum Informationsaustausch. Hier werden Markeneinheiten in Kollegien zur gemeinsamen Strategieberatung zusammengeführt, wobei das Kollegium auf Empfehlungsbasis arbeitet, deren Vorschläge für die Linien allerdings unverbindlich bleiben.

- Beauftragung von Brandmanagern für strategische und operative Markenangelegenheiten. Spezifische Markenmanager sind für einzelne Portfoliomarken verantwortlich, wobei im Falle eines beschränkt funktionalen Weisungsrechts jedoch keine Durchsetzung des Markenkonzepts garantiert ist.

- Bildung von organisatorischen Markeneinheiten, die sich hinsichtlich ihrer Kompetenz in Form einer Matrix mit der Linienorganisation überschneiden. Neben der Linie haben auch die Markeneinheiten Verantwortung für Produkt und Marktentscheidungen.

- Ausrichtung und Anpassung der Linienorganisation an die Struktur der Markeneinheiten, wodurch letztlich eine markenspezifische Ausrichtung der Organisation des Gesamtunternehmens bezweckt wird.

Aufgrund der zahlreichen, funktionsbereichsübergreifenden Komponenten und Wechselbeziehungen bei der Entstehung der Markenidentität und der damit einhergehenden Notwendigkeit einer institutionellen Bündelung aller markenprägenden Funktionen und Prozesse erweist es sich in Mehrmarkenstrategien als sinnvoll, die verschiedenen Portfoliomarken in separaten organisatorischen Einheiten mit einer entsprechenden organisatorischen Anpassung der Gesamtunternehmensorganisation zu führen. ${ }^{205}$ Ein solcher struktureller Rahmen vermag die

203 Während die Primärorganisation alle dauerhaften Organisationseinheiten beinhaltet, die durch hierarchische Beziehungen miteinander verknüpft sind, umfasst die Sekundärorganisation alle hierarchieergänzenden und hierachieübergreifenden Organisationsstrukturen. Vgl. hierzu Schulte-Zurhausen, M., Organisation, a.a.O., S. 221 und 247. Vgl. in diesem Zusammenhang auch Brockhoff, K., Hauschildt, J., Schnittstellen-Management - Koordination ohne Hierarchie, a.a.O., S. 401.

Vgl, etwa Bühner, R., Betriebswirtschaftliche Organisationslehre, a.a.O., S. $201 f$.

In der Unternehmenspraxis findet sich neben einer organisatorischen Verselbstständigung überdies häufig auch eine rechtliche Selbstständigkeit der einzelnen Portfoliomarken (etwa Automobilmarken), wobei die Führung vielfach unter dem gemeinsamen Dach einer Manage- 
Individualität als konstitutives Identitätsmerkmal der jeweiligen Portfoliomarken am ehesten sicherzustellen, wobei es naheliegt, die so entstandenen organisatorischen Einheiten als Träger der Marken so eigenständig und von den anderen Marken bzw. deren Organisationseinheiten getrennt wie möglich zu führen. ${ }^{206}$

Die organisatorische Eigenständigkeit der Marken geht mit einer Entlastung der Portfolioleitung einher, da Entscheidungen auf die Markenebenen delegierbar sind. Zudem begünstigt eine organisatorische Trennung der Marken die Entwicklung differenzierter Markenkulturen, welche die Identifikation und Motivation der Mitarbeiter mit der jeweiligen Marke fördert und ein verstärktes eigenverantwortliches Verhalten im Sinne eines Entrepreneurship zu realisieren vermag. Gleichzeitig erhöht sich die Flexibilität im Markenauftritt sowie die Marktnähe der jeweiligen Portfoliomarken zu den Bedürfnissen der Nachfrager und Wettbewerber nach auBen.

Allerdings wird bei der Verfolgung einer Mehrmarkenstrategie weniger eine Optimierung des Marktauftritts einzelner Marken als vielmehr eine bestmögliche Marktbearbeitung durch das Gesamtportfolio zur Sicherstellung übergeordneter Portfoliozielsetzungen angestrebt, so dass eine zu umfassende Dezentralisierung und Verlagerung der Verantwortung auf einzelne Markeneinheiten auch spezifische Gefahren in sich birgt. Eine wesentliche Gefahr ist in einer fehlenden Berücksichtigung markenübergreifender Synergievorteile zur Produktivitätssicherung des Gesamtportfolios zu sehen. Da Strategieentwicklung durch das Portfoliomanagement und Strategiedurchführung durch die markenspezifischen Organisationseinheiten auseinanderfallen, besteht zudem das Risiko einer mangelnden Konzentration der einzelnen Marken auf ihre Kernfähigkeiten bzw. einer unzureichenden Realisierung ihrer Mission im Markenportfolio. Unter Portfoliogesichts-

ment-Holding erfolgt. Wesentliche Merkmale der Management-Holding sind die Trennung strategischer Aufgaben der Unternehmensleitung von operativen Aufgaben der geschäftsführenden Bereiche sowie die Identität von Rechts- und Organisationsstruktur. Vgl. etwa Schulte-Zurhausen, M., Organisation, a.a.O., S. $238 \mathrm{ff}$.

206 Überdies ist die Aufgabe der Markenführung heute stärker als bisher als Tätigkeit im Rahmen einer gesamtunternehmerischen Mitentscheidungs- und Mitverantwortungsfunktion aufzufassen, so dass etwa eine Stabstelle ohne bzw. mit erheblich eingeschränkten Kompetenzen dieser Aufgabe nur schwer gerecht werden kann. Vgl. Haedrich, G., Tomczak, T., Strategische Markenführung: Planung und Realisierung von Marketingstrategien für eingeführte Produkte, a.a.O., S. 72. Zur Effizienz der Organisationsstruktur mit Marken- bzw. Produktmanagern vgl. auch Low, G. S., Fullerton, R. A., Brand, Brand Management, and the Brand Manager System: A Critical-Historical Evaluation, in: Journal of Marketing Research, Vol. 31, May 1994, S. $181 \mathrm{ff}$. 
punkten sind Entscheidungen einzelner Markenmanager überdies vielfach wenig zielführend, da Leiter markenspezifischer Einheiten gerade im Falle kurzfristig orientierter Erfolgsbeurteilungen häufig risikoscheu bzw. markenegoistisch handeln. $^{207}$

Zur Sicherstellung der übergeordneten Portfolioziele sind die separaten Markeneinheiten somit als Elemente eines komplementären, synergetischen Portfolios zu steuern. Die Führung des Portfolios „aus der Einheit des Ganzen heraus ${ }^{\text {208 }}$ verlangt eine Zentralisierung spezifischer Entscheidungen wie etwa der Festlegung strategischer Rollen und Positionierungen einzelner Marken. Durch eine Vernetzung der organisatorisch selbstständigen Markeneinheiten sowie deren Geschäftsprozesse ist die gemeinsame Nutzung zentraler Ressourcen durch möglichst viele Marken sicherzustellen. Hierdurch reduziert sich der Grad markenspezifischer Autonomie entlang der vollständigen Wertschöpfungskette, wobei weder das Selbstbild noch das Fremdbild der Markenidentitäten untergraben werden darf. ${ }^{209}$ Die organisatorische Verankerung von Mehrmarkenstrategien muss somit auf eine Optimierung des Autonomiegrades der selbstständigen Markeneinheiten bzw. der Eingriffstiefe der Portfolioleitung ausgerichtet sein. ${ }^{210}$

Abb. 10 zeigt in diesem Zusammenhang ein Kontinuum der Mehrmarkenführung zwischen Zentralisation und Dezentralisation mit entsprechend differierenden Autonomiegraden der markenspezifischen Einheiten bzw. Eingriffstiefen der Portfolioleitung. $^{211}$

Vgl. auch Prahalad, C. K., Hamel, G., The Core Competence of the Corporation, a.a.O., S. $88 \mathrm{ff}$.

Bühner, R., Betriebswirtschaftliche Organisationslehre, a.a.O., S. 127.

Meffert, H., Burmann, Ch., Identitätsorientierte Markenführung - Konsequenzen für die Handelsmarke, a.a.O., S. 56; Vgl. Ghoshal, S. S., Bartlett, Ch., Building the Entrepreneurial Organisation: New Organisational Processes, New Managerial Tasks, in: European Management Journal, Vol. 13, Heft 2, 1995, S. 148.

Der Grad der Entscheidungsautonomie gibt hier an, in welchem Umfang die Markeneinheiten bei der Lösung von Entscheidungsproblemen autonom handeln können und nicht durch vorgegebene Beschränkungen gebunden sind.

211 Die Sichtung der Organisationsliteratur zeigt, dass die Begriffe Zentralisation und Dezentralisation unterschiedlich definiert werden. ALBACH spricht „von Dezentralisation dann, wenn der Entscheidungsspielraum entscheidungsgebundener Abteilungen größer als Null ist, von Zentralisation dagegen, wenn die weisungsgebundenen Abteilungen keinen eigenen Ermessensspielraum bei ihren Entscheidungen haben." Albach, H., Die Koordination der Planung im Großunternehmen, in: Rationale Wirtschaftspolitik und Planung in der Wirtschaft von heute, 


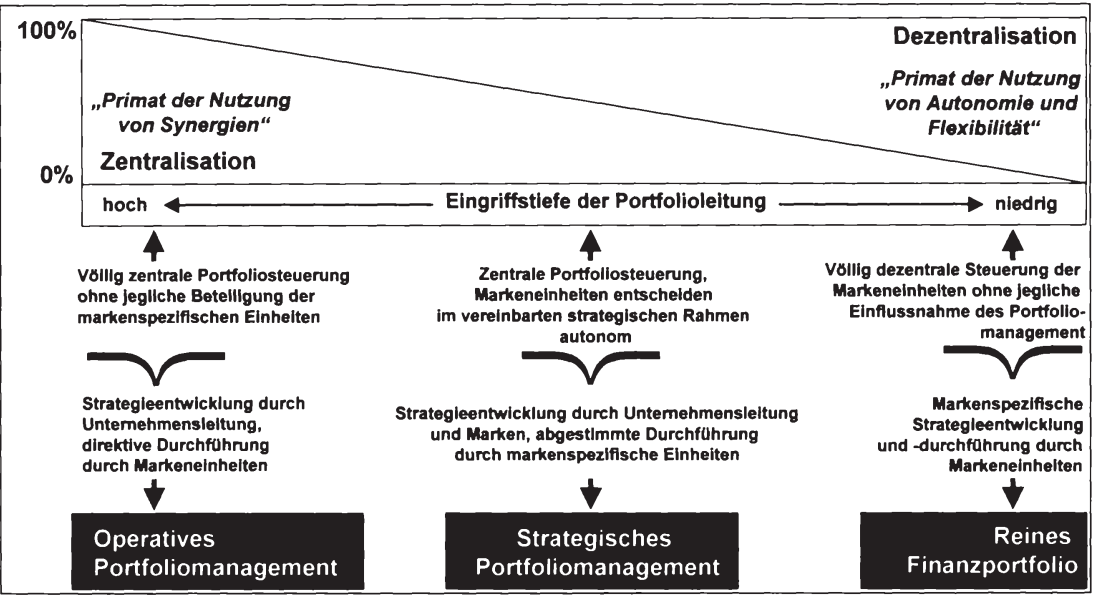

Abb. 10: Ausprägungen alternativer Zentralisationsgrade und Eingriffstiefen im Markenportfolio

Die idealtypischen Portfolioausprägungen lassen sich entsprechend der Position auf dem Kontinuum anhand der Eingriffstiefe der Portfolioleitung in die Markeneinheiten klassifizieren. ${ }^{212}$ Während die Unternehmensleitung in dem hier als operatives Portfoliomanagement bezeichneten Markenportfolio auch operative Aufgaben der Markenführung wahrnimmt bzw. diese notfalls direktiv durchsetzt, beschränkt sich das strategische Portfoliomanagement auf die Schaffung des strategischen Rahmens, innerhalb dessen die einzelnen Portfoliomarken weitgehend autonom agieren. In einem reinen Finanzportfolio fungieren die einzelnen Marken schließlich ausschließlich als Investitionsobjekt des Unternehmens, wobei das Portfoliomanagement keinerlei Einfluss auf markenspezifische Strategien und Durchsetzung ausübt, sondern lediglich Renditeaspekte fokussiert. Bei nicht hin-

Schriften des Vereins für Sozialpolitik, Band 45, Schneider, E. (Hrsg.), Berlin 1967, S. 342. $\mathrm{Vgl}$. auch Frese, E., Grundlagen der Organisation: Konzept - Prinzipien - Strukturen, 6. Aufl., Wiesbaden 1995, S. 84.

212 Auch die verschiedenen Varianten der Holdingorganisation werden durch das Ausmaß der Funktionen geprägt, die von der Konzernzentrale durchgeführt werden. Dabei wird zwischen den drei Formen der Operativen Holding, der Management Holding und der Finanz Holding unterschieden. Vgl. Bleicher, K., Organisation. Strategien - Strukturen - Kulturen, 2. Aufl., Wiesbaden 1991, S. $652 \mathrm{ff}$. 
reichender Erfüllung der Renditeerwartung werden die Marken in Analogie zum Wertpapierportfolio veräußert. ${ }^{213}$

Die rein dezentrale Führung eines Markenportfolios als Finanzportfolio stellt indes primär ein theoretisches Konzept dar, da die zentrale Festlegung strategischer Rollen und Positionierungen sowie die Realisierung markenübergreifender Synergien als Notwendigkeit einer gleichermaßen effektiven wie effizienten Mehrmarkenstrategie zu interpretieren sind. Demgegenüber geht ein rein operatives Portfoliomanagement je nach Größe des Markenportfolios vielfach mit einer Überlastung der Unternehmensleitung einher. Ausdruck einer solchermaßen postulierten Überlastung der Portfolioleitung ist das empirisch beobachtbare "GRESHAMsche

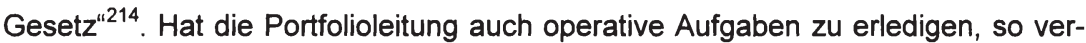
schieben sich bei besonderer Belastung der Portfolioleitung die Gewichte zu Lasten der strategischen Rahmenplanung. Zur Sicherstellung der Portfoliozielsetzungen erscheint vor diesem Hintergrund ein strategisches Portfoliomanagement gemäß der Leitmaxime „so viel Dezentralität wie möglich, so wenig Zentralität wie nötig“ als zweckmäßig. So führt auch PAUSENBERGER an: „Die Frage: Zentralisation oder Dezentralisation? ist eine Frage des Maßes; ein Entweder-Oder wäre hier falsch am Platze. “215

213 Allerdings bestehen zwischen dem Portfoliomanagement bei Wertpapieren und dem Portfoliomanagement in der Unternehmensplanung wesentliche Unterschiede. So lässt sich ein Wertpapierportfolio auch kurzfristig umschichten, wohingegen sich Unternehmen vielfach in einer historisch gewachsenen Situation mit Aktivitäten befinden, die sich nicht beliebig auswechseln lassen. Vgl. hierzu etwa Gälweiler, A., Strategische Unternehmensführung, Frankfurt a. M., New York, 1987, S. 76f. Vgl. auch Neubauer, F.-F., Portfolio-Management: Erfolgspotentiale vor Planungsritualen, 3. Aufl., Neuwied 1989, S. $111 \mathrm{ff}$.

Vgl. zum GreShamschen Gesetz Pfohl, H.-Ch., Zettelmeyer, B., Strategisches Controlling: in ZfB, 57. Jg., Heft 2, 1987, S. 146.

Pausenberger, E., Der Konzernaufbau. Versuch einer Morphologie des Konzerns, München 1957, S. 120. Es ist nur selten der Versuch unternommen worden, Zentralisations- und Dezentralisationsmaße operational zu definieren. FESLER stellt in diesem Zusammenhang fest, dass weder ein Begriff vorliegt, der das gesamte Kontinuum zwischen den beiden Polen Zentralisation und Dezentralisation bezeichnet, noch einer, der den mittleren Bereich benennt, in dem Zentralisations- und Dezentralisationstendenzen sich die Waage halten. Vgl. Fesler, J. W., Approaches to the Understanding of Decentralization, in: The Journal of Politics, Vol. 27, 1965, S. 537. Vgl. zu den messtheoretischen Strukturen von (De-)zentrailsationsmaßen die Ansätze von WHISLER und der ASTON-GRUPPE dargestellt bei Frese, E., Grundlagen der Organisation: Konzept - Prinzipien - Strukturen, a.a.O., S. $84 \mathrm{ff}$. 
Die Führung ${ }^{216}$ des Markenportfolios erfolgt in einem solchen Portfolio im Gegenstromverfahren. ${ }^{217}$ Während die Portfolioleitung den strategischen Rahmen der Mehrmarkenführung vorgibt und top-down-gerichtet im Sinne einer retrograden, von einem gewünschten Endergebnis ausgehenden Planung die Stoßrichtungen des Marktauftritts festlegt, sind die dezentralen Markeneinheiten mit der leitbildgerechten Ausgestaltung der Instrumentalstrategien betraut. ${ }^{218}$ Die Ausgestaltung der Mehrmarkenführung vollzieht sich damit auf der Grundlage markenspezifischer Zielvereinbarungen. Eventuelle Abweichungen von den Zielvereinbarungen können nach dem Prinzip der progressiven Planung (bottom-up) eine entsprechende Anpassung der strategischen Rahmenplanung bewirken. Auf diese Weise lassen sich vor dem Hintergrund bestehender Informationsasymmetrien im Portfolio die Detailkenntnisse der Markeneinheiten mit den Portfoliozielen verbinden.

Dieser Abgleichungsprozess erfordert einen hohen Kommunikations-, Koordinations- und Zeitaufwand, der sich in häufigen formellen und informellen Gesprächen zwischen Portfolioleitung und Markeneinheiten äußert. Das organisatorische $\mathrm{Zu}$ sammenspiel der verschiedenen Marken und der Portfolioleitung lässt sich dabei in verschiedenen hierachieergänzenden Abstimmungsgremien und Komitees wie etwa Produktstrategie-, Preis- und Länderkomitees institutionalisieren. ${ }^{219}$ Die Portfolioleitung als übergeordnete Führungsinstanz kann hierarchisch auf der Ebene der Unternehmensleitung verankert bzw. dieser auch als Stabsstelle un-

In Analogie zU ULRICH/FLURI bezeichnet Führung hier einerseits Einfluss auf die Marken auszuüben, der sie veranlasst, die erwarteten Beiträge zur Erreichung der Portfolioziele zu erbringen (Produktivitäts-/Leistungsaspekt), andererseits Bedingungen zu schaffen, die es zugleich ermöglichen, dass die Marken auch die markenspezifischen Ziele zu realisieren in der Lage sind (Zufriedenheitsaspekt). Vgl. Ulrich, P., Fluri, E., Management. Eine konzentrierte Einführung, 5. Aufl., Stuttgart 1988.

Das Gegenstromverfahren ist auch das vorherrschende Verfahren in der Konzernpraxis. Nur Top-Down- oder Bottom-Up-Ansätze widersprechen hier der Ausnutzung des kreativen Spannungsfeldes von Vielheit und Einheit. Vgl. auch Hamprecht, M., Controlling von Konzernplanungssystemen: theoretische Ableitung und betriebliche Realität führungsstrukturabhängiger Ausprägungsmuster, Wiesbaden 1996, S. 129 f.

Ähnlich argumentiert BARTLETT in seinen Ausführungen zur Steuerung multinationaler Konzerne: "It soon becomes evident that primary responsibility of top management is not to manage such activities directly, but to create and manage an organizational context that will provide the means for the appropriate resolution of decision and coordination of activities. "Bartlett, Ch. A., Building and Managing the Transnational: The New Organizational Challenge, in: Competition in Global Industries, Porter, M. E. (Hrsg.), Boston 1986, S. 386.

Vgl. hierzu auch Brockhoff, K., Hauschildt, J., Schnittstellen-Management - Koordination ohne Hierarchie, a.a.O., S. 403. 
mittelbar unterstellt werden. Eine solche Anordnung hängt indes von der bisherigen Organisationsstruktur, der Anzahl der Marken im Portfolio und damit der Entscheidungs- und Koordinationskomplexität, dem Diversifikationsgrad des Unternehmens (werden beispielsweise mehrere Mehrmarkenstrategien in unterschiedlichen Produktbereichen verfolgt) oder auch der Größe des Unternehmens etc. ab. Die Entscheidung kann letztlich nur im Einzelfall vor dem Hintergrund des unternehmensspezifischen Kontextes getroffen werden. Tendenziell lässt sich für die Verankerung einer solchen Zentralstelle konstatieren: Je kleiner das Unternehmen ist und je weniger Hierarchieebenen vorhanden sind, desto eher bietet sich eine unmittelbare Verankerung auf der Ebene der Geschäftsleitung an. Hierdurch wird eine unnötige Verlängerung der Entscheidungswege vermieden. Je komplexer das zu führende Portfolio und je höher die erforderliche Koordinationsintensität ist, desto eher ist eine Verankerung der strategischen Portfoliosteuerung in einer eigenständigen Organisationseinheit vorzuziehen, die der Geschäftsleitung unmittelbar unterstellt ist. Durch die Bündelung von Know-how und personellen Ressourcen in einer Abteilung kann den Anforderungen eines komplexen Markenportfolios besser entsprochen werden. Mitglieder der Unternehmensleitung bzw. der eigenständigen Organisationseinheiten können dabei nach dem Prinzip der Leiteridentität auch die jeweiligen Leiter der Marken sein. ${ }^{220}$

Zur Fixierung der markenspezifischen Freiheitsgrade auf dem Kontinuum zwischen Zentralisation und Dezentralisation bietet sich die Formulierung portfolioübergreifender Leitsätze des Marktauftritts an, in denen die zentralen Grundsätze der Führung des gesamten Markenportfolios sowie des innerorganisationalen $\mathrm{Zu}$ sammenspiels der dezentralen Markeneinheiten zu definieren sind. ${ }^{221}$ Marktstrategische Leitsätze stellen somit allgemeine Grundsätze im Sinne einer Verfassung des Portfolios dar. Im Mittelpunkt steht dabei die Aufgabenverteilung zwischen

Zum Prinzip der Leiteridentität vgl. auch Abegglen, W. E., Die Organisation der Unternehmensleitung. Versuch einer Theorie, Zürich 1966, S. $66 \mathrm{ff}$.

$221 \mathrm{Zu}$ bedenken ist in diesem Zusammenhang allerdings, dass das Handeln der Unternehmensleitung in praxi nicht ausschließlich auf bewusst technokratische Formulierungen rekurriert, sondern ebenso auf unternehmenskulturell geprägte Dimensionen und Ideale der Unternehmensleitung abstellt. Die Kultur lässt sich als Grundgesamtheit aller Werte- und Normenvorstellungen sowie Denk- und Verhaltensmuster beschreiben, die Entscheidungen, Handlungen und Aktivitäten der Unternehmensmitglieder prägen. Vgl. etwa Heinen, E., Dill, H., Unternehmenskultur aus betriebswirtschaftlicher Sicht, in: Herausforderung Unternehmenskultur, Simon, H. (Hrsg.), Stuttgart 1990, S. 12 ff. 
Portfoliomanagement und dezentralen Markeneinheiten. Die Leitsätze sollten dabei insbesondere in Bezug auf folgende Aspekte ausgestaltet werden:

- Grad des eigenständigen Marktauftritts der übergeordneten, portfoliohaltenden Unternehmung unter eigener Markierung,

- Grad der Eigenständigkeit der jeweiligen Portfoliomarken im Marktauftritt, d. $\mathrm{h}$ eigenständige vs. kombinierte Markierung,

- Grad der innerorganisationalen Eigenständigkeit der einzelnen Portfoliomarken insbesondere hinsichtlich der funktionalen Organisationsstruktur,

- Grad der eigenständigen Ergebnisverantwortung der Portfoliomarken,

- Grad der Markenbindung an innerorganisationale Ressourcen wie Technologien, Plattformen, Lieferanten etc.,

- Grad der Markenbindung an übergeordnete Steuerungs- und Koordinationsinhalte wie etwa zentrale Positionierungsvorgaben,

- Steuerungs- und Koordinationsinhalte zwischen den Marken sowie

- Prozess und Instrumente der Zielvereinbarung.

Die konkrete Aufgabenabgrenzung zwischen den Markeneinheiten und dem übergeordneten Portfoliomanagement ist letztlich im jeweiligen situativen bzw. historisch gewachsenen Kontext zu treffen, wobei die in Abb. 11 dargestellte Aufgabenteilung zusammenfassend als zweckmäßig anzusehen ist.

\begin{tabular}{|c|c|}
\hline Portfolioleitung & \\
\hline 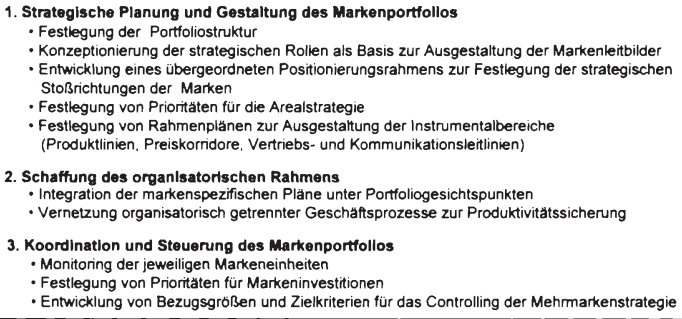 & $\begin{array}{l}\text { vn- } \\
\text { en }\end{array}$ \\
\hline Führung im Gegenstromverfahren & Mehrstufiger Abgleich \\
\hline $\begin{array}{l}\text { 1. Umsetzung des Markenauftrags Im Rahmen des Portfollos nach innen und außen } \\
\text { - Planung fü die jeweilige Marke } \\
\text { - Feinbudgetierung } \\
\text { - Operative Ausgestaltung und Umsetzung des Instrumente-Mix entsprechend } \\
\text { der instrumentalstrategischen Rahmenpläne } \\
\text { - Identithatsgestaltung } \\
\text { - Koordination der funktionalen Einheiten } \\
\text { 2. Monhtoring der Entwicklung der Jewelllgen Marke } \\
\text { - Monitoring zentraler Steuerungsgrößen } \\
\text { - Kontrolle und Nachweis des Erfolgsbeitrags der jeweiligen Marke zum Erfolg des } \\
\text { Gesamtportfolios }\end{array}$ & $\begin{array}{l}\text { n-Up- } \\
\text { hlage }\end{array}$ \\
\hline Markenmanagement & \\
\hline
\end{tabular}

Abb. 11: Aufgabenverteilung im Markenportfolio

Da die Markeneinheiten bzw. die von innen getroffenen Entscheidungen und Handlungen miteinander verknüpft sind, darf keine isolierte Betrachtung der Marken erfolgen, sondern es gilt, die Beziehungen zwischen den Marken zu berück- 
sichtigen. Nur dann, wenn keine Wechselwirkungen zwischen den Marken bestünden, würde die isolierte Ausführung markenspezifischer Teilaufgaben zu einer optimalen Erfüllung der Gesamtaufgabe des Portfolios führen. Der Fall vollkommener Isolierbarkeit einer Marke ist jedoch letztlich nur bei vollkommener Dezentralisierung gegeben, der im Rahmen von Mehrmarkenstrategien den obigen Ausführungen zur Folge kaum Relevanz besitzt. Betrachtet man - wie in Kapitel A dargestellt - die spezifische Problemstellung des Mehrmarkencontrolling in der Koordination des Führungssystems von Mehrmarkenstrategien, stellen Interdependenzen im Markenportfolio den zentralen Gegenstand des Mehrmarkencontrolling dar. Für seine Fundierung sind folglich die im Führungssystem des Portfolios bestehenden Interdependenzen sowie die sie beeinflussenden Interdependenzen im Ausführungssystem herauszuarbeiten. ${ }^{222}$ Betont sei an dieser Stelle, dass die Koordination im Führungssystem des Markenportfolios prinzipiell dem Management und mithin der Portfolioleitung selbst obliegt, der Unterstützungsbedarf des Management in diesem Zusammenhang indes als besonders groß gilt, so dass das Mehrmarkencontrolling hier vornehmlich aufgerufen ist, Unterstützungsleistungen zu erbringen. AHLERT folgend "würde es eine erhebliche Unschärfe bedeuten, wenn gesagt würde, die Sekundärkoordination [also die Abstimmung zwischen unterschiedlichen Managementaufgaben, Anm. d. V.] obliege dem Controlling. [...] Denn schon Erich Gutenberg hat die Koordination der großen Unternehmensteilbereiche als Aufgaben der obersten Managementebene bezeichnet. [...] Dafür Sorge zu tragen, dass die notwendigen Abstimmungsprozesse rechtzeitig und mit der erforderlichen Sorgfalt stattfinden und dass sie effizient moderiert und professionell abgewickelt werden, kann als die wichtigste Aufgabe einer unternehmensweiten Controllingorganisation bezeichnet werden. [...] Plakativer ausgedrückt: Controlling ist 'Management von Schnittstellen' im Auftrag der Linieninstanzen. “223

Vgl. Küpper, H.-U., Controlling: Konzeptionen, Aufgaben und Instrumente, a.a.O., S. 31.

Ahlert, D., Grundlagen des Controlling, a.a.O., S. 23 f. 


\section{Identifikation des Controllingbedarfs und Ableitung einer Controllingkonzeption in Markenportfolios}

Der Controllingbedarf in Mehrmarkenstrategien wird vom Ausmaß der Interdependenzen und Zielkonflikte im Markenportfolio determiniert und hängt somit in hohem Maße vom situativen Bedingungsrahmen ab. Dabei lassen sich interne und externe Kontextfaktoren abgrenzen, die unmittelbar den Grad der Interdependenzen und Zielkonflikte im Portfolio beeinflussen (vgl. Abb. 12). Wenngleich zwischen internen und externen Faktoren starke Interdependenzen bestehen, soll ihr Einfluss auf den Controllingbedarf zunächst getrennt diskutiert werden, da nur so die Einflüsse klar erkennbar und im Hinblick auf die Gestaltung der Controllingkonzeption operational sind. Anschließend werden zusammenfassend die Interdependenzen zwischen den Kontextfaktoren aufgezeigt.

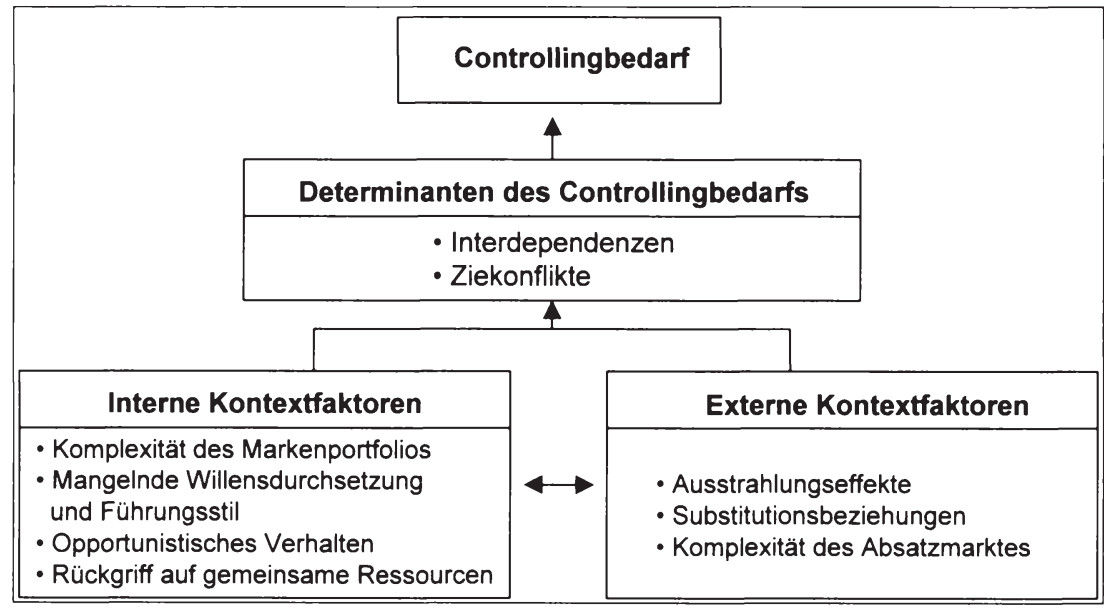

Abb. 12: Controllingbedarf in Mehrmarkenstrategien

\section{Controllingbedarf in Mehrmarkenstrategien}

\subsection{Determinanten des Controllingbedarfs}

Im Markenportfolio erfolgt eine Arbeitsteilung dergestalt, dass jede Portfolioeinheit - also Portfolioleitung und Markeneinheiten - spezifische Aufgaben innerhalb des Portfolios übernimmt. Hierdurch entstehen unmittelbare Wirkungszusammenhänge zwischen den Entscheidungen der einzelnen Marken. Die Erreichung der Portfoliogesamtzielsetzung macht somit eine Abstimmung der interdependenten 
Markeneinheiten erforderlich. Zur Gewährleistung einer effizienten Arbeitsteilung werden den Portfoliomarken überdies je nach Grad der Zentralisierung im Portfolio Entscheidungsspielräume eingeräumt. Der Controllingbedarf resultiert somit aus den Abhängigkeiten zwischen den Marken und den Entscheidungsspielräumen, wobei die Höhe des Controllingbedarfs von der Art und Intensität zielbezogener Interdependenzen zwischen den Marken sowie dem Ausmaß der den Marken eingeräumten Entscheidungsspielräumen bestimmt wird. ${ }^{224}$

Interdependenzen führen dazu, dass Tatbestände nicht isoliert voneinander festgelegt werden können, ohne die Zielerreichung zu vermindern. Entsprechend sind die Wirkungen auf den jeweils anderen Tatbestand und deren Rückwirkungen bei der Einzelentscheidung zu berücksichtigen. ${ }^{225}$ Zur näheren Kennzeichnung ist es für die vorliegende Problemstruktur zweckmäßig, mit THOMPSON nach dem Kriterium unterschiedlicher Intensität zwischen gepoolten, sequentiellen und reziproken Interdependenzen zu unterscheiden, ${ }^{226}$ wobei Gegenstand der Austauschbeziehungen sowohl materielle als auch informationelle Interdependenzen sein können:

- Gepoolte Interdependenzen: die Einheiten sind indirekt voneinander abhängig, z. B. Konkurrenz der Portfoliomarken um knappes Kapital, gemeinsame Nutzung von Ressourcen, Infrastrukturen und Dienstleistungsangebot im Portfolio, Bedienung gleicher Segmente und damit Konkurrenz um Kunden und

Vgl. Hoffmann, F., Führungsorganisation, Band 1, Stand der Forschung und Konzeption, Tübingen 1980, S. 308. Vgl. auch Benkenstein, M., F\&E und Marketing: eine Untersuchung zur Leistungsfähigkeit von Koordinationskonzeptionen bei Innovationsentscheidungen, Wiesbaden 1987, S. 36

Entsprechend liegen Interdependenzen erst dann vor, wenn sich mindestens zwei Tatbestände gegenseitig beeinflussen. Vgl. Küpper, H.-U., Controlling: Konzeptionen, Aufgaben und Instrumente, a.a.O., S. 31. Interdependenzen existieren immer nur bei gemeinsamen, extremalen Zielen für die Entscheidungsbereiche. Eine solche typische extremale Zielfunktion im vorliegenden Problemfeld ist etwa die Gewinnmaximierung des Gesamtportfolios. Interdependenzen zwischen den Portfoliomarken führen dazu, dass markenspezifische Gewinne keine alleinige Funktion markenbezogener Entscheidungen sind, sondern von den Entscheidungen anderer Marken beeinflusst werden. Das Ziel der Gewinnmaximierung des Gesamtportfolios führt dazu, dass Entscheidungen über die den Gewinn beeinflussenden Variablen für einzelne Marken nur simultan getroffen werden können. Vgl. Adam, D., Planung und Entscheidung: Modelle - Ziele - Methoden; Fallstudien und Lösungen, a.a.O., S. 148.

Vgl. Thompson, J. D., Organizations in Action, New York u. a. 1967, S. 54f. Vgl. auch Frese, E., Grundlagen der Organisation: Konzept - Prinzipien - Strukturen, a.a.O., S. 54f. Im Falle sequentieller Beziehungen sind organisatorische Einheiten im Leistungsprozess hintereinander geschaltet, so dass hier auch Dependenzen vorliegen können ( $B$ ist von $A, A$ aber nicht von B abhängig), allerdings beeinflussen Entscheidungen auf der nachgelagerten Stufe zumeist auch Entscheidungen auf der vorgelagerten. Vgl. auch Picot, A., Dietl, H., Franck, E., Organisation: Eine ökonomische Perspektive, Stuttgart 1997, S. 73. 
Marktanteil etc. ${ }^{227}$ FRESE unterscheidet in diesem Zusammenhang zwischen Ressourcen- und Marktinterdependenzen. Ressourceninterdependenzen ergeben sich bei der gemeinsamen Verwendung knapper Faktoren wie etwa der Budgetallokation zwischen den Marken bzw. dem gemeinsamen Rückgriff auf standardisierte Produktkomponenten bzw. Bauteile im Portfolio. Marktinterdependenzen liegen vor, wenn die auf ein bestimmtes Marktsegment ausgerichteten Aktivitäten einer Marke die auf ein anderes Segment gerichteten Aktivitäten der gleichen oder einer anderen Marke beeinflussen, wobei die Beeinflussung auch in zukünftige Perioden hineinreichen kann (zeitliche Interdependenzen). Überdies werden Marktinterdependenzen dadurch verursacht, dass verschiedenen Marken des Portfolios ihre Aktivitäten auf dasselbe Marktsegment ausrichten.

- Sequentielle Interdependenzen: der Output einer Einheit wird zum Input einer anderen Einheit, z. B. stellt die übergeordnete strategische Rahmenplanung der Portfolioleitung den Input der untergeordneten Markeneinheiten dar. $^{228}$

- Reziproke Interdependenzen: gegenseitiger Leistungsaustausch zwischen den Einheiten. Sie beruhen auf einer Veränderung der Ausgangslage markenpolitischer Entscheidungen einer Marke durch Entscheidungen anderer Marken. So kann die Preisentscheidung oder die Art der Kommunikation für eine Marke A daran gebunden sein, welche Preis- oder Kommunikationsentscheidungen für die Marke $B$ getroffen werden et vice versa.

Die Interdependenzenkategorien bilden dabei in der genannten Reihenfolge zunehmend komplexere Abhängigkeiten zwischen den Portfolioeinheiten. ${ }^{229}$ Wie aufgezeigt, bedingt die begrenzte qualitative und quantitative Kapazität der Portfolioleitung zur gleichzeitigen Steuerung aller markenrelevanten Variablen und $\mathrm{Pa}$ rameter eine Dezentralisierung von Entscheidungen und Handlungen auf die einzelnen Markeneinheiten. ${ }^{230}$ Durch Delegation erwächst aber die Gefahr ineffizienter Verhaltensweisen aufgrund der zu beobachtenden Tendenz einer organisatori-

Vgl. Frese, E., Grundlagen der Organisation: Konzept - Prinzipien - Strukturen, a.a.O., S $53 \mathrm{ff}$

228 In diesem Zusammenhang finden sich auch Unterscheidungen in horizontale und vertikale Interdependenzen. Vgl. etwa Mag, W., Planung, in: Vahlens Kompendium der Betriebswirtschaftslehre, Bitz, M. et al. (Hrsg.). Band 2, 3. Aufl., München 1993, S. 45; Ahlert, D., Franz, K.-P., Kaefer, W., Grundlagen und Grundbegriffe der Betriebswirtschaftslehre, 5. Aufl., 1990, S. $218 f$.

229 Vgl. auch Hoffmann, F., Führungsorganisation, a.a.O., S. 310.

230 Traditionell wird der Vorgang der Zerlegung von Teilaufgaben mit KOSIOL als Aufgabenanalyse und ihre Verknüpfung mit Aufgabenträgern zu zielwirksamen Strukturen als Aufgabensynthese bezeichnet. Vgl. Kosiol, E., Organisation der Unternehmung, a.a.O. Vgl. zur Zerschneidung von Interdependenzen auch Küpper, H.-U., Controlling: Konzeptionen, Aufgaben und Instrumente, a.a.O., S. 34. 
schen Verselbstständigung und der damit verbundenen Entwicklung eigener Zielsysteme und Normengefüge mit der Konsequenz möglicher Zielkonflikte zwischen den Portfolioeinheiten. Zur Maximierung der Zielerreichungsgrade im Gesamtportfolio bedarf es somit einer integrierenden Abstimmung zwischen den separierten Einheiten. Zusammenfassend begründet die Aufspaltung des Markenführungssystems in partielle Entscheidungsfelder mit der Folge von Interdependenzen und möglichen Zielkonflikten den Koordinations- und damit Controllingbedarf in Mehrmarkenstrategien.

Im Folgenden ist zu fragen, welche Faktoren auf das Mehrmarkencontrolling wesentlichen Einfluss nehmen. Als externe Faktoren sollen jene Faktoren verstanden werden, auf die das Unternehmen nur indirekt Einfluss nehmen kann, wohingegen interne Faktoren im direkten Einflussbereich des Management liegen. ${ }^{231}$

\subsection{Interne Kontextfaktoren des Controllingbedarfs}

\subsection{Komplexität von Markenportfolios}

Der spezielle Charakter des Markenportfoliomanagement lässt sich durch den Übergang der Führung einer Marke hin zur Steuerung einer Vielzahl von Markeneinheiten begründen. Betrachtet man das Markenportfolio als System und die jeweiligen Marken als Elemente, so kann das Portfolio auch als eine geordnete Menge von miteinander verbundenen Elementen bezeichnet werden. Der Portfolioleitung kommt hier die Aufgabe zu, die jeweiligen Markeneinheiten in einem ausgewogenen System zu führen. Das Grundproblem kann dabei in der „Einheit des Mannigfaltigen“232 gesehen werden, wie das Phänomen der Komplexität in einem ersten Ansatz von LUHMANN bezeichnet wird.

231 Allerdings zeigt sich, dass eine genaue Abgrenzung von unternehmensexternen und -internen Auslösern des Controllingbedarfs nicht immer möglich, aber auch nicht notwendig ist, da ein solches Abgrenzungsproblem die Zuordnung von Bedarfsfaktoren in keiner Weise beeinträchtigt.

Vgl. Luhmann, N., Komplexität, in: Handwörterbuch der Organisation, Grochla, E. (Hrsg.), 2. Aufl., Stuttgart 1980, Sp. 1064. 
Die Portfoliokomplexität umschreibt dabei die immanente Vielschichtigkeit sowie dynamische Veränderlichkeit des Portfolios (vgl. Abb. 13). ${ }^{233}$ Die Vielschichtigkeit eines Markenportfolios resultiert aus der Anzahl und Heterogenität der Marken (sog. Elementekomplexität) und deren Verknüpfung untereinander (sog. Relationenkomplexität). So erschwert eine große Anzahl und Hierarchie von Marken und Submarken (etwa Dachmarken mit entsprechenden Produktmarken) mit ihren Relationen die Erfassung und Steuerung der Systemgesamtheit des Markenportfolios. Die Vielschichtigkeit des Portfolios ergibt sich dabei vor allem aus der Tatsache, dass sich die Identität der Marken und Submarken immer aus einer widerspruchsfreien Summe von essentiellen und akzidentiellen Merkmalen einer Marke ergibt, die es sowohl innerhalb einer Marke (Intramarkenkomplexität) als auch zwischen den Marken (Intermarkenkomplexität) abzustimmen gilt (vgl. Abb. 13).

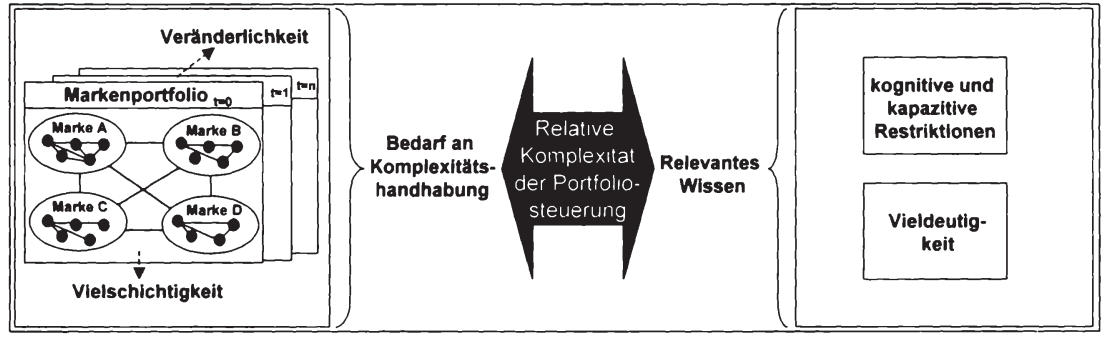

Abb. 13: Relative Komplexität der Portfolioführung

Neben einer solchen statischen Portfoliokomplexität unterliegen Markenportfolios zudem einer dynamischen Komplexität, da Marken realiter durch Veränderungen im Zeitablauf in eine veränderliche Beziehung zueinander gesetzt werden. Je häufiger, stärker und weniger regelmäßig Veränderungen des Zustands von Marken und Relationen in einem Portfolio auftreten, desto dynamischer und infolgedessen komplexer ist ein solches Markenportfolio zu bewerten. ${ }^{234}$

Je vielschichtiger und dynamischer das Markenportfolio, desto höher ist der Controllingbedarf.

233 Vgl. Meffert, H., Marketing: Grundlagen marktorientierter Unternehmensführung: Konzepte Instrumente - Praxisbeispiele, a.a.O., S. 954ff.; Bliss, Ch., Management von Komplexität: Ein integrierter, systemtheoretischer Ansatz zur Komplexitätsreduktion, Wiesbaden 2000, S. $91 \mathrm{ff}$.

234 Vgl. auch Roters, M., Komplexität und Dynamik als Einflußgrößen der Effizienz von Organisationen. Eine empirische Untersuchung, Frankfurt a. M., 1989, S. 23. 
Die zentrale Herausforderung hinsichtlich der Führung eines Markenportfolios besteht in der Reduktion der Komplexität sowohl innerhalb als auch zwischen den Marken. Dabei stellt sich ein solches Portfolio je nach dem Wissen, das die Portfolioleitung über die systemkonstituierenden Elemente und Relationen des Markenportfolios besitzt, in unterschiedlichen Graden von Komplexität dar. Schon MARCH/SIMON definierten die Komplexität einer Aufgabe als relativ zu den individuellen Fähigkeiten des mit der Aufgabe betrauten Individuums. ${ }^{235}$ Die Komplexität der Führung eines Markenportfolios hängt somit neben der Vieldeutigkeit des Systems von den unterschiedlichen kognitiven und kapazitiven Restriktionen ${ }^{236} a b$, welche die Erfassung und Beherrschung der objektiven Komplexität des zu steuernden Markenportfolios begrenzen. ${ }^{237}$ Mit WEBER ET AL kann die Menge der Daten als Repräsentanten von Elementen und Beziehungen, welche eine vollständige Beschreibung und Steuerung des Portfolios erlauben, auch als relevantes Wissen bezeichnet werden ( $\mathrm{vgl}$. Abb. 13). ${ }^{238}$ Der Mangel eines solchen Wissens führt zu Wissensdefiziten und mithin zu einer Begrenzung der Handhabung der Portfoliokomplexität.

Je geringer das relevante Wissen bei der Portfolioleitung, als desto höher kann der Controllingbedarf in Mehrmarkenstrategien bezeichnet werden.

Wie aufgezeigt, erfolgt im Markenportfolio zur Komplexitätsbewältigung eine strukturelle Differenzierung des Gesamtsystems. ${ }^{239}$ So kommt es zum Aufbau von

Vgl. March, J., G., Simon, H. A., Organizations, New York, London, Sydney, 1958, S. 55. Vgl. auch Bleicher, der die Handhabung von Komplexität als Kern der Managementaufgabe beschreibt und auf die Schwierigkeit seiner Erfassung und Beherrschung verweist. Vgl. Bleicher, K., Das Konzept integriertes Management, a.a.O., S. 31.

Zu berücksichtigen sind die Erkenntnisse der Kognitionswissenschaft, wonach die Datentransformationsfähigkeit des Menschen begrenzt und seine Rationalität beschränkt ist. MILLER zeigt, dass die Kapazität für die bewusste Unterscheidung, Aufnahme und Verarbeitung gleichzeitig eintreffender Daten auf ca. sieben Einheiten begrenzt ist. „...mit derart vielen Kennzahlen [...] werden die kognitiven Grenzen der Akteure überschritten und die Energie und Aufmerksamkeit des Managements "in alle Winde" zerstreut". Weber, J., Schäffer, U., Entwicklung von Kennzahlensystemen, WHU- Forschungspapier Nr. 62, Koblenz 1998, S. 14.

Entsprechend argumentiert HAMPRECHT für die relative Komplexität der Konzernführungsaufgabe. Vgl. Hamprecht, M., Controlling von Konzernplanungssystemen: theoretische Ableitung und betriebliche Realität führungsstrukturabhängiger Ausprägungsmuster, a.a.O., S. 58ff.

Vgl. Weber, J. et al, Grundgedanken zur Entwicklung einer Theorie der Unternehmensführung, WHU-Forschungspapier Nr. 30, Koblenz 1995, S. 10

Vgl. die Ausführungen zum organisatorischen Rahmen der Mehrmarkenstrategie. Vgl. auch Bircher, B., Langfristige Unternehmensplanung. Konzepte, Erkenntnisse und Modell auf systemtheoretischer Grundlage, Bern, Stuttgart, 1976, S. 49. 
hierarchischen Ebenen, die sich auf unterschiedliche Aufgaben beziehen und damit zu einer Reduktion der Gesamtaufgabe beitragen. ${ }^{240}$ Aufgrund der Aufgabenteilung zwischen Portfolioleitung und den organisatorisch getrennten Markeneinheiten nimmt die Entfernung zwischen dezentraler Informationsentstehung und zentraler Informationsverarbeitung zu. Diese Entfernung reduziert das entscheidungsrelevante Wissen zur Handhabung der Portfoliokomplexität, so dass die strategische Rahmenplanung durch die Portfolioleitung trotz hoher Anforderungen an Informations-Input große Informationslücken besitzt. Gemessen an den strengen Maßstäben der traditionellen Entscheidungslogik stellt die Steuerung von Mehrmarkenstrategien durch die Portfolioleitung somit auch ein schlechtstrukturiertes Entscheidungsproblem dar. ${ }^{241}$

\subsection{Mangelnde Willensdurchsetzung und Führungsstil}

Verhaltenssteuerung im Markenportfolio ist primär ein Problem der Durchsetzung zentraler Vorgaben durch die Portfolioleitung bzw. ein Problem fehlender Verknüpfung zwischen strategischer und operativer Planung. Eine fehlende Verbindung zwischen Strategie und Umsetzung stellt zwar grundsätzlich kein rein mehrmarkeninhärentes Spezifikum dar, sondern ist ein empirisch gestütztes Problem

In der Literatur finden sich unterschiedliche Ebenenkonzepte zur stufenweisen Komplexitätsreduktion von Systemen. Vgl. etwa Zahn, E., Mehrebenenansatz der Planung, in: Handwörterbuch der Planung, Szyperski, N., Winand, U. (Hrsg.), Band 9, Stuttgart 1989, Sp. 1081. Aus kybernetischer Sicht bedeutet dies, dass das Gesamtsystem in Subsysteme unterteilt wird, die jeweils eine geringere Varietät aufweisen als das Gesamtsystem. Vgl. Ackoff, R. L., Towards a System of System Concepts, in: Management Science, Vol. 17, Heft 11, 1971, S. 667.

In diesem Zusammenhang sind neben Lösungs- und Zielsetzungsdefekten insbesondere Bewertungs- und Wirkungsdefekte von besonderer Relevanz. Entsprechend bieten sich anstatt algorithmischer Verfahren zur Lösung des Strategieproblems heuristische Verfahren an, deren Ergebnisse Approximationen an eine idealtypische Lösung unter der Annahme vollkommener Information darstellen und mehr grundlegende Verhaltensrichtungen als detaillierte Ziel- und Mittelvorgaben sind. Vgl. zu den Defekten Adam, D., Planung und Entscheidung: Modelle Ziele - Methoden; Fallstudien und Lösungen, a.a.O., S. 10ff. Bewertungsdefekte liegen vor, wenn die erwarteten Strategiefolgen hinsichtlich ihres ökonomischen Erfolges nicht bewertet werden können oder allgemein anhand der strategischen Ziele nicht bewertbar sind. So stellt sich etwa die Frage, wie sich die Verbesserung des Images einer Automobilmarke bezüglich der Dimension Sportlichkeit auf das Ziel "Steigerung der Kundenzufriedenheit" oder "Erhöhung des Marktanteils ${ }^{u}$ auswirkt. Wirkungsdefekte liegen vor, wenn nicht bekannt ist, mit welchen strategischen Entscheidungen die angestrebten Strategiefolgen erreicht werden können oder keine Vorstellung darüber existiert, welches Niveau der Handlungsparameter zur Erreichung der gewünschten Strategiefolgen notwendig ist (in welchem Umfang muss etwa das Werbebudget erhöht werden, um den Bekanntheitsgrad einer Automobilmarke um 2 Prozent zu steigern?). Vgl. Meffert, H., Marketing: Grundlagen marktorientierter Unternehmensführung: Konzepte - Instrumente - Praxisbeispiele, a.a.O., S. $293 f$. 
für den Erfolg jeglicher Unternehmensführung. ${ }^{242}$ Die trennscharfe Abstimmung und Umsetzung der Schlüsselelemente der strategischen Rahmenplanung in Mehrmarkenstrategien bildet jedoch die Voraussetzung zur Schaffung differenzierter Markenidentitäten und damit den entscheidenden Ordnungsrahmen für einen intern wie extern differenzierten Marktauftritt der Portfoliomarken. Der Verknüpfung von strategischer und operativer Ebene in Mehrmarkenstrategien kommt vor diesem Hintergrund nicht nur eine zentrale Bedeutung für den Erfolg, sondern für die Existenz der Mehrmarkenstrategie zu. Bei unzureichender Verbindung der Ebenen sollten somit Instrumente und Kennzahlen entwickelt werden, die zur Schließung der Implementierungslücke beitragen. ${ }^{243}$

Je unzureichender die Verknüpfung zwischen strategischer Rahmenplanung und operativer Umsetzung, um so größer ist der Controllingbedarf.

Zentrale Entscheidungen und Rahmenpläne werden allerdings nicht unmittelbar zu Entscheidungsprämissen aller dezentralen Markeneinheiten, sondern haben eine gewisse Schwelle der politischen Akzeptanz bei diesen zu überwinden, da die einzelnen Marken unmittelbar im Hinblick auf ihre Positionierungsfreiräume oder die Konfiguration ihrer Leistungen eingeschränkt werden. Auf diese Weise lassen sich für die einzelnen Marken Chancen im Wettbewerb wie etwa die Wahl

242 So gaben in einer in 1998 vom CFO-Magazin durchgeführten Untersuchung 57 Prozent der Befragten an, dass ihr Unternehmen über keine klare Strategie verfügte, 53 bemängelten eine fehlende Verbindung von Strategie mit operativer Planung, 45 die fehlende persönliche Verantwortung für Ergebnisse und 34 die fehlende Präsenz von aussagefähigen Leistungskennzahlen. Für Deutschland merkten RAFFÉE/FRITZ bereits 1989/1990 an, dass im Bereich des normativen Management kunden- bzw. absatzmarktbezogene Grundhaltungen und Unternehmensziele zwar die führende Rolle spielen, im Gegensatz dazu jedoch in den meisten Strategien ein Innovations- bzw. Technologiedenken sowie Produktions- und Kostendenken vorherrsche. RAFFEE/FRITZ folgern hieraus ein Defizit in der Umsetzung strategischer Ziele und Grundhaltungen. WEBER/GOELDEL/SCHÄFFER kommen auf der Grundlage eines Vergleichs der Planungsprozesse von acht Konzernen ebenfalls zum Ergebnis einer mangelnden Verbindung zwischen strategischer und operativer Planung. Nach ihren Angaben ist eine wesentliche Ursache hierfür die mangelnde Kommunikation, wodurch die Brücke zur Umsetzung der Ziele in die operative Planung und die sich anschließende Ausführung fehle. Vgl. zu einer Darstellung der genannten Studien mit entsprechenden Auffindungshinweisen vgl. Weber, J., Schäffer, U., Balanced Scorecard - Gedanken zur Einordnung des Konzeptes in das bisherige Controlling-Instrumentarium, WHU-Forschungspapier, S. 18.

Vgl. zur Implementierungsproblematik Krink, J., Kracht-Müntz, B., Operative und strategische Planung - Aufgaben, Interdependenzen und Koordinationsproblematik (I), in: Das Wirtschaftsstudium, Heft 1, 1984, S. 24 ff; Hilker, J., Marketingimplementierung: Grundlagen und Umsetzung am Beispiel ostdeutscher Unternehmen, Wiesbaden 1993 sowie Reuter, J., Komplexität und Dynamik der Implementierung von Wettbewerbsstrategien, Wiesbaden 1998. Zum Implementierungscontrolling vgl. Grimmeisen, M., Implementierungscontrolling: wirtschaftliche Umsetzung von Change-Programmen, Wiesbaden 1998. 
kostengünstigerer Lieferanten oftmals nicht aufgreifen bzw. können Risiken nicht rechtzeitig durch entsprechende Handlungsweisen begegnet werden. ${ }^{244}$ Eine den zentralen Entscheidungen und Rahmenplänen inhärente Logik reicht somit vielfach nicht aus, eine Akzeptanz bei den die Marke führenden Personen zu schaffen. ${ }^{245}$ Mit SCHOLL ist in Organisationen auch kein perfekt rationales Verhalten per se zu erwarten, sondern das Verhalten ist stets unter bereichs- bzw. interessengebundener Perspektive zu betrachten. Die Frage lautet letztlich stets: Rational und effizient für wessen Interessen? ? $^{246}$

Vor diesem Hintergrund bedarf es zur Steuerung der Marken im Portfolio der Konsensbildung im Rahmen von Partizipation sowie der Erzeugung von Motivation ${ }^{247}$ durch die Zuordnung von Entscheidungsfreiräumen. Hiermit eng verknüpft ist die Unterscheidung alternativer Führungsstile, in denen sich die Art und Weise ausdrückt, in der Führung im Markenportfolio ausgeübt wird. Mögliche Ausprägungen eines Führungsstils, die üblicherweise in einem Kontinuum zwischen autoritär oder partizipativ beschrieben werden, unterscheiden sich im Rahmen von Mehrmarkenstrategien vor allem darin, in welchem Umfang das Portfoliomanagement die einzelnen Markeneinheiten in den Willensbildungsprozess einbezieht. ${ }^{248}$

Bedingt durch einen hohen Formalisierungsgrad des Entscheidungsprozesses, Zentralisierung der Entscheidungen und Willensbildung und bilaterale Willens-

Vgl. Meffert, H., Perrey, J., Mehrmarkenstrategie - Ein Beitrag zum Management von Markenportfolios, a.a.O., S. 632.

245 РІСОт beschreibt in diesem Zusammenhang das Koordinationsproblem als Überwindung des Nichtwissens, das Motivationsproblem hingegen als Problem des Nichtwollens. Vgl. Picot, A., Dietl, H., Franck, E., Organisation: Eine ökonomische Perspektive, Stuttgart 1997, S. 8.

Scholl, W., Politische Prozesse in Organisationen, in: Handwörterbuch der Organisation, Frese, E. (Hrsg.), 3. Aufl., Stuttgart, Sp. 2002.

247 Motivation wird hier als Aktivierung eines Fähigkeitspotenzials in Richtung auf die Markenziele und mithin als Leistungsmotivation verstanden. Schon in der MASLOWschen Bedürfnispyramide wird das Selbstverwirklichungsbedürfnis als am höchsten entwickelter Motivator eingeschätzt. Vgl. Maslow, A. H., Motivation and Personality, 3. Aufl. New York 1989, S. 16f. Auch in HERzBERgs "Zwei-Faktoren-Theorie" wird die Verantwortung bzw. Selbstverwirklichung als bedeutender Motivator angeführt. Vgl. Herzberg, F., Mausner, B., Snyderman, B., The Motivation to Work, 2. Aufl., New York 1959, S. 44ff.

Zur Verdeutlichung einzelner Führungsstile wird vielfach auf das Schema von TANNENBAUM/SCHMIDT verwiesen. Es unterscheidet mit dem autoritären, patriarchalischen, informierenden, beratenden, kooperativen, partizipativen und demokratischen Führungsstil sieben unterschiedliche Ausprägungen alternativer Stile. Vgl. Tannenbaum, R., Schmidt, W., How to choose a Leadership Pattern, in: Harvard Business Review, Vol. 36, 1958, S. 95ff. 
durchsetzung hat die autoritäre Führung des Markenportfolios grundsätzlich einen eher geringeren Controllingbedarf der Marken zur Folge. Der geringe Bedarf ist darauf zurückzuführen, dass sowohl die Interdependenzen als auch die Entscheidungsspielräume der Marken bei dieser Form des Führungsstils äußerst gering sind. Auf der anderen Seite ist der Koordinationsbedarf in partizipativ geführten Markenportfolios eher hoch, da sich eine Vielzahl von Markenmitgliedern multilateral koordinieren müssen und damit die Anzahl der Interdependenzen sowie die Entscheidungsspielräume und Zielkonflikte zwischen den Marken anwachsen.

Je autoritärer der Führungsstil in Markenportfolios, um so geringer ist der Controllingbedarf.

\subsection{Opportunistisches Verhalten im Markenportfolio}

Ausgehend von der Gesamtzielsetzung des Portfolios stellt sich die Frage, wie weit der Handlungsspielraum der dezentralen Markeneinheiten in der Portfoliosteuerung zu bemessen und wie intensiv seine Ausfüllung zu überwachen ist. Wird den Markeneinheiten ein zu großer Entscheidungsspielraum gewährt, können diese eigene opportunistische Verhaltensmuster entwickeln, welche ihre eigenen Zielerreichungsgrade maximieren, hingegen die Oberziele bzw. die im Rahmen des Gesamtportfolios angestrebte Mehrwertgenerierung beschränken.

Dieses Problem lässt sich auch mit Hilfe der Prinzipal-Agenten-Theorie veranschaulichen: Die Portfolioleitung als schlecht informierter Prinzipal delegiert Aufgaben an die infolge ihrer Marktnähe besser informierten dezentralen Markeneinheiten als Agenten. Aufgrund bestehender Informationsasymmetrien eröffnen sich für den Agenten Handlungsspielräume, die er zum Schaden des Prinzipals ausnutzen kann. So auch JENSEN/ MECKLING: „If both parties to a relationship are utility maximizers there is good reason to believe that the agent will not always act in the best interest of the principal." 249 Der Prinzipal weiß nicht, ob ein schlechtes Ergebnis durch Versäumnis des Agenten oder andere Umweltfaktoren bedingt ist. Der Agent kann somit durch seine Handlungen die Wohlfahrt des Prinzipals beeinflussen, der damit verschiedenen Typen von Verhaltensrisiken ausgesetzt ist.

249 Jensen, M, Meckling, W. H., Theory of the Firm: Managerial Behaviour, Acency Costs and Ownership Structure, in: Journal of Financial Economocs, 3. Vol., S. 308. 
Die Prinzipal-Agenten-Theorie leitet entsprechende Anreizmechanismen zur Eingrenzung dieser Verhaltensrisiken ab. Zur Lösung der Agency-Probleme schlägt die Theorie zum einen Anreizsysteme vor (Incentive Structures). Die Markeneinheiten sollen unter Berücksichtigung ihres Eigeninteresses Entscheidungen finden, die auch die Interessen des Gesamtportfolios miteinbeziehen. Zum anderen sollen Überwachungsmechanismen (Monitoring Structures) die Aktivitäten der dezentralen Markeneinheiten transparenter machen. Ziel der Anreiz- und Überwachungsmechanismen ist es, den Wohlfahrtsverlust des Gesamtportfolios zu reduzieren, der sich im Vergleich zu einem idealisierten Zustand ohne AgencyProbleme ergibt. ${ }^{250}$

Je größer der Grad der Markenautonomie und je größer die Gefahr des opportunistischen Verhaltens, desto höher ist der Controllingbedarf in Mehrmarkenstrategien.

\subsection{Rückgriff auf gemeinsame Ressourcen}

Zur Nutzbarmachung von Economies of Scale greifen insbesondere Volumenhersteller auf innerbetriebliche Synergiepotenziale in Produktion und Entwicklung zurück, die zu einer Standardisierung und einheitlichen Verwendung ganzer Baugruppen über Markengrenzen hinweg führen. Um möglichst viel von dem sich aus der Erfahrungskurve ergebenden Produktivitätsgewinn zu profitieren, wird der Differenzierungsmoment im Produktionsablauf jedoch häufig soweit hinausgezögert, dass den einzelnen Marken die technische Differenzierungsbasis zu Gunsten von Kostenvorteilen entzogen wird. ${ }^{251}$

Die objektive Angleichung hängt vor allem vom Grad der Standardisierung als Ergebnis unterschiedlicher Anpassungsprozesse in der Automobilindustrie ab. Die von den Automobilherstellern angewandten strategischen Optionen zur Beherr-

250 Vgl. Picot, A., Böhme, M., Controlling in dezentralen Unternehmensstrukturen, München 1999, S. 6

251 In diesem Zusammenhang wird auch vom sog. ${ }_{n}$ Freezing Point" gesprochen. Vgl. Rommel, G. et al., Einfach überlegen. Das Unternehmen, das die Schlanken schlanker macht und die Schnellen schneller macht, Stuttgart 1993, S. 37. Vgl. auch Kapferer, J.-N., Die Marke - Kapital des Unternehmens, a.a.O., S. 212. Eine ausreichende Differenzierung ist dem Volkswagen-Konzern beispielsweise bei der Platzierung des VW-Lupo parallel zum Seat Arosa nicht gelungen. Da beide Automobile des Kleinwagensegments nahezu baugleich sind, sich im Preis jedoch unterscheiden, hat die Einführung des Lupo den Absatz des Arosa erheblich angegriffen. 
schung variantenabhängiger Kosten bewegen sich dabei im Spannungsfeld zwischen der Sicherstellung eines möglichst hohen Differenzierungsgrades zwischen den Modellen und Marken einerseits sowie der Realisierung möglichst hoher Kostensenkungseffekte andererseits, welche wiederum direkt vom Grad der Standardisierung abhängen. Die Aufgabe eines Automobilherstellers stellt sich entsprechend als Wahl einer geeigneten Fahrzeugkosten-ProduktdifferenzierungsKombination. Den Zusammenhang zwischen Herstellkosten und Differenzierungspotenzial verdeutlicht Abb. 14, wobei offensichtlich wird, dass Differenzierung Kosten verursacht. ${ }^{252}$

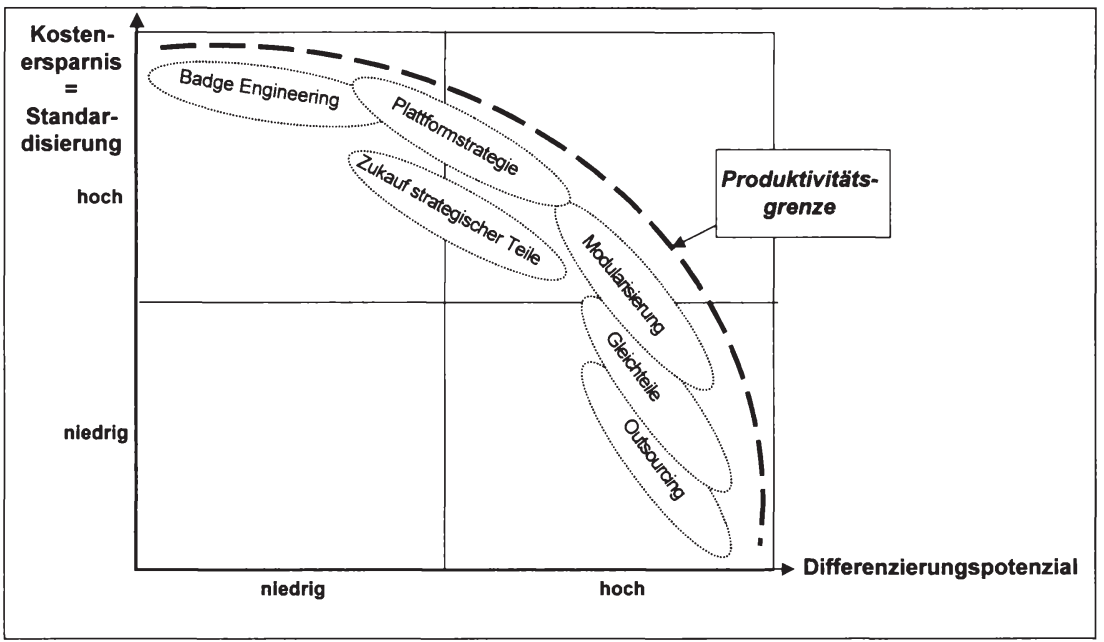

Abb. 14: Auswirkungen der Produktionskonzepte auf Kosten und Differenzierungsposition

Die dargestellte Produktivitätsgrenze kennzeichnet maximale Werte von Standardisierungs-Differenzierungs-Kombinationen, die Automobilhersteller momentan zu realisieren in der Lage sind. Werte oberhalb dieser Linie, d.h. eine kostengünstigere Produktion mit gleichem Grad an Differenzierung et vice versa sind nicht möglich. Die Nutzung von Synergien im Markenportfolio soll bewirken, dass das Portfolioergebnis der Mehrmarkenstrategie mehr ist als die Summe der markenspezifi-

252 Vgl. Porter, M. E., What is Strategy, a.a.O., S. 62. Ähnliche Darstellungen mit anderer Achsenbezeichnung finden sich bei Dudenhöffer, F., Marken-Management bei Produktkonvergenz - Neue Ansätze im Automobilmarketing, a.a.O., S. 11. 
schen Einzelergebnisse. Ob die Nutzung innerbetrieblicher Synergien jedoch immer ein höheres Ergebnis gewährleistet, lässt sich a priori nicht eindeutig festlegen, da die Bewertung der objektiven Angleichung letztlich durch den Markt erfolgt.

Grundsätzlich führt eine übermäßige Synergienutzung bei essentiellen Merkmalen der Marken zu einer Schwächung der Markenidentitäten des Portfolios und mithin zur Erosion vormals konturierter Marken in der Wahrnehmung der externen Anspruchsgruppen und erhöht damit aufgrund fehlender Trennschärfe die Austauschbarkeit der Marken, welche grundsätzlich durch den getrennten Markenauftritt minimiert werden soll. ${ }^{253}$

Je höher die Standardisierung in Mehrmarkenstrategien, um so größer ist der Controllingbedarf zur Überwachung eines differenzierten Markenauftritts der Portfoliomarken.

\subsection{Externe Kontextfaktoren des Controllingbedarfs}

\subsection{Substitution}

Werden die charakteristischen Unterschiede zwischen den verschiedenen Portfoliomarken nicht mehr differenziert wahrgenommen bzw. wird der Marktauftritt zunehmend homogenisiert, steigt die Gefahr einer Bearbeitung der gleichen Nachfragersegmente. Im externen Marktauftritt stellt somit die Kannibalisierung der Marken durch gegenseitige Marktanteilssubstitution das zentrale Risiko von Mehrmarkenstrategien dar. In diesem Fall gehen die Umsatzerfolge einer Marke zu Lasten der Umsätze anderer Marken des Portfolios.

Diesen Sachverhalt verdeutlicht Abb. 15. Im Volkswagen-Konzern geht die fehlende technische Differenzierung der Marken aufgrund der intensiven Verwendung von Gleichteilen im Konzern mit einer zunehmenden Homogenisierung des Marktauftritts einher und hat zur Konsequnez, dass die Marke WW zunehmend Käufer an die preislich niedriger positionierten Marken SKODA und SEAT verliert.

253 Vgl. Meffert, H., Burmann, Ch., Identitätsorientierte Markenführung - Grundlagen für das Management von Markenportfolios, a.a.O., S. 15. 
Insbesondere gegenüber der Preismarke SKODA haben sich die Verlust in 1999 gegenüber 1997 nahezu verfünffacht. ${ }^{254}$

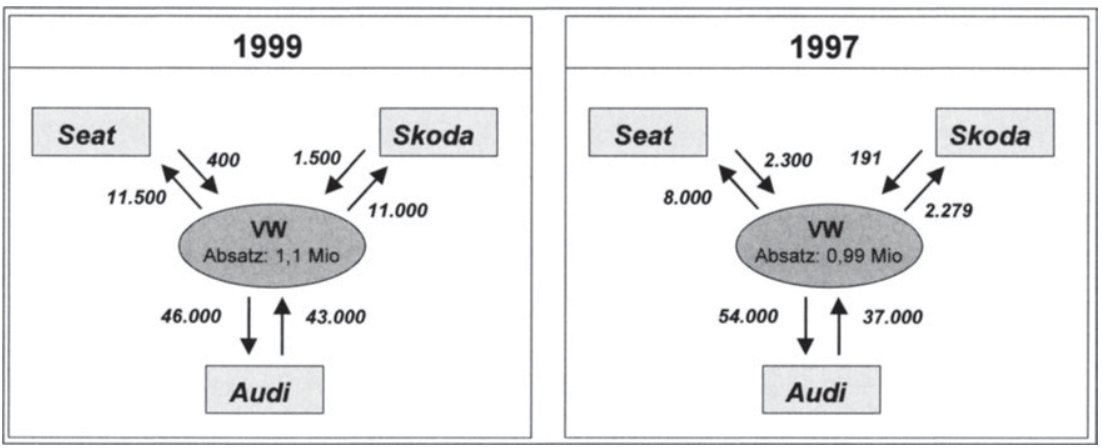

Abb. 15: Substitution im Volkswagen-Konzern (nach Anzahl der Kaufverträge) (Quelle: Wirtschaftswoche vom 1.6.2000, Heft 23, S. 12.)

Portfolioübergreifende Substitution zwischen Marken verschiedener Hersteller kann mit GUTENBERG als Partizipationseffekt bezeichnet werden. ${ }^{255}$ Hiermit wird ausgedrückt, dass durch die Marken eines Portfolios eine Absatzpartizipation zu Lasten anderer Anbieter eines anderen Portfolios in einem Markt erreicht wird. Die Untersuchung bzw. Überlagerung von Partizipations- und Substitutionseffekten stellt somit einen zentralen Ansatzpunkt zur Analyse der Wirksamkeit einer Mehrmarkenstrategie dar. Im Spannungsfeld zwischen Substitution und Partizipation gilt es, eine bestmögliche Marktabdeckung unter Minimierung von Substitutionseffekten innerhalb des Markenportfolios sicherzustellen.

Je höher die Substitutionsbeziehungen im Markenportfolio, desto größer ist der Controllingbedarf.

Substitutionseffekte können durch eine andere Positionierung einer bereits eingeführten Marke verhindert oder zumindest abgeschwächt werden. Die Umsatzwir-

254 Vgl. hierzu O.V., Piechs Sündenbock, in: Wirtschaftswoche vom 1.6.2000, Heft 23, S. 12.

255 Vgl. Gutenberg, E., Grundlagen der Betriebswirtschaftslehre, Zweiter Band, Der Absatz, 16. Aufl., Berlin, Heidelberg, New York 1979, S. 548; Vgl. Jacob, H., Der Absatz, in: Allgemeine Betriebswirtschaftslehre: Handbuch für Studium und Prüfung, Jacob, H. (Hrsg.), 5. Aufl., Wiesbaden 1988, S. 358; Meffert, H., Marketing: Grundlagen marktorientierter Unternehmensführung: Konzepte - Instrumente - Praxisbeispiele, a.a.O., S. $433 \mathrm{ff}$. 
kungen einer Mehrmarkenstrategie erhöhen sich dabei, wenn jede Marke auf ein anderes Segment abzielt.

\subsection{Ausstrahlungseffekte}

Die Markenportfolios von Automobilherstellern sind überwiegend dadurch gekennzeichnet, dass sie mehrere Firmen- bzw. Dachmarken beinhalten, unter denen wiederum einzelne, unterschiedlich markierte Produktlinien geführt werden. Entsprechend werden die Vorstellungsbilder externer Anspruchsgruppen von einer konkreten Produktmarke (z. B. WW Golf) maßgeblich durch die Einstellungen gegenüber der Dachmarke (Volkswagen) sowie den sonstigen Produktmarken geprägt. Gleichzeitig kann aber auch die betrachtete Produktmarke zum Image der anderen Modelle sowie zum gesamten Dachmarkenimage beitragen. Falls die $\mathrm{Zu}$ gehörigkeit der Dachmarken zum gleichen Unternehmen gegenüber dem Endverbraucher nicht verborgen oder besonders hervorgehoben wird, ist überdies davon auszugehen, dass sich die Images der Dachmarken gegenseitig beeinflussen und schließlich sämtliche Dachmarken einen Beitrag zum Image des Gesamtunternehmens leisten. Die skizzierten Ausstrahlungseffekte sind in Abb. 16 systematisiert.

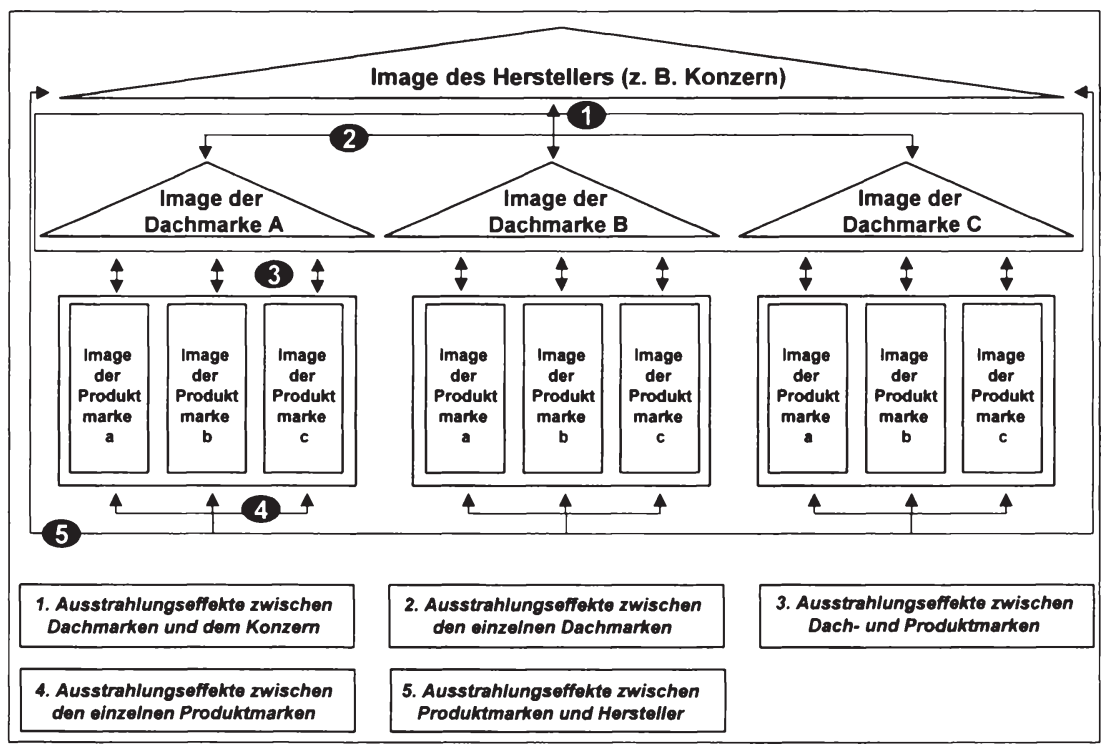

Abb. 16: Ausstrahlungseffekte zwischen Produkt- und Dachmarken 
Wichtig ist in diesem Zusammenhang vor allem

- die Richtung der Ausstrahlungseffekte, d. h. in welche Richtung dominierende Beziehungen verlaufen,

- die Stärke der Ausstrahlungseffekte, d. h. in welchem Maße die Images der jeweiligen Marken tatsächlich durch andere Vorstellungsbilder geprägt werden sowie

- die Qualität der Ausstrahlungseffekte, d. h. welche Eigenschaftsdimensionen in positiver oder negativer Weise von den Verbundwirkungen betroffen sind. ${ }^{256}$

Die Identifikation positiver (Goodwill-Transfer) oder negativer (Badwill-Transfer) Ausstrahlungseffekte im Rahmen von Mehrmarkenstrategien ist mit Schwierigkeiten behaftet, da sie nur schwer meßbar sind und vielfach erst langfristig in späteren Perioden auftreten. ${ }^{257}$ Ein zentrales Problem besteht in der Isolierbarkeit der einzelnen Effekte, da sowohl direkte als auch indirekte Ausstrahlungseffekte zu berücksichtigen sind. So kann das externe Vorstellungsbild gegenüber einer Produktmarke 1 direkt durch das Dachmarkenimage A determiniert sein, welches wiederum wesentlich durch eine andere Produktmarke 2 geprägt wird, so dass 2 eine indirekte Wirkung auf 1 ausübt. ${ }^{258}$ Ebenso kann im Portfolioverbund das Dachmarkenimage A direkten Einfluss auf das Herstellerimage ausüben, welches wiederum positiv auf sonstige Dachmarken im Portfolio abstrahlt (z. B. VWSkoda).

Vgl. auch Waltermann, B., Internationale Markenpolitik und Produktpositionierung: markenpolitische Entscheidungen im europäischen Automobilmarkt, Wien 1989, S. $174 \mathrm{ff}$.

SULLIVAN wies beispielsweise einen Badwill-Transfer in einer empirischen Studie nach, in deren Zentrum ein von einzelnen Fahrern eines AUDI 5000 in den USA bemängelter Defekt stand. Amerikanische Medien zweifelten daraufhin die Sicherheit des AUDI 5000 an. Berichten von Unfallopfern begegnete man seitens AUDI mit Widerruf. Statt des vorgeworfenen technischen Defekts beim Leerlauf des Motors sahen die Verantwortlichen die Verwechslung von Gaspedal und Bremse als Ursache des Problems an. Als Konsequenz sanken die Absatzzahlen von AUDI auf dem amerikanischen Markt rapide. Der Rückgang beschränkte sich jedoch keineswegs auf das in die Kritik geratene Modell, sondern auch die Modell AuDI 4000 und AUDI QUATTRO verloren nachhaltig Marktanteile. Obwohl der beschriebene Defekt nur bei der Automatikversion des AUDI 5000 lag, strahlte der Badwill somit auch auf Fahrzeuge mit Schaltgetriebe aus. Vgl. Sullivan, M., Measuring Image Spillovers in Umbrella-branded Products, in: Journal of Business, 63. Jg., Heft 3, 1990, S. 309ff.

So wird die Dachmarke Volkswagen maßgeblich durch das Image des VW-Golf geprägt, da Konsumenten die Dachmarke Volkswagen unmittelbar mit dem Golf in Verbindung bringen. Der Golf kann mithin auch als Träger des Volkswagen-Images bezeichnet werden. 
Die Stärke der Ausstrahlungseffekte ist typischerweise zwischen den Dachmarken und ihren jeweiligen Produktmarken am größten. Zum einen ist zu erwarten, dass Erfahrungen mit den Produkten positive oder negative Vorstellungsbilder über die entsprechende Dachmarke beim Konsumenten festigen, zum anderen werden Einstellungen aufgrund des aus der Psychologie bekannten Generalisationsprinzips von einem Produkt auf ein anderes Produkt der gleichen Dachmarke übertragen, da beide Produkte aufgrund des gemeinsamen Dachs eine wahrnehmbare Gemeinsamkeit aufweisen. ${ }^{259}$ Eine solche Gemeinsamkeit zwischen den Dachmarken eines Portfolios liegt nur dann vor, wenn die Zugehörigkeit der Marken zum Portfolio und mithin zum gleichen Hersteller den externen Anspruchsgruppen nicht verborgen bleibt. Die Dachmarke schränkt die Freiheitsgrade bei der Produktpositionierung somit ein, da die Dachmarke auf das Image der Einzelprodukte ausstrahlt. Je stärker die Dachmarke dabei in den Vordergrund rückt, desto ähnlicher wird die Wahrnehmung der Produkte ausfallen. ${ }^{260}$

Aus einer dynamischen Perspektive dienen Erfahrungen mit einer Produktmarke auch als Indikator für die zukünftige Qualität der gleichen oder anderer Produktmarken der gleichen Dachmarke. Die Informationsinhalte über ein Produkt bzw. eine Marke können sowohl aus eigenen Erfahrungen als auch aus fremden Erfahrungen (Informationen Dritter) wie auch aus Aussagen stammen, die vom Anbieter mit Hilfe von Marketinginstrumenten übermittelt werden. So erhöht die Werbung für eine bestimmte Produktmarke den Bekanntheitsgrad der Dachmarke, so dass im Laufe der Zeit sämtliche unter dem Dach der Marke zusammengefassten Produkte von der Werbemaßnahme profitieren.

Analoge Beziehungen bestehen auch im Portfolioverbund zwischen den Dachmarken. Preiserhöhungen einer Portfoliomarke beeinflussen nicht nur den Absatz dieser Marke, sondern können - insbesondere unter dem Bewusstsein technischer Homogenität der Marken im Portfolio - den heutigen oder zukünftigen Absatz anderer Marken des Portfolios maßgeblich beeinflussen. In Abb. 17 sind

259 Beispielsweise schließt die Markenpolitik von PROCTER \& GAMBLE einen Transfer auf Konsumentenebene weitgehend aus, da die Zugehörigkeit der Marken zum Firmennamen und dieser selbst kaum bekannt sind. Vgl. Simon, H., Goodwill und Marketingstrategie, Wiesbaden 1985, S. $25 \mathrm{ff}$.

Eine Schlussfolgerung könnte hier lauten, dass eine Einzelmarkenstrategie um so eher angezeigt scheint, je stärker sich die Präferenzstrukturen der von den Produkten anzusprechenden Marktsegmente unterscheiden. 
für den einfachsten Fall zweier Produktmarken $a$ und $b$ einer Dachmarke $A$ sämtliche Wirkungseffekte einer auf Produktmarke a bezogenen Marketingmaßnahme veranschaulicht. Die Wirkungen lassen sich anhand der Überschreitung der Linien $Y$ und $Z$ wie folgt klassifizieren: ${ }^{261}$

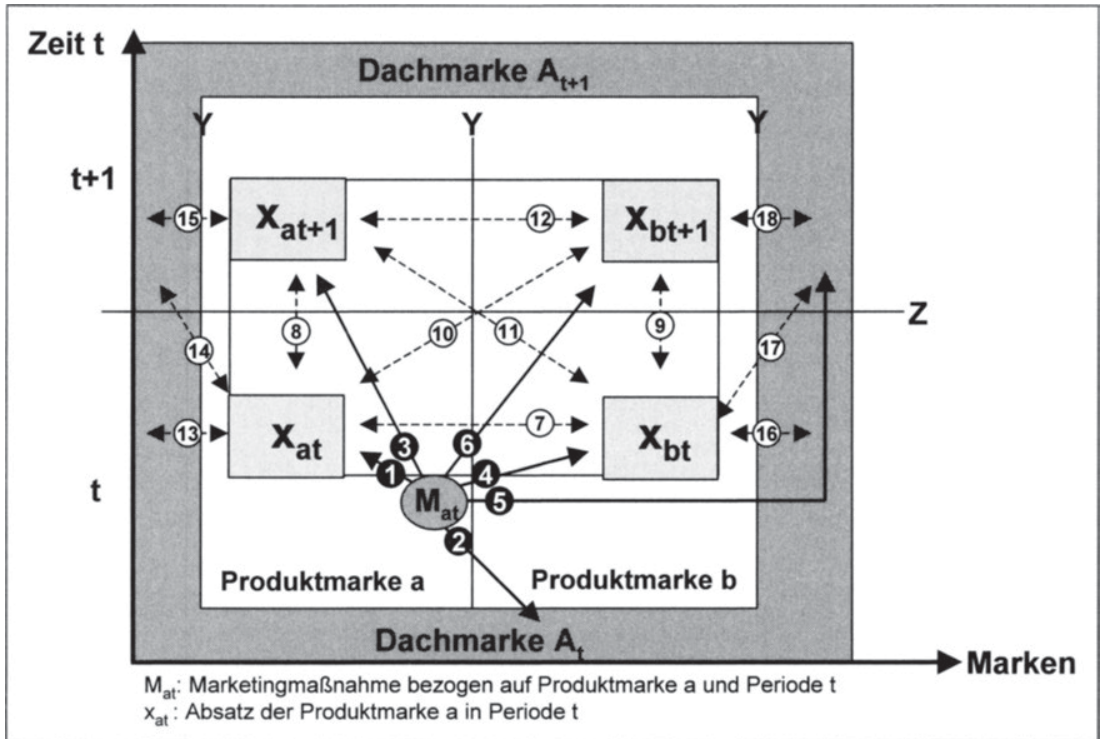

\begin{tabular}{|l|c|c|}
\hline Unmittelbare Wirkung (weder $Y$ noch $Z$ wird überschritten) & \multicolumn{2}{|c|}{1} \\
\hline Ausstrahlungseffekte & Direkt & Indirekt \\
\hline Dynamische: nur Z wird überschritten & 3 & 7,8 \\
\hline Statisch-markenübergreifende: nur $Y$ wird überschritten & 2,4 & $6,11,12$, \\
\hline Dynamisch-markenübergreifende: $Y$ und Z werden überschritten & 5 & $9,10,13,16$ \\
\hline
\end{tabular}

Abb. 17: Dynamische und markenübergreifende Ausstrahlungseffekte (Quelle: Aufbauend auf den Überlegungen von Simon, H., Goodwill und Marketingstrategie, a.a.O., S. 21.)

Neben der unmittelbaren Auswirkung [1] $]^{262}$ auf die Produktmarke a in der gleichen Periode (kein Ausstrahlungseffekt) kann die Maßnahme auch erst in einer nächsten Periode wirken [3, dynamisch] bzw. direkte Ausstrahlungseffekte auf die

Vgl. zur Strukturierung des Goodwill-Transfers auch Simon, H., Goodwill und Marketingstrategie, a.a.O., S. $19 \mathrm{ff}$. 
Dachmarke A bzw. Produktmarke $b$ in der gleichen $[2,4$, statisch-markenübergreifend] bzw. der nächsten Periode [5, 6, dynamisch-markenübergreifend] nach sich ziehen. ${ }^{263}$ Überdies ergeben sich statische und dynamische indirekte Wirkungen aufgrund von Erfahrungen mit den Produktmarken a und b sowohl zwischen den Produktmarken [7-12] als auch mit Bezug auf die Dachmarke A [1318].

Die Abbildung verdeutlicht letztlich prägnant die zahlreichen markenübergreifenden und dynamischen Interdependenzen, die im Markenportfolio selbst im einfachsten Fall zweier Produkte einer Dachmarke zu berücksichtigen sind. Idealtypisch gilt es, positive Ausstrahlungseffekte zu stärken bzw. negative Effekte zu vermeiden.

Je höher die Ausstrahlungseffekte zwischen den Portfoliomarken, desto größer ist der Controllingbedarf in Mehrmarkenstrategien.

Wie aufgezeigt, steht das Image der Marken als Fremdbild der Markenidentität in einer wechselseitigen Beziehung zu den aktiven, marktorientierten Handlungen des Unternehmens selbst (Selbstbild der Markenidentität). Ursachen von Ausstrahlungseffekten zwischen den Marken sind somit - neben wenig beeinflussbaren unternehmensexternen Gründen - primär in der Art der Formulierung bzw. Umsetzung und Abstimmung der Selbstbilder der Portfoliomarken zu vermuten.

Da nur das Selbstbild vom Management direkt gesteuert werden kann, stellt es auch den Ausgangspunkt einer Steuerung markenspezifischer Merkmalsausprägungen zur Verstärkung oder Schwächung von Ausstrahlungseffekten zwischen den Portfoliomarken dar. Bei der Führung von Markenportfolios ergibt sich dabei grundsätzlich die Notwendigkeit, die Identitäten der Portfoliomarken wie folgt abzustimmen (vgl. Abb. 18). ${ }^{.64}$

263 Während bei Carry-over-Effekten Wirkungen von Markenaktivitäten nicht nur in der Periode ihres Einsatzes, sondern auch in späteren Perioden auftreten, wirken bei Time-lag-Effekten Markenaktivitäten nicht in der Periode ihres Einsatzes, sondern treten erst in späteren Perioden auf. Vgl. Sander, M., Die Bestimmung und Steuerung des Wertes von Marken. Eine Analyse aus Sicht des Markeninhabers, a.a.O., S. 247.

Vgl. hierzu Meffert, H., Burmann, Ch., Identitätsorientierte Markenführung - Grundlagen für das Management von Markenportfolios, a.a.O., S. $48 f$. 


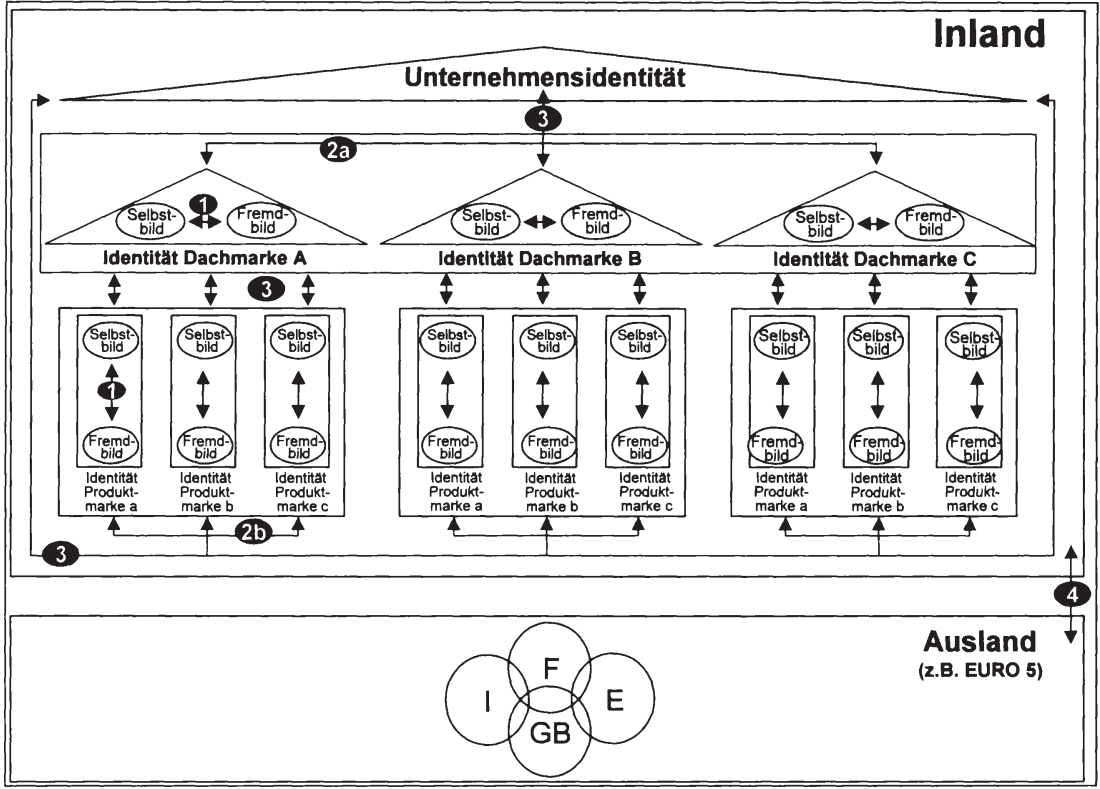

Abb. 18: Spannungsfelder der Markenidentitäten

1. Abstimmung des Selbst- und Fremdbildes der Identität jeder Marke.

2. Ebenenspezifische wechselseitige Abstimmung der Identitäten zwischen den Marken des Portfolios, d.h. zwischen

- 2a) den Dachmarken untereinander, wobei mit zunehmender Heterogenität der Dachmarkenidentitäten im internen Markenverbund die notwendige Differenzierungsfähigkeit zwischen den Dachmarken steigt.

- 2b) den Produktmarken untereinander, wobei mit zunehmender Homogenität der Produktmarkenidentitäten die Prägnanz der jeweiligen Dachmarke steigt, denn „entspricht die Substanz der Produkte [bzw. der Produktmarken, Anm. d. V.] nicht den Ansprüchen der [Dach-, Anm. d. V.]Marke, fehlt die „Erdung“ und die Marke wird unglaubwürdig ". ${ }^{265}$

3. Ebenenübergreifende Abstimmung der einzelnen Markenidentitäten.

4. Abstimmung der unterschiedlichen Identitätswahrnehmungen in verschiedenen Ländermärkten. 
Da in Mehrmarkenstrategien vor allem die Dachmarken im Fokus der Betrachtung stehen, bilden diese auch den zentralen Ansatzpunkt der Identitätssteuerung. ${ }^{266}$ Weicht das formulierte Selbstbild vom wahrgenommenen Fremdbild der Dachmarken $a b$, gilt es, die Ursachen hierfür zu identifizieren und entsprechende Anpassungen zu initiieren, um ungewollte Ausstrahlungseffekte zwischen den Marken zu vermeiden.

Je größer die Diskrepanz zwischen Selbstbild und Fremdbild der Portfoliomarken, desto größer ist der Controllingbedarf in Mehrmarkenstrategien.

\subsection{Marktliche Interdependenzen}

Der Komplexitätsgrad des Absatzmarktes ergibt sich aus der Anzahl der Marktsegmente, aus der Verschiedenartigkeit der Segmentbedürfnisse sowie aus der Vielschichtigkeit der Beziehungen zwischen den Anbietern und den Segmenten. ${ }^{267}$ Zur Identifikation marktbezogener Interdependenzprobleme bietet es sich an, die einzelnen Marken des Portfolios hinsichtlich ihrer Breite, Höhe und Tiefe im Marktauftritt zu kennzeichnen (Abb. 19). ${ }^{268}$ Die Breite des Marktauftritts bezieht sich auf die Zahl der unter einer Marke angebotenen Produkte. Für Automobilhersteller stellt sie einen Ausdruck dafür dar, für welche Größen- und Karosserievarianten die jeweilige Marke grundsätzlich eingesetzt werden kann (Markenausdehnung). Die Höhe des Markenauftritts umfasst hingegen die aus den strategischen Rollen abgeleitete Grundpositionierung der einzelnen Marken (Premiummarke versus Preismarke etc.). ${ }^{269}$ Die Tiefe des Marktauftritts beinhaltet schließlich die geographische Reichweite der Portfoliomarken. Zwischen Breite, Höhe und Tiefe des Marktauftritts bestehen vielfältige Interdependenzen.

Vgl. hier auch Meffert, H., Perrey, J., Mehrmarkenstrategie - Ein Beitrag zum Management von Markenportfolios, a.a.O., S. $626 f$.

So auch BENKENSTEIN in Anlehnung an LUHMANN. Vgl. Benkenstein, M., F\&E und Marketing: eine Untersuchung zur Leistungsfähigkeit von Koordinationskonzeptionen bei Innovationsentscheidungen, a.a.O..

BECKER bezeichnet die Festlegung der Kompetenzbreite, -höhe und -tiefe auch als markenpolitische Grundsatzentscheidungen. Vgl. hierzu Becker, J., Typen von Markenstrategien, a.a.O., S. $468 f$.

269 So ist für eine klar erkennbare Umsetzung eines Positionierungskonzepts vor allem entscheidend, ob die für die Marketinginstrumente geplanten Maßnahmen auch entsprechend von den Konsumenten wahrgenommen werden. Vgl. Esch, F.-R., Markenpositionierung als Grundlage der Markenführung, a.a.O., S. 254. 


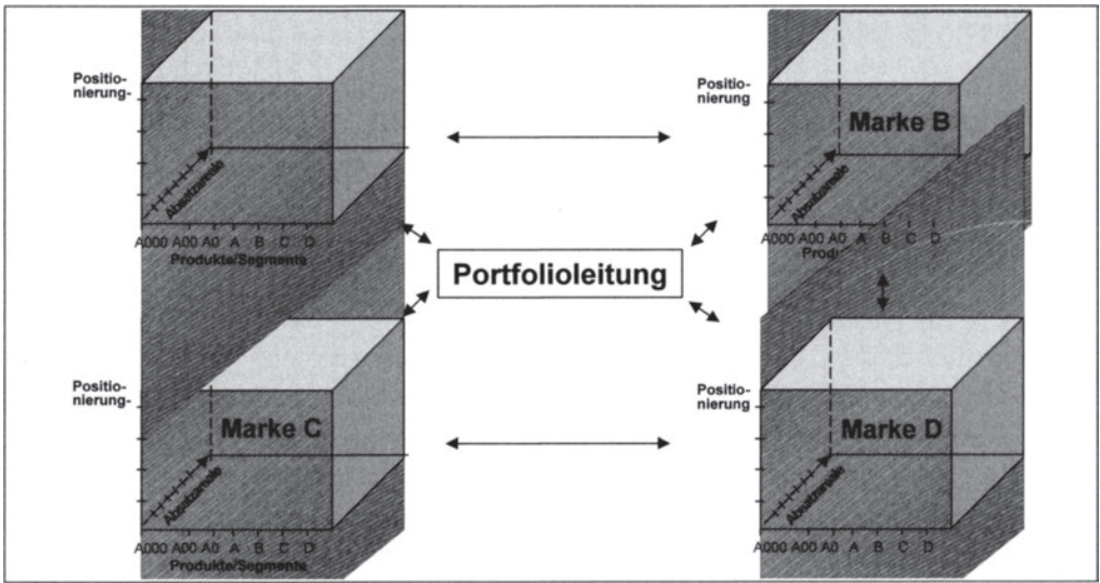

Abb. 19: Breite, Höhe und Tiefe des Marktauftritts der Portfoliomarken

Zur Sicherstellung der markenpolitischen Schlüssigkeit sind die Ebenen somit sowohl innerhalb einer Marke aufeinander abzustimmen als auch markenübergreifend im Portfolio und gegenüber den Wettbewerbern zu koordinieren. Neben der

- Gefahr einer Markenüberdehnung aufgrund mangelnder Abstimmung zwischen der Kompetenzhöhe und -breite einer Marke, die sich darin ausdrücken kann, dass zu viele Segmente mit einer Marke abgedeckt werden, ${ }^{270}$ besteht das

- Risiko einer zunehmenden Substitution im Portfolio bei paralleler Ausrichtung der jeweiligen Markenaktivitäten auf die gleichen Marktsegmente.

Folglich bedarf es hier der Bestimmung eines geeigneten Grads der Segmentabdeckung durch die jeweiligen Portfoliomarken. Insbesondere in der Automobilindustrie ist dabei eine zunehmende Ausdehnung der Produktprogramme zu beobachten, wie Tab. 5 für die Marken AUDI, BMW, MERCEDES-BENZ und VW verdeutlicht.

Im Kontext der Bildung von Zielsegmenten für die einzelnen Marken ist darüber hinaus das Problem der mangelnden zeitlichen Stabilität einer Segmentlösung zu berücksichtigen. Vielfach degeneriert eine erzielte Zielgruppenlösung aufgrund der zunehmend individualisierten Konsumwünsche und der Inkonsistenz des Nachfra-

270 Vgl. auch Smith, D. C., Parc, W. C., The Effects of Brand Extensions on Market Share and Advertising Efficiency, in: Journal of Marketing Research, Vol. 29, Nr. 1, S. $296 \mathrm{ff}$. 
geverhaltens nur noch zu einer Momentaufnahme. ${ }^{271}$ Veränderte Präferenzen oder strukturelle Nachfrageverschiebungen können dabei mit einem Verlust an wahrgenommener Trennschärfe zwischen den Marken einhergehen und damit eine differenzierte Positionierung (Höhe des Markenauftritts) der Marken im Portfolio gefährden.

\begin{tabular}{|c|c|c|c|c|c|c|c|}
\hline \multicolumn{2}{|c|}{ Audi } & \multicolumn{2}{|c|}{ WW } & \multicolumn{2}{|c|}{ BMW } & \multicolumn{2}{|c|}{ Mercedes-Benz } \\
\hline $\begin{array}{l}\text { - Audi quattro } \\
\text { - Audi 100/20ф } \\
\text { - Lim } \\
\text { - Audi } 80 / 90 \\
\text { - Lim }\end{array}$ & $\begin{array}{l}\text { - Audi A8 } \\
\text { - Audi A6 } \\
\text { - Lim } \\
\text { - Avant } \\
\text { - Audi TT } \\
\text { - Coupé } \\
\text { - Roadster } \\
\text { - Audi Cabrio } \\
\text { - Audi A4 } \\
\text { - Lim } \\
\text { - Avant } \\
\text { - Audi A3 }\end{array}$ & $\begin{array}{l}\text { - Passat } \\
\text { - Lim } \\
\text { - Kombi } \\
\text { - Corrado } \\
\text { - Jetta } \\
\text { - Lim } \\
\text { - Golf } \\
\text { - Lim } \\
\text { - Cabrio } \\
\text { - Polo } \\
\text { - Lim } \\
\text { - Coupé }\end{array}$ & $\begin{array}{l}\text { - Passat } \\
\text { - Lim } \\
\text { New Beetle } \\
\text { - Lim } \\
\text { - Kombi } \\
\text { - Golf } \\
\text { - Lim } \\
\text { - Kombi } \\
\text { - Cabrio } \\
\text { - Polo } \\
\text { - Lim } \\
\text { - Kombi } \\
\text { - Lupo }\end{array}$ & $\begin{array}{l}\text { - 8er } \\
\cdot 7 \mathrm{er} \\
\cdot \text {-5er } \\
\text { - Lim } \\
\text { - 3er } \\
\text { - Lim } \\
\text { - Touring } \\
\text { - Cabrio }\end{array}$ & $\begin{array}{l}\text { - 8er } \\
\cdot 7 \mathrm{er} \\
\cdot \text { X5 } \\
\text { - Z3 } \\
\text { - Coupé } \\
\text { - Roadster } \\
\text { - 5er } \\
\text { - Lim } \\
\text { - Touring } \\
\text { - 3er } \\
\text { - Lim } \\
\text { - Touring } \\
\text { - Cabrio } \\
\text { - Coupé } \\
\text { - Compact }\end{array}$ & $\begin{array}{l}\text {-S-Klasse } \\
\text { - Lim } \\
\text { - Coupé } \\
\text { - SL } \\
\text { - } 200-300 \\
\text { - Lim } \\
\text { - Kombi } \\
\text { - Coupé } \\
\text { - 190er } \\
\text { - Lim }\end{array}$ & $\begin{array}{l}\text { - S-Klasse } \\
\text { - Lim. } \\
\text { - Coupé } \\
\text {-V-Klasse } \\
\text { - M-Klasse } \\
\text { - E-Klasse } \\
\text { - Lim } \\
\text { - Kombi } \\
\text { - CLK } \\
\text { - Coupé } \\
\text { - Cabrio } \\
\text { - SLK } \\
\text { - C-Klasse } \\
\text { - Lim. } \\
\text { - Kombi } \\
\text {-A-Klasse }\end{array}$ \\
\hline 1990 & 1999 & 1990 & 1999 & 1990 & 1999 & 1990 & 1999 \\
\hline
\end{tabular}

Tab. 5: Markenausdehnung ausgewähiter deutscher Automobilhersteller

Schließlich ist die parallele Führung mehrerer Marken mit der Gefahr einer zu feinen Parzellierung und damit der Übersegmentierung des Gesamtmarktes verbunden. ${ }^{272}$ Auch ist die Ausrichtung auf zu viele Teilsegmente mit separaten Marken wenig zweckmäßig, wenn deren Bearbeitung keine hinreichende Profitabilität garantiert oder sonstige wettbewerbsstrategische Funktionen für das Markenportfolio erfüllt. ${ }^{273}$

Mit zunehmender marktlicher Interdependenz (fehlender Unabhängigkeit) steigt der Controllingbedarf bei der parallelen Marktbearbeitung mit mehreren Marken.

271 Vgl. Perrey, J., Nutzenorientierte Marktsegmentierung - Ein integrativer Ansatz zum Zielgruppenmarketing im Verkehrsdienstleistungsbereich, Wiesbaden 1998, S. $227 \mathrm{ff}$.

$272 \mathrm{Vgl}$. Becker, J., Marketing-Konzeption: Grundlagen des strategischen und operativen Marketing-Management, a.a.O., S. 291.

273 Vgl. Kotler, Ph., Bliemel, F., Marketing-Management: Analyse, Planung, Umsetzung und Steuerung, a.a.O., S. 711. 


\subsection{Interdependenzen zwischen den Kontextfaktoren des Controllingbe- darfs}

Die vorangegangenen Überlegungen verdeutlichen, dass die Kontextfaktoren nicht isoliert auf den Controllingbedarf von Mehrmarkenstrategien einwirken, sondern zwischen den Faktoren Wechselwirkungen bestehen bzw. diese nicht überschneidungsfrei sind. Infolgedessen wird der Controllingbedarf auch weniger von Einzelfaktoren als vielmehr von Situationsclustern bestimmt.

Bezüglich ihrer Wirkungen auf den Controllingbedarf kann zwischen komplementären und substitutiven Beziehungen differenziert werden. So erhöht beispielsweise eine markenübergreifende Angebotsstandardisierung die Ähnlichkeit zwischen den Marken und damit die Gefahr einer fehlenden Trennschärfe bzw. negativer Ausstrahlungseffekte, wodurch das Risiko der Substitution im Portfolio steigt und der Controllingbedarf in Mehrmarkenstrategien zunimmt. Dieses Beziehungsgeflecht ist komplementärer Natur, da sich die Einflussfaktoren hinsichtlich ihrer Wirkungen auf den Controllingbedarf gegenseitig verstärken können. Beinhaltet das Portfolio dabei nur wenige Marken und verfügt die Portfolioleitung über ein ausreichend relevantes Wissen hinsichtlich der Beziehungsstrukturen im Portfolio, sinkt der Controllingbedarf in Mehrmarkenstrategien.

Unabhängig davon, welche einzelnen Kontextfaktoren bzw. welche situativen Cluster die Höhe des Controllingbedarfs bestimmen, ist davon auszugehen, dass die Wirkungen des situativen Bedingungsrahmens auf den Controllingbedarf in der Regel indirekter Natur sind. Die zentral intervenierenden Variablen bilden letztlich

- die Art und Intensität der Interdependenzen zwischen den Marken sowie

- die sich aus den Entscheidungsspielräumen ergebenden Zielkonflikte zwischen den Marken sowie der Portfolioleitung (vgl. Abb. 20).

Das Ziel des Mehrmarkencontrolling liegt - wie aufgezeigt - in der umfassenden Unterstützung der Portfolioleitung im Hinblick auf eine Ergebnisoptimierung des externen und internen Zusammenspiels der Portfoliomarken. In diesem Zusammenhang hat es vor allem die Angemessenheit von führungsrelevanten Daten, 
Methoden und Systemen im Rahmen eines geeigneten Controllingansatzes sicherzustellen. $^{274}$

\begin{tabular}{|r|r|r|r|r|}
\hline Interdependenzintensität \\
im Portfolio
\end{tabular}

Abb. 20: Einfluss der Kontextfaktoren auf den Controllingbedarf in Mehrmarkenstrategien

2. Controllingkonzeption zur Deckung und Reduktion des Controllingbedarfs

\subsection{Controllingprozess und Anforderungen an das Controlling von Mehr- markenstrategien}

Zur Erfüllung der Koordination im Markenportfolio sind Informationen von hoher Bedeutung, da ohne gezielten Informationsfluss eine Abstimmung arbeitsteilig vollzogener Handlungen nicht möglich ist. Infolgedessen erweist sich für das Mehrmarkencontrolling die Schaffung eines gezielten, entscheidungsrelevanten Informationsflusses als notwendige Bedingung zur Erfüllung der Koordinationsfunktion. ${ }^{275}$ Informa-

274 Vgl. Weber, J., Schäffer, U., Sicherstellung der Rationalität von Führung als Aufgabe des Controlling?, in: DBW, 59. Jg., Heft 6, 1999, S. 743.

275 Schon 1978 prägte HORVATH den Ausdruck "Koordination durch Information“. Horváth, P., Controlling - Entwicklung und Stand einer Konzeption zur Lösung der Adaptions- und Koordi- 
tionen werden dabei stets durch die zweckorientierte Aggregation von Daten generiert, so dass der zur Koordination notwendige Controllingprozess in Mehrmarkenstrategien auch als „Beschaffung, Aufbereitung und Analyse von Daten zur Vorbereitung zielsetzungsgerechter Entscheidungen“276 im Markenportfolio beschrieben werden kann (vgl. Abb. 21).

In Abgrenzung zum Informationsmanagement, das nur die informationswirtschaftliche Bereitstellung der führungsunterstützenden Informationen umfasst, bedeutet die Informationsversorgung im Sinne des Controlling überdies die inhaltliche Bestimmung der führungsunterstützenden Informationen im Prozess der Mehrmarkenführung. ${ }^{277}$ Die Verwendung derartiger Informationen trägt zur Koordination innerhalb des Führungssystems des Portfolios bei. ${ }^{278}$ Ermöglicht wird die Koordination durch Controllinginstrumente, in deren Mittelpunkt eine systematische Bestandsaufnahme der Marken des Markenportfolios steht. Dabei nehmen zeitablaufbezogene Analysen der Positionierung im Rahmen von Tracking-Studien und Wanderungsbilanzen eine zentrale Bedeutung ein. Abb. 21 verdeutlicht durch die beiden aus den Prozessstufen Datenbeschaffung sowie Datenaufbereitung und -analyse gerichteten Pfeile überdies, dass ein Teil der beschafften Daten auch ohne eine anschließende Aufbereitung weitergegeben werden kann. Aufgrund ihres Charakters als übergreifende Koordinationsinstrumente sollten die Informationen der dritten Prozessstufe insbesondere zur Koordination zwischen den Führungsteilsystemen im Markenportfolio beitragen. ${ }^{279} \mathrm{Da}$ dem Aufgabenspektrum des Mehrmarkencontrolling jedoch auch die Koordination innerhalb einzelner Subsysteme der Führung (also auch innerhalb

nationsprobleme der Führung, in: ZfB, 48. Jg., Heft 3, 1978, S. 195. Information kennzeichnet dabei zweckorientiertes Wissen, wobei der Zweck in der Vorbereitung des Handelns liegt.

Berens, W., Hoffjan, A., Strack, M., Okologiebezogenes Controlling - umweltorientierte Koordination in kommunalen Versorgungsunternehmen, in: Zeitschrift für öffentliche und gemeinwirtschaftliche Unternehmen, 18. Jg., Heft 2, 1995, S. 144.

277

Zur Abgrenzung von Informationsmanagement und Controlling vgl. Ahlert, D., Olbrich, R., Controlling und Informationsmanagement im Prozeß des strategischen Managements - Ein Diskussionsbeitrag zur Abgrenzung der Zuständigkeiten, Arbeitspapier Nr. 16 des Instituts für Handelsmanagement der Westfälischen Wilhelms Universität Münster, Ahlert, D. (Hrsg.), 2. Aufl., Münster 1996, S. $1 \mathrm{ff}$

Vgl. Köhler, R., Marketing-Controlling: Konzepte und Methoden, in: Marketing-Controlling, Reinecke, S., Tomczak, T., Dittrich, S. (Hrsg.), St. Gallen 1998, S. 10.

279 Vgl. auch Küpper, H.-U., Controlling: Konzeptionen, Aufgaben und Instrumente, a.a.O., S. 24ff. 
einzelner Marken) innewohnt, bedarf es im Controllingprozess zusätzlich isolierter Koordinationsinstrumente.

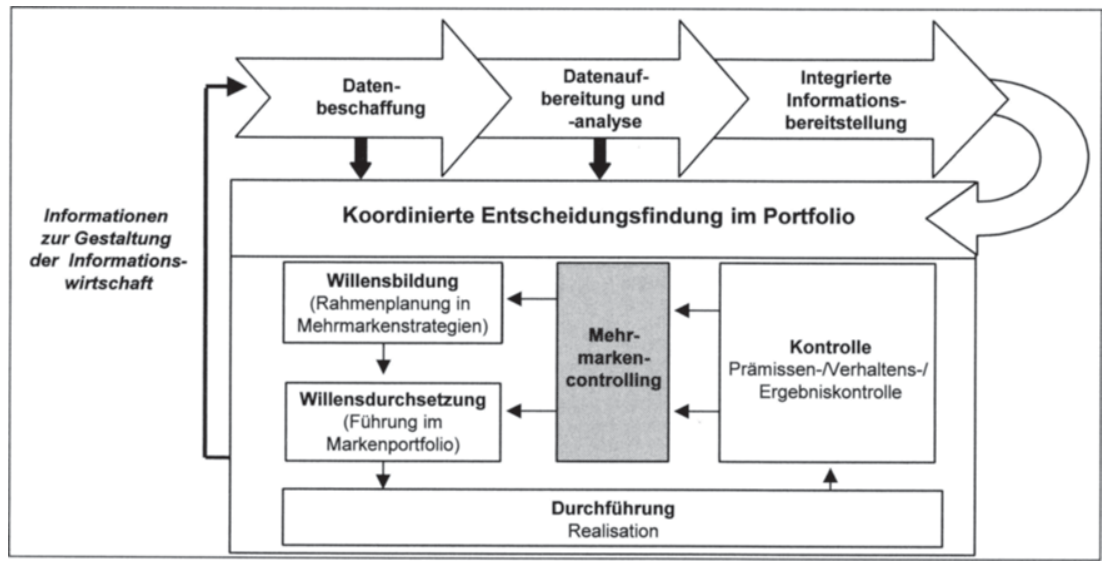

Abb. 21: Mehrmarkencontrolling im Prozess der Mehrmarkenführung

Eine integrierte Informationsbereitstellung lässt sich insbesondere durch den Aufbau eines Kennzahlensystems für das Markenportfolio gewährleisten. ${ }^{280}$ Kennzahlensysteme lassen sich mit REICHMANN definieren als „eine Zusammenstellung von quantitativen Variablen [...], wobei die einzelnen Kennzahlen in einer sachlich sinnvollen Beziehung zueinander stehen, einander ergänzen oder erklären und insgesamt auf ein gemeinsames übergeordnetes Ziel ausgerichtet sind." 281 Dabei tragen Kennzahlen und Kennzahlensysteme dann zur Koordination im Markenportfolio bei, „wenn sie als Ziel zur Steuerung genutzt werden“. ${ }^{282}$

Ein Beispiel für ein Kennzahlensystem, das sich allerdings auf monetäre und rein vergangenheitsorientierte Größen fokussiert, ist das DuPont-System of Financial Control, welches bereits 1919 von der amerikanischen Firma I. E. DuPont de Nemours \& Co. entwickelt wurde. Vgl. hierzu auch Heigl, A., Controlling - Interne Revision, Stuttgart, New York 1978, S. 103.

Reichmann, Th., Controlling mit Kennzahlen und Managementberichten: Grundlagen einer systemgestützten Controlling-Konzeption, a.a.O., S. 23.

Küpper, H.-U., Controlling: Konzeptionen, Aufgaben und Instrumente, a.a.O., S. 340. Mit PALLOKS lassen sich drei Einsatzbereiche betriebswirtschaftlicher Kennzahlen nennen, die sich entsprechend auf das Mehrmarkencontrolling übertragen lassen. Im Rahmen von kennzahlengestützten Strukturanalysen wird das jeweilige Untersuchungsobjekt aufgeschlüsselt und strukturiert, um so Hinweise auf Schwachstellen zu liefern. So können bei einer Strukturanalyse des Markenwertes die einzelnen wertbestimmenden Dimensionen einer Marke (je nach Definition des Markenwertes) untersucht werden und auf evtl. Positionierungsmängel im 
Mit dem Anspruch, einen wesentlichen Beitrag zur Koordination und Steuerung der Marken im Markenportfolio zu leisten, sind als Ausgangspunkt der Controllingkonzeption Gestaltungsanforderungen zu formulieren. Diese lassen sich deduktiv aus dem Zweck des Mehrmarkencontrolling ableiten (vgl. Abb. 22).

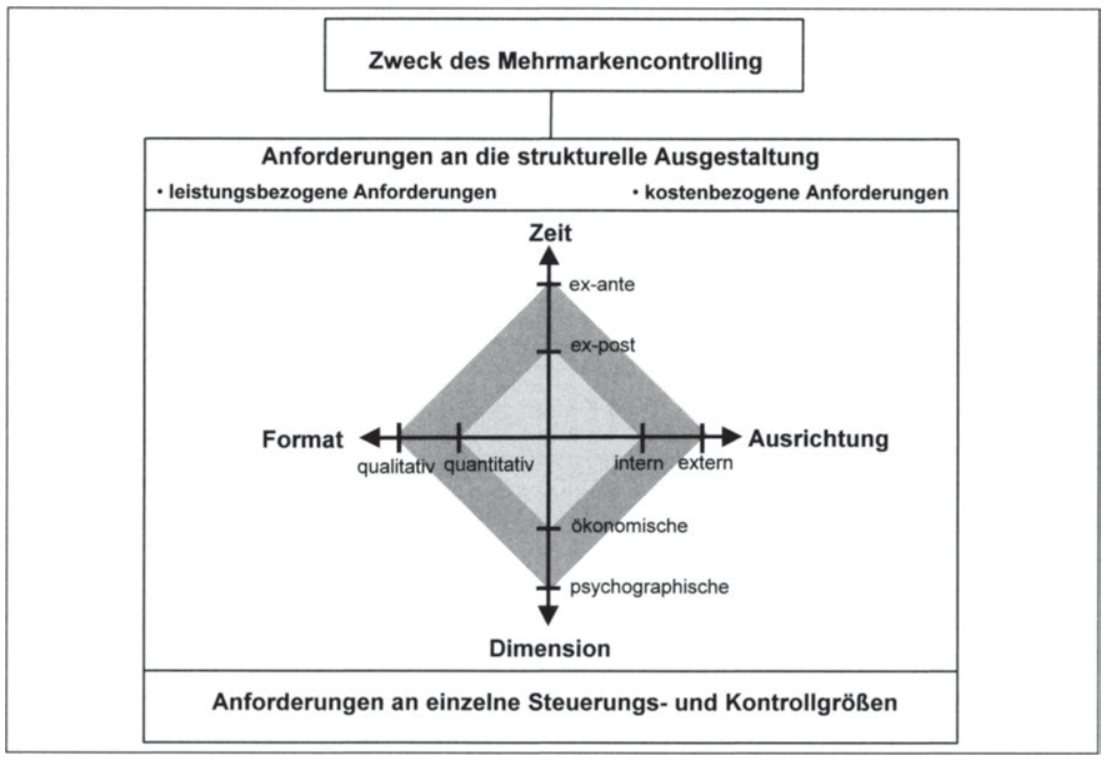

\section{Abb. 22: Anforderungen an das Mehrmarkencontrolling}

Zur Erfüllung der Koordinationsfunktion greift das Controlling auf unterschiedliche Daten zurück, so dass neben den allgemeinen Anforderungen an die Konzeption eines Mehrmarkencontrolling spezifische Anforderungen an die zu verwendenden Größen zur Sicherstellung einer hohen Aussagefähigkeit des Mehrmarkencontrolling

Marktauftritt überprüft werden. Da Kennzahlen eine eindeutige Formulierung von Zielen und Zielvorgaben und gleichzeitig eine einfache Kontrolle der Zielerreichung gestatten, beruhen kennzahlengestützte Abweichungsanalysen auf einer Gegenüberstellung von Plan- und Istwerten zur laufenden Ergebnis- und Erfolgskontrolle in Mehrmarkenstrategien. Kennzahlengestützten Lageanalysen kommt schließlich die Aufgabe zu, die Marktsituation der Portfoliomarken zu analysieren und zu vergleichen, frühzeitige Marktentwicklungen zu erkennen sowie Maßnahmen zur Anpassung an veränderte Bedingungen des Marktes zu initiieren. Vgl. Palloks, M., Kennzahlen, absatzwirtschaftliche, in: Handwörterbuch des Marketing, Tietz, B., Köhler, R., Zentes, J. (Hrsg.), 2. Aufl., Stuttgart 1995, Sp. 1138f. Vgl. hierzu auch BekmeierFeuerhahn, S., Marktorientierte Markenbewertung: eine konsumenten- und unternehmensbezogene Betrachtung, a.a.O., S. 106. 
zu stellen sind. Hinsichtlich der strukturellen Ausgestaltung eines Mehrmarkencontrolling sind leistungs- und kostenorientierte Anforderungen festzulegen. Zu den leistungsbezogenen Anforderungen zählen Vollständigkeit, Anpassungsfähigkeit und Verwendungsbezogenheit. Die Forderung nach Vollständigkeit bezieht sich auf die möglichst umfassende Berücksichtigung aller für die Mehrmarkenstrategie relevanten Entscheidungstatbestände, situativen Faktoren, Wirkungsbeziehungen und Zielgrößen. Zur Sicherstellung einer hohen Informationsqualität

- umfasst das Mehrmarkencontrolling sowohl ökonomische (Kernergebnisgrößen) als auch psychographische (Leistungstreiber ${ }^{283}$ ) Größen,

- nimmt das Mehrmarkencontrolling gleichermaßen einen ex-post wie ex-ante Fokus ein,

- beinhaltet das Controlling sowohl quantitative als auch qualitative Beschreibungen und Interpretationen, da sich nicht alle Sachverhalte über Zahlen vermitteln lassen,

- berücksichtigt das Mehrmarkencontrolling interne (Inside-Out) und externe (Outside-In) Informationen.

Entscheidend ist, dass sich die Kennzahlen zu einem kohärenten System ergänzen, komplementär zueinander sind und dazu dienen, eine klar definierte Strategie zu unterstützen. Zur Erfüllung seiner Zwecksetzung als Führungsunterstützungssystem bedarf die konkrete Ausgestaltung des Mehrmarkencontrolling einer laufenden Anpassung entsprechend der konkreten Ausgestaltung der Mehrmarkenstrategie. Das Mehrmarkencontrolling hat eine abgestimmte und in sich konsistente Informationsversorgung unterschiedlicher Aggregationsebenen des Portfolios zu gewährleisten und dabei die Wechselwirkungen zwischen den Marken des Portfolios zu berücksichtigen. Zur Sicherstellung der Akzeptanz bei den Mitarbeitern sollte sich das Unternehmen auf wenige, aber relevante Größen fokussieren, die entsprechend beeinflussbar sind und damit eine hohe Handlungsorientierung aufweisen. ${ }^{284}$ Dabei sollte der Zielerreichungsgrad der festzulegenden Soligrößen auf ein realisierbares Ausmaß festgelegt werden, um motivierend zu wirken. Zur Bestimmung der Sollvorgaben ist die Erfassung eines Vergleichsmaßstabes der

283 Als Leistungstreiber werden Größen bezeichnet, welche die Erfolgspotenziale der Zukunft darstellen. Vgl. Horváth, P., Kaufmann, L., Balanced Scorecard - ein Werkzeug zur Umsetzung von Strategien, in Harvard Business Manager, Heft 5, 1998, S. 41.

So sind Konjunkturdaten zwar wichtig; sie gehören aber aufgrund ihrer mangelnden Beeinflussbarkeit in der Regel nicht in ein Mehrmarkencontrollingsystem. Vgl. hierzu auch Reineke, S., Tomczak, T., Aufgabenorientiertes Marketingcontrolling, in: Marketing-Controlling, Reinekke, S., Tomczak, T., Dittrich, S. (Hrsg.), St. Gallen 1998, S. 102. 
gewählten Größen sinnvoll, wobei insbesondere Vergleichsgrößen der relevanten strategischen und operativen Wettbewerber als Benchmarks heranzuziehen sind. ${ }^{285}$

Um einen hohen Verwendungsbezug der Informationen aufzuweisen, ist der eigenständige Informationsbedarf dezentraler Einheiten des Portfolios in die Controllingkonzeption miteinzubeziehen. Das Mehrmarkencontrolling hat dabei einen Beitrag zur Abstimmung der strategischen und operativen Planungsebenen im Markenportfolio zur Vermeidung opportunistischen Verhaltens zu leisten. Hierzu bedarf es der Verdeutlichung von Ursache-Wirkungs-Zusammenhängen zwischen strategischer und operativer Planungsebene im Markenportfolio. ${ }^{286}$ Mit dem Kriterium der Wirtschaftlichkeit wird schließlich gefordert, dass die Kosten eines Mehrmarkencontrolling (insb. Datenerfassung und -aufbereitung) in einem angemessen Verhältnis zur Verbesserung des Portfolioergebnisses stehen. Zur Berücksichtigung der Wirtschaftlichkeit der Informationsbeschaffung sollte das Mehrmarkencontrolling im Wesentlichen auf solche Größen zurückgreifen, die zyklisch erhoben werden oder ohne großen Aufwand gemessen werden können. Entsprechend hat das Mehrmarkencontrolling dem Grenznutzen der Informationsbeschaffung Rechung zu tragen. Zusammenfassend kann die möglichst vollständige und mit mess- bzw. kontrollierbaren Steuerungsgrößen verknüpfte Abbildung sowie Vernetzung des Planungsprozesses von Mehrmarkenstrategien als zentrale Voraussetzung eines wirksamen Mehrmarkencontrolling angesehen werden.

Wird das Mehrmarkencontrolling als betriebswirtschaftliche Kennzahlenforschung betrachtet, ist ähnlich dem Erfolgsfaktorenansatz davon auszugehen, dass wenige, aber grundlegende Einflussfaktoren auf den Erfolg oder Misserfolg der Portfoliomarken wirken. ${ }^{287} \mathrm{Im}$ Folgenden ist zu untersuchen, welche Größen auf den

285 In diesem Zusammenhang erscheint es zweckmäßig, sowohl ein langfristiges theoretisches Soll, definiert durch den strategischen Wettbewerber (Potenzialität), als auch ein eher mittelfristiges realistisches Soll, definiert durch den operativen Wettbewerber (Kapabilität), im Vergleich zum eigenen vorhandenen Ist (Aktualität) zu unterscheiden. Vgl. hierzu auch Lütke Schwienhorst, R., Strategische Kontrolle, Rahmenbedingungen, Aufgaben und Methoden, Diss., Wiesbaden 1989, S. 182.

Kaplan, R. S., Norton, D. P., Strategieumsetzung mit Hilfe der Balanced Scorecard, in: Gleich, R., Seidenschwarz, W. (Hrsg.): Die Kunst des Controlling, 1997, S. $330 f$.

Vgl. zu dieser Argumentation auch Bekmeier-Feuerhahn, S., Marktorientierte Markenbewertung: eine konsumenten- und unternehmensbezogene Betrachtung, a.a.O., S. 107. Der Erfolgsfaktorenansatz basiert auf der Überlegung, dass für jede betriebliche Einheit wenige, aber grundlegende erfolgsentscheidende Einflussfaktoren existieren. 
Erfolg der Portfoliomarken Einfluss nehmen und infolgedessen als Steuerungsund Kontrollgrößen im Rahmen des Mehrmarkencontrolling grundsätzlich zur Verfügung stehen.

\subsection{Zielsystem des Mehrmarkencontrolling}

Mit ADAM setzt jede Koordination voraus, dass sich die Unternehmensleitung Klarheit über ihr Unternehmensziel und damit auch über ihren anzustrebenden Unternehmenserfolg verschafft. ${ }^{288}$ Vielfach besteht jedoch nicht einmal bei der Unternehmensspitze eine klare Vorstellung über das zu verfolgende Ziel, so dass die dezentralen Markeneinheiten ihre Entscheidungen weitgehend an Kriterien ausrichten, die nie hinsichtlich ihrer Zielkonsistenz überprüft wurden. Entsprechend ist in dieser Situation eine dem „Portfolioziel" gerecht werdende Koordination bereits der Sache nach unmöglich.

Zur Festlegung geeigneter Erfolgsmaßgrößen empfehlen TOLLE/STEFFENHAGEN, sich an „übergeordneten unternehmerischen Wertmaßstäben" zu orientieren. ${ }^{289}$ Sie weisen darauf hin, sich bei der Auswahl nicht auf die Betrachtung einzelner Erfolgsmaße zu beschränken, sondern mehrere Indikatoren zu einem integrierten Erfolgsindex (Spitzenkennzahl) zusammenzuführen. Eine solche Spitzenkennzahl stellt im Rahmen von Mehrmarkenstrategien der Portfoliowert dar, der sich in die Wertindikatoren Markenstärke und Markengewinn der einzelnen Portfoliomarken aufspalten lässt (vgl. Abb. 23). ${ }^{290}$

Vgl. Adam, D., Koordinationsprobleme bei dezentralen Entscheidungen, in: ZfB, 39. Jg., Heft 10,1969, S. $620 f f$.

Vgl. Tolle, E., Steffenhagen, H., Kategorien des Markenerfolges und einschlägige Meßmethoden, in: Handwörterbuch Markenartikel, Bruhn, M. (Hrsg.), Band 2, Stuttgart 1994, S. 1301f; Riedel, F., Die Markenwertmessung als Grundlage strategischer Markenführung, Heidelberg 1996, S. 32.

290 Zu dieser die Konsumenten- und Herstellerperspektive umfassenden Betrachtung des Markenwertes vgl. auch Sander, M., Die Bestimmung und Steuerung des Wertes von Marken. Ejne Analyse aus Sicht des Markeninhabers, a.a.O., S. 48f; Bekmeier-Feuerhahn, S., Marktorientierte Markenbewertung: eine konsumenten- und unternehmensbezogene Betrachtung, a.a.O., S. $221 \mathrm{ff}$. 


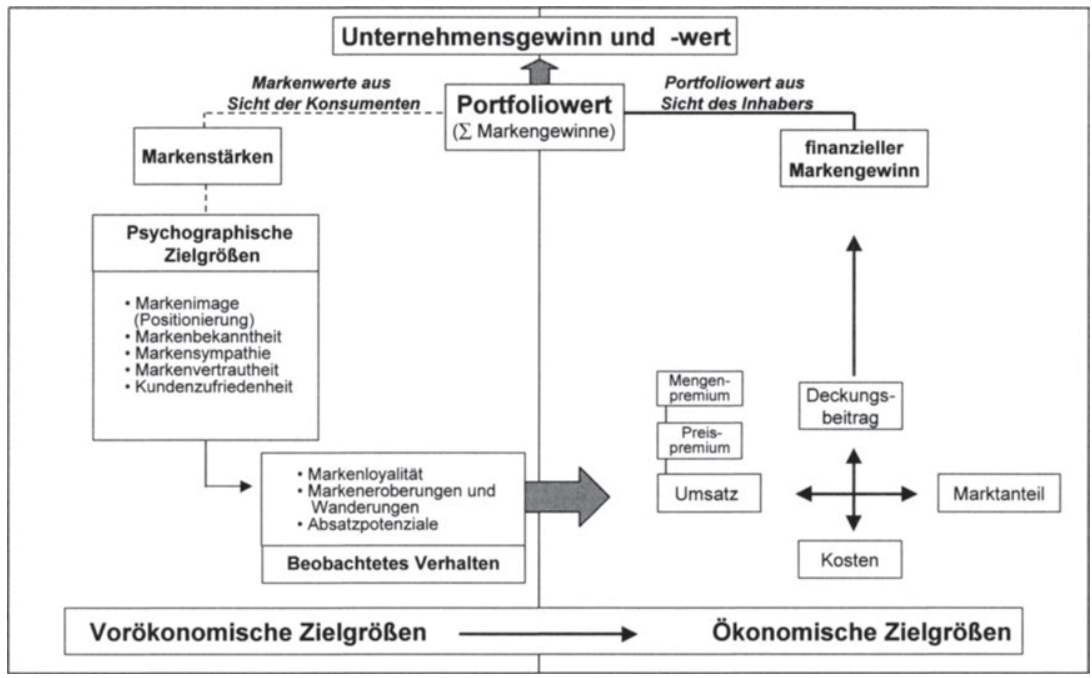

\section{Abb. 23: Zielgrößen des Mehrmarkencontrolling}

Die Markenstärke repräsentiert den Markenwert aus Kundensicht und wird auch als psychographischer Markenwert bezeichnet. ${ }^{291} \mathrm{Er}$ ist Voraussetzung für die Entstehung von Marken- respektive Portfoliogewinn beim Hersteller. Eine solche Differenzierung zwischen Konsumenten- und Herstellerperspektive ist für die vorliegende Untersuchung sinnvoll, da das Mehrmarkencontrolling sowohl eine gesteigerte externe Wirkungseffizienz der Portfoliomarken als auch eine höhere interne Planungs- und Umsetzungseffizienz im Portfolio anstrebt. Als Quelle der einzelnen Markengewinne wird die Wahrnehmung der Marken durch den Konsumenten gesehen, wobei seine rational und emotional bedingten Assoziationen und Vorstellungen gegenüber einer Marke seine Wertschätzung für diese Marke prägen. Markengewinn beim Hersteller kann somit erst entstehen, wenn sich beim Konsumenten Gedächtnisstrukturen zur Marke gebildet haben, denn „a brand name adds value for the manufacture [... ] only because it adds value for the consumer." 292

291 Vgl. Farquhar, P.H., Managing Brand Equity, a.a.O., S. 7ff.; Meffert, H., Burmann, Ch., Markenbildung und Markenstrategien, a.a.O., S. 6.

Crimmins, J. C., Better Measurement and Management of Brand Value, in: JoAR, Heft 4, 1992, S. 11. 
So sind Konsumenten vielfach bereit, für bestimmte Marken einen höheren Preis zu zahlen als für andere. Beispielsweise war der VW SHARAN 1997 ausstattungsbereinigt im Durchschnitt ca. 3.500 DM teurer als der FORD GALAXY bzw. der SEAT ALHAMBRA, obwohl sich die baugleichen Fahrzeuge (Badge Engineering) im Wesentlichen nur durch das Markenemblem unterscheiden. Dennoch verzeichnete der VW SHARAN die höchsten Verkaufszahlen: von Januar bis August 1997 wurden vom SHARAN 22.288, vom FORD GALAXY 11.498 und vom SEAT ALHAMBRA 2.284 Fahrzeuge zugelassen. ${ }^{293}$ Begründen lässt sich dies vor allem durch die unterschiedliche Wahrnehmung und Präferenz der potenziellen Käufer für die Marke. Das Ansehen der Marke schlägt sich dabei zum einen in einem höheren Verkaufspreis (Preispremium der Marke) und zum anderen in höheren Verkaufszahlen (Mengenpremium der Marke) nieder. Da die Produktionskosten im beschriebenen Beispiel nahezu identisch sind, hat die Markenwahrnehmung somit direkte Auswirkungen auf den Umsatz und damit auf übergeordnete Unternehmensziele.

Die Identifikation der Wahrnehmung und Akzeptanz der von einer Unternehmung offerierten Marken setzt dabei eine Analyse hinsichtlich deren Verankerung in der Psyche der Nachfrager voraus. Einen zentralen Stellenwert nimmt eine regelmäBige Ermittlung der ungestützten Markenbekanntheiten (Share of Mind) ein, die als notwendige Bedingung markenspezifischer Assoziationen durch die externen Anspruchsgruppen fungieren und somit letztlich die Grundvoraussetzung für den möglichen Kauf der Markenprodukte darstellen. ${ }^{294}$ Überdies ist eine dezidierte Analyse der Markenimages vorzunehmen, da diese das Ergebnis der nachfragerseitigen Wahrnehmung, Dekodierung und Akzeptanz der von den Marken ausgehenden Impulse darstellen und - wie aufgezeigt - als Fremdbild der Markenidenti-

293 Vgl. O.V., Marktbericht August 1997, in: Auto, Motor und Sport, Heft 21, 1997, S. 10. Alle drei Fahrzeuge werden baugleich in Palmela in Portugal gefertigt. VW bietet vier, SEAT drei und FORD fünf Motorisierungen an. Außer einem vergleichbaren Basismotor und einem nur bei Ford angebotenem Motor sind die Fahrzeuge nahezu identisch (leicht unterschiedliche Innenausstattung sowie geringe äußerliche Unterschiede wie Gestaltung des Kühlergrills). Vgl. hierzu ausführlich Meffert, H., Koers, M., Positionierung im Wettbewerb a.a.O., S. $18 \mathrm{ff}$.

Laut AAKER wachsen mit größerer Markenbekanntheit Vertrauen und Zuneigung des Konsumenten zur Marke. Vgl. Aaker, D. A., Managing Brand Equity, Capitalizing on the Value of a Brand Name, New York u.a. 1991, S. 56ff; Keller, K. L., Conzeptualizing, Measuring, and Managing Customer-Based Brand Equity, in: Journal of Marketing, Vol. 57., No.1, 1993, S. $3 \mathrm{ff}$. 
täten zu interpretieren sind. ${ }^{295}$ Dabei ist der Einfluss des Markenimages auf die Markenstärke als um so größer zu werten, je eigenständiger das jeweilige Image gegenüber den sonstigen Portfoliomarken bzw. Konkurrenzmarken ist. ${ }^{296}$

Bedingt durch die hohe Komplexität des Markenimages, in das Aspekte der mit einer Marke assoziierten Eigenschaften sowie allgemeine Einstellungen einflieBen, sollte die Erfassung des Markenimages auf disaggregierter Ebene erfolgen. Zwar stellt in praxi häufig das aggregierte Image im Wettbewerbsumfeld eine wesentliche Zielgröße des Markenmanagement dar, jedoch lässt sich die angestrebte Differenzierung der Marken im Rahmen von Mehrmarkenstrategien nur bedingt anhand dieser inhaltlich wenig konkreten Globalimages analysieren. Stattdessen ist zu überprüfen, ob sich die angestrebte Kompetenzdifferenzierung im Wettbewerbsumfeld sowie im internen Markenvergleich in entsprechenden Detailimages widerspiegelt. ${ }^{297}$ So sollte die Automobilmarke SKODA über ein preisorientiertes Image verfügen, wohingegen dies für die etablierte Marke VW keine notwendige Voraussetzung darstellt und für die Premiummarke AuDı nur wenig förderlich wäre.

Über die zentralen Größen der Markenbekanntheit und des Markenimages hinaus werden in der Literatur zahlreiche weitere Konstrukte zur Analyse der Wahrnehmung und Akzeptanz von Marken angeführt, die im Einzelfall wertvolle Hinweise für den Markenerfolg liefern können, ${ }^{298}$ allerdings nicht immer scharf voneinander abgrenzbar und zudem aufgrund ihrer vielschichtigen Operationalisierungen oftmals nur wenig objektivierbar sind. Als Beispiele seien hier stellvertretend die häu-

Im Rahmen einer 1996 durchgeführten Befragung von 42 Marketingspezialisten gab über die Hälfte der Befragten an, dass besonders das Markenimage die Verkaufszahlen beeinflusst. Die Studie zeigt jedoch auch, dass nur bei einem Viertel der Befragten das Markenimage Teil eines Markencontrolling ist. Vgl. Biel, A. L., Grundlagen zum Markenwertaufbau, a.a.O., S. 90.

Auch KOHLER bezeichnet die Eigenständigkeit einer Marke bezogen auf ihre Produkteigenschaften und ihre emotional wahrnehmbare Originalität als die wesentliche Bedingung für den Markenerfolg. Vgl. Köhler, R., Marke und Verbraucher - Zu den Grundlagen lebenslanger Bindungen, in: Markenartikel, Heft 7, 1993, S. 342.

297 Vgl. Meffert, H., Perrey, J., Mehrmarkenstrategie - Ein Beitrag zum Management von Markenportfolios, a.a.O., S. 636.

298 Vgl. etwa Campbell, K., Researching Brands, in: Brands: The New Wealth Creators, Hart, S., Murphy, J. (Hrsg.), Houndmills 1998, S. 56ff. Tolle, E., Steffenhagen, H., Kategorien des Markenerfolges und einschlägige Meßmethoden, in: Handwörterbuch Markenartikel, Bruhn, M. (Hrsg.), Band 2, Stuttgart 1994, S. $1283 \mathrm{ff}$. 
fig eingesetzten Größen wie Markenvertrautheit oder Markensympathie angeführt. ${ }^{299}$

Neben den markenspezifischen Konstrukten sind im Rahmen des Mehrmarkencontrolling ferner psychographische Größen wie Kundenzufriedenheit oder Kaufgründe zu analysieren. Die Zufriedenheit der Konsumenten mit der Marke stellt dabei das Ergebnis des Vergleichs zwischen subjektiver Enwartung und wahrgenommener Leistung dar. ${ }^{300}$ Durch Präferenzbildung räumt der Konsument einer Marke aufgrund objektiver Leistungsmerkmale oder emotionaler Erlebnisse eine Vorzugsstellung beim Kaufentscheidungsprozess ein. ${ }^{301}$ Dabei bildet ein hoher Zufriedenheitsgrad die Voraussetzung zum Wiederkauf und damit einer hohen Markenloyalität bzw. Markentreue, welche als beobachtbare Resultate markenspezifischer Investitionen die ökonomischen Markenziele (Marktanteile, Umsätze, Erträge etc.) direkt beeinflussen. ${ }^{302}$

So resultieren aus der Markentreue zum einen geringere Marketingkosten für den Hersteller, da das Halten aktueller Kunden vergleichsweise weniger Kosten verursacht als die Akquisition neuer Kunden. ${ }^{303}$ Zum anderen können aus der positiven Mund-zu-Mund Propaganda der angestammten Kunden sowie einem unterbundenen Markenwechsel höhere Absatzmengen realisiert werden. ${ }^{304}$ Überdies bietet

Vgl. Esch, F.-R., Andresen, Th., Messung des Markenwerts, in: Erfolgreiches Markenmanagement, MTP e. V. Alumni, Hauser, U. (Hrsg.), Wiesbaden 1997, S. 19.

Vgl. Meffert, H., Bruhn, M., Beschwerdeverhalten und Zufriedenheit von Konsumenten, in: DBW, 41. Jg., Heft 4, 1981, S. 597. Hierbei handelt es sich um das sogenannte Confirmation/Disconfirmation-Paradigma (kurz: C/D-Paradigma).

Vgl. auch Aaker, D. A., Managing Brand Equity, Capitalizing on the Value of a Brand Name, New York u.a. 1991, S. 34ff. Letztlich entspricht die dauerhafte Befriedigung der Kundenbedürfnisse zur Verwirklichung der Unternehmensziele dem Grundverständnis des Marketing. Vgl. Meffert, H., Marketing: Grundlagen marktorientierter Unternehmensführung: Konzepte Instrumente - Praxisbeispiele, a.a.O., S. 7.

Die Kosten der Neukundenakquisition betragen im Schnitt etwa das Fünffache jener Kosten, die für die Pflege von Altkunden aufzubringen sind. Vgl. hierzu Müller, W., Riesenbeck, H.J., Wie aus zufriedenen auch anhängliche Kunden werden, in: Harvard Manager, 13. Jg., Heft 3, 1991, S. 69

In diesem Zusammenhang kommen zahlreiche empirische Untersuchungen zu dem Resultat, dass Pkw-Hersteller ihre Gewinne erhöhen können, wenn es ihnen gelingt, die Treue ihrer Abnehmer zu steigern. Vgl. hierzu Bauer, H. H., Hermann, A., Hubert, F., Die Bestimmungsgrößen der Markentreue beim Pkw-Kauf: Ergebnisse einer empirischen Untersuchung, in: der 
sich dem Hersteller ein größerer preispolitischer Spielraum, da zufriedene Kunden einen Markenwechsel zumeist aufgrund des mit einem neuen Produkt verbundenen Risikos sowie der Wechselkosten eher meiden.

Im Falle des Markenwechsels wird im Rahmen von Mehrmarkenstrategien der Aufbau von Loyalität zur einzelnen Marke durch die Maximierung der Portfolioloyalität erweitert. Vor dem Hintergrund, dass von einer Unternehmung durchaus mehrere Markenportfolios in unterschiedlichen Produktbereichen geführt werden können, ist die Portfolioloyalität begrifflich von der übergeordneten Unternehmensloyalität zu trennen. Aus den Entwicklungen der Markenloyalitäten im Portfolio sowie unter Berücksichtigung möglicher Veränderungen in den Rahmenbedingungen (ökonomisch, technologisch, sozial und politisch) können wertvolle Hinweise zur zukünftigen strategischen Ausrichtung des Markenportfolios (Anzahl der Marken, Zielsegmente etc.) abgeleitet werden. Eine Besonderheit der Markentreue beim Automobilkauf kann überdies darin gesehen werden, dass sie eine Händler- bzw. Händlernetztreue impliziert, infolgedessen die ökonomischen Konsequenzen des Treueverhaltens über das eigentliche Produkt hinausreichen.

Zusammenfassend zeichnet sich das Zielsystem im Markenportfolio durch ein komplexes Beziehungsgeflecht zwischen psychographischen und ökonomischen Größen aus. Insbesondere der Marken- respektive Portfoliowert kann dabei als anzustrebendes Idealziel im Sinne ADAMs bezeichnet werden, dessen Maximierung jedoch von vielfältigen Faktoren abhängt und daher wenig operational ist. ${ }^{305}$ Vor diesem Hintergrund erscheint es zweckmäßig, das Idealziel der einzelnen Marken durch ein leichter zu erreichendes Realziel zu ersetzen. Die Koordination der Marken erfolgt somit bei Verfolgung von Realzielen. Die Realziele offenbaren dabei zentrale Steuerungsgrößen für das Mehrmarkencontrolling.

Wie aufgezeigt, nimmt aufgrund der Dezentralisierung von Entscheidungen im Markenportfolio insbesondere die Abstimmung der verschiedenen Planungsebenen und hier insbesondere der strategischen Rahmenplanung mit der operativen Instrumentalebene eine besondere Bedeutung im Rahmen von Mehrmarkenstra-

markt, Heft 4, 1994, S. 165; Reichheld, F. F., Sasser, W. E., Zero-Migration: Dienstleister im Sog der Qualitätsrevolution, in: Harvard Manager, 13. Jg., Heft 4, 1991, S. $108 \mathrm{ff}$.

Vgl. Adam, D., Koordinationsprobleme bei dezentralen Entscheidungen, in: ZfB, 39. Jg., Heft 10, 1969, S. 620. 
tegien ein. ${ }^{306}$ Ein zuletzt in den Fokus der Diskussion gerückter Ansatz zur Verknüpfung von strategischer und operativer Planungsebene stellt die von KAPLAN/NORTON entwickelte Balanced Scorecard dar. ${ }^{307}$ Im Folgenden soll das Konzept der Balanced Scorecard dahingehend analysiert werden, inwieweit es einen Beitrag für das Mehrmarkencontrolling leisten kann.

\subsection{Das Balanced Scorecard Konzept}

Hintergrund der Balanced Scorecard (BSC) ist die Kritik in Literatur und Praxis an der einseitigen Finanz- und Vergangenheitsorientierung gängiger Kennzahlensysteme in Unternehmen. ${ }^{308}$ Zentrale Idee der BSC ist die Berücksichtigung unterschiedlicher Perspektiven bei der Leistungsbeurteilung eines Unternehmens oder Geschäftsbereichs als Grundlage zu deren Steuerung, unter Beachtung der perspektivenübergreifenden Zusammenhänge und unter Hinzuziehung perspektivenspezifischer Maßgrößenbündel. ${ }^{309}$ „Balanced“ indiziert dabei eine integrierte und

Vgl. Köhler, R., Beiträge zum Marketing-Management. Planung, Organisation, Controlling, a.a.O., S. $102 \mathrm{ff}$.

Der Balanced Scorecard ist aus einem von KAPLAN/NORTON durchgeführten Forschungsprojekt entstanden, bei dem die Performancemessung in einer Auswahl amerikanischer Großunternehmen untersucht worden ist. Vgl. Hoffmann, O., Performance Management, Systeme und Implementierungsansätze, Bern, Stuttgart, Wien 1999, S. 49

So beklagt DRUCKER, dass in der Informationsversorgung der Führung noch immer eine buchhalterische Vergangenheitsorientierung dominiert. Vgl. Drucker, P., Umbruch im Management, Düsseldorf 1996, S. 113. Zur Produktion von Zahlenfriedhöfen vgl. Homburg, Ch. et al., Interne Kundenorientierung der Kostenrechnung - Ergebnisse der Koblenzer Studie, Band 7 der Reihe Advanced Controlling, Koblenz 1998, S. 19f. Der Bedarf zusätzlicher nichtmonetärer Informationen lässt sich empirisch belegen. Vgl. hierzu Eccles, R. G., Mavrinac, S., Improving the Corporate Disclosure Process, in: Sloan Management Review, Vol. 36, Heft 4, S. 20. Vgl. auch Neely, A., Gregory, M., Platts, K., Performance Measurement System Design, in: International Journal of Operations \& Production Management, Heft 4, 1995, S. 106

309 Vgl. Kaplan, R. S., Norton, D. P., In Search of Excellence - der Maßstab muss neu definiert werden, in: Harvard manager, Heft 4, 1992, S. 37ff.

DIE VON KAPLAN/NORTON propagierte Idee ist letztlich nicht neu, sondern wurde schon in den fünfziger Jahren gefordert. Vgl. hierzu die Ausführungen bei Weber, J., Schäffer, U., Balanced Scorecard - Gedanken zur Einordnung des Konzeptes in das bisherige ControllingInstrumentarium, a.a.O., S. 4f. und die dort genannte Literatur. So zeigen ECCLES/NORIAH Inhalte eines Kennzahlenprojektes bei General Electric im Jahre 1951, in dem verschiedene Kennzahlentypen vorgeschlagen werden. Eccles, R. G., Noriah, N., Beyond the Hype - Rediscovering the Essence of Management, Boston 1992, S. 156ff. Das bekannte, von LAUZELCILBERT 1959 konzipierte Tableau de Bord stellt ein System finanzieller und nichtfinanzieller Kennzahlen dar. Vgl. hierzu Epstein, M., Manzoni, J.-F., Implementing Corporate Strategy: From Tableaux de Bord to Balanced Scorecard, in: European Management Journal, Vol. 16, No. 2, 1997, S. 191. Schott strukturiert bereits 1951 ein Kennzahlensystem nach 
ausgewogene Darstellung sowohl qualitativer als auch quantitativer vergangenheits- wie zukunftsbezogener Informationen unternehmensinterner (z. B. Mitarbeitermotivation) und -externer Natur (Kundenzufriedenheit) und damit die explizite Berücksichtigung von Ergebniskennzahlen und Leistungstreibern. ${ }^{310}$ KAPLAN/NORTON beleuchten in ihrem Konzept der BSC vier miteinander verkettete Perspektiven, welche die Wünsche der drei wichtigsten Stakeholder ${ }^{311}$ (Shareholder, Kunden und Mitarbeiter) berücksichtigen (vgl. Abb. 24): ${ }^{312}$

- Die finanzielle Perspektive verdeutlicht, inwiefern die Implementierung der verfolgten Strategie zur Ergebnisverbesserung beiträgt. Dabei definieren finanzielle Kennzahlen einerseits die von der Strategie zu erwartende finanzielle Leistung, anderseits fungieren sie als Endziele für andere Perspektiven, deren Kennzahlen grundsätzlich über Ursache-Wirkungsbeziehungen mit den finanziellen Zielen verbunden sein sollten. ${ }^{313}$

- Fokus der Kundenperspektive ist eine spezifische Zusammenstellung von Größen, die für den Erwerb einer Leistung entscheidend sind (Zeit, Qualität, Produktwert aus Kundensicht, Preiseinschätzung etc.). Als Ergebnisgrößen werden etwa die Kundenzufriedenheit, Kundenbindung, Kundenprofitabilität etc. genannt. ${ }^{314}$

Struktur-, Leistungs-, Ergebnis- und Entwicklungskennzahlen. Vgl. Schott, G., Kennzahlen Instrument der Unternehmensführung, 4. Aufl., Wiesbaden 1991, S. 294ff. Auch das von WEBER entwickelte System selektiver Kennzahlen setzt an dem Problem der ungenutzten Zahlenfriedhöfe an. Vgl. Weber, J. Einführung in das Controlling, a.a.O., S. $201 \mathrm{ff}$.

Zunehmend wird auch von den Eignern eines Unternehmens ein Anspruch auf eine Veröffentlichung - meist nicht monetärer - Größen erhoben, mit denen eine verbesserte Beurteilung der zukünftigen Unternehmensentwicklung ermöglicht wird. Vgl. hier etwa den vom Special Committee on Finacial Report veröffentlichten Compehensive Report, dessen MusterUnternehmensbericht eine deutlich erweiterte Aufnahme von nicht-monetären Unternehmensinformationen zeigt. Vgl. Klingenbiel, N., Performance Measurement: Grundlagen - Ansätze - Fallstudien, a.a.O., S. 2 und 8.

311 Zum Stakeholder-Konzept vgl. auch Wagner, H., Marktorientierte Unternehmensführung versus Orientierung an Mitarbeiterinteressen, Shareholder-Value und Gemeinwohlverpflichtung, in: Marktorientierte Unternehmensführung: Reflexionen-Denkanstöße-Perspektiven, Bruhn, M., Steffenhagen, H. (Hrsg.), Wiesbaden 1997, S. 87ff.

Vgl. Kaplan, R. S., Norton, D. P., Balanced Scorecard: Strategien erfolgreich umsetzen, a.a.O., S. 8.

313 Beim Scorecard-Ansatz besteht keine zwingende Voraussetzung, die finanziellen Größen aufeinander abzustimmen, wie dies bei klassischen finanziellen Kennzahlensystemen wie etwa dem Du-Pont-Schema der Fall ist. Von größerer Bedeutung ist ein bestehender logischer Zusammenhang zwischen den Messgrößen der Perspektiven bezüglich der Strategieumsetzung.

314 Vgl. Kaplan, R. S., Norton, D. P., Strategieumsetzung mit Hilfe der Balanced Scorecard, a.a.O., S. 321. 
- Zur Befriedigung der Kundenansprüche sind intern die hierfür notwendigen Prozesse zu beherrschen. Infolgedessen richtet die interne Prozessperspektive den Fokus auf jene Abläufe und deren spezifische Massgrößen, die maßgeblich zur Befriedigung der Kundenwünsche beitragen (Fertigkeit der Mitarbeiter, Qualitätstandards etc. ) ${ }^{315}$

- Zur Befriedigung auch zukünftiger Kundenbedürfnisse sowie zur Induzierung langfristiger Verbesserungen von Wachstum und Wertsteigerungen bedarf es der ständigen Weiterentwicklung der Produkte und Verfahren. Im Rahmen der Lern- und Wachstumsperspektive sind hierzu schließlich Maßstäbe für die Verbesserung der existierenden Fähigkeiten der Mitarbeiter, der Systeme und Abläufe zu generieren.

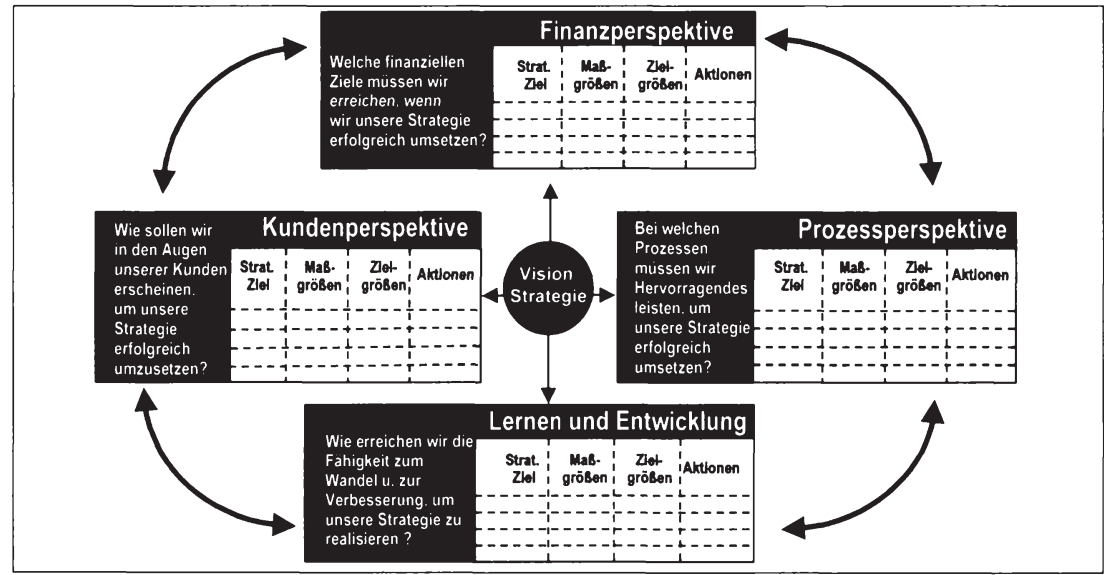

Abb. 24: Perspektiven und Managementprozess der Balanced Scorecard

(Quelle: Kaplan, R., Norton, D., Using the Balanced Scorecard as a Strategic Management System, in: HBR, Jan.-Feb. 1996, S. 76.

Die Perspektiven der BSC sind auf die Unternehmensziele abzustimmen, deren Erreichen über definierte Leistungsmaßstäbe überprüft wird. Ausgangspunkt stellt die Vision des Unternehmens dar, auf welche die Strategie- und Zielformulierung sowie die Leistungsplanung und -messung ausgerichtet ist. Die BSC bildet die Strategie des Unternehmens durch die Verknüpfung von Zielen über die Perspek-

315 Zur Förderung der Leistungsorientierung wird auch ein Benchmarking der Messgrößen der internen Prozessperspektive mit vergleichbaren Organisationen empfohlen. 
tiven sowie deren Ergebnis- und Leistungstreiber mittels der zu Grunde liegenden Ursache-Wirkungs-Beziehungen ab. ${ }^{316}$

Die Gestaltung der BSC nach KAPLAN/NORTON mit vier Scorecards (Ergebnistafeln) ist dabei lediglich als Vorschlag zu verstehen, um strategierelevante Informationen zu strukturieren. Die BSC-Konzeption ist grundsätzlich auf die Informationsbedürfnisse der Unternehmung auszurichten, welche wiederum durch die verfolgten strategischen Ziele, Organisationsform etc. determiniert werden, so dass die Perspektiven der BSC je nach Informationsbedarf eine Kürzung bzw. Ergänzung erfahren. ${ }^{317}$ Überdies ist bei der Scorecardgestaltung die Hierarchiestufe inrer Anwender zu berücksichtigen. So gehen die in den Perspektiven dargestellten Kennzahlen auf Unternehmensebene vielfach aus einer Verdichtung von Kennzahlen unterer Ebenen mittels Indizes hervor. Auf tieferen Hierarchiestufen kann sich mithin der Aufbau von Scorecards deutlich von einer Top-Management Scorecard unterscheiden, da nur eine Teilsicht von operationalisierbaren Indikatoren relevant sein kann. Letztlich handelt es sich somit beim Ansatz von KAPLAN/NORTON somit um einen Gestaltungsrahmen, der einer organisationsspezifischen Anpassung bedarf. ${ }^{318}$

316 Problematisch stellt sich die Verknüpfung von Messgrößen dar, wenn eine sehr lange Feedback-Dauer zwischen einzelnen Kennzahlen besteht. In diesem Fall ist eine eindeutige Zuordnung von Ursache-Wirkungs-Zusammenhängen nur schwer zu realisieren, da vielfältige Störfaktoren kompensierende Wirkungen entfalten können. Entsprechend kann der Nutzen einer BSC in Frage gestellt werden, wenn keine finanziellen Auswirkungen innerhalb einer überschaubaren Zeit feststellbar sind. Vgl. Hoffmann, O., Performance Management, Systeme und Implementierungsansätze, a.a.O., S. 55.

WEBER/SCHÄFER weisen entsprechend explizit darauf hin, dass eine zu enge Orientierung im Prozess der individuellen Gestaltung der BSC am Basisbeispiel von Kaplan/Norton keine eigenständige Lösungen erwarten lässt. Vgl. Weber, J., Schäffer, U., Balanced Scorecard Gedanken zur Einordnung des Konzeptes in das bisherige Controlling-Instrumentarium, a.a.O., S. 17. Beispielsweise ergänzte die Firma FISCHERWERKE inre Balanced Scorecard um eine explizite Mitarbeiterperspektive und die Kundenperspektive wurde zur Marktperspektive erweitert. Vgl. zu den Gründen Fink, C.A., Grundler, Ch., Strategieimplementierung im turbulenten Umfeld - Steuerung der Firma fischerwerke mit der Balanced Scorecard, in: Controlling, Heft 4, 1998, S. 228f. Ausführliche Informationen zur Gestaltung der BSC bietet ABB (Schweden). Vgl. Ewing, P., Lundahl, L. The Balanced Scorecards at ABB Sweden - The EVITA Project, 0.O., 1996

So existieren in Literatur und Praxis zahlreiche weitere Ansätze mit ähnlichem Fokus bzw. die auf der Struktur der BSC aufbauen. Vgl. z.B. die "Performance Pyramid" bei Lynch, R. L., Cross, K. F., Measure Up! Yardsticks for Continuous Improvement, Cornwall 1995, S. 65; das "Quantum Performance Measurement Modell" bei Hronec, S. M., Vital Signs, New York u.a. 1993, S. 25. Vgl. zu weiteren Beratermodellen Klingebiel, N., Performance Measurement: Grundlagen - Ansätze - Fallstudien, Wiesbaden 1999, S. 63ff. 
KAPLAN/NORTON zur Folge fungiert die BSC dabei nicht nur als Kennzahlensystem, sondern stellt als umfassendes Managementsystem das Bindeglied zwischen der Entwicklung einer Strategie und ihrer Umsetzung dar, so dass die BSC offensichtlich einen Beitrag zur Schließung der Implementierungslücke in Mehrmarkenstrategien leisten kann: ${ }^{319}$

- Schon der Entwicklungsprozess einer BSC soll gemäß KAPLAN/NORTON zur Klärung sowie zum Konsens im Hinblick auf die strategischen Ziele führen.

- Die BSC soll zur einheitlichen Zielausrichtung der Handlungsträger im Unternehmen beitragen.

- Durch die Anwendung der BSC soll die strategieförderlichste Allokation von Ressourcen sichergestellt werden.

- Nach KAPLAN/NORTON ist der traditionell hierachische Prozess zur Strategieformulierung und -implementierung durch einen mangelhaften FeedbackProzess gekennzeichnet. Die Rückkopplung erfolgt nur auf der operativen Ebene als "Single-Loop-Lernen". Durch die BSC soll dagegen die Rückkopplung auf die Strategie bezogen werden und einen durch "Double-LoopLernen" ${ }^{320}$ charakterisierten strategischen Lernprozess fördern.

Für das Controlling von Mehrmarkenstrategien liefert das Konzept der BSC somit wertvolle Hinweise, da auch hier gilt, aus der Vielzahl der in der Literatur existierenden Kennzahlen und Instrumente ein leistungsfähiges System zu konstruieren. Das Controllingsystem soll den Managern von Markenportfolios in Analogie zur Portfolioperformance im Aktienmanagement ein umfassendes Bild über die Markenperformance vermitteln und als ein integratives dimensions- und bereichsübergreifendes Planungs- und Steuerungskonzept zur Optimierung des Marktauftritts der Portfoliomarken beitragen, um so den Wert des Gesamtportfolios zu erhöhen. Ausschlaggebend für den Markenerfolg ist dabei sowohl die Berück-

Vgl. Kaplan, R. S., Norton, D. P., Using the Balanced Scorecard as a Strategic Management System, in: Harvard Business Review, No. 1, 1996, S. 77. Siehe auch Schmidt, U., Balanced Scorecard - Von Kennzahlen zur Handlungssteuerung, in: Organisationsentwicklung, Heft 2, 1998, S. 30ff.

Die Unterscheidung zwischen Single-Loop-Lernen (Einkreislernen oder Anpassungslernen), durch das einzelne Personen oder Gruppen ihr Verhalten an feststehende Ziele, Normen oder Annahmen anpassen, und Double-Loop-Lernen (Zweikreislernen oder Veränderungslernen), in dem sowohl die Ziele, Normen und Annahmen als auch das Anpassungsverhalten selbst offen für Adaption sind, hat ihre Wurzeln bei ARGYRIS/SCHON. Zweikreislernen bedeutet danach das Sichtbarmachen ("surfacing") und Hinterfragen tiefsitzender Überzeugungen und Normen einer Organisation, die bisher nicht zugänglich waren, entweder weil sie unbekannt oder tabuisiert waren. Vgl. Argyris, C., Schön, D., Organizational Learning: A Theory of Action Perspective, Mass. 1978, S. $4 \mathrm{ff}$. 
sichtigung unternehmensinterner als auch -externer Informationen. ${ }^{321}$ Ebenso sind neben monetären Ziel- bzw. Ergebnisgrößen auch solche nicht-monetärer Art (z. B. psychographische Größen wie Bekanntheitsgrad, Image) zu berücksichtigen. ${ }^{322}$ Auch fördert die BSC durch die Berücksichtigung unterschiedlicher Perspektiven die verlangte Fokussierung einer Inside-Out-Perspektive und mithin einer Ergänzung der dominierenden Outside-In-Perspektive der Markenführung, ${ }^{323}$ so dass die BSC eine Vielzahl der oben genannten Anforderungen an ein Mehrmarkencontrolling zu erfüllen vermag.

Schließlich kann ein zentraler Nutzen des Scorecard-Ansatzes in seiner Eignung als Kommunikationsinstrument bei der Operationalisierung von Unternehmensstrategien gesehen werden. ${ }^{324}$ Denn eine solche Operationalisierung bedingt neben klaren Zielsetzungen ein Verständnis über das Zusammenwirken portfoliointerner Prozesse und einen Konsens divergierender Interessen. Gerade vor diesem Hintergrund liefert die BSC einen entscheidenden Beitrag dazu, strategisches Denken und Handeln auf allen Ebenen eines Markenportfolios zu fördern und damit den strategischen Führungsprozess im Rahmen von Mehrmarkenstrategien zu unterstützen bzw. als Handlungsrahmen für diesen Prozess zu dienen. ${ }^{325}$

\subsection{Ableitung eines integrativen Controllingansatzes}

Die vorhergehenden Ausführungen haben verdeutlicht, dass mit Hilfe der Balanced Scorecard eine notwendige Fokussierung und Reduktion auf entscheidende Größen im Markenportfolio erreicht werden kann. Damit wird einerseits Steue-

Vgl. Meffert, H., Markenführung in der Bewährungsprobe, in: Markenartikel, Heft 10, 1994, S. $479 \mathrm{ff}$.

322 Vgl. hierzu auch Becker, J., Marketing-Konzeption: Grundlagen des strategischen und operativen Marketing-Managements, a.a.O., S. 863.

323 Vgl. Kaplan, R. S., Norton, D. P., Balanced Scorecard: Strategien erfolgreich umsetzen, a.a.O., S. 35; Hoffecker, J., Goldenberg, C., Using the Balanced Scorecard to Develop Companywide Performance Measures, in: Journal of Cost Management, 8. Jg., Heft 3, 1994, S. 57 .

324 Vgl. Norton, D. P., Use Strategy Maps to Communicate Your Strategy, in: Balanced Scorecard: Insight, Experience \& Ideas for Strategy Focused Organizations (http//umw.hbsp.harvard.edu./products/bsr/index.html [5.5.2000]).

ECCLES/NORIAH sehen gerade die Rhetorik solcher „Konzepte“ positiv und erblicken in ihr gar das Wesentliche für das Management. Vgl. Eccles, R. G., Noriah, N., Beyond the Hype Rediscovering the Essence of Management, a.a.O., S. 29. 
rungsklarheit geschaffen, andererseits wird durch die Darstellung von Führungsgrößen auf den verschiedenen Ebenen des Portfolios und die Anbindung der Steuerungsgrößen an die strategischen Ziele die Operationalität von Strategien über mehrere Steuerungsebenen erhöht. Der BSC-Ansatz besticht dabei auf den ersten Blick durch seine intuitive Eingänglichkeit, welche jedoch irreführend sein kann: So liefern KAPLAN/NORTON bei der Operationalisierung von Strategien nur in begrenztem Umfang Hinweise darüber, wie Messgrößen gefunden und auf tiefere Hierarchiebenen heruntergebrochen werden können. In diesem Zusammenhang weist MüLlER-HAGEDORN zu Recht darauf hin, „dass der Aufbau von Kennzahlensystemen keine triviale Angelegenheit ist." 326

Im Rahmen des hier verfolgten Controllingansatzes wird es als zweckmäßig erachtet, sich an den differenzierten Informationsansprüchen des strategischen und operativen Portfoliomanagement zu orientieren ${ }^{327}$ Differenzierte Informationsansprüche verlangen dabei auch differenzierte Analyseinstrumente als zentrale $\mathrm{Be}$ standteile eines Mehrmarkencontrolling. Insofern sind entsprechend der Führungsstruktur im Portfolio adressaten- und damit entscheidungsorientiert zwei Ebenen des Mehrmarkencontrolling zu unterscheiden. ${ }^{328}$ Während das strategi-

Müller-Hagedorn, L., Bausteine eines Management-Informationssystems: Balanced Scorecard - Benchmarking - Betriebsvergleich, in: Beisheim, O., Distribution im Aufbruch - Bestandsaufnahme und Perspektiven, München 1999, S. 738. Überdies sind Kennzahlen und Indikatoren vielfach nicht selbsterklärend, sondern bedürfen einer zusätzlichen Interpretation. Vgl. Klingebiel, N., Performance Measurement: Grundlagen - Ansätze - Fallstudien, a.a.O., S. 1.

327 In diesem Zusammenhang konstatiert AHLERT zweckmäßig, dass die Verwendungszwecke von Informationen aus einem Managementinformationssystem sehr unterschiedlich sind. Ein Informationssystem in eine strategische und operative Variante einzuteilen erscheint nach seiner Ansicht jedoch nicht sinnvoll, da ein und dasselbe Informationssystem die Basis für sämtliche Managementaufgaben bildet. Der Einsatz eines marktpolitischen Instrumentes oder einer Kennzahl ist somit nicht per se strategisch oder operativ, sondern kann sowohl der Realisierung eines strategischen Prozesses im Rahmen des Positionierungsmanagement als auch der Abwicklung des operativen Geschäftes oder gleichzeitig beiden Aufgaben dienen. Das Mehrmarkencontrolling weist hingegen sehr unterschiedliche Ausprägungen auf, je nachdem, ob es der Unterstützung des strategischen oder operativen Portfoliomanagement dient. Die Trennung zwischen einer operativen und einer strategischen Controllingkonzeption kann daher in der Regel als zweckmäßig erachtet werden. Vgl. Ahlert, D., Anforderungen an Handelsinformationssysteme aus Nutzersicht - Auswertungspotentiale für das Handels- und Wertschöpfungsprozeß-Management, in: Ahlert, D. et al. (Hrsg.), Informationssysteme für das Handelsmanagement: Konzepte und Nutzung in der Unternehmenspraxis, Berlin et al. 1998, S. 23.

Zum entscheidungsorientierten Ansatz vgl. Heinen, E., Einführung in die Betriebswirtschaftslehre, 9. Aufl., Wiesbaden 1985, S. 259ff. Zur Methodenbewertung vgl. Meffert, H., Die Leistungsfähigkeit der entscheidungs- und systemorientierten Marketingtheorie, in: Wissen- 
sche Mehrmarkencontrolling die einzelnen Phasen der strategischen Rahmenplanung in Mehrmarkenstrategien analysiert, fokussiert das operative Mehrmarkencontrolling die Aussgestaltung der Instrumentalstrategien im Portfolio. Eine Integration der mit Hilfe der Instrumente generierten Steuerunggrößen erfolgt sodann in einem Konzept der Balanced Scorecard, das für die hier betrachtete Problemstruktur eine Modifikation erfährt. ${ }^{329}$

Gemäß WEBER/SCHÄFFER fordern KAPLAN/ NORTON durch die Abbildung von strategischen Kennzahlen und der sie verbindenden Ursache-Wirkungsbeziehungen in der BSC letztlich nichts anderes als die Abbildung der betrieblichen Wertschöpfungskette, welche sich in den entsprechenden Perspektiven der Scorecard spiegelt. So fokussiert

- die Lern- und Wachstumsperspektive letztlich auf die Mitarbeiter und damit einen wesentlichen Input,

- die Prozessperspektive auf den zugrundeliegenden Transformationsprozess,

- die Marktperspektive auf den Output der Produktionsfunktion und

- die finanzielle Perspektive schließlich auf den aus unternehmerischer Sicht entscheidenden Outcome. ${ }^{330}$

Einem solchen Wertschöpfungsgedanken Rechnung tragend, ist die Balanced Scorecard in der Lage, den auch im Rahmen der Arbeit postulierten Fokus einer Inside-Out-Perspektive als Ergänzung der bisher betonten Outside-In-Perspektive der Markenführung nachzukommen. KAPLAN/NORTON zur Folge kann die Balanced Scorecard ,jeden Ansatz zur Formulierung einer Strategie für Geschäftseinheiten

schaftsprogramm und Ausbildungsziele der Betriebswirtschaftslehre, Tagungsberichte des Verbandes der Hochschullehrer für Betriebswirtschaft e.V., Band 1, Kortzfleisch, G. v. (Hrsg.), Berlin 1971, S. 167ff. Vgl. auch Witt, F. H., Theorietradition der betriebswirtschaftlichen Forschung, Wiesbaden 1985, S. $29 \mathrm{ff}$.

Eine Anpassung der Perspektiven erscheint je nach Zielsetzung und Bezugsobjet der BSC zweckmäßig. Zur Anpassung einer Scorecard vgl. auch Martinsons, M., Davison, R., Tse, D., The Balanced Scorecard: a Foundation for the Strategic Management of Information Systems, in: Decision Support Systems, Vol. 25, 1999, S. 71-88.

330 Damit ist WEBER/SCHAFFER zur Folge die Forderung nach einer ausgewogenen Abbildung der Perspektiven und ihre Verknüpfung über Ursache-Wirkungsbeziehungen nichts anderes als die Forderung der herkömmlichen Kostenrechnung, den Zusammenhang zwischen eingesetzten Produktionsfaktoren und ausgebrachten Leistungen der vorgelagerten Realprozesse gemäß GUTENBERG zum Ausgangspunkt und zur Grundlage der Analyse zu machen. Vgl. Weber, J., Schäffer, U., Entwicklung von Kennzahlensystemen, a.a.O., S. 5f. Vgl. auch Chernatony, L. de, Dall'Ollmo Riley, F., Defining A "Brand": Beyond the Literature with Experts' Interpretations, a.a.O., S. 435. 
nutzen - mit der Kundenperspektive oder den besonderen internen Prozesspotenzialen angefangen."331 Um zu verdeutlichen, dass der markenspezifische Erfolg seinen Ursprung sowohl in den externen Marktanforderungen als auch in den internen Fähigkeiten und Kompetenzen hat, ist es sinnvoll,

- mit einer internen Perspektive Informationen im Rahmen des Aussagenkonzepts der Markenführung zu erfassen,

- mit einer Markt-Perspektive notwendige Kennzahlen des Akzeptanzkonzepts der Markenführung zu betrachten und

- im Rahmen einer Ergebnisperspektive schließlich die angestrebte Ergebniswirkung der Markenstrategie abzubilden.

Folglich beinhaltet die hier zu entwickelnde BSC drei Perspektiven, wobei die einzelnen Perspektiven eine Ergänzung erfahren sollen. Damit auch wettbewerbsrelevante Aspekte abgebildet werden können, bedarf es zum einen einer Erweiterung der von KAPLAN/ NORTON vorgeschlagenen Kundenperspektive zur Marktperspektive. Zum anderen fokussiert die interne Perspektive alle unternehmensintern relevanten Informationen im Umsetzungsprozess der Markengestaltung. Die BSC ist somit zugleich kapitalmarkt-, absatzmarkt- und ressourcenorientiert und ermöglicht damit "als Steuerungsinstrument" eine Synthese des market- und ressourcebased view. Ergebniskennzahlen der Vergangenheit werden um die treibenden Faktoren künftiger Ergebnisse ergänzt. ${ }^{332}$ Eine Erweiterung der hier vorgeschlagenen Perspektiven um zusätzliche Perspektiven ist dabei durchaus denkbar, jedoch stets unter dem Primat einer managementseitigen Akzeptanz zu würdigen. ${ }^{333}$

Kaplan, R. S., Norton, D. P., Balanced Scorecard: Strategien erfolgreich umsetzen, a.a.O., S. 35. Vgl. zum Inside-Out-Fokus einer BSC auch Hoffecker, J., Goldenberg, C., Using the Balanced Scorecard to Develop Companywide Performance Measures, a.a.O., S. 57.

Vgl. Galli, A., Wagner, M., Balanced Scorecard an der Schnittstelle Profit - Non-Profit?, in: Der Betrieb, 52. Jg., Heft 39, 1999, S. 1966.

So darf eine Integration der bisher aufgezeigten Teilerkenntnisse nicht um der Integration willen erfolgen, denn je stärker integriert wird, desto komplexer werden die Systeme. Je komplexer sich aber das Mehrmarkencontrolling erweist, desto weniger Akzeptanz findet es erfahrungsgemäß in der Praxis. So lassen sich im Bereich des klassischen Rechnungswesens zahlreiche Negativbeispiele dafür finden, dass die Wissenschaft neue Ansätze etwa in den Bereichen der Prozesskostenrechnung oder wertorientierten Unternehmensführung hervorgebracht hat, weiche jedoch nur selten tatsächlich implementiert werden bzw. deren Nutzen zu weilen angezweifelt wird. So formulieren HORVATH/ KAUFMANN trefflich: „Durch seine Berechnung allein hat bis heute noch kein Unternehmen den Shareholder Value steigem können. " Vgl. Horváth, P., Kaufmann, L., Balanced Scorecard - ein Werkzeug zur Umsetzung von Strategien, a.a.O., S. 43. 
Die Struktur eines solchen vorwärts- und rückwärtsgekoppelten Mehrmarkencontrolling ist in Abb. 25 zusammenfassend aufgeführt.

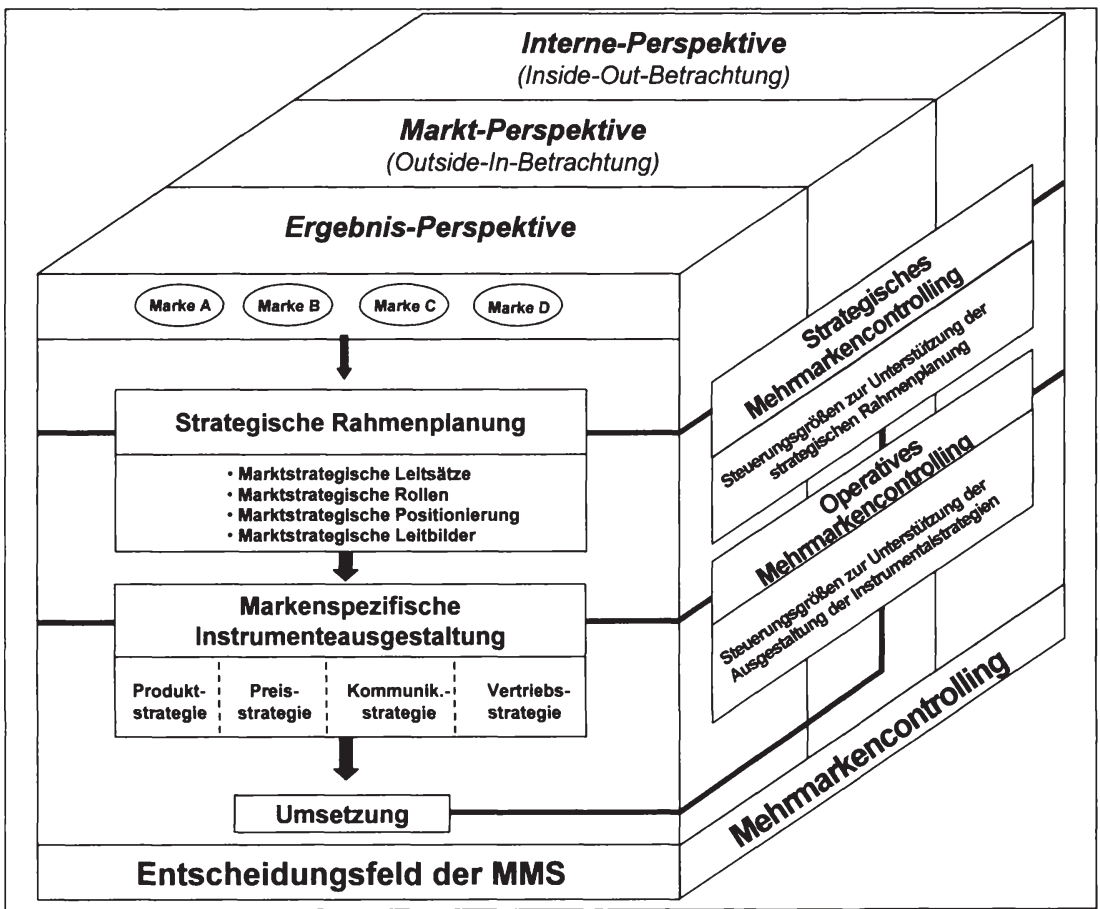

Abb. 25: Struktur eines Mehrmarkencontrolling

Vor dem Hintergrund der verfolgten Controllingstruktur werden im Folgenden zentrale Instrumente und Kennzahlen des Mehrmarkencontrolling auf strategischer und operativer Ebene abgeleitet und diskutiert. Neben einer grundsätzlichen Bewertung der Mehrmarkenstrategie als Grundsatzentscheidung des Unternehmens (Vorteilhaftigkeitsanalyse) nehmen dabei im Rahmen des strategischen Mehrmarkencontrolling vor allem Wanderungsanalysen und dezidierte Markenwahrnehmungsanalysen einen zentralen Stellenwert ein. Demgegenüber stehen beim operativen Mehrmarkencontrolling zentrale Instrumente und Steuerungsgrößen für die operative Ausgestaltung der Mehrmarkenstrategie im Vordergrund. Zur Sicherstellung eines abgestimmten Verhaltens der verschiedenen Ebenen im Portfolio erfolgt schließlich eine Integration der Steuerungsgrößen im Rahmen einer Scorecard-Ausgestaltung. 


\section{Mehrmarkencontrolling zur Deckung des Controllingbe- darfs am Beispiel der Automobilwirtschaft}

\section{Informationsgrundlagen der Analyse}

Das Mehrmarkencontrolling ist auf das Engste mit der zur Verfügung stehenden Datenbasis und dem situativen Kontext verbunden. Zum Zweck der vorliegenden Untersuchung kann auf unterschiedliche Marktforschungsstudien und unternehmensinterne Statistiken der Automobilindustrie zurückgegriffen werden.

Differenzierte Informationen zum beobachtbaren und nicht-beobachtbaren Käuferverhalten liefern europaweit durchgeführte Studien, die im Auftrag verschiedener Automobilhersteller jährlich erstellt werden. Dabei handelt es sich insbesondere um

- den New Customer Buying Survey (NCBS) zur Analyse von Strukturverschiebungen im Neuwagenkäufermarkt,

- die European Customer Satisfaction Study (ECS) zur Messung der Zufriedenheit der Kunden bzw.

- die Dealer Relation Study zur Messung der Zufriedenheit von Händlerorganisationen mit ihren Herstellern/ Importeuren sowie

- den Bekanntheits- und Imagecheck (sog. Car-Park-Studie) zur Messung der Marken- und Modellimages im Wettbewerbsvergleich. ${ }^{334}$

Obwohl die verwendeten Daten auch auf der Modellebene vorliegen, bezieht sich die Analyse im Folgenden vornehmlich auf die Ebene der Dachmarken. Ein solches Vorgehen erscheint zweckmäßig, da Dachmarken zum einen die spezifischen Charakteristika der einzelnen Marken zum Inhalt haben und zum anderen die dargestellten Instrumente konzeptionell analog auf Modellebene übertragen werden können. Bei dem der Analyse zugrundeliegendem Markenportfolio handelt es sich um ein Portfolio eines weltweit tätigen Volumenherstellers mit mehreren

334 Insofern greift die Untersuchung auf zyklische Sekundärstudien zurück. Die Marktforschungsdaten werden dabei den zentralen Anforderungen des Mehrmarkencontrolling gerecht. So sind die Daten regelmäßig (meist jährlich) verfügbar, erweisen sich aufgrund langjähriger $\mathrm{Er}$ fahrungen als reliabel und weisen eine entsprechende Akzeptanz in der Praxis auf. 
Marken. ${ }^{335}$ Zum besseren Verständnis der intendierten Portfoliostruktur sind die strategischen Rollen der hier betrachteten Marken in Tab. 6 dargestellt.

\begin{tabular}{|c|c|c|c|}
\hline \multicolumn{2}{|c}{ Marktstrategische Rollen } \\
\hline Marke & $\begin{array}{c}\text { Auftrag im } \\
\text { Portfolio }\end{array}$ & $\begin{array}{c}\text { Zentrale Elemente der } \\
\text { Marken }\end{array}$ & Externe Ausrichtung \\
\hline A & Leit-/ Hauptmarke & Zuverlässigkeit und Sicherheit & $\begin{array}{c}\text { Befriedigung der breiten Volumensegmente } \\
\text { [Absicherung und Ausbau] }\end{array}$ \\
\hline B & Trendmarke & Expressivität & $\begin{array}{c}\text { Emotions- und trendorientierte Automobilkäufer } \\
\text { [Angreifer Trendsegmente ] }\end{array}$ \\
\hline C & Preismarke & Robuste Qualität zu attraktiven \\
Preisen & $\begin{array}{c}\text { Funktions- und preisorientierte Automobilkäufer } \\
\text { [Verteidiger Preissegmente] }\end{array}$ \\
\hline D & Premiummarke & Fortschrittl. Technologie & $\begin{array}{c}\text { Befriedigung gehobener Automobilansprüche } \\
\text { [Eroberung Premium-/ Aufsteigersegmente] }\end{array}$ \\
\hline
\end{tabular}

Tab. 6: Struktur des betrachteten Markenportfolios

335 Die im Rahmen der Arbeit verwendeten Marken werden aus Gründen der Vertraulichkeit einzelner Marktforschungsdaten nicht explizit genannt, sondern durch Buchstabenbezeichnungen verfremdet. 
2. Strategisches Mehrmarkencontrolling:

Deckung des Informationsbedarfs bei der Ausgestaltung der strategischen Rahmenplanung

\subsection{Analyse der Vorteilhaftigkeit von Mehrmarkenstrategien}

Die Bewertung der Vorteilhaftigkeit von Mehrmarkenstrategien stellt ein vergleichsweise komplexes Problem dar. ${ }^{336}$ Vor diesem Hintergrund erfolgt im Folgenden im Sinne eines heuristischen Konzepts ${ }^{337}$ eine zweistufige Bewertung der Mehrmarkenstrategie, wobei es in einem ersten Schritt zu analysieren gilt,

- inwieweit bzw. unter welchen unternehmensspezifischen Bedingungen die Mehrmarkenstrategie die geeignete markenstrategische Option darstellt (qualitative Bewertung), um darauf aufbauend

- eine ökonomische Bewertung der Mehrmarkenstrategie vorzunehmen (quantitative Bewertung).

In diesem Zusammenhang stellt sich auch die Frage, wieviele Marken das Portfolio umfassen sollte. Das grundsätzliche Hinterfragen der Markenanzahl im Portfolio taucht z. B. regelmäßig nach der Akquisition eines Unternehmens auf, wenn sich Überschneidungen zwischen den Marken der Unternehmen ergeben, die eine Umstrukturierung und ggf. Bereinigung des Portfolios durch Eliminierung bzw. Reduktion von Marken notwendig machen (Reduktion des Markenportfolios). Die Entscheidung, welche Marke aus dem bestehenden Portfolio zu eliminieren ist, erfordert dabei eine umfassende Eliminierungsanalyse im Kontext des Gesamtportfolios und der markenübergreifenden Zielsetzungen. Andererseits können Ak-

Zu den allgemeinen Anforderungen an ein Konzept der Strategieauswahl vgl. Köhler, R., Marketing-Entscheidungen als Anwendungsgebiet der quantitativen Planung, in: Entscheidungshilfen im Marketing, Köhler, R., Zimmermann, H.-J. (Hrsg.), Stuttgart 1977, S. $2 \mathrm{ff}$.

Mit ADAM wird ein Lösungsverfahren als heuristisch bezeichnet, wenn a) es den Umfang komplexer Probleme reduziert, indem die Heuristiken einen Teil der potenziellen Lösungen durch Abstraktion von bestimmten Beziehungen zwischen den Elementen des Planungsproblems aus der Analyse ausschließen, b) nicht bewiesen werden kann, dass die eingesetzten Entscheidungsoperatoren zur optimalen Lösung konvergieren und somit nur eine zulässige Năherungslösung des Problems erzeugt wird und c) die verwendeten Entscheidungsoperatoren nicht zufällig bestimmte Lösungsmengen des Problems aus der Analyse ausschließen, sondern die Entscheidungsoperatoren aus der Problemstellung heraus mehr oder weniger plausibel begründbar sind. Vgl. Adam, D., Planung und Entscheidung: Modelle - Ziele - Methoden; Fallstudien und Lösungen, a.a.O., S. 415. 
quisitionen jedoch auch Folge der Entscheidung sein, das Portfolio bewußt um zusätzliche Marken zu ergänzen (Ausdehnung des Markenportfolios).

\subsection{Qualitative Vorteilhaftigkeitsanalyse}

Wie in Kapitel B aufgezeigt, werden mit der Mehrmarkenstrategie verschiedene Ziele verfolgt. Gleichzeitig hat insbesondere die Analyse des Controllingbedarfs gezeigt, dass dieser marktstrategischen Option auch vielfältige Gefahren inhärent sind. Für einen Anbieter kommt es somit darauf an, vor dem Hintergrund der realen Gegebenheiten die situativ beste Markenstrategie zu identifizieren. Hierzu sind die grundsätzlich zur Verfügung stehenden Strategieoptionen eines Herstellers anhand geeigneter Kriterien zu bewerten. Die Auswahl der Bewertungskriterien stellt dabei eine zentrale Aufgabe im Rahmen der Strategieauswahl dar, da sie letztlich den Maßstab bilden, an dem die Güte der einzelnen Strategiealternativen gemessen wird. ${ }^{338}$

Nahezu alle Automobilhersteller verfügen über eine mehr oder weniger ausgeprägte Markenhierarchie, in der alle markenstrategischen Optionen anzutreffen sind. Kaum ein Hersteller (z. B. SMART) führt seine Marken allerdings ohne Bezug auf den Firmennamen, so dass Dachmarken auf der Mikroebene eine besondere Stellung einnehmen, die auf Marktebene zumeist um spezifische Produkt- bzw. Modellmarken ergänzt werden. Vor diesem Hintergrund sollen im Folgenden die Ein-(Dach)-Markenstrategie (z. B. PORSCHE) und die Mehr-(Dach)-Markenstrategie (z. B. FORD) als zur Verfügung stehende Strategieoptionen eines Automobilherstellers gegenübergestellt werden.

Hinsichtlich der Beurteilungskriterien kann zwischen externen und internen Evaluierungsfaktoren unterschieden werden. Kriterien, die auf externe Situationsmerkmale rekurrieren, sind unabdingbar, da die Markenstrategie den Anbieter in die Lage versetzen soll, adäquat auf die für die Markenführung relevanten Umweltentwicklungen zu reagieren, um so Wettbewerbsvorteile zu gewährleisten. Da sich dies im Beziehungsgeflecht Konkurrenten, Händler und Nachfrager abspielt, sind wettbewerbs-, händler- und abnehmerbezogene Kriterien gleichermaßen zu berücksichtigen. Daneben bedarf es zur Ableitung einer tragfähigen Markenstrategie

Vgl. zum Vorgehen auch Schiele, Th. P., Markenstrategien wachstumsorientierter Unternehmen, Wiesbaden 1999, S. $251 \mathrm{ff}$ 
einer Beurteilung der unternehmensinternen Ausgangslage (Feasibility-Test) bzw. der Formulierung unternehmensspezifischer Anforderungen an eine Markenstrategie. Abb. 26 (linke Spalte) zeigt auf der Grundlage der erarbeiteten Zielsetzungen in Kapitel B. 2 sowie des Controllingbedarfs von Mehrmarkenstrategien in Kapitel C. 1 mögliche relevante interne und externe Beurteilungskriterien der Strategieoptionen.

\begin{tabular}{|c|c|c|c|c|c|c|c|c|c|c|}
\hline \multirow[b]{2}{*}{ Beurteilungskriterium } & \multicolumn{5}{|c|}{$\begin{array}{l}\text { Wichtlgkelt des Kriteriums aus der } \\
\text { Sicht des Unternehmens }\end{array}$} & \multicolumn{5}{|c|}{$\begin{array}{l}\text { Erfullungsgrad des Kriteriums aus der } \\
\text { Sicht des Unternehmens }\end{array}$} \\
\hline & $\begin{array}{c}\text { Unwichtig } \\
0\end{array}$ & $\begin{array}{c}\text { weniger } \\
\text { wichtig } \\
0.25\end{array}$ & $\begin{array}{c}\text { weder } \\
\text { noch } \\
0.5\end{array}$ & $\begin{array}{c}\text { wichtig } \\
0.75\end{array}$ & $\begin{array}{c}\text { sehr } \\
\text { wichtig } \\
1\end{array}$ & $\begin{array}{l}\text { Unge- } \\
\text { eignet } \\
0\end{array}$ & $\begin{array}{l}\text { weniger } \\
\text { geeignet } \\
0.25\end{array}$ & $\begin{array}{c}\text { woder } \\
\text { noch } \\
0.5\end{array}$ & $\begin{array}{c}\text { goeigner } \\
0.75\end{array}$ & $\begin{array}{c}\text { sehr } \\
\text { geeignet } \\
1\end{array}$ \\
\hline 1. Vermeidung von Kannibalisierung & & & & & & 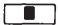 & & & tri & \\
\hline 2. Sicherung einer günstigen Kostenpositio & & & & & & & & & & 可 \\
\hline 5. Forderung eines intemen Wettbewerbs & & & & & & & 工 & & & \\
\hline 6. Ausmaß der Komplexitát & & & & & & E & & & & \\
\hline 7. Ausmaß $\beta$ des Koordinationsbedar's & & & & & & $\mathbf{D}$ & & & & \\
\hline 8. Möglichkeit der Marktausschöpfung & & & & & & & & & & \\
\hline 9. Moglichkeit der Marktabdeckung & & & & & & & & & & 8 \\
\hline 13. Kundengewinnung bzw -bindung & & & & & & & & 잔 & & \\
\hline 14. Vermeidung von Ausstrahlungseffekten & & & & & & & & & $\mathbf{D}$ & \\
\hline 15. Gewăhrleistung von Positionierungsfreir & & & & & & & & & & E \\
\hline 16. Vermeidung einer „Zwischen-d.-Stühlen & & & & & & & st & & & 里 \\
\hline 17. Abkoppein vom Preiswettbewerb & & & & & & & $x$ & & a & \\
\hline 18. Gewăhrleistung von Strategieflexibilitatt & & & & & & & 5 & & $\overline{0}$ & \\
\hline 19. Akzeptanz bei Stakeholdern & & & & & & & & FE & & \\
\hline 20. Abkopplung vom Preiswettbewerb & & & & & & 난 & & & D & \\
\hline 21. Auffangen von Markenwechslern & & & & & & & tr & & & $\mathbf{D}$ \\
\hline
\end{tabular}

Abb. 26: Ermittlung der Bedeutungsgewichte und Erfüllungsgrad im Hinblick auf Anforderungen an eine Ein- und Mehrmarkenstrategie

Um die angeführten grundsätzlichen Strategieoptionen in einer Vorauswahl qualitativ vergleichen zu können, bietet es sich an, auf ein Punktbewertungsverfahren zurückzugreifen. ${ }^{339}$ Hier wird in einem ersten Schritt die Wichtigkeit der einbezogenen Beurteilungskriterien ermittelt, da je nach Ausgangslage eines Anbieters die Anforderungen an eine Markenstrategie variieren. Die Gewichtung der Be-

Zum Punktbewertungsverfahren vgl. Adam, D., Planung und Entscheidung: Modelle - Ziele Methoden; Fallstudien und Lösungen, a.a.O., S. 357ff; ein besonderes Interesse hat das gewichtete Punktbewertungsmodell von O'MEARA gefunden. Vgl. O'Meara, J. T., Selecting Profitable Products, in: Harvard Business Review, Vol. 39, Heft 1, 1961, S. 83ff; vgl. auch Dreyer, A., Scoring-Modelle bei Mehrfachzielsetzungen - Eine Analyse des Entwicklungsstandes von Scoring-Modellen, in: ZfB, 44. Jg., 1974, S. 255ff. 
wertungskriterien spiegelt deshalb wider, welchen individuellen Chancen und Risiken sich ein Automobilhersteller ausgesetzt sieht. Die mittlere Spalte der Abb. 26 zeigt ein fiktives Profil der Kriteriengewichte, wie es von jedem Anbieter zu erstellen wäre. Aufbauend auf der Fixierung der Bedeutungsgewichte folgt in einem zweiten Schritt die Einschätzung, inwieweit die beiden Strategieoptionen die gestellten Anforderungen erfüllen (vgl. hierzu die fiktiven Werte in der rechten Spalte der Abb. 26).

Die Multiplikation eines jeden Beurteilungswertes mit dem jeweiligen Bedeutungsgewicht sowie die anschließende Addition der so errechneten Einzelscores lassen einen dimensionslosen Index der Vorteilhaftigkeit einer Option entstehen:

$$
x=\sum_{i=1}^{n} g_{i} y_{i}
$$

i: $\quad$ Beurteilungskriterium $(i=1,2 \ldots, n)$

g: Bedeutungsgewicht von Beurteilungskriterium i

$y_{i}: \quad$ Grad, mit dem die Strategieoption die Anforderung i erfüllt

$\mathrm{x}$ : Indexwert der beurteilten Strategieoption

Dieser Index muss für jede Strategieoption festgelegt werden. Allerdings sind die so erzielten Vorteilhaftigkeiten kritisch zu reflektieren. So führen eine Vernachlässigung relevanter Beurteilungskriterien und interessengefärbte Beurteilungsgewichte zu einem verzerrten Ergebnis. ${ }^{340}$ Probleme ergeben sich ferner hinsichtlich der fehlenden Überschneidungsfreiheit der Faktoren. So besteht $z$. B. zwischen den Kriterien elf und dreizehn ein enger Zusammenhang, so dass Verbundeffekte indirekt doppelt gewichtet werden. Hier würde sich somit eine faktoranalytische Überprüfung der Kriterien anbieten, um die Unabhängigkeit der Faktoren sicherzustellen. Positiv zu werten ist jedoch, dass ein Scoring-Modell die am Entscheidungsprozess Beteiligten dazu zwingt, die Vor- und Nachteile einer Strategie abzuwägen und systematisch offenzulegen, infolge dessen eine auf Fakten basierende Diskussion geführt werden kann. Überdies trägt ein Vergleich der ausgewählten Strategieoptionen anhand ausgewählter Prüfkriterien erheblich zur Reduktion der Komplexität des Entscheidungsproblems bei. Die so generierten $\mathrm{Er}$ kenntnisse über das Nutzenpotenzial einer Strategieoption und deren unterneh-

340 HAEDRICH/TOMCZACK sehen hier die Gefahr einer subtilen Manipulation, vgl. Haedrich, G., Tomczak, T., Produktpolitik, Stuttgart 1996, S. 219. Zur sonstigen Kritik an Scoring-Modellen vgl. auch Meffert, H., Marketing: Grundlagen marktorientierter Unternehmensführung: Konzepte - Instrumente - Praxisbeispiele, a.a.O., S. 384ff; Adam, D., Planung und Entscheidung: Modelle - Ziele - Methoden; Fallstudien und Lösungen, a.a.O., S. 357ff. 
mensspezifische Relevanz erscheinen vor diesem Hintergrund letztlich bedeutsamer als die Gesamtscores.

\subsection{Quantitative Vorteilhaftigkeitsanalyse}

Markenstrategien sind immer mit Markeninvestitionen verbunden, die einen Markenwert erzeugen oder verändern. Mithin können Markenstrategien auch als Investitionsprojekte verstanden werden. ${ }^{341}$ So fordert auch MEFFERT, markenpolitische Entscheidungen als Investitionsproblem zu begreifen und „die langfristigen Auswirkungen der ergriffenen Maßnahmen auf den Markenwert im Auge [zu] behalten. ${ }^{342}$

Grundsätzlich haben sich in Wissenschaft und Praxis je nach Zweck eine Vielzahl verschiedener Ansätze zur Bewertung von Marken herausgebildet. ${ }^{343}$ Bisher konnte sich indes noch keine allgemein akzeptierte Auffassung zur Markenbewertung durchsetzen. So stellt KELLER fest: „...no common viewpoint has emerged as to how brand equity should be conzeptualized and measured." 344 Die derzeit vorherrschenden Ansätze lassen sich im wesentlichen zwei Forschungsrichtungen zuordnen. Zum einen existieren finanzwirtschaftliche Ansätze, die den Markenwert als „Banwert aller zukünftigen Einzahlungsüberschüsse, die der Eigentümer aus der Marke erwirtschaften kann “345 definieren. Dabei wird zur Berechnung des mo-

341 Vgl. Sattler, H., Markenstrategien für neue Produkte, in: Moderne Markenführung: Grundlagen - innovative Ansätze - praktische Umsetzung, Esch, F.-R. (Hrsg.), Wiesbaden 1999, S. 339; Meffert, H., Strategien zur Profilierung von Marken, a.a.O., S. 135.

Meffert, H., Markenführung in der Bewährungsprobe, a.a.O., S. 481.

Einen aktuellen Überblick über Ansätze und Modell der Markenwertermittlung sowie ihre Bedeutung in der deutschen Praxis gibt Drees, N., Markenerfolgsforschung und Markenberatung in Deutschland - Ergebnisse einer empirischen Studie, in: Werbeforschung \& Praxis, Heft 4, 1999, S. 24ff; vgl. auch Bekmeier-Feuerhahn, S., Marktorientierte Markenbewertung: eine konsumenten- und unternehmensbezogene Betrachtung, a.a.O., S. 60ff.; Meffert, H., Burmann, Ch., Abnutzbarkeit und Nutzungsdauer von Marken, a.a.O., S. 244ff.; Zu Untersuchungen des Markenwertes in der amerikanischen Marketingforschung vgl. etwa Srivastava, R. K., Shocker, A. D., Brand Equity: A Perspective on Its Meaning and Measurement, MSI Technical Working Paper, Report No. 91-124, Cambridge 1991;

344 Keller, K. L., Strategic Brand Management: Building, Measuring, and Managing Brand Equity, a.a.O., S. 42.

Kaas, K.P., Langfristige Werbewirkung und Brand Equity, in: Werbeforschung und Praxis, Heft 3, 1990, S. 48; vgl. zur gleichen Richtung auch Kern, W., Bewertung von Warenzeichen, in: BfuP, Heft 1, 1962, S. 17ff; Simon, C. J., Sullivan, M. W., A Financial Approach to Estimating Firm-Level Brand Equity and Measuring the Impact of Marketing Events, MSI Working 
netären Markenwertes in einer Vorstufe vielfach auf nicht monetäre Markenwertdeterminanten zurückgegriffen. ${ }^{346} \mathrm{Im}$ Gegensatz hierzu betrachten konsumentenorientierte bzw. verhaltenswissenschaftliche Ansätze den Markenwert aus Sicht des Konsumenten. Hier werden primär nicht monetäre, psychographische Indikatoren herangezogen, so dass der Markenwert nicht unbedingt in Geldeinheiten ausgedrückt werden muss. ${ }^{347}$

Für die hier interessierende investitionstheoretische Betrachtung der Mehrmarkenstrategie erweisen sich konsumentenorientierte Ansätze somit als wenig geeignet. Statt dessen ist ein zahlungsstromorientierter Markenwert gefragt. Im Sinne des Kapitalisierungsansatzes entspricht dieser den abgezinsten markenspezifischen Einzahlungsüberschüssen. ${ }^{348}$ Der Begriff "Markeninvestition" impliziert dabei, dass es sich bei monetär wirksamen Markenaktivitäten stets um Auszahlungen handelt, denen unsichere Nettoerlöse gegenüberstehen (vgl. Abb. 27).

Paper Report No. 92-116, Cambridge 1992; Sander, M., Die Bestimmung und Steuerung des Wertes von Marken. Eine Analyse aus Sicht des Markeninhabers, a.a.O

Insbesondere die auf einer Vielzahl von Indikatoren aufbauenden Scoring-Modelle der Marktforschungsunternehmen NIELSEN und INTERBRAND haben hier eine weite Verbreitung gefunden. Im NIELSEN-ANSATZ wird zunächst anhand eines Scoring-Modells sowie 19 Markenwertdeterminanten die sog. Markenstärke bestimmt, in einer zweiten Stufe wird dann der monetäre Markenwert berechnet. Dabei werden die zukünftigen Erträge, die auf die Marke zurückzuführen sind, prognostiziert und anschließend abdiskontiert. Der INTERBRAND-ANSATZ ermittelt ähnlich dem Nielsen-Ansatz zunächst die Markenstärke anhand von 80-100 Unterkriterien, die als Determinanten des Markenwerts potenziell in Betracht kommen, um dann in der zweiten Stufe einen ermittelten Markenstärkepunktwert anhand einer Transformationsfunktion in einen Multiplikator zu überführen. Bei beiden ist jedoch kritisch die teilweise willkürliche Auswahl und Gewichtung der verwendeten Markenwertdeterminanten anzumerken. Vgl. Franzen, $\mathbf{0 .}$ Trommsdorff, V., Riedel, F., Ansätze der Markenbewertung und Markenbilanz, in: Markenartikel, Heft 8, 1994, S. 372ff, Hamman, P., Der Wert einer Marke aus betriebswirtschaftlicher und rechtlicher Sicht, in: Marke und Markenartikel als Instrumente des Wettbewerbs, Dichtl, E., Eggers, W. (Hrsg.), München 1992, S. 220ff.

347 Vgl. etwa Keller, K. L., Conzeptualizing, Measuring, and Managing Customer-Based Brand Equity, a.a.O., S. 1ff.; Esch, F.-R., Andresen, Th., Messung des Markenwerts, a.a.O., S. $11 \mathrm{ff}$.

$348 \mathrm{Vgl}$. in diesem Zusammenhang zur Kapitalwertmethode Perridon, L., Steiner, M., Finanzwirtschaft der Unternehmung, 9. Aufl., München 1997, S. 61ff. Aus der Kritik an der Kapitalwertmethode kann das Markenportfolio auch als Portfolio von Realoptionen betrachtet werden. Vgl. etwa Luehrman, T. A., Strategy as a Portfolio of Real Options, in: Harvard Business Review, Sept.-Oct. 1998, S. $89 \mathrm{ff}$. 


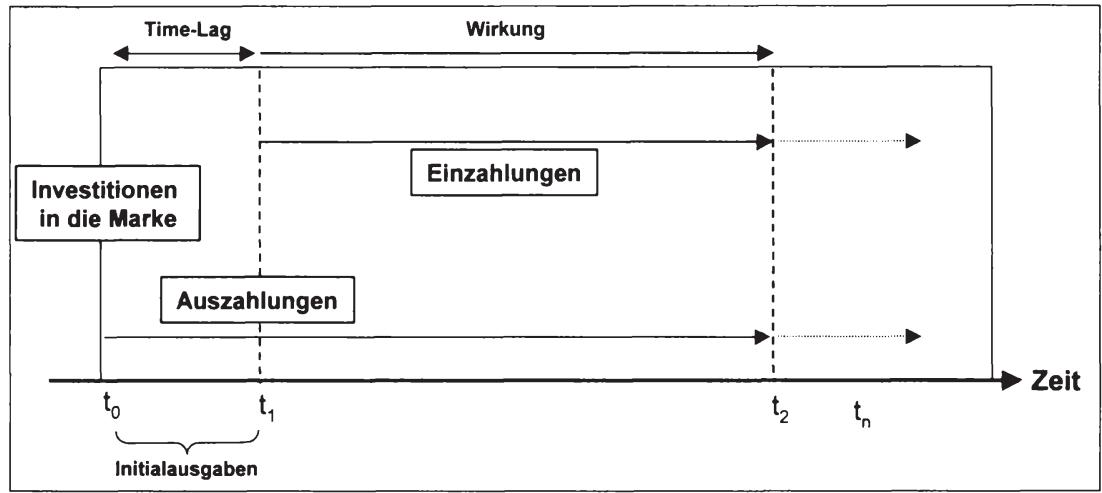

Abb. 27: Marke als Investitionsobjekt

Markenauszahlungen sind dabei einerseits solche Auszahlungen, die durch den Aufbau oder Kauf einer Marke entstehen und als Initialausgaben bezeichnet werden können sowie andererseits Auszahlungen, die durch die kontinuierliche Pflege der Marke im Rahmen von Marketingaktivitäten des Herstellers verursacht werden (z. B. im Bereich der Kommunikationspolitik die Bekanntmachung und Profilierung der Marke) und damit laufend anfallen. Als Markeneinzahlungen können die gesamten Einzahlungen bezeichnet werden, die während des Lebenszyklus der Marke anfallen und vom Nachfrager ab einem bestimmten Zeitpunkt (hier $t_{1}$ ) aufgrund erzielter Bekanntheit, positiver Einstellungen, Kaufpräferenzen, Vertauen gegenüber der Marke etc. geleistet werden.

Unter Festlegung einer an anderweitigen Verwendungsmöglichkeiten orientierten Mindestverzinsung $^{349}$ (Kalkulationszinsfuß) ergibt sich ein so berechneter Markenwert als Summe der diskontierten Ein- und Auszahlungen:

349 Für die Wahl des Zinssatzes werden eine Reihe von Vorschlägen unterbreitet. So wird $\mathbf{z}$. B. ein Zinssatz empfohlen, der die Finanzierungskosten abdeckt, der den Finanzierungskosten für die Risikoklasse der Sachinvestition entspricht (Basiszins plus Risikoaufschlag), der dem Habenzins einer gewünschten Mindestverzinsung entspricht oder der für den Ausschüttungsbedarf zur Bedienung des Kapitaleinsatzes der Eigentümer ausreicht. Bei diesen Vorschlägen handelt es sich jeweils um die Wahl anderer Opportunitäten als Vergleichsmaßstab für die zu beurteilende Investition. Entsprechend der Wahl des Vergleichsmaßstabes kann eine Investition somit als vorteilhaft oder unvorteilhaft erscheinen. Vgl. hierzu Adam, D., Investitionscontrolling, a.a.O., S. $67 \mathrm{ff}$. 


$$
\begin{aligned}
M W & =\sum_{t=0}^{T}\left(E_{m_{t}}-A_{m_{t}}\right) \frac{1}{(1+q)^{t}} \\
& =\sum_{t=0}^{T}\left(e_{m_{t}}{ }^{\star} x_{t}-A_{m_{t}}\right) \frac{1}{(1+q)^{t}}
\end{aligned}
$$

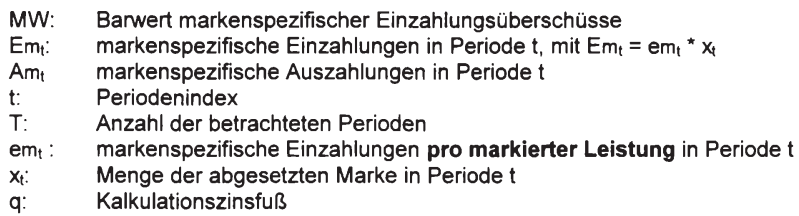

Zur Bestimmung des Portfoliowerts müssten die Markenwerte der einzelnen Marken gemessen und darauf aufbauend zu einem übergeordneten Portfoliowert aggregiert werden. Allerdings ist bereits die Ermittlung des Markenwertes einer einzelnen Marke mit zentralen Problemen behaftet. So resultieren Einzahlungsüberschüsse aus einer Vielzahl von Einflussfaktoren, wobei es bis dato praktisch kaum gelungen ist, markenspezifische Ein- und Auszahlungen von solchen zu isolieren, die nicht auf die Marke zurückzuführen sind. ${ }^{350}$ Nur so wäre jedoch eine Zuordnung der Einzahlungsüberschüsse auf den Markenwert möglich. Wirft in diesem Zusammenhang bereits die Ermittlung des Markenwertes nur einer Marke besondere Probleme auf, stellt um so mehr die Ermittlung eines Portfoliowertes unter Berücksichtigung der Interdependenzbeziehungen im Markenportfolio ein bisher nicht lösbares Problem dar. Vor diesem Hintergrund ist auf alternative Bewertungsverfahren zurückzugreifen.

Eine Beurteilung der Vorteilhaftigkeit einer Mehrmarkenstrategie kann auch mit Hilfe einer Deckungsbeitragsanalyse vorgenommen werden. ${ }^{351}$ Hierzu empfiehlt

350 Zur Problematik der Isolierung der reinen Markenein- und -auszahlungen vgl. etwa Schweiger, G., Friederes, G., Vom Markenmythos zum Markenwert, a.a.O., S. 28f. Insbesondere Conjoint-Analysen (Teilnutzen der Marke) ist zur Lösung des Zurechnungsproblems markenspezifischer Einzahlungen eine besondere Bedeutung zuzusprechen. SANDER bewertet die Preis-Eigenschaftsbeziehungen anhand der hedonischen Theorie mit Hilfe hedonischer PAF. Vgl. Sander, M., Die Bewertung internationaler Marken auf Basis der hedonischen Theorie, in: Marketing ZFP, Heft 4, 1994, S. 234ff. Als weitere Schwierigkeit lassen sich Indikatorenprobleme, d.h. Probleme bei der Bestimmung von angebots- und nachfragebezogenen Indikatoren für die Prognose der Ein- und Auszahlungen, sowie Monetarisierungsprobleme, d.h. Probleme bei der Überführung der Indikatorausprägungen in monetäre Wertgrößen, nennen.

351 Nach RIEBEL ist der Deckungsbeitrag der Überschuss der Einzelerlöse über die Einzelkosten eines sachlich und zeitlich begrenzten Kalkulationsobjekts bspw. einer Marke. Vgl. Riebel, P., Einzelkosten- und Deckungsbeitragsrechnung, 6. Aufl., Wiesbaden 1990, S. $46 f$. 
auch SCHIELE, in einem ersten Schritt auf Basis der Bestimmung der Größe eines jeden Marktsegments eine Berechnung der wertmäßigen Segmentvolumina vorzunehmen. $^{352}$ Dem in jedem Segment mit den einzelnen Marken angestrebten Marktanteil bzw. Umsatz sind in einem zweiten Schritt die Kosten einer Mehrmarkenstrategie gegenüberzustellen. Auf Grundlage des geplanten Umsatzes und der geschätzten variablen Kosten kann die Höhe des in einem Marktsegment mit einer Marke erzielbaren Deckungsbeitrages (DB) ermittelt werden. ${ }^{353}$ Die Bearbeitung eines weiteren Segments mit einer eigenen Marke ist dann sinnvoll, wenn der hierdurch zu erwartende Deckungsbeitrag positiv ausfält, wobei allerdings das Entscheidungskriterium DB > 0 nur eingeschränkte Bedeutung besitzt. Denn beim Wechsel von einer Einzel- zu einer Mehrmarkenstrategie ist davon auszugehen, dass zum einen zusätzliche Fixkosten anfallen, zum anderen werden von der bisherigen Marke Nachfrager zur neu eingeführten Marke wechseln (Substitutionseffekte), so dass die Entscheidungsregel zu erweitern ist. In einem solchen Fall hat der erzielbare Deckungsbeitrag mindestens die zusätzlichen Fixkosten sowie die durch die Substitution verursachte Erlösschmälerung zu kompensieren. Überdies ist davon auszugehen, dass ein Anbieter nicht in der Lage ist, auf beliebig viele Ressourcen (z. B. finanzielle Mittel) zurückzugreifen, so dass die Entscheidungsregel durch die Maximierung $D B \rightarrow$ max! zu ergänzen ist. In dem Falle, dass mehrere Marktsegmente die Voraussetzung DB > Erlösminderung durch Kannibalisierungseffekt plus zusätzliche Fixkosten erfüllen, der Anbieter aber aufgrund beschränkter Ressourcen nur noch eine Marke einführen kann, so ist diese in jenem Segment zu platzieren, welches im Vergleich zu den anderen Optionen den höchsten zusätzlichen Deckungsbeitrag liefert.

Die Mehrmarkenstrategie setzt somit ein Abwägen der Mehrerlöse und -kosten voraus. In Analogie zum klassischen Produktdifferenzierungsmodell kann davon ausgegangen werden, dass mit jeder zusätzlich eingeführten Marke im gleichen Produktbereich der zu erzielende Mehrerlös sinkt und die Kosten steigen. ${ }^{354}$ Fol-

\footnotetext{
352 Vgl. Schiele, Th. P., Markenstrategien wachstumsorientierter Unternehmen, a.a.O., S. 188.

353 Vgl. hierzu auch Freter, H., Markenpositionierung. Ein Beitrag zur Fundierung markenpolitischer Entscheidungen auf der Grundlage psychologischer und ökonomischer Modelle, (unveröffentlichte) Habilitationsschrift, Münster 1977, S. 281.

Vgl. Meffert, H., Marketing: Grundlagen marktorientierter Unternehmensführung: Konzepte Instrumente - Praxisbeispiele, a.a.O., S. 433ff.; Kilger, W., Optimale Produktions- und Absatzplanung, Opladen 1973; Brockhoff, K. Produktpolitik, 4. Aufl., Stuttgart 1999, S. 303ff.
} 
gende Überlegungen des klassischen Differenzierungsmodells sollen diese Annahme verdeutlichen, wobei die Führung nur einer Marke auch als "Standardisierung", die Führung mehrerer Marken als "Differenzierung" interpretiert werden kann.

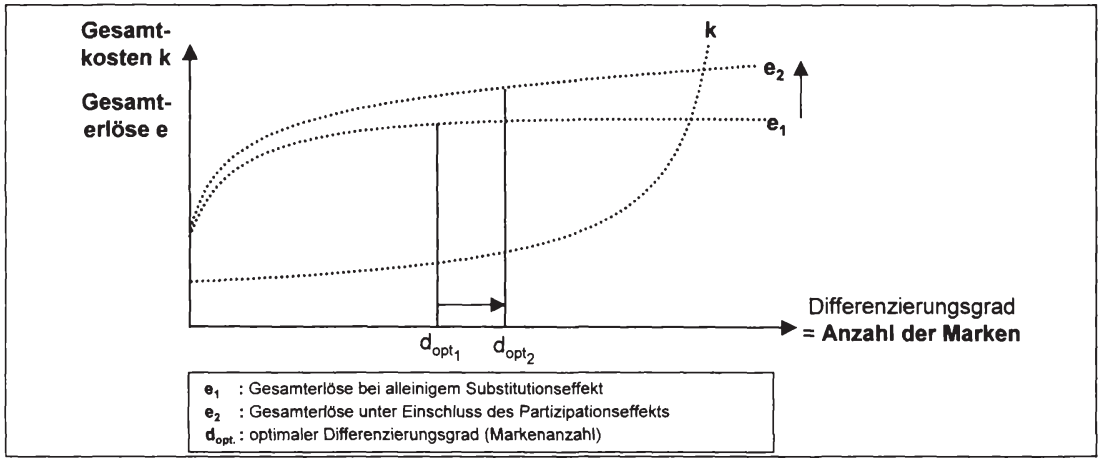

Abb. 28: Optimierungsmodell zur Bestimmung der Markenanzahl

Unter der zunächst gesetzten Prämisse, dass die Absatzmengen konstant bleiben - die Nachfrage also ausschließlich zu Lasten einer eigenen bestehenden Marke geht (Substitutionseffekt) - beeinflusst die "Anzahl der Marken“, also der Differenzierungsgrad, als zu optimierende Variable die erzielten Erlöse in der in Abb. 28 dargestellten Weise. Die Gesamtkosten k eines Herstellers für Entwicklung, Produktion, Vertrieb etc. steigen mit steigender Anzahl der Marken (Übergang von Einmarken- zur Mehrmarkenstrategie) und damit dem Differenzierungsgrad überproportional an. Dies scheint plausibel, da mit zunehmender Differenzierung durch zunehmende markenspezifische Varianten die Gesamtkosten direkt beeinflusst werden.

Der steigende Differenzierungsgrad wirkt sich demgegenüber positiv auf die Gesamterlöse aus, was auf eine steigende Zahlungsbereitschaft der Nachfrage für eine bessere Anpassung der Marken an eine heterogene Präferenzstruktur zurückzuführen ist. Allerdings wird die zusätzliche Zahlungsbereitschaft der Nachfrager immer geringer, da Nachfrager keine unendlich hohe Präferenzheterogenität aufweisen und eine maximale Zahlungsbereitschaft eines jeden Konsumenten 
existiert. ${ }^{355}$ Folglich liegt der optimale Differenzierungsgrad $d_{\text {opt } 1}$ dort, wo bei streng monotonen Kosten- und Erlösfunktionen die Grenzkosten der Differenzierung den Grenzerlösen entsprechen. Hier ist die Differenz zwischen Differenzierungskosten und -erlösen maximal. ${ }^{356}$ Zusätzlich zu der oben gesetzten Prämisse eines ausschließlichen Substitutionseffektes ist in praxi davon auszugehen, dass eine Erhöhung der Anzahl der Marken einen zusätzlichen positiven Effekt auf die Absatzmenge hat, da der Markt die bessere Anpassung an die Präferenzen der Nachfrager aufgrund der Differenzierung durch eine Steigerung seiner Nachfrage bei diesem Anbieter honoriert (Partizipationseffekt). Diese Annahme stellt letztlich ein zentrales Ziel der Mehrmarkenstrategie dar. Dabei ist das Ausmaß der Absatzsteigerung durch die Differenzierung von dem Ausmaß der Präferenzen der Nachfrager gegenüber Konkurrenzmarken abhängig. Es ist davon auszugehen, dass bei gegebenen Präferenzen gegenüber den Konkurrenzmarken ceteris paribus zwar die Kaufwahrscheinlichkeit mit der Anzahl der Marken steigt, allerdings nicht unbegrenzt, sondern mit positiv-abnehmender Steigung bis zum Erreichen eines Grenzwertes, der den bei bestimmter Wettbewerbssituation erreichbaren Maximalwert - abhängig von der Basisloyalität der Fremdmarken - oder aber die vollständige Abschöpfung des Gesamtmarktes reflektiert. Mit steigendem Partizipationseffekt erhöhen sich damit die Erlöse $\left(e_{1} \rightarrow e_{2}\right)$ und somit auch der optimale Differenzierungsgrad ( $d_{\mathrm{opt} 1} \rightarrow d_{\mathrm{opt} 2}$, vgl. Abb. 28). Je größer somit der Partizipationseffekt ist, desto stärker wird der Differenzierungsgrad über das bei konstanter Absatzmenge (Substitutionseffekt) optimale Maß hinaus steigen.

Das Optimierungsmodell gibt somit Ansatzpunkte zur Festlegung der optimalen Anzahl der Marken in einem Portfolio. Grundsätzlich setzt eine marginalanalytische Bestimmung des Modells jedoch voraus, dass der Differenzierungsgrad in stetiger Weise operationalisierbar ist (Stetigkeit der Funktionen). Die Markenanzahl ist indes nur diskret messbar (also als "Zahl" der möglichen Marken), wobei jedoch die exakt gleichen Überlegungen gelten. Die Bestimmung des Optimums

Zur Messung der Zahlungsbereitschaften kann z. B. die Conjoint-Analyse herangezogen werden.

Vgl. hierzu auch Backhaus, K., Büschken, J., Voeth, M., Internationales Marketing, a.a.O., S. $174 \mathrm{ff}$. 
erfolgt dabei nicht marginalanalytisch, sondern durch den vollständigen Vergleich aller möglichen Zustände in Bezug auf deren Kosten- und Erlöswirkungen. ${ }^{357}$

Die bisherigen Überlegungen haben gezeigt, dass der Gesamtgewinn eines Markenportfolios letztlich keine lineare Funktion der mit den einzelnen Marken erzielten Gewinne ist, da sich die markenspezifischen Erträge aufgrund wechselseitiger Marktanteilssubstitution gegenseitig bedingen. Eine Mehrmarkenstrategie ist ökonomisch dann sinnvoll, wenn den zusätzlichen Kosten eine entsprechend höhere Zahlungsbereitschaft der Nachfrage bzw. eine zusätzliche mengenmäßige Nachfrage gegenübersteht. Eine für eine bestimmte Zielgruppe zusätzlich eingeführte Marke muss somit entsprechend eine hohe zusätzliche wertmäßige Nachfrage bei der Zielgruppe hervorrufen.

Bei Auftreten von Substitutionseffekten lässt sich in Analogie zu JACOB für den Gewinn einer zusätzlich zur Marke A eingeführten Marke B - also beim Übergang von einer Einmarken- zu einer Mehrmarkenstrategie - unter Vernachlässigung der Fixkosten folgendes Entscheidungskalkül aufstellen ${ }^{358}$ :

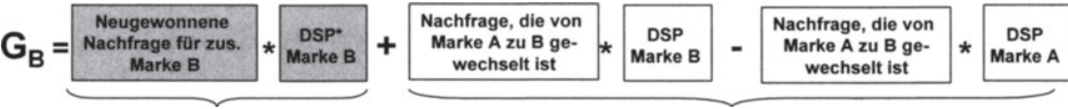

$$
\begin{aligned}
& \begin{array}{ll}
\text { Partizipationseffekt Substitutionsseffekt } & \text { St }
\end{array} \\
& G_{B}=\left(X_{B p}{ }^{*} D S P_{B}\right)+\left(X_{B s}{ }^{*} D S P_{B}\right)-\left(X_{B s}{ }^{*} D S P_{A}\right) \\
& =\left(X_{B p}{ }^{*} D S P_{B}\right)-X_{B s} *\left(D S P_{A}-D S P_{B}\right) \gtreqless 0 \text { (Entscheidungskriterium) }
\end{aligned}
$$

\footnotetext{
mit

$X_{B p}$ : neugewonnene Nachfrage für zusätzliche Marke B (Partizipationseffekt)

$X_{B s}$ : Nachfrage, die von Marke $A$ zu $B$ gewechselt ist (Substitutionseffekt)

DSPA: Deckungsspanne Marke A

$\mathrm{DSP}_{\mathrm{B}}$ : Deckungsspanne Marke $\mathrm{B}$
}

Ist DSP $_{\mathrm{B}}$, also die Deckungsspanne der Marke $\mathrm{B}$ als Differenz zwischen dem der Marke B eindeutig zurechenbaren Erlös und den dieser Marke eindeutig zurechenbaren variablen Kosten, größer als die Deckungsspanne der Marke A, so

357 Vgl. Backhaus, K., Büschken, J., Voeth, M., Internationales Marketing, a.a.O., S. 179.

358 Vgl. Jacob, H., Der Absatz, a.a.O., S. 358; Gutenberg, E., Grundlagen der Betriebswirtschaftslehre, a.a.O., S. 548; Meffert, H., Marketing: Grundlagen marktorientierter Unternehmensführung: Konzepte - Instrumente - Praxisbeispiele, a.a.O., S. 434. 
dass das zweite Glied des Entscheidungskalküls einen positiven Wert annimmt, so ist der Gewinn der Marke B auf jeden Fall positiv und die Einführung der Marke B und damit der Übergang von der Einmarken- zur Mehrmarkenstrategie ökonomisch positiv zu werten. Ist die Deckungsspanne $D_{S P_{B}}$ hingegen kleiner als $D_{S P_{A}}$, nimmt das zweite Glied des Entscheidungskalküls einen negativen Wert an. Der Gewinn der Marke B ist dann negativ, wenn die Beziehung

$$
\left(\mathrm{X}_{\mathrm{BP}}{ }^{*} \mathrm{DSP}_{\mathrm{B}}\right)<\mathrm{X}_{\mathrm{BS}}{ }^{*}\left(\mathrm{DSP}_{\mathrm{A}}-\mathrm{DSP}_{\mathrm{B}}\right)
$$

gilt, wenn also die neu hinzugewonnene wertmäßige Nachfrage durch den Partizipationseffekt kleiner ist als der wertmäßige Substitutionseffekt. In einem solchen Fall ist es für das Unternehmen günstiger, auf die Einführung der zusätzlichen Marke B zu verzichten. Falls der Gewinn größer als Null ist, ist zu überprüfen, ob die Gewinne sämtlicher zukünftiger Perioden, in denen die Marke B angeboten wird, gegebenenfalls diskontiert auf den Kalkulationszeitpunkt, größer oder kleiner sind als die auf den gleichen Zeitpunkt diskontierten Kosten zuzüglich der sonstigen fixen Kosten, die durch die Einführung der Marke B entstehen würden. ${ }^{359}$

Wie die bisherigen Überlegungen zusammenfassend zeigen, nehmen sowohl Substitutions- als auch Partizipationseffekte einen besonderen Stellenwert zur quantitativen Bewertung der Mehrmarkenstrategie ein. Vor diesem Hintergrund soll im Folgenden eine genauere Analyse dieser bisher in der Literatur nur unzureichend behandelten Effekte im Rahmen von Mehrmarkenstrategien vorgenommen werden:

- Dabei geht es zunächst darum, das Gesamtportfolio ex-post zu beurteilen, indem Substitutions- und Partizipationseffekte anhand von Markttransaktionen bestimmt werden, so dass auf beobachtbare Ergebnisse des Kaufentscheidungsprozesses Bezug genommen wird.

In dem Fall, dass mit einer zusätzlichen Marke B ausschließlich ein Substitutionseffekt eintritt, bewirkt das Angebot der Marke $B$ eine alleinige Aufspaltung des Firmenmarktes in mehrere Käuferschichten. Sind die Käufer der bisherigen Marke A gewillt, unterschiedlich hohe Preise für die bisherige Marke A zu zahlen, offeriert die neue Marke B die Möglichkeit, statt der bisherigen Preisspanne für die bisher angebotene Marke $A$ eine andere zu verlangen, um sich damit an die Kaufkraft bzw. Kaufwilligkeit der einzelnen Käuferschichten anzupassen. Hierdurch wird zweierlei erreicht: 1. Abschöpfung der Konsumentenrente bei jenen Käuferschichten, die gewillt gewesen wären, für die Marke A einen höheren als den verlangten Preis zu zahlen. 2. Erhöhung der Ausbringung, ggf. bessere Auslastung der Kapazität, in dem durch Niedrigpreismarke auch solche Käuferschichten angesprochen werden, denen bislang die Marke $A$ zu teuer war. 
- Zur Erklärung von Substitution und Partizipation im Markenportfolio erfolgt in einem umfassenden zweiten Schritt eine Beurteilung des Portfolios aus vorökonomischer Perspektive, wobei hier insbesondere psychographische Indikatoren für die Beurteilung der Mehrmarkenstrategie herangezogen werden. Die hier zu identifizierenden Indikatoren liefern dabei auch Anhaltspunkte für die zukünftige Entwicklung des Portfolios im Sinne einer ex-ante Betrachtung.

\subsection{Substitutions- und Partizipationsanalysen als Elemente der Wande- rungsanalyse}

Die Wanderungsanalyse kann als zentrales Element des Mehrmarkencontrolling bezeichnet werden, da durch eine wertmäßige Gegenüberstellung möglicher Wanderungsbewegungen innerhalb eines Markenportfolios (Substitutionseffekt) mit den Wanderungen zwischen den eigenen Marken und dem Wettbewerb (Partizipationseffekt) letztlich das Ergebnis der markenpolitischen Aktivitäten offenbart wird. Detaillierte Untersuchungen zum Thema Partizipation und Substitution im Rahmen der Mehrmarkenführung sind indes nicht vorhanden, gleichwohl die Begriffe häufig genannt werden. Im Folgenden wird daher zunächst der Grundgedanke der Analyse in Anlehnung an MASON/MILNE erläutert. ${ }^{360}$

\subsection{Konzeptioneller Bezugsrahmen der Wanderungsanalyse}

MASON/MILNE gehen zweckmäßig von einer festen Position jeder Marke im Wahrnehmungsraum der Konsumenten aus. Dies verdeutlicht die Abb. 29 anhand eines fiktiven Marktes mit drei Marken, wobei die Marken A und B von dem gleichen Hersteller produziert werden und somit dem gleichen Markenportfolio angehören. Die Kleinbuchstaben (Idealpunkte) kennzeichnen einzelne Konsumenten und verdeutlichen, welche Marke erworben wurde. Je zentrierter sich ein Konsument in einem bestimmten Markenbereich befindet, desto eher entspricht dieser der Zielgruppe der Marke bzw. kann dieser als "typisch" für die Marke bezeichnet werden. So kaufen etwa die meisten der im Markenbereich $A$ vorzufindenden Konsumenten die Marke A, andere jedoch die Marken B und C. Die tatsächlichen Markenkäufer einer Marke innerhalb der zugehörigen Markengrenze können als Kern-

360 Vgl. im Folgenden Mason, Ch. H., Milne, G. R., An Approach for Identifying Cannibalization within Product Line Extensions and Multi-Brand Strategies, a.a.O., S. 163ff. Vgl. allg. auch Moorthy, K. S., Png, I. P. L., Market Segmentation, Cannibalization and the Timing of Product Introduction, in: Management Science, 38. Jg., Heft 3, 1992, S. 345ff. 
konsumenten bezeichnet werden, die Gesamtzahl der Konsumenten innerhalb der Markengrenze als Markenpotenzial, so dass der Quotient aus tatsächlichen Käufern und dem Potenzial als Nischenanteil (Anteil im Markenraum) definiert werden kann. Die Buchstaben außerhalb der einzelnen Markenbereiche beschreiben schließlich Konsumenten, die zwar eine Marke kaufen, sich allerdings nicht im Zielmarkt der Marke entsprechend der gewünschten Positionierung befinden und somit als Randkonsumenten bezeichnet werden können.

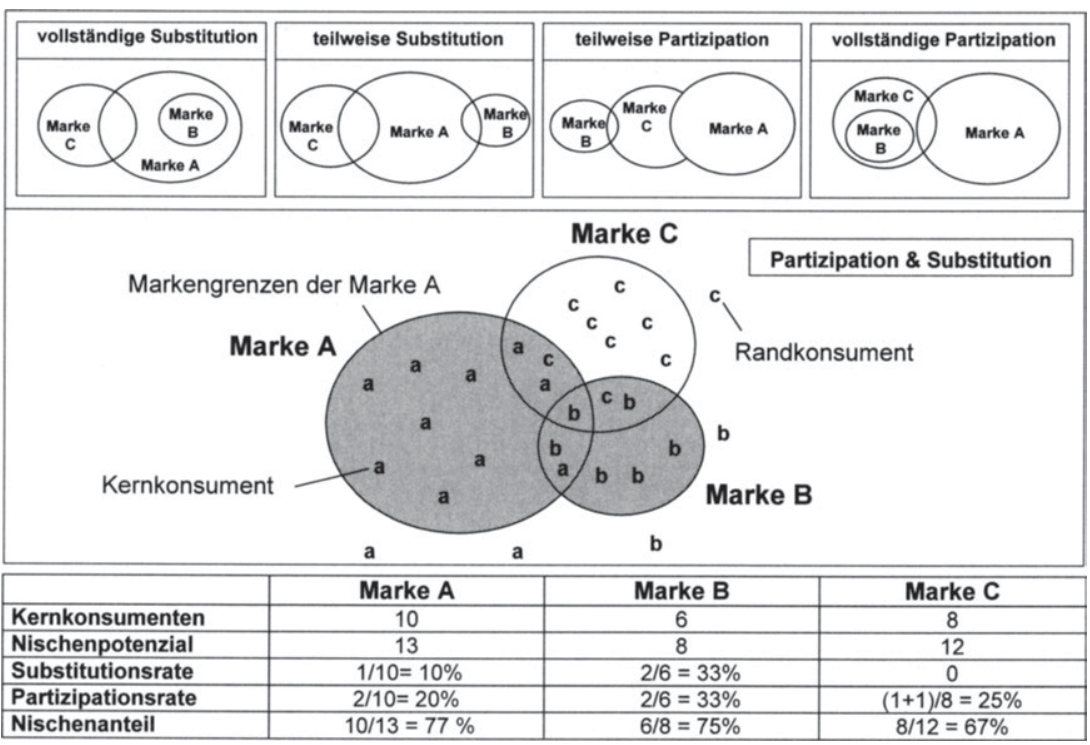

Abb. 29: Grundmodell der Substitution und Partizipation

(Quelle: In Anlehnung Mason, Ch. H., Milne, G. R., An Approach for Identifying Cannibalization within Product Line Extensions and Multi-Brand Strategies, a.a.O., S. 165)

Alle Konsumenten, die in Abb. 29 im Bereich der Marke A liegen, diese aber nicht gekauft haben, sind entweder durch die portfolioeigene Marke B substituiert worden oder zur Konkurrenzmarke C gewandert (Partizipation der Konkurrenz). ${ }^{361}$

Der Begriff der Substitution wird in der Praxis aktivisch und passivisch verwendet. Passive Interpretation der Substitution: Zwei potenzielle Käufer der Marke A werden von der Marke B substituiert (die Käufer befinden sich zwar im Grenzbereich der Marke A, kaufen diese aber nicht); aktive Interpretation wie in Tabelle: Die Marke A substituiert einen Käufer der Marke B, da der Konsument, der Marke A kauft, potenziell auch die Marke B gekauft hätte, da er sich auch im Grenzbereich der Marke B befindet und diese somit erwogen hat. 
Marke A hat somit einen Konsumenten von Marke B substituiert (ein „a“ liegt im Überschneidungsbereich zur Marke $B$ ) und zwei Konsumenten von Marke $C$ abgezogen (Partizipation: zwei "c" liegen im Überschneidungsbereich mit der Marke C). Die Ergebnisse der Substitution und Partizipation des fiktiven Beispiels lassen sich der Tabelle entnehmen.

Bei Einführung einer neuen Marke B sind letztlich neben dem in realiter vorherrschenden gleichzeitigen Substitutions- und Partizipationseffekt theoretisch sämtliche Szenarios

- von vollständiger Substitution (sämtliche Käufer der neuen Marke B gehen zu Lasten der portfolioeigenen Marke A),

- über teilweiser Substitution (ein Teil der Käufer geht zu Lasten der Marke A, ansonsten zieht die Marke B neue Konsumenten an und erweitert damit das Marktvolumen),

- und teilweiser Partizipation (ein Teil der Käufer geht zu Lasten der Marke C, ansonsten erweitert die Marke B das Marktvolumen),

- bis hin zur vollständigen Partizipation (z. B. sämtliche Käufer der Marke B gehen zu Lasten der Fremdmarke $C$ ) etc.

denkbar (vgl. Abb. 29). ${ }^{362}$ Hohe Substitutionsraten deuten dabei auf eine starke portfoliointerne Konkurrenz zwischen den Portfoliomarken, hohe positive Partizipationsraten auf eine hohe Wettbewerbsstärke hin. Ein wesentlicher Grund hoher Substitutionsraten kann in der mangelnden Differenzierung und der damit einhergehenden hohen Austauschbarkeit der Portfoliomarken in der Wahrnehmung der Konsumenten liegen. Verdeutlicht an Abb. 29 bedeutet dies, dass sich letztlich zu viele Konsumenten im Überschneidungsbereich der Marken befinden. Das Ausmaß der Substitution hängt dabei wesentlich von der Stellung der Marken im Preis- und Qualitätsfeld des Marktes ab. Der Substitutionseffekt wird letztlich um so geringer (größer) sein, je größer (geringer) der Preis- und Qualitätsunterschied zwischen den Marken ist.

Bei gleicher Qualität wird es ceteris paribus nur schwer möglich sein, höhere Preise durchzusetzen. Vor dem Hintergrund der Verwendung von Gleichteilen, Plattformen etc. lässt sich jedoch gerade in der Automobilindustrie eine Qualitätsangleichung feststellen. Falls die charakteristischen Unterschiede zwischen den Marken nicht mehr differenziert wahrgenommen werden bzw. der Marktauftritt der Marken zunehmend homogenisiert wird, steigt die Gefahr einer Bearbeitung der

Vgl. Traylor, M. B., Cannibalism in Multibrand Firms, in: Journal of Consumer Marketing, Vol. 3, No. 2, Spring 1986, S. 72. 
gleichen Nachfragersegmente. Die dennoch vorgenommene Preisdifferenzierung bei den auf die gleichen Kundensegmente ausgerichteten Modellen im Rahmen eines Markenportfolios (vgl. Tab. 7) lässt entsprechend stärkere Substitutionseffekte zugunsten von Preismarken und zu Lasten von Premiummarken erwarten ${ }^{363}$, denn „mit dem Argument „AUDI-Technik zu SKODA Preisen" lässt sich besser Geschäfte machen als gegen den Vonwurf „SKODA-Technik zu AUDI-Preisen“" ${ }^{664}$

\begin{tabular}{|l|c|c|}
\hline & Preise (UPE) in DM & Differenz Min.-Max.in DM \\
\hline Kompaktklasse Kurzheck & 33.400 & $\Delta 4.150$ \\
- Audi A3 1,6 & 29.250 & \\
- VW Golf 1,6 & 39.440 & \\
\hline Mittelklasse Stufenheck & 37.500 & $\Delta 9.990$ \\
- Audi A4 1,6 & 33.400 & \\
- VW Passat 1,6 & 31.400 & \\
Kompaktklasse Stufenheck & 29.450 & \\
- WW Bora1,6 & 41.760 & \\
- Seat Toledo & 39.450 & \\
- Skoda Octavia 1,6 & 34.400 & \\
\hline Mittelklasse Kombi & \\
- Audi A4 Avant 1,6 & 28.350 & \\
- WW Passat Variant 1,6 & & \\
Kompaktklasse Kombi & - WW Bora Variant 1,6 & \\
- Skoda Octavia 1,6 & & \\
\hline
\end{tabular}

Tab. 7: Preisdifferenzierung strategischer Wettbewerbsmodelle im VolkswagenKonzern

Nach PORTER ersetzt ein Produkt ein anderes, wenn es dem Abnehmer unter Berücksichtigung seiner Substitutionsneigung einen Substitutionsanreiz bietet, der stärker ist als die Substitutionskosten. Eine Ersatzmarke bietet dabei einen Substitutionsanreiz, wenn sie im Vergleich zu ihrem Preis dem Abnehmer einen hö-

Grundsätzlich gilt: je unelastischer die Nachfrage reagiert, desto vorteilhafter erscheint ein hoher Preis für den Anbieter. In der Regel reagiert die Nachfrage aber nur dann unelastisch, wenn es nur wenige oder überhaupt keine Substitutionsprodukte gibt. Dies ist offensichtlich in der Automobilindustrie nicht der Fall.

Diez, W., Plattform-Strategien: Irrweg oder Königsweg in der Modellpolitik, a.a.O., S. 32. So musste der im Juni 1996 auf der A-Plattform des Volkswagen Konzerns in den Markt eingeführte AUDI A3 im Jahr 1998 einen Absatzrückgang um 7,7 Prozent hinnehmen, wobei er insbesondere gegenüber seinem auf der gleichen Plattform beruhenden konzerninternen Wettbewerber GOLF IV verloren hat, der bei einer gleichen Motorisierung um 4.150 DM niedriger eingepreist ist. Auch beim AuDI A4 waren die Zulassungszahlen im ersten Halbjahr 1999 rückläufig, während in der gleichen Klasse andererseits der SKODA OCTAVIA ein Zulassungsplus von 78,3 Prozent und der SEAT TOLEDO von 117,9 Prozent verzeichneten. Die positive Entwicklung von AUDI in 1999 wird letztlich allein vom AUDI A6 mit einem Zulassungsplus von 9,4 Prozent und dem neu eingeführten AUDI TT getragen. Bemerkenswerter Weise handelt es sich bei beiden Fahrzeugen um Modelle ohne konzerninternen Wettbewerber. 
heren Wert als die bislang bezogene Marke bietet. Die Substitutionskosten sind hingegen eine Analogie zu den Kosten, die beim Wechsel von einer Marke zu einer anderen entstehen. Im beschriebenen Falle liegt der die Substitutionskosten überwiegende Substitutionsanreiz darin, dass die vergleichbare Qualität einer Marke zu niedrigeren Preisen erworben werden kann. ${ }^{365}$

Mikroökonomisch kennzeichnet die Wirtschaftswissenschaft den Begriff „enge Substitutionsprodukte" auch als Produkte mit hoher Kreuzpreiselastizität ${ }^{366}$ der Nachfrage. Analog kann von engen Substitutionsmarken gesprochen werden. Wenn bei Unveränderlichkeit aller übrigen Einflussfaktoren der Nachfrage, z.B. der Kommunikationspolitik, der Preis einer Marke steigt und dies zu einer Erhöhung der Nachfrage für eine andere Marke führt, sind die beiden Marken enge Substitutionsmarken. ${ }^{367}$ Steigt somit der Preis für die Modelle der Marke AuDI und werden die Konsumenten dadurch veranlasst, vermehrt Modelle der Marke WW nachzufragen, sind dies enge Substitutionsmarken.

Allerdings ist in Markenportfolios durchaus eine gewisse Substitution erwünscht: Unter der Prämisse ansteigender Einzeldeckungsbeiträge sowohl entlang der Portfoliomarken (z. B. von SKODA zu Aud im VOLKSWAGEN-KONZERN) als auch der Modelle innerhalb einer Marke (vom Kleinwagen zur Oberklasse) ergibt sich als Konsequenz eine gewünschte Substitution entlang der ansteigenden Einzeldeckungsbeiträge, da sich hierdurch letztlich der Portfoliogesamtgewinn erhöhen lässt. Folglich ist TRAYLOR zuzustimmen, wenn er sagt: „The real task of management, then, is not to avoid cannibalism [Hervorhebung $d$. Verf.], but to know when and where it happens, be aware of its likely competitive and profitability effects, and take the appropriate marketing action that serves the best strategic inte-

365 PORTER spricht entsprechend von Substitutionsökonomie. Vgl. Porter, M. E., Wettbewerbsvorteile: Spitzenleistungen erreichen und behaupten, a.a.O., S. 361

366 Bei der Kreuzpreiselastizität $T$ werden die relative Preisänderung des Anbieters $A$ und die daraus resultierende Absatzänderung des Anbieters B zueinander in Beziehung gesetzt. Die Konkurrenzintensität lässt sich dabei mittels des sogenannten Triffinischen Koeffizienten bestimmen: $T=\left(d x_{B} / x_{B}\right) /\left(d p_{A} / p_{A}\right)$, mit $x=$ Nachfragemenge und $p=$ Preis. Anhand des Vorzeichens der Kreuzpreiselastizität lässt sich feststellen, ob zwischen Gütern eine Substitutionsoder Komplementaritätsbeziehung besteht.

367 Vgl. auch Kotler, Ph., Bliemel, F., Marketing-Management: Analyse, Planung, Umsetzung und Steuerung, a.a.O., S. 392. Vgl. zu den "Cross-elasticities" im Rahmen der Kannibalisierungsanalyse auch Bultez. A. et al., Asymmetric Cannibalism in Retail Assortements, in: Journal of Retailing, Vol. 65, Nr. 2, Summer 1989, S. 155. 
rests of the enterprise. " ${ }^{368}$ Abb. 30 verdeutlicht das Grundschema der im Folgenden zu behandelnden Wanderungsanalyse. Hierauf aufbauend folgt in einem zweiten Schritt die Bestimmung des markenspezifischen Absatzpotenzials.

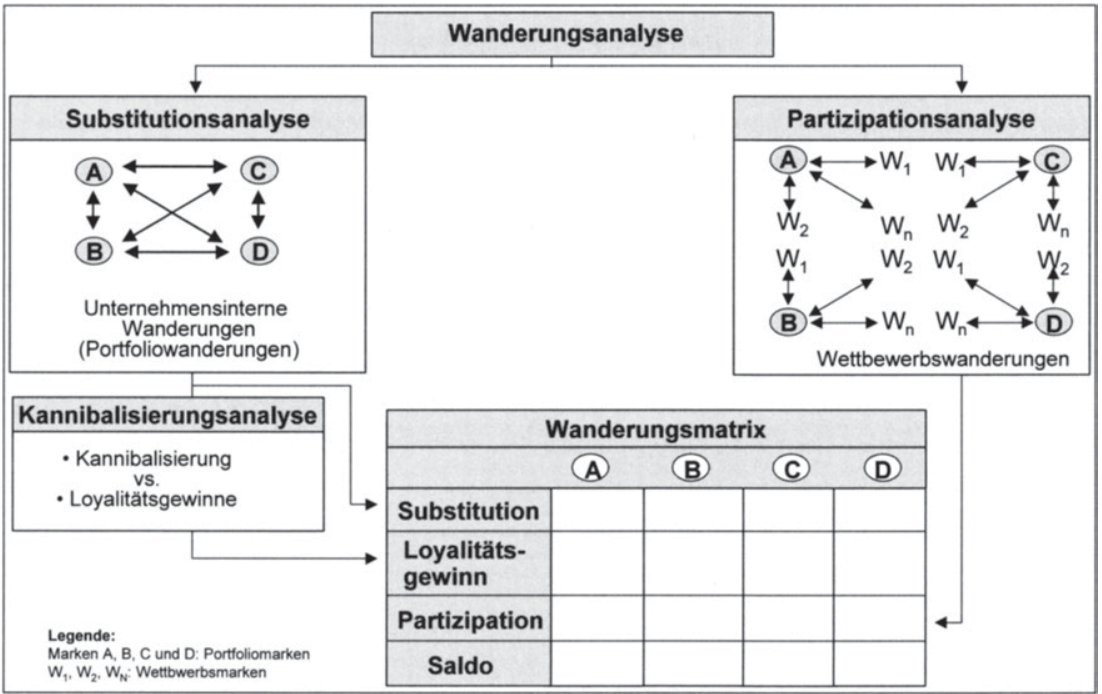

Abb. 30: Grundschema der Wanderungsanalyse

Während im Rahmen der Partizipationsanalyse sowohl die Nachfrage der durch die Marken A-D neu hinzugewonnenen Käufer (Zuwanderungen), die bislang Konkurrenzmarken erworben haben, als auch die Abwanderungen aktueller Käufer zu den Wettbewerbern untersucht wird, kommt der Substitutionanalyse ${ }^{369}$ die Aufgabe zu, Wechsel der Kunden von einer Marke des Portfolios zu einer anderen aufzudecken und mithin eine mögliche "interne Konkurrenz" der Marken innerhalb

BROCKHOFF nimmt eine andere Systematisierung vor, indem er unter der Substitution die Partizipation und Kannibalisierung subsumiert. Denn grundsätzlich wird bei der Substitution beobachtet, dass eine vermehrte Nachfrage nach einer Marke A mit einer verminderten Nachfrage nach Marke B einhergeht, ohne dass diese Beziehung durch andere Einflüsse zu erklären ist. Ergebnisse einer Substitution innerhalb eines Portfolios können dann als Kannibalisierungseffekt, diejenigen zwischen den Portfolios verschiedener Hersteller als Partizipationseffekt bezeichnet werden. Vgl. Brockhoff, K. Produktpolitik, a.a.O., S. 85. Dieser durchaus sinnvollen terminologischen Trennung soll allerdings im Folgenden nicht gefolgt werden, da der Begriff Substitution noch eine nähere Konkretisierung erfahren wird, die entsprechend als Kannibalisierung bezeichnet werden soll. 
des Markenportfolios zu analysieren. Hierbei ist zwischen „echter" Kannibalisierung einerseits sowie Loyalitätsgewinnen des Gesamtportfolios andererseits zu unterscheiden. Wanderungsbewegungen innerhalb einer Marke, also die Betrachtung möglicher Auf- und Absteiger, sind nicht der Wanderungsanalyse des Portfolios, sondern der Wanderungsanalyse einer Marke zuzurechnen. Die Ergebnisse der Partizipations- und Substitutionsanalyse lassen sich schließlich in einer Wanderungsmatrix bzw. -bilanz zusammenfassen.

\subsection{Substitutions- versus Kannibalisierungseffekt}

Die Analyse der Markensubstitution kann auf der Grundlage unterschiedlicher Messkonzepte erfolgen. Grundsätzlich besteht die Möglichkeit, Informationen über das tatsächliche Markenwechselverhalten als Grundlage zur Beurteilung der Austauschbeziehungen heranzuziehen. Hierzu werden in der Automobilindustrie regelmäßig die Vorbesitz- und Abwanderungsstrukturen einer Marke erhoben. Dabei gibt die Vorbesitzstruktur Auskunft darüber, welchen Vorwagen die Besitzer einer bestimmten Marke gefahren haben. Im Gegensatz dazu zeigt die Abwanderungsstruktur, zu welchem Modell die ehemaligen Besitzer einer Marke überwechseln. ${ }^{370}$ In Abb. 31 ist die portfoliointerne Abwanderungsstruktur der vier betrachteten Marken beispielhaft dargestellt.

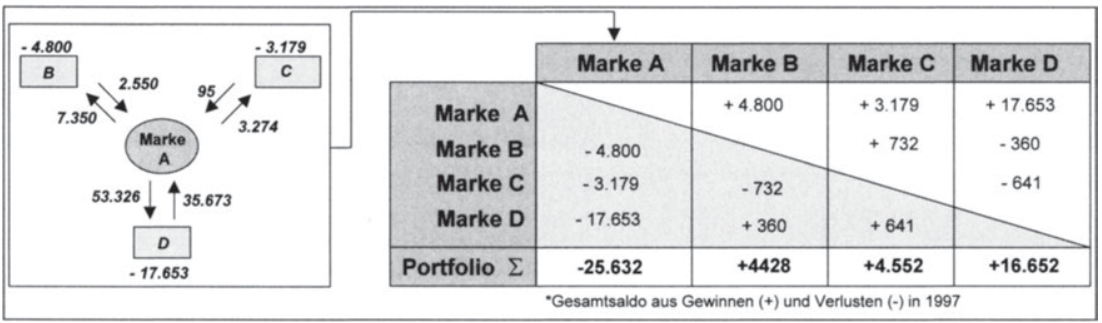

Abb. 31: Portfoliointerne Wanderungen

Die Wanderungsbewegungen zeigen eine positive mengenmäßige Substitution für die Marken B und C. So hat die Marke B beispielsweise 7.091 Mengeneinhei-

370 BAUER/HANNIG beschreiben die Erfassung der Substitutionsbeziehungen anhand des Verwendungszwecks eines Produktes. Vgl. Bauer, H. H., Hannig, U., Die Erfassung der Substitutionswirkung eines neuartigen Elektrowerkzeuges für den Heimwerker-Markt, in: Marketing ZfP, Heft 1, 1987, S. 5-15. 
ten $(\mathrm{ME})$ von $\mathrm{A}$ und $886 \mathrm{ME}$ von $\mathrm{D}$ gewonnen, hingegen $664 \mathrm{ME}$ an $\mathrm{C}$ verloren. Da die Marken $A$ und $D$ preislich höher positioniert sind als $B$ und $C$, bestätigt sich offensichtlich vor dem Hintergrund einer portfolioübergreifenden Verwendung von Gleichteilen im betrachteten Portfolio die oben aufgestellte These einer mangelnden Durchsetzbarkeit höherer Preise bei gleicher Qualität mit der Konsequenz hoher Substitutionseffekte im Portfolio.

Entscheidend für die Beurteilung der Substitutionseffekte sind letztlich die wertmäßigen Substitutionsbeziehungen im Portfolio. Die zugrunde gelegten Marken lassen sich anhand der jeweiligen Deckungsbeiträge nach abnehmender Größe bzw. relativer Bedeutung für das Gesamtportfolio nach dem Prinzip der ABCAnalyse $^{371}$ ordnen. Unter der Prämisse steigender Deckungsbeiträge entlang der Preismarke $C$ über $B$ und $A$ bis zur Premiummarke $D$ - eine solche Annahme erscheint aufgrund der hohen Verwendung von Gleichteilen und Plattformen und damit ähnlichen Kostenstrukturen ${ }^{372}$ im betrachteten Markenportfolio plausibel erweisen sich die dargestellten mengenmäßigen Wanderungsbewegungen in Abb. 31 um so nachteiliger für das Gesamtportfolioergebnis. Denn grundsätzlich sind Substitutionsbeziehungen bei gleichen Kostenstrukturen zu Lasten preislich höher positionierter Marken negativ, Substitution entlang steigender Einzeldeckungsbeiträge hingegen für den Portfoliogesamtgewinn positiv zu werten.

Die wertmäßigen Auswirkungen der Wanderungsbeziehungen seien an folgendem fiktiven Beispiel verdeutlicht, wobei aufgrund mangelnder Zugänglichkeit realer Deckungsbeiträge die Beiträge der einzeinen Marken zum Gesamtumsatz des Portfolios als Wertmaßstäbe verwendet werden. Grundsätzlich ist der Umsatz zur Bewertung zwar schlechter geeignet als der Deckungsbeitrag der einzelnen Marken, da hohe Umsätze letztlich nichts über Gewinne aussagen, d. h. eine Marke mit hohen Umsätzen kann dennoch defizitär sein. Vor dem Hintergrund der extensiven Verwendung von Gleichteilen im Markenportfolio kann jedoch von ähnlichen variablen Kosten ausgegangen werden, so dass sich der Überschuß (=Deckungsbeitrag) der Einzelerlöse über die Einzelkosten einzelner Marken insb.

Bei der den Scoringmodellen zuzurechnenden ABC-Analyse handelt es sich um eine Methode zur Analyse der Konzentration von Verteilungsstrukturen. Grundgedanke ist, dass ein relativ kleiner Mengenanteil einen relativ hohen Wertanteil einer Gesamteinheit verkörpert. Das Prinzip besteht darin, Objekte (Produkte, Marken oder auch Kunden etc.) bezüglich eines bestimmten Kriteriums, z. B. Umsatz, nach abnehmender Größe zu ordnen. Vgl. Becker, J., Marketing-Konzeption: Grundlagen des strategischen und operativen MarketingManagements, a.a.O., S. $886 \mathrm{ff}$.

372 Allerdings ist im Beispiel von geringeren Lohnkosten bei den Marken B und C aufgrund der Produktion in Ländern mit entsprechend geringerem Lohnniveau auszugehen. 
über den Umsatz bestimmt. Die Umsatzerlöse des betrachteten Portfolios betrugen im Jahr 1998 134.234 Mio. DM. Davon entfielen 79.745 Mio. DM auf Marke A, 13.535 Mio. DM auf Marke B, 6.114 Mio. DM auf C und auf Marke D 27.222 Mio. DM (sonstiges 7.627 Mio. DM). Entsprechend erzielt Marke $A$ einen prozentualen Beitrag zu den Umsatzerlösen in Höhe von ca. 59 Prozent, B von 10, C von 5 und $\mathrm{D}$ von 20 Prozent (Tab. 8). Aufgrund der Wanderungsbewegungen in Richtung Preismarken ergibt sich somit in dem Beispiel ein negativer wertmäßiger Saldo (der mengenmäßige Saldo ist aufgrund portfoliointerner Wanderungen gleich Null) in Höhe von $-5113,85$ (GE) [Wert 1]. Wären die Wanderungsbewegungen genau in umgekehrter Richtung verlaufen oder wären die Wert-Sätze vertauscht, ergäbe sich ein entsprechend spiegelbildliches Ergebnis (im letzten Fall bei $+5.494,31$ (GE) [Wert 2]).

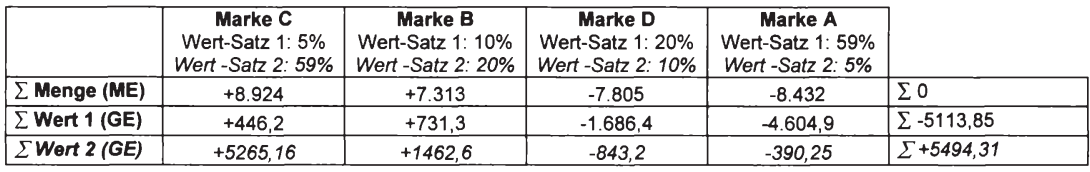

Tab. 8: Fiktives Beispiel des wertmäßigen Substitutionseffektes

Die bisher betrachtete und in der Automobilindustrie weit verbreitete Analyse der Wanderungsstrukturen erweist sich jedoch als nur bedingt geeignet für eine dezidierte Analyse der Substitutionsbeziehungen:

- So kann der gewünschte Wechsel zu einer preislich höher positionierten Marke bzw. der im Lebenszyklus des Konsumenten typische Aufstieg in eine höhere Modellklasse zu Fehlinterpretationen bei der Analyse der Wettbewerbsbeziehungen führen.

- Die zeitlichen Abstände zwischen dem Kauf der vorherigen Marke A und der jetzigen Marke $B$ betragen gerade in der Automobilindustrie zumeist mehrere Jahre. Mithin können sich die Präferenzen der Konsumenten geändert haben, so dass diese nicht mehr zur Kernzielgruppe einer Marke gehören. Die Informationen geben somit aufgrund der seit dem letzten Kauf entstandenen Zeitspanne keinen Aufschluss darüber, welche Marken in welchem Ausmaß unmittelbar vor der Kaufentscheidung tatsächlich als Kaufalternativen in Erwägung gezogenen wurden.

Eine Verbesserung der Ergebnisse lässt sich erreichen, wenn von den berechneten Wanderungen von einer Marke A zu einer Marke B für die Bestimmung der Substitution nur diejenigen Konsumenten berücksichtigt werden, die als Alternative für den Kauf der Marke B die frühere Marke A erwogen haben (sog. SecondChoice-Ansatz). Denn streng genommen

- lässt sich im Markenportfolio nur bei solchen Wanderbewegungen von echter Substitution - im Folgenden als Kannibalisierung bezeichnet - sprechen, bei denen Nachfrager von einer Marke des Portfolios zur anderen wechseln, dabei jedoch als Alternativmarke die vorher besessene - und damit die Marke des Portfolios - und nicht eine Wettbewerbsmarke in Betracht gezogen haben. Relevant sind somit die Erwägerraten. Zur Bestimmung der Enwägerrate wird der 
Käufer eines Automobils unmittelbar nach seiner Kaufentscheidung befragt, für welche Marke er sich entschieden hätte, wenn das tatsächlich erworbene Modell nicht erhältlich gewesen wäre. ${ }^{373}$

- Markenwechsler innerhalb des Portfolios ohne Präferenz für die vorher besessene Marke können hingegen den Loyalitätsgewinnen des Portfolios zugerechnet werden, d.h. ohne Präsenz weiterer Marken im Portfolio wären sie zu Fremdmarken abgewandert.

Dabei gilt: Je höher die Erwägerraten zwischen den Portfoliomarken, desto austauschbarer werden diese empfunden bzw. je höher der Loyalitätsgewinn, desto effektiver ist die Mehrmarkenstrategie trotz Substitution, da Markenwechsler nicht zu Fremdmarken abwandern, sondern zu portfolioeigenen Marken. Tab. 9 veranschaulicht diesen Sachverhalt.

\begin{tabular}{|c|c|c|c|c|c|c|c|c|c|c|}
\hline \multirow[b]{2}{*}{ Marke A } & \multicolumn{2}{|c|}{ Marke A } & \multicolumn{2}{|c|}{ Marke B } & \multicolumn{2}{|c|}{ Marke C } & \multicolumn{2}{|c|}{ Marke D } & \multirow{2}{*}{$\begin{array}{c}\begin{array}{l}\text { Abwander-. } \\
\text { ungen } \Sigma \\
\left(C_{\text {nur Porttolo })}\right.\end{array} \\
-58.810 \\
\end{array}$} & \multirow{2}{*}{$\begin{array}{c}\text { Absatz } \Sigma \\
624.036\end{array}$} \\
\hline & & & $\begin{array}{l}\text { davon: } \\
\text { A-Erwager } \\
\text { Nichterwager }\end{array}$ & $\begin{array}{r}+10.097 \\
+5.943 \\
+4.154 \\
\end{array}$ & $\begin{array}{l}\text { davon: } \\
\text { A-Enwager } \\
\text { Nichtenwager }\end{array}$ & $\begin{array}{r}+7071 \\
+4100 \\
+2971 \\
\end{array}$ & $\begin{array}{l}\text { davon: } \\
\text { A-Enwager } \\
\text { Nichterwager }\end{array}$ & $\begin{array}{r}+41.642 \\
+22.593 \\
+19.049 \\
\end{array}$ & & \\
\hline Marke B & $\begin{array}{l}\text { davon: } \\
\text { B-Enwager } \\
\text { Nichterwager }\end{array}$ & $\begin{array}{r}+3-006 \\
+554 \\
+2.452\end{array}$ & & & $\begin{array}{l}\text { davon: } \\
\text { B-Erwagger } \\
\text { Nichterwager }\end{array}$ & $\begin{array}{l}+916 \\
+246 \\
+670\end{array}$ & $\begin{array}{l}\text { davon: } \\
\text { B-Erwager" } \\
\text { Nichterwager" }\end{array}$ & $\begin{array}{r}+384 \\
+190 \\
+194\end{array}$ & -4.306 & 60.931 \\
\hline Marke C & $\begin{array}{l}\text { davon: } \\
\text { C-Enwager } \\
\text { Nichterwager: }\end{array}$ & $\begin{array}{r}+125 \\
+61 \\
+64\end{array}$ & $\begin{array}{l}\text { davon: } \\
\text { C-Erwager } \\
\text { Nichterwager }\end{array}$ & $\begin{array}{r}+252 \\
+118 \\
+134 \\
\end{array}$ & & & $\begin{array}{l}\text { davon: } \\
\text { C-Erwager } \\
\text { Nichterwager" }\end{array}$ & $\begin{array}{r}+129 \\
+65 \\
+64\end{array}$ & -506 & 38.462 \\
\hline Marke D & $\begin{array}{l}\text { davon: } \\
\text { D-Erwager } \\
\text { Nichterwager }\end{array}$ & $\begin{array}{l}+47.247 \\
+27.323 \\
+19.924\end{array}$ & $\begin{array}{l}\text { davon: } \\
\text { D-Enwager } \\
\text { Nichterwager }\end{array}$ & $\begin{array}{r}+1.270 \\
+209 \\
+1.061\end{array}$ & $\begin{array}{l}\text { davon: } \\
\text { D-Erwăger } \\
\text { Nichterwager }\end{array}$ & $\begin{array}{r}+1.443 \\
+696 \\
+747\end{array}$ & & & -49.960 & 233.352 \\
\hline $\begin{array}{l}\text { Zuwander- } \mathbf{~ u n g e n * " ~} \\
\text { (" nur Portlolos) }\end{array}$ & & 50.378 & & +11.619 & & +9.430 & & +42.155 & $\begin{array}{r}-113.582 \\
+113.582 \\
\end{array}$ & $\Sigma+956.781$ \\
\hline $\begin{array}{l}\text { Kanniball- } \\
\text { sierung }\end{array}$ & & +27.938 & & +6.270 & & +5.042 & & +22.848 & $\Sigma+62.098$ & \\
\hline $\begin{array}{l}\text { Loyalităts- } \Sigma \\
\text { gewinn }\end{array}$ & & +22.440 & & +5.349 & & +4.388 & & +19.307 & $\Sigma+51.484$ & \\
\hline
\end{tabular}

Tab. 9: Kannibalisierungseffekt der Mehrmarkenstrategie

So haben beispielsweise von 7.071 Markenwechslern, die von der Marke A zu C gewandert sind, 2.971 Konsumenten die Marke A nicht mehr als Kaufalternative

Die Frage "Wenn ihr jetziger Wagen zum Zeitpunkt Ihres Kaufs nicht auf dem Markt gewesen wäre: Welches Modell welcher Marke hätten Sie dann am ehesten gekauft? wird regelmäßig im Rahmen der sog. Neuwagenkäufer-Studie (NCBS-Studie: New Customer Buying Study) erhoben. Ziel der Studie ist die Analyse von Strukturverschiebungen im Neuwagenkäufermarkt sowie die Ermittlung der Ursachen hierfür. Zu diesem Zweck werden regelmäßige (i.d.R. jährliche) Neuwagenkäufer schriftlich befragt, die ihr Fahrzeug ca. 4-6 Monate im Besitz hatten. 
enwogen, wohl aber eine Fremdmarke und können deshalb als verloren gelten, wären sie nicht von einer anderen Portfoliomarke - in diesem Fall von C - aufgefangen und somit im Portfolio gehalten worden. Dagegen hätten sich $4.100 \mathrm{Kon}-$ sumenten wieder für die Marke $A$ entschieden, wäre das tatsächlich erworbene Modell der Marke $C$ nicht erhältlich gewesen. Diese Konsumenten können damit als kannibalisiert durch die Marke $C$ bezeichnet werden. Summiert über die Marke C ergibt sich bei einer Zuwanderung von den Marken A, B und D in Höhe von 9.430 eine Kannibalisierung von 5.042 und ein Loyalitätsgewinn von 4.388. Die Differenz aus $\mathrm{Zu}$ - und Abwanderungen ergibt entsprechend 7.313. Von 113.582 Wanderungen im Markenportfolio insgesamt sind somit theoretisch 62.098 als echte Kannibalisierung, hingegen 51.484 als Loyalitätsgewinn des Portfolios zu interpretieren. Somit eröffnet die Mehrmarkenstrategie die Möglichkeit, den Aufbau von Loyalität zur einzelnen Marke durch die Maximierung der Portfolioloyalität zu erweitern, um so der zunehmenden Fragmentierung des Automobilmarktes und der damit einhergehenden wachsenden Markenwechselbereitschaft zu begegnen.

Betrachtet man den Gesamtabsatz des Portfolios in Höhe von 956.781 Einheiten, lässt sich der Substitutionsindex im Portfolio berechnen als

$$
\text { Substitutionsindex }=\frac{\text { Portfoliointerne Wanderungen }}{\text { Neuwagenkäufer insgesamt }} \text {. }
$$

Damit ergibt sich ein Index in Höhe von 11,9 Prozent (113.582 / 956.781), d.h. 11,9 Prozent des Gesamtabsatzes beruhen auf portfoliointernen Wanderungen. Je höher letztlich der Substitutionsindex, desto mehr gehen die Umsatzerfolge einer Marke zu Lasten anderer Marken im Portfolio.

Analog zum Substitutionsindex lässt sich der Kannibalisierungindex berechnen als

$$
\text { Kannibalisierungsindex }=\frac{\text { "echte" Substitution }}{\text { Neuwagenkäufer insgesamt }} .
$$

Je höher der Kannibalisierungsindex, desto eher kann eine Marke aus dem Portfolio eliminiert werden, da als Alternativmarken jeweils andere Marken des Portfolios genannt werden. In diesem Zusammenhang ist insbesondere eine dynamische Veränderung der Kennzahl im Zeitablauf von Interesse: Sinkt der Kannibalisierungsindex im Zeitablauf bei steigendem Substitutionsindex, so stellt dies ein Indiz einer effektiven, Life-Cycle-gerichteten Mehrmarkenstrategie dar, da Markenwechsler entsprechend der Zielsetzung der Mehrmarkenstra- 
tegie nicht zu Fremdmarken abwandern, sondern im Markenportfolio gehalten werden.

Eine weitere Analyse der markenspezifischen Wanderungssalden lässt sich vornehmen, indem der Beitrag der einzelnen Modelle zu den Markensalden, d.h. die Substitutionsbeziehungen zwischen den einzelnen Modellen der Marken, nach dem gleichen Vorgehen bestimmt werden. Die Summe der einzelnen Modellsalden erklärt dabei die Markensalden. ${ }^{374}$ Insbesondere aus zeitlich-dynamischer Perspektive bietet eine disaggregierte Analyse auf Modellebene einen hohen Erkenntniswert zur Erklärung der markenspezifischen Wanderungssalden. Neue Modelle bringen Bewegung und Unruhe in den Markt, da sie Nachfrage auf sich ziehen. Da nahezu alle Innovationsprodukte einen Substitutionsprozess auslösen, indem sie eine bisherige Lösung ganz oder teilweise substituieren, ist auch davon auszugehen, dass neue Modelleinführungen einer Marke positive Substitutionseffekte zu Lasten der sonstigen Portfoliomarken bewirken. In der Regel werden sich die Modelle der einzelnen Portfoliomarken dabei in verschiedenen Produktlebenszyklusphasen ${ }^{375}$ befinden. ${ }^{376}$ Die Erzielung eines ausgewogenen „Life-Cycle-

Gründe für negative Markensalden können dann darin bestehen, wenn ein Modell einer Dachmarke technische Defekte aufweist und als Konsequenz nicht nur das betrachtete Modell, sondern quasi als "Kettenreaktion" auch die sonstigen Modelle der Dachmarke aufgrund negativer Imagetransfer-Effekte Absatzeinbußen erleiden. Genauso können umgekehrt einzelne Modellerfolge aufgrund des Goodwill-Transfers erhöhte Absatzeffekte bei den sonstigen Modellen der Dachmarke bewirken.

375 Die grundlegenden Aussagen des Lebenszyklusmodells sind, dass jedes Produkt zunächst steigende und dann sinkende Umsätze erzielt und ganz bestimmte Phasen durchläuft, unabhängig davon, ob und wie lange die absolute Lebensdauer ist. Vgl. Meffert, H., Marketing: Grundlagen marktorientierter Unternehmensführung: Konzepte - Instrumente - Praxisbeispiele, a.a.O., S. 330 .

Betrachtet man die Marktanteilsentwicklung eines Modells während seiner Lebensdauer, so sind zwar grundsätzlich unterschiedliche Kurvenverläufe denkbar, jedoch kann zumeist von einem ertagsgesetzlichen, s-förmigen Verlauf ausgegangen werden. In folgender Tabelle sind beispielhaft die Marktanteile des Golfs in Deutschland wiedergegeben, wobei die grauunterlegten Kästen die Einführung einer neuen Golf Generation symbolisieren. \begin{tabular}{|l|l|l|l|l|l|l|l|l|l|l|l|l|l|l|l|}
\hline 1983 & 1984 & 1985 & 1986 & 1987 & 1988 & 1989 & 1990 & 1991 & 1992 & 1993 & 1994 & 1995 & 1996 & 1997 & 1998 \\
\hline
\end{tabular}

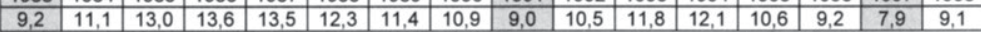

Die Veränderung der Marktanteile spiegelt letztlich den Golf-LZ in Deutschland wider. Bei einer Analyse der Marktanteilsentwicklung des Golfs ist die verstärkte Ausdifferenzierung des Automobilmarktes zu beachten. Betrachtet man die Produktpolitik der deutschen Hersteller, so ist in den 90er Jahren eine produktpolitische Offensive deutlich erkennbar, die sich sowohl in der Einführung technischer Neuerungen in bestehenden Produktprogrammen, als auch besonders in der Erweiterung der Programme um neue Modelle und Varianten ausdrückt. Gab es 1990 beispielsweise neben der Golf Limousine (Schrägheck) noch ein Golf Cabrio, so existieren mittlerweile mit der Einführung des Golf Variant (Kombiversion) in 1993 schon drei Golf-Varianten, die den Marktanteil des Golfs mitbegründen. 
Mixes" der Modelle nimmt somit einen zentralen Stellenwert bei der Mehrmarkenführung ein und ist weitgehend kongruent mit der Realisierung eines gut ausbalancierten Markenportfolios.

Nach GÄLWEILER haben in diesem Zusammenhang vielfältige empirische Untersuchungen ergeben, dass die meisten Substitutionen auch bei sehr unterschiedlichen Substitutionszeiten nach einem nahezu gleichförmigen Verlaufsmuster erfolgen. ${ }^{377}$ Die Gleichförmigkeit besteht darin, dass eine einmal begonnene Substitution, sobald sie nur einen gewissen Marktanteil erreicht und entsprechend ihre Einsatzfähigkeit bewiesen hat, mit dieser anfänglichen Substitutionsgeschwindigkeit weiterläuft, bis sie den gesamten Markt erreicht hat, es sei denn, dass sie zwischendurch durch eine neue Substitution abgelöst wird. ${ }^{378}$ In Anlehnung an die Überlegungen von GALWEILEIER lassen sich etwa die steigenden positiven Substitutionssalden der Marke C erklären (vgl. Abb. 32).

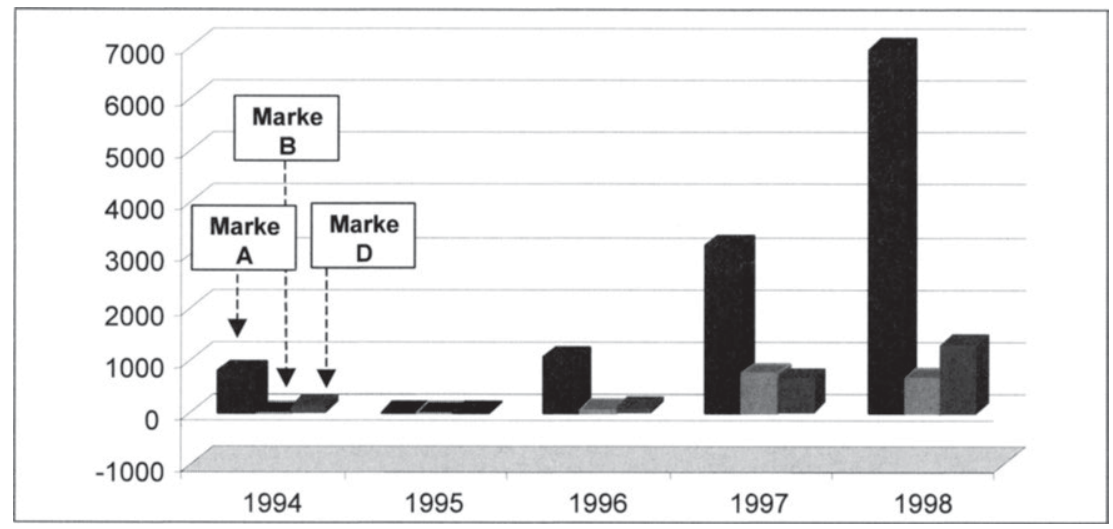

Abb. 32: Substitutionssalden der Marke $C$ in dynamischer Betrachtung

GÄLWEILER spricht in diesem Zusammenhang von der Substitutionszeitkurve. Vgl. Gälweiler, A., Strategische Unternehmensführung, a.a.O., S. $49 \mathrm{ff}$.

378 Diffusionsmodelle sind für Prognosen über das Wachstum neuer Marken und über die Technologiediffusionsrate häufig angewendet worden. Wichtige Diffusionsmodelle sind die von Mansfield, E., Technological Change and the Rate of Imitation, in: Econometrica, Vol. 29, Heft 4, S. 741ff; Fisher, J.C., Pry, R.H., A Simple Substitution Model of Technological Change, in: Technological Forecasting and Social Change, Vol. 2, May 1971, S. 75ff. 
Die Marke $C$ zeigt seit Anfang der neunziger Jahre eine zunehmende Präsenz mit neuen Modellen auf dem deutschen Markt zu Lasten der sonstigen Portfoliomarken. Die hohe Akzeptanz der Modelle beruht dabei im Wesentlichen auf ihrer bewiesenen Einsatzfähigkeit, welche maßgeblich durch den Rückgriff auf portfolioübergreifende Komponenten und Bauteile bei gleichzeitig geringerem Preis im Portfoliovergleich bestimmt ist. Gleichzeitig fördert ein positiver Goodwill-Transfer aufgrund der Integration in den Konzern die Akzeptanz der Modelle bei den Konsumenten. Wie die Wanderungssalden zeigen, geht der Substitutonseffekt insbesondere zu Lasten der Marken A und D und impliziert damit ein Trading-Down im Portfolio. Insbesondere unter Beachtung der hohen Kannibalisierungsraten (ca. 60 Prozent der Zuwanderer von Marke A zu C hätten beispielsweise in 1998 wiederum die Marke $A$ gekauft, wäre die Marke $C$ nicht am Markt, vgl. Tab. 9) stellt sich somit unter dem Gesichtspunkt der Gewinnmaximierung die Frage, ob die Aufnahme der Marke $C$ in das Markenportfolio den übergeordneten Portfoliozielen dient, wenn davon ausgegangen wird, dass die Marke $C$ den niedrigsten Dekkungsbeitrag erzielt. ${ }^{379}$ Eine Beurteilung, ob die Einführung einer Marke positiv oder negativ zu werten ist, lässt sich indes nur vornehmen, wenn neben dem Substitutionseffekt auch der Partizipationseffekt betrachtet wird.

\subsection{Partizipationseffekt}

In Analogie zur Berechnung der Substitutionsbeziehungen im Markenportfolio kann der Partizipationseffekt der Mehrmarkenstrategie durch Bestimmung der Wanderungen zwischen den Portfolio- und den Wettbewerbsmarken ermittelt werden. Zur Ausrichtung der Portfoliomarken im Wettbewerb erweist sich dabei eine gesonderte Analyse der Wanderungsbewegungen zwischen den eigenen Marken und den im Rahmen der Ausgestaltung der Mehrmarkenstrategie definierten strategischen und operativen Wettbewerbern als zweckmäßig, da die Wanderungsrichtungen Aussagen über das Konkurrenzverhalten der einzelnen Portfoliomarken im Wettbewerb ermöglichen. Da sich die Definition strategischer Wettbewerber am langfristigen, visionären Kompetenzanspruch der Marken im Wettbewerbsumfeld orientiert, ermöglichen die Wanderungsstrukturen zwischen den beitragsstarker Ford-Modelle ging. Vgl. Copulsky, W., Cannibalism in the Marketplace, Journal of Marketing, 40. Jg., Heft 10, 1976, S. 104 
strategischen Wettbewerbern und den einzelnen Portfoliomarken im Zeitablauf eine Überprüfung der Realisation eines solchen Kompetenzanspruchs.

Abb. 33 zeigt den Partizipationseffekt für die betrachteten vier Marken. Hier wird deutlich, dass in Summe sämtliche Marken des Portfolios mit Ausnahme von Marke A eine Absatzpartizipation zu Lasten anderer Anbieter im Markt erzielen. Über alle Marken hinweg ergibt sich jedoch aufgrund der hohen Abwanderungen der Marke A ein Verlust in Höhe von $36.358 \mathrm{ME}^{380}$

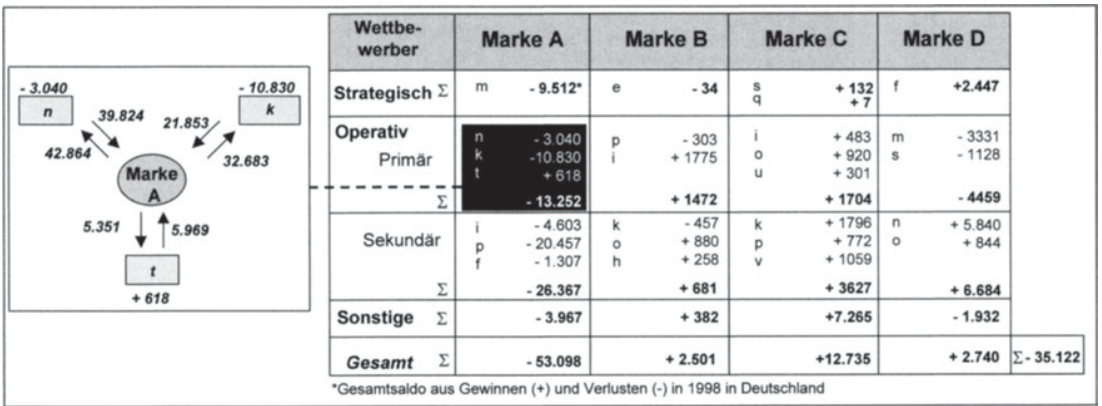

Abb. 33: Partizipationseffekt der Mehrmarkenstrategie

Analog zum Substitutionsindex lässt sich der Partizipationsindex berechnen als

$$
\text { Partizipationsindex }=\frac{\text { Portfolioexterne Zuwanderungen }}{\text { Neuwagenkäufer insgesamt }} \text {. }
$$

Dabei gilt: Je höher der Partizipationsindex, desto mehr gehen die Umsatzerfolge der Portfoliomarken zu Lasten von Fremdmarken. Ein hoher Partizipationsindex kann somit Indiz einer hohen Wettbewerbsfähigkeit der Marken sein.

380 Ein wichtiges Indiz bei der Beantwortung der Frage, an wen die Marktanteile innerhalb der einzelnen Segmente verloren gehen, ergibt sich aus der Betrachtung der Wettbewerbsaktivitäten in den einzelnen Segmenten. Beispielsweise sah sich der Golf mit den Jahren aufgrund des großen Volumenspotenzials des A-Segments einer immer stärkeren Konkurrenz gegenüber. Heute gibt es über 20 Wettbewerbsmodelle mit durchschnittlich drei Karosserievarianten in diesem Fahrzeugsegment. Durch Downsizing-Strategien erschließen auch Premiumhersteller zunehmend das durch den Golf definierte A-Segment. Das prägnanteste Beispiel ist hier die Einführung der A-Klasse von Mercedes-Benz. 


\subsection{Wanderungsbilanzen}

Unter Zusammenführung des Substitutions- und Partizipationseffektes lässt sich der Gesamteffekt der Mehrmarkenstrategie im Rahmen einer Wanderungsbilanz oder synonym Wanderungsmatrix ermitteln ( $\mathrm{vgl}$. Tab. 10). ${ }^{381}$

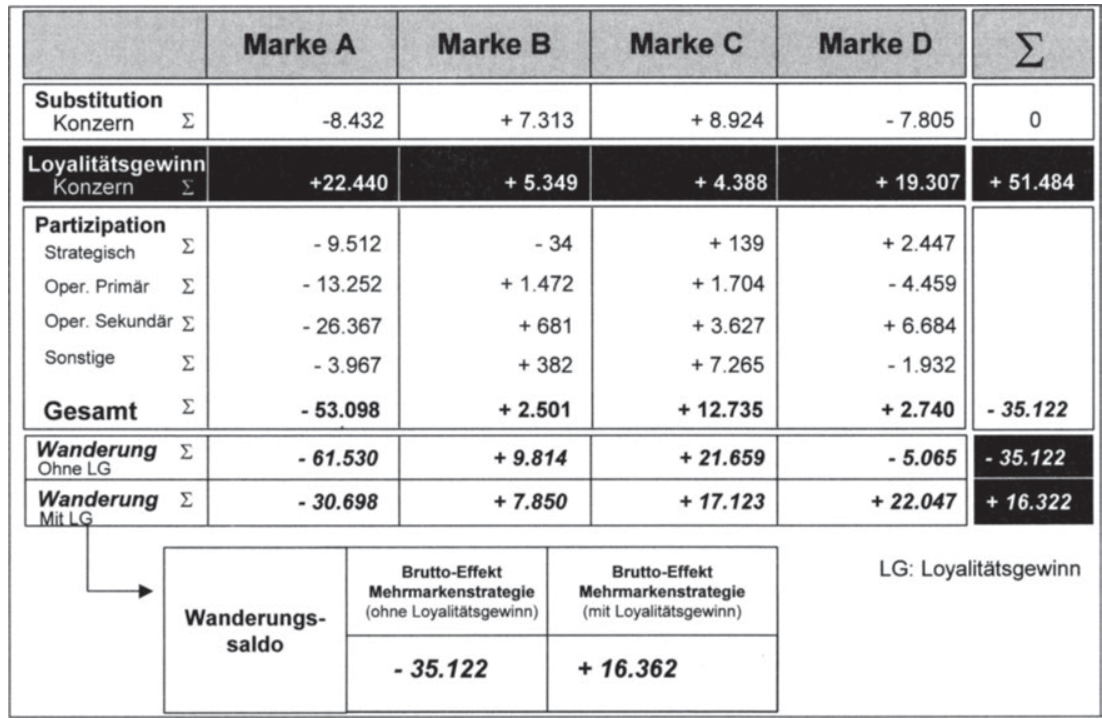

Tab. 10: Wanderungsbilanz

381

Die Überlagerung von Substitutions- und Partizipationseffekten kann auch im Rahmen von Markow-Ketten untersucht werden. Bei einem Markowschen Modell handelt es sich um ein mathematisches Modell, dessen grundlegende Begriffe die des Zustandes eines Systems und des Übergangs von einem Zustand $i$ in einen anderen Zustand j sind. So lässt sich der Kauf bestimmter Marken als möglicher Zustand eines Systems interpretieren, der Folgekauf als Veränderung des Zustands. Die oben dargestellten Wanderungen können mit den Begriffen Markowscher Ketten als Marken-Bewegungsmatrix oder auch Käufer-Bewegungsmatrix bezeichnet werden. Aus einer solchen Bewegungsmatrix kann formal eine stochastische Matrix der Übergangswahrscheinlichkeiten abgeleitet werden. Die Elemente einer solchen Matrix geben an, mit welcher Wahrscheinlichkeit ein Käufer einer bestimmten Marke in der nächsten Periode eine andere oder wieder die gleiche Marke kaufen wird. Mit den gegebenen Angaben ist es möglich, das dynamische Käuferverhalten und damit die Marktentwicklung in der Zeit als endliche Markowsche Kette darzustellen, um so die möglichen Partizipations- und Substitutionseffekte zu beschreiben. Solche Modelle sind in der amerikanischen Literatur ganz allg. unter Namen wie "Brand-Switching-Models" oder "Consumer Flow Models" bekannt. Vgl. zu Markow-Ketten Dynkin, E. B., Die Grundlagen der Theorie der Markoffschen Prozesse, ins Deutsche übertragen von J. Wloka, Berlin u.a. 1961; Sabel, H., Produktpolitik in absatzwirtschaftlicher Sicht - Grundlagen und Entscheidungsmodelle, Wiesbaden 1971, S. 201ff. sowie die dort umfangreiche Literatur. 
Der hier als Bruttoeffekt bezeichnete Wanderungssaldo der Mehrmarkenstrategie ohne Loyalitätsgewinn berechnet sich als Summe von Substitution und Partizipation über alle Marken. Er weist ein negatives Ergebnis in Höhe von - 35.122 ME aus, wobei die Marken $A$ und $D$ einen negativen, $B$ und $C$ hingegen einen positiven Ergebnisbeitrag leisten. Wie ersichtlich, entspricht der Gesamtsaldo des Portfolios somit der Summe der Partizipationseffekte, da sich gesetzmäßig die Substitutionssalden im Portfolio gegenseitig aufheben.

Der Partizipationseffekt allein hat allerdings nur eine vergleichsweise geringe Aussagekraft bezüglich der Wirksamkeit der Mehrmarkenstrategie. Zentrales Ziel der parallelen Markenführung ist es, Markenwechsler innerhalb des eigenen Portfolios zu halten. Der Bruttoeffekt der Mehrmarkenstrategie ignoriert jedoch eine Differenzierung der Substitution in "Kannibalisierung" einerseits und "Loyalitätsgewinn" andererseits und nimmt somit eine "verkürzte" Bewertung vor. Vor diesem Hintergrund errechnet sich der Nettoeffekt als Partizipation plus Loyalitätsgewinn (im vorliegenden Beispiel + 16.322). Dieser gibt gemäß dem Second-Choice-Ansatz somit einen Wanderungssaldo unter Berücksichtigung derjenigen Markenwechsler an, welche die in $t_{0}$ besessene Portfoliomarke nicht mehr erwogen haben und damit für das Portfolio als verloren gelten würden, wären sie nicht tatsächlich im Portfolio von einer anderen Marke in $t_{1}$ aufgefangen worden. ${ }^{382}$ Letztlich stellt somit der Loyalitätsgewinn die zentrale Größe zur Bewertung von Wanderungsbewegungen im Portfolio dar: Je größer der Loyalitätsgewinn, desto effektiver ist die Mehrmarkenstrategie, da Markenwechsler nicht zu Fremdmarken abwandern, sondern zu portfolioeigenen Marken und damit dem Gesamtportfolio erhalten bleiben.

In Bezug auf das Markenwahlverhalten findet letztlich sowohl beim Substitutionseffekt als auch beim Partizipationseffekt ein Markenwechsel statt. Substitutionsund Partizipationseffekte zeigen somit unmittelbare Auswirkungen auf den markenspezifischen Absatz einer Marke. Je höher die markenspezifischen positiven Substitutions- und Partizipationssalden, desto höher erweist sich der markenspezifische Absatz. Hier offenbart sich letztlich das „Dilemma der Mehrmarkenführung": Stellen positive Substitutionssalden aus Sicht einer Marke grundsätzlich

382 Allerdings ist die Frage, welches Gesamtergebniss ohne diese „Auffangmarke" erzielt worden wäre, nur mit einem Bündel von Annahmen möglich. Fundierte Aussagen im Sinne entgangener Gewinne bei Reduktion der Mehrmarkenstrategie lassen sich somit nur schwer treffen. 
anzustrebende Vorzugszustände dar, da sie letztlich die Wettbewerbsstärke der Marke indizieren und ihren Absatz positiv beeinflussen, sind sie aus Gesamtportfolioperspektive insbesondere bei Wanderungen von deckungsbeitragsstarken zu deckungsbeitragsschwachen Marken negativ zu beurteilen, deuten sie doch vor allem auf eine hohe Austauschbarkeit der Marken im Portfolio hin.

\subsection{Analyse des markenspezifischen Absatzpotenzials als Indikator der Wettbewerbsstärke}

Lag der Analyse bisher primär eine Portfolioperspektive zugrunde, sollen im Folgenden Kennzahlen aus einer Markenperspektive als Indikatoren der Wettbewerbsstärke einer Marke im Portfolio betrachtet werden. Zur Analyse der Wettbewerbsstärke einer Marke dient neben der Identifikation der absoluten Absatzströme bzw. Marktanteile die Bestimmung des Absatzpotenzials der einzelnen Portfoliomarke. Das Absatzpotenzial ${ }^{383}$ stellt dabei grundsätzlich eine fiktive Größe dar, die aufgrund entsprechender Schätzverfahren möglichst realistisch sein soll. Grundsätzlich ergibt sich der markenspezifische Absatz A der Marke a in einer Periode $t\left(A_{a}{ }^{t}\right)$ als Summe aus

- Erstkäufern: Käufern von a, die bisher noch keinen Pkw besessen haben (EK) sowie

- Zusatzkäufern: Käufern von a, die schon einen Pkw egal welcher Marke (ZK) besitzen und einen Pkw der betrachteten Marke als Zweitwagen anschaffen sowie

- Ersatzkäufern: Käufern von a, die zuvor schon einen Pkw der betrachteten (ESK) Marke besessen haben und als Neuwagen wiederum die Marke kaufen (Markenloyale), als auch aus

Käufern, die von den sonstigen Portfoliomarken (Substitutionseffekt) oder Fremdmarken (Partizipationseffekt) zu der betrachteten Marke wechseln (Eroberungen), samtheit möglicher Absatzmengen eines Marktes für eine bestimmte Produktgattung und damit die Aufnahmefähigkeit des Markts. Vgl. Becker, J., Marketing-Konzeption: Grundlagen des strategischen und operativen Marketing-Management, a.a.O., S. 393. 
so dass $A_{a}{ }^{t}=E K^{t}+Z K^{t}+E S K^{t}$. Abb. 34 zeigt den konzeptionellen Rahmen der Absatzanalyse am Beispiel der Marke A.

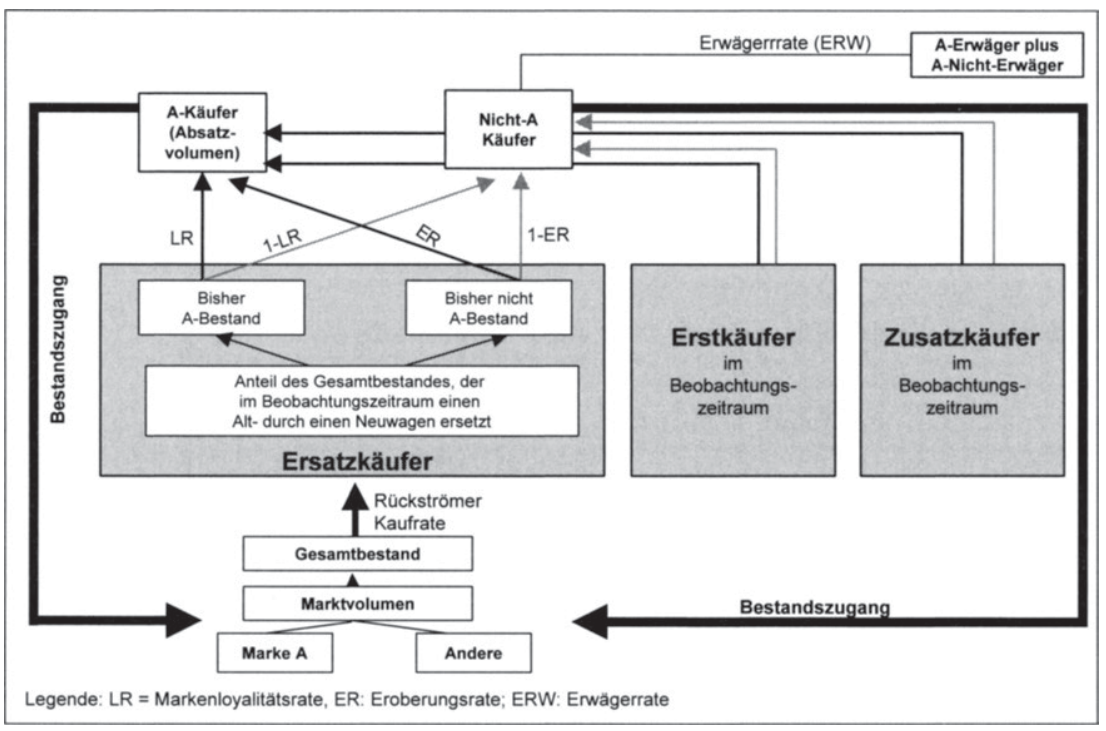

Abb. 34: Konzeptioneller Rahmen der Absatzanalyse

Aus einem Pkw-Gesamtbestand fragt ein gewisser Anteil an Pkw-Haltern (je nach Kaufrate) einen neuen Pkw nach (sog. Rückströmer in den Neuwagenmarkt). Die Rückströmer erweisen sich entweder als markenloyal (entsprechend der Loyalitätsrate LR) und kaufen die im Vorbesitz befindliche Marke A oder wandern zu einer anderen Marke ab (1-LR). Werden zusätzlich Eroberungen (entsprechend der Eroberungsrate [ER]) von Fremdmarken sowie Auf- und Absteiger sonstiger Modelle der Marke a bzw. Portfoliomodelle berücksichtigt, ergibt sich die Zahl der Ersatzkäufer. Unter Hinzuziehung der Erstkäufer sowie Zusatzkäufer erschliest sich letztlich das markenspezifische Absatzvolumen.

Insbesondere der Bestimmung des Ersatzbedarfs kommt in gesättigten und durch Verdrängungswettbewerb gekennzeichneten Märkten wie dem Automobilmarkt eine hohe Bedeutung für den Gesamtabsatz zu. Zentrale Indikatoren für die Position einer Marke im Wettbewerbsumfeld und deren Entwicklung sind dabei die Marken- und Modelloyalitätsraten (LR) sowie die Eroberungsrate (ER). 
- Während die beiden Loyalitätsraten angeben, wie viele Pkw-Besitzer bei einem Folgekauf wieder das entsprechende Modell (Modelloyalität) beziehungsweise ein sonstiges Modell der Marke (Markenloyalität) erwerben ${ }^{384}$,

- zeigt die Eroberungsrate ${ }^{385}$, wieviel Prozent der Rückströmer in den Neuwagenmarkt mit einem Fremdfahrzeug im Vorbesitz durch die Marke erobert werden konnten. Steigende Eroberungsraten einer Marke in einem Portfolio deuten somit Substitutions- und Partizipationseffekte im Wettbewerbsumfeld an.

Fasst man unter Eroberungen entgegen der automobilen, aber sonst gängigen Praxis vereinfacht sämtliche Käufer zusammen, die bisher die Marke nicht gekauft haben, also Wanderungskäufer (Partizipation und Substitutionskäufer) sowie Erstund Zusatzkäufer, so lassen sich Eroberung und Loyalität auch wie folgt bestimmen:

Geht man von der Überlegung aus, dass in jeder Periode t ein Anteil $\alpha$ der bisherigen Käufer zu Wiederholungskäufern werden (Markenloyale) und ein bestimmter Prozentsatz $\beta$ der bisherigen Nichtkäufer zu der betrachteten Marke wechselt ( $\mathrm{Er}$ oberungen im eben interpretierten Sinne), lässt sich der Absatz $a_{t}$ auch aus $a_{t}=\alpha$ $a_{t-1}+\beta\left(A-a_{t-1}\right)$ bestimmen, wobei $A$ die gesamte Nachfrage symbolisiert. ${ }^{386}$ Nach einer Umformung gilt: $a_{t}=\beta A+(\alpha-\beta) a_{t-1}$

Definition laut Neuwagenkäuferstudie (NCBS): „Im Modelljahr $t$ kauften X Prozent aller Besitzer eines neuen oder gebrauchten $X$, die in den Neuwagenkäufermarkt zurückströmten, wiederum einen neuen $X$. "Die solchermaßen verwendete Definition der Markenloyalität stellt allerdings eine gewisse Vereinfachung dar. In der Literatur wird vielfach darauf hingewiesen, dass es bei der Definition von Markenloyalität weniger darum geht, zufällige Wiederholungskäufe eines Kunden zu erfassen. Hingegen sollte ein Kunde als loyal gelten, wenn er aus Überzeugung eine Marke bewusst wiederholt nachfragt und auch zukünftig nachzufragen beabsichtigt. NEWMANWERBEL führen hier an: „... repurchase is not sufficient evidence of brand loyalty. If the measure is to be meaningful to management, it should reflect buyer resistence to persuasion to switch brands. "Newman, J. W., Werbel, R. A., Multivariate Analysis of Brand Loyalty for Major Household Appliances, in: Journal of Marketing Research, 10. Jg., November 1973, S. 404. Die Überlegung geht letztlich auf DAY zurück. Vgl. Day, G. S., A Two Dimensional Concept of Brand Loyalty, in: Journal of Advertising Research, Vol. 9, 1969, Heft 3, S. 29ff.; Homburg, Ch., Giering, A., Messung von Markenzufriedenheit und Markenloyalität, in: Moderne Markenführung: Grundlagen - innovative Ansätze - praktische Umsetzung, Esch, F.-R. (Hrsg.), Wiesbaden 1999, S. 1094. Die Markenbeziehungsqualität wird neuerdings als Alternative zum Konstrukt der Markenloyalität gesehen. Beide Konstrukte versuchen, die Stärke der Bindung zwischen Konsument und Marke festzuhalten. Vgl. Fournier, S. M., Consumers and Their Brands: Developing Relationship Theory in Consumer Research, a.a.O., S. $343 \mathrm{ff}$.

Definition laut NCBS: „Im Modelljahr $t$ kauften $X$ Prozent aller Nicht-X-Besitzer, die in den Neuwagenkäufermarkt zurückströmten, einen neuen $X . "$

Vgl. auch Simon, H., Goodwill und Marketingstrategie, a.a.O., S. 63 
$\alpha$ und $\beta$ sind bedingte Wahrscheinlichkeiten und können aus Marktforschungsstudien erhoben werden. Hierzu interpretiert man $a_{t}$ als Marktanteil, so dass die Gesamtnachfrage $A$ gesetzmäßig eins ergibt $(A=1)$. Der Parameter $\alpha$ entspricht der Wiederkaufswahrscheinlichkeit:

$$
\mathrm{a}=\frac{\text { Anzahl der Käufer, die die Marke in } \mathrm{t}-1 \text { und } \mathrm{t} \text { kaufen }}{\text { Anzahl der Käufer, die die Marke in } \mathrm{t}-1 \text { kaufen }}
$$

Indem $\alpha$ in die obige Funktion eingesetzt, wird ergibt sich

$$
\beta=\frac{a_{t}-\alpha a_{t-1}}{1-a_{t-1}}
$$

Zur Ermittlung des Absatzpotenzials in einer Periode bietet es sich an, markenspezifische Erwägerraten heranzuziehen, da Markenerwäger aufgrund der postulierten „Zweitpräferenz" auch als potenzielle Käufer der Marke interpretiert werden

\begin{tabular}{|c|c|c|c|c|c|}
\hline $\begin{array}{c}\text { Deutschland } \\
1998\end{array}$ & Marke A & Marke B & Marke C & Marke D & $\Sigma$ \\
\hline $\begin{array}{l}\text { Anzahl der } \\
\text { tats. Kunden }\end{array}$ & 624.036 & 60.931 & 38.462 & 233.352 & 956.781 \\
\hline $\begin{array}{c}\text { Nicht ausgeschöpftes } \\
\text { Potenzial }\end{array}$ & 778.386 Erwăger & 31.415 Erwäger & 27.924 Erwäger & 380,467 Erwäger & \\
\hline Gesamtpotenzial * & $\sum 1.402 .422^{* *}$ & $\sum 92.346$ & $\sum 66.386$ & $\sum 613.819$ & 2.174 .973 \\
\hline $\begin{array}{c}\text { Potenzialaus- } \\
\text { schöpfung }\end{array}$ & $44,5 \%$ & $66 \%$ & $58 \%$ & $38,0 \%$ & $44 \%$ \\
\hline \multicolumn{3}{|c|}{$\begin{array}{l}\text { "Kundenpotenzial: } \\
\text { Enwäger aus NCBS-Studie" + Anzahl der tatsăchlichen Kunden } \\
\text { (Def: Erwäger: Jetztwagen Fremdmarke - Alternativmodell Portfolio-Group) }\end{array}$} & $\begin{array}{l}\text { piel: } \\
\text { (A-Erwägerrate Gesa } \\
386 \text { (A-Erwäger) + } 62\end{array}$ & $\begin{array}{l}\text { tmarkt) } \times 3.490 .519(\mathrm{Ma} \\
36 \text { (tats. A-Kunden) }=1 .\end{array}$ & $\begin{array}{l}\text { rktvolumen 1998)) } \\
402.422\end{array}$ \\
\hline
\end{tabular}
können (vgl. Tab. 11).

Tab. 11: Absatzpotenzialanalyse

Durch Addition des tatsächlich realisierten Absatzes und der Markenerwäger ergibt sich das markenspezifische, nicht realisierte Absatzpotenzial einer Periode. So beträgt das Potenzial der Marke A 1.402.422 Einheiten, wenn man von einer markenspezifischen Erwägerrate bezogen auf das Gesamtmarktvolumen ( $=3.490 .519)$ in Höhe von 22,3 Prozent und damit 778.386 Einheiten und einem tatsächlich realisierten Absatz in Höhe von 624.036 Einheiten ausgeht. Die Ausschöpfung des möglichen Potenzials lässt sich dann definieren als

$$
\text { Potenzialausschöpfung }=\frac{\text { Anzahl der tatsächlichen Kunden }}{\text { Kundenpotenzial }},
$$

d.h. das der Marke A zur Verfügung stehende Potenzial wird nur zu 44,5 Prozent

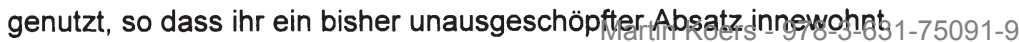


Unter Hinzuziehung der oben betrachteten Größen lässt sich die Absatzstruktur im Sinne einer Absatzspaltung („Sources of Sales“) einer jeden Portfoliomarke identifizieren. In Abb. 35 ist beispielhaft die Absatzstruktur der Marke C im Zeitablauf wiedergegeben.

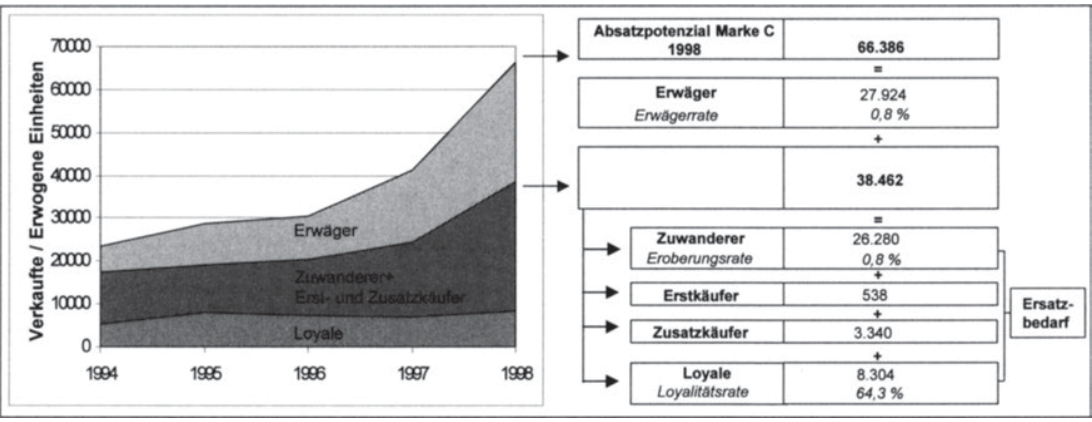

Abb. 35: Absatzstrukturanalyse in dynamischer Betrachtung

Die betrachtete Marke ist durch eine positive Entwicklung aller Bestimmungsgrößen gekennzeichnet, wobei die steigende Erwägerrate als Indikator weiteren Wachstums interpretiert werden kann. Je höher dabei die Erwägerrate, desto höher kann der potenziell mögliche (die Marke ist im evoked set des Konsumenten), aber unrealisierte Absatz einer Marke bezeichnet werden. Über den Zeitvergleich können Entwicklungen sichtbar gemacht werden, wobei insbesondere auffällige Verlagerungen zwischen den Absatzquellen von Interesse sind. Steigt beispielsweise der Anteil der Erstkäufer oder Eroberungen, sinkt hingegen die Zahl der Markenloyalen, so kann dies ein Indiz dafür sein, dass die betreffende Marke von Nicht- bzw. Fremd-Markenbesitzern besser beurteilt wird als von den Käufern der Marke selbst et vice versa.

In Tab. 12 sind abschließend die bisher analysierten ex-post Kennzahlen und Indikatoren des Mehrmarkencontrolling zusammengefasst. Während der obere Teil der Tabelle solche Kennzahlen umfasst, die nur aus Gesamtportfolioperpektive generiert werden können, beinhaltet der untere Teil Größen, die sich aus Einzelmarkenperspektive errechnen. 


\begin{tabular}{|c|c|c|}
\hline Kennzahl & Definition & Hypothese \\
\hline \multicolumn{3}{|c|}{ Mehrmarkenfokus } \\
\hline $\begin{array}{l}\text { Substitutions- } \\
\text { Index }\end{array}$ & $\begin{array}{l}\text { Ausmaß der portfoliointernen Wan- } \\
\text { derungsbewegungen. }\end{array}$ & $\begin{array}{l}\text { Je höher der Substitutionsindex, desto mehr gehen } \\
\text { die Umsatzerfolge einer Marke zu Lasten anderer } \\
\text { Marken im Portfolio. Zu vermeiden: Substitu- } \\
\text { tionseffekte entlang sinkender Deckungsbeitrăge. }\end{array}$ \\
\hline $\begin{array}{l}\text { Kannibalisierungs- } \\
\text { Index }\end{array}$ & $\begin{array}{l}\text { Ausmaß der portfoliointernen Wan- } \\
\text { derungsbewegungen, bei denen Nach- } \\
\text { frager von einer Portfoliomarke zur } \\
\text { anderen wechseln, dabei jedoch als } \\
\text { Alternativmarke die vorher besessene } \\
\text { angeben. }\end{array}$ & $\begin{array}{l}\text { Je höher der Kannibalisierungssindex, als desto aus- } \\
\text { tauschbarer werden die Portfoliomarken aus Sicht der } \\
\text { Konsumenten wahrgenommen. Folglich gilt: bei } \\
\text { hohem Kannibalisierungsindex kann eine Marke um } \\
\text { so eher aus dem Portfolio eliminiert werden, da als } \\
\text { Alternativmarken jeweils andere Portfoliomarken } \\
\text { genannt werden. }\end{array}$ \\
\hline $\begin{array}{l}\text { Loyalitäts- } \\
\text { Gewinn } \\
\text { (LG) }\end{array}$ & $\begin{array}{l}\text { Portfoliointerne Wanderungen minus } \\
\text { Kannibalisierung. }\end{array}$ & $\begin{array}{l}\text { Je höher der LG, desto effektiver ist die Mehrmarken- } \\
\text { strategie, da Markenwechsler nicht zu Fremdmarken } \\
\text { abwandern, sondern zur portfolioeigenen Marken. }\end{array}$ \\
\hline \multicolumn{3}{|c|}{ Einzelmarkenfokus } \\
\hline $\begin{array}{l}\text { Erwäger- } \\
\text { Rate } \\
\text { (ERW) }\end{array}$ & $\begin{array}{l}\text { Gibt an, inwieweit eine Marke von } \\
\text { Fremdmarkenbesitzern als Kaufalter- } \\
\text { native in Erwägung gezogenen wurde } \\
\text { (Second-Choice). }\end{array}$ & $\begin{array}{l}\text { Je höher die Erwăgerrate, desto höher der potenziell } \\
\text { mögliche, aber unrealisierte Absatz einer Marke; } \\
\text { dabei gilt: } \\
\text { - je höher die Erwägerraten zwischen den Portfo- } \\
\text { liomarken, desto austauschbarer werden diese } \\
\text { empfunden. } \\
\text { - Je höher die Erwägerraten zwischen den Portfo- } \\
\text { liomarken und Fremdmarken, desto höher erweist } \\
\text { sich der potenzielle Absatz des Portfolios. }\end{array}$ \\
\hline
\end{tabular}

Tab. 12: Zentrale Ex-Post-Kennzahlen des Mehrmarkencontrolling

Die bisherige Beurteilung des Portfolios erfolgte auf Basis quantitativer Größen des beobachtbaren Kaufverhaltens. ${ }^{387}$ Zur Erklärung des Kaufverhaltens bzw. zur Antizipation des zukünftigen Verhaltens bedarf es in einem weiteren Schritt einer Beurteilung des Portfolios aus vorökonomischer Perspektive. Dabei gilt es, die nachfragerseitige Wahrnehmung der Portfoliomarken (Akzeptanzkonzept) anhand von Indikatoren des nicht beobachtbaren Kaufverhaltens dem vom Hersteller intendierten Aussagenkonzept gegenüberzustellen, um hierdurch mögliche Positionierungsdefizite als Ursache realisierter oder zukünftiger negativer Wande-

387 Zur prognostischen Relevanz der eher vergangenheitsbezogenen Größen vgl. allg. Meffert, H., Marketing: Grundlagen marktorientierter Unternehmensführung: Konzepte - Instrumente Praxisbeispiele, a.a.O., S. 165. Wie in FN 381 erwähnt, ist es mit den gegebenen Angaben grundsätzlich möglich, das dynamische Käuferverhalten und damit die Marktentwicklung in der Zeit als endliche Markowsche Kette darzustellen. 
rungssalden zu bestimmen. Denn aus der Perspektive des Gesamtportfolios ermöglicht eine differenzierte Positionierung der einzelnen Portfoliomarken

- die mit der Mehrmarkenstrategie zwangsläufig entstehenden absatzmäßigen Verbundwirkungen zwischen den Marken, die auf ihr in der Regel substitutionales Verhältnis zurückzuführen sind, zu senken und damit hohe Substitutionseffekte im Portfolio weitgehend zu vermeiden,

- Markenwechsler innerhalb des Portfolios zu halten und mithin den Loyalitätsgewinn zu erhöhen,

- eine verstärkte Eroberung von Wettbewerbern bzw. Neukunden zu realisieren,

so dass Positionierungsentscheidungen in einem unmittelbaren Zusammenhang zum realisierten und zukünftigen markenspezifischen Absatz stehen. So wird etwa im Falle zweier Marken A und B durch eine differenzierte Positionierung der Marke $B$ gegenüber $A$ die Wahrscheinlichkeit erhöht, dass Käufer der Marke $B$ von Konkurrenten abgezogen werden (positiver Partizipationseffekt), so dass die Abwanderung von der Marke $A$ auf diese Weise begrenzt werden kann. ${ }^{388}$ Mit COPULSKY kann auch gesagt werden: „The lesson to be learned is that cannibalism results from too close identification of a [...] product with the launching company's older products and established markets. New appeals to new market segments will avoid eating one's own market share". ${ }^{389}$ Im Folgenden gilt es, die Markenpositionierung der betriebswirtschaftlichen Steuerung und Kontrolle zugänglich zu machen.

Vgl. Sander, M., Die Bestimmung und Steuerung des Wertes von Marken. Eine Analyse aus Sicht des Markeninhabers, a.a.O., S. 33.

Copulsky, W., Cannibalism in the Marketplace, a.a.O., S. 105. 


\subsection{Markenwahrnehmungsanalysen zur Aufdeckung von Profilierungs- und Differenzierungsdefiziten im Markenportfolio}

Während im Rahmen der strategischen Ausgestaltung der Mehrmarkenstrategie die angestrebte Positionierung der einzelnen Portfoliomarken festgelegt wird (Positionierungsstrategie), umfasst das Mehrmarkencontrolling die Analyse der realisierten Positionierung (Positionierungsanalyse). ${ }^{390}$ Um Positionierungsdefizite systematisch zu analysieren (Soll- versus Ist-Positionierung), sei im Folgenden das Grundkonzept der Gap-Analyse in Anlehnung an das aus dem Qualitätsmanagement bekannten Gap-Modell von PARASURAMAN/ZEITHAML/BERRY erläutert. ${ }^{391}$ Das GAP-Modell soll dabei im weiteren Verlauf der Arbeit als zentraler Analyserahmen zur Identifikation von Soll-Ist-Abweichungen bei der Positionierung fungieren.

\subsection{Das GAP-Modell der Markenidentität als konzeptioneller Bezugsrah- men der Markenwahrnehmungsanalyse}

Wie aufgezeigt, bedarf es zur Schaffung einer starken Markenidentität einer hohen Übereinstimmung zwischen dem internen Selbstbild und dem externen Fremdbild einer Marke. Für eine Analyse möglicher Positionierungsdefizite ist es daher zweckmäßig, sowohl das Selbstbild als auch das Fremdbild einer Marke in eine jeweilige Soll- und Ist-Komponente zu zerlegen:

- Das Soll-Selbstbild basiert auf der strategischen Rolle der Marke und beschreibt den Kern der anzustrebenden "Zielidentität".

- Das Ist-Selbstbild hingegen repräsentiert die tatsächliche Umsetzung der vorgegebenen Inhalte einer Marke in eine marktfähige Leistung.

390 Markenpositionierung beinhaltet somit grundsätzlich zwei Sichtweisen: Zum einen die für das Markenmanagement notwendige Abbildung des im Wettbewerb verbundenen Markensystems auf Basis subjektiver Wahrnehmungsurteile (Akzeptanzkonzeption, Positionierungsanalyse), zum anderen die Strategien und Maßnahmen des Markenmanagement, die zur gezielten Veränderung dieses Systems führen (Aussagekonzeption, Positionierungsstrategie). Grundlage hierfür ist die Informationsbasis der analytischen Komponente. Unter Positionierung kann folglich sowohl eine Analyse als auch eine Strategie verstanden werden. Im Zuge der Ausgestaltung der Mehrmarkenstrategie wurde Positionierung demnach als Strategiekonzept aufgefasst, im Folgenden steht im Rahmen des Mehrmarkencontrolling spiegelbildlich das Analysekonzept im Vordergrund.

391 Vgl. Parasuram, A., Zeithaml, V., Berry, L.L., A Conceptual Model of Service Quality and its Implications for Future Research, in: Journal of Marketing, Vol. 49, 1985, S. 41ff. Zum Grundgedanken der Übertragung auf Marken vgl. Meffert, H., Burmann, Ch., Identitätsorientierte Markenführung - Grundlagen für das Management von Markenportfolios, a.a.O., S. 62. 
- Während das Ist-Fremdbild schließlich die externe Wahrnehmung der tatsächlichen Marktleistung als Realimage umfasst,

- beinhaltet das Soll-Fremdbild das von den externen Anspruchsgruppen als ideal empfundene Image einer Marke.

Langfristig ist eine hohe Kongruenz zwischen dem Soll-Selbstbild und dem SollFremdbild anzustreben. Für das hier zur Analyse möglicher Positionierungsdefizite betrachtete GAP-Modell ergibt sich damit der in Abb. 36 dargestellte Aufbau, welcher eine Modifikation des bisher in der Literatur bestehenden GAP-Modells der Markenidentität vornimmt, indem das Soll-Selbstbild im Modell explizit ausgewiesen wird. ${ }^{392}$

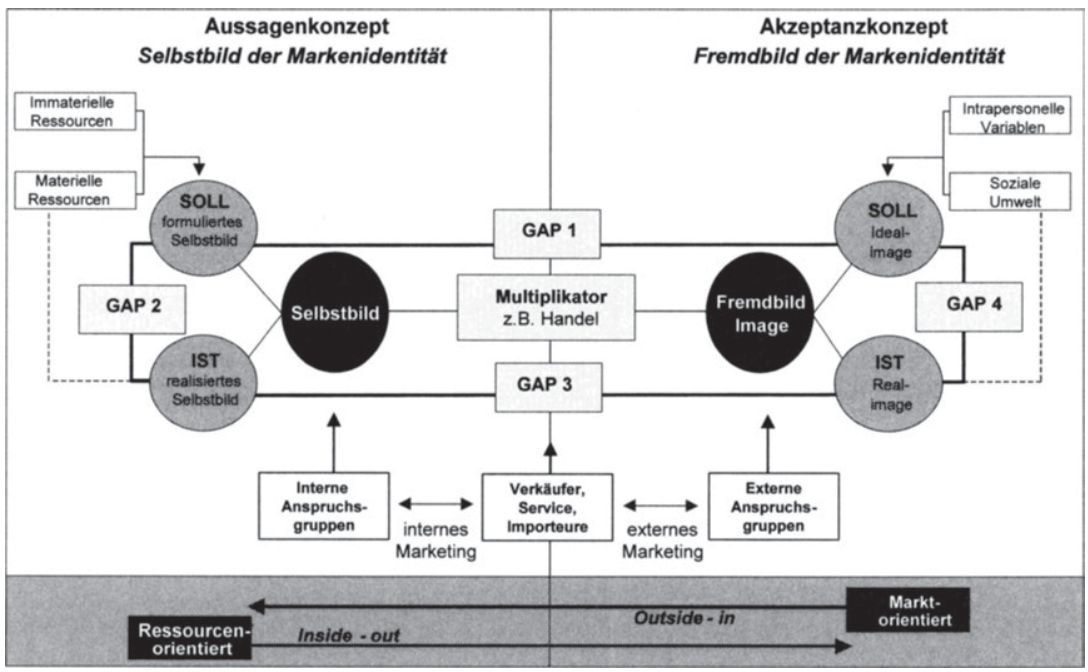

Abb. 36: GAP-Modell der Markenführung

GAP 1 kennzeichnet als Wahrnehmungsgap die Abweichung zwischen den Erwartungen der Konsumenten gegenüber einer Marke (Ideal-Image) und der WahrManagement von Markenportfolios, a.a.O., S. 60ff. Vgl. zu weiteren Anwendungen des GAPModells etwa Corsten, H., Stuhlmann, St., Das GAP-Modell als Orientierungsrahmen für ein Kapazitätsmanagement in Dienstleistungsunternehmungen, in: Kapazitătsmanagement in Dienstleistungsunternehmungen: Grundlagen und Gestaltungsmöglichkeiten, Corsten, $H$., Stuhlmann, St. (Hrsg.), Wiesbaden 1997, S. 11ff.; Bieger, Th., Dienstleistungsmanagement: Einführung in Strategien und Prozesse bei persönlichen Dienstleitungen, Bern, Stuttgart, Wien, 1998, S. $172 \mathrm{ff}$. 
nehmung dieser Erwartung auf Seiten des Markenmanagement als Grundlage zur Formulierung des Markenselbstbildes. ${ }^{393}$ Vielfach wird diese Abweichung als Ausgangspunkt der GAP-Analyse betrachtet. Ob allerdings das Idealimage der Konsumenten tatsächlich Ausgangspunkt der Betrachtung ist, hängt letztlich von der Art und Weise der Formulierung des Selbstbildes ab, d.h. in wie weit sich die Formulierung des Selbstbildes tatsächlich an den artikulierten Konsumentenwünschen orientiert oder aber primär von eigenen Erfahrungen, herstellerspezifischen Ressourcen bzw. Know-how dominiert wird ${ }^{394}$ Hier stellt sich somit die Frage, ob zur Erlangung eines KKV

- eine reaktive Positionierung verfolgt wird, bei der sich die Positionierungen insbesondere an den artikulierten Wünschen der Konsumenten orientieren (primär Outside-In-Betrachtung), oder

- eine aktive Positionierung vorgenommen wird, indem dem Kunden bisher unbekannte, für seine Kaufentscheidung aber wichtige Eigenschaftsdimensionen besetzt werden (primär Inside-Out-Betrachtung).

Wie aufgezeigt entsteht die Markenidentität erst durch eine wechselseitige Beziehung zwischen internen und externen Zielgruppen der Marke, so dass sie nicht isoliert aus der Perspektive des Marktes oder des Unternehmens betrachtet werden darf, sondern im Spannungsfeld zwischen dem Unternehmen und seiner Umwelt steht. Zur Sicherstellung einer starken Markenidentität besteht ungeachtet reaktiver oder aktiver Positionierung langfristig das Ziel einer Übereinstimmung zwischen dem Idealimage der Konsumenten und dem formulierten Selbstbild des Unternehmens und damit - wie bei allen GAPs - eine Schließung der Lücke. Vor diesem Hintergrund liefert die Identifikation der Ideal-Images sowohl für eine reak-

In Anlehnung an das "consumer expectation - management perception gap" bei Parasuram, A., ZeithamI, V., Berry, L.L., A Conceptual Model of Service Quality and its Implications for Future Research, a.a.O., S. 44.

In diesem Zusammenhang ist die Äußerung eines TOP-Managers eines großen, weltweit tätigen Automobilkonzerns zu verstehen: „Grundsätzlich gilt, dass die Manager und besonders die Top-Manager der einzelnen Marken noch am besten über die wünschbare und mögliche Zukunft der Marke Bescheid wissen und daher ein Soll-Image (verstanden als Soll-Selbstbild, Anm. d. Verf.) viel klarer und deutlicher festlegen können als dies auch gut informierten Kunden möglich wäre. [...] Es hätte zum Beispiel nie einen [das Modell X, Anm. d. Verf.] gegeben, wenn das Soll-Image vom Markt her bestimmt worden wäre." Und weiter: „Die Menschen sind eben nicht in der Lage zu artikulieren, was sie wirklich fasziniert. [...] Es gilt festzuhalten, dass große wirtschaftliche Erfolge häufig gegen große Widerstände durchgesetzt werden mussten und dass sie trotz eines großen Fehlschlag-Risikos zustande kamen. Hier gilt nach wie vor die Auffassung des österreichischen Nationalökonomen Schumpeter: „Der Markt ist immer schon voll, was neu hinzukommt, muss sich gegen die Logik des Marktes durchsetzen." 
tive als auch aktive Positionierung wichtige Anhaltspunkte zur Formulierung des Selbstbildes. Maßnahmen, die zur Schließung der Divergenz zwischen den von den Nachfragern artikulierten und vom Anbieter wahrgenommenen Erwartungen beitragen, konzentrieren sich dabei insbesondere auf die Marktforschung, um dem Markenhersteller die Wünsche und Bedürfnisse der Nachfrager transparent zu machen.

Eine Diskrepanz zwischen dem unter Beachtung der Kundenerwartung formulierten Selbstbild durch das Portfoliomanagement und seiner tatsächlichen Realisierung zeigt ein Umsetzungsgap (GAP 2). ${ }^{395}$ Dabei lassen sich im Grunde zwei getrennte Problembereiche identifizieren:

- Diskrepanz zwischen dem zentral formulierten Markenselbstbild (Planung) und seiner Spezifikation hinsichtlich bestimmter Markenmerkmale durch das dezentrale Markenmanagement (strategische Ebene). Beispielsweise ist die Spezifikation der im Selbstbild formulierten Merkmale „Sportlichkeit und Dynamik" in konkrete Markeneigenschaften hinsichtlich Höchstgeschwindigkeit, Beschleunigung, Fahrwerksabstimmung oder Innenausstattung etc. mit entsprechenden Schwierigkeiten verbunden.

- Diskrepanz zwischen den geplanten Markenmerkmalen und der tatsächlich erbrachten Markenleistungen auf operativer Ebene der jeweiligen Marken. So kann es trotz spezifizierter Markenmerkmale im Lastenheft eines Automobilherstellers in Bezug auf die Markenqualität zu Fahrzeugmängeln bei der Auslieferung kommen.

Bei den hier aufgeführten Abweichungen handelt es sich somit um unternehmensinterne Gaps. Ursachen für die aufgeführten GAPs sind insbesondere unzureichende Potenzialfaktoren, die in Form mangelnder Mitarbeiterqualifikation oder einer ungeeigneten unternehmensinternen Infrastruktur verhindern, dass formulierte Markenmerkmale eingehalten werden können. Zur erfolgreichen Umsetzung des formulierten Selbstbildes in eine tatsächliche Marktleistung sind neben der Sicherstellung einer geeigneten Infrastruktur bestimmte Voraussetzungen bei den Mitarbeitern der jeweiligen Markenorganisationen zu erfüllen. So ist die Kenntnis ("Kennen“) und das "Verstehen“ des Soll-Selbstbildes zu gewährleisten und die Betroffenen sind mit der entsprechenden Kompetenz ("Können") auszustatten. Überdies ist mit der Akzeptanz ("Wollen") des Selbstbildes bei den internen An- 
spruchsgruppen die Voraussetzung für die Leistungs- und Einsatzbereitschaft der Mitarbeiter zu erreichen. ${ }^{396}$

GAP 3 des Modells bezieht sich auf die Abweichung zwischen der tatsächlich erstellten und der in der marktgerichteten Kommunikation versprochenen Leistung des Herstellers. ${ }^{397}$ So kann beispielsweise die besondere Wirtschaftlichkeit als wesentliches Kennzeichen der Marke herausgestellt werden, ohne dass der Kunde dies bei seinem tatsächlichen Benzinverbrauch oder den Ersatzteilpreisen nachvollziehen kann. Ein Kommunikationsgap tritt somit im Rahmen des externen Marketing dann auf, wenn Erwartungen bei dem Kunden aufgebaut werden, die später vom Markenprodukt aus Sicht des Konsumenten nicht erfüllt werden.

Das letzte GAP kennzeichnet schließlich eine Abweichung zwischen dem IdealMarkenimage und dem tatsächlichen Markenimage und entsteht aus der subjektiven Bewertung der Marke aus Sicht der externen Anspruchsgruppen. Ursache für ein Identifikationsgap sind entweder die GAPS 1-3, also GAP $4=f(G a p 1$, Gap2, Gap3), oder der Nachfrager ist nicht in der Lage, die tatsächliche Eignung der Marke zu beurteilen. ${ }^{398}$ Existiert eine solches GAP, wobei auch eine positive Lükke möglich ist, d.h. die Erwartungen des Nachfragers werden übertroffen, so ist zu unterscheiden, ob die Abweichung noch im Toleranzbereich des Nachfragers liegt oder ob er diese Diskrepanz als unakzeptabel einstuft. Ein GAP zwischen Ist- und Soll-Image führt letztlich wiederum zu einer Anpassung der zukünftigen Erwartungen der Nachfrager, wodurch der dynamische Charakter des GAP-Modells verdeutlicht werden kann.

Neben sonstigen Elementen der Prozesskette zwischen Hersteller und Kunden kann insbesondere der Automobilhandel als „Multiplikator" möglicher GAPs zwischen dem Selbstbild des Herstellers und dem Fremdbild externer Anspruchsgruppen interpretiert werden (mangelnde Realisierung des Markenleitbildes über

396 Vgl. hier auch Kolks, V., Strategieimplementierung: Ein anwendungsorientiertes Konzept, Wiesbaden 1990, S. $110 \mathrm{ff}$.

397 In Anlehnung an das "expected service - perceived service gap" bei Parasuram, A., Zeithaml, V., Berry, L.L., A Conceptual Model of Service Quality and its Implications for Future Research, a.a.O., S. 46; vgl. hier auch Meffert, H., Burmann, Ch., Identitätsorientierte Markenführung - Grundlagen für das Management von Markenportfolios, a.a.O., S. 64.

Vgl. Parasuram, A., ZeithamI, V., Berry, L.L., A Conceptual Model of Service Quality and its Implications for Future Research, a.a.O., S. 45. 
die gesamte Prozesskette). ${ }^{399}$ Zum einen können unterschiedliche Auffassungen von Hersteller und Händler über die Ausprägung der die Markenidentität beeinflussenden Produkt- und POS-Merkmale existieren, worin sich letztlich eine mangelhafte Verankerung der Markenidentität nach innen ausdrückt. Zum anderen beeinflussen die Vertragshändler aufgrund ihrer hohen Bedeutung in der Automobilbranche unmittelbar das Fremdbild der externen Anspruchsgruppen durch konkrete POS-Standards, erbrachte Serviceleistungen vor und nach dem Kauf oder aber die handelseigene Markenkommunikation.

Wie bereits bei der Ermittlung des Controllingbedarfs aufgezeigt, besteht in Mehrmarkenstrategien die Herausforderung nicht nur darin, das Spannungsfeld zwischen dem Selbst- und Fremdbild der Identität jeder Marke zu überbrücken, sondern darüber hinaus auch die verschiedenen Markenidentitäten im Portfolio untereinander im nationalen und internationalen Kontext abzustimmen. Zur innen- und außengerichteten Festigung der Markenidentität und damit zur Schließung möglicher Gaps nehmen Markenleitbilder eine zentrale Bedeutung im Rahmen des internen und externen Marketing ein. Markenleitbilder mögen in ihrer Charakterisierung jedoch noch so eindeutig sein, für den weiteren Fortgang ist ein Mess- und Kontrollinstrumentarium efforderlich, mit dem geprüft werden kann, wie weit die Realimages von der innen zugewiesenen Soll-Position entfernt sind und ob die Bewegung in die gewünschte Richtung verläuft. Das GAP-Modell erscheint dabei zum einen für eine Identifikation und Strukturierung möglicher Leistungsfähigkeitsdefizite und zum anderen als Ansatzpunkt zur Formulierung konkreter Gestaltungsempfehlungen geeignet. Hierzu sei im Folgenden auf Basis des vorgestellten Bezugsrahmens in folgenden Schritten vorgegangen:

- In einem ersten Schritt gilt es zunächst, die Fremdbilder der einzelnen Portfoliomarken als realisierte Ist-Positionierungen zu analysieren. Denn um das Ausmaß der Substitution in einem Markenportfolio abschätzen zu können, empfiehlt auch FRETER, die von den Nachfragern wahrgenommene Ähnlichkeit der unternehmenseigenen Marken zu prüfen, welche sich graphisch mit Hilfe eines auf Konsumentenurteilen basierenden Positionierungsmodells verdeutlichen lässt. ${ }^{400}$

Da der Handel zum einen als innerorganisationale Institution des Herstellers interpretiert wer-

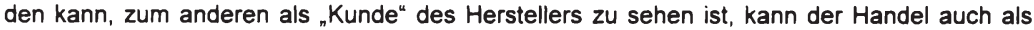
Bindeglied zwischen Aussage- und Akzeptanzkonzept zwischen Hersteller und Endkunde verstanden werden.

Vgl. Freter, H., Marktsegmentierung, a.a.O., S. 122 
- Darauf aufbauend sind in einem zweiten Schritt die Selbstbilder der Marken im Sinne der angestrebten Soll-Positionierung in die Analyse zu integrieren, um etwaige GAPs zwischen den einzelnen Selbst- und Fremdbildern zu identifizieren. Dabei lassen sich Gesamtabweichungen wiederum mit Hilfe von Positionierungsmodellen verdeutlichen. Zur Identifikation möglicher Abweichungsursachen gilt es sodann, mögliche interne und externe GAPs weiter aufzuspalten.

\subsection{Analyse der marktseitigen Markenwahrnehmung: Outside-In- Betrachtung}

Um zunächst die marktseitige Wahrnehmung der Marken zur Bestimmung der portfoliointernen Substitution zu erfassen, bietet es sich an, auf klassische Positionierungsmodelle zurückzugreifen. Positionierungsmodelle verkörpern die zwischen einzelnen Marken bestehenden Relationen, die sich in Form von Ähnlichkeiten, Präferenzen und Austauschbeziehungen konkretisieren lassen. Um die Struktur aus Marktobjekten und Relationen zu erfassen, verwendet man das Konzept der räumlichen Nähe. Dabei werden die Marken mit Hilfe mathematischer Verfahren derart in einem zwei- oder mehrdimensionalen Raum abgebildet, dass diese um so näher beieinander liegen, je ähnlicher sie wahrgenommen werden. Positionierungsansätze verknüpfen die Markenwahrnehmung somit mit einer Verhaltensrelevanz: Eine geringe Distanz zwischen zwei Marken im Wahrnehmungsraum deutet darauf hin, dass diese als austauschbar empfunden werden et vice versa. In einer solchen Situation ist aufgrund der gegenseitigen $\mathrm{Be}$ einträchtigung der Einzugsgebiete durch zu starke Überlappungen mit einer wechselseitig hohen Substitution zu rechnen. Gleiche oder ähnliche Markenwahrnehmungen, die zu Substitutionseffekten führen können, müssen daher im Rahmen des Mehrmarkencontrolling aufgedeckt und analysiert werden, um darauf aufbauend entsprechende Gegenmaßnahmen (z. B. Umpositionierungen) ergreifen zu können.

Grundsätzlich lassen sich zur Analyse der Ist-Positionierung direkte bzw. komponierende und indirekte bzw. dekomponierende Verfahren unterscheiden. Ziel beider Verfahren ist es, grundlegende Merkmalsdimensionen aufzudecken, anhand derer Konsumenten die Marken wahrnehmen und beurteilen.

- Bei direkten Verfahren werden Einzelbewertungen explizit vorgegebener Markeneigenschaften zu Imagedimensionen verdichtet.

- Bei indirekten Verfahren werden zunächst Globalurteile wie Ähnlichkeiten oder Präferenzrangfolgen der Marken erhoben, die dann in die dimensionalen Beiträge einzelner Merkmale zum Globalurteil zerlegt werden. 


\subsection{Analyse auf Basis von Einzelurteilen}

Bei der direkten Positionierung mittels vorgegebener Eigenschaften hängt der Aussagewert der sich ergebenden Markenkonfiguration von der Auswahl geeigneter Eigenschaften und insbesondere von der richtigen Skalierung ab. ${ }^{401}$ In einem ersten Schritt sind somit Imagedimensionen zu generieren, welche bestimmte Anforderungen zu erfüllen haben: ${ }^{402}$

- Verhaltensrelevanz: die selektierten Markeneigenschaften sollen Markenpräferenzen und Kaufverhalten beeinflussen ${ }^{403}$

- Instrumentalbezug: die Wahrnehmung der Markeneigenschaften soll durch Marketinginstrumente gut beeinflusst werden können und

- Diskriminanzfähigkeit: die Eigen- und Fremdmarken sollen nach diesen Merkmalen subjektiv unterscheidbar sein.

Sind relevante Eigenschaften bestimmt worden, so haben Konsumenten die wahrgenommen Eigenschaftsausprägungen der einzelnen Marken auf vorgelegten Ratingskalen direkt anzugeben. Durch individuell zugeordnete Werte in den einzelnen Eigenschaften ergibt sich die individuell wahrgenommene Position einer jeden Marke. ${ }^{404}$ Die wahrgenommenen Ausprägungen der relevanten Merkmale können mittels Mittelwertbildung über alle Befragten der Zielgruppe als Profil abgebildet werden, so dass Profilvergleiche je Merkmal die Positionierungsunter-

Zum formalen Aufbau von Ratingskalen sowie zur semantischen Steuerung von Skalenbezeichnungen vgl. Trommsdorff, V., Die Messung von Produktimages für das Marketing. Grundlagen und Operationalisierung, Köln u. a. 1975, S. 81ff; Andritzky, K., Der Zusammenhang zwischen Skalenbreite und Urteilsverhalten, in: Der Marktforscher, Heft 3, 1976, S. $60 f f$.

Zur Ermittlung positionierungsrelevanter Dimensionen kann kundenorientiert (Welche Dimensionen sind in den Köpfen?) oder wettbewerbsorientiert vorgegangen werden (Wie profiliert sich die Konkurrenz?). Vgl. Trommsdorff, V., Paulssen, M., Messung und Gestaltung der Markenpositionierung, in: Moderne Markenführung: Grundlagen - innovative Ansätze - praktische Umsetzung, Esch, F.-R. (Hrsg.), Wiesbaden 1999, S. 1074.

SHOCKER, SRINIVASAN sprechen von "want satisfiers"; vgl. Shocker, A. D., Srinivasan, V., A Consumer-Based Methodology for the Identification of New Product Ideas, in: Management Science, Vol. 20, No. 6, 1974, S. 922.

Mit FRETER kann die Gegebenheit, dass die Ausprägung einer Marke in den einzelnen Eigenschaften nicht genau bekannt ist bzw. dass der Konsument nur ungenaue Vorstellungen besitzt, dadurch berücksichtigt werden, dass von den Konsumenten pro Marke und Eigenschaft eine subjektive Wahrscheinlichkeitsverteilung (Glaubwürdigkeitsziffern) bezüglich der Eigenschaftsausprägungen angegeben wird. Vgl. Freter, H., Markenpositionierung. Ein Beitrag zur Fundierung markenpolitischer Entscheidungen auf der Grundlage psychologischer und ökonomischer Modelle, a.a.O., S. 104. 
schiede der Marken indizieren. ${ }^{405}$ Abb. 37 zeigt die Imageprofile der hier betrachteten vier Marken. ${ }^{406}$

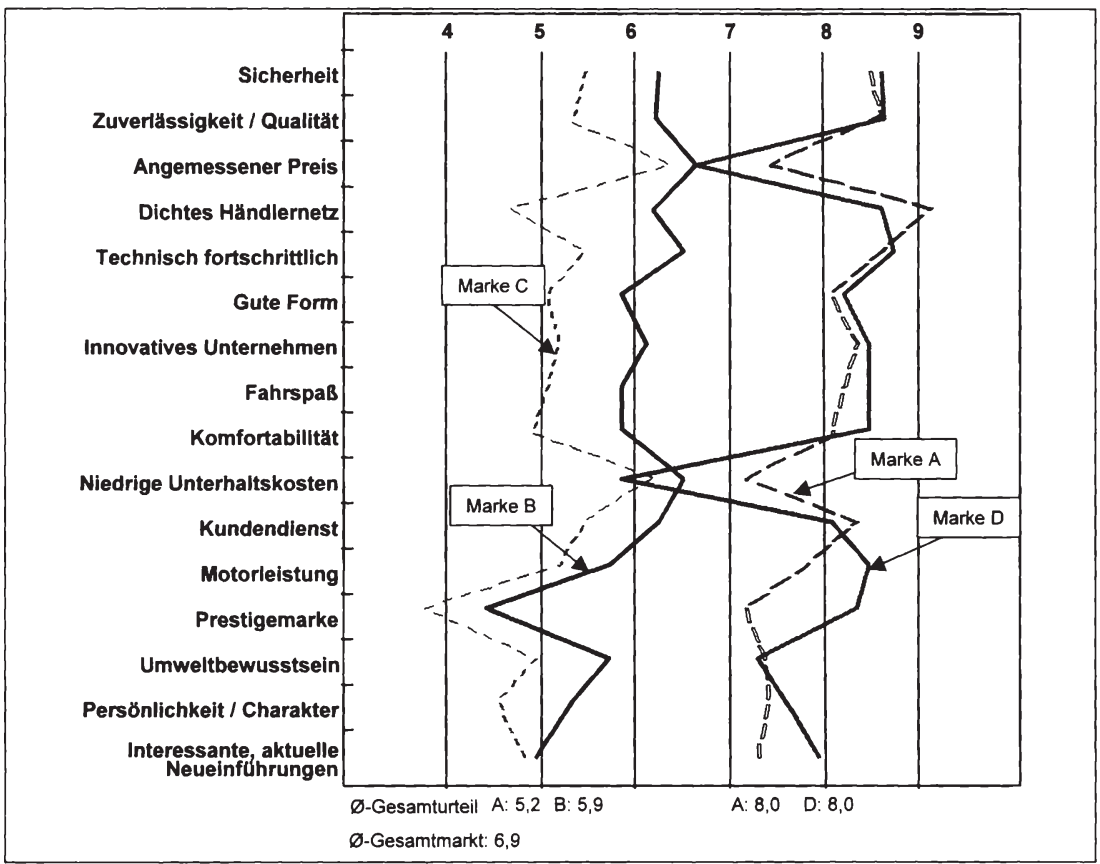

Abb. 37: Mehrdimensionale Imageanalyse

Die dargestellten Markenprofile zeigen kaum mehr als eine Zweiteilung im Markenportfolio: während die Marken $A$ und $D$ über ein vergleichsweise hohes Image verfügen, wird den Marken $B$ und $C$ ein eher (unter-)durchschnittliches Image beigemessen (Marktdurchschnitt $=6,9$ ). Die Marken $A$ und $D$ liegen auf fast gleichem Niveau mit einem Vorsprung bei der Marke D in typischen Merkmalen einer Pre-

Einen Überblick über die möglichen methodischen Varianten, verhaltenstheoretischen Modelle und auftretenden Messprobleme gibt Trommsdorff, V., Positionierung, in: Handwörterbuch des Marketing, 2. Aufl., Tietz, B, Köhler, R., Zentes, J. (Hrsg.), Stuttgart 1995, Sp. 2055ff.

Mittels mündlicher Befragung im März/ April 1998 in Deutschland wurden private Pkw Neuwagenkäufer der letzten fünf Jahre gebeten, die genannten Items für die jeweiligen Marken auf einer Skala von 1 (schlechteste Note) und 10 (beste Note) auf vorgegebenen Karten zu beurteilen. Basis aller Befragten: 2607. 
miummarke. Marke $C$ liegt auf unterem Niveau mit positiven Wertungen nur beim Preis. Das Image-Profil der Marke B liegt auf mittlerem Niveau und hat im Verlauf Ähnlichkeiten mit dem Profil der Marke C.

Das Ausmaß, in dem sich eine Marke über alle Eigenschaften hinweg in der Wahrnehmung der Konsumenten gegenüber dem Marktdurchschnitt (auf Modellebene auch Segmentdurchschnitt) differenzieren kann, ist mit einem Profilierungsindex operationalisierbar. Dieser ergibt sich durch die über alle Dimensionen $\mathrm{j}$ aufsummierten Distanzen zwischen dem Realimage der jeweiligen Marke i und den durchschnittlichen Ausprägungen aller relevanten Marken des Marktes (bzw. des Segments):

$$
\begin{aligned}
& P I_{i}=\sum_{i=1}^{n}\left|e_{j i}-\bar{e}_{j}\right| \\
& \text { Ausprägung einer Eigenschaft } \\
& \text { Eigenschaftsindex } \\
& \text { Markenindex }
\end{aligned}
$$

Je höher sich der Profilierungsindex erweist, desto profilierter und damit konturierter stellt sich die Marke im (durchschnittlichen) Wettbewerbsumfeld dar. Der wahre KKV der einzelnen Marken wird allerdings über so ermittelte Profile bzw. Indizes nur unzureichend deutlich, da nichts darüber ausgesagt wird, ob und wie sich diese Positionierung als Wettbewerbsvorteil gegenüber welchen anderen Marken auswirkt.

Zur Erhöhung der Aussagefähigkeit könnten theoretisch mit Hilfe einer Faktorenanalyse aus der Vielzahl der zugrundegelegten Items die wesentlichen, voneinander unabhängigen Faktoren extrahiert werden, um die in den Items enthaltenen Überschneidungen zu eliminieren. ${ }^{407}$ Durch eine Verdichtung der zentral abgefragten Merkmale auf zentrale Imagedimensionen lässt sich ein Wahrnehmungsraum erzeugen, so dass die betrachteten Marken als Positionen im gemeinsamen Raum dargestellt werden können. Hierdurch lässt sich ein Vergleich auf jeder Imagedimension mit den Eigen- und Fremdmarken vornehmen (ImageBenchmarking).

407 Dabei können alle Items auf alle Faktoren laden. Die Zusammenfassung zu Faktoren ergibt sich aus ihrer empirischen Korrelation, womit die explorative Faktorenanalyse strukturentdekkenden Charakter hat. Sie geht davon aus, dass Korrelationen zwischen Items auf hinter diesen stehende Items zurückgeführt werden können. 
Allerdings weisen BAUER/HANNIG explizit darauf hin, dass die Frage, ob ein Markenraum auch einen Wettbewerbsraum im Sinne mehr oder weniger substitutiver Marktobjekte verkörpert, von der Art der verwendeten Relationen abhängt. ${ }^{408}$ Physikalisch-technische Merkmale von Automobilen zeigen beispielsweise nur das Ausmaß ihrer diesbezüglichen Gleichartigkeit an, ohne dass sich daraus zwingend Wettbewerbsbeziehungen ableiten lassen. Denn eine solchermaßen festgestellte Ähnlichkeit impliziert zwar eine Austauschbarkeit der Marken, jedoch können Marken auch trotz physikalisch-technischer Unähnlichkeit austauschbar sein, wenn sie den gleichen Nutzen besitzen. Die hier theoretisch zu extrahierenden Imagedimensionen mittels Faktorenanalyse erlauben damit keine Aussagen darüber, wie stark sie den Kauf oder Nichtkauf einer Marke tatsächlich beeinflussen.

Als weitere Kritik weisen TROMMSDORFF/PAULSSEN daraufhin, dass klassische Positionierungsstudien formal zwar Fremdmarken mit einbeziehen, dass aber über globale Wettbewerbsrelationen hinaus häufig nicht bekannt ist, welche Beziehungen zwischen bestimmten Wettbewerberimage-Dimensionen bestehen und wie sie zur Stärkung der eigenen Wettbewerbsposition verändert werden können. ${ }^{409}$ Überdies unterstellen klassische Image-Wettbewerbserfassungen, dass eine Imagedimension bei allen Wettbewerbern die gleiche Bedeutung hat. Auch werden pauschale Imagewirkungen zwischen Wettbewerbern unterstellt, indem Merkmale einer Marke nur die eigene Position bestimmen, aber nicht deren Wirkung auf den Erfolg oder Misserfolg von Fremdmarken abbilden.

Zur Ermittlung der Ähnlichkeiten der hier untersuchten Automobilmarken empfiehlt es sich vor dem dargestellten Hintergrund, auf die subjektiv empfundene Austauschbarkeit der Marken aus Sicht der Konsumenten abzustellen. Entsprechend soll die Positionierungsanalyse im Folgenden nicht auf Basis der den Imageprofilen zugrunde gelegten Einzelitems erfolgen, sondern mit Hilfe von Erwägerraten

Entsprechend stellen BAUER/HANNIG bei der Konstruktion von Marktmodellen mit dem Ziel, die Substitutionsbeziehungen eines neuen Erzeugnisses zu bereits eingeführten Erzeugnissen aufzudecken, auf die Eignung der Marktobjekte für die Erfüllung verschiedener Verwendungszwecke ab. Vgl. Bauer, H. H., Hannig, U., Die Erfassung der Substitutionswirkung eines neuartigen Elektrowerkzeuges für den Heimwerker-Markt, a.a.O., S. $5 \mathrm{ff}$.

Als Alternative nennen sie die Wettbewerbs-Image-Struktur-Analyse (WISA) als Analyse vernetzter Wettbewerbsverhältnisse. Vgl. Trommsdorff, V., Paulssen, M., Messung und Gestaltung der Markenpositionierung, in: Moderne Markenführung: Grundlagen - innovative Ansätze - praktische Umsetzung, a.a.O., S. 1078. 
als Maß subjektiv empfundener Austauschbarkeit und Bedürfnisbefriedigung bzw. gleich wahrgenommenen Nutzens und damit auf Basis von Globalurteilen. ${ }^{410}$

\subsection{Analyse auf Basis von Globalurteilen}

Zur Bestimmung von Ähnlichkeitsbeziehungen im betrachteten Markenportfolio eines Automobilherstellers bietet es sich an, auf die bereits diskutierten Erwägerraten zurückzugreifen. ${ }^{411}$ Hierdurch kann zum einen dem gestellten Primat der Wirtschaftlichkeit der Informationsbeschaffung im Rahmen eines Mehrmarkencontrolling Rechnung getragen werden, da die Daten regelmäßig erhoben werden. Noch wichtiger aber wird zum anderen der Anforderung genüge getan, einen angemessenen Situationsbezug vorzunehmen. So postulieren auch DAY/SHOCKER/SRIVASTAVA: „A useful variant of the free-response question asks respondents what they would do if they were unable to buy their preferred brand. One advantage of this question is that it can realistically be tailored to specific situations [Hervorhebung d. Verf.]. For example, one study asked scotch drinkers what they would do if scotch were not available in a variety of situations [...]. Evidently, there were some situations where white wine was the preferred alternative. ${ }^{412}$

Die ermittelten Erwägerraten in der Automobilindustrie spiegeln genau die von DAY/SHOCKER/SRIVASTAVA als useful variant bezeichnete Frage wider, da hier Konsumenten kurze Zeit nach ihrem Kauf nach der Alternativmarke befragt werden. Aufgrund der direkten Frage der Konsumenten nach der Kaufalternative besitzt die Messung der Alternativerwägung im Vergleich zur Abfrage der wahrge-

410 Auf die subjektive Substituierbarkeit rekurriert das Konzept der verwenderorientierten subjektiven Austauschbarkeit, das auch als Konzept des ${ }_{n}$ evoked set" bezeichnet wird. Ein erweiterter Ansatz hierfür kann im Substitution-in-use-Ansatz gesehen werden, der auf die Erkenntnis abstellt, dass die Austauschbarkeit von Produkten nur unter Berücksichtigung einer spezifischen Verwendungssituation erfasst werden kann. Entsprechend sind es nicht ähnliche, den Produkten inhärente Nutzenkomponenten, die deren Austauschbarkeit bedingen, sondern der in einer bestimmten Verwendungssituation vom Nachfrager gewünschte Nutzen. Vgl. Srivastava, R. K., Alpert, M. J., Shocker, A. D., A Customer-oriented Approach for Determining Market Structures, in: Journal of Marketing, Vol. 48., Heft 2, S. 32ff.

411 Eine andere Möglichkeit wäre mit Hilfe der Wanderungsstruktur bzw. der Vorbesitzstruktur gegeben. Als Ergebnis erhielte man eine Häufigkeitsmatrix auf Basis von tatsächlichen Wanderungen.

412 Day, S. D., Shocker, A. D., Srivastava, R. K., Customer-Oriented Approaches To Identifying Product-Markets, in: Journal of Marketing, Vol. 43, Fall 1979, S. 15. 
nommenen Ähnlichkeiten (z. B. Paarvergleiche) $)^{413}$ somit eine höhere Kaufverhaltensrelevanz. So auch TROMmSDORFF/ZELLERHOFF: „Die Substituierbarkeit sagt etwas über die relative Attraktivität der Marken aus. Damit ist diese Methode noch näher am Markenwahlverhalten orientiert als es schon die Präferenzdatenmethodik gegenüber der Ähnlichkeitsdatenmethodik ist. “414

In der Datenmatrix, die man mit Hilfe der Erwägerraten erhält, sind die paarweisen absoluten Häufigkeiten der Nennungen abgetragen, die eine Marke als Substitution einer anderen sieht. Tab. 13 gibt einen Ausschnitt der dieser Untersuchung zugrundeliegenden Erwägermatrix wieder, in der die vier betrachteten Marken A-D sowie weitere Fremdmarken abgetragen sind. ${ }^{415}$

\begin{tabular}{|c|c|c|c|c|c|c|c|}
\hline Second-Choice ${ }^{\text {First-Choice }}$ & A & B & C & D & e & f & $\ldots$ \\
\hline Portfoliomarke A & 1.660 & 1.162 & 200 & 600 & 600 & 600 & $\ldots$ \\
\hline Portfoliomarke B & 237 & 555 & 64 & 3 & 3 & 3 & $\ldots$ \\
\hline Portfoliomarke C & 7 & 27 & 71 & 1 & 1 & 1 & $\ldots$ \\
\hline Portfoliomarke D & 1.183 & 164 & 17 & 531 & 531 & 531 & $\ldots$ \\
\hline Fremdmarke e & 74 & 65 & 5 & 32 & 382 & 68 & $\ldots$ \\
\hline Fremdmarke f & 398 & 41 & 4 & 1.077 & 405 & 1.340 & $\ldots$ \\
\hline$\ldots$ & $\ldots$ & $\ldots$ & & $\ldots$ & & $\ldots$ & ... \\
\hline
\end{tabular}

Tab. 13: Ausschnitt aus der Erwägermatrix

Die Spalten repräsentieren die tatsächlich erworbenen Marken (First-Choice-Car), die Zeilen die angegebenen Alternativerwägungen (Second-Choice-Car). Da zur Illustration 19 Marken in die Analyse einbezogen wurden, ergibt sich insgesamt

413 Grundsätzlich lassen sich hierzu Global- und Partialmodelle unterscheiden. Während in Partialmodellen Probanden hinsichtlich einzelner Eigenschaften der Objekte befragt werden, durch deren Aggregation dann Ähnlichkeitsbeziehungen ermittelt werden, erfolgt im Rahmen von Globalmodellen eine Beurteilung von Objekten in ihrer Gesamtheit. In der Literatur werden zahlreiche Methoden diesbezüglich vorgestellt (z. B. Rangreihung, Ankerpunktmethode, Ratingmethode). Vgl. Backhaus, K. et al., Multivariate Analysemethoden, 8. Aufl., Berlin u.a. 1996, S. 439.

414 Trommsdorff, V., Zellerhoff, C., Produkt- und Markenpositionierung, in: Handwörterbuch Markenartikel, Bruhn, M. (Hrsg.), Band 1, Stuttgart 1994, S. 358.

415 Die vollständige Matrix befindet sich im Anhang I. Die Erwägerraten lassen sich mittels Kreuztabellierung aus der NCBS-Studie EURO 5 errechnen. Hier wurden 140.000 Neuwagenkäufer schriftlich befragt, die ihr Fahrzeug ca. 4-6 Monate im Besitz hatten. 
eine 19X19-Matrix ${ }^{416}$, deren Verwendung als Dateninput für multivariate Analysemethoden dient. ${ }^{417}$

Da durch Erwägerraten globale Urteile hinsichtlich Austauschbeziehungen zwischen den Marken dargestellt werden, ohne dabei einzelne Dimensionen zu betrachten (wie z. B. Sicherheit des Fahrzeugs) und damit das empfundene Ausmaß der Ähnlichkeit nicht begründet wird, bietet sich insbesondere die Multidimensionale Skalierung als Analyseinstrument an. ${ }^{418}$ Die Verwendbarkeit der ermittelten Häufigkeiten als Ähnlichkeitsmaß für das Verfahren der Multidimensionalen Skalierung ist dabei grundsätzlich unstrittig. ${ }^{419}$

416 Grundsätzlich liegen die Daten auch auf Modellebene vor. Das hier verwendete Aggregationsniveau erscheint jedoch zweckmäßig, da erstens mit Blick auf die Zielsetzung der Arbeit primär Ähnlichkeiten der Dachmarken festzustellen sind und zweitens für eine detaillierte Untersuchung nicht ausreichend hohe Fallzahlen vorliegen. Bei der Analyse von Produktmarken wie $z$. B. Golf etc. entsteht ein wesentlich umfangreichere Matrix, so dass durch den Anstieg der Anzahl der Paarbeziehungen, für die keine Werte vorliegen, die Datenanalyse mit gängigen Statistikprogrammen nicht mehr möglich ist bzw. die Genauigkeit der Ergebnisse sehr stark abnimmt.

Vgl. Bauer, H. H., Marktabgrenzung, Berlin 1989, S. 136; Shocker, A. D., Srivastava, R. K., Customer-Oriented Approaches To Identifying Product-Markets, a.a.O., S. 15; Müller, W., Strategische Marktforschung - dargestellt am Beispiel der Automobilindustrie, in: Wettbewerbsvorteile erkennen und sichern: Erfahrungsberichte aus der Marketingpraxis, Neuwied 1994, S. 47.

418 Grundsätzlich sind fast alle multivariaten Analyseverfahren bei der Markenpositionierung nützlich. So werden mit der Clusteranalyse Zielkunden oder Marken zusammengefaßt, mit der Faktorenanalyse können Imageitems zu Dimensionen verdichtet werden, mit der Reggressionsanalyse können Beziehungen zwischen Image- und Einstellungs- oder Verhaltenswerten quantifiziert werden, die Conjointanalyse kann zur experimentellen Untersuchung der Beziehung zwischen Imageausprägungen und Einstellungs- oder Präferenzwerten genutzt werden u.s.w. Vgl. Trommsdorf, V., Zellerhoff, C., Produkt- und Markenpositionierung, a.a.O., S. $360 \mathrm{ff}$

419 Vgl. Trommsdorff, V., Zellerhoff, C., Produkt- und Markenpositionierung, a.a.O., S. 357; Day, S. D., Shocker, A. D., Srivastava, R. K., Customer-Oriented Approaches To Identifying Product-Markets, a.a.O., S. 15; Dichtl, E., Schobert, R., Grundzüge der Multidimensionalen Skalierung, in: Multidimensionale Skalierung: Methodische Grundlagen und betriebswirtschaftliche Anwendungen, München 1979, S. 6.

Kritisch zu bemerken ist hingegen die Skalenaufwertung, da die Daten wie metrisch skalierte Werte behandelt werden, obwohl der Dateninput aus nominal skalierten Werten besteht (der Proband gibt nur die beiden Zustände Alternativerwägung - Nicht Alternativerwägung an), d.h. die Probanden haben jeweils nur eine Beziehung zwischen zwei Marken hergestellt. Auch gibt das Aggregationsniveau der Untersuchung Anlass zur Kritik, da die Probanden als Erwägung andere Varianten einer Produktmarke nennen können (Golf 3-Türer versus Golf 5-Türer). Die verwendete Matrix enthält somit einen bestimmten Anteil an Kannibalisierung, d.h. Erwägungen innerhalb derselben Marke. Diese Substitutionen werden bei der Berechnung der Distanzmatrix allerdings nur im Zuge der Standardisierung beachtet, nicht aber bei der Berech- 
Ziel der Multidimensionalen Skalierung (MDS) ist es, aus Ähnlichkeits-, Präferenz oder Substitutionsdaten einen möglichst nur zwei- oder dreidimensionalen Positionierungsraum aufzuspannen, in dem Objekte (z. B. Marken) so abgebildet werden, dass die geometrische Nähe (Distanzen) die von den Befragten wahrgenommene Ähnlichkeit der Objekte wiedergibt. ${ }^{420}$ Die Rangfolge der Distanzen entspricht somit der Rangfolge der ermittelten Ähnlichkeiten. ${ }^{421}$

Zur Lösung der Aufgabe, eine Konfiguration zu erzeugen, bei der die Rangfolge der Distanzen die Rangfolge der Ähnlichkeiten zwischen den Marken widerspiegelt, bedient sich der MDS-Algorithmus eines iterativen Vorgehens. ${ }^{422}$ Auf Basis einer willkürlich gewählten Startkonfiguration wird versucht, die Markenkonfiguration so lange zu verbessern, bis die geforderte Übereinstimmung der Rangfolgen erfüllt ist. Hierzu werden mittels eines geeigneten Maßes ${ }^{423}$ die Distanzen der Markenpaare [ $d_{k l}$ (für Marken $k$ und $I$ )] aus den Ähnlichkeiten bzw. Unähnlichkeiten der Marken [ $\left.u_{k l}\right]$ berechnet. ${ }^{424}$ Als Maß für die Güte einer Konfiguration und damit als Zielkriterium für deren Optimierung dient das sog. STRESS-Maß. ${ }^{425} \mathrm{Je}$ größer

nung der eigentlichen Distanzen. Überdies können bei der Überführung der asymmetrischen Ausgangsmatrix in eine symmetrische Ähnlichkeitsmatrix zum einen wichtige Informationen verlorengehen, zum anderen besteht die Gefahr, dass die berechnete symmetrische Matrix die tatsächliche Beziehung der Objekte zueinander nicht mehr exakt beschreiben kann.

Vgl. Dichtl, E., Schobert, R., Grundzüge der Multidimensionalen Skalierung, a.a.O., S. $1 \mathrm{ff}$

Vgl. Nommensen, J. N., Die Prägnanz von Markenbildern, Reihe Konsum und Verhalten, a.a.O. Esch, F.-R., Kontrolle der Eigenständigkeit von Markenauftritten, in: Moderne Markenführung: Grundlagen - innovative Ansätze - praktische Umsetzung, Esch, F.-R. (Hrsg.), Wiesbaden 1999, S. 1057.

Für eine Erläuterung des Kruskal-Algorithmus vgl. Backhaus, K. et al., Multivariate Analysemethoden, a.a.O., S. $453 \mathrm{ff}$.

Die gebräuchlichsten Distanzmaße sind die sog. Minkowski-Metrik und als spezielle Form davon die Euklid-Metrik. Vgl. Ahrens, H. J., Multidimensionale Skalierung, Basel 1974, S. $78 \mathrm{ff}$.

Dabei wird dem ähnlichsten Paar die kürzeste, dem unähnlichsten Paar die längste Distanz zugeordnet, so dass die Monotoniebedingung 'wenn $u_{k l}>u_{i j}$, dann $d_{k l}>d_{i j}$ ' erfüllt ist. Eine perfekte Erfüllung der Monotoniebedingung ist in der Regel nicht möglich, so dass mit den Disparitäten $D_{k l}$ weitere Größen eingeführt werden. Hier wird aus den zusammenliegenden Objektpaaren, welche die Monotoniebedingung verletzten, das arithmetische Mittel gezogen, wobei auch die Bedingung 'wenn $u_{k l}>u_{i j}$, dann $D_{k l}>D_{k l}$ ' erfüllt sein muss.

425 Die beiden gebräuchlichsten STRESS-Kriterien sind Kruskals Stress 1 und das von Young entwickelte S-Stress.

$$
\text { Stress - } 1=\sqrt{\frac{\sum_{k} \sum_{1}\left(\mathrm{~d}_{w}-D_{x}\right)^{2}}{\sum_{k} \sum_{1} d_{w}{ }^{2}}} \quad S \text {-Stress }=\sqrt{\frac{\sum_{k} \sum_{1}\left(d_{w}{ }^{2}-D_{w i}{ }^{2}\right)^{2}}{\sum_{k} \sum_{1} D_{w}{ }^{2}}}
$$


der STRESS ausfällt, desto schlechter ist die Anpassung der Markendistanzen im Wahrnehmungsraum an die Markenähnlichkeiten (sog. badness of fit). In der Literatur finden sich Erfahrungswerte zur Beurteilung des STRESS-Maßes und damit zur Anpassungsgüte der erreichten Konfiguration. Zur endgültigen Darstellung des Markenwahrnehmungsraums ist schließlich dessen Dimensionalität festzulegen. Die Zahl der Dimensionen sollte dabei zum einen der "wahren“ Dimensionalität der Wahrnehmung entsprechen, zum anderen muss die gefundenen Lösung auch interpretierbar bzw. visuell darstellbar sein, so dass sich die Anzahl der Dimensionen unter diesem Aspekt auf zwei bzw. drei beschränkt. ${ }^{426}$

Kritisch anzumerken an dem hier verfolgten Ansatz der MDS ist allerdings, dass jede Konfiguration "designabhängig" ist, d.h. neben der Art der Datenerhebung (direkte oder abgeleitete Ähnlichkeitsdaten) und der Anzahl einbezogener Marken beeinflussen auch die Wahl der Metrik sowie die vorzugebende Anzahl der Dimensionen das Resultat einer MDS. ${ }^{427}$ Vor diesem Hintergrund ist das Ergebnis in hohem Maße von den subjektiven Eingriffen des Anwenders abhängig, so dass es sich anbietet, im Sinne eines Methodendualismus die gefundenen Lösung anhand eines weiteren Auswertungsverfahrens zu überprüfen. ${ }^{428}$

Wie ersichtlich, wird die Größe des STRESS-Maßes durch die Differenzen von $d_{k l}$ und $D_{k \mid}$ bestimmt. Sind diese Unterschiede gleich Null, liegt eine perfekte monotone Anpassung der Daten vor. Es ist leicht ersichtlich, dass auch triviale oder ungültige Konfigurationen einen STRESS von Null erzielen können, $z$. B. wenn alle Objekte die gleichen Koordinaten haben. $\mathrm{Vgl}$. Backhaus, K. et al., Multivariate Analysemethoden, a.a.O., S. 452ff; Böhler, H., Methoden und Modell der Marktsegmentierung, Stuttgart 1977, S. 255; Young, F., Day, G., Multidimensional Scaling, New York 1974, S. $65 f$.

Als formales Kriterium zur Bestimmung der Anzahl der Dimensionen kann auch das STRESSMaß herangezogen werden. Mit zunehmender Zahl der Dimensionen nimmt der STRESS ab, so dass bei nur geringfügiger Änderung des STRESS die Lösung mit geringerer Anzahl von Dimensionen vorgezogen werden sollte. Vgl. zum Elbow-Kriterium Backhaus, K. et al., Multivariate Analysemethoden, a.a.O., S. 458

Zu einer grundsätzlichen Verfahrenskritik der MDS vgl. auch die Ausführungen bei Tennagen, U., Produktrelaunch in der Konsumgüterindustrie: Diagnosekonzept zur Auswahl, Ermittlung und Bewertung von Informationen, Wiesbaden 1993, S. 130ff.

Dabei ist vor allem sicherzustellen, dass die MDS zuverlässige Lösungen generiert, $d$. h. bei einer zeitlich versetzten wiederholten Messung unter identischen Bedingungen - hierzu zăhlen insb. die Konstanz der Erhebungsverfahren und die Stichprobenhomogenität - darf eine Veränderung der im Wahrnehmungsraum enthaltenen Komponenten nicht auf methodische Artefakte und damit auf mangelnde Zuverlässigkeit zurückzuführen sein, sondern auf reale Entwicklungen im Markt. 
In Abb. 38 sind zur Veranschaulichung die der Erwägermatrix zugrundeliegenden Marken in einem zwei- bzw. dreidimensionalen Raum eingeordnet. ${ }^{429}$ Obwohl der Optimierungsprozess ${ }^{430} \mathrm{im}$ ein- bis vierdimensionalen Raum vorgenommen wurde, wird eine Begrenzung auf zwei Dimensionen als zweckmäßig erachtet: Zum einen werden sowohl die zwei- als auch dreidimensionale Lösung als befriedigende Güteanpassung kategorisiert, zum anderen ergeben sich bei der dreidimensionalen gegenüber der zweidimensionalen Lösung zusätzliche Interpretationsschwierigkeiten.

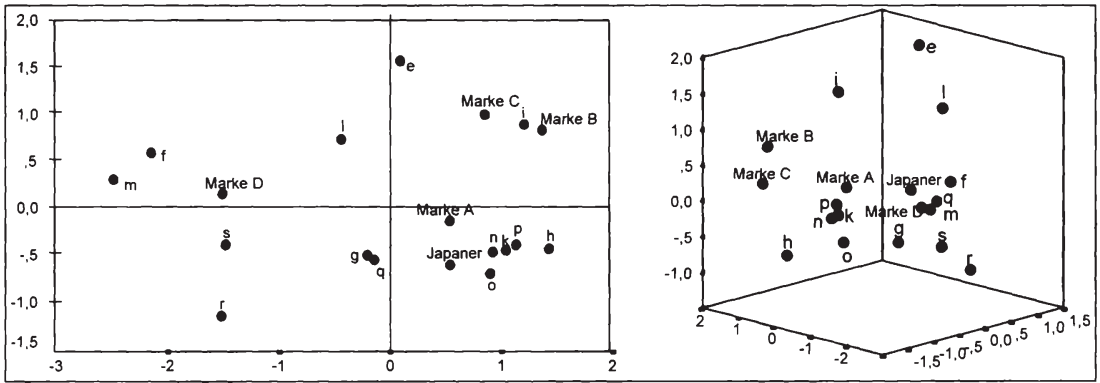

Abb. 38: Zwei- und dreidimensionaler Wahrnehmungsraum auf Basis von SecondChoice-Daten

Eine Validierung der im Rahmen der MDS gefundenen Positionierungsergebnisse kann mit Hilfe der Clusteranalyse erfolgen. ${ }^{431}$ In Abb. 39 wurde in den Ergebnisraum der zweidimensionalen Lösung der Fusionierungsprozess der Clusteranalyse eingetragen: Je dunkler die Kreise dargestellt sind, desto eher wurde das ent-

Für die dargestellten Untersuchungen kam das Softwarepaket SPSS für Windows Version 10.0 zum Einsatz.

Distanzen wurden aus den Häufigkeiten mit Hilfe des Phi-Quadrat-Maßes errechnet. Als Zielvorgaben für den Optimierungsprozess wurde jeweils ein STRESS von Null bzw. eine Verbesserung des STRESS-Wertes < 0,000 vorgegeben. Das STRESS-1 als Gütekriterium erreicht mit 0,1962 eine befriedigende Anpassung.

Im Rahmen einer clusteranalytischen Untersuchung fungieren die Zeilen der in Tab. 13 dargestellten Erwägermatrix als Variablen, die der Beschreibung der Objekte in den Spalten dienen. Die Häufigkeiten sind entsprechend als Merkmalsausprägungen der Marken zu verstehen. Aufrund der umfangreichen Verwendung dieses Verfahrens in Wissenschaft und Praxis sowie deren Dokumentation in einer Vielzahl wissenschaftlicher Beiträge soll auf eine Darstellung ihrer methodischen Vorgehensweise verzichtet werden. Vgl. stellvertretend Backhaus, $\mathbf{K}$. et al., Multivariate Analysemethoden, a.a.O., S. $261 \mathrm{ff}$. 
sprechende Objektpaar zu einem Cluster zusammengefasst. ${ }^{432}$ Das Ergebnis zeigt, dass Marken mit geringer Distanz im Wahrnehmungsraum auch im Rahmen der Clusteranalyse vergleichsweise früh zu Gruppen zusammengefaßt werden. Zur Interpretation der raumaufspannenden Dimensionen bietet der Fusionierungsprozess der Clusteranalyse sowie die aufgezeigte mehrdimensionale Imageanalyse zentrale Ansatzpunkte. ${ }^{433}$

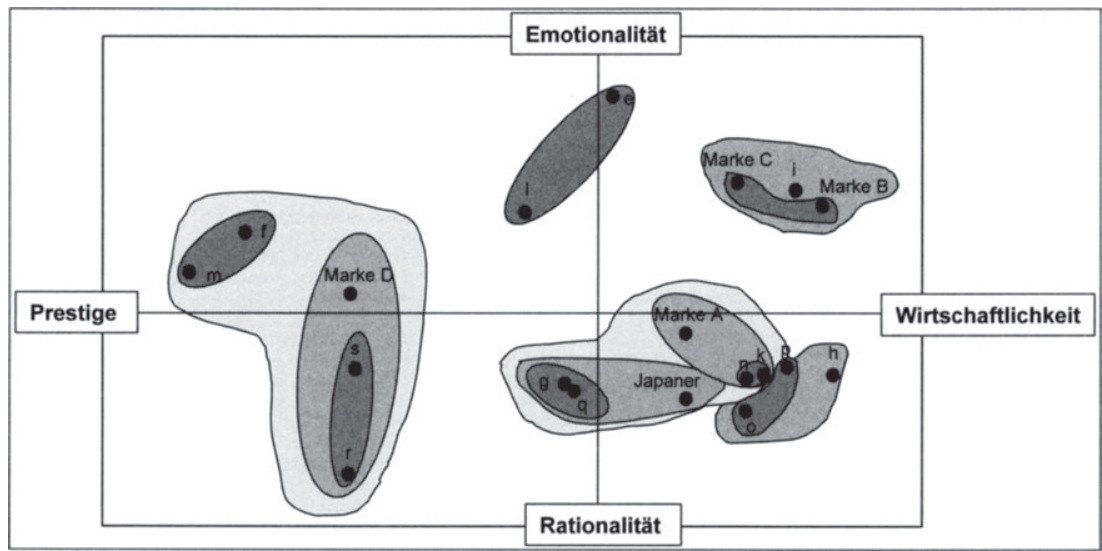

Abb. 39: Interpretation der zweidimensionalen Lösung

Bei den im linken Cluster liegenden Marken handelt es sich ausschließlich um Premiumanbieter (Marke D, s, r, m, f), die Marken in der rechten Hälfte des Wahrnehmungsraums stellen hingegen vorwiegend Preismarken (z.B. Marke $C, h, 0$ ) dar. Dennoch spiegelt die Abszisse keine einfache Preisleiter wider, sondern

432 Vgl. zum Vorgehen auch Dannath, U. M., Ansätze und Gestaltungsmöglichkeiten des strategischen Markenmanagements in der Automobilindustrie, unveröffentlichte Diplomarbeit am Lehrstuhl für Handel und Marketing der Universität Hamburg, Hamburg 1998.

Grundsätzlich besteht auch die Möglichkeit, nachträglich erhobene Eigenschaftsbeurteilungen der Marke in den Wahrnehmungsraum einzubeziehen (sog. Property Fitting). Dabei wird versucht, für jede Eigenschaft einen Vektor so in den Schwerpunkt des Wahrnehmungsraums zu projizieren, dass die Eigenschaftsurteile und die Projektion der Markenposition auf den entsprechenden Vektor hoch korrelieren. Vgl. Miller, J. E., Shepard, R. N., Chang, J. J., An Analytical Approach to the Interpretation of Multidimensional Scaling Solution, in: American Psychologist, Vol. 19, 1964, S. 579f. Vgl. auch Srinivasan, V., Shocker, A. D., Linear Programming Techniques for Multidimensional Analysis of Preferences, in: Psychometrica, Vol. 38, 1973, S. 337ff. Der postulierten Anforderung der Wirtschaftlichkeit entsprechend greift das Mehrmarkencontrolling jedoch vor allem auf solche Daten zurück, die zyklisch erhoben werden und keine nachtäglichen Sonderstudien erfordern. 
spannt ein Kontinuum von einer starken Betonung der "Wirtschaftlichkeit" bis zum Prestige /Luxus auf. Die Ordinate kann demgegenüber primär als Kontinuum von "Emotionalität" und „Rationalität" charakterisiert werden. Gerade die im oberen Cluster zu findenden Marken e und I wurden im Rahmen der Imageanalyse durchweg als emotional oder auch sportlich/dynamisch eingestuft, wohingegen Marken unterhalb der Abszisse verstärkt als robust, konservativ bzw. zurückhaltend bezeichnet wurden. Insgesamt fällt bei der Betrachtung eine relative Zwei- bis Dreiteilung des Marktes auf, welche als Indiz für die beschriebene Tendenz zur Marktpolarisierung gewertet werden kann.

Mit der genannten Zielsetzung, Substitutions- und Partizipationsbeziehungen anhand der Distanzen im Wahrnehmungsraum zu antizipieren, sind grundsätzlich die hier gefundenen Ergebnisse den im Rahmen der Wanderungsanalyse identifizierten Partizipations- und Substitutionseffekten gegenüberzustellen. Weisen die Marken eine geringe Distanz zueinander auf, ist mit einer wechselseitig hohen Substitution bzw. Partizipation zu rechnen.

In Abb. 40 beinhalten die grau schraffierten Flächen operative Wettbewerber der einzelnen Portfoliomarken. Aufgrund der hier dargestellten räumlichen Nähe sind entsprechend hohe Partizipationseffekte zu erwarten. Genau diese Vermutungen lassen sich durch die Wanderungsergebnisse weitgehend bestätigen. Abb. 40 zeigt beispielhaft die Wettbewerbspartizipation der Marke A mit den Marken n, p, o und $h$, die auch im Wahrnehmungsraum eine geringe Distanz zueinander aufweisen. Hinsichtlich des Substitutionseffektes zeigen die Wanderungsbewegungen allerdings ein etwas anderes, tatsächliches Kaufverhalten als die Distanzen im Wahrnehmungsraum vermuten ließen. Aufgrund der räumlichen Nähe sowie der frühzeitigen Zusammenfassung zu einem Cluster weisen insbesondere die Marken $B$ und $C$ eine hohe Substitutionswahrscheinlichkeit auf. Wanderungsbewegungen zwischen den Marken $B$ und $C$ lassen sich zwar durchaus feststellen, jedoch geringer als antizipiert. Hingegen findet primär eine Substitution zu Lasten der höherpreisigen Marken im Portfolio statt. Eine Erklärung hierfür kann insbesondere darin gesehen werden, dass die Marken $B$ und $C$ im europäischen Raum durchaus substitutiv wahrgenommen werden, ihre marktliche Präsenz aber eine umfassende Substitution verbietet, da die Marke $B$ insbesondere den südeuropäischen, die Marke $C$ hingegen primär den osteuropäischen Raum bearbeitet. Nur die Marken A und D sind sowohl in Süd- als auch Osteuropa präsent. Genau hier zeigen die Wanderungsstrukturen Abwanderungen zur Lasten der höherpreisigen Marken (vgl. beispielhaft Marke A in Abb. 40), welche in physikalisch-technischer

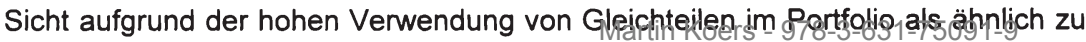


den Marken B und C einzustufen sind (Ausmaß physikalisch-technischer Gleichartigkeit). Für eine Erklärung der Abweichung des erwarteten vom tatsächlichen Kaufverhalten sind letztlich eine Vielzahl von situativen Faktoren heranzuziehen. Wie dargelegt, lösen beispielsweise neue Modelle der jeweiligen Marken einen Substitutionsprozess zwischen den Marken aus, so dass die Modellpolitik der einzelnen Hersteller einen wesentlichen Einfluss auf die Substitutionszeiten haben kann.

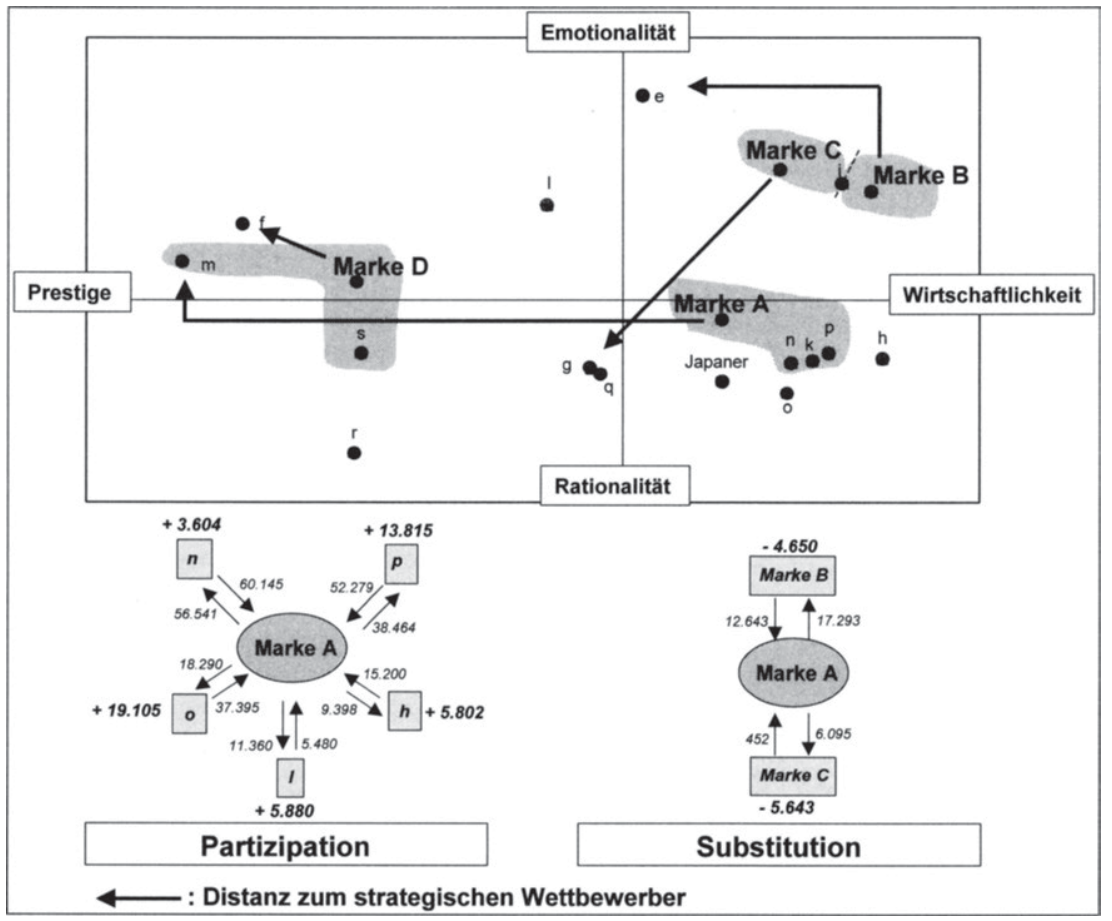

Abb. 40: Erklärung von Wanderungseffekten aufgrund der Positionierung im Wahrnehmungsraum

Kritisch anzumerken an den hier verwendeten Erwägerraten zur Positionierung der Marken ist die künstliche Reduktion des gesamten evoked sets auf zwei Alternativen. ${ }^{434}$ Hierdurch erhält man keinen Aufschluss über den Abstand der First- 
Choice Marke zur Second-Choice Marke und erlangt keine Vergleichsurteile zu weiteren Marken. Überdies erfolgt eine Vermischung von Kaufverhaltens- und Wahrnehmungsdaten, so dass aufgrund der Budgetrestriktionen bzw. der Festlegung eines Preisrahmens vor dem Kauf trotz des grundsätzlich dimensionslosen Ansatzes eine Betonung der Preisdimension zu befürchten ist.

Wie aufgezeigt, orientiert sich die Definition strategischer Wettbewerber am langfristigen, visionären Kompetenzanspruch der Marken im Wettbewerbsumfeld. In Abb. 40 zeigen die Pfeile die relative Lage der hier betrachteten Portfoliomarken zu den definierten strategischen Wettbewerbern an. Der Abbildung zur Folge weisen insbesondere die Marken $A$ und $C$ einen großen Abstand $z u$ den definierten Wettbewerbern auf. Ob die aufgezeigte relative Lage der strategischen Wettbewerber den tatsächlich angestrebten Kompetenzanspruch der Portfoliomarken widerspiegelt, erscheint allerdings insbesondere aus zwei Gründen fraglich:

- Zum einen repräsentieren die hier angeführten Markenpositionen IstPositionen sowohl der Eigen- als auch Fremdmarken. Eine Orientierung an dem von den Konsumenten wahrgenommenen Ist-Zustand der Fremdmarken für den Kompetenzanspruch der eigenen Portfoliomarken kann jedoch zu fehlerhaften strategischen Entscheidungen führen. ${ }^{435}$ Ist-Images lassen zwar Schwachstellen eines Images und Ansatzpunkte für Verbesserungsmaßnahmen erkennen, machen aber die strategischen Einzelziele zur Erreichung eines abgestimmten Gesamtaufmarschs der Portfoliomarken noch nicht deutlich. Erst wenn die Soll-Vorstellungen bestimmt und für verbindlich erklärt worden sind, lässt sich eine abgestimmte Mehrmarkenstrategie langfristig verfolgen.

- Zum anderen handelt es sich bei dem hier aufgespannten Wahrnehmungsraum um eine ausschließliche Darstellung der Markenpositionierung auf Basis von Konsumentenurteilen und damit um eine ausschließliche Outside-In Betrachtung. Eine solche fremdbildorientierte Betrachtung ist jedoch um eine Inside-Out-Perspektive im Sinne einer identitätsorientierten Markenführung zu ergänzen.

Zur Identifikation der vom Portfoliomanagement angestrebten Soll-Positionierung der einzelnen Portfoliomarken sind im Folgenden die jeweiligen Selbstbilder der Marken zu analysieren und den wahrgenommenen Fremdbildern gegenüberzu-

435 So weisen die strategischen Wettbewerber $f$ und $m$ der Marken $A$ und $D$ in dem in Abb. 40 dargestellten Wahrnehmungsraum nur geringe Distanzen auf. Eine gleichzeitige Orientierung $\operatorname{der}$ Marken $A$ und $D$ in die Richtung der beschriebenen Wettbewerbsmarken würde somit dem Ziel der Gesamtmarktabdeckung im Sinne der Mehrmarkenstrategien widersprechen. 
stellen. Als mögliches Analyseinstrument dient dabei das Semantische Differential als direktes Erhebungsverfahren.

\subsection{Analyse der angestrebten Markenwahrnehmung: Inside-Out- Betrachtung}

\subsection{Analyse auf Basis von Einzelurteilen}

Das auf OSGOOD/SUCI/TANNENBAUM beruhende Semantische Differential (SD) stellt eine bekannte Methode mehrdimensionaler Imagemessung dar, welche ursprünglich zur Messung von Wortbedeutungen entwickelt und später für seine Anwendung im Marketing modifiziert bzw. ausgeweitet wurde. ${ }^{436}$ Der Grundgedanke des Semantischen Differentials ist die Existenz eines semantischen Raums, in dem jedes Wort eine konkrete Position einnimmt. Die Erhebung von Wortassoziationen findet dabei mittels einer zweipoligen Ratingskala mit adjektivischen Gegensatzpaaren statt:

Die Bedeutung eines Wortes wird durch die mit dem Wort verbundenen Assoziationen gemessen, wozu den Befragten eine Menge von Assoziationen vorgegeben werden, mit denen sie das jeweilige Stimuluswort zu beurteilen haben. Das klassische SD verwendet hierzu adjektivische Gegensatzpaare wie "laut-leise“, „schönhässlich" etc. Um die Stärke der Assoziationen zu messen, sind die Adjektive auf einer Rating-Skala abzutragen, so dass der Befragte angeben kann, inwieweit ein vorgegebenes Adjektiv seine Assoziationen zum Stimuluswort wider gibt. Wichtig ist, dass die gegensätzlichen Eigenschaftspaare nicht wörtlich, sondern metaphorisch zu interpretieren sind, so dass sie sich auf verschiedene Konzepte beziehen lassen. Im Marketing werden allerdings häufig Eigenschaftswörter (Items) verwendet, die im wörtlichen Sinne zu verstehen sind und sich direkt auf den Meinungs-

Vgl. Osgood, C. E., Suci, G. J., Tannenbaum, P. H., The Measurement of Meaning, University of Illinois Press, Chicago u. a 1957. Eine kurze Zusammenfassung findet sich bei Osgood, C. E., Eine Entdeckungsreise in die Welt der Begriffe und Bedeutungen, in: Grundfragen der Kommunikationsforschung, Schramm, W. (Hrsg.), 5. Aufl., München 1973, S. 39ff. Zu den Modifizierungen und Ausweitungen vgl. etwa Trommsdorf, V., Die Messung von Produktimages für das Marketing, a.a.O., S. 27ff.; Bergler, R., Das Eindrucksdifferential - Theorie und Technik, Bern u. a., 1975. Die Semantik als linguistische Disziplin erforscht als Teildisziplin der Sprachwissenschaft die Bedeutung von Wörtern, Sätzen und Texten und kann auch als Bedeutungslehre verstanden werden. SPIEGEL baut auf das von OSGOOD entwickelte Modell auf. Vgl. Spiegel, B., Die Struktur der Meinungsverteilung im sozialen Feld: Das psychologische Marktmodell, Bern, Stuttgart 1961, S. $19 \mathrm{ff}$. 
gegenstand beziehen. Zwar wird dann häufig noch vom „Semantischen Differential" gesprochen, allerdings handelt es sich hier im Grunde um Multi-AttributModelle. Beim SD kann hingegen von lauten oder leisen Menschen, lauten oder leisen Produkten aber auch lauten und leisen Marken gesprochen werden. Neben wertenden Eigenschaftswörtern werden dabei auch Wörter einbezogen, die sich auf emotionale (z. B. temperamentvoll-gelassen) oder sachliche Eindrücke (z. B. langlebig-kurzlebig) beziehen. Eine besondere Bedeutung hat hier die PleasureScale nach MEHRABIAN/RUSSEL erlangt, deren Gegensatzpaare zur Messung der Gefallenswirkung herangezogen werden. ${ }^{437}$ Durch Verbindung der Mittelwerte der von den Befragten angekreuzten Skalen lässt sich ein Vorstellungsprofil der Konsumenten erzeugen. Durch die Berechnung von Ähnlichkeiten mittels DistanzmaBen oder Korrelationen kann schließlich festgestellt werden, inwieweit sich Images ähneln oder wie groß die Distanz zum Image einer idealen Marke ist. ${ }^{438}$

Während bei der Markenpositionierung auf Basis von Erwägerraten eine globale Bewertung der Marken vorgenommen wurde (indirektes Verfahrten), geht das hier angewandte Verfahren analog zur Ermittlung von Imageprofilen von Einzeleindrücken aus (direktes Verfahren). Für die im Rahmen der Arbeit betrachteten Marken wurden die konkreten Ausprägungen bezüglich einer gegenüber dem klassischen Semantischen Differential verfeinerten, speziell auf die Marken zugeschnittenen Polaritätenbatterie ermittelt. ${ }^{439}$ Bei der Erhebung bestanden die verwendeten Polpaare aus "Begriffswolken", die hier jeweils nur durch ein einzelnes Adjektiv benannt sind ${ }^{440}$ Mittels Mittelwertbildung über alle Befragten entsteht für jede Marke ein Image-Profil aus Sicht der Befragten. Ebenso wie ein solchermaßen erhobenes Marken-Image (als Ist-Image) kann auch das formulierte Selbstbild

Vgl. Mehrabian, A., Russel, J. A., An Approach to Environmental Psychology, MIT-Press, Cambridge, Mass., 1974. Die hohe Validität und Reliabilität der Pleasure-Scale wurde in zahlreichen Studien belegt. Vgl. Esch, F.-R., Langner, T., Potenzialanalyse der Markennamen FIRST, Hapag-Lloyd, Thomas Cook und TUI, Gießen 1999, S. 22.

Statt der Eigenschaftswörter besteht die Möglichkeit, visuelle Zeichen, Farben oder Symbole zu benutzen, die die gleiche Bedeutung wie die Eigenschaftswörter haben, so dass man zu non-verbalen Skalen gelangt, die auch für interkulturelle Vergleiche verwendet werden können. Vgl. Kroeber-Riel, W., Weinberg, P., Konsumentenverhalten, 7. Aufl., München 1999, S. 197ff; Trommsdorff, V., Konsumentenverhalten, a.a.O., S. $170 \mathrm{ff}$.

439 Im Rahmen der hier betrachteten Studie (1998) wurden vom Spiegel Institut sowohl das Management des betrachteten Markenportfolios als auch Konsumenten $(n=1000)$ für alle Marken A-D in 1998 gebeten, die Marken den jeweiligen Adjektivpaaren zuzuordnen.

Die Begriffswolken des Spiegel Instituts Mannheim sind im Anhang II. aufgeführt. 
(Soll) als gebündelte Willenserklärung der Portfolioleitung in ein solches Schema eingetragen werden. Abb. 41 zeigt das Ist-Profil aus Sicht der externen Konsumenten sowie das Soll-Profil aus Sicht der Portfolioleitung beispielhaft für die Marke $A{ }^{441}$ Wie ersichtlich, weicht das Ist-Image vom formulierten Selbstbild der Marke $A a b$.

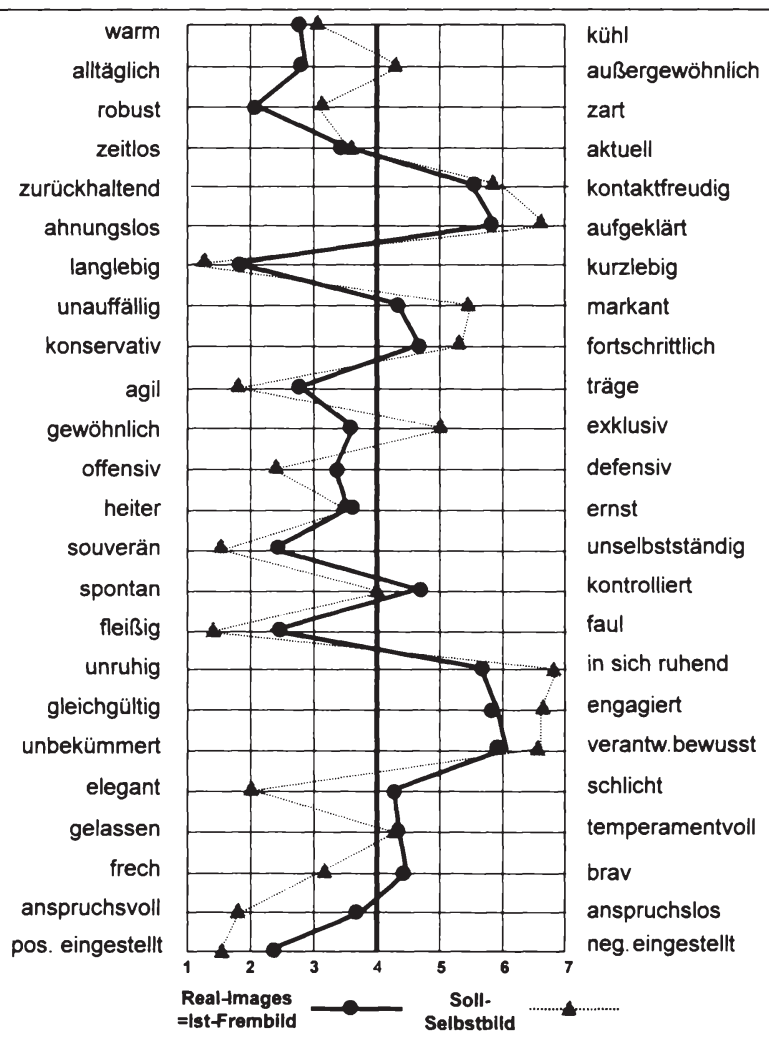

Abb. 41: Polaritätenprofil Soll-Selbstbild und Ist-Fremdbild Marke A(n=1000) 
Zur verdichteten Darstellung der Soll-Ist-Positionierungen sämtlicher Portfoliomarken und damit zur Darstellung der Globaldistanzen bietet es sich im Folgenden an, die einzelnen Marken wiederum mit Hilfe des bereits vorgestellten Verfahrens der Multidimensionalen Skalierung in einen mehrdimensionalen Wahrnehmungsraum zu projizieren. Bei einer solchen Untersuchung geht es weniger um einen zu erstellenden Wahrnehmungsraum mit anschließender Interpretation der Dimensionen, sondern um die Generierung einer anschaulichen Konfiguration, welche die Lage der Marken und damit die Distanzen zwischen Soll- und IstPositionierung punktuell und nicht profilhaft darstellt.

\subsection{Ermittlung der Gesamtabweichung}

Zur Abbildung der Selbst- und Fremdbilder der Marken in einem mehrdimensionalen Wahrnehmungsraum sind die von der Portfolioleitung vorgegebenen Sollzustände als "fiktive Marken" zu betrachten, d.h. das Selbstbild der Marke A wird als fiktive eigenständige Marke gesehen, so dass der Untersuchung formal-analytisch - verdeutlicht für die Marke A - zwei Marken, nämlich die Realmarke a und die Idealmarke a' zugrunde gelegt werden können. Für die anschließende Interpretation des Wahrnehmungsraums ist diese gedankliche Trennung wieder aufzugeben, indem a als Ist-Positionierung der Marke A (Realimage), a' hingegen als SollPositionierung (Selbstbild) zu interpretieren ist. Entsprechend beinhaltet das Portfolio formal-analytisch nicht vier, sondern acht Marken, nämlich a-a', b-b', c-c' sowie $d-d$ ', deren Distanzen zueinander wichtige Anhaltspunkte für das Management liefert. Die Gesamtdistanz zwischen Ideal- und Realposition kann dabei aus den Einzeldistanzen aller Dimensionen gebildet werden: ${ }^{442}$

$$
D_{i}=\sqrt{\sum_{i=1}^{24}\left(c_{i i}-e_{i i}\right)^{2}} \rightarrow \min !
$$

$D_{i}:$

c: e:
Gesamtdistanz zwischen Soll-Selbstbild und Ist-Fremdbild Idealausprägung von Eigenschaft j bei Marke i aus Sicht der Portfolioleitung (Soll-Selbstbild) Realausprägung von Eigenschaft $\mathrm{j}$ bei Marke $\mathrm{i}$ aus Konsumentensicht (Ist-Fremdbild) schaften eine exakt gleich hohe Bedeutung zuschreiben, müssten die einzelnen Eigenschaftsausprägungen zur Vermeidung einer Gleichgewichtung zusätzlich mit Bedeutungsgewichten versehen werden. 
Wie aufgezeigt, hängt die Stärke der Markenidentität wesentlich vom Ausmaß der Übereinstimmung zwischen Selbst- und Fremdbild der Marken ab. Je größer folglich die Distanz zwischen der Soll- und Ist-Position einer Marke im Wahrnehmungsraum ist, desto schwächer ist die Identität dieser Marken et vice versa. Langristig bedarf es somit einer hohen Übereinstimmung zwischen dem SollSelbstbild und dem Ist-Fremabild.

Da geringe Distanzen hohe Ähnlichkeiten zwischen den Marken verdeutlichen, sind auf der anderen Seite hohe Distanzen zwischen den Realpositionen der Portfoliomarken (also zwischen den einzelnen Fremdbildern der Portfoliomarken untereinander) anzustreben, wenn davon ausgegangen wird, dass hierdurch die Wahrscheinlichkeit hoher Substitutionseffekte zwischen den Marken sinkt. Für die Marke A und B ergibt sich dabei formal:

$$
\begin{array}{ll} 
& D_{A, B}=\sqrt{\sum_{j=1}^{24}\left(e_{j A}-e_{j B}\right)^{2}} \rightarrow \text { max! } \\
D_{A, B}: & \text { Gesamtdistanz von Marke A zu Marke B } \\
\text { e: } & \text { Realausprägung von Eigenschaft j bei Marke i (Ist-Fremdbilder) }
\end{array}
$$

Auf diese Weise lässt sich eine Distanzmatrix erstellen, die Aufschluss über die Ähnlichkeiten der Ideal- und Realmarken untereinander gibt. Die verfügbaren Informationen seien die semantischen Distanzen, welche sich aus den 24 Polpaaren zwischen den Marken ableiten lassen. Eine solche Distanztabelle gibt nicht die räumliche Lage der Marken an, sondern lediglich die paarweisen Distanzen zwischen den Marken. ${ }^{443}$

Abb. 42 zeigt die mit Hilfe der MDS ermittelte Konfiguration der Lösung. Neben den betrachteten Portfoliomarken wurden zusätzlich die Marken $f, k$ und $n$ in die Analyse einbezogen. ${ }^{444}$ Wie die Konfiguration verdeutlicht, weichen die IstPositionierungen von den jeweiligen Soll-Positionierungen ab, so dass die bestehenden Distanzen als GAPs zwischen dem Soll-Selbstbild und dem Ist-Fremdbild interpretiert werden können. Dabei weist insbesondere die vergleichsweise neu

\footnotetext{
443

Die hier verwendete Distanzmatrix findet sich im Anhang IV.

444

Distanzen im Wahrnehmungsraum wurden mit Hilfe der quadrierten Euklidischen Distanz errechnet. Als Zielvorgaben für den Optimierungsprozess wurde jeweils ein STRESS von Null bzw. eine Verbesserung des STRESS-Wertes $<0,000$ vorgegeben. Das STRESS- 1 als Gütekriterium erreicht mit 0,0336 eine gute bis hervorragende Anpassung.
} 
akquirierte Marke B eine hohe Diskrepanz zwischen Selbst- und Fremdbild auf, so dass bei dieser Marke auf eine vergleichsweise geringe Identität geschlossen werden kann.

Die räumliche Lage der Realimages zeigt überdies eine unzureichende Differenzierung der Portfoliomarken in der Wahrnehmung der Konsumenten. Aufgrund der wahrgenommenen Ähnlichkeit der Marken sind entsprechende Wanderungsbewegungen im Portfolio zu erwarten. Die Lage der angestrebten Soll-Positionierung zeigt hingegen eine von der Portfolioleitung angestrebte breite Abdeckung des Wahrnehmungsraums zur Vermeidung zu großer Überschneidungsbereiche zwischen den Marken. Eine Positionierung der Marken im grau hinterlegten unteren Quadranten des Wahrnehmungsraums, aus Sicht der Konsumenten durch die Marken $B$ und $C$ besetzt, soll dabei gänzlich vermieden werden. Gründe hierfür offenbart die Interpretation der zugrunde gelegten Raumdimensionen.

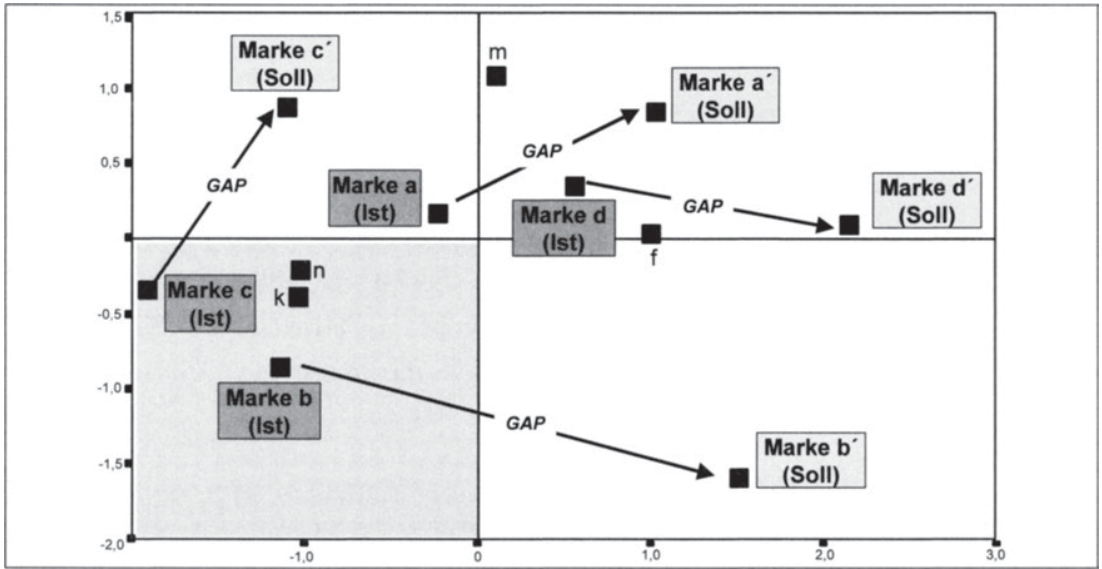

Abb. 42: Soll-Selbstbild und Ist-Fremdbild im zweidimensionalen Wahrnehmungsraum

Zur Interpretation der Dimensionen des Wahrnehmungsraums ist es möglich, die hier verwendeten Eigenschaftsbeurteilungen mit in den Wahrnehmungsraum einzubeziehen. Hierzu erfolgt eine Eigenschaftsregression, d.h. eine Regression der Eigenschaften auf die Dimensionen des Wahrnehmungsraums. Die Lage der 
Eigenschaftsvektoren lässt sich dabei graphisch mit Hilfe der Regressionskoeffizienten bestimmen. ${ }^{445}$

In Abb. 43 sind die in der vorliegenden Untersuchung ermittelten Eigenschaftsvektoren im Wahrnehmungsraum zusammen mit der Konfiguration der betrachteten Marken dargestellt: Je näher eine Marke der vorliegenden Konfiguration an der Spitze eines Pfeiles eingeordnet ist, desto mehr stimmen die Befragten dem entsprechenden Item zu. Letztlich zeigt sich illustrativ, dass die Dimensionen des Wahrnehmungsraums komplexer Natur sind und sich nur unzulänglich mit einem einzigen Begriff umreißen lassen. ${ }^{446}$

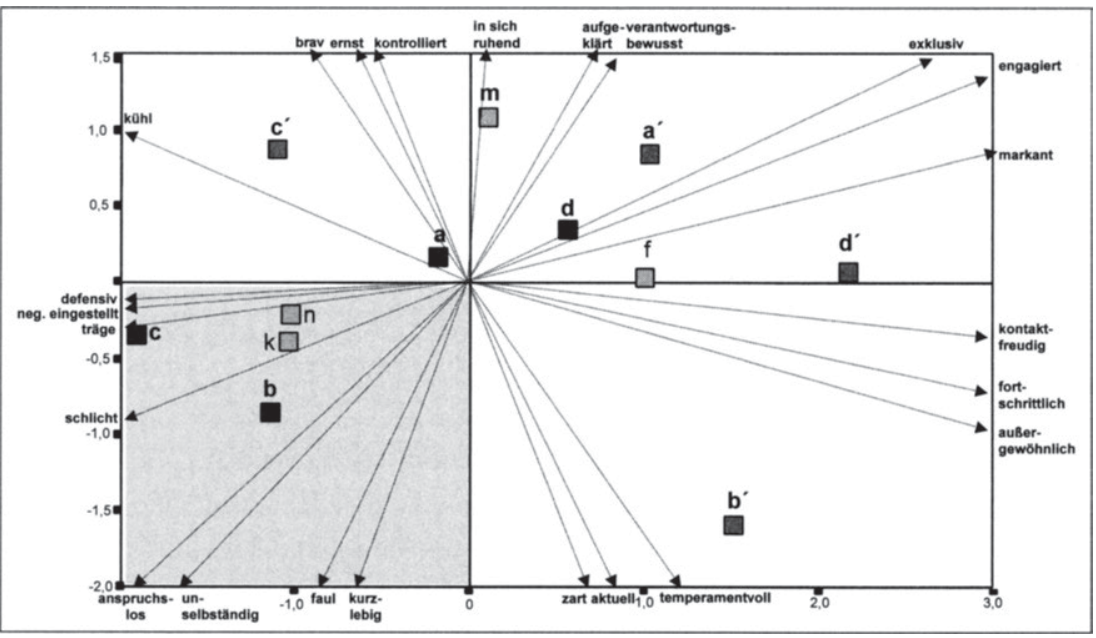

Abb. 43: Marken und Eigenschaften im Wahrnehmungsraum

Mittels der Beta-Werte der Regressionskoeffizienten lässt sich überdies aussagen, welche unterschiedliche Wichtigkeit die Dimensionen des Wahrnehmungsraumes haben. Vgl. hierzu Backhaus, K. et al., Multivariate Analysemethoden, a.a.O., S. 473.

446 Bei einem Versuch der Klassifizierung lassen sich die Eigenschaftsvektoren im positiven Bereich der Ordinate sprachlich primär als traditionell, die im negativen Bereich als unkonventionell zusammenfassen. Die Vektoren im negativen Bereich der Abszisse kennzeichnen vor allem rationale, die im positiven Bereich emotionale Eigenschaften. 
Die Betrachtung des linken unteren Quadranten verdeutlicht, dass die dort positionierten Marken mit "negativen" Eigenschaften - verdeutlicht durch die dem semantischen Differential zugrunde gelegten Adjektive defensiv, neg. eingestellt, träge, schlicht, anspruchslos, unselbstständig, faul und kurzlebig - assoziiert werden. Wie ersichtlich, zeigen die jeweiligen Soll-Positionen der einzelnen Portfoliomarken hingegen eine inhaltliche Konsistenz zwischen den dimensionsbeschreibenden Eigenschaftsvektoren und den entsprechenden Markenleitbildern. Dies sei beispielhaft für die Marke B verdeutlicht: Die Ist-Position der Marke B äußert sich in den oben beschriebenen Eigenschaften (insb. schlicht und anspruchslos), die Soll-Position wird durch die fiktive Marke $b^{\prime}$ ' dargestellt, welche anhand der umliegenden Eigenschaftsvektoren als temperamentvoll, aktuell und außergewöhnlich charakterisiert wird. Eine solche Charakterisierung trägt dem Markenleitbild „Automobile Lebensfreude" der Marke B Rechnung. Die hier gefundene Lösung trägt somit zusammenfassend dazu bei,

- zum einen durch Identifikation möglicher GAPs zwischen dem Selbstbild und dem Fremdbild einer Marke den Grad der Umsetzung der Markenleitbilder zu analysieren sowie

- zum anderen die realisierte und angestrebte Differenzierung zwischen den Portfoliomarken zur Vermeidung von Substitutionseffekten im Portfolio zu indizieren.

Werden GAPs zwischen den Soll-Selbstbildern (Zielniveau) und den IstFremdbildern (Ergebnisniveau) und damit eine mangelnde Umsetzung der Markenleitbilder festgestellt, sind zur Identifikation der Abweichungsursachen die "Erklärungsbeiträge" innen- und außengerichteter Abweichungen im Sinne einer Abweichungsaufspaltung weiter zu differenzieren. Abb. 44 zeigt den hier verfolgten formalen Zusammenhang der Abweichungsaufspaltung zur Erklärung der Gesamtabweichung. ${ }^{447}$

Die hier vorgeschlagene kumulative Abweichungsaufspaltung ist dadurch gekennzeichnet, dass bei den Einzelabweichungen die Erklärungsbeiträge der bereits analysierten Faktoren wieder eliminiert werden, und beim Übergang von einer Analysestufe zur nächsten nur der zusätzliche Beitrag des jeweils neu einbezogenen Faktors berücksichtigt wird. Zur Abgrenzung von kumulativer und alternativer Abweichungsaufspaltung vgl. Schwienhorst, R., Strategische Kontrolle, Rahmenbedingungen, Aufgaben und Methoden, a.a.O., S. 189. 


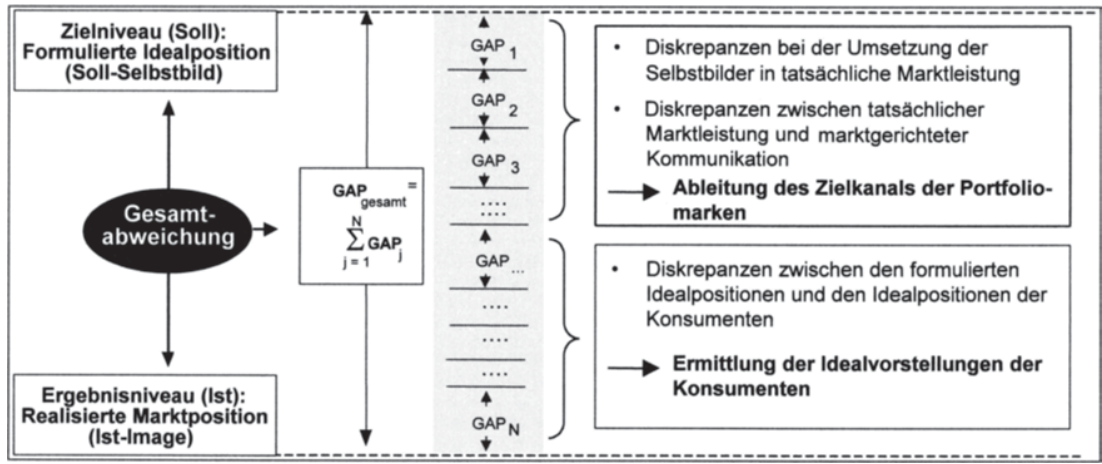

Abb. 44: Grundidee der Abweichungsaufspaltung

Zur Analyse der Abweichungsursachen ist somit zu fragen,

- inwieweit bei der Ausgestaltung der Marketing-Instrumente im Rahmen des internen und externen Marketing den Markenleitbildern der einzelnen Portfoliomarken zur innen- und außengerichteten Festigung der Markenidentität entsprochen wird, d.h.

- inwieweit Diskrepanzen bei der Umsetzung und Spezifikation der formulierten Selbstbilder in tatsächlich erstellte Marktleistungen bestehen (Umsetzungsgap) sowie

- inwieweit Diskrepanzen zwischen der tatsächlich erstellten und der in der marktgerichteten Kommunikation versprochenen Leistung des Herstellers vorliegen (Kommunikationsgap)

- inwieweit die jeweiligen Soll-Selbstbilder als formulierte Idealpositionen der Marken mit den Idealmarken der Konsumenten übereinstimmen (Wahrnehmungsgap). 


\subsection{Abweichungsaufspaltung}

Umsetzungs- und Kommunikationsgaps entstehen vor allem durch den Einsatz des Marketing-Instrumentariums. Zielsetzung des Instrumenteeinsatzes muss es sein, die angestrebte Soll-Wahrnehmung im Markt bzw. in der Zielgruppe zu etablieren und damit die Gesamtabweichungen zu minimieren. Konkrete Ansatzpunkte ergeben sich dabei aus den Einzelabweichungen der Polaritätenbatterie.

Wie aufgezeigt, ermöglicht das Semantische Differential die Markenwahrnehmung anhand von beschreibenden Attributen und Assoziationen zu messen. Durch Gegensatzpaare kann sowohl das Ist- als auch das Soll-Profil einer Marke dargestellt werden. Zur Messung der Einzelabweichungen ist das Ist-Profil einer Marke mit dem Soll-Profil, das sich aus der definierten Markenphilosophie ableitet, abzugleichen. Das Resultat eines solchen Abgleichs durch Inversion und Konversion der Profile ergibt einen sogenannten Zielkanal. In der linken Hälfte der Abb. 45 ist der Zielkanal für die Marke $A$ dargestellt, die rechte Hälfte repräsentiert die aus den Soll-Ist-Profilen ermittelten Einzelabweichungen für sämtliche Portfoliomarken.

So ergibt sich die Gesamtabweichung der Marke A als Summe der Absolutbeträge, wobei sich das Realimage der Marke $A$ im Vergleich zum angestrebten Selbstbild aus Sicht der Portfolioleitung vor allem als zu schlicht, anspruchslos und gewöhnlich darstellt. Dem gegenüber erweist sich die Marke B als zu unauffällig, brav und zu wenig temperamentvoll, Marke $\mathrm{C}$ als zu kurzlebig und wenig robust und die Marke $D$ als zu kontrolliert und konservativ. Unter Hinzuziehung der gesamten Begriffswolken wird ersichtlich, dass die wesentlichen Einzelabweichungen der Marken $A$ und $C$ eher rationale Dimensionen der Marke (Marke als Produkt), die der Marken $B$ und $D$ eher emotionale Dimensionen (Marke als Person bzw. Symbol) kennzeichnen. 


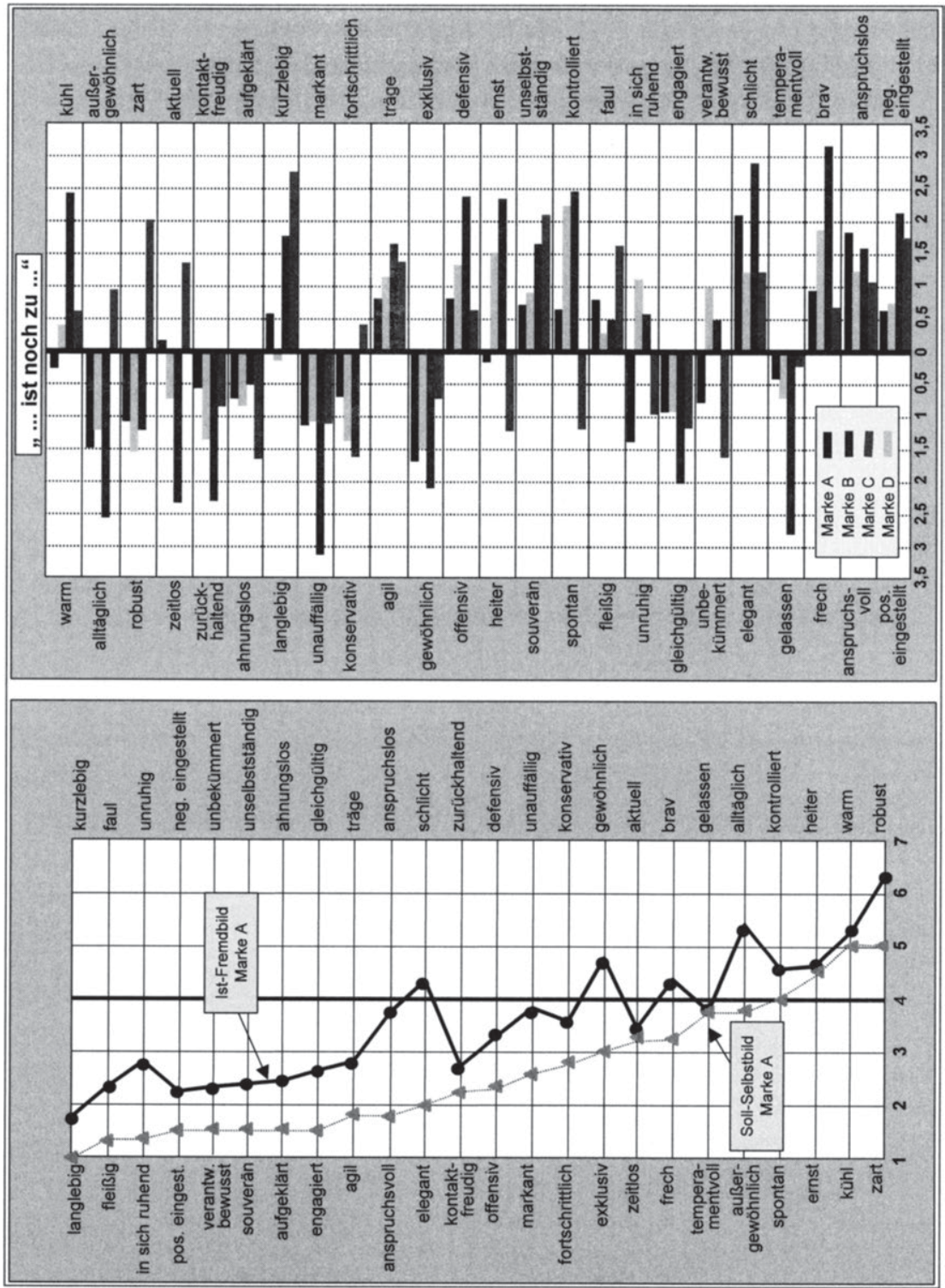

Abb. 45: Ermittlung des Zielkanals durch Abgleichung der Markenprofile 
Zielsetzung ist, das Delta im Zielkanal durch entsprechende Marketingmaßnahmen zu minimieren. Dabei können aus den ermittelten Zielkanälen und den Einzelabweichungen Anhaltspunkte für markenspezifische Maßnahmen zur SchlieBung von Diskrepanzen zwischen Selbstbild und Fremdbild gewonnen werden:

- Weist die Marke objektiv nachvollziehbare Mängel auf (Umsetzungsgap), können gezielte Verbesserungen vorgenommen werden. Für Marke B kann beispielsweise angestrebt werden, der fehlenden Auffälligkeit durch ein entsprechend markanteres Modelldesign oder dem mangelnden Temperament durch eine leidenschaftlichere Botschaftsgestaltung zu begegnen.

- Bei Wahrnehmungsverzerrungen, die nicht den tatsächlichen Markeneigenschaften entsprechen (Kommunikationsgap), kann vor allem durch Maßnahmen der Kommunikationspolitik eine Veränderung erreicht werden. So ist die Robustheit und Langlebigkeit der Marke $\mathrm{C}$ aufgrund der intensiven Verwendung von Gleichteilen im Markenportfolio objektiv nicht wesentlich weniger ausgeprägt als bei den sonstigen Portfoliomarken, so dass hier vor allem Wahrnehmungsdefizite zu vermuten sind. Hier könnte die Wahrnehmung der Robustheit und Langlebigkeit der Marke $C$ durch explizite Herausstellung spezifischer Modellmerkmale (zusätzliche Versteifung der Karosserie, hochwertige Kunststoffteile im Innenraum, etc.) verbessert werden.

Die Polaritätenprofile können hierzu gezielt in Bezug auf bestimmte MarketingMaßnahmen eingesetzt werden. So lassen sich alle Maßnahmen für eine Marke, die in irgendeiner Weise Image-beeinflussend sind, testen (z. B. Anzeigenserien, ein Slogan, ein Facelifting oder neues Modell etc.). Dazu hat eine geeignete Stichprobe den fraglichen Meinungsgegenstand anhand der gleichen Polaritätenbatterie zu bewerten: Je größer die Zahl der Polaritäten, bei denen die Bewertung im Zielkanal liegt, desto besser ist diese Maßnahme geeignet, das Ist-Image an das definierte Selbstbild anzunähern. Dabei können Maßnahmen eine gewisse Zeit auch bewusst das Selbstbild übertreffen, um so zu einer Beschleunigung der angestrebten Image-Bewegung beitragen zu können. ${ }^{448}$

Zur Ermittlung von Wahrnehmungsgaps ist zu analysieren, inwieweit die jeweiligen Soll-Selbstbilder als formulierte Idealpositionen der Marken mit den Idealmarken der Konsumenten übereinstimmen bzw. inwieweit die Kundenerwartungen bei

448 Wenn das formulierte Selbstbild weltweit gleich ist, die Realimages aber länderspezifisch verschieden sind, kann davon ausgegangen werden, dass es länderindividuell unterschiedliche Barrieren zu überwinden gilt. Wenn das Realimage nun dem formulierten Selbstbild angenähert werden soll, lässt sich länderspezifisch testen, ob die vorgesehene Maßnahme ( $z$. B. Werbung) in die richtige Richtung wirkt. 
der Selbstbildformulierung antizipiert werden. Ungeachtet reaktiver oder aktiver Positionierung besteht langfristig das Ziel einer hohen Übereinstimmung zwischen den Idealimages der Konsumenten und den formulierten Soll-Selbstbildern des Unternehmens.

Hierzu ist das Soll-Image aus Sicht der Konsumenten zu erheben und dem SollSelbstbild gegenüberzustellen. Ein solches Konzept eines hypothetischen Ideals erlaubt im Markenverbund vor allem Aussagen über die markenspezifischen Erwartungen der Konsumenten gegenüber einer Marke. Ebenso wie bei der $\mathrm{Be}$ stimmung der Ist-Images können Soll-Images direkt oder indirekt erhoben werden:

- Bei direkten Verfahren wird nach den Ausprägungen einer Wunsch- oder idealen Marke gefragt, wozu der Befragte die Idealpunkte in den einzelnen Eigenschaften auf gleichen Skalen angeben muss, auf den auch die Realausprägungen abgetragen werden (bspw. das hier verwendete Semantische Differential).

- Bei indirekten Methoden wie der Multidimensionalen Skalierung werden von den Befragten keine direkten Angaben über Idealmarken verlangt, sondern durch die Erhebung von Präferenzdaten wird im Nachhinein auf die Ausprägungen der Idealmarken geschlossen, die den Präferenzen implizit zugrunde liegen. ${ }^{449}$

Bei direkten Verfahren ist allerdings davon auszugehen, dass Konsumenten stets eine hohe oder auch extremale Ausprägung für jede Marke auf der verwendeten Ratingskala präferieren, so dass eventuell bestehende Unterschiede der Idealvorstellungen zwischen den Marken nicht transparent werden. Extremal gewünschte Ausprägungen sind insbesondere bei solchen Rating-Skalen zu vermuten, bei denen sich die Eigenschaftsausprägungen konkret-wörtlich auf den Meinungsgegenstand beziehen (der Befragte möchte beispielsweise eine möglichst hohe Qualität zu einem möglichst niedrigen Preis), so dass es in einem solchen Fall einer geeigneten Operationalisierung der konsumentenseitigen Soll-Images bedarf. Dabei kann angenommen werden, dass Konsumenten nicht allen Eigenschaften eine exakt gleich hohe Bedeutung beimessen. Vor diesem Hintergrund besteht ein Operationalisierungsansatz der Idealimages der Konsumenten darin,

449 Vgl. Freter, H., Markenpositionierung. Ein Beitrag zur Fundierung markenpolitischer Entscheidungen auf der Grundlage psychologischer und ökonomischer Modelle, a.a.O., S. 114. 
die Extremausprägungen mit zu erhebenden markenspezifischen Bedeutungsgewichten der einzelnen Eigenschaftsausprägungen zu verknüpfen, also: ${ }^{450}$

$$
\hat{c}_{j i}=w_{j i}{ }^{*} e_{j i} \max
$$

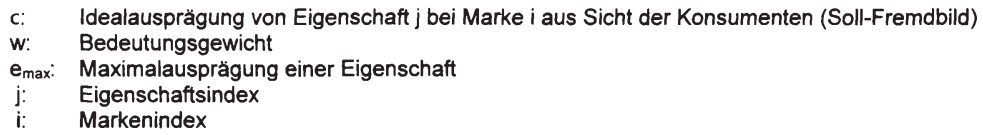

Da bei dem gewählten Semantischen Differential nicht von konkret-wörtlichen, sondern metaphorischen Eigenschaftsausprägungen ausgegangen wird, lassen sich Extremalausprägungen bei der Bestimmung der zu erhebenden SollVorstellungen im Gegensatz zur Verwendung direkt auf den Meinungsgegenstand bezogener Ausprägungen zwar weitgehend vermeiden. Allerdings ist dann davon auszugehen, dass Konsumenten bei der Erhebung eines Soll-Images im Vergleich zum Ist-Image aufgrund der Metaphorik schnell überfordert werden.

Liegen Idealimages der Konsumenten vor, sind diese den Idealvorstellungen der Portfolioleitung gegenüberzustellen. ${ }^{451} \mathrm{Im}$ Falle einer Erhebung der Idealimages auf Basis des vorgestellten Semantischen Differentials sind somit die Abweichungen zwischen den konsumenten- und herstellerseitigen Idealvorstellungen langfristig in Einklang zu bringen:

$$
D_{i}=\sqrt{\sum_{j=1}^{24}\left(c_{j i}-\hat{c}_{j i}\right)^{2}} \rightarrow \min !
$$

$D_{\mathrm{i}}$ : Gesamtdistanz zwischen Idealimage und Soll-Selbstbild

c: Idealausprägung von Eigenschaft j bei Marke i aus Sicht der Portfolioleitung (Soll-Selbstbild)

c: Idealausprägung von Eigenschaft j bei Marke i aus Sicht der Konsumenten (Soll-Fremdbild)

Werden Abweichungen zwischen den Idealvorstellungen identifiziert, ist das Selbstbild entweder seitens der Portfolioleitung zu korrigieren (insb. bei reaktiver Positionierung) oder langfristig im Markt zu etablieren (insb. bei aktiver Positionierung), was letztlich eine Anpassung der Idealimages im Zeitablauf seitens der

Vgl. hier auch Waltermann, B., Internationale Markenpolitik und Produktpositionierung: markenpolitische Entscheidungen im europäischen Automobilmarkt, a.a.O., S. 118ff.; Freter, H., Markenpositionierung. Ein Beitrag zur Fundierung markenpolitischer Entscheidungen auf der Grundlage psychologischer und ökonomischer Modelle, a.a.O., S. 113. 
Konsumenten erfordert. Betont sei an dieser Stelle nochmals, dass kurzfristig durchaus eine Diskrepanz zwischen den anbieter- und nachfragerseitigen Idealvorstellungen bewusst "toleriert" werden kann, falls Eigenschaftsdimensionen besetzt werden sollen, die dem Kunden bisher unbekannt sind, die von der Portfolioleitung aber als relevant angesehen werden.

Zusammenfassend ist die Ermittlung der Idealvorstellungen mit besonderen methodischen Problemen verbunden. Während Befragte bei direkter Erfassung hinsichtlich ihres Abstraktionsvermögens schnell überfordert werden, erweist sich der Einsatz komplexer indirekter Verfahren häufig als problematisch. ${ }^{452}$ Unter Berücksichtigung der festgestellten Diskrepanzen zwischen Soll-Selbstbildern und IstFremdbildern der hier betrachteten Portfoliomarken sollen vor diesem Hintergrund weitere Einflussgrößen auf die Markenpositionierung dahingehend untersucht werden, in welcher Weise sie die ermittelten Ist-Positionen erklären und gleichzeitig Ansatzpunkte bzw. Restriktionen für die Ableitung einer Soll-Positionierung aufzeigen können.

\subsection{Analyse ausgewählter Einflussgrößen auf die Markenpositionierung}

Für die Analyse der Ideal- und Realpositionen sind eine Reihe psychographischer Variablen relevant, die durch die Beeinflussung der Wahrnehmung (kognitive Komponente) und Bewertung (affektive Komponente) eine prädisponierende Wirkung (konative Komponente) auf den Konsumenten ausüben. ${ }^{453}$ Überdies sind die zentralen Kaufmotive im Hinblick auf eine potentielle Beeinflussung der Ideal- und Realpositionen zu analysieren. Dabei liegt es nahe, dass sich Abweichungen im Kaufverhalten der Käufer auch aufgrund unterschiedlicher Besitzverhältnisse und Einstellungen zum Automobil ergeben.

452 Zu einer kritischen Würdigung vgl. Shocker, A. D., Srinivasan, V., Multiattribute Approaches for Product Concept Evaluation and Generation: A Critical Review, in: Journal of Marketing Research, Vol. 16, May 1979, S. 168ff. Zur Verwendung von Bedeutungsgewichten vgl. Waltermann, B., Internationale Markenpolitik und Produktpositionierung: markenpolitische Entscheidungen im europäischen Automobilmarkt, a.a.O., S. $116 \mathrm{ff}$.

$\mathrm{Vgl}$. zur Affektion, Kognition und Konation Meffert, H., Marketing: Grundlagen marktorientierter Unternehmensführung: Konzepte - Instrumente - Praxisbeispiele, a.a.O., S. 114 


\subsection{Markenbekanntheit und -wissen}

Eine Marke wird nur dann als Kaufalternative in Frage kommen, wenn diese von vornherein oder aufgrund aktiver Suchprozesse bekannt ist. Dabei liegt der passiven Bekanntheit (Recognition-Messung) einer Marke die Fähigkeit des Konsumenten zugrunde, die Marke durch ein Signal wiederzuerkennen, wohingegen die aktive Markenbekanntheit (Recall-Messung) die ungestützte Erinnerung des Konsumenten an die Marke misst und entscheidend für die Bildung des evoked-set beim Konsumenten ist. ${ }^{454}$ Dass die Kenntnis einer Marke die Markenstärke fördert, ist von vielen Autoren belegt worden. ${ }^{455}$ Allerdings reicht für die konkrete Beurteilung einer Marke die alleinige Kenntnis nicht aus, vielmehr muss der Konsument auch die Ausprägungen der Marke in den relevanten Eigenschaften bewerten können, so dass er über ein entsprechendes Wissen - ausgedrückt als Markenvertrautheit - bezüglich der Marke verfügen muss. ${ }^{456}$ Ist der Konsument mit den

Insbesondere bei Low-involvement-Produkten (Zigaretten, Benzin) steigt mit relativ höherem Bekanntheitsgrad die Wahrscheinlichkeit, dass diese Marken anderen bevorzugt werden. In diesem Zusammenhang ist zu berücksichtigen, dass die Markenbekanntheit und das Wissen über eine Marke neben den kommunikativen Maßnahmen maßgeblich durch die Dauer der Marktpräsenz einer Marke im Markt determiniert wird. So weisen Marken in fortgeschrittenen Stadien ihres Lebenszykluses häufig überlegene Bekanntheitsgrade aus, wobei jedoch bisher weniger bekannte, innovative Marken Modelle etablierter Marken in ihren wahrgenommenen Eigenschaftsausprägungen durchaus übertreffen können. WALTERMANN führt an, dass man in diesem Zusammenhang bei Automobilen von einem Kumulationseffekt sprechen kann, da sich der Bekanntheitsgrad des jeweiligen Modells während des Lebenszyklus häufig bis zum Ausscheiden aus dem Markt kontinuierlich erhöht. Vgl. Waltermann, B., Internationale Markenpolitik und Produktpositionierung: markenpolitische Entscheidungen im europäischen $\mathrm{Au}$ tomobilmarkt, a.a.O., S. 166.

455 So bezieht sich OWEN auf eine empirische Studie, in der ein Marken-Ranking in 14 unterschiedlichen Ländern nach dem Markenwert vorgenommen wurde. Er kommt zu dem Resultat, dass ein Markenwert vor allem durch einen hohen Bekanntheitsgrad und eine positive Wertschätzung des Produktes gekennzeichnet ist. Vgl. Owen, S., The Landor Image Power Survey: A Global Assessment of Brand Strength, in: Brand Equity \& Advertising. Advertising's Role in Buliding Strong Brands, Aaker, D. A., Biel, A. L. (Hrsg.), Hillsdale 1993, S. $11 \mathrm{ff} ;$ KELLER führt an, dass Bekanntes Unbekanntem vorgezogen wird, selbst wenn keine weiteren Markenassoziationen vorliegen. Keller, K. L., Conzeptualizing, Measuring, and Managing Customer-Based Brand Equity, a.a.O., S. 3.

Die Bedeutung rationaler Produktinformationen wird durch die Anzahl der Automobilfachzeitschriften, Messen und dem öffentlichen Interesse bei Produktneueinführungen bestätigt. Die Intensität der Nutzung rationaler Produktinformationen kann in den verschiedenen Phasen des Pkw-Kaufentscheidungsprozesses modellhaft verdeutlicht werden: 1. Phase der unstrukturierten, passiven Informationsaufnahme [gelegentliche, situative Nutzung von Autozeitschriften]; 2. Phase der strukturierten und gezielten Informationssuche [bewusster Kontakt mit Autozeitschriften]; 3. Phase der Entscheidungsfindung und Kaufabschluss [Starke personale Kommunikation, Studium von Prospekten, Händlerkontakte]; 4. Phase des Wartens auf Auslieferung [Nutzung von Testberichten zur Kaufbestätigung]; 5. Phase der ersten konkreten 
Marken nicht ausreichend vertraut, kann er die Marken auch nur unzulänglich beurteilen.

Kennt der Konsument die Marke hingegen, können sich Einstellungen als innere Bereitschaft (Prädisposition) eines Individuums, auf eine bestimmte Marke positiv oder negativ zu reagieren, bilden. Die abstraktesten Markenassoziationen sind die globalen Markeneinstellungen, die u.a. in der Markensympathie zum Ausdruck kommen und die übergreifenden, wertenden Globalüberzeugungen gegenüber einer Marke kennzeichnen. Abb. 46: stellt die genannten Größen für die hier betrachteten Portfoliomarken mit den jeweiligen Hauptwettbewerbern dar. ${ }^{457}$

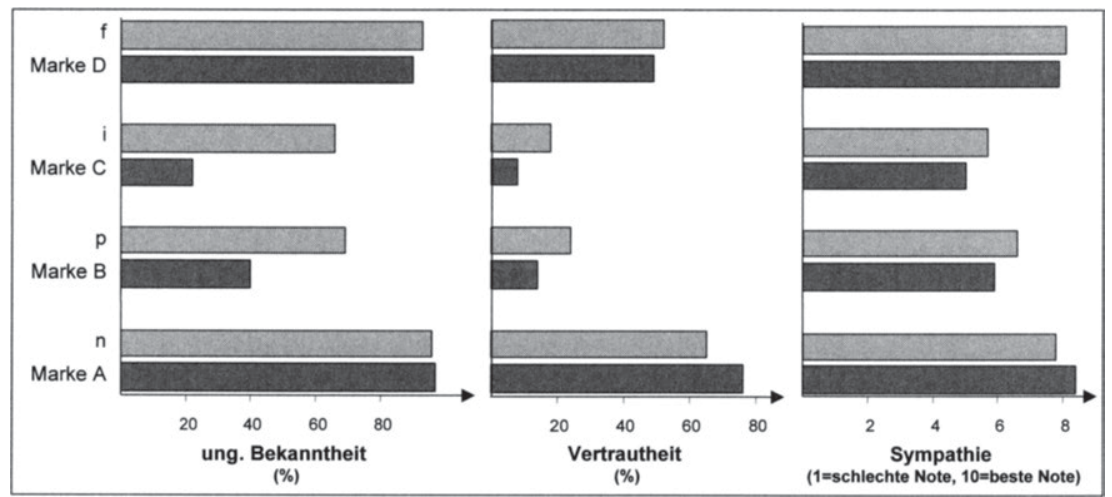

Abb. 46: Markenbekanntheit, - vertrautheit und -sympathie

Im Hinblick auf die ungestützte Markenbekanntheit weist die Marke A mit 97 Prozent den höchsten Wert auf. Auffällig sind die vergleichsweise geringen Werte bei C (22 Prozent) und B (40 Prozent). Während die Bekanntheitsgrade von A und D mit denen der Hauptwettbewerber vergleichbar sind, werden die Marken B und C

Erfahrung; 6. Phase der Produktnutzung im Alltag [Nutzung von Massen- und ZielgruppenMedien; gelegentliche situative Nutzung von Autozeitschriften]. Vgl. hierzu o.V., autofahren in deutschland 1999, a.a.O., S. 56f. Vgl. auch Tomczak, T., Müller, F., Kommunikation als zentraler Erfolgsfaktor der strategischen Markenführung, in: Thexis, Heft 6, 1992, S. 20.

Hierzu wurden 19982.607 Personen mündlich befragt. Dabei wurden folgende Fragen gestellt: ungestützte Bekanntheit: ${ }_{n}$ Nennen Sie mir bitte alle Automobilmarken, die ihrer Meinung nach hier in der BRD verkauft werden. Und welche noch? Gibt es weitere?"; Vertrautheit: "Welche der folgenden Feststellungen beschreibt am besten, wie gut sie die [Marke X] kennen?"; Markensympathie: „Welchen Gesamteindruck haben Sie von jeder der Marken?" (Studie: Bekanntheits- und Image Check). 
von den jeweiligen Wettbewerbern weit übertroffen. Betrachtet man überdies das bekundete Wissen der Befragten, so wird deutlich, dass im portfoliointernen Vergleich die Resultate überwiegend in Übereinstimmung mit der entsprechenden Markenbekanntheit stehen. Trotz wesentlich höherer ungestützter Bekanntheit der Hauptwettbewerber weisen die Marken B und C jedoch nahezu gleich hohe Sympathiewerte auf, was letztlich Ausdruck eines hohen zukünftigen Markenpotenzials sein kann.

Im Portfolioverbund liefern die Markenkenntnis bzw. Gesamteindrücke letztlich wichtige Anhaltspunkte zur Erklärung der Realimages und damit Fremdbilder der Markenidentitäten. So setzt Markensympathie Bekanntheit voraus, wobei die Sympathiewerte als Zeichen einer Globalüberzeugung gegenüber einer Marke ein positives Image zu fördern vermögen. ${ }^{458} \mathrm{Im}$ Umkehrschluss deuten somit vergleichsweise geringe Sympathiewerte auch auf unterdurchschnittliche Ausprägungen der Markenimages hin. ${ }^{459}$

\subsection{Produkt- und Händlerzufriedenheit}

Grundsätzlich sollten bei Positionierungsentscheidungen solche Markeneigenschaften besondere Beachtung finden, die im Vergleich zum Wettbewerb eine hohe Zufriedenheit bei den Besitzern auslösen können. Denn zentrale Ursache von Kommunikationsgaps sind Produktversprechen, die letztendlich nicht realisiert werden können. So weisen auch verschiedene Autoren auf die Gefahr hin, im Rahmen der Markenpositionierung ein Versprechen zu formulieren, welches hohe Erwartungen beim Käufer auslöst, die durch das Produkt letztlich aber nicht erfüllt werden können. ${ }^{460}$ Weisen die der Positionierung zugrunde gelegten Eigenschaf-

Zur Markensympathie als Bestandteil des Fremdbilds einer Marke vgl. Meffert, H., Burmann, Ch., Identitätsorientierte Markenführung - Grundlagen für das Management von Markenportfolios, a.a.O., S. 36 .

Vgl. hier die mehrdimensionale Imageanalyse auf S. 174. Auch COLVIN, HEELER, THORPE haben in einem Experiment eine Korrelation zwischen Produktkenntnis und Beurteilung nachgewiesen. Die Autoren bildeten zwei Versuchsgruppen, von denen der einen lediglich Fotos und der Markenname eines neuen Automobils vorgelegt wurde, der anderen darüber hinaus detailliertes Informationsmaterial. Die ausführlich informierte Gruppe gab später in allen Eigenschaften eine durchschnittlich positivere Beurteilung des Produktkonzepts ab als die befragten mit geringerem Informationsniveau. Vgl. Colvin, M., Heeler, R., Thorpe, J., Developing International Advertising Strategy, in: Journal of Marketing, Vol. 44, Heft 3, 1980, S. 73ff.

Vgl. Hamman, P., Schuchard-Fischer, Chr., Die Verhaltensrelevanz von Nachkaufdissonanzen im Automobilmarkt, in: Marktforschung im Automobilsektor, Dichtl, E., Raffée, H., Potucek 
ten hohe Zufriedenheitswerte auf, vermag der Käufer das Markenversprechen auch in geeigneter Weise nachzuvollziehen.

Die Markenzufriedenheit verkörpert somit das Ausmaß, in dem der Konsument seine Erwartungen bzw. Ansprüche durch die Marke erfüllt sieht. ${ }^{461}$ BAUER und später auch BURMANN haben für den Automobilsektor empirisch belegt, dass eine hohe Zufriedenheit der Besitzer hinsichtlich verschiedener Eigenschaften zur Stärkung der Markenloyalität führt. ${ }^{462}$ BURMANN stellt dabei heraus, dass fast die Hälfte (45,9 Prozent) der Produktzufriedenheit auf die Zufriedenheit mit den Leistungen des Händlers zurückgeführt werden kann, der Rest (54,1 Prozent) auf die Zufriedenheit mit spezifischen Fahrzeugeigenschaften, wie z. B. Benzinverbrauch oder Motorleistung. Für diese und eine Reihe anderer Kriterien sind in Abb. 47 die Zufriedenheitswerte von Besitzern der verschiedenen Portfoliomarken gegenübergestellt. $^{463}$

Die Analyse der Produktzufriedenheiten im Portfoliovergleich verdeutlicht die höchsten Zufriedenheitswerte für die Marke D, die geringsten bei der Marke B. Bemerkenswert im Vergleich zu den Imagewerten ist die hohe Zufriedenheit der Käufer mit der Marke C. Dabei weist die Marke C insbesondere in rationalen Eigenschaften sehr hohe Werte auf, so dass diese als Grundlage der Positionierung dienen können. Interessante Resultate ergeben sich insbesondere in der Bewertung der Preiswürdigkeit. Hier stehen die Urteile der Fahrer der verschiedenen Marken im Einklang mit den Images der jeweiligen Marken. Ein etwas anderes

V., Schriftenreihe des Verbandes der Automobilindustrie e.V. (VDA), Nr. 40, Frankfurt 1983, S. 69.

Vgl. Bauer, H. H., Die Determinanten der Markentreue beim Automobilkauf, in: Marktforschung im Automobilsektor, Dichtl, E., Raffée, H., Potucek, V., Schriftenreihe des Verbandes der Automobilindustrie e.V. (VDA), Nr. 40, Frankfurt 1983, S. 26.

Vgl. Bauer, H. H., Die Determinanten der Markentreue beim Automobilkauf, a.a.O., S. 26ff; Burmann, Ch., Konsumentenzufriedenheit als Determinante der Marken- und Händlerloyalität - Das Beispiel der Automobilindustrie, a.a.O., S. 249-258. Vgl. hier auch Korte, Ch., Customer Satisfaction Measurement, Kundenzufriedenheitsmessung des Hersteller- und Handelsmarketing am Beispiel der Automobilwirtschaft, Frankfurt a. M. 1995; hierzu Bauer, H. H., Hermann, A., Hubert, F., Die Bestimmungsgrößen der Markentreue beim Pkw-Kauf: Ergebnisse einer empirischen Untersuchung, a.a.O., S. 165ff.

Die Zufriedenheitswerte wurden durch einer schriftlichen Befragung im Rahmen der NCBSStudie (1998) erhoben. Basis: Marke A: 3.917, Marke B: 1.358 , Marke C: 523 ; Marke D: 1.827; Gesamtmarkt: 41.363. Die Gesamtzufriedenheit wurde dabei vor der detaillierten Analyse einzelner Eigenschaften erhoben. 
Bild offenbart die Händlerzufriedenheit, bei der die Marke B den weitaus höchsten Wert aufzeigt. Für die sonstigen Portfoliomarken ergibt sich hier auch im Vergleich zum Gesamtmarkt eine eher unterdurchschnittliche Beurteilung. Ein Vergleich der Produktgesamtzufriedenheitswerte gegenüber den operativen Wettbewerbern offenbart schließlich, dass die im deutschen Markt noch jungen Marken $B$ und $C$ das Niveau ihrer Hauptkonkurrenten erreicht haben, die Marke $C$ den Konkurrenten i sogar übertroffen hat.

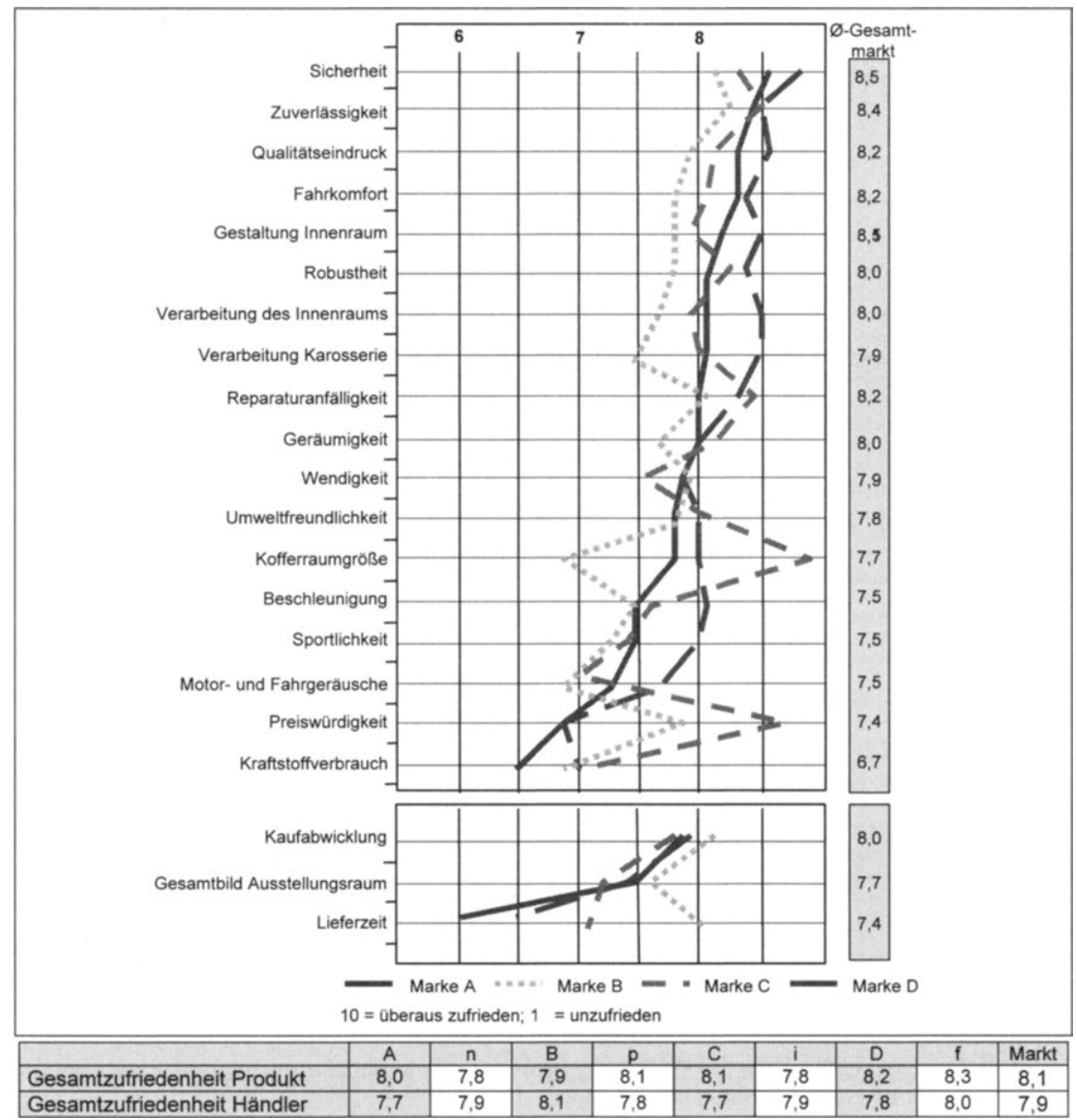

Abb. 47: Produkt- und Händlerzufriedenheit

Letztlich offenbart die Analyse der Zufriedenheitswerte prägnant eine Diskrepanz zwischen den Image- und Zufriedenheitswerten bei den Marken B und C: die allgemeine Wahrnehmung steht im Widerspruch zur eigentlichen Mayktleistung 
der Marken. Dies ist in erster Linie auf den Einfluss der Fremdimages zurückzuführen. Bei Marke $C$ korrespondieren die eher negativen Vorstellungsbilder der Fremdmarkenbesitzer offensichtlich nicht mit den auf der Produkterfahrung beruhenden positiven Urteilen der Käufer der Marke $C .{ }^{464}$ Hingegen wird die Marke B von den Fremdmarkenbesitzern besser beurteilt als von den Käufern der Marke selbst.

\subsection{Kaufgründe}

Die unmittelbar nach dem Erwerb des Fahrzeugs erhobenen Kaufgründe reflektieren die aus Sicht des Käufers wahrgenommenen Stärken und Kompetenzen des Modells, die zur Entscheidung für die betreffende Marke geführt haben. Allerdings ist eine Analyse der Kaufgründe gerade im Automobilbereich nicht zuletzt unter dissonanztheoretischen Gesichtspunkten vorzunehmen. ${ }^{465}$ Allgemein kommen Dissonanzen darin zum Ausdruck, dass Konsumenten nach dem Kauf eines Produktes an der Richtigkeit ihrer Entscheidung zweifeln und den Kauf als eine Störung des inneren Gleichgewichtes empfinden: Es ist das Wissen, durch die Entscheidung die Nachteile der gewählten Alternative hinzunehmen und nicht in den Genuss der Vorteile der ausgeschlagenen Alternativen zu kommen. Entsprechend sucht der dissonante Käufer nach Argumenten, die seine Entscheidung rechtfertigen, so dass beim erworbenen Produkt die positiven Aspekte besonders betont, bei den nicht gekauften Erzeugnissen negative Aspekte stärker in den Vordergrund gerückt werden. ${ }^{466}$

WALTERMANN weist darauf hin, dass das Fremdimage das Eigenimage in seiner Wirkung auf das Realimage immer überlagern wird, es sei denn, dass eine Marke in ihrem Segment über 50 Prozent Marktanteil auf sich vereinigen kann. Waltermann, B., Internationale Markenpolitik und Produktpositionierung: markenpolitische Entscheidungen im europäischen Automobilmarkt, a.a.O., S. 170.

Zur Einführung in die Dissonanztheorie und zugleich als Leitfaden durch die Literatur vgl. Frey, D., Die Theorie der kognitiven Dissonanz, in: Theorien der Sozialpsychologie, Frey, D. Irle, M. (Hrsg.), Band 1, Bern u.a. 1993, S. 243ff. Unter den gleichgewichtstheoretischen Ansätzen nimmt die Dissonanztheorie von FESTINGER einen besonderen Stellenwert ein. Vgl. Festinger, L., Theorie der kognitiven Dissonanz, Bern 1978; Hamman, P., Schuchard-Fischer, Chr., Die Verhaltensrelevanz von Nachkaufdissonanzen im Automobilmarkt, a.a.O., S. $59 f f$

Eine gängige Alternative, den Konsumenten zur Höherschätzung des gekauften Produktes zu bewegen, ist die Nachkauf-Werbung, welche die Besonderheiten des gekauften Produktes hervorheben kann (z. B. „Sie haben ein Produkt gewählt, dessen mattschwarze Oberflăche eine besonders schöne Wirkung hat. Damit Sie sich lange hieran erfreuen können, achten Sie bitte darauf, ..." (Montblanc VIP-Quickpen). Im einem Experiment von DONELLY und 
Bezüglich der Positionierung der Portfoliomarken können sich hieraus dennoch wichtige Anhaltspunkte darüber ergeben, welche Schlüsselfaktoren bislang die Kaufentscheidung zugunsten einer konkreten Marke determiniert haben. Aus diesem Grund sollen die in Abb. 48 verzeichneten Argumente beleuchtet werden, die nach Auskunft der Befragten zum Kauf der jeweiligen Marke geführt haben. ${ }^{467}$

\begin{tabular}{|c|c|c|c|c|c|c|c|c|c|c|}
\hline \multirow[b]{2}{*}{ Image, Prestige } & \multicolumn{2}{|c|}{ Marke A } & \multicolumn{2}{|c|}{ Marke B } & \multicolumn{2}{|l|}{ Marke C } & \multicolumn{2}{|l|}{ Marke D } & \multicolumn{2}{|c|}{ Ø-Markt } \\
\hline & 1 & 1,3 & a & 4,5 & 1 & 0,5 & I & 0,9 & 回 & 2,6 \\
\hline Komfort & $\mathbf{n}$ & 2,2 & $\mathbf{m}$ & 4,1 & 回 & 2,5 & $\mathbf{p}$ & 2,0 & $\square$ & 3,2 \\
\hline Wirtschaftlichkeit & - & 3,8 & $\mathbf{1}$ & 3,3 & 国 & 5,5 & I & 2,2 & 回 & 3,1 \\
\hline Høchstgeschw., sportl. & n & 4,3 & m & 7,4 & 国 & 5,2 & r & 2,9 & $\square$ & 5,2 \\
\hline Rat von Freunden & & 5,1 & ש & 7,0 & En & 8,3 & Wan & 13,6 & $\square$ & 5,1 \\
\hline Werkstatt/ KD & & 6,1 & w & 5,6 & mand & 9,8 & D & 4,7 & $\square$ & 6,9 \\
\hline Gegenwert für's Geld & $=$ & 6,8 & $=$ & 5,8 & mannan & 16,0 & Eanganas & 408 & 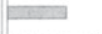 & 10,6 \\
\hline Preis & & 7,1 & - & 4,4 & Ex-2an & 31.7 & 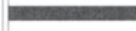 & 34,5 & $\square$ & 15,3 \\
\hline Verbrauch & & 9,6 & & 11,0 & 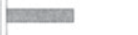 & 13,6 & 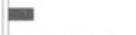 & 6,6 & $\square$ & 8,5 \\
\hline Ausstattungsniveau & = & 9,8 & $=$ & 11,3 & 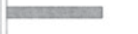 & 19,4 & Enanang & 26,4 & 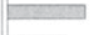 & 13,9 \\
\hline Gerăumigkeit & 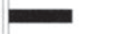 & 12,9 & a & 6,0 & एक्ष & 10,2 & Ea & 10,4 & $\square$ & 10,6 \\
\hline Styling & 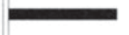 & 21,9 & & 36. & 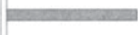 & 26,5 & 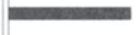 & 32,6 & & 26,6 \\
\hline Loyalitalt & . & $32 \sqrt{3}$ & E. & 29,2 & स्या & 15,8 & Ena & 13,9 & & 26.5 \\
\hline
\end{tabular}

\section{Abb. 48: Kaufgründe}

Die einzelnen Portfoliomarken werden aus unterschiedlichen Gründen gekauft. Bei Marke A wird insbesondere die Loyalität zur Marke betont. Die Loyalität ist jedoch für die Kennzeichnung einer bestimmten Markenposition nur von begrenztem Aussagewert, da sie auf keine konkrete Eigenschaftsdimension Bezug nimmt. Bei Marke D steht - trotzt hochpreisiger Positionierung - der Gegenwert für's Geld im Vordergrund. Die Verwendung von Gleichteilen im Portfolio scheint somit keine Auswirkungen auf die Wahrnehmung der Marke durch die Konsumenten zu haben. Die wesentlichen Anreize für die Marke B liegen im Styling und im Preis, wohingegen für Marke $C$ der Anschaffungspreis dominiert. Die Nennung des Preises als Kaufgrund korrespondiert mit den für die Marken ermittelten Assoziationen

IVANCEVICH war der Anteil von Konsumenten, die (ohne große Verluste) vom Kauf eines Autos zurücktraten, erheblich geringer, wenn sie nach dem Kauf einer Werbung mit Informationen über die Vorteile des gekauften Autos ausgesetzt waren. Die Rückgabequote bei den Käufern, die gezielt einer Nachkauf-Werbung ausgesetzt wurden, betrug 2,4 gegenüber 5,2 Prozent für Käufer, die keine Werbung erhielten. Vgl. Kroeber-Riel, W., Weinberg, P., Konsumentenverhalten, a.a.O., S. $187 f$.

Die Kaufgründe wurden durch einer schriftlichen Befragung im Rahmen der NCBS-Studie (1998) erhoben. Basis: Marke A: 3.908, Marke B: 1.351, Marke C: 522; Marke D: 1.827; Gesamtmarkt: 40.930 . 
(primär rationale Eigenschaftsdimensionen). Ein überraschend hoher Stellenwert für die Kaufentscheidung bei Marke $\mathrm{C}$ kommt dem Styling zu, welches in einem gewissen Widerspruch zum Selbstbild der Marke steht. Auffällig hoch ist die Nennung des After-Sales Services als Kaufgrund bei der Marke B, ein Ergebnis, das in Übereinstimmung mit der bekundeten Händler-Zufriedenheit dieser Marke steht.

Die Analyse der Kaufgründe vermag insgesamt wertvolle Hinweise für erfolgsversprechende Positionierungsansätze im Portfolioverbund zu liefern. Die Kaufgründe reflektieren die zentralen Stärken und Kompetenzen der Marken, welche auch von den Konsumenten nachvollzogen werden können. Zentrales Ziel im Portfolioverbund muss es sein, möglichst differenzierte Kaufgründe als Indikator verschiedenartiger Kompetenzen der Portfoliomarken sicherzustellen.

\subsection{Käuferstruktur und einstellungen}

In gesättigten Märkten kommt aufgrund der hohen Bedeutung des Ersatzbedarfs der Sicherung der Markenloyalität ein zentraler Stellenwert zu. Hieraus leitet sich das Bestreben ab, der demographischen Käuferstruktur des bestehenden Besitzerstamms der einzelnen Portfoliomarken bei der Formulierung der strategischen Rollen der Marken im Wettbewerb weitgehend Rechnung zu tragen. Vor diesem Hintergrund sind im Rahmen des Mehrmarkencontrolling die Käuferstrukturen über die verschiedenen Portfoliomarken hinweg zu vergleichen, um Unterschiede der Besitzerstrukturen herauszuarbeiten (vgl. Abb. 49). ${ }^{468}$

Ein besonderer Stellenwert kommt dabei den Einkommensverhältnissen und damit korrelierend dem Ausbildungsgrad bzw. der Berufsstruktur zu. So stützt beispielsweise der hohe Anteil weiblicher Käufer, das vergleichsweise geringe Durchschnittsalter sowie der hohe Anteil an Mehrwagenhaushalten - offenbar findet die Marke $B$ insbesondere vor dem Hintergrund des niedrigen Preises als Zweitwagen Verwendung - das Bestreben einer eher emotionalen Positionierung der Marke B. Hingegen indiziert bei Marke $C$ der geringe Anteil an Mehrwagenhaushalten in Verbindung mit einem niedrigen Preis eine Nutzung als Hauptwagen, wobei ein

468 Demographika entsprechend einer schriftlichen Befragung im Rahmen der NCBS-Studie (1998). Basis: Marke A: 3.908, Marke B: 1.351, Marke C: 522; Marke D: 1.827; Gesamtmarkt: 40.930 . 
hoher Anteil an Arbeitern verbunden mit geringeren Einkommensverhältnissen eine rationale Positionierung der Marke durchaus plausibel erscheinen lässt.

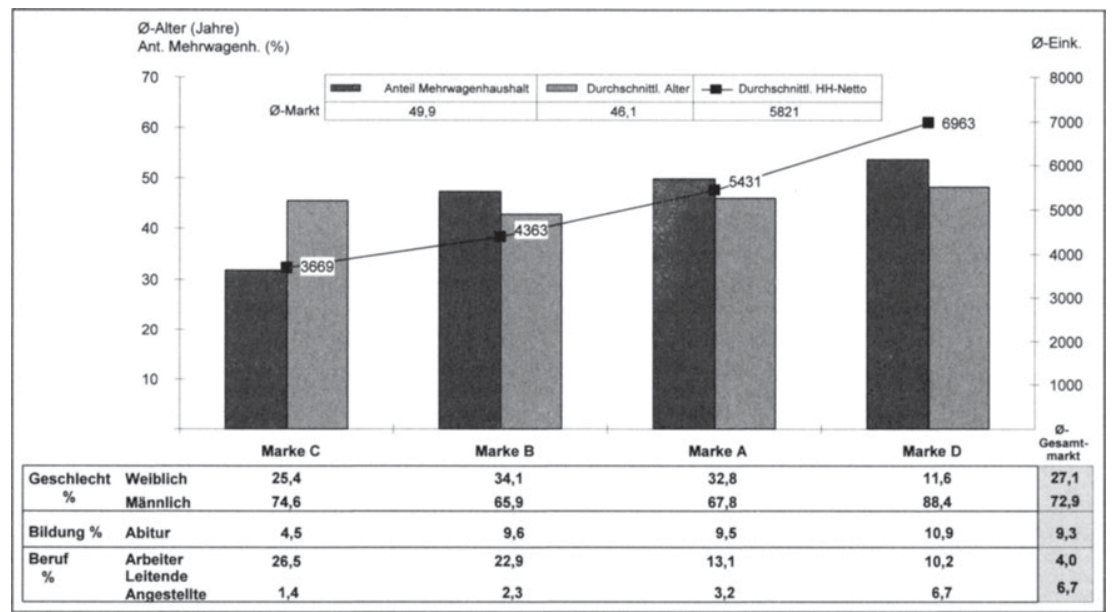

Abb. 49: Käuferstruktur der Portfoliomarken

Die individuelle Kaufbereitschaft eines Konsumenten hängt nicht zuletzt davon ab, inwieweit sich der potenzielle Käufer mit den typischen Besitzern einer konkreten Marke identifizieren kann. „Den unterschiedlichen Marken-Images eines Produktbereichs entsprechen im Idealfall psychologisch relativ reine charakterologische Typen, die aufgrund ihrer spezifischen Erlebnisweise eben zu jener bestimmten Markenpersönlichkeit hingefunden haben. ${ }^{4469}$ Letztlich strebt der Konsument in Bezug auf das Markenwahlverhalten grundsätzlich eine Kongruenz von Markenimage und Selbstimage bzw. Selbstvorstellung an, wobei sich die Selbstvorstellungen aus Werten, Einstellungen, Emotionen und Bedürfnissen zusammensetzen, die der Konsument gelernt hat und welche wesentlich durch sein gesellschaftliches Umfeld geprägt werden.

In diesem Zusammenhang ist es Zielsetzung von Lebensstiltypologien, die Lebenswelt von Zielgruppen unter Berücksichtigung sich wandelnder Einstellungen

469 Beck zitiert bei Freter, H., Markenpositionierung. Ein Beitrag zur Fundierung markenpolitischer Entscheidungen auf der Grundlage psychologischer und ökonomischer Modelle, a.a.O. S. 107. 
und Wertorientierungen möglichst adäquat zu erfassen. ${ }^{470}$ Eine der bekanntesten Lebensstiltypologien stellt der Milieu-Ansatz dar, der eine Segmentierung der bundesdeutschen Bevölkerung in kombinierte Werte- und Sozialschichtgruppen vornimmt.

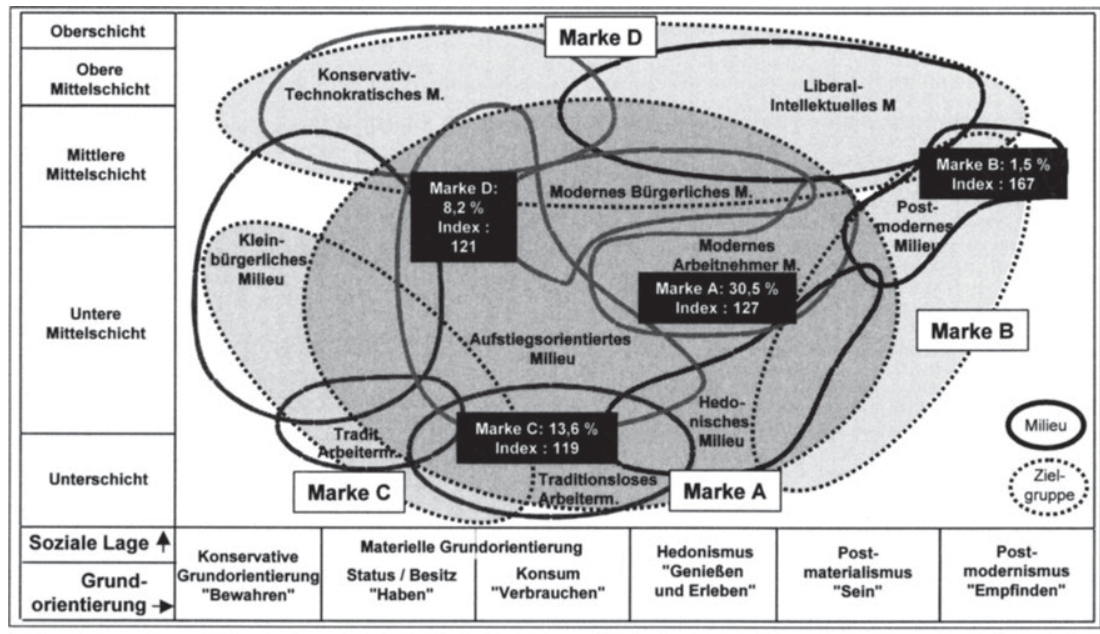

\begin{tabular}{|l|c|c|c|c|c|c|c|c|}
\hline \multicolumn{1}{|c|}{ Milieu } & \multicolumn{2}{c|}{ Marke A } & \multicolumn{2}{c|}{ Marke B } & \multicolumn{2}{c|}{ Marke C } & \multicolumn{2}{c|}{ Marke D } \\
\hline & $\%$ & Index & $\%$ & Index & $\%$ & Index & $\%$ & Index \\
\hline Konservativ-Technokratisches & 26,8 & 112 & 0,9 & 100 & 10,4 & 101 & 7,1 & 104 \\
\hline Kleinbürgerliches & 17,5 & 73 & 0,3 & 33 & 14,6 & 102 & 7,1 & 104 \\
\hline Traditionelles Arbeitnehmer & 18,7 & 78 & 0,7 & 78 & 5,5 & 122 & 4,3 & 63 \\
\hline Traditionsloses Arbeitnehmer & 20,1 & 84 & 0,8 & 89 & 13,6 & 119 & 5,6 & 82 \\
\hline Aufstiegsorientiertes & 27,2 & 113 & 1,3 & 144 & 18,2 & 100 & 8,2 & 121 \\
\hline Modernes Arbeitnehmer & 30,5 & 127 & 1,3 & 144 & 13,3 & 175 & 6,5 & 96 \\
\hline Modernes Bürgerliches & 19,9 & 110 & 1,2 & 133 & 10,4 & 135 & 8,0 & 118 \\
\hline Hedonistisches & 22,6 & 94 & 0,7 & 78 & 3,6 & 33 & 6,7 & 99 \\
\hline Postmodernes & 24,6 & 103 & 1,5 & 167 & 1,6 & 34 & 5,7 & 84 \\
\hline Liberal-Intellektuelles & 26,2 & 109 & 0,7 & 78 & 8,8 & 83 & 6,0 & 88 \\
\hline Lesebeispiel: Am Pkw-Bestand im knservativ-technkratischen Milieu in Deutschland hat die Marke A $1996 / 97$ einen \\
Anteil von 26,8 Prozent, uber alle Milieus einen von 24 Prozent; Index: 26,8/24=112
\end{tabular}

Abb. 50: Die Marken in den sozialen Milieus in Deutschland

470 Unter Lebenswelt werden dabei alle relevanten Erlebnisbereiche verstanden, mit denen die Personen täglich zu tun haben, wie etwa Arbeit, Freizeit, Konsum etc. und die wesentlich zur Entwicklung und Veränderung von Einstellungen, Werthaltungen und Verhaltensmustern beitragen. Vorreiter bei der Nutzung des SINUS-Milieus waren Hersteller der Automobilindustrie, die bereits seit Mitte der achtziger Jahre versuchten, ihre Modellpolitik auf die relevanten Abnehmergruppen auszurichten. 
Durch eine Verknüpfung der Milieus mit Daten des beobachteten Kaufverhaltens lässt sich für Automobilhersteller in einfacher Form eine Verteilung und Beschreibung der Marken durch die jeweiligen Milieus erzielen. ${ }^{471}$ Abb. 50 verdeutlicht in diesem Zusammenhang die realisierten (schwarz hinterlegt) sowie die im Rahmen der Leitbildformulierung angestrebten Positionen (weiß hinterlegt) der betrachteten Marken in den sozialen Milieus Deutschlands. ${ }^{472}$

Grundsätzlich finden sich die einzelnen Marken in allen Milieus wieder, wobei sich allerdings durchaus Schwerpunkte identifizieren lassen. Die Darstellung erweist sich dabei weitgehend konsistent gegenüber den erörterten demographischen Daten. Besitzer der Marke $C$ finden sich überwiegend im preissensiblen Arbeitermilieu wieder, die der Marke $D$ vor allem im kaufkräftigen aufstiegsorientierten bzw. modern-bürgerlichen Milieu. ${ }^{473}$ Auffällig ist überdies die breite Milieuabdekkung der Marke A, welche Überschneidungen zu allen anderen Marken aufweist. Dies deutet darauf hin, dass sich die Marke A nicht unbedingt mit einem konkreten Fahrertyp in Verbindung bringen lässt, was als Ausdruck einer gewissen Statusneutralität gewertet werden kann. Schwerpunkt der Marke B ist schließlich das Postmoderne Milieu, welches sich durch Originalität, Authentizität und Expressivität beschreiben lässt und damit dem Charakter der Marke B grundsätzlich gerecht wird. ${ }^{474}$ Die Abb. 50 verdeutlicht letztlich prägnant die Überschneidungen der Marken in den sozialen Milieus und damit die Gefahr möglicher Substitutionseffekte im Portfolio.

471 Allerdings bleibt der Milieu-Ansatz aufgrund einer vielfach postulierten mangelnden Aussagekraft für das Marketinginstrumentarium nicht unkritisiert. Vgl. hierzu etwa Stegmülier, B., Internationale Marktsegmentierung als Grundlage für internationale Marketingkonzeptionen, Bergisch-Gladbach u. a., 1995, S. 269ff; o. V., Lifestyle-Typologien helfen dem Marketing kaum, in: w\&v, Heft 3, 1992, S. $12 \mathrm{ff}$.

472 Der Untersuchung aus 1996/97 liegt für die Marken A, B und D eine Fallzahl in Höhe von 16.588 zugrunde. Im Gegensatz dazu wurden bei Marke $C$ keine Bestandsgrößen, sondern Neuwagenkäufer eines Modells betrachtet. Aus diesem Grund sind die Zahlen im Vergleich zu Bestandsgrößen größer. So sind 10,4\% aller X-Modell Neuwagenkäufer dem konservativtechnokratischen Milieu zuzurechnen, welches einen Anteil von 10,3\% am deutschen MilieuModell hat. Index: 10,4/10,3=101. Überdies liegt die betrachtete Fallzahl der Marke $C$ nur bei 311.

$473 \mathrm{Zu}$ beachten ist, dass sich einige Milieus wie etwa das traditionslose Arbeitermilieu auch als typische Gebrauchtwagenkäufer-Milieus bezeichnen lassen.

474 Zur inhaltlichen Konkretisierung der einzelnen Milieus vgl. auch Meffert, H., Marketing: Grundlagen marktorientierter Unternehmensführung: Konzepte - Instrumente - Praxisbeispiele, a.a.O., S. 194. 
Wichtige Erklärungshinweise liefern in diesem Zusammenhang abschließend auch die Einstellungen der Konsumenten zum Automobil. In Abb. 51 sind die Einstellungen der Neuwagenkäufer zum Auto in aggregierter Form dargestellt. ${ }^{475}$

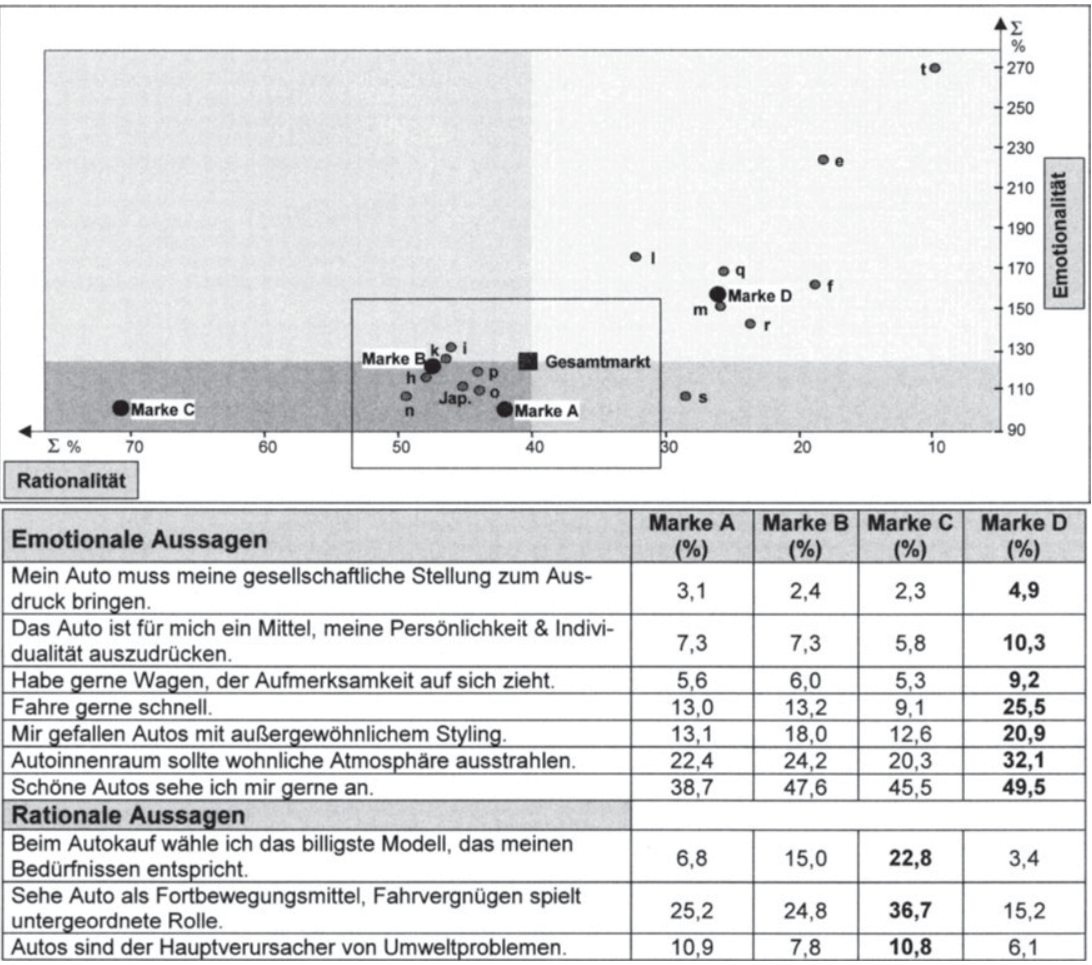

Abb. 51: Positionierungsportfolio aufgrund der Einstellungen der Neuwagenkäufer zum Auto

Hierzu wurden die von den Neuwagenkäufern geäußerten Einstellungen nach emotionalen und rationalen Äußerungen kategorisiert. Besonders deutlich wird die rationale Einstellung der Käufer der Marke $C$, welche das Auto vor allem als Fortbewegungsmittel sehen und dem Fahrvergnügen eine untergeordnete Bedeutung

475 Die Einstellungen zum Automobil wurden durch eine schriftliche Befragung im Rahmen der NCBS-Studie (1998) erhoben. Basis: Marke A: 3.917, Marke B: 1.358, Marke C: 523; Marke D: 1.827; Gesamtmarkt: 40.930. 
zukommen lassen. Die Käufer des strategischen Wettbewerbers (Marke s) lassen sich analog durch eine geringe Emotionalität charakterisieren, weisen jedoch eine weitaus geringere Preissensibilität auf. Fast spiegelbildlich zur Marke $C$ kennzeichnet die Käufer der Marke D eine eher emotionale Einstellung, wohingegen rationale Einstellungsdimensionen eine untergeordnete Rolle spielen. Die Nähe der Marke A zum Marktdurchschnitt indiziert den Volumencharakter der Marke. Da für emotionsbetonte Käufer der Preis zumeist nicht die kaufentscheidende Rolle spielt, offenbart die Analyse der Marke B das Spannungsfeld, als preisorientierte Trendmarke verstärkt emotionsbetonte Autofahrer anzusprechen.

Fasst man die Ergebnisse der Analyse zusammen, lässt sich zeigen, inwieweit die jeweiligen Soll-Selbstbilder als formulierte Idealpositionen der Marken mit den derzeit realisierten demographischen, psychographischen und verhaltensbezogenen Strukturen der Zielgruppen korrespondieren. Aufbauend auf den Informationen des Mehrmarkencontrolling ist dann zu entscheiden, inwieweit Umpositionierungen bzw. Positionsfestigungen zur Verbesserung der Zielerreichungsgrade des Gesamtportfolios vorgenommen werden sollten. Die Einzelergebnisse liefern somit nicht nur wertvolle Erklärungshinweise für die im Rahmen der Positionierungsanalyse ermittelten Ist-Positionen, sondern auch Ansatzpunkte für die Ableitung gewünschter Soll-Positionierungen.

Da sich die Entscheidungen der dezentralen Markeneinheiten im Portfolio auf ihre kontrollierten Variablen (insb. Produkt, Preis, Kommunikation und Distribution) beziehen, kommt es unabhängig der Positionierungen auch mit FRETER darauf an, „....Verbindungen zwischen den sich ergebenden Dimensionen [der Positionierung, Anm. d. Verf.] und dem Einsatz der Marketing-Instrumente herzustellen . ${ }^{4476}$ Dabei gilt es im Rahmen des Mehrmarkencontrolling generell zu überprüfen, welchen Einfluss die Marketing-Instrumente auf die Markenwahrnehmung bzw. das Ergebnis des Gesamtportfolios nehmen können.

476 Freter, H., Markenpositionierung. Ein Beitrag zur Fundierung markenpolitischer Entscheidungen auf der Grundlage psychologischer und ökonomischer Modelle, a.a.O., S. 124. 
3. Operatives Mehrmarkencontrolling: Zentrale Aspekte zur Deckung des Informationsbedarfs bei der Ausgestaltung der Instrumentalstrategien

Bei der Analyse der Ausgestaltung der Instrumentalstrategien im Markenportfolio stehen im Folgenden zentrale Problembereiche des Marketing-Mix im Vordergrund:

- bei den produktpolitischen Entscheidungen die Identifikation der zur Sicherstellung einer starken Markenidentität von jeder Marke selbstständig zu produzierenden Leistungskomponenten,

- bei den preispolitischen Entscheidungen die Preispositionierung sowie die Ableitung von Preiskorridoren im Portfolio,

- bei den kommunikationspolitischen Entscheidungen die Einflussnahme auf die Dimensionen des Wahrnehmungsraums zur Profilierung der Portfoliomarken sowie die Verteilung des Kommunikationsbudget im Portfolio und

- bei den distributionspolitischen Entscheidungen die Überprüfung der Markenidentifikation des Handels zur Sicherstellung eines leitbildgerechten Auftritts der Händlerorganisation als wesentliche Determinante der Markenwahrnehmung am POS.

\subsection{Leistungsvereinheitlichungs- und -differenzierungsanalyse}

Im Rahmen von Mehrmarkenstrategien besteht die Herausforderung, für jede Portfoliomarke ein markenspezifisches Leistungsbündel zu schaffen, das die Individualität jeder Marke widerspiegelt und sich damit von den übrigen Portfoliomarken eindeutig differenziert. Das konstitutive Identitätsmerkmal der Individualität legt es dabei einerseits nahe, die technische Ausgestaltung der Marke unabhängig von den sonstigen Marken vorzunehmen, um auf diese Weise die Ausprägung einer eigenständigen Markenpersönlichkeit zu unterstützten. Andererseits stellt im Markenportfolio jedoch die Identifikation und Nutzung möglicher Synergien eine unabdingbare Voraussetzung zur Sicherstellung der übergeordneten Rentabilitätsziele dar. Im Portfolio ergibt sich somit das Optimierungsproblem einer bestmöglichen Synergienutzung, ohne den auf eine Eigenprofilierung abstellenden Marktauftritt der einzelnen Marken nachhaltig zu gefährden.

Vor diesem Hintergrund stellt die Identifikation der zur Sicherstellung einer starken Markenidentität von jeder Marke selbstständig zu produzierenden Leistungskom- 
ponenten bzw. autonom auszuführenden Geschäftsprozesse eine zentrale Aufgabe dar. ${ }^{477}$ Im Spannungsfeld zwischen erforderlicher Differenzierung und kosteninduzierter Standardisierung ist das Controlling aufgefordert, konkrete Anhaltspunkte für produktpolitische Entscheidungen aufzuzeigen. Hierzu sind für jede Marke entsprechend den formulierten Selbstbildern massgebliche Unterscheidungsmerkmale festzulegen. In diesem Zusammenhang erweist es sich als zweckmäßig, auf Grundlage der Produktprogrammstrategie der einzelnen Portfoliomarken Differenzierungs- und Vereinheitlichungspläne im Portfolio festzulegen: $^{478}$

- Mit Differenzierungsplänen werden für jede Marke maßgebliche Unterscheidungsmerkmale bestimmt, in welchen sich die für den Kunden ausschlaggebenden Markendimensionen konkretisieren. Ein Differenzierungsplan enthält dabei Zielwerte für maßgebliche physische Unterscheidungsmerkmale einer jeden Marke.

- Der Vereinheitlichungsplan beschreibt den Umfang, in dem Marken und Modelle aus denselben physischen Elementen bestehen und umfasst explizite $\mathrm{Be}$ rechnungen der Entwicklungs- und Herstellkosten für jedes Produkt. Der Vereinheitlichungsplan enthält dabei jene Elemente, die aus der Wahrnehmung der Konsumenten markenübergreifend standardisiert werden können.

Da die subjektive Wahrnehmung der Konsumenten als Kriterium einer Differenzierung bzw. Standardisierung fungiert, ist für die Fundierung produktpolitischer Entscheidungen im Markenportfolio entscheidend, inwieweit bzw. in welcher Form Beziehungen zwischen objektiven Ausprägungen der einzelne Marken und den subjektiven Wahrnehmungen existieren. In diesem Zusammenhang hängt es vom jeweiligen Wahrnehmungsverhalten der Konsumenten $a b$, welche Verbindungen zwischen den Wahrnehmungsdimensionen und den objektiven Markeneigenschaften als Handlungsvariable der Produktpolitik bestehen.

Zur Bestimmung maßgeblicher Unterscheidungsmerkmale im Differenzierungsplan ist zu überprüfen, welche objektive Produkteigenschaft als Variable der Pro-

477 DIEZ bezeichnet die Produktqualität in diesem Zusammenhang auch als wichtigsten PenaltyFaktor im Automobilmarketing, da produktpolitische Schwächen kaum korrigierbar sind. Vgl. Diez, W., Das Handbuch für das Automobilmarketing - Strategien, Konzepte, Instrumente, 3. Aufl., Landsberg/Lech 1997, S. 38.

Vgl. Robertson, D., Ulrich, K., Produktplattformen: Was sie leisten, was sie erfordern in: Harvard Business Manager, No. 4, 1999, S. 80. 
duktpolitik mit welcher Wahrnehmungsdimension korrespondiert. Soll die Marke A als verantwortungsbewußte, innovative Marke wahrgenommen werden, Marke $B$ hingegen als jugendlich-dynamisch, sind die diesen Wahrnehmungsdimensionen zugrundeliegenden objektiven Produkteigenschaften zu konkretisieren, d.h. es sind konkrete Maßeinheiten zu bestimmen. Dabei hängt es vor allem von der jeweils betrachteten Eigenschaft $a b$, ob sich die objektiven und subjektiven Maßeinheiten entsprechen oder nicht.

Eine Übereinstimmung zwischen den Maßeinheiten liegt im Automobilbereich beispielsweise bei der Eigenschaft Höchstgeschwindigkeit vor, da sowohl die tatsächliche als auch die wahrgenommene Geschwindigkeit in $\mathrm{km} / \mathrm{h}$ angegeben werden kann. Die Maßeinheiten entsprechen sich allerdings nicht, wenn auf der Wahrnehmungsdimension z. B. das Ausmaß "Robustheit" auf einer 5-er Skala erfasst wird, bei der korrespondierenden objektiven Eigenschaft hingegen die "Torsionssteifigkeit" (gemessen in $\mathrm{Hz}$ ). Hier ist es nicht möglich, Konsumenten direkt die Steifigkeit in $\mathrm{Hz}$ beurteilen zu lassen. Liegen Eigenschaften vor, in denen Hersteller und Konsumentensicht zu divergierenden Maßeinheiten führen, ergibt sich die Aussagefähigkeit für den Hersteller aus der Kenntnis abzuleitender Transformationskurven. ${ }^{479} \mathrm{Im}$ Beispiel ist es die Torsionssteifigkeit, die dem Automobil eine bestimmte Robustheit verleiht. ${ }^{480}$

In einer explorativen Befragung von 400 Autofahrern hinsichtlich der identitätsprägenden Bestandteile einer Automobilmarke wurden die in Abb. 52 dargestellten

479 Eine Transformationskurve stellt ein Erklärungsmodell des Wahrnehmungsverhaltens dar, welches den Zusammenhang zwischen objektiven Produkteigenschaften als unabhängige und der Wahrnehmung dieser Eigenschaften als abhängige Variable abbildet. Vgl. hierzu ausführlich Freter, H., Markenpositionierung. Ein Beitrag zur Fundierung markenpolitischer Entscheidungen auf der Grundlage psychologischer und ökonomischer Modelle, a.a.O., S. 125ff.

In diesem Zusammenhang liefern Car-Clinics wichtige Hinweise hinsichtlich des Differenzierungs- und Standardisierungspotentials einer Marke. Car Clinics stellen Produkttests in der Automobilindustrie dar, bei der etwa hundert Personen, welche die Zielgruppe repräsentieren, nach bestimmten Merkmalen und anschließend nach ihren subjektiven Wahrnehmungen und Beurteilungen bzgl. des getesteten Automobils als Ganzes bzw. einzelner Bestandteile befragt werden. Neben dem Gesamteindruck Styling, Bewertung der Fahrzeugkonstruktion, Produkterlebnis und Gebrauchsnutzen kann die Markentypizität (Wird das Modell der richtigen Marke zugeordnet, oder hat es Merkmale, die zu einer Verwechslung mit anderen Merkmalen führen könnten?) als wichtiges Erkenntnisziel einer Car Clinic gesehen werden. Vgl. Diez, W., Das Handbuch für das Automobilmarketing - Strategien, Konzepte, Instrumente, a.a.O., S. 48. 
Ergebnisse erzielt. ${ }^{481}$ Hiernach konnten insbesondere der Motor mit 74 Prozent sowie die Karosserie des Fahrzeugs mit 66 Prozent als solche Produktmerkmale ermittelt werden, die wesentlich zur Eigenständigkeit von Automobilen in der Wahrnehmung der Nachfrager beitragen.

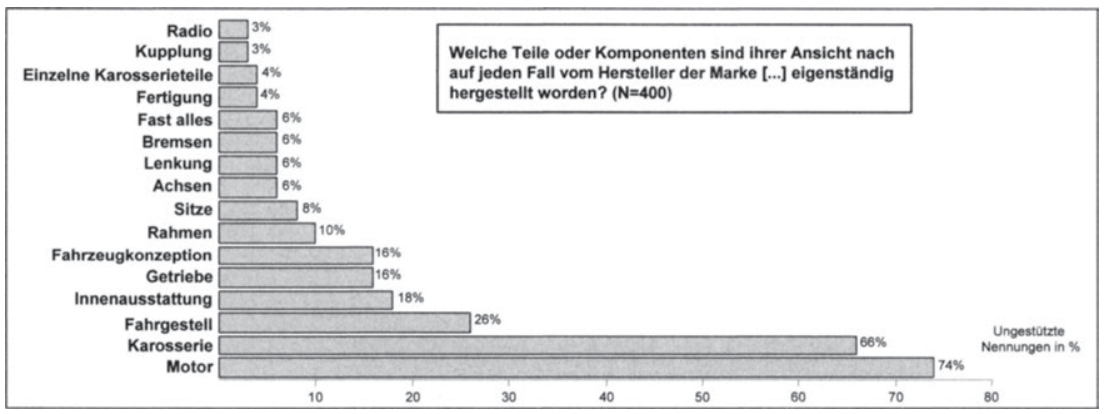

Abb. 52: Nachfragerwahrnehmung der Eigenständigkeit von Automobilen

Der Untersuchung entsprechend unterstreichen der Motor und die Karosserie die Individualität einer Marke. Folglich können die jeweiligen Bauteile als maßgebliche Unterscheidungsmerkmale fungieren. Die im betrachteten Portfolio praktizierte, markenübergreifende Verwendung gleicher Motoren konterkariert damit im Falle einer nachfragerseitigen Transparenz der Gleichteilestrategie den differenzierten Marktauftritt der verschiedenen Marken und fördert die Verwässerung der Markenidentitäten innerhalb des Portfolios.

So zeigen die Untersuchungsergebnisse, dass für 57 bzw. 58 Prozent der Befragten das Auto nicht mehr ein Fahrzeug der jeweiligen Marke wäre und damit seine Identität verloren hätte, würde der Motor bzw. die Karosserie nicht vom Hersteller der entsprechenden Marke gefertigt werden (im Falle einer völligen Fremdfertigung 73 Prozent). 56 bzw. 60 Prozent der Befragten würden die jeweilige Marke bei Fremdherstellung von Motor bzw. Karosserie nicht mehr kaufen (vgl. Tab. 14).

Eines der Hauptziele der Untersuchung war es festzustellen, inwieweit die Markenidentität durch Outsourcing-Aktivitäten untergraben wird. Hierzu wurden 400 Autofahrer mittels persönlicher Interviews in Deutschland (West) befragt, die ihr Auto in den letzen 12 Monaten gekauft haben bzw. planen ein neues Auto zu kaufen. Vgl. zu weiteren Ergebnissen der Untersuchung Meffert, H., Burmann, Ch., Towards an Identity-Oriented Approach of Branding, University of Cambridge, Research Papers in Management Studies, Nr.18, 1996, S. $31 \mathrm{ff}$. 


\begin{tabular}{|c|c|c|}
\hline $\begin{array}{l}\text { Wenn der Motor bzw. die Karosserie nicht vom He } \\
\text { Hersteller produziert wäre, würde ihr Auto immer }\end{array}$ & $\begin{array}{l}\text { arke }[. . .], \text { sc } \\
\text { zeug der } \mathrm{M}\end{array}$ & $\begin{array}{l}\text { on einem andere } \\
\text { sein? }\end{array}$ \\
\hline Fremdherstellung von ... & Motor (in \%) & Karosserie (in \%) \\
\hline Würde noch immer ein Fahrzeug der Marke [...] sein & 27 & 23 \\
\hline Würde nicht mehr ein Fahrzeug der Marke [...] sein & 57 & 58 \\
\hline Kommt drauf an & 16 & 19 \\
\hline Würde im Falle der völligen Fremdfertigung ihr Aut & h ein Fahrzeu & Marke [...] sein? \\
\hline Ja & 27 & \\
\hline Nein & 73 & \\
\hline Würden Sie das Auto Im Falle der Fremdherstellung & Karosserie $\mathbf{n}$ & nmal kaufen? \\
\hline Wiederkauf des Autos, wenn Fremdherstellung von [... & Motor (in \%) & Karosserie (in \%) \\
\hline $\mathrm{Ja}$ & 44 & 40 \\
\hline Nein & 56 & 60 \\
\hline
\end{tabular}

Tab. 14: Auswirkungen der Fremdherstellung auf Markenidentität und Markenloyalităt

Sind vor dem dargestellten Hintergrund diejenigen Produktmerkmale ermittelt, die wesentlich zur Eigenständigkeit von Automobilen in der Wahrnehmung der Nachfrager beitragen, lassen sich schließlich maßgebliche Unterscheidungsmerkmale im Rahmen des Differenzierungsplans festlegen sowie die aus kosteninduzierten Gründen zu standardisierenden Bauteile im Vereinheitlichungsplan bestimmen.

Abb. 53 stellt abschließend beispielhaft einen Differenzierungs- und Vereinheitlichungsplan sowie das Verhältnis zwischen differenzierenden und standardisierten Merkmalen dar. Im dargestellten Differenzierungsplan werden die wesentlichen Unterscheidungsmerkmale zweier zu produzierender Modelle der Marken A und B aufgeführt. Der Vereinheitlichungsplan enthält den Umfang der standardisierbaren Elemente mit entsprechenden Berechnungen für Entwicklungs-, Werkzeug- und Herstellungskosten. So kann beispielsweise die Klimaanlage weitgehend standardisiert werden, erfordert aber bei Marke A andere Sonderteile als bei Marke B. Der Zusammenhang zwischen Differenzierungs- und Standardisierungsplan läßt sich in einer Matrix darstellen, in der Unterscheidungsmerkmale nach ihrem abnehmenden Wert für den Kunden und die standardisierten Elemente nach abnehmenden Kosten der Differenzierung geordnet werden. Insbesondere sollten jene Elemente, die keine Beziehung zu wichtigen maßgeblichen Unterscheidungsmerkmalen haben, konsequent standardisiert werden.

Neben der eigentlichen Leistung ist der Preis als eine verhaltensbeeinflussende Determinante bei der Erklärung des Markenwahlverhaltens zu berücksichtigen und kann als eine der relevanten Wahrnehmungsdimensionen des Eigenschaftsraums betrachtet werden. Da er sowohl die mengen- als auch wertmäßige Komponente des Umsatzes beeinflusst, kommt ihm bei der Bestimmung der ökonomischen Konsequenzen der Markenpositionierung im Portfolio eine herausragende Bedeutung zu. 


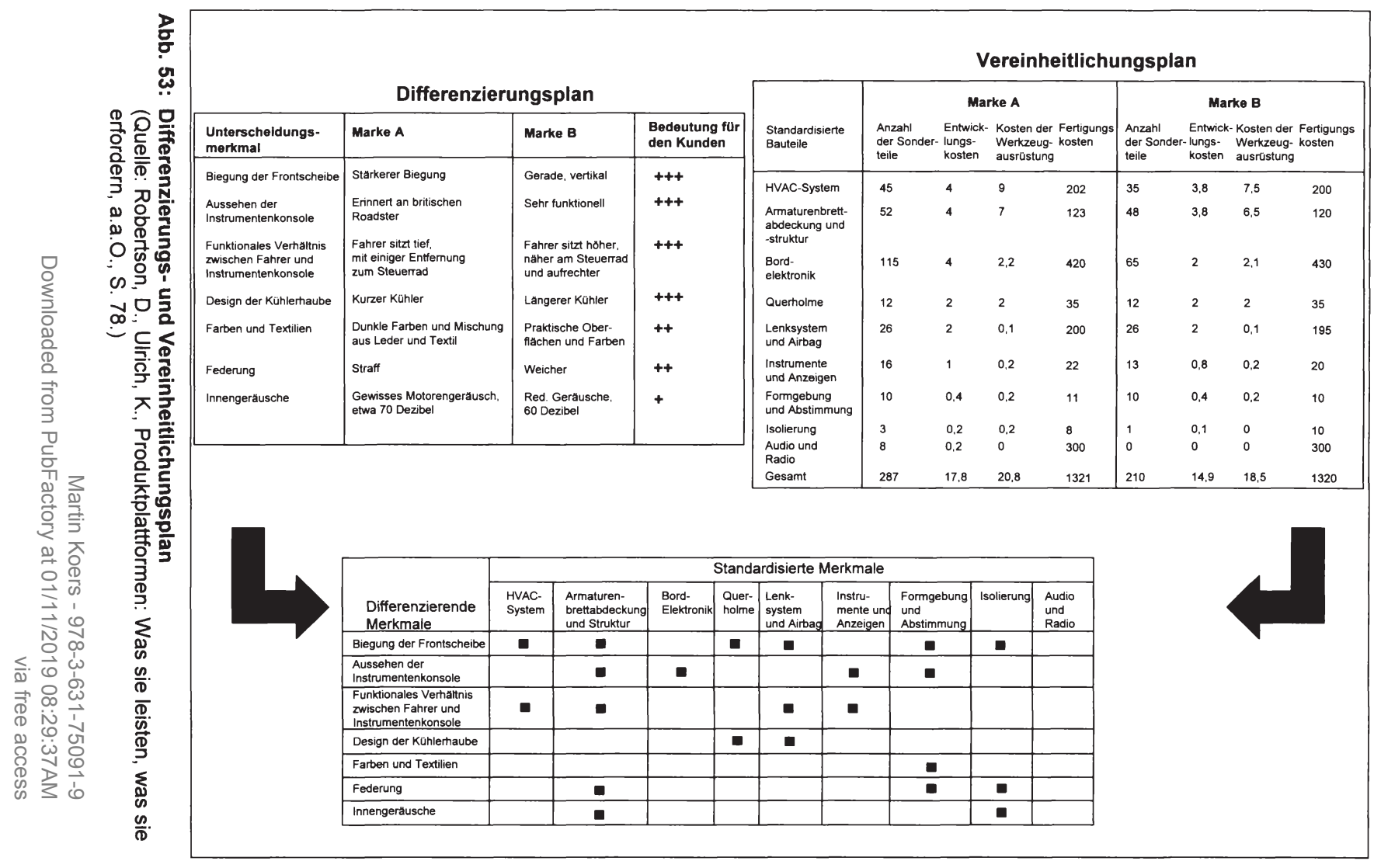




\subsection{Preispositionierung und Ableitung von Preiskorridoren}

Alle preispolitischen Probleme, die sich in einem Markenportfolio gegenüber einer Einzelmarkenstrategie zusätzlich ergeben, resultieren aus Interdependenzen zwischen den Marken. ${ }^{482}$ Bestehen keine Interdependenzen, so vollzieht sich die Preisbildung für jede Marke wie in einer Einzelmarkenstrategie. Im Folgenden gilt es, eine Überprüfung der relativen Preispositionierung und Preiswahrnehmung vorzunehmen sowie Preiskorridore zur Gewinnmaximierung im Gesamtzusammenhang des Portfolios abzuleiten.

Konsumenten werden grundsätzlich als Käufer gewonnen, wenn eine Marke zum einen bei den nicht-preislichen Eigenschaften ihren Vorstellungen entspricht und zum anderen der vom Hersteller geforderte Preis für die Marke dem akzeptierten Preis genügt oder darunter liegt. ${ }^{483}$ Die Positionierung einer Marke in ein Segment, das eine bestimmte Preishöhe akzeptiert, zieht dabei auch diejenigen Segmente an, die für die sonstigen Markeneigenschaften auch einen höheren Preis akzeptieren würden. Liegen mehrere Marken im Akzeptanzbereich des Konsumenten, so wird die zu prognostizierende Markenwahl von den Idealvorstellungen des Konsumenten hinsichtlich der sonstigen Markeneigenschaften bestimmt. Abb. 54 verdeutlicht die Marktanteile der hier betrachteten Portfoliomarken in den unterschiedlichen Preissegmenten.

Grundsätzlich spiegelt die Preispositionierung zunächst prägnant die unterschiedliche Modellpolitik der Marken wieder. In einzelnen Segmenten jedoch überschneiden sich die Marken des hier betrachteten Portfolios, so dass mehrere Portfoliomarken mit Modellen zugleich im gleichen preislichen Akzeptanzbereich der Konsumenten liegen. Hieraus läßt sich wie aufgeführt folgern, dass bei gegebenem Preis die Distanzen in den sonstigen Eigenschaften einer Marke zur Idealmarke eines Konsumenten den Kauf einer Marke bestimmen muss. Denn nur

Zur Preispolitik der Mehrproduktunternehmung allgemein vgl. Theisen, P., Preispolitik der Mehrproduktunternehmung, in: Wist, 4. Jg., Heft 6, S. 273ff. Zum Automobilpricing vgl. Mengen, A., Tacke, G., Methodengestütztes Automobil-Pricing mit Conjoint Measurement, in: Reuss, H., Müller, W. (Hrsg.), Wettbewerbsvorteile im Automobilhandel, Frankfurt, New York 1995 , S. $220 \mathrm{ff}$

Neben dem Anschaffungspreis können die sog. Life Cycle Costs als ${ }_{n}$ Preis ${ }^{4}$ des Automobils interpretiert werden, welche sich aus den Betriebskosten und dem Wertverlust des Fahrzeugs zusammensetzen. Vgl. Diez, W., Das Handbuch für das Automobilmarketing - Strategien, Konzepte, Instrumente, a.a.O., S. $105 f$. 
hierdurch läßt sich erklären, dass etwa im Preissegment 30.000-36.000 DM sämtliche Marken des Portfolios mit eigenen Modellen vertreten sind. Im gleichen preislichen Akzeptanzbereich entscheidet somit der Akzeptanzbereich in den sonstigen Eigenschaften über die Vorziehenswürdigkeit einer Marke im Portfolio. Je niedriger dabei die Distanz in den sonstigen Eigenschaften der Marke, desto höher wird der zugehörige maximal akzeptierte Preis et vice versa.

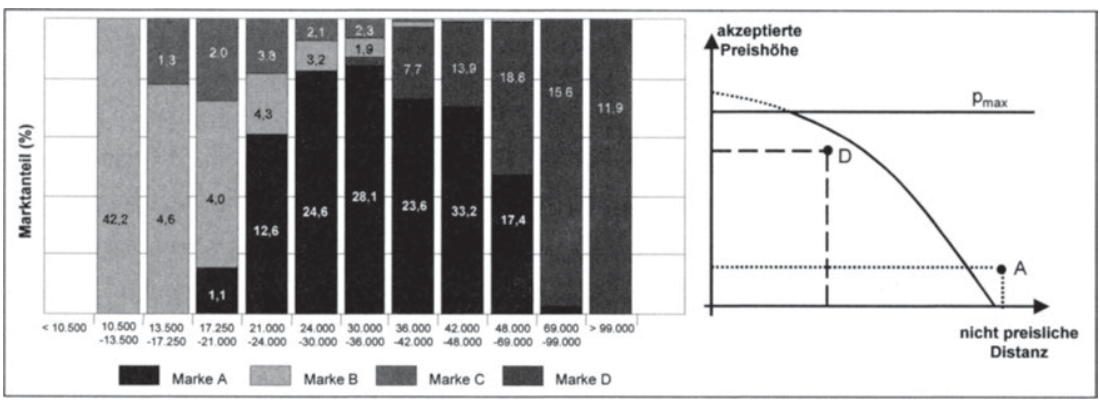

Abb. 54: Preissegmente und Zusammenhang zwischen akzeptiertem Preis und nicht preislicher Distanz

Die rechte Seite in Abb. 54 zeigt den postulierten funktionalen Zusammenhang. ${ }^{484}$ Fallen die Marken $A$ und $D$ in einen maximal akzeptierten Preisbereich (also $p_{A}$ bzw. $p_{D}<p_{\max }$ [für das betrachtete Portfolio bspw. $<42.000 \mathrm{DM}$ ]), so würde $z$. B. Marke A - obwohl sie billiger ist - nicht gekauft, weil sie unter Berücksichtigung von Preishöhe und Abweichungen in den sonstigen Eigenschaften außerhalb des Akzeptanzbereichs liegt. Marke D würde hingegen trotz höherem Preis wegen geringerer Distanzen in den sonstigen Eigenschaften gekauft werden. Hierdurch läßt sich das unterschiedliche Wahlverhalten in den Preissegmenten erklären.

Allerdings bestehen zwischen der Wahrnehmung des Preises und der Wahrnehmung der übrigen Eigenschaften einer Marke zumeist Interdependenzen. Vielfach werden Zusammenhänge zwischen Preis und sonstigen Eigenschaften vereinfacht auch als Preis-Qualitäts-Zusammenhang gekennzeichnet. ${ }^{485}$ Aufgrund der hohen

484 Zum Verlauf des Akzeptanzbereichs vgl. Freter, H., Markenpositionierung. Ein Beitrag zur Fundierung markenpolitischer Entscheidungen auf der Grundlage psychologischer und ökonomischer Modelle, a.a.O., S. $152 \mathrm{ff}$.

BECKER führt an, dass strenggenommen gar nicht isoliert über Preise, sondern immer nur über Preis-Leistungsverhältnisse entschieden werden kann. Vgl. Becker, J., Marketing-Konzeption: 
Korrelation zwischen Preis und sonstigen Eigenschaften der Marken bildet der Preis somit ein zentrales Instrument zur Imagesteuerung der Marken im Portfolio. Ein bekanntes Beispiel einer fehlenden Preis-Image-Konsistenz war etwa die Einführung des Sportwagens Corrado unter der Marke Volkswagen. ${ }^{486}$

Zur Beurteilung der relativen Preisposition einer Marke im Portfolio kann der sogenannte Preisindex herangezogen werden, wobei mit bereinigten und unbereinigten Preisindices zwei verschiedene Varianten existieren. Bedingung eines sinnvollen Preisvergleichs ist dabei, dass nur Modelle einer Klasse in Beziehung gesetzt werden. Während die unbereinigte Variante die jeweiligen Marktpreise der zu beurteilenden Marken ins Verhältnis setzt, beinhaltet die bereinigte Variante eine Neutralisierung und damit Preiskorrektur von Unterschieden zwischen den zu vergleichenden Marken hinsichtlich bestimmter Eigenschaften oder Komponenten. Eine solche Neutralisierung beruht dabei auf der Ableitung von Preisen, wie sie real am Markt für bestimmte Ausstattungsmerkmale bestehen, so dass eine solche Bereinigung auch nur für solche Eigenschaften und Komponenten der Marke erfolgen kann, für die Marktpreise tatsächlich existieren.

Da für die Kaufentscheidung der Konsumenten jedoch weniger der objektive Preis einer Marke, sondern die subjektive Bewertung des wahrgenommenen Preises entscheidend ist, stellt die Preiswahrnehmung der Marke letztlich die relevante Größe dar. Dabei wird die Wahrnehmung der Marken auch von nicht technischenobjektiven Merkmalen einer Marke bestimmt. ${ }^{487}$ Zur Preiskorrektur auch hinsichtlich sog. "weicher Faktoren" einer Marke wie etwa der Umweltverträglichkeit können Kundenwertmethoden herangezogen werden. Sie beruhen auf einer Bestimmung von Nutzenwerten und geben Aufschluss über die individuelle Preisbereitschaft der Konsumenten für einzelne Komponenten der Marke. Somit können auch für solche Marken- bzw. Produkteigenschaften monetäre Größen herangezogen werden, für die keine Marktpreise bestehen oder abgeleitet werden können. Beispielsweise kann so beim Automobil der vom Konsumenten akzeptierte Preis-

Grundlagen des strategischen und operativen Marketing-Management, a.a.O., S. 488. Vgl. hierzu auch Diller, H., Preispolitik, 2. Aufl., Stuttgart u. a. 1991, S. 20.

Vgl. hierzu Meffert, H., Marketing: Grundlagen marktorientierter Unternehmensführung: Konzepte - Instrumente - Praxisbeispiele, a.a.O., S. 488. mensführung: Konzepte - Instrumente - Praxisbeispiele, a.a.O., S. 474. 
aufschlag für eine höhere Endgeschwindigkeit ermittelt werden. Anders als Marktpreise spiegeln Kundenwerte keinen objektiven, sondern einen subjektiven Nutzenzuwachs wieder.

Da auch psychographische Faktoren der Preiswahrnehmung durch eine relative Betrachtung an Aussagekraft gewinnen, kann der „Preiswahrnehmungsfit" - also die Übereinstimmung zwischen der realisierten Preispositionierung und der Beurteilung der Preiswürdigkeit der Portfoliomarken aufgrund der sonstigen, imageprägenden Markeneigenschaften - aus dem Verhältnis von Preispositionierungsindex und Preiswahrnehmungsindex abgeleitet werden:

$$
\text { Preiswahrnehmungsfit }=\frac{\text { Preispositionierungsindex }}{\text { Preiswahrnehmungsindex }}
$$

So indiziert ein Index $<=1$ einen preispolitischen Spielraum, da die Preiswürdigkeit der Marken und damit auch die Preisbereitschaft der Kunden größer ist als die aktuelle Abschöpfung. ${ }^{488}$ Vice versa verdeutlicht ein Index >= 1 eine geringere individuelle Preisbereitschaft als derzeit angenommen wird. Bei einem Index $=1$ entspricht die Positionierung den Vorstellungen der Konsumenten. Abb. 55 verdeutlicht den Preisfit der betrachteten Portfoliomarken für die Jahre 1997 und $1998 \mathrm{im}$ Vergleich zu den jeweiligen relevanten Wettbewerbern.

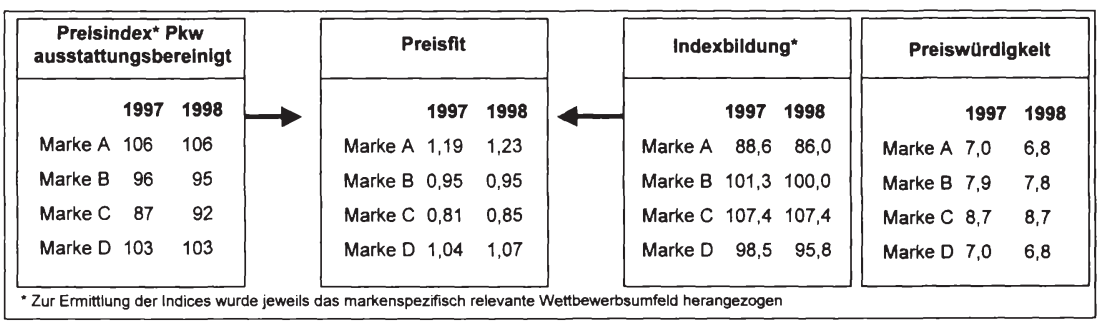

\section{Abb. 55: Preisfit}

Die Abbildung zeigt einen deutlichen Preisfit der Marke $D$ im Vergleich zu den operativen Wettbewerbern. Hier entspricht somit die Preispositionierung der von den Konsumenten angegebenen Preiswürdigkeit. Die Marke $A$ weist demgegenüber eine über dem Richtwert liegende Preisabschöpfung auf, Marken $B$ und $C$ 
hingegen eine in Relation zu den ihnen zugespochenen Preiswürdigkeiten aufgrund der sonstigen Eigenschaften der Marken eine vergleichsweise niedrige.

Neben Fragen der relativen Preispositionierung kommt der gewinnmaximalen Preisermittlung im Portfolio eine besondere Bedeutung zu. Eine optimale Preisermittlung im Markenportfolio bedeutet letztlich, markenspezifische Preise im Gesamtzusammenhang und nicht isoliert voneinander zu ermitteln. Bei Verwendung von Plattformen und sonstigen Gleichteilen bieten Automobilhersteller vielfach objektiv nahezu gleiche Leistungen unter unterschiedlicher Markierung an, so dass die Marken von einer zunehmenden absatzmäßigen Interdependenz gekennzeichnet sind. Preispolitisch relevante Koordinationsprobleme entstehen dann bei Existenz von Preisdifferenzen, da Konsumenten zumeist nicht bereit sind, für die physisch gleiche Leistung einen höheren Preis zu bezahlen. Die Identifikation gewinnoptimaler Preise im Markenportfolio wird dabei grundsätzlich von folgenden Faktoren beeinflußt:

- markenspezifische Zahlungsbereitschaft der Konsumenten entsprechend der zugesprochenen Preiswürdigkeit.

- Wechselneigung der Konsumenten (Abwanderungen zu anderen Marken) aufgrund bestehender Preisdifferenzen.

- Kosten der Produktion der Automobile.

Sind die markenspezifischen Zahlungsbereitschaften trotz hohem Standardisierungsgrad der Automobile sehr unterschiedlich, ergibt sich bei isolierter Betrachtung ein hoher Grad an Preisdifferenzierung. Je höher jedoch die Wechselneigung der Nachfrager ist, bei einem hohem Standardisierungsgrad also nicht die Hochpreismarke, sondern die Niedrigpreismarke zu kaufen, desto stärker wird der Druck auf eine Verringerung der Preisdifferenzen zwischen den Portfoliomarken sein. Da die Preise der Marken somit gegenseitig voneinander abhängen, ist für die Identifikation gewinnoptimaler Preise im Markenportfolio grundsätzlich ein simultaner Planungsansatz notwendig, um die Wechselwirkungen zwischen den Marken adäquat abbilden zu können. Die Ergebnisse eines solchen Ansatzes hängen dabei vor allem von den Annahmen bzgl. des Wechselverhaltens der Nachfrager ab, wobei zu bestimmen ist, welches Ausmaß an Markenwechsel bei gegebenen Preisdifferenzen entsteht. ${ }^{489}$

489

Das grundsätzliche marginalanalytische Prinzip der Preiskoordination bei hoher absatzmäßiger Interdependenz der Marken erfolgt in Analogie zur optimalen Preiskoodination im Rahmen 
Grundsätzlich ist von variabler Wechselneigung auszugehen, d.h. der Substitutionseffekt zwischen den Marken nimmt mit zunehmender Differenz zum Einheitspreis (beide Marken werden unter dem gleichen Preis angeboten) zu. Bei Erhöhung der Preisdifferenzen wechseln nicht alle Nachfrager "sprunghaft" zur Niedrigpreismarke, sondern ein gewisser Anteil wird weiterhin die Hochpreismarke nachfragen. Hieraus folgt, dass Preisdifferenzen in einem bestimmten, durch die Wechselneigung determinierten Maße aufrecht erhalten werden können, um unterschiedliche Zahlungbereitschaften - als zentrales Ziel der Mehrmarkenstrategie - abzuschöpfen. In Abb. 56 ist die Wechselneigung b linear von steigenden Preisdifferenzen abhängig. Ab einer gewissen Preisdifferenz wird vollständige Substitution $(S=1)$ erreicht.

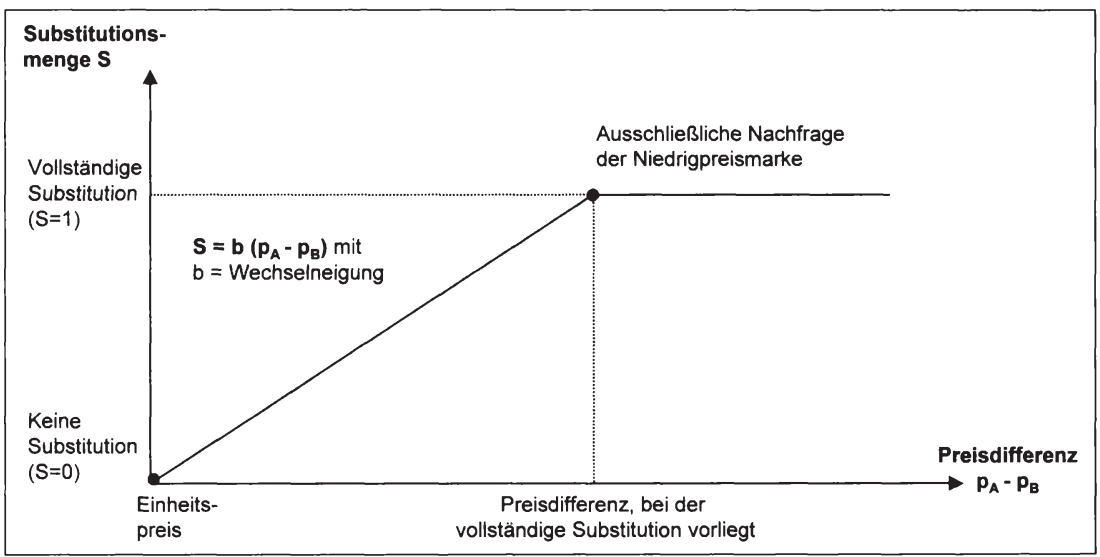

Abb. 56: Lineare Wechselneigung in Abhängigkeit von der Preisdifferenz (rein preisliche Substitution)

(Quelle: In Anlehnung an Backhaus, K., Büschken, J., Voeth, M., Internationales Marketing, a.a.O., S. 191ff.)

Entsprechend wird bei geringer Wechselneigung $b$ die koordinationsoptimale Lösung mit der Lösung bei isolierter Preissetzung nahezu identisch sein. Nehmen zwei fiktive Marken A (Hochpreismarke) und B (Niedrigpreismarke) eines Portfolios die Preissetzung isoliert vor, bedeutet dies, dass eine mögliche Substitution

des internationalen Marketing aufgrund von Arbitrageeffekten. Vgl. hierzu etwa Backhaus, K., Büschken, J., Voeth, M., Internationales Marketing, a.a.O., S. 191ff.; Kucher, E., Simon, H., Schwierige Balanceakte: Die Preispolitik in Europa, in: Harvard Business Manager, No. 4, 1993, S. $46 \mathrm{ff}$. 
im Portfolio nicht beachtet wird. Die markenspezifischen Preise werden isoliert voneinander geplant, so dass sich der Portfoliogesamtgewinn ergibt, indem die Markeneinheiten ihre Gewinne entsprechend ihrer markenspezifischen PAF maximieren, so dass $G_{\text {porttolio }}=G\left(p_{A}\right)+G\left(p_{B}\right)$. In Abbildung Abb. 57 ist diese Situation durch die jeweiligen Maxima der Kurven gekennzeichnet.

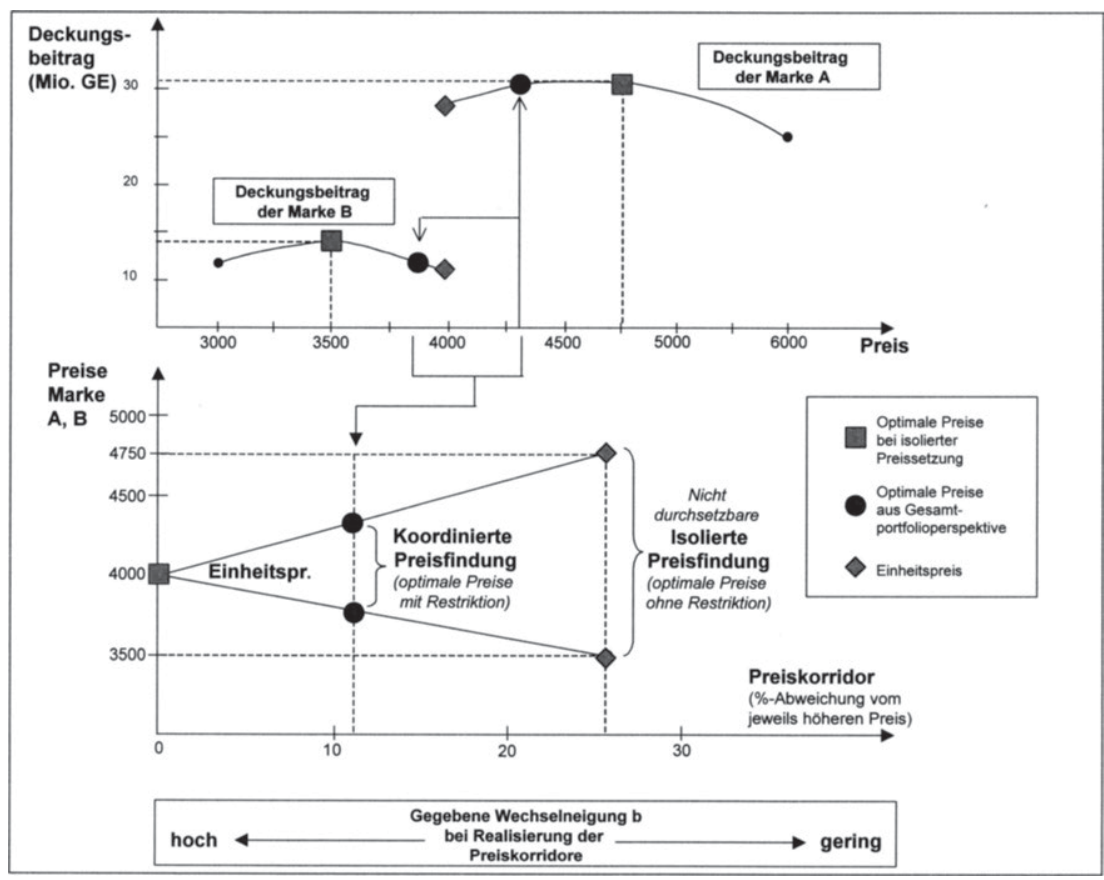

Abb. 57: Bestimmung des Preiskorridors in Abhängigkeit von der Wechselneigung

Mit zunehmender Wechselneigung ergibt sich die Notwendigkeit, die Preise der betrachteten Marken stärker anzugleichen. Eine absolute Angleichung spiegelt der Einheitspreis wider. Eine koordinationsoptimale Preispolitik im Markenportfolio bei variabler Wechselneigung entspricht letztlich einem Preiskorridor für die betrachteten Marken, welcher mit zunehmender Wechselneigung einen sinkenden Differenzierungsgrad der markenspezifischen Preise aufweist. Bei koordinierter Planung der Preispolitik und damit unter Berücksichtigung der Wechselwirkungen zwischen den Marken sind nicht die isolierten Gewinnfunktionen der Marken, sondern die Gewinnfunktion $G\left(p_{A}, p_{B}\right)$ des Gesamtportfolios zu maximieren. Je nach Wechselneigung der Konsumenten ist dann ein bestimmtes Maß an Preisdifferenzierung koordinationsoptimal. Je enger dabei der Preiskorridor angesetzt wird, 
desto mehr Gewinn geht im Vergleich zur isolierten, aber aufgrund der Substitution nicht durchsetzbaren Preissetzung verloren. ${ }^{490}$

Vor dem dargestellten Hintergrund bietet es sich zur Optimierung der Preise im Portfolio an, in drei Schritten vorzugehen:

- Optimalen Preis für jedes Modell einer Marke bestimmen.

- Abschätzen, in welchem Ausmaß bei diesen Preisen Substitutionseffekte zwischen den Modellen der verschiedenen Marken auftreten.

- Bei Notwendigkeit einer Anpassung, markenspezifische Preiskorridore unter Beachtung der jeweiligen Preis-Image-Konsistenzen sowie Minimierung der Substitution festlegen.

Letztlich ist es nicht das Ziel, Substitutionen im Markenportfolio restlos zu vermeiden, sondern den Gewinn des Gesamtportfolios zwischen Einheitspreis (totale Unterdrückung der rein preislichen Substitution) und markenspezifisch optimalen Preisen zu maximieren.

\subsection{Kommunikative Positionierung und Aufteilung des Kommunikations- budgets}

Die Bedeutung der Kommunikationspolitik im Rahmen von Mehrmarkenstrategien ergibt sich daraus, dass durch sie die Wahrnehmung der einzelnen Portfoliomarken und deren Eigenschaften stark beeinflusst wird. Denn die Wirkung objektiver Eigenschaftsausprägungen der einzelnen Marken kommt erst durch die Marktkommunikation zur Geltung. Wie aufgezeigt, kann ein Konsument eine Marke erst dann beurteilen, wenn er die Marke ausreichend kennt. Kennt der Konsument die Marke nicht, kann dies Ursache möglicher Kommunikations- oder Identifikationsgaps im Portfolio sein:

- Je weniger der Konsument auf der einen Seite über die objektive Beschaffenheit einer Marke informiert ist, desto stärker ist eine Diskrepanz zwischen den subjektiv wahrgenommenen und den tatsächlich vorliegenden Eigenschaften einer Marke zu vermuten.

- Je weniger offensichtlich auf der anderen Seite die objektiven Eigenschaften der Marken sind bzw. je weniger bedeutende Unterschiede die einzelnen Portfoliomarken in den objektiven Eigenschaften aufweisen (z. B. aufgrund des 
Badge Engineering), desto größere Bedeutung erlangt die rein kommunikative Positionierung für die Markenpositionierung im Portfolio.

In beiden Fällen trägt die Marktkommunikation maßgeblich zur Profilierung der Portfoliomarken bei:

- Im ersten Fall übt die Marktkommunikation vor allem Einfluss auf die Marktpositionen im Eigenschaftsraum aus. Hier sind die objektiven Eigenschaften zu betonen und damit einer subjektiven Bewertung zugänglich zu machen. Aufgabe der Kommunikationspolitik kann es dabei sein,

- Konsumenten anzusprechen, deren Idealposition einer Marke in der Nähe der von der Portfolioleitung angestrebten Idealposition liegt. Da die Konsumenten in diesem Fall zum Kauf der Marke prädisponiert sind, muss keine Einstellungs- oder Bedürfnisänderung bewirkt werden, sondern die Absatzchancen der Marken steigen allein mit der verstärkten Bekanntmachung der Marke.

- Fehlwahrnehmungen durch eine Konkretisierung der Markeneigenschaften zu korrigieren, um so die wahrgenommenen Distanzen zwischen Selbstbild und Fremdbild zu verkürzen.

- Im zweiten Fall übt die Marktkommunikation vor allem Einfluß auf die Struktur des Eigenschaftsraums aus, da die Ausprägungen einer Marke allein durch kommunikative Maßnahmen erreicht werden. Hier lässt sich die Positionierung durch kommunikative Maßnahmen auf einer fiktiven Dimension erreichen, bei der die Marktkommunikation nicht an die objektiv vorgegebenen Eigenschaften gebunden ist.

Unterscheiden sich die Portfoliomarken in relevanten objektiven Eigenschaften, so fällt es vergleichsweise leicht, diese Unterschiede zu kommunizieren. Die zunehmende objektive Homogenität der Automobile hat jedoch zur Folge, dass konkurrierende Produktangebote verstärkt durch eine emotions- und erlebnisbetonte Kommunikationspolitik profiliert werden müssen. Hierzu werden vermehrt neue Kommunikationsformen wie die Multimedia-Kommunikation und das EventManagement zur Schaffung von Markenerlebniswelten eingesetzt. ${ }^{491}$ Dabei gilt es im Rahmen des Mehrmarkencontrolling insbesondere, eine Überprüfung des "Brand-Fits" eines Werbemittels vorzunehmen bzw. eine konsistente und kontinuierliche Kommunikation des Markenleitbildes in allen Kommunikationsinstrumenten sicherzustellen. ${ }^{492}$ Hierzu existieren in der Praxis eine Vielzahl von Tracking-

So kommt dem Aufbau einer Markenerlebniswelt im Internet mit zunehmender Nutzerzahl eine immer wichtigere Rolle für Automobilhersteller zu. Clef, U., Mehrmarkenstrategie für die PolePosition, a.a.O., S. 79 .

Zur Umsetzung der gewählten Positionierung ist Kontinuität unabdingbar, da es sich beim Aufbau von Gedächtnisstrukturen für Marken um einen Lernprozess handelt. Wird für eine 
Instrumenten. ${ }^{493}$ Letztlich muss es Aufgabe der Kommunikationspolitik sein, eine feste Handlungsabsicht im Sinne einer Kauf-Prädisposition zu bewirken, wodurch die engen Interdependenzen zwischen psychographischen und ökonomischen Kommunikationszielen deutlich werden.

So ist heute auch in vielen Produktmärkten ein starker Zusammenhang zwischen Marktanteil und Werbeanteil zu beobachten. Dieser Beziehung liegt die Überlegung zugrunde, dass aus einem hohen Werbeanteil ein hoher Marktanteil resultiert, beide Größen mithin positiv korreliert sind. ${ }^{494}$ Insbesondere in kommunikativ umkämpften Märkten wie dem Automobilmarkt übt dabei der Werbedruck einer Marke - gemessen am Anteil des Werbebudgets dieser Marke zu den Werbeausgaben aller Marken in der Produktart - einen wesentlichen Einfluss auf das Markenwahlverhalten ausübt. ${ }^{495} \mathrm{Da}$ folglich der Marktanteil der einzelnen Portfoliomarken durch das zugeteilte Werbebudget entscheidend determiniert wird, kommt der Aufteilung des Kommunikationsbudgets im Markenportfolio eine besondere Bedeutung zu. ${ }^{496}$

Ein in der Automobilindustrie verbreitetes heuristisches Vorgehen stellt in diesem Zusammenhang die Werbeanteil-Marktanteil-Methode dar. Hier werden die Kommunikationsausgaben einer Marke in Relation zu den gesamten Kommunikationsausgaben einer Branche gesetzt (Share of Voice) und mit dem eigenen Marktan-

Marke mit mehreren Kommunikationskonzepten geworben, treten Störungen in der Beziehung zwischen Marke und Kunden auf. Bei einer empirischen Untersuchung von 37 Unternehmen der Konsumgüterindustrie in 1997 hinsichtlich der Qualität der Markenführung wurde auf einer Skala von 1 (unwichtig) - 6 (sehr wichtig) die Produktqualität an erster Stelle $(\varnothing-4,86)$ genannt, gefolgt vom Kommunikationsauftritt $(\varnothing-4,58)$. Ferner Design $\varnothing-4,56$, Distributionskanäle $\varnothing$ 4,44, Kommunikationsintensität $\varnothing-4,11$ und Endverbraucherpreis $\varnothing-4,06$. Vgl. Puhlmann, $\mathbf{M}$., Semlitsch, B., Wie geht das Management mit der Marke um?, a.a.O., S. 26.

Vgl. in diesem Zusammenhang den Überblick bei Schwaiger. M., Wirkungskontrolle kommunikationspolitischer Maßnahmen, in: Marketingcontrolling, Reinecke, S., Tomczak, T., Dittrich, S. (Hrsg.), St. Gallen 1998, S. 210ff; siehe für den Automobilmarkt auch die Ausführungen bei Landwehr, R., Standardisierung der internationalen Werbeplanung - Eine Untersuchung der Prozeßstandardisierung am Beispiel der Werbebudgetierung im Automobilmarkt, Frankfurt a. M. 1988 .

Vgl. hierzu sowie zu alternativen Werbebudgetierungsmethoden Landwehr, R., Standardisierung der internationalen Werbeplanung - Eine Untersuchung der Prozeßstandardisierung am Beispiel der Werbebudgetierung im Automobilmarkt, a.a.O., S. 141ff.

Vgl. hierzu die Golffallstudie zum Golf III bei Meffert, H., Marketing: Grundlagen marktorientierter Unternehmensführung: Konzepte - Instrumente - Praxisbeispiele, a.a.O., S. $1287 \mathrm{ff}$.

Vgl. Low, G.S., Mohr, J.J. Setting Advertising and Promotion Budgets in Multi-Brand Companies, in: JoAR, No. 1,1999, S. $67 \mathrm{ff}$. 
teil verglichen. Der Heuristik liegt der Gedanke zu Grunde, dass Unternehmen durch ihre Werbung in einem bestimmten Stärkeverhältnis zum Wettbewerb auftreten und mit KOTLER/BLIEMEL einen bestimmten „Anteil am Stimmenkonzert ${ }^{497}$ zu erlangen versuchen. Zur Aufrechterhaltung des Marktanteils einer Marke ist somit ein entsprechender Anteil an den Ausgaben der Branche durch ein Unternehmen zu tätigen. Wird eine Absatzsteigerung der einzelnen Marken angestrebt, so sollte der Share of Voice größer sein als der Marktanteil. ${ }^{498}$

Abb. 58 stellt beispielhaft den Share of Voice der betrachteten Portfoliomarken sowie einiger Fremdmarken ins Verhältnis zum Marktanteil dar und identifiziert diejenigen Marken mit höheren anteilsmäßigen Werbeaufwendungen, als dies der Marktanteil erwarten ließe. Anhand der Position im Koordinatensystem kann somit das Ausgabenverhalten der einzelnen Marken gemessen werden. So impliziert SoV/MA>1, dass die Werbeausgaben bezogen auf den damit erreichten Marktanteil überproportional hoch sind (Marken B und C). Umgekehrt zeigen SoV/MAWerte $<1$ unterproportionale Werbeaufwendungen bezogen auf den Marktanteil an (Marke $D$ und insb. A). Allerdings ist die SoV/SoM-Relation abhängig vom Lebenszyklus der Marken, da zu Beginn vielfach ein überproportionaler Werbeaufwand erforderlich ist, um der Marke ein entsprechendes "Gehör" bzw. eine Position am Markt zu erkämpfen (hier etwa die Marken $B$ und $C$ auf dem betrachteten Markt). ${ }^{499}$

Kotler, Ph., Bliemel, F., Marketing-Management: Analyse, Planung, Umsetzung und Steuerung, a.a.O., S. 952.

Zur Beschreibung der positiven Beziehung zwischen Markt- und Werbeanteil lassen sich drei grundlegende Regressionsfunktionen beschreiben: 1. MA $=a+b{ }^{*} W$ : direkter positiver Zusammenhang von Werbeaufwendungen (W) und Marktanteil (MA), a und $b$ sind konstanten der Regressionsanalyse; $2 . \Delta M A_{1}=a+b \Delta W_{t-1}$ : direkte Beziehung zwischen der Veränderung der Werbeaufwendungen in der Periode $t-1$ und dem Marktanteil in der Periode $t ; \Delta M A_{1}=a+$ b $\Delta\left(W_{t}-M A_{1-1}\right)$ : Differenz zwischen dem Werbeanteil in $t$ abzüglich des Marktanteils der Vorperiode bestimmt Werbedruck, den ein Anbieter relativ zur Konkurrenz ausübt. Vgl. hierzu Landwehr, R., Standardisierung der internationalen Werbeplanung - Eine Untersuchung der Prozeßstandardisierung am Beispiel der Werbebudgetierung im Automobilmarkt, a.a.O., S. $153 f f$.

Vgl. Pepels, W., Kommunikations-Management: Marketing-Kommunikation vom Briefing bis zur Realisation, 2. Aufl., Stuttgart 1996, S. $314 f$. 


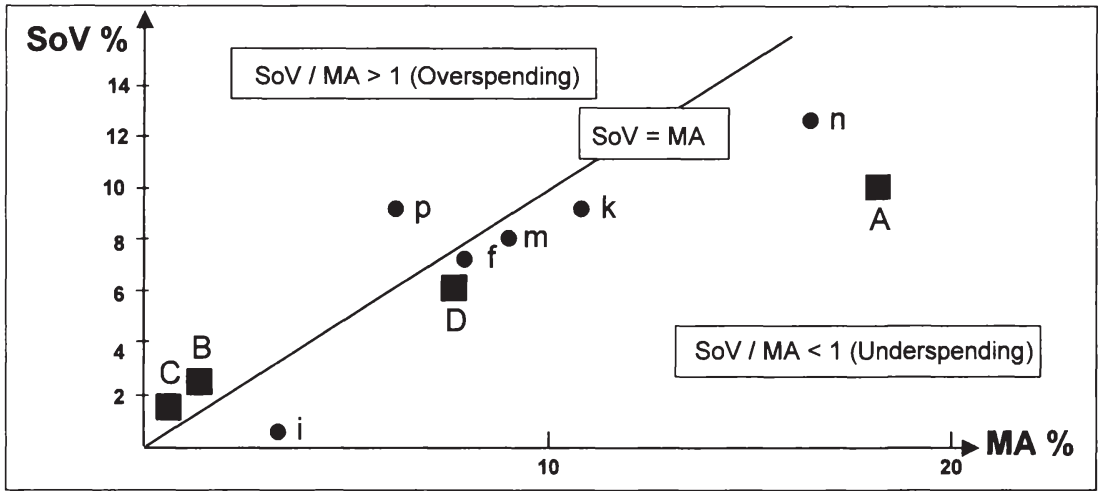

Abb. 58: Beziehung Share of Voice - Marktanteil

Die Marken D und insbesondere A weisen zwar einen Marktanteil auf, der höher ist, als der SoV erwarten ließe, allerdings sind hier die bereits vorhandenen akquisitorischen Wirkungen der Marken unabhängig von den Kommunikationsausgaben zu berücksichtigen. So herrscht in der Literatur Einigkeit darüber, dass mit der Zunahme der Marktanteile die Werbeintensität abnimmt. Dies erklärt sich durch Präferenzvorteile für Produkte mit hohem Marktanteilen oder auch Schwellenwerte der Werbeintensität. ${ }^{500}$

\subsection{Markenidentifikation des Handels}

Im Rahmen von Mehrmarkenstrategien bedarf die eigenständige Positionierung der Portfoliomarken insbesondere auch einer Markendifferenzierung auf Handelsseite durch einen eigenständigen Markenauftritt am Point of Sale. ${ }^{501}$ Die zentrale

500 Generell wird die These vertreten, dass ältere Marken mit einer geringeren Werbeintensität auskommen. Vgl. hierzu Karnani, A., Strategic Implications of Market Share Attraction Models, in: Management Science, 1985, S. 536ff.

Die Distributionspolitik nimmt in der Automobilbranche einen besonderen Stellenwert ein, da es sich beim Automobil um ein komplexes Gebrauchsgut handelt, das sowohl einen intensiven persönlichen Verkauf erfordert, als auch wartungs- und reparaturbedürftig ist, so dass neben dem Verkaufsaspekt auch dem Serviceaspekt eine hohe Relevanz beizumessen ist. Zudem kommt der Vertriebsorganisation von Automobilherstellern eine bedeutende präferenzbildende Funktion zu, da die Zufriedenheit mit der Verkaufs- und Kundendienstleistung eines Händlers als eine wesentliche Komponente in die Gesamtzufriedenheit eines Kunden während sowie nach dem Automobilkauf eingeht. Vgl. hierzu etwa Burmann, Ch., Konsumentenzufriedenheit als Determinante der Marken- und Händlerloyalität - Das Beispiel der Automobilindustrie, a.a.O., S. 249ff.; Hoffmeister, M., Multi-Franchise-Konzepte im Automobileinzelhandel: Entwicklungen und Auswirkungen auf die Absatzkanalpolitik der Automobilhersteller, a.a.O., S. 1. 
Herausforderung besteht darin, für jede Marke ein leitbildgerechtes Markenbild über das gesamte Händlernetz der jeweiligen Marken sicherzustellen und damit einen markenexklusiven Vertrieb von Neufahrzeugen zu gewährleisten. ${ }^{502}$

Grundsätzlich sind bei der Analyse des Einflusses distributionspolitischer Entscheidungen auf die Markenwahrnehmung die Besonderheiten des indirekten und direkten Vertriebs zu berücksichtigen. Dabei erfolgt der Vertrieb der Leistungen im Rahmen der indirekten Distribution über verschiedene Absatzmittler, wohingegen die Distributionsaufgabe beim Direktvertrieb vom Hersteller selbst übernommen wird. ${ }^{503}$ Während

- der direkte Vertrieb über werkseigene Niederlassungen den Vorteil bietet, ein homogenes Serviceniveau unter einheitlicher Markierung anzubieten, wodurch der Aufbau eines markenexklusiven Einkaufsstättenimages erleichtert und die Gefahr möglicher Gaps zwischen Selbst- und Fremdbild begrenzt wird ${ }^{504}$,

Vor diesem Hintergrund kann die handelsbezogene Trennung der Marken Audi und Volkswagen des Volkswagen-Konzerns gesehen werden, welche bisher über V.A.G.-Partner gemeinsam vertrieben wurden. Durch strikte räumliche Trennungen sollen „Markenwelten" geschaffen werden, mittels derer ein die Markenpositionierung förderndes Erscheinungsbild vor dem Kunden erzeugt werden kann. Hierfür investierten Händler mehr als 7 Milliarden Mark, weltweit standen über 11.000 Audi- und WW-Betriebe zur Umrüstung an. Vgl. Büchelhofer, R., Markenführung im Volkswagen-Konzern im Rahmen der Mehrmarkenstrategie. Unveröffentlichte Fallstudie 1999, S. 13

503 In jüngster Zeit zeichnen sich neue Entwicklungslinien ab, welche insbesondere in einer Erhöhung des Autokaufs via Internet und mithin dem Entstehen zusätzlicher Vertriebskanäle (Mehrkanalsystem) sowie der Vergrößerung der Betriebe und Marktverantwortungsgebiete zur Verbesserung der Renditesituation der Automobilhändler zu sehen sind. Die in 1995 in Kraft getretene neue Gruppenfreistellungsverordnung (GVO) läßt zwar das selektive Vertriebssystem in der Automobilbranche weiterhin zu, räumt jedoch den Handelsbetrieben den Vertrieb von mehreren Marken ein, sofern diese dem Kunden beispielsweise durch getrennte Schauräume oder Kundendienstannahme optisch getrennt präsentiert werden. Nach Art. 85 Abs. 1 des EWG-Vertrages sind Vereinbarungen zwischen Unternehmen verboten, welche geeignet sind, den Handel zwischen Mitgliedstaaten zu beeinträchtigen. Hiervon wurden die Vertragshändlerverträge im Rahmen der GVO zunächst mit einer Dauer von zehn Jahren (GVO Nr. 123/85) im Jahr 1985 ausgenommen, da der selektive Vertrieb für alle Beteiligten vorteilhaft sei. 1995 wurde die GVO in veränderter Form bis 2002 verlängert (GVO Nr. 1475/95), wobei es insb. zu einer Aufweichung der bisherigen Regelung hinsichtlich der Markenexklusivität sowie der Gebietsbindung kam. Vgl. zu den rechtlichen Grundlagen des Vertragshändlersystems Diez, W., Das Handbuch für das Automobilmarketing - Strategien, Konzepte, Instrumente, a.a.O., S. $171 f$.

504 Allerdings ist eine solche flächendeckende Vertriebsform gleichzeitig mit hohen herstellerseitigen Investitionen verbunden, so dass Vertragshändlern als selbständige, vom Hersteller autorisierte Handelsunternehmen eine größere Bedeutung zukommt, welche in der Regel nur eine Marke führen und sich vor allem durch ihre höhere Flexibilität und Marktnähe auszeichnen. Überdies ist der Handel mit Neuwagen untrennbar mit der Bereitstellung umfassender Kundendienstleistungen verbunden ist, so dass die Verknüpfung von Neuwagen- und Kunden- 
- verfolgen die auf die Profilierung des eigenen Autohauses achtenden Händler vielfach eigenständige Ziele, welche sich maßgeblich von den Interessen des auf die Markenprofilierung ausgerichteten Herstellers unterscheiden können, infolgedessen sich die Wahrscheinlichkeit etwaiger Gaps multipliziert. ${ }^{505}$

Da der Handel einen starken Einfluß auf die Position einer Herstellermarke im Wahrnehmungsraum des Konsumenten ausüben kann, bedarf es zur Sicherstellung eines leitbildgerechten Auftritts der Händlerorganisation einer hohen Identifikation des des Handels mit der Marke. Dies setzt eine handelsgerichtete Profilierung der Marke durch klare Kompetenznachweise und institutionalisierte Instrumente des Beziehungsmanagement voraus, wobei die Händlerzufriedenheit - also die Zufriedenheit der Händler mit dem Hersteller - als Basis für eine erfolgreiche Gestaltung der Hersteller-Handels-Beziehung im Sinne einer gemeinsamen Vertriebskultur verstanden werden kann. Auf einer anderen Ebene steht die Händlerzufriedenheit des Kunden - also die Zufriedenheit des Kunden mit dem Händler welche als „Markt-Feed-Back-Konzept" ${ }^{506}$ die Einlösung des Markenversprechens am PoS bewertet und damit erlaubt, die Vertriebsaktivitäten aus einer ganzheitlichen Kundenperspektive zu betrachten.

Aufgrund der vertraglichen Bindung handelt es sich bei der Händlerzufriedenheit um eine Zufriedenheit in einer auf Dauer angelegten Geschäftsbeziehung, so dass sie nicht nur von der direkten Beziehung zwischen Hersteller-Händler beeinflußt wird, sondern auch von situativen Faktoren wie der aktuellen Ertragssituation und dem Grad des Intra-Brand Wettbewerbs sowie strukturellen Faktoren wie der Be-

dienstgeschäft bei den meisten Marken primär über vertragliche Vertriebssysteme organisiert ist. Darüber hinaus findet sich neben dem Vertragshändler („Verkauf von Waren im eigenen Namen und auf eigene Rechnung") bei einigen Herstellern wie Mercedes-Benz der Agent als Absatzmittler, bei dem es sich rechtlich betrachtet um einen Handelsvertreter nach $\S 84 \mathrm{HGB}$ handelt („Verkauf von Waren in fremdem Namen und für fremde Rechnung“). Vgl. hierzu auch Creutzig, J., Die zukünftige Regelung des Automobilvertriebs in Europa, in: Automobilwirtschaft, Meinig, W. (Hrsg.), Wiesbaden 1993, S. $65 \mathrm{ff}$.

Aufgrund unmittelbarer Erfolgskopplungen zwischen dem Hersteller und den angeschlossenen Handelsbetrieben können auftretende Konflikte zwischen den Systempartnern nicht kurzfristig durch eine Trennung beider Partner gelöst werden, so dass einem geeignetem Konfliktund Kooperationsverhalten innerhalb vertraglicher Vertriebssysteme unter Anwendung eines professionellen Konfliktmanagement ein besonderer Stellenwert zukommt. Zum Konfliktverhalten vgl. Meffert, H., Wöllenstein, St., Burmann, Ch., Erfolgswirkungen des Konflikt- und Kooperationsverhaltens in vertraglichen Vertriebssystemen des Automobilhandels, in: Marketing ZFP, Heft 4, 1996, S. 279ff.

Burmann, Ch., Konsumentenzufriedenheit als Determinante der Marken- und Händlerloyalität - Das Beispiel der Automobilindustrie, a.a.O., S. 258. 
triebsgröße und der Dauer des Händlervertrages. ${ }^{507}$ Die Adäquanz einer Steigerung der Händlerzufriedenheit mit den allgemeinen vertriebspolitischen Zielen des Herstellers läßt sich - neben dem häufig angeführten Zusammenhang zwischen Händler- und Kundenzufriedenheit - auch auf motivationstheoretischer Basis ableiten. So hat sich in empirischen Studien gezeigt, dass

- sowohl die Leistungsbereitschaft der Händler, im Sinne der Herstellerziele tätig zu werden,

\section{- als auch der Leistungserfolg zufriedener Händler}

höher ist als der unzufriedener. ${ }^{508}$ Vor diesem Hintergrund stellt die Händlerzufriedenheit gewissermaßen einen "Frühindikator" für die Markenidentifikation des Handels dar. Mit MEINIG kann auch gesagt werden: „Considering the way in which a dealer is treated by ist manufacturer has decisive influence on the dealer's commitment to the brand as well as on the dealer's business success, an examination of dealer satisfaction has become strinktly important [...]. ${ }^{\text {“509 }}$ Abb. 59 zeigt die Händlerzufriedenheit der hier betrachteten Portfoliomarken.

Aus Händlersicht stehen im Portfolio dem als vorteilhaft angesehen Produktprogramm größere Schwächen bei der Belieferung mit Neuwagen sowie im Verkauf und Marketing gegenüber. In der Detailbetrachtung verdeutlicht die Abbildung, dass die Händler der Marke $A$ insgesamt am wenigsten zufrieden sind. Schwerpunkte der Unzufriedenheit sind insbesondere Aspekte, die den Verkaufs- und Marketing-Sektor betreffen. Die allgemeine Zufriedenheit der Händler mit der Marke $D$ liegt deutlich über dem Niveau von $A$ und im Schnitt aller Händlerorganisationen, wobei insbesondere Kriterien der Belieferung mit Neuwagen für das positive Stimmungsbild sorgen. Die Händler der Marke B sind in nahezu allen Details

507 Vgl. auch Meinig, W., Dealer Satisfaction and its Significance with Regard to the Relationsship between authorized Car Dealers and Manufacturers/Importers, in: der markt, Heft 1, 1998, S. $12 \mathrm{ff}$.

508 Vgl. Diez, W., Prozeßoptimierung im Automobilvertrieb: Auf dem Weg zu einem integrierten Kunden- und Kostenbindungsmanagement, Wiesbaden 1999, S. 148. DIEZ weist allerdings darauf hin, dass die kausale Wirkungsrichtung des Zusammenhangs zwischen Händler- und Kundenzufriedenheit nicht eindeutig ist. Denn so plausibel wie die Behauptung, eine hohe Händlerzufriedenheit führe zu einer hohen Kundenzufriedenheit, ist die These, eine hohe Kundenzufriedenheit trage (via einer hohe Markentreue) zu einer hohen Händlerzufriedenheit bei. Mutmaßlich besteht ein sich gegenseitig verstärkender Zusammenhang, so dass die Steigerung der Händlerzufriedenheit ohne Zweifel ein wichtiges vertriebspolitisches Ziel darstellt.

Meinig, W., Dealer Satisfaction and its Significance with Regard to the Relationship between authorized Car Dealers and Manufacturers/Importers, a.a.O., S. 12. 
unzufrieden mit der Marke. Die C-Händler weisen im Portfoliovergleich insgesamt das höchste Zufriedenheitsniveau auf, wobei allerdings die Erwartungen hinsichtlich der Neuwagenlieferung weit verfehlt werden.

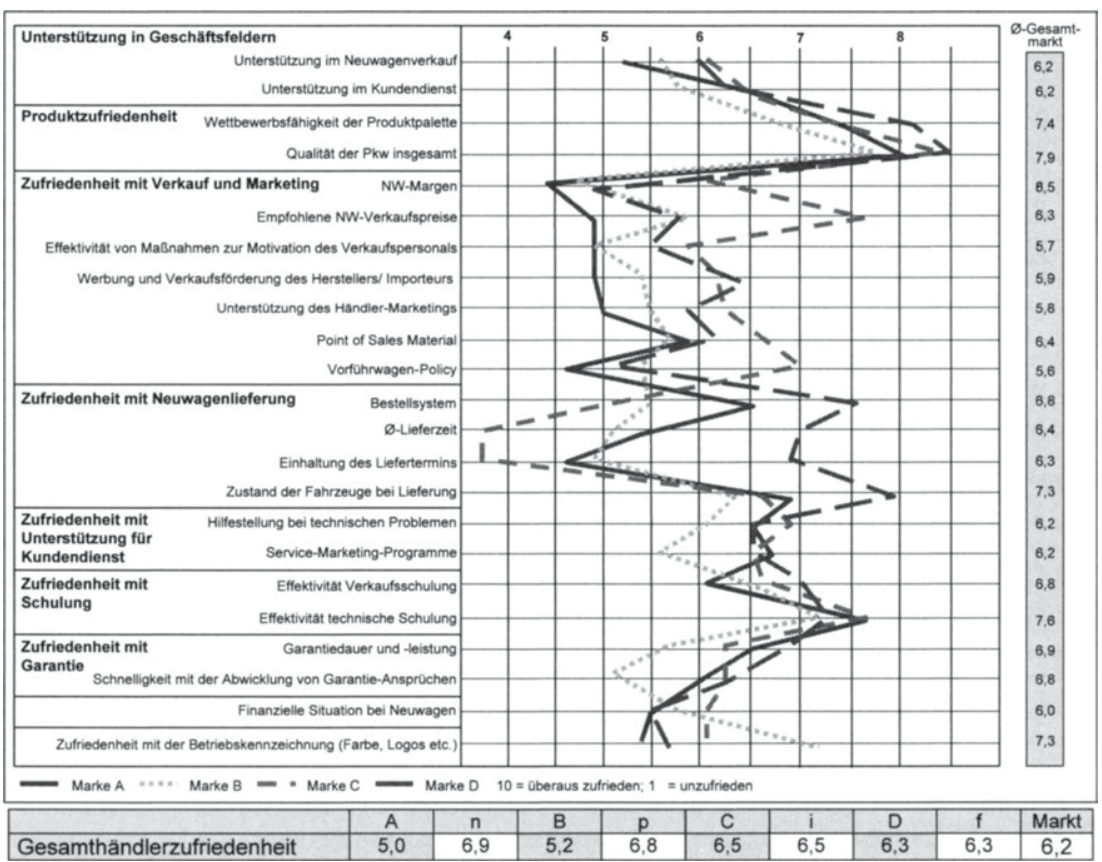

\section{Abb. 59: Händlerzufriedenheit ${ }^{510}$}

Vor dem Hintergrund der Händlerzufriedenheiten offenbart die Partnerschaft und Bindung der Händler zur jeweiligen Marke interessante Ergebnisse (Abb. 60). So fühlen sich im Portfoliovergleich lediglich die Händler der Marke $C$ überdurchschnittlich als echte Partner des Herstellers. Die Händler der sonstigen Marken beurteilen die Beziehung zum Hersteller eher unterdurchschnittlich. Bemerkenswert ist, dass sich nur 37,3 Prozent der A-Händler als echte Partner des Herstel-

510 Die Zufriedenheiten der Händler mit dem Hersteller werden in der DSS-Studie regelmäßig mit Hilfe einer 10er Skala (1=absolut unzufrieden, 10 voll und ganz zufrieden) telefonisch erhoben. Das Interview wird mit dem Inhaber/Geschäftsführer der Handelsorganisation geführt. Netto-Stichproben im vorliegenden Fall: Marke A: 212; Marke B:152; Marke C: 151; Marke D: 211. 
lers sehen, sich dennoch 81 Prozent im Falle eine Neubeginns trotzdem wieder für die gleiche Marke entscheiden würden. Hervorzuheben ist schließlich die Konkurrenzmarke $h$, deren Position auf eine nicht hinreichende partnerschaftliche Beziehung zwischen Hersteller und Händler schließen läßt.

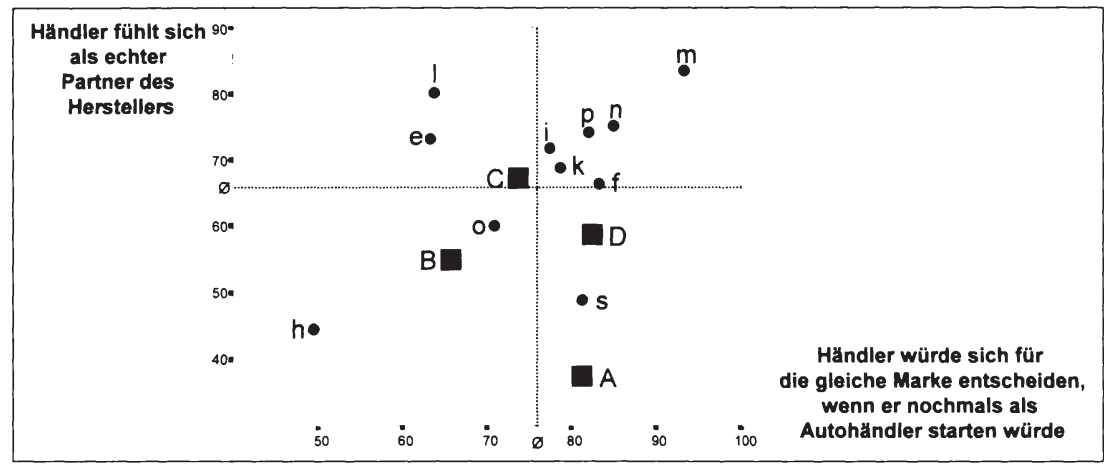

Abb. 60: Partnerschaft und Bindung zur Marke

Insgesamt verdeutlicht die Abbildung die bislang noch gering ausgeprägten vertikalen Kooperationsbeziehungen bei den hier betrachteten Portfoliomarken. Dies mag zum einen damit zusammenhängen, dass die bewusste Entwicklung und Gestaltung einer Organisations- und Vertriebskultur im Automobilvertrieb vor dem Hintergrund der Stabilität und Routine der vertraglichen Vertriebssysteme bisher kaum Tradition besitzt. ${ }^{511}$ Zum anderen kann dies als ein Indiz für die übermäßige Inanspruchnahme der als Charakteristikum der Branche geltenden Marketingführerschaft des Hersteller gesehen werden. ${ }^{512}$

511 Während im Rahmen der Hersteller-Zulieferer-Beziehungen vergleichsweise schnell vertrauensbildende Maßnahmen zur Förderung der Beziehungen mit den Lieferanten etabliert wurden, wie beispielsweise das Picos-Programm bei OPEL oder das Tandem-Konzept bei DAIMLERCHRYSLER, fehlt ein solcher Ansatz im Vertriebsbereich bislang völlig. Vgl. Diez, W., Prozeßoptimierung im Automobilvertrieb: Auf dem Weg zu einem integrierten Kunden- und Kostenbindungsmanagement, a.a.O., S. 145. Vgl. Meyer, M., Bartelt,A. Okonomische Analyse von Vertrauen in Zuliefernetzwerken der Automobilindustrie, Arbeitspapier Nr. 5 der Reihe Marketingtheorie und -management des LS für BWL und Marketing der Universität Würzburg, Würzburg 1999.

512 Die ${ }_{n}$ Macht" des Herstellers drückt sich prägnant im folgenden Sachverhalt aus: Aufgrund der Verwendnung von Gleichteilen etc. muss die herstellerseitig induzierte sinkende produktpolitische Differenzierung durch eine verstärkte Differenzierung am Point of Sale seitens der Händler kompensiert werden. Eine wachsende Differenzierung am POS führt jedoch zu steigenden Kosten. In der Konsequenz bedeutet dies, dass Kostensenkungseffekte in der Pro- 
In einer Gesamtwürdigung der bisherigen Analyse wurde die Vielfalt relevanter Informationen aus den Bereichen des strategischen und operativen Mehrmarkencontrolling deutlich. Um in diesem Zusammenhang allerdings der Gefahr von „Informationsinseln" bzw. der Suboptimierung des Mehrmarkencontrolling auf der Ebene einzelner Instrumente und Kennzahlen zu entgehen, bedarf es statt der isolierten Betrachtung der einzelnen Größen einer Integration der Informationen in ein Gesamtsystem. Vor diesem Hintergrund erfolgt im Folgenden eine Integration der Steuerungsgrößen in ein Balanced-Scorecard Konzept.

\section{Integration mehrmarkenspezifischer Steuerungsgrößen in ein Kon- zept der Balanced Scorecard}

Grundsätzlich ist die Forderung nach einem integrierenden System bzw. einem Gesamtmodell des Unternehmens in der Betriebswirtschaftslehre nicht neu. Bereits in den fünfziger Jahren wurde versucht, Unternehmen im Gesamtzusammenhang zu modellieren, um damit bessere Aussagen über betriebliche Zusammenhänge gewinnen zu können. Bis heute ist es jedoch nicht gelungen, ein solches Modell ausreichend zu formulieren, so dass der Jahresabschluss sowie die Finanzplanung noch am ehesten dem Anspruch eines unternehmerischen Gesamtmodells gerecht werden. ${ }^{513}$ Auch die Forderung nach ManagementInformationssystemen in den siebziger Jahren, die es ermöglichen sollten, für jede Entscheidungssituation die adäquaten Daten und Kennzahlen in einem „Totalan-

duktion durch Kostensteigerungen im Vertrieb und Handel zumindest teilweise konterkariert werden. DIEZ spricht in diesem Zusammenhang von zwangsläufig entstehenden asymmetrischen Kosteneffekten zwischen Herstellern und Händlern: während die kostensenkenden Effekte einer Plattform-Strategie in Entwicklung und Produktion voll dem Hersteller zugute kommen, muss der Handel die Kosten der Plattform-Strategie in einem nicht unerheblichen Umfang mittragen. Vgl. Diez, W., Plattform-Strategien: Irrweg oder Königsweg in der Modellpolitik, a.a.O., S. 34ff.

In einer empirischen Analyse von PERLITZ wurde untersucht, welche strategischen Kennzahlen regelmäßig Vorständen und Geschäftsführern in Deutschland und den USA vorgelegt werden. Dabei offenbarte sich folgendes Bild: Finanzkennzahlen [Deutschland 87 Prozent/ USA 98 Prozent], Betriebliche Leistungskennzahlen [64/82], Kundenzufriedenheit [47/ 76], Mitarbeiterbelange [29/57], Innovation [35/33], Prozessqualität [37/ 44]. Beim Vergleich wird deutlich, dass in den USA die betrachteten strategischen Kennzahlen weit mehr Beachtung fanden als in Deutschland. Vor diesem Hintergrund ist es auch nicht verwunderlich, dass Performance Measurement-Konzepte in den USA eine höhere Relevanz beigemessen wird als in Deutschland. Vgl. Perlitz, M., Unternehmen durch „Performance Measurement" erfolgreich machen, in: Gablers Magazin, Heft 2, 1999, S. $7 \mathrm{ff}$. 
satz" zu generieren, scheiterte am Problem der Modellierung aller Entscheidungssituationen für das Topmanagement. ${ }^{514}$

Da Markenführung und somit auch dessen Planung und Kontrolle situativ ausgestaltet werden sollten, erscheint eine allgemeingültige Systemintegration im Rahmen einer Balanced Scorecard allerdings wenig sinnvoll. Grundsätzlich sind Branchenbesonderheiten wie auch portfoliointern vorhandene Rahmenbedingungen und Infrastrukturen (Berichtswesen, Informatikunterstützung) zu berücksichtigen. ${ }^{515}$ Dennoch gibt es Zusammenhänge, die unabhängig von Branche und Unternehmenssituation im Rahmen der Mehrmarkenführung zu reflektieren sind. Von daher kann die folgende logisch-deduktive Herleitung einer BSC als Referenzansatz unternehmensindividuell zu erstellender Scorecards im Rahmen der Mehrmarkenführung fungieren.

Wie aufgezeigt, geht der Aufbau einer Balanced Scorecard von systematischen Wirkungszusammenhängen zwischen Leistungstreibern und Ergebnisgrößen und damit von einer inneren Logik zwischen Kennzahlen aus. ${ }^{516}$ Leistungstreiber als Frühindikatoren erklären Ergebnisgrößen als Spätindikatoren und zeigen Ansatzpunkte zu deren Beeinflussung auf verschiedenen Ebenen auf. Entsprechend sind Führungskräfte angehalten, Wirkungszusammenhänge der Kennzahlen im Portfolio zu reflektieren.

Grundsätzlich lassen sich bei den einzelnen Portfoliomarken Wirkungszusammenhänge zwischen den beobachtbaren Ergebnisgrößen und den nicht beobachtbaren, erklärenden Vorsteuerungsgrößen des Kaufverhaltens identifizieren, welche Ansatzpunkte zur Steigerung einzelner Markenergebnisse liefern. Ziel im Markenportfolio ist jedoch nicht die isolierte Steigerung der einzelnen Markenperformance, sondern die koordinierte Erhöhung der markenspezifischen Ergebnisse,

514 Vgl. Meffert, H., Computergestützte Marketing-Informationssysteme und Marketing-Modelle, in: Computergestützte Marketing-Planung, Hansen, H. R., München 1974, S. 11; zur historischen Bewertung vgl. Hornung, K., Mayer, J. H., Erfolgsfaktoren-basierte Balanced Scorecards zur Unterstützung einer wertorientierten Unternehmensführung, in: Controlling, Heft 8/9, 1999, S. 389

515 Vgl. hierzu auch Krulis-Randa, J. S., Theorie und Praxis des Marketing-Controlling, in: Siegwart, H. et al, Management Controlling, Meilensteine im Management, Basel, Frankfurt 1990, S. 269; Reineke, S., Tomczak, T., Aufgabenorientiertes Marketingcontrolling, a.a.O., S. 91.

516 Vgl. Horváth, P., Kaufmann, L., Balanced Scorecard - ein Werkzeug zur Umsetzung von Strategien, a.a.O., S. 48. 
da aufgrund der im Rahmen der Arbeit identifizierten Wechselbeziehungen zwischen den Marken davon auszugehen ist, dass sich das Ergebnis des Gesamtportfolios bei koordinierter Vorgehensweise steigern läßt. Vor diesem Hintergrund ist zu fragen,

- welche grundsätzlichen Kennzahlen die zu entwickelnde BSC zur Erfassung von Leistungstreibern und Ergebnisgrößen umfassen sollte. Eine solche Frage stellt sich grundsätzlich auch im Rahmen isolierter Markenführung, da es auch hier für jede Marke zu bestimmen gilt, welche Leistungstreiber die markenspezifischen Ergebnisse beeinflussen.

- wie die Wechselwirkungen zwischen den Marken zur Abbildung der Gesamtportfolioperformance zu erfassen sind. Eine solche Betrachtung erweist sich als mehrmarkenspezifisch und berücksichtigt die Interdependenzen zwischen den Marken im Portfolio.

Während somit im Rahmen von Einzelmarkenstrategien nur die erste Frage zu lösen ist, bedarf es im Rahmen von Mehrmarkenstrategien der Beantwortung beider Fragen. Mithin erweist sich der Aufbau einer BSC im letzten Fall als wesentlich komplexer. $^{517}$

\subsection{Erfassung von Leistungstreibern und Ergebnisgrößen im Rahmen von Scorecards}

Den verschiedenen Anspruchsgruppen des Unternehmens Rechnung tragend fokussieren KAPLAN/ NORTON die Wünsche verschiedener Anspruchsgruppen in den Perspektiven ihrer BSC. Auch die Markenführung hat sich aufgrund der innen und außengerichteten Wechselseitigkeit der Markenidentität an verschiedenen externen und internen Bezugsgruppen auszurichten, die es im Rahmen der BSCGestaltung zu berücksichtigen gilt.

So geht es kundengerichtet bei der Gestaltung der Markenidentität um den Nachweis einer echten Nutzenstiftung bzw. Problemlösungskompetenz des Markenproduktes. Zentrales Ziel ist dabei, eine hohe Markenloyalität der Konsumenten durch ein klares Leistungsprofil, die Sicherung der Zufriedenheit und des Vertrauens in die Marke aufzubauen. Wettbewerbsgerichtet ist eine starke Markenidenti-

517 Anhaltspunkte zur Implementierung einer BSC finden sich auch bei Fischer, Th., Implementierung von Balanced Scorecards in Handelsunternehmen, in: HHL-Arbeitspapier Nr. 32, Leipzig 2000; Olve, N., Roy, J., Wetter, M., Performance Drivers - A Practical Guide to Using the Balanced Scorecard, Chichester U. a. 1999. 
tät an eine differenzierte Markenpositionierung gebunden, welche der Marke einen dauerhaften, wahrgenommenen und aus Sicht der Zielgruppe relevanten KKV verschafft. Mitabeitergerichtet geht es um die Vermittlung eines klaren Markenverständnisses als Identifikations- und Motivationsbasis, um hierdurch ein leitbildgerechtes Verhalten auf allen Hierarchieebenen zu bewirken. Handelsgerichtet erfordert der Aufbau einer starken Markenidentität die Identifikation des Händlers mit der Marke, um entsprechende, auf die Markenidentität abgestimmte Leistungen und Verhaltensweisen der Händler sicherzustellen. Shareholdergerichtet geht es schließlich darum, einen hohen Markenwert als Basis eines hohen Unternehmenswertes zu realisieren. Da sich das Ansehen einer Marke sowohl in einem Preispremium als auch einem Mengenpremium der Marke und damit unmittelbar in höherem Umsatz niederschlägt, kann die Marke auch als Werttreiber des Shareholder Value interpretiert werden, dessen Höhe von unterschiedlichen Werttreibern wie der Wachstumsrate des Umsatzes oder der Betriebsgewinnmarge beeinflußt wird. ${ }^{518}$

Die verschiedenen internen und externen Zielgruppen finden ihren Niederschlag in den drei vorgeschlagenen Perspektiven der BSC, welche im Folgenden aufgezeigt werden (vgl. Abb. 61 mit beispielhaften Maßgrößen). Die Ergebnisperspektive zeigt, ob die verfolgte Markenstrategie ex-post zu einer Ergebnisverbesserung beiträgt. Kennzahlen, die dieser Perspektive zugrunde liegen, nehmen dabei eine Doppelrolle ein: Zum einen definieren sie die Leistung, die von der Strategie erwartet wird, zum anderen fungieren sie als Endziele für die weiteren Perspektiven der Scorecard. Denn jede für eine Scorecard gewählte Kennzahl ist Teil einer Ursache-Wirkungskette, die inr Ende in der Ergebnisperspektive und damit in einem Ergebnisziel findet. Scheinbar sinnvolle Zielwerte bei den anderen Perspektiven erweisen sich auf Dauer wenig nützlich, wenn angestrebte ökonomische Ergebnisgrößen zur Befriedigung der Kapitalgeber nicht erreicht werden.

518 Vgl. hierzu auch Rappaport, A., Creating Shareholder Value. The New Standard for Business Performance, New York 1986, S. 76. 
Die externe Marktperspektive bildet die Markenleistung so ab, wie sie vom Kunden wahrgenommen wird und sich vom Wettbewerb differenziert und umfasst damit alle wettbewerbsentscheidenden Größen, welche die marktseitige Akzeptanz und Wettbewerbsfähigkeit der Marke widerspiegeln (im Rahmen des GAPModells: Identifikationsgap). Für den Markterfolg ist die Kenntnis der kaufentscheidenden Faktoren aus Sicht der Konsumenten von entscheidender Bedeutung, da sie für den Kunden greifbare Faktoren darstellen, welche bewusst oder unbewusst die Entscheidungsfindung beeinflussen.

Im Rahmen der internen Perspektive geht es darum, die Kernkompetenzen einer Marke so abzubilden, dass die vom Kunden gewünschten bzw. vom Management intendierten Leistungen marktgerecht erfüllt werden können. Hierzu sind die für die markenspezifische Leistungserstellung kritischen Prozesse zu identifizieren. Im „Innovationsprozess" sind die artikulierten bzw. latent vorhandenen Wünsche der Konsumenten zu erforschen, um sodann Leistungen zu schaffen, die diesen Wünschen entsprechen (Wahrnehmungsgap). Im "Erstellungsprozess" als zweiter Stufe der internen Wertkette werden die angestrebten Leistungen produziert (Umsetzungsgaps). In der dritten Stufe erfolgt schließlich der "Markttransfer" der erstellten Leistung, wobei Abweichung zwischen tatsächlich erstellter und der in der marktgerichteten Kommunikation versprochenen Leistung des Herstellers Kommunikationsgaps anzeigen. Störungen im Prozess der Markenerstellung liegen unter anderem in einer unzureichenden internen Identifikation der Beteiligten mit der Marke begründet. Als Indikatoren einer innengerichteten Markenidentifikation lassen sich analog zur Hersteller-Handels-Identifikation eine hohe Mitarbeiterzufriedenheiten anführen. ${ }^{519}$

Zusammenfassend schafft die Verbindung der hier genannten Perspektiven die Voraussetzung dafür, dass die Fokussierung einer Perspektive nicht zum Nachteil

Die Mitarbeiterzufriedenheit kann dabei in Anlehnung an das Konstrukt der externen Kundenzufriedenheit als Ergebnis eines komplexen Informationsverarbeitungsprozesses verstanden werden, in dessen Mittelpunkt die kontextgebundene Bewertung aktueller Erfahrungen (Ist) der Mitarbeiter mit den Leistungen des Arbeitgebers anhand eines spezifischen An spruchsniveaus (Soll) steht. Insofern handelt es sich auch bei der Mitarbeiterzufriedenheit um eine Zufriedenheit in einer auf Dauer angelegten Geschäftsbeziehung. Vgl. hier auch Schwetje, Th., Kundenzufriedenheit und Arbeitszufriedenheit bei Dienstleitungen: Operationalisierung und Erklärung der Beziehungen am Beispiel des Handels, Wiesbaden 1999. 
anderer erfolgt. ${ }^{520}$ Eine solchermaßen geschaffene Scorecard stellt somit sicher, dass Inside-Out- und Outside-In-Perspektive über Ursache-Wirkungsketten stärker interagieren und damit nicht isoliert betrachtet werden. Wurden bisher die elementaren Perspektiven der BSC einer Marke zur Erfassung von Leistungstreibern und Ergebnisgrößen erläutert, gilt es im Folgenden, die Wechselwirkung zwischen den Marken zur Abbildung der Gesamtportfolioperformance zu erfassen.

\subsection{Erfassung von Wechselwirkungen zwischen den Marken durch Score- card-Hierarchien}

Zur Erfassung der Wechselwirkungen im Markenportfolio ist es zweckmäßig, neben einer Portfolio-Scorecard je nach Markenzahl weitere markenspezifische Scorecards zu unterscheiden. Da im Markenportfolio den dezentralen Markeneinheiten ein Teil der Führungsaufgaben übertragen wird, liegt es aufgrund der unterschiedlichen Wissensbasen nahe, sich in der Portfolioleitung - wie aufgezeigt - auf eine strategische Rahmenplanung zu beschränken. In einem solchermaßen geschaffenen Rahmen sind für die dezentralen Markeneinheiten in Abhängigkeit ihrer Kernfähigkeiten eigenständige Scorecards zu entwerfen.

Dabei erweisen sich die jeweiligen Leistungstreiber der Marken auf dieser Ebene als hochgradig markenspezifisch und bilden jene wettbewerbsentscheidenden Zielgrößen, welche die einzelnen Marken besonders gut zu erfüllen haben. Vor diesem Hintergrund ist zur Operationalisierung der Leistungstreiber auf die im Rahmen der strategischen Rollen formulierten Markenleitbilder und deren Konkretisierung zurückzugreifen. ${ }^{521}$ Während die generischen Größen der Ergebnisperspektive (z. B. Marktanteil) für alle Marken Gültigkeit besitzen, spiegeln die Leistungstreiber als Frühindikatoren die Besonderheiten der Marke wieder, mit der sich diese von den sonstigen Portfoliomarken bzw. Fremdmarken unterscheiden

520 Zur kritischen Diskussion der BSC vgl. auch Mooraj, St., Oyon, D., Hostettler, D., The Balanced Scorecard: a Necessary Good or an Unnecessary Evil?, in: European Management Journal, Vol. 17, Heft 5, 1999, S. 481ff.

Vgl. hierzu auch Horváth, P., Kaufmann, L., Balanced Scorecard - ein Werkzeug zur Umsetzung von Strategien, a.a.O., S. 42. Werden Scorecards in diesem Zusammenhang undifferenziert auf alle Marke ausgerichtet, so wirkt dieses auf die Steuerung der Marken wie der "Haarschneideautomat von Kirsch/Roventa/Trux". Vgl. Weber, J., Schäffer, U., Balanced Scorecard \& Controlling: Implementierung - Nutzen für Manager und Controller - Erfahrungen in deutschen Unternehmen, a.a.O., S. 64. 
möchte. Die Leistungstreiber reflektieren somit die Einzigartigkeit der Strategie einer Marke.

Die BSC der Portfolioleitung führt die dezentralen Markenscorecards über Kennzahlen zusammen und ergänzt sie um Kennzahlen,

- die sich aus der Gesamtbetrachtung der Mehrmarkenstrategie ergeben (z. B. Wanderungssaldo des Gesamtportfolios) bzw.

- die sich auf die spezifische Wertschöpfung der Portfolioleitung in der Realisierung finanzieller und immaterieller Synergien beziehen (z. B. gemeinsame Nutzung von Technologien oder zentrale Dienstleitungen).

Die Kennzahlen der Portfolioscorecard erhalten dabei den Charakter von Richtzahlen, welche den Rahmen abstecken für die eigenständigen Entscheidungen der dezentralen Markeneinheiten. Auf dieser Basis erarbeiten die Markeneinheiten ihre eigenen Scorecards, so dass im Ergebnis ein "Netz" über die Portfolioscorecard miteinander verwobener Marken-Scorecards entsteht. ${ }^{522}$ Die auf der Ebene der Portfoliosteuerung erarbeiteten Steuerungsgrößen sind auf die jeweiligen Markenebenen herunterzubrechen ("Cascading-Effekt ${ }^{4523}$ ), da die eigentliche Wertschöpfung durch die einzelnen Markeneinheiten erbracht wird. ${ }^{524}$ Grundsätzlich kann die vertikale Ausdehnung der Scorecards auch weiter auf Modellebene bzw. sonstige nachgelagerte Ebenen erfolgen. Der formale Aufbau einer solchen Scorecard-Hierarchie ist der Abb. 62 zu entnehmen. ${ }^{525}$

Vgl. hier auch Friedag, H. R., Schmidt, W., Balanced Scorecard. Mehr als ein Kennzahlensystem, Freiburg i. Br., Berlin, München 1999, S. 238.

Vgl. Brunner, J., Value-Based Performance Management, Wertsteigernde Unternehmensführung: Strategien, Instrumente, Praxisbeispiele, a.a.O., S. 100. 


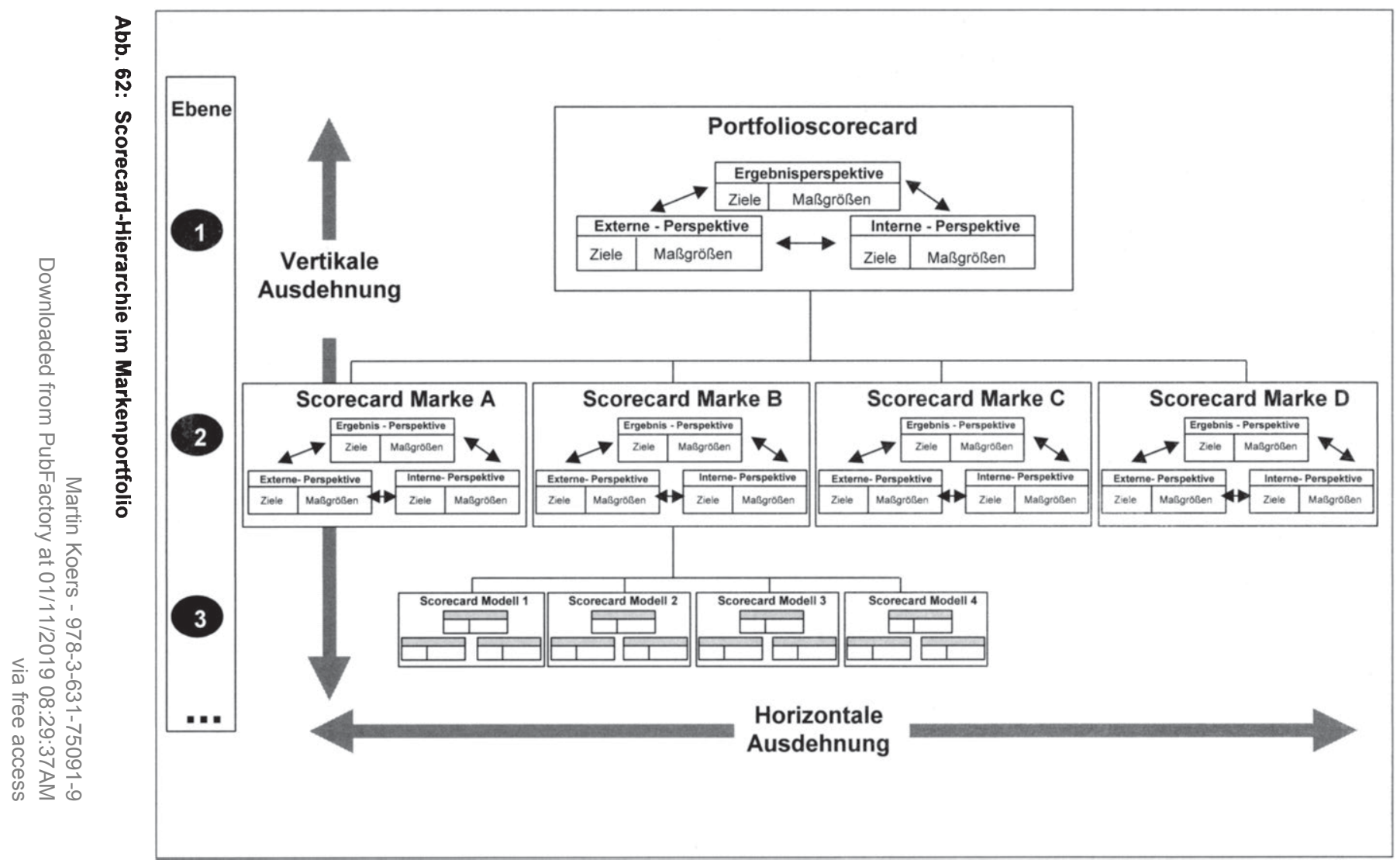


Das Ziel der Hierarchisierung einer Balanced Scorecard in einem umfassenden Balanced Scorecard-System besteht somit in einer an den jeweiligen Aufgaben der einzelnen Markeneinheiten abgestimmten Informationsversorgung sowie der Ausrichtung der untergeordneten Markeneinheiten an die Strategie des Gesamtportfolios. Anstatt das gesamte Portfolio mit all seinen Informationen in einem Informationssystem abzubilden (Totalansatz des Gesamtportfolios), wird hier somit ein empfängerorientierter Totalansatz verfolgt, wobei die strategieorientierte Gestaltungsausrichtung als konstitutives Merkmal des Balanced Scorecard Aufbaus bezeichnet werden kann. ${ }^{526}$ Ein solcher Aufbau ermöglicht es, die strategische Heterogenität der Marken in den Scorecards abzubilden.

Das Herunterbrechen der Scorecards kann je nach Führungsstil und Detaillierungsgrad der Entscheidungen im Portfolio grundsätzlich auf unterschiedliche Art und Weise erfolgen:

- Sind die einzelnen Einheiten sehr heterogen, sind auch unterschiedliche Scorecards zu entwerfen. Die Balanced Scorecard der übergeordneten Einheit dient als strategischer Rahmen, innerhalb dessen die einzelnen untergeordneten Markeneinheiten eigenständige Scorecards formulieren. Strategische Ziele und Aktionen der übergeordneten Einheiten, die der nachgelagerten Markeneinheit zugeordnet wurden, werden aufgenommen. Das Ergebnis ist eine eigenständige, aber kompatible BSC für jede Einheit. Heterogenität zwischen den Einheiten liegt grundsätzlich auf Markenebene vor, da die Markeneinheiten unterschiedliche Strategien in unterschiedlichen Marktsegmenten verfolgen und sich per definitionem anhand zentraler Merkmale unterscheiden sollen.

- Weisen die einzelnen Einheiten eine große Ähnlichkeit auf, d.h. agieren sie beispielsweise mit vergleichbaren Strategien in denselben Marktsegmenten, so können die Scorecards standardisiert werden. In der Balanced Scorecard stehen nur Ziele, die für alle Einheiten gelten, so dass jede Scorecard grundsätzlich gleich aussieht, allerdings unterschiedliche Zielwerte beinhaltet. Die Scorecard-Standardisierung erleichtert letztlich die Vergleichbarkeit der Einheiten untereinander. Ähnlichkeiten zwischen den Einheiten liegen primär auf Mo-

526 Vgl. auch Butler, A., Letza, St, Neale, B., Linking the Balanced Scorecard to Strategy, in: Long Range Planning, Vol. 30, Heft 2, 1997, S. 242ff. 
dellebene, wenn Modelle unterschiedlicher Marken bei standardisiertem Ausstattungsgrad ähnliche Marktsegmente ansprechen.

Die Erstellung von Scorecards für die einzelnen Markeneinheiten im Portfolio gestaltet sich wie folgt: Die strategische Rahmenplanung gibt ein Bündel von Zielen für die einzelnen Perspektiven der Scorecards vor. Dies sind auf der Portfolioebene Ziele wie z. B. die Erreichung eines bestimmten Marktanteils, eines bestimmten Umsatzes, die Position in einem bestimmten Markt, etc. Diese werden für die einzelnen Markeneinheiten in Abhängigkeit von ihrer Rolle im Portfolio konkretisiert. Die Konkretisierung erfolgt sowohl durch die Spezifizierung einzelner Werte als auch durch die Überführung der Messgrößen in beeinflussbare und verantwortbare Größen für die Markeneinheiten. Durch die Konkretisierung entlang der Portfoliohierarchie wird die vertikale Durchdringung der Ziele und damit auch der Maßnahmen erreicht. Manche Größen sind dabei nur auf der Portfolioebene zu steuern, andere werden von den unteren Ebenen bis auf die Portfolioebene aggregiert und in der Portfolioscorecard ausgewiesen (z. B. Kundenzufriedenheit). In den jeweiligen untergeordneten Scorecards sind die Einzelwerte in verschiedenen Detaillierungen vorhanden (z. B. Zufriedenheit mit Kundenservice).

Abb. 63 zeigt eine beispielhafte Darstellung vor- und nachgelagerter Scorecards im Markenportfolio anhand des in Kapitel B aufgezeigten Markenleitbildes der Volumensmarke $\mathrm{VW}$. Während die Oberziele der hier verfolgten Mehrmarkenstrategie inren Niederschlag in der Portfolioscorecard finden, deren Zielerreichungsgrad mit Hilfe geeigneter Steuerungsgrößen gemessen wird, beinhaltet die dargestellte Markenscorecard die spezifischen Ziele der Marke VW auf Basis des definierten Markenleitbildes bzw. der strategischen Rolle im Portfolio. 


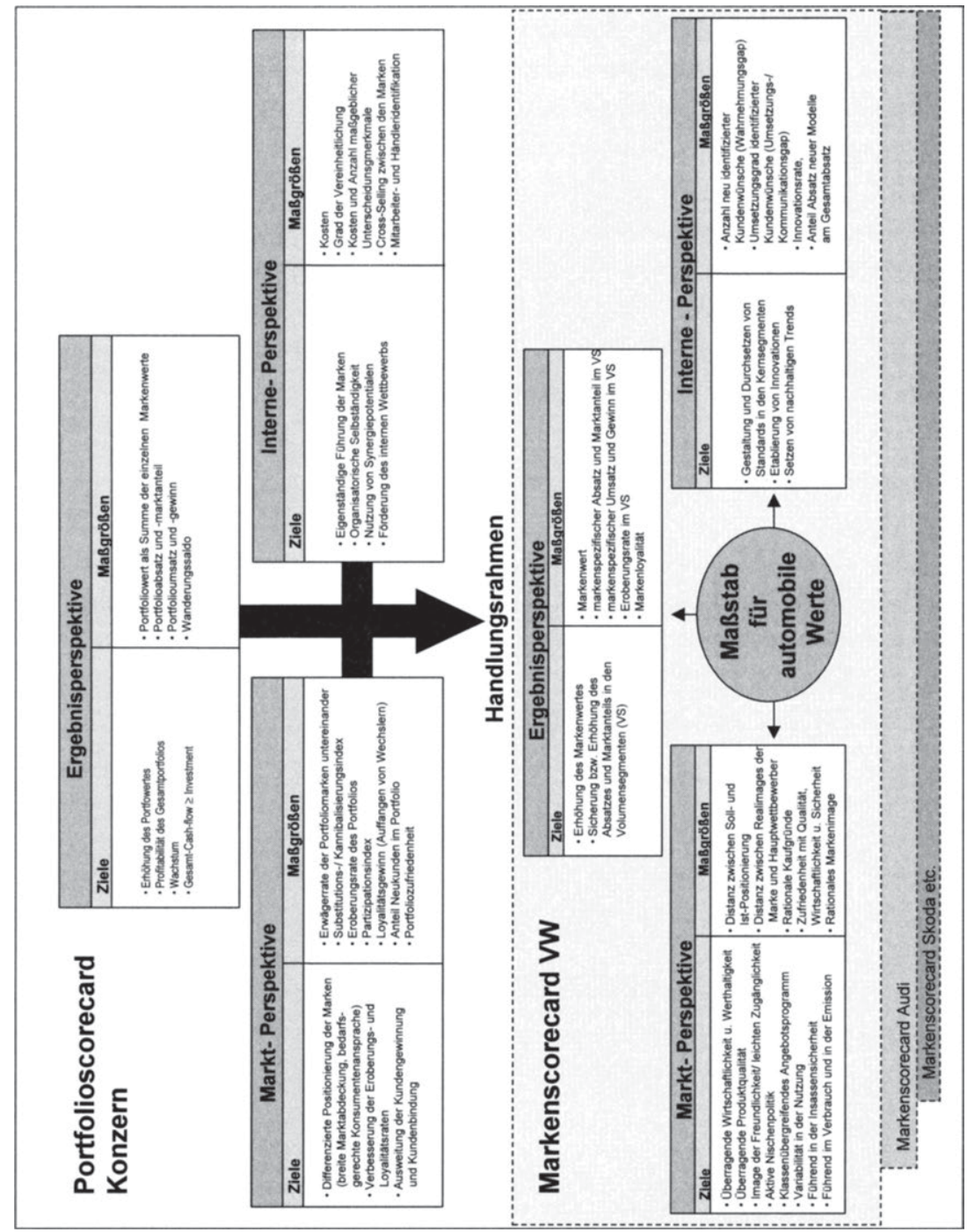

Abb. 63: Beispielhafte Darstellung einer Scorecard-Hierarchie 
Zusammenfassend stellt die BSC ein hilfreiches Instrument der Planung, Steuerung und Kontrolle der Markeneinheiten im Portfolio dar. Keinesfalls soll die Balanced Scorecard die differenzierten Kontrollstrukturen im Portfolio ersetzen, vielmehr dient sie dazu,

- die qualitativen und quantitativen Ziel der Mehrmarkenstrategie in konkrete Vorgaben zu konkretisieren und zu übersetzen,

- die Prioritäten zu kommunizieren, um so Anhaltspunkte für den Marketing-Mix zu generieren sowie

- den Feedback zu erhöhen und damit die Möglichkeit für Lernprozesse zu verbessern.

Ein Prozess des strategischen Lernens und der strategischen Anpassung stellt letztlich eine zentrale Grundlage für die erfolgreiche Umsetzung der Mehrmarkenstrategie dar. ${ }^{527}$ Infolge der ganzheitlichen Betrachtung, der Orientierung an langfristigen Strategien und des permanenten Abgleichs aller Bezugsgruppen der Marken gewährleistet die BSC schließlich, dass Markenleitbilder nicht eine abstrakte Konstruktion bleiben, sondern konkret steuerbar werden.

527 Vgl. hierzu auch Jenner, Th., Markenführung als Lernprozeß, in: Harvard Business Manager, No. 5,1999 , S. $20 \mathrm{ff}$. 


\section{E. Schlussbetrachtung und Ausblick}

\section{Zusammenfassende Würdigung der Untersuchungsergebnisse}

Ausgehend von der Beobachtung einer zunehmenden Marktbearbeitung mit mehreren, parallel auf den Absatzmarkt ausgerichteten Marken und damit dem vermehrten Auftreten von Mehrmarkenstrategien als Antwort auf die Polarisierung des Konsumentenverhaltens war es die grundsätzliche Aufgabe der vorliegenden Arbeit, einen Beitrag zur Gestaltung eines Controllingsystems zur Planung, Koordination und Steuerung von Markenportfolios zu leisten. Ausgangspunkt der Untersuchung bildete die Überlegung, dass eine koordinierte Führung von Markenportfolios durch eine gegenseitige Abstimmung der Portfoliomarken einen höheren Portfoliogesamtgewinn erwarten läßt als die unkoordinierte. In diesem Sinne sind die Ergebnisse der Untersuchung sowohl für die Praxis des Managements von Markenportfolios als auch für die weitere Forschung im Bereich der Mehrmarkenführung und des Mehrmarkencontrolling relevant.

Unter Bezugnahme auf die eingangs formulierten Zielsetzungen der Untersuchung lassen sich die zentralen Untersuchungsergebnisse wie folgt zusammenfassen:

- Als Ausgangspunkt der Analyse galt es zunächst, die Ziele bei der Verfolgung von Mehrmarkenstrategien zu erarbeiten, wobei mit marktgerichteten und unternehmensgerichteten Zielen der Mehrmarkenstrategie zwei grundsätzliche Zielsetzungen aufgezeigt werden konnten. Gerade im Automobilmarkt als situativem Kontext der Untersuchung befinden sich Hersteller im Spannungsfeld einer kostenorientierten Standardisierung einerseits und einer zunehmenden Individualisierung der Nachfrage anderseits. Hier konnte gezeigt werden, dass die Mehrmarkenstrategie einen gangbaren Weg darstellt, durch eine bedarfsgerechte Ausweitung des Angebotsspektrums der zunehmenden Fragmentierung bislang homogener Marktsegmente gerecht zu werden und gleichzeitig wettbewerbsinduziert die Wirtschaftlichkeit der Marktbearbeitung sicherzustellen.

- Zur Durchdringung der Mehrmarkenstrategie als Objekt des Controlling wurden aufbauend auf der Zielanalyse die entscheidungsfeldabhängigen planerischen und organisatorischen Aspekte eines Markenportfolios umfassend erarbeitet. Zur Berücksichtigung des Wechselspiels zwischen Inside-Out- und Outside-In-Perspektive und damit zur Schaffung einer Synthese der markt- und ressourcenorientierten Sichtweise erwies sich der identitätsorientierte Ansatz 
der Markenführung als geeignete theoretische Grundlage der Mehrmarkenführung.

- Im Entscheidungsfeld des Portfolios wurden mit der strategischen Rahmenplanung und der marktstrategischen Instrumenteausgestaltung zwei Ebenen der Planung unterscheiden, deren Ausgestaltung im Sinne des identitätsorientierten Ansatzes sowohl auf eine Outside-In- als auch eine Inside-Out-Perspektive zu stellen war. Im Mittelpunkt der strategischen Rahmenplanung steht dabei die Definition strategischer Rollen, um darauf aufbauend Markenpositionierungen abzuleiten, welche es innen- und außenwirksam durch Markenleitbilder zu fördern gilt. Auf der Grundlage dieser übergeordneten Stoßrichtungen des Marktauftritts erfolgt auf der Ebene der marktstrategischen Instrumenteausgestaltung die Planung des zielgerichteten und auf die relevanten Absatzmärkte abgestimmten Einsatzes der absatzpolitischen Instrumente.

- Hinsichtlich der organisatorischen Verankerung von Mehrmarkenstrategien wurde es als zweckmäßig erachtet, das Führungssystem im Portfolio unter der Maxime "so viel Dezentralität wie möglich, so wenig Zentralität wie nötig" auf eine Optimierung des Handlungsspielraums der selbständigen Markeneinheiten bzw. der Eingriffstiefe der Portfolioleitung auszurichten. Unter der Prämisse der Ergebnismaximierung des Gesamtportfolios zielt die Mehrmarkenstrategie dabei weniger auf die Optimierung des Marktauftritts einzelner Marken ab, sondern strebt eine bestmögliche Marktbearbeitung durch das gesamte Portfolio an. Die Führung des Markenportfolios sollte letztlich im Gegenstromverfahren erfolgen, wobei die Portfolioleitung im Sinne einer Top-DownPlanung die Formulierung der Stoßrichtungen des Marktauftritts dominiert, während die dezentralen Markeneinheiten mit der Umsetzung der Rahmenplanung auf der Grundlage spezifischer Zielvereinbarungen betraut sind.

- Die Untersuchung des Controllingbedarfs ließ erkennen, dass insbesondere die Aufspaltung des Markenführungssystems in partielle Entscheidungsfelder mit der Folge von Interdependenzen und möglichen Zielkonflikten den Koordinations- und damit Controllingbedarf in Mehrmarkenstrategien begründen. Interdependenzen zwischen den Marken desselben Produktbereichs führen da$\mathrm{zu}$, dass die Freiheitsgrade bei der Strategieplanung und -umsetzung und damit auch die Ergebnisse einzelner Marken nicht unabhängig voneinander sind. Durch die separate Führung der Markeneinheiten erwächst überdies die Gefahr der Entwicklung eigener Zielsysteme mit der Konsequenz möglicher Zielkonflikte zwischen den Einheiten. In diesem Zusammenhang hat sich die de-

Martin Koers - 978-3-631-75091-9 
tailanalytische Diskussion der internen und externen Kontextfaktoren des Controllingbedarfs als zweckmäßig erwiesen. Durch die disaggregierte Analyse des Controllingbedarfs gelang es, die vielschichtigen Einflüsse, denen die Abstimmung im Markenportfolio ausgesetzt ist, differenziert zu erfassen, ohne die Interdependenzen zwischen den Einflussfaktoren zu vernachlässigen. Gleichzeitig wurde durch diesen kontigenztheoretischen Ansatz ein Konzept zur empirischen Erfassung des Controllingbedarfs erarbeitet.

- Da ohne gezielten Informationsfluss eine Abstimmung arbeitsteilig vollzogener Handlungen zur Erreichung der übergeordneten Zielsetzungen der Mehrmarkenstrategie nicht möglich ist, kommt der Beschaffung, Aufbereitung und Analyse von Daten zur Vorbereitung zielsetzungsgerechter Entscheidungen im Portfolio eine besondere Bedeutung zu. Zur Sicherstellung eines abgestimmten Verhaltens im Markenportfolio und damit zur Schaffung von "Steuerungsklarheit" wurde die Balanced Scorecard als zweckmäßiges integratives Controllingkonzept erachtet. Durch eine Anbindung der Steuerungsgrößen an die strategischen Ziele erlaubt die Balanced Scorecard, die Operationalität von Strategien über mehrere Steuerungsebenen zu erhöhen. In Abwandlung zur Balanced Scorecard im Sinne KAPLAN/NORTONS wurde dabei eine Modifikation des Konzepts vorgenommen. Um auch im Controlling dem Wechselspiel zwischen Inside-Out und Outside-In-Perspektive der Markenführung Rechnung tragen zu können, bot es sich an, mit einer internen Unternehmens-Perspektive, einer externen Marktperspektive und einer Ergebnisperspektive drei Perspektiven einer Scorecard zu unterscheiden. Zur Analyse relevanter Kennzahlen für eine solche Scorecard erwies es sich hingegen als zweckmäßig, entsprechend der Führungsstruktur im Portfolio mit einer strategischen und einer operativen Ebene zwei Ebenen des Mehrmarkencontrolling zu differenzieren. Eine solche Unterscheidung erlaubt, den differenzierten Informationsansprüchen des strategischen und operativen Portfoliomanagement mittels differenzierter Instrumente nachzukommen.

- Auf einer Metaebene des strategischen Controlling wurden zunächst Instrumente aufgezeigt, welche eine grundlegende Revision der bisherigen Markenpolitik erlauben. Während zur qualitativen Bewertung der Mehrmarkenstrategie ein Scoring-Modell mit geeigneten Evaluierungsfaktoren herangezogen wurde, lieferte die quantitative Bewertung insbesondere Hilfestellungen zur Festlegung der optimalen Markenzahl in einem Portfolio. 
- Zur weiteren quantitativen Beurteilung der Mehrmarkenstrategie erwiesen sich Wanderungs- und Absatzanalysen auf Basis des beobachtbaren Kaufverhaltens als geeignet. Durch eine wertmäßige Gegenüberstellung von Wanderungsbewegungen innerhalb des Portfolios (Substitutionseffekt) mit den Wanderungen zwischen den Eigen- und Fremdmarken (Partizipationseffekt) konnten die Ergebnisse markenpolitischer Aktivitäten verdeutlicht werden. Dabei erschien es sinnvoll, nur bei solchen internen Wanderbewegungen von Kannibalisierung in Abgrenzung zur Substitution zu sprechen, bei denen Nachfrager von einer Marke des Portfolios zur anderen wechseln, als Alternativmarke jedoch die vorher besessene Marke des Portfolios und nicht eine Wettbewerbsmarke in Betracht gezogen haben. Markenwechsler innerhalb des Portfolios, ohne Präferenz für die vorher besessene Portfoliomarke, wurden im vorgestellten Ansatz den Loyalitätsgewinnen des Portfolios zugerechnet.

- Zur Identifikation und Strukturierung möglicher Positionierungsmängel im Portfolio als Ursache ungewollter Wanderungsbewegungen stellte das GAPModell einen tragfähigen Orientierungsrahmen der Positionierungsanalyse dar. Die Analyse zeigte, dass das aufgestellte Modell insbesondere deshalb eine geeignete Heuristik für das Portfoliomanagement ist, weil es die Möglichkeit eröffnet, dem postulierten Grundgedanken der Markenidentität folgend nicht nur eine nachfragerorientierte Sichtweise der Problemstellung, sondern darüber hinaus auch eine angebotsorientierte Sicht als gleichberechtigte Perspektive zu verfolgen.

- Um das Ausmaß der Wanderungsbewegungen im Markenportfolio abschätzen zu können, galt es zunächst, die von den Nachfragern wahrgenommene Ähnlichkeit der unternehmenseigenen Marken zu prüfen (Fremdbildbetrachtung). Insbesondere mit Hilfe der Multidimensionalen Skalierung auf Basis von Erwägerraten konnte die subjektiv empfundenen Austauschbarkeit der Marken verdeutlicht werden, wobei die in diesem Zusammenhang ermittelten Distanzen im Wahrnehmungsraum zur Beleuchtung der Substitutions- und Partizipationsbeziehungen zwischen den Marken herangezogen wurden. Zur Analyse möglicher Abweichungen zwischen intendierten Soll- und realisierten IstPositionierungen wurden in einem zweiten Schritt mit Hilfe des Semantischen Differentials die Selbstbilder der Marken analysiert und den wahrgenommenen Fremdbildern gegenübergestellt.

- Zur Erklärung der Gesamtabweichung zwischen Selbst- und Fremdbild der einzelnen Marken liefert eine Abweichungsaufspaltung probate Informatio- 
nen, die den Ausgangspunkt für konkrete Gestaltungsmaßnahmen zur SchlieBung bestehender GAPs bilden. Überdies ermöglicht die Analyse ausgewählter Einflussfaktoren auf die Markenpositionierung zusätzliche Erkenntnisse zur Erklärung der ermittelten Realpositionen der Marken. Gleichzeitig stellen sie eine Grundlage für die Ermittlung einer situationsgerechten und sinnvollen SollPositionierung der Marken dar.

- Im Rahmen des operativen Mehrmarkencontrolling standen zentrale Controllingaspekte bei der Ausgestaltung der Instrumentalstrategien im Fokus der Betrachtung. So bedarf es zur Unterstützung produktpolitischer Entscheidungen der Identifikation derjenigen Leistungskomponenten, die von jeder Marke zur Sicherstellung einer starken Markenidentität selbständig zu produzieren sind. Zur Ermittlung koordinierter, optimaler Preise zur Gewinnmaximierung im Gesamtportfolio gilt es, entsprechend der Wechselneigung der Konsumenten spezifische Preiskorridore im Portfolio zu fixieren. Neben Werbebudgetierungsentscheidungen stellt schließlich die Analyse der Markenidentifikation des Handels zur Sicherstellung eines leitbildgerechten Auftritts der Händlerorganisation ein zentrales Element des operativen Mehrmarkencontrolling dar.

- Zur Vermeidung einzelner, mosaiksteinartiger Kennzahlen und Instrumente und damit zur Gewährleistung einer abgestimmten und in sich konsistenten Informationsversorgung unterschiedlicher Aggregationsebenen des Portfolios erfolgte schließlich eine hierarchische Informationsintegration relevanter Steuerungsgrößen. Indem das Portfolio aus verschiedenen Perspektiven in vor- und nachgelagerten Scorecards betrachtet wird, lässt sich insgesamt eine adressatenorientierte Unterstützungsleistung als zentrale Aufgabe des Mehrmarkencontrolling gewährleisten.

\section{Implikationen für weiterführende Forschungsarbeiten}

Der vorliegenden Arbeit kommt der Charakter einer Basisuntersuchung zu, die den generellen Rahmen der Mehrmarkenführung und des Mehrmarkencontrolling unter besonderer Berücksichtigung des Automobilsektors aufspannt. Gemäß der formulierten Untersuchungsziele wurde dabei der Versuch unternommen, einen Beitrag zur Planung, Koordination und Steuerung von Markenportfolios zu leisten. Aufgrund der Breite des Untersuchungsspektrums und der dadurch notwendigen Konzentration auf zentrale Instrumente und Zusammenhänge zeichnen sich neben den diskutierten und weiter auszuführenden Aspekten der Balanced Scorecard verschiedene Ansatzpunkte für weiterführende Forschungsarbeiten ab: 
- Grundlage der Erkenntnisgewinnung in der vorliegenden Untersuchung waren vor allem literaturgestützte Plausibilitätsüberlegungen, welche durch einzelfallstudienhafte Informationen ergänzt wurden. Diese informatorische Basis liess bezüglich des Erkenntnisobjekts häufig nur Tendenzaussagen zu. Die Ergebnisse der Untersuchung sind deshalb im Rahmen empirischer Studien zu validieren. Dies gilt insbesondere für die Ermittlung des Controllingbedarfs, wobei hier unter Einbezug möglichst vieler und heterogener Unternehmen auf Basis von Dependenz- und Interdependenzanalysen gesicherte Aussagen über die Zusammenhänge zwischen dem situativen Umfeld und der Koordination von Markenportfolios zu erarbeiten sind. Daneben gilt es, die angedeuteten kausalen Zusammenhänge innerhalb des Controlling-Instrumentariums dezidiert zu überprüfen.

- Die vorliegende Untersuchung konzentrierte sich ausschließlich auf Markenportfolios in der Automobilindustrie und damit auf langlebige Gebrauchsgüter. Um Parallelen oder Unterschiede zu anderen Branchen bezüglich der Auswirkungen der Mehrmarkenführung aufzudecken, wäre die Ausdehnung des Branchenfokus, insbesondere auf kurzlebige Konsumgüter, wünschenswert. Hierbei ist zu untersuchen, inwieweit Aspekte des unterschiedlichen Involvement die Übertragbarkeit des vorgestellten Controlling-Instrumentariums zulassen bzw. die Anpassung der Konzeption erfordern.

- Es wurde deutlich, dass das Mehrmarkencontrolling in einem unmittelbaren Zusammenhang mit der zur Verfügung stehenden Datenbasis steht. Folglich kommt der informationstechnologischen Infrastruktur im Rahmen des Controlling eine zentrale Bedeutung zu. Hier sind weitere Forschungsbemühungen auf der Schnittstelle zwischen Marketing und Informatik erforderlich, welche die Umsetzung der vorgestellten Konzeption eines Mehrmarkencontrolling in funktionsfähige IT-Systeme zum Inhalt haben. Dies gilt zum einen hinsichtlich der Schaffung einer integrierten und konsistenten Datenbasis, welche die Erfassung und Organisation aller benötigten Daten unter mehrmarkenspezischen Gesichtspunkten sicherstellt. Hier gilt es primär, Ansätze des Data Warehousing ${ }^{528}$ auf ihre Übertragbarkeit in den Mehrmarkenkontext hin zu

528 Das Datawarehouse-Konzept wurde ursprünglich entwickelt, um das Integrationsproblem heterogener Datenquellen zu lösen und die ungenügenden Abfragemöglichkeiten von Datenbanken für Benutzer zu umgehen. Mittlerweile hat sich dieses Konzept zu einem integralen Bestandteil einer effizienten IT-Struktur für die Analyse der historischen, aggregierten, sub- 
überprüfen bzw. den benötigten Anpassungsbedarf festzustellen. Darüber hinaus bedarf es der Entwicklung geeigneter Verfahren der Informationsmodellierung und -auswertung, welche eine effiziente und effektive Unterstützung des Controllers gewährleisten. Weiterführende Ansatzpunkte können sich hier insbesondere aus Arbeiten über dynamische Datenanalysen wie z.B. OLAP ${ }^{529}$ im Kontext der Balanced Scorecard ergeben.

- Bei der Operationalisierung des Markenwertes als Idealziel der Markenführung stehen zahlreiche (vorwiegend in der Praxis entwickelte) finanzwirtschaftliche und verhaltenswissenschaftliche Konzepte relativ isoliert nebeneinander. Es ist eine zentrale Aufgabe der Marketingwissenschaft, diese Konzepte zu evaluieren und in ein leistungsfähiges Mehrmarkencontrolling zu integrieren. Hierbei ist auch nach Möglichkeiten zu suchen, den Abnutzungseffekt des Wertes einzelner Portfoliomarken in Folge alternativer Konfigurationen von Mehrmarkenstrategien zu quantifizieren.

- Damit die für die einzelnen Portfoliomarken entwickelten Markenleitbilder dauerhaft zielgerichtet realisiert werden können, ist die konsequente Anbindung der dezentralen Markenmanager an eine spürbar variable, leistungsorientierte Vergütung unverzichtbar, so dass Fragen der Leistungsvergütung von Markenmanagern eine erheblich erweiterte Bedeutung zukommt. Dies umfasst nicht nur die Ebene der Portfolioleitung, sondern ist in ihrem Anspruch deutlich auf die nachfolgenden Hierarchieebenen auszulegen. Hier haben verstärkt Forschungsbemühungen anzusetzen, inwiefern ein ausreichend hoher Dekkungsgrad von aussagefähigen Leistungsindikatoren zur Portfoliosteuerung erreicht werden kann, welche zur effektiven Einbindung in ein Anreizsystem benötigt werden. Bei Anreizsystemen auf der Grundlage der Balanced Scorecard kann die variable Vergütung an wesentliche Kennzahlen und damit an Fakto-

jektorientierten und integrierten Geschäftsdaten aus den Transaktionssystemen entwickelt. Vgl. hierzu etwa Immon, W. H., Building the Datawarehouse, New York 1996, S. 1ff.

Der Begriff OLAP (On-Line Analytical Processing) kennzeichnet Verfahren zur multidimensionalen Strukturierung bzw. Modellierung von Daten in Datenbanken. Diese können im Vergleich zu klassischen relationalen Datenbanken wesentlich flexibler, d.h. aus mehreren frei definierbaren Dimensionen, und schneller analysiert werden. Zudem wird die unternehmensweite Verteilung der Analysekompetenz auf einzelne Anwender durch Anbindung von OLAPStandardmodulen durch alle führenden Datenbankanbieter wie z.B. ORACLE oder IBM unterstützt. Vgl. für einen Überblick Schinzer, H.D./Bange, C./Mertnes, H., Data Warehouse und Data Mining: Marktführende Produkte im Vergleich, 2. Aufl., München 1999, S. 38 ff. 
ren gebunden werden, die dem Aufbau und der Sicherung strategischer Erfolgspotenziale dienen kann. Die Forschungsbemühungen sollten dabei auch eine Verknüpfung der Erfolgsmessung und -vergütung von Managern mit dem langfristigen Markenwert der einzelnen Portfoliomarken fokussieren.

- Da insbesondere Kultmarken für den Konsumenten einen starken emotionalen Zusatznutzen durch die Vermittlung sinnhafter Lebensziele, Weltbilder und Werthaltungen schaffen, gewinnt die mystifizierende Inszenierung von Marken insbesondere in der Automobilbranche zunehmend an Bedeutung. In diesem Zusammenhang ist das Controlling aufgefordert, verstärkt emotionalaffektive sowie symbolische Werte und inre Wechselwirkungen untereinander zu berücksichtigen. Hierzu sind im Rahmen des Mehrmarkencontrolling verstärkt Informationen aus qualitativen Frühwarnsystemen, wie sie z.B. die Trendforschung darstellt, einzubeziehen. Auch Ansätze der Means-EndForschung ${ }^{530}$ können für das Controlling wertvolle Hinweise, insbesondere im Bereich von Positionierungsanalysen liefern. Dabei sollten die Forschungsbemühungen allerdings in dem Bewusstsein vorangetrieben werden, dass sich die Modellqualität nicht zuletzt am Kriterium der Akzeptanz durch das Management zu messen hat.

- Schließlich stellt auch das Mehrmarkencontrolling ein Mittel zum Zweck eines übergreifenden ökonomischen Unternehmenserfolges dar. Vor diesem Hintergrund sind die Kosten und der Nutzen eines Mehrmarkencontrolling zu spezifizieren und damit die Erfolgswirksamkeit des Mehrmarkencontrolling zu überprüfen, um hierdurch zur Ermittlung und Realisierung einer optimalen Controllingintensität beizutragen. Darüber hinaus sind Ansatzpunkte für eine organisatorische Verankerung des Mehrmarkencontrolling im Rahmen zukünftiger Forschungsaktivitäten zu generieren um damit die Frage „Selbst- versus Fremdcontrolling" zu spezifizieren.

Die zentrale Aussage der Means-End-Theorie stützt sich auf die These, dass Produkte von Konsumenten als Mittel ("means") zur Erreichung erwünschter, oftmals abstrakter Ziele ("ends") betrachtet werden. Durch eine verfahrenstechnische Integration qualitativer und quantitativer Forschungsmethoden wird versucht, Kausalitäten zwischen konkreten Produktattributen und subjektiv damit verbundenen Nutzenerwartungen und Werthaltungen abzubilden. Einen aktuellen Überblick über den Forschungsstand und Anwendungsfelder der MeansEnd-Theorie liefern Herrmann, A., Huber, F., Braunstein, C., Gestaltung der Markenpersönlichkeit mittels der "means-end"-Theorie, in: Moderne Markenführung: Grundlagen - innovative Ansätze - praktische Umsetzung, Esch, F.-R. (Hrsg.), Wiesbaden 1999, S. $103 \mathrm{ff}$. 
Insgesamt zeichnet sich ab, dass Fragen der Mehrmarkenführung im Allgemeinen und des Mehrmarkencontrolling im Besonderen in Zukunft weiter an Bedeutung gewinnen werden. Die mit dieser Aufgabe verbundene Komplexität wird im Rahmen der weiteren Diffusion des Internet als Vertriebsplattform (E-Commerce) zunehmen, da den Unternehmen speziell in der Automobilindustrie in Zukunft weitere Vertriebswege i.S.v. von Mehrkanalsystemen zur Verfügung stehen werden. Daneben geben die zu beobachtende Entwicklung in der Aufgabenumwelt und dabei insbesondere die kostengetriebene Angleichung von Produkten, die weitere Fragmentierung der Märkte und damit das Spannungsfeld von Standardisierung und Individualisierung zu der Erwartung Anlass, dass bei vielen Markenartiklern weitere Wachstumschancen nur durch Führung von MehrMarken zu realisieren sind.

Vor diesem Hintergrund muss sich die Unternehmenspraxis darauf einstellen, dass die Führung von Markenportfolios und damit verbunden das Mehrmarkencontrolling immer mehr zu einem zentralen Erfolgsfaktor werden. Indessen ist die Marketing-Wissenschaft angesichts der damit verbundenen Herausforderungen aufgefordert, die genannten Anregungen aufzugreifen und die Forschungsaktivitäten in diesem noch weitgehend unergründeten Problemfeld zu intensivieren. 
Martin Koers - 978-3-631-75091-9

Downloaded from PubFactory at 01/11/2019 08:29:37AM

via free access 


\section{Anhangsverzeichnis}

$\begin{array}{ll}\text { Anhang I: Erwägermatrix } 263 & 263\end{array}$

Anhang II: Begriffswolken des Semantischen Differentials 264

Anhang III: Polaritätenprofil Soll-Selbstbild und Ist-Fremdbild Marke B 266

Polaritätenprofil Soll-Selbstbild und Ist-Fremdbild Marke C 267

Polaritätenprofil Soll-Selbstbild und Ist-Fremdbild Marke D 268

Anhang IV: Semantische Distanzen zwischen den Marken 269 
Martin Koers - 978-3-631-75091-9

Downloaded from PubFactory at 01/11/2019 08:29:37AM

via free access 


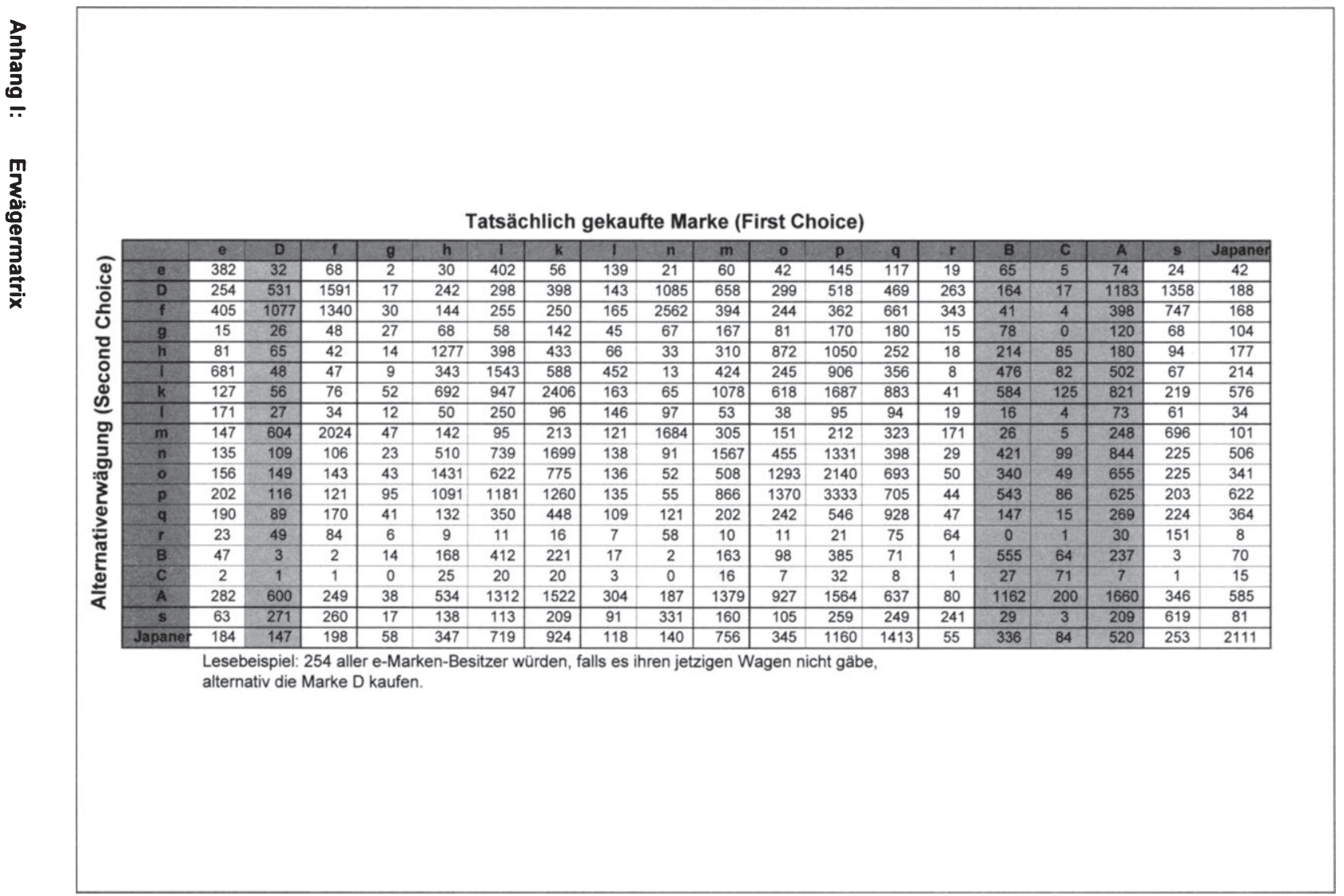




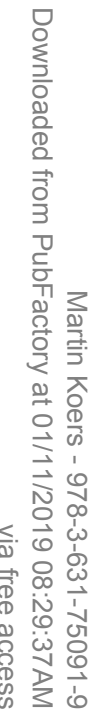

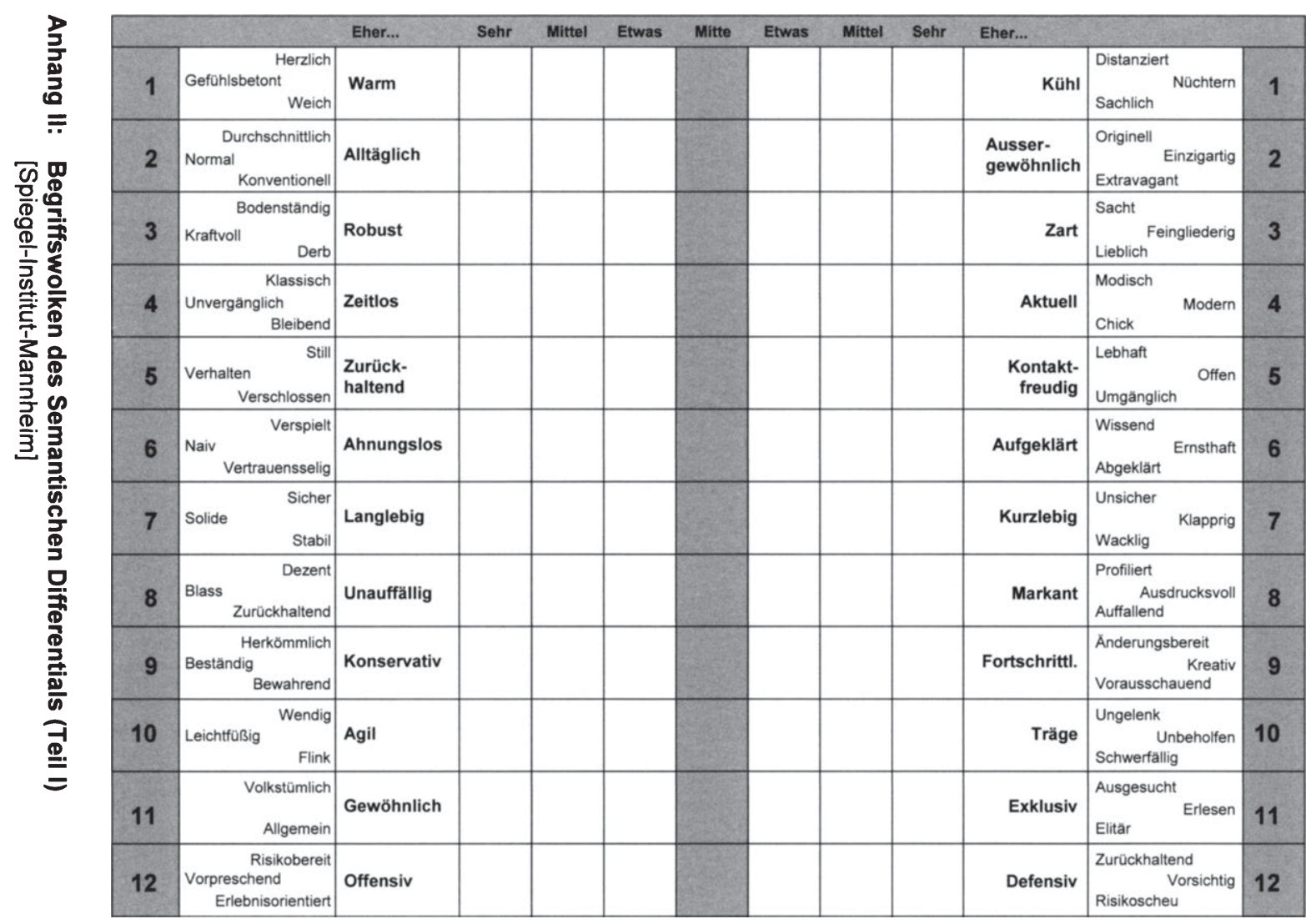




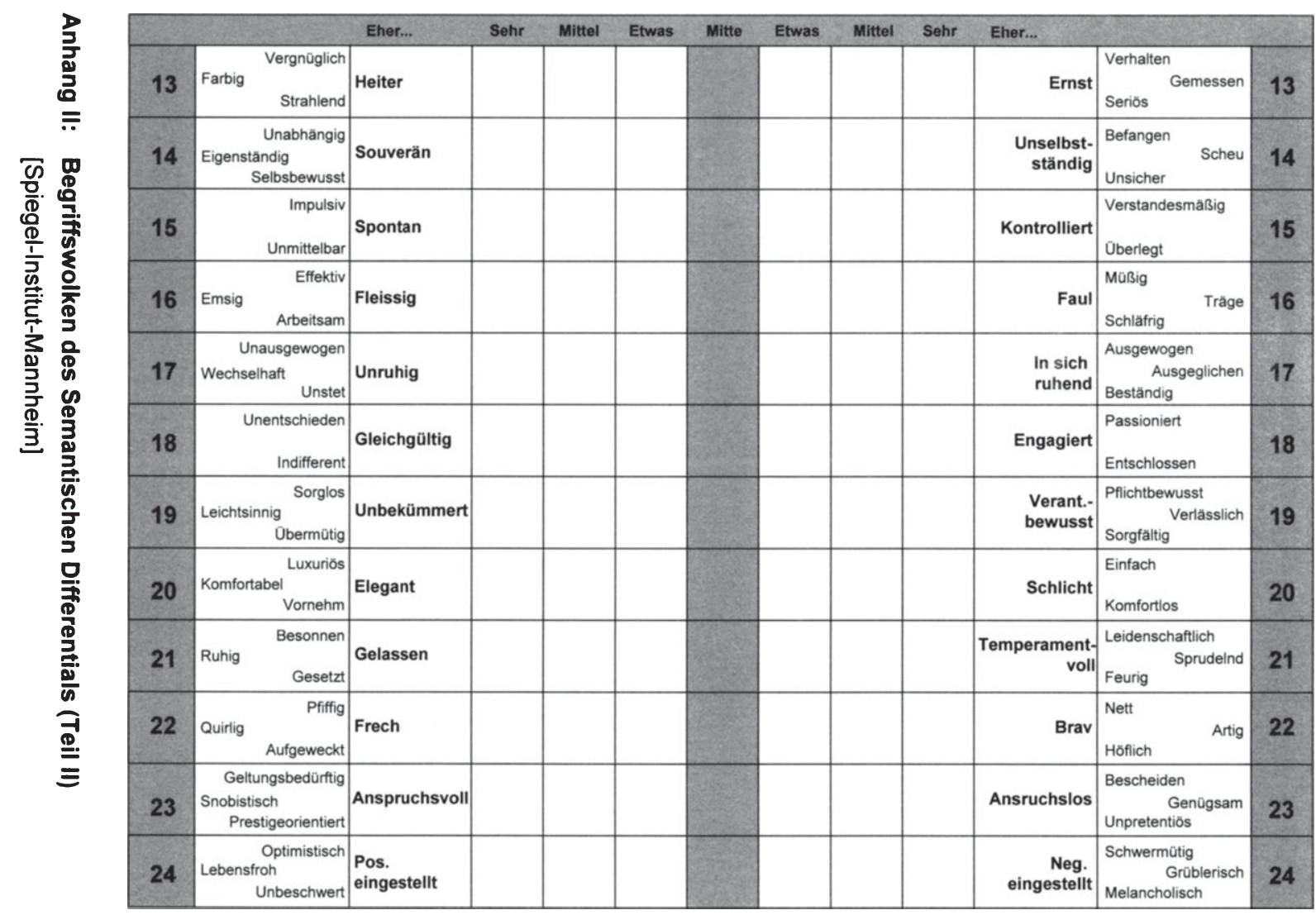




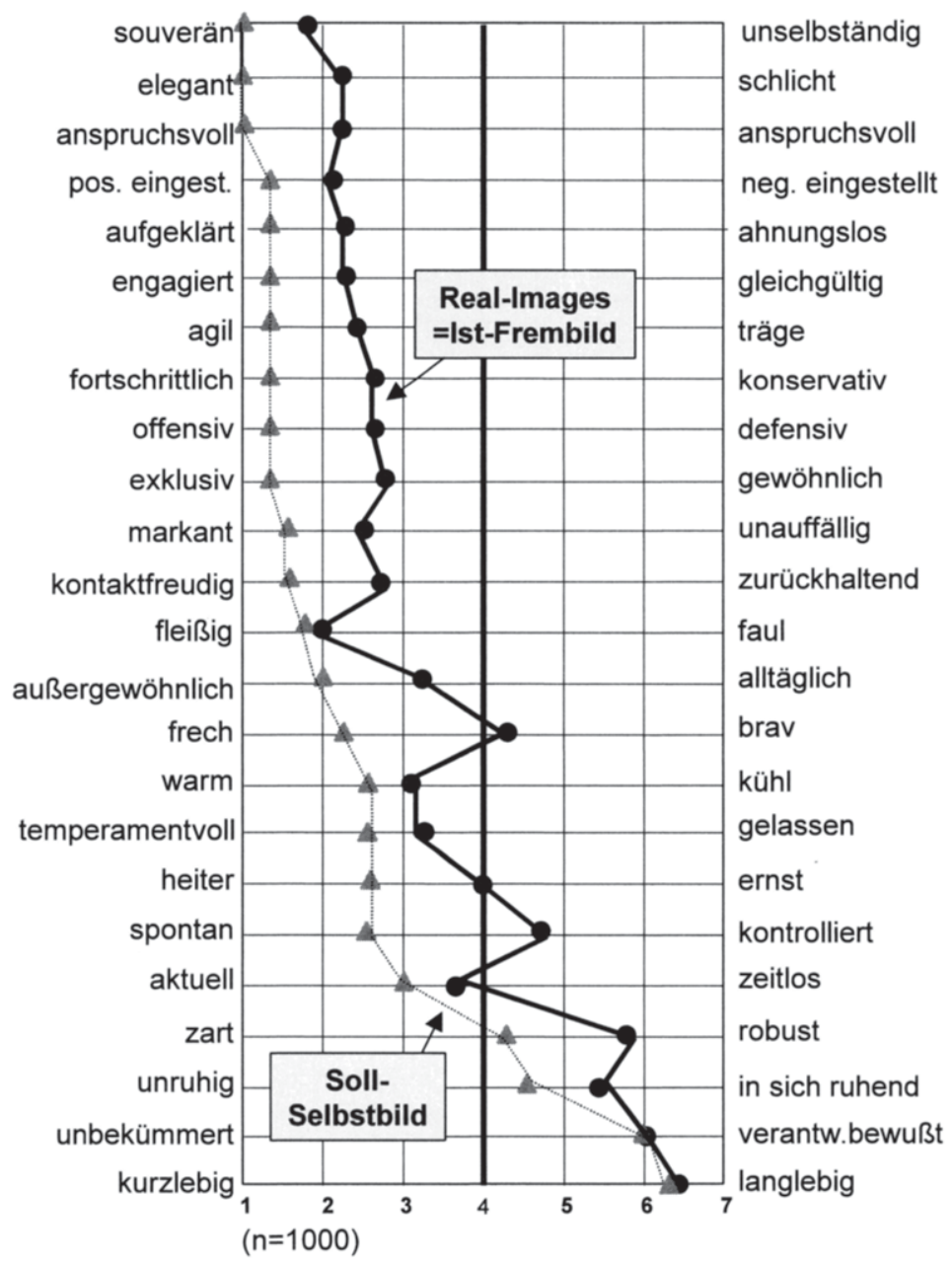

Anhang III: Polaritätsprofil Soll-Selbstbild und Ist-Fremdbild Marke B 


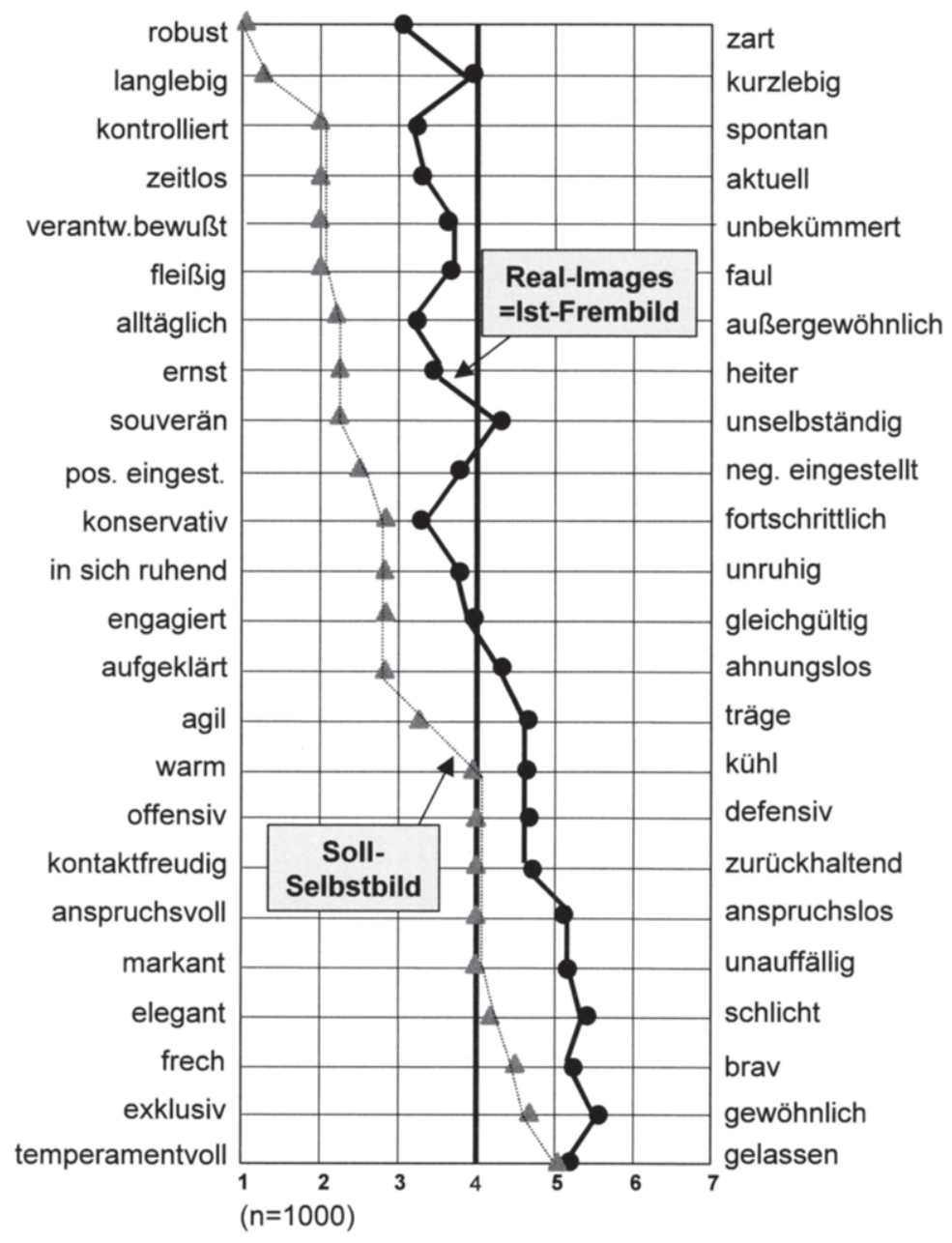

Anhang III: Polaritätsprofil Soll-Selbstbild und Ist-Fremdbild Marke C 


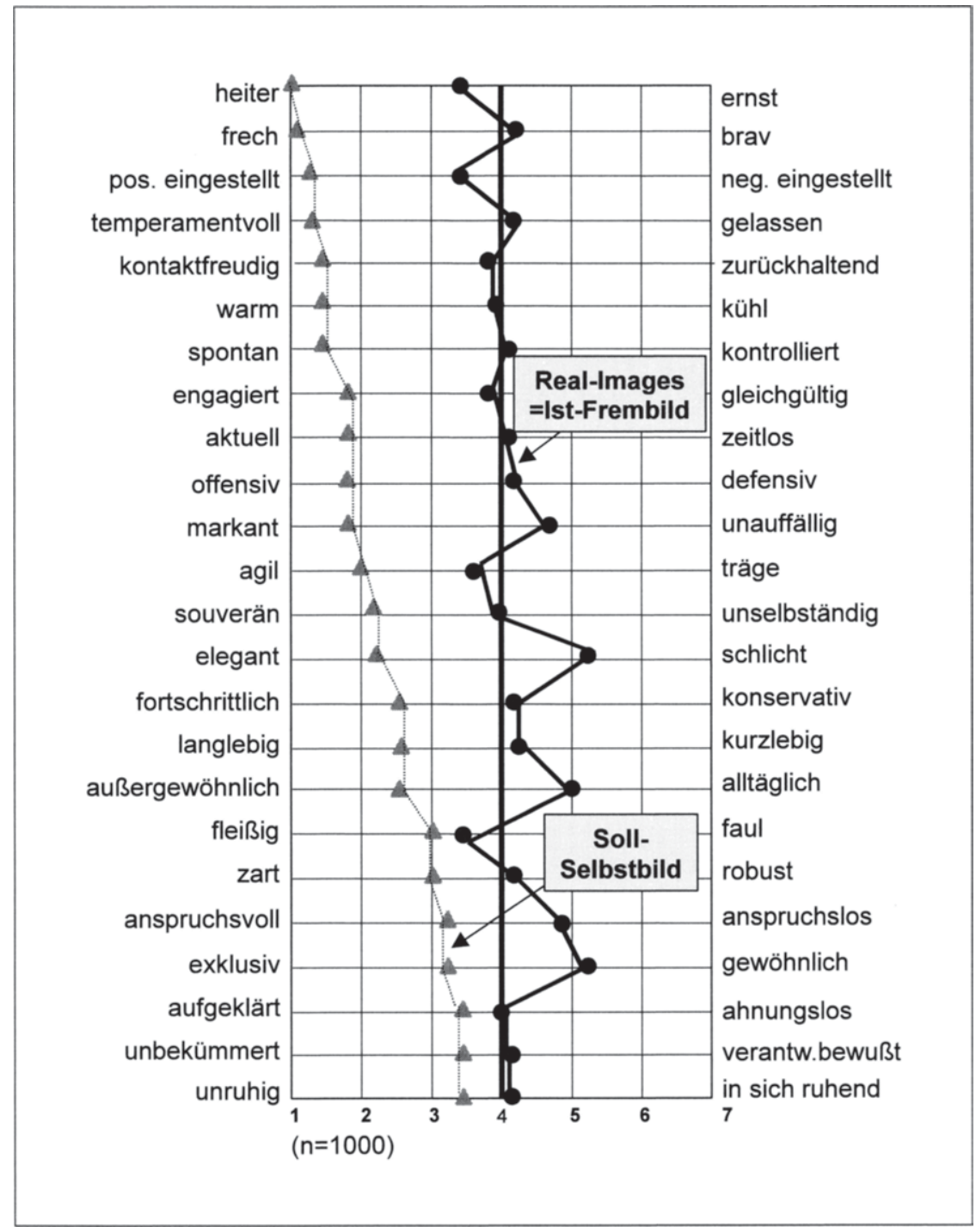

Anhang III: Polaritätsprofil Soll-Selbstbild und Ist-Fremdbild Marke D 


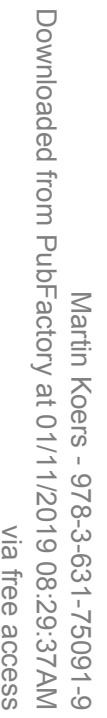

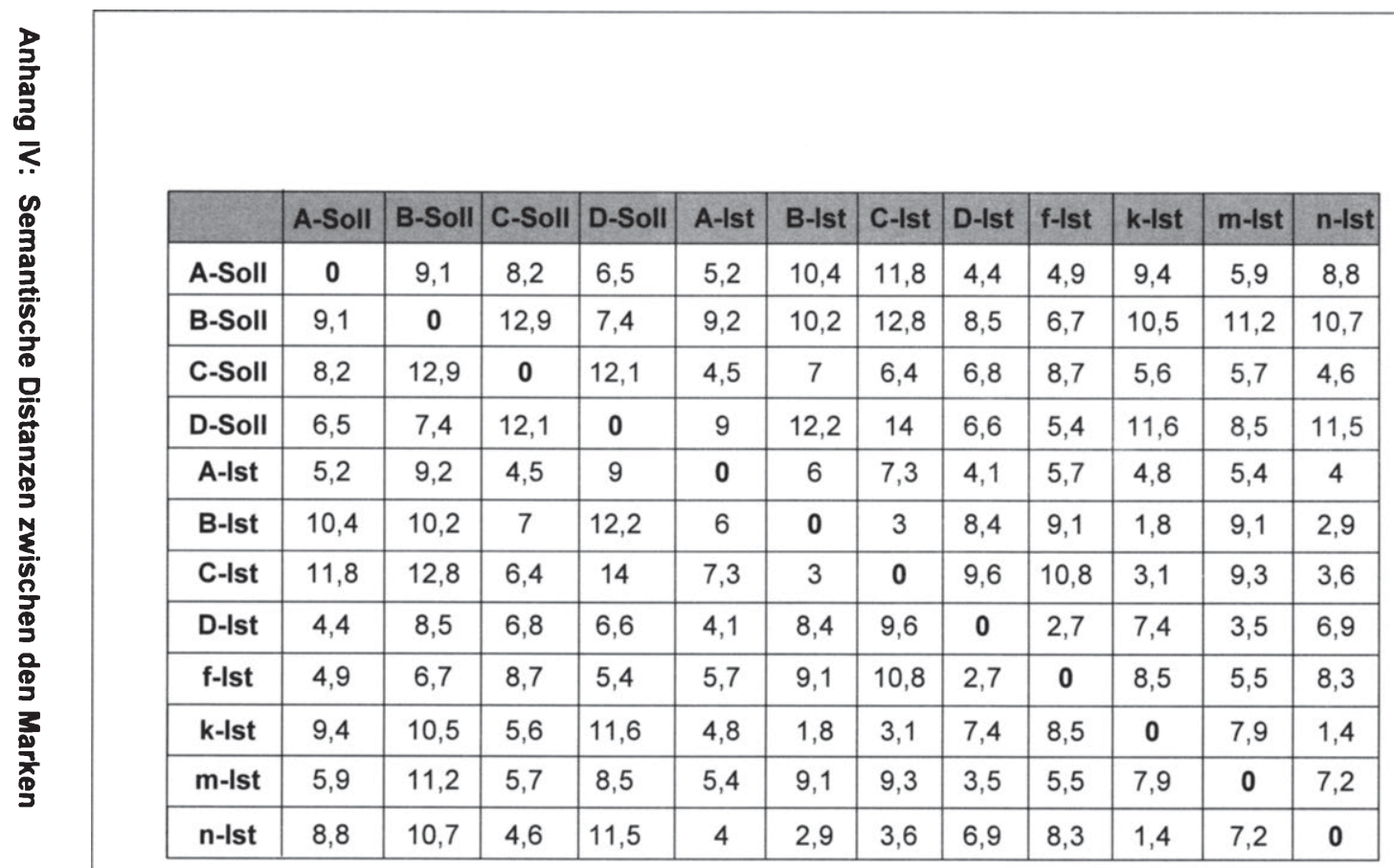


Martin Koers - 978-3-631-75091-9

Downloaded from PubFactory at 01/11/2019 08:29:37AM

via free access 


\section{Literaturverzeichnis}

Aaker, D. A., Brand Extensions: The Good, the Bad, and the Ugly, in: Sloan Management Review, Summer 1990, S. 47-56.

Aaker, D. A., Managing Brand Equity, Capitalizing on the Value of a Brand Name, New York u.a. 1991.

Aaker, D. A., Building Strong Brands, New York 1996.

Aaker, D. A., Should you take your Brand where the Action is?, in: Harvard Business Review, September-October 1997, S. 135-143.

Aaker, D. A., Joachimsthaler, E., The Lure of Global Branding, in: Harvard Business Review, No. 6, Nov.-Dec. 1999, S. 137-144.

Aaker, D. A., Joachimsthaler, E., Brand Leadership, New York u. a 2000.

Abegglen, W. E., Die Organisation der Unternehmensleitung. Versuch einer Theorie, Zürich 1966.

Abell, D. F., Defining the Business. The Starting Point of Strategic Planning , Englewood Cliffs 1980.

Ackoff, R. L., Towards a System of System Concepts, in: Management Science, Vol. 17, Heft 11, 1971, S. 661-671.

Adam, D., Investitionscontrolling, München, Wien, Oldenbourg 1994.

Adam, D., Planung und Entscheidung: Modelle - Ziele - Methoden; Fallstudien und Lösungen, 3. Aufl., Wiesbaden 1993.

Adam, D., Koordinationsprobleme bei dezentralen Entscheidungen, in: ZfB, 39. Jg., Heft 10, 1969, S. 615-632.

Ahlert, D., Grundlagen des Controlling, Münsteraner Schriften zur Distributionsund Handelsforschung, Ahlert, D. (Hrsg.), 3. Aufl., Münster 1999.

Ahlert, D., Anforderungen an Handelsinformationssysteme aus Nutzersicht Auswertungspotentiale für das Handels- und WertschöpfungsprozeßManagement, in: Informationssysteme für das Handelsmanagement: Konzepte und Nutzung in der Unternehmenspraxis, Ahlert, D. et al. (Hrsg.), Berlin et al. 1998.

Ahlert, D., Franz, K.-P., Kaefer, W., Grundlagen und Grundbegriffe der Betriebswirtschaftslehre, 5. Aufl., 1990.

Ahlert, D., Olbrich, R., Controlling und Informationsmanagement im Prozeß des strategischen Managements - Ein Diskussionsbeitrag zur Abgrenzung der Zuständigkeiten, Arbeitspapier Nr. 16 des Instituts für Handelsmanagement der Westfälischen Wilhelms Universität Münster, Ahlert, D. (Hrsg.), 2. Aufl., Münster 1996.

Ahrens, H. J., Multidimensionale Skalierung, Basel 1974.

Albach, H., Die Koordination der Planung im Großunternehmen, in: Rationale Wirtschaftspolitik und Planung in der Wirtschaft von heute, Schriften

Martin Koers - 978-3-631-75091-9 
des Vereins für Sozialpolitik, Band 45, Schneider, E. (Hrsg.), Berlin 1967, S. 332-438.

Albach, H., Der dispositive Faktor in Theorie und Praxis, in: ZfB, Heft 5/6, 1990, S. 533-548.

Andresen, Th., Esch, F.-R., Messung der Markenstärke durch den Markeneisberg, in: Moderne Markenführung: Grundlagen - innovative Ansätze praktische Umsetzung, Esch, F.-R. (Hrsg.), Wiesbaden 1999, S. 10111033.

Andresen, Th., Nickel, O., Führung von Dachmarken, in: Moderne Markenführung: Grundlagen - innovative Ansätze - praktische Umsetzung, Esch, F.-R. (Hrsg.), Wiesbaden 1999, S. 575-604.

Andritzky, K., Der Zusammenhang zwischen Skalenbreite und Urteilsverhalten, in: Der Marktforscher, Heft 3, 1976, S. 60-73.

Ansoff, H. I., Management-Strategie, München 1966.

Argyris, C., Schön, D., Organizational Learning: A Theory of Action Perspective, Mass. 1978.

Arnold, D., Modernes Markenmanagement, Wien 1992.

Backhaus, K. Investitionsgütermarketing, 6. Aufl., München 1999.

Backhaus, K., Büschken, J., Voeth, M., Internationales Marketing, 3. Aufl., Stuttgart 2000.

Backhaus, K. et al., Multivariate Analysemethoden, 8. Aufl., Berlin u.a. 1996.

Baetge, J., Überwachung, in: Vahlens Kompendium der Betriebswirtschaftslehre, Bitz, M. et al. (Hrsg.), Band 2, 3. Aufl., München 1993, S. 175-218.

Bain, J., Industrial Organization, 2. Aufl., New York 1968.

Baldwin, C. Y., Clark, K. B., Modularisierung: Ein Konzept wird universell, in: Harvard Business Manager, Heft 2, 1998, S. 39-48.

Barney, J. B., Gaining and Sustaing Competitive Advantage, Mass. u.a. 1997.

Barney, J. B., Firm Resources and Sustained Competitive Advantage, in: Journal of Management, Vol. 17, Heft 1, 1991, S. 99-120.

Bartlett, Ch. A., Building and Managing the Transnational: The New Organizational Challenge, in: Competition in Global Industries, Porter, M. E. (Hrsg.), Boston 1986, S. 367-404.

Barwise, P., Robertson, Th., Brand Portfolio, in: European Management Journal, Vol. 10, Nr. 3, September 1992, S. 277-285.

Bauer, H. H., Die Determinanten der Markentreue beim Automobilkauf, in: Marktforschung im Automobilsektor, Dichtl, E., Raffée, H., Potucek, V., Schriftenreihe des Verbandes der Automobilindustrie e.V. (VDA), Nr. 40, Frankfurt 1983, S. 15-37.

Bauer, H. H., Marktabgrenzung, Berlin 1989. 
Bauer, H. H., Hannig, U., Die Erfassung der Substitutionswirkung eines neuartigen Elektrowerkzeuges für den Heimwerker-Markt, in: Marketing ZfP, Heft 1, 1987, S. 5-15.

Bauer, H. H., Hermann, A., Hubert, F., Die Bestimmungsgrößen der Markentreue beim Pkw-Kauf: Ergebnisse einer empirischen Untersuchung, in: der markt, Heft 4, 1994, S. 165-171.

BBDO, Auswege aus der kommunikativen Katastrophe, Düsseldorf 1993.

Becker, J., Typen von Markenstrategien, in: Handwörterbuch Markenartikel, Bruhn, M. (Hrsg.), Band 1, Stuttgart 1994, S. 463-498.

Becker, J., Marketing-Konzeption: Grundlagen des strategischen und operativen Marketing-Management, 6. Aufl., München 1998.

Bekmeier-Feuerhahn, S., Marktorientierte Markenbewertung: eine konsumentenund unternehmensbezogene Betrachtung, Wiesbaden 1998.

Benkenstein, M., F\&E und Marketing: eine Untersuchung zur Leistungsfähigkeit von Koordinationskonzeptionen bei Innovationsentscheidungen, Wiesbaden 1987.

Berekoven, L., Internationales Marketing, 2. Aufl., Berlin 1985.

Berens, W., Hoffjan, A., Strack, M., Ökologiebezogenes Controlling - umweltorientierte Koordination in kommunalen Versorgungsunternehmen, in: Zeitschrift für öffentliche und gemeinwirtschaftliche Unternehmen, 18. Jg., Heft 2, 1995, S. 143-160.

Bergler, R., Das Eindrucksdifferential - Theorie und Technik, Bern u. a., 1975.

Berthon, P., Hulbert, J.M., Leyland F.P., Brand Management Prognostications, in: Sloan Management Review, Winter 1999, S. 53-65.

Bhargava, M., Dubelaar, Ch., Ramaswami, S., Reconciling Diverse Measures of Performance. A Conceptual Framework and Test of Methodology, in: JoBR, 1994, S. 1-10.

Bieger, Th., Dienstleistungsmanagement: Einführung in Strategien und Prozesse bei persönlichen Dienstleitungen, Bern, Stuttgart, Wien, 1998.

Biel, A. L., Grundlagen zum Markenwertaufbau, in: Moderne Markenführung: Grundlagen - innovative Ansätze - praktische Umsetzung, Esch, F.-R. (Hrsg.), Wiesbaden 1999, S. 61-90.

Bircher, B., Langfristige Unternehmensplanung. Konzepte, Erkenntnisse und Modell auf systemtheoretischer Grundlage, Bern, Stuttgart, 1976.

Bleicher, K., Organisation. Strategien - Strukturen - Kulturen, 2. Aufl., Wiesbaden 1991.

Bleicher, K., Leitbilder: Orientierungsrahmen für eine integrative ManagementPhilosophie, Stuttgart 1992.

Bleicher, K., Das Konzept integriertes Management, 4. Aufl., Frankfurt a. M., New York, 1996.

Bliss, Ch., Management von Komplexität: Ein integrierter, systemtheoretischer Ansatz zur Komplexitätsreduktion, Wiesbaden 2000. 
Böcker, F., Marketing-Kontrolle, in: Köhler, R., Meffert, H. (Hrsg.), KohlhammerEdition Marketing, Stuttgart et al. 1988.

Böhler, H., Methoden und Modell der Marktsegmentierung, Stuttgart 1977.

Bohne, F., Komplexitätskostenmanagement - Konzepte zur Identifizierung und Gestaltung vielfaltsinduzierter Kosten in der Automobilbranche, Augsburg 1998.

Botta, V., Kennzahlensysteme als Führungsinstrumente. Planung, Steuerung und Kontrolle der Rentabilität im Unternehmen, 5. Aufl. 1999.

Brabeck-Letmathe, P., Marketing ist Chefsache, mehr denn je, in: asw, Heft 1, 2000, S. 32-33.

Brandmeyer, K., Schmidt, M., Der "Genetische Code der Marke ${ }^{u \circledast}$ als Management-Werkzeug, in: Brandmeyer, K., Deichsel, A. (Hrsg.), Jahrbuch Markentechnik 2000/2001: Markenwelt, Markentechnik, Markentheorie, Forschungsbericht, Horizonte, Frankfurt a. M. 1999, S. 271-289.

Brockhoff, K. Produktpolitik, 4. Aufl., Stuttgart 1999.

Brockhoff, K., Hauschildt, J., Schnittstellen-Management - Koordination ohne Hierarchie, in: Zeitschrift für Organisation, Heft 6, 1993, S. 396-403.

Brüne, G., IMAGERY 2 - strategische Markenführung in gehobenen Zielgruppen, in: Geo Extra, Anzeigen-Ticker, G+J (Hrsg.), Heft 7, 1998.

Brunner, J., Value-Based Performance Management, Wertsteigernde Unternehmensführung: Strategien, Instrumente, Praxisbeispiele, Wiesbaden 1999.

Büchelhofer, R., Markenführung im Volkswagen-Konzern im Rahmen der Mehrmarkenstrategie. Unveröffentlichte Fallstudie 1999.

Bühner, R., Betriebswirtschaftliche Organisationslehre, 7. Aufl., München, Wien, Oldenbourg 1994.

Bukhari, I., Europäisches Brand Management. Entwicklung und Umsetzung erfolgreicher Marketingkonzepte, Wiesbaden 1999.

Bultez. A. et al., Asymmetric Cannibalism in Retail Assortements, in: Journal of Retailing, Vol. 65, Nr. 2, Summer 1989, S. 153-192.

Burmann, Ch., Konsumentenzufriedenheit als Determinante der Marken- und Händlerloyalität - Das Beispiel der Automobilindustrie, in: Marketing ZFP, Heft 4, 1991, S. 249-258.

Burmann, Ch., Strategiewechsel in turbulenten Märkten - Neuere theoretische Ansätze zur Unternehmensflexibilität, in: Arbeitspapier Nr. 134 der Wissenschaftlichen Gesellschaft für Marketing und Unternehmensführung e.V., Meffert, H., Backhaus K., Becker, J., (Hrsg.), Münster 2000.

Burmann, G., Marktarealstrategien der internationalen Automobilhersteller, in: Internationales Automobilmarketing, Hünerberg, R., Heise, G., Hoffmeister, M., (Hrsg.), Wiesbaden 1995, S. 121-141.

Butler, A., Letza, St, Neale, B., Linking the Balanced Scorecard to Strategy, in: Long Range Planning, Vol. 30, Heft 2, 1997, S. 242-253. 
Campbell, K., Researching Brands, in: Brands: The New Wealth Creators, Hart, S., Murphy, J. (Hrsg.), Houndmills 1998, S. 56-62.

Carbon, M., Leitbilder, in: Effizientes Informationsmanagement in dezentralen Organisationsstrukturen, Bullinger, H.-J. (Hrsg.), Berlin u.a., S. 87-94.

Chernatony, L. de, Categorizing Brands: Evolutionary Processes Underspinned by Two Key Dimensions, in: Journal of Marketing Management, Heft 9, 1993, S. 173-188.

Chernatony, L. de, Dall'Ollmo Riley, F., Defining A „Brand“: Beyond the Literature with Experts' Interpretations, in: Journal of Marketing Management, Heft 14, 1998, S. 417-443.

Clef, U., Mehrmarkenstrategie für die Pole-Position, in asw, Sondernummer Oktober 1999, S. 72-80.

Cochran, E. B., What is a Controller?, Journal of Accountancy, July 1955, S. 4653.

Collins, J., Porras, J., Werkzeug Vision - Wie Unternehmensphilosophie und Leitbilder visionäre Unternehmen prägen, in: Harvard Business Manager, Heft 4, 1992, S. 108-118.

Colvin, M., Heeler, R., Thorpe, J., Developing International Advertising Strategy, in: Journal of Marketing, Vol. 44, Heft 3, 1980, S. 73-79.

Copulsky, W., Cannibalism in the Marketplace, in: Journal of Markting, October 1976, S. 103-105.

Corsten. H., Stuhlmann, St., Das GAP-Modell als Orientierungsrahmen für ein Kapazitätsmanagement in Dienstleistungsunternehmen, in: Kapazitätsmanagement in Dienstleistungsunternehmen: Grundlagen und Gestaltungsmöglichkeiten, Corsten, H.,Stuhlmann, St. (Hrsg.), Wiesbaden 1997, 3-54.

Creutzig, J., Die zukünftige Regelung des Automobilvertriebs in Europa, in: Automobilwirtschaft, Meinig, W. (Hrsg.), Wiesbaden 1993, S. 43-73

Crimmins, J. C., Better Measurement and Management of Brand Value, in: JoAR, Heft 4, 1992, S. 11-19.

D'Aveni, Hyperwettbewerb: Strategien für die Dynamik der Märkte, Wiesbaden 1995.

Dacin, P. A., Smith, D. C., The Effect of Brand Portfolio Characteristics on Consumer Evaluations of Brand Extensions, in: JoMR, Vol. XXXI, May 1994 , S. 229-242.

Dannath, U. M., Ansätze und Gestaltungsmöglichkeiten des strategischen Markenmanagements in der Automobilindustrie, unveröffentlichte Diplomarbeit am Lehrstuhl für Handel und Marketing der Universität Hamburg, Hamburg 1998.

Day, G. S., A Two Dimensional Concept of Brand Loyalty, in: Journal of Advertising Research, Vol. 9, 1969, Heft 3, S. 29-35. 
Day, S. D., Shocker, A. D., Srivastava, R. K., Customer-Oriented Approaches To Identifying Product-Markets, in: Journal of Marketing, Vol. 43, Fall 1979, S. 8-19.

Decker, R., Klein, T., Wartenberg, F., Marketing und Internet - Markenkommunikation im Umbruch?, in: Markenartikel, Heft 10 1995, S. 468-473.

Dellmann, K., Eine Systematisierung der Grundlagen des Controlling, in: Controlling. Grundlagen - Informationssysteme - Anwendungen, Spremann, K., Zur, E. (Hrsg.), Wiesbaden 1992, S. 113-140.

Demuth, A., Corporate Branding als Herausforderung an das Marketing, in: Kongressdokumentation vom Deutschen Marketing Verband des 27 . Deutschen Marketing-Tag am 29. Oktober 1999 in Frankfurt unter dem Thema "Chancen im 3. Jahrtausend. Information managen - Zukunft gestalten", S. 42.

Demuth, A., Markenidentität durch Corporate Branding, in: HORIZONT, Nr. 43, 1999, S. 38.

Dichtl, E., Andritzky, K., Schobert, R., Ein Verfahren zur Abgrenzung des „relevanten Marktes" auf der Basis von Produktperzeptionen und Präferenzurteilen, in: WiSt, 6. Jg., Heft 6, 1977, S. 290-301.

Dichtl, E., Schobert, R., Grundzüge der Multidimensionalen Skalierung, in: Multidimensionale Skalierung: methodische Grundlagen und betriebswirtschaftliche Anwendungen, München 1979, S. 1-13.

Dichtl, E., Schobert, R., Mehrdimensionale Skalierung - Methodische Grundlagen und betriebswirtschaftliche Anwendungen, München 1979.

Diekhof, R., Preisgekröntes Marketing, in: W\&V, Heft 29, 1999, S. 80-81.

Diez, W., Das Handbuch für das Automobilmarketing - Strategien, Konzepte, Instrumente, 3. Aufl., Landsberg/Lech 1997.

Diez, W., Plattform-Strategien: Irrweg oder Königsweg in der Modellpolitik, Arbeitspapier Nr. 2 des Instituts für Automobilwirtschaft, Gelsenkirchen 1999.

Diez, W., Prozeßoptimierung im Automobilvertrieb: Auf dem Weg zu einem integrierten Kunden- und Kostenbindungsmanagement, Wiesbaden 1999.

Domizlaff, H., Die Gewinnung des öffentlichen Vertrauens. Ein Lehrbuch der Markentechnik, 2. Aufl., Hamburg 1951.

Doyle, P., Building Successful Brands. The Strategic Options, in: Journal of Marketing Management, No. 1, 1989, S. 77-95.

Doyle, P., Marketing-Management and Strategy, New York 1994.

Drees, N., Markenerfolgsforschung und Markenberatung in Deutschland - Ergebnisse einer empirischen Studie, in: Werbeforschung \& Praxis, Heft 4, 1999, S. 24-28.

Dreyer, A., Scoring-Modelle bei Mehrfachzielsetzungen - Eine Analyse des Entwicklungsstandes von Scoring-Modellen, in: ZfB, 44. Jg., 1974, S. 255274. 
Droege, W.P.J., Kricsfalussy, A., Marketingaudit: Check up the strategic fit!, in: Marketingcontrolling, Reinecke, S./Tomczak, T./ Dittrich, S. (Hrsg.), St. Gallen 1998, S. 70-78.

Drosten, M., Wo die Märkte aufreißen, in: asw, 39. Jg., Nr. 6, 1996, S. 32-37.

Drucker, P., Umbruch im Management, Düsseldorf 1996.

Dudenhöffer, F., Marken-Management bei Produktkonvergenz - Neue Ansätze im Automobilmarketing, in: GfK JdAV, Heft 1, 1997, S. 4-24.

Dudenhöffer, F., Dittler, Th., Thies, C., Auto-Markt 2005 und danach, in: Marketing Journal, Heft 4, 1998, S. 216-220.

Dudenhöffer, F., Dittler, Th., Thies, C., Schlüssel-Trends im Automobilgeschäft Europas, in: Internationales Verkehrswesen, Heft 10, 1998, S. 441-447.

Duncan, T. R., Moriarty, S. E., Driving Brand Value using Integrates Marketing to manage profitable Stakeholder Relationships, New York 1997.

Dyer, J. H., Specialized Supplier Networks as a Source of Competitive Advantage: Evidence from the Auto Industry, in: Strategic Management Journal, Vol. 17, 1996, S. 271-291.

Dynkin, E. B., Die Grundlagen der Theorie der Markoffschen Prozesse, ins Deutsche übertragen von J. Wloka, Berlin u.a. 1961.

Ealey, L., Troyano-Bermúdez, L., Are Automobiles the next Commodity?, in: The McKinsey Quarterly, No. 4, 1996, S. 62-75.

Eccles, R. G., Mavrinac, S., Improving the Corporate Disclosure Process, in: Sloan Management Review, Vol. 36, Heft 4, S. 11-25.

Eccles, R. G., Noriah, N., Beyond the Hype - Rediscovering the Essence of Management, Boston 1992.

Epstein, M., Manzoni, J.-F., Implementing Corporate Strategy: From Tableaux de Bord to Balanced Scorecard, in: European Management Journal, Vol. 16, No. 2, 1997, S. 190-203.

Errichiello, O. C., Groß, A., Pirck, P., Forschungsbericht 2000/2001, in: Brandmeyer, K., Deichsel, A. (Hrsg.), Jahrbuch Markentechnik 2000/2001: Markenwelt, Markentechnik, Markentheorie, Forschungsbericht, Horizonte, Frankfurt a. M. 1999, S. 417-456.

Esch, F.-R., Andresen, Th., 10 Barrieren für eine erfolgreiche Markenpositionierung und Ansätze zu deren Überwindung, in: Positionierung: Kernentscheidung des Marketing, Tomczak, T., Rudolph, Th., Roosdorp, A. (Hrsg.), St. Gallen 1996, S. 78 - 94.

Esch, F.-R., Andresen, Th., Messung des Markenwerts, in: Erfolgreiches Markenmanagement, MTP e. V. Alumni, Hauser, U. (Hrsg.), Wiesbaden 1997, S. 11 - 37.

Esch, F.-R., Kontrolle der Eigenständigkeit von Markenauftritten, in: Moderne Markenführung: Grundlagen - innovative Ansätze - praktische Umsetzung, Esch, F.-R. (Hrsg.), Wiesbaden 1999, S. 1053-1068. 
Esch, F.-R., Markenpositionierung als Grundlage der Markenführung, in: Moderne Markenführung: Grundlagen - innovative Ansätze - praktische Umsetzung, Esch, F.-R. (Hrsg.), Wiesbaden 1999, S. 231-265.

Esch, F.-R., Wicke, A., Herausforderungen und Aufgaben des Markenmanagements, in: Moderne Markenführung: Grundlagen - innovative Ansätze praktische Umsetzung, Esch, F.-R. (Hrsg.), Wiesbaden 1999, S. 3-55.

Ewing, P., Lundahl, L. The Balanced Scorecards at ABB Sweden - The EVITA Project, 0.O., 1996.

Fankart, P., Widmer, H., Rezept - Corporate Identity. Leitbild, Erscheinungsbild, Kommunikation, Zürich, Wiesbaden 1987

Farquhar, P.H., Managing Brand Equity, in: Journal of Advertising Research, Aug./ Sept. 1990, S. 7-12.

Fesler, J. W., Approaches to the Understanding of Decentralization, in: The Journal of Politics, Vol. 27, 1965, S. 536-566.

Festinger, L., Theorie der kognitiven Dissonanz, Bern 1978.

Fink, C.A., Grundler, Ch., Strategieimplementierung im turbulenten Umfeld Steuerung der Firma fischerwerke mit der Balanced Scorecard, in: Controlling, Heft 4, 1998, S. 226-235.

Fischer, Th., Implementierung von Balanced Scorecards in Handelsunternehmen, in: HHL-Arbeitspapier Nr. 32, Leipzig 2000.

Fisher, J.C., Pry, R.H., A Simple Substitution Model of Technological Change, in: Technological Forecasting and Social Change, Vol. 2, May 1971, S. 7588.

Fournier, S. M., Consumers and Their Brands: Developing Relationship Theory, in: Journal of Consumer Research, Vol. 24., March 1998, S. 343-373.

Franzen, O., Marken-Controlling effizient gestalten, in: Markenartikel, Heft 2, 1995, S. 57-62.

Franzen, O., Trommsdorff, V., Riedel, F., Ansätze der Markenbewertung und Markenbilanz, in: Markenartikel, Heft 8, 1994, S. 372-376.

Frese, E., Grundlagen der Organisation: Konzept - Prinzipien - Strukturen, 6. Aufl., Wiesbaden 1995.

Freter, H., Markenpositionierung. Ein Beitrag zur Fundierung markenpolitischer Entscheidungen auf der Grundlage psychologischer und ökonomischer Modelle, (unveröffentlichte) Habilitationsschrift, Münster 1977.

Freter, H., Marktsegmentierung, Stuttgart 1983.

Frey, D., Die Theorie der kognitiven Dissonanz, in: Theorien der Sozialpsychologie, Frey, D., Irle, M. (Hrsg.), Band 1, Bern u.a. 1993, S. 243-292.

Friedag, H. R., Schmidt, W., Balanced Scorecard. Mehr als ein Kennzahlensystem, Freiburg i. Br., Berlin, München 1999.

Friederes, G., Priemer, V., Dachmarke versus Einzelmarke, in: der markt, 37 Jg, Heft: 2, 1998, S. 47-58. 
Funaki, Y., Produktentwicklung zwischen kundenorientierter Individualisierung und kostenorientierter Standardisierung, in: Markt für Mobilität, Meinig, W., Mallad, H. (Hrsg.), Darmstadt 1996, S. 247-257.

Galli, A., Wagner, M., Balanced Scorecard an der Schnittstelle Profit - NonProfit?, in: Der Betrieb, 52. Jg., Heft 39, 1999, S. 1965-1969.

Gälweiler, A., Strategische Unternehmensführung, Frankfurt a. M., New York, 1987.

Gerberich, C. W., Wohin führt der Weg des Controllers? in: FAZ, Nr. 123 vom 31.5.1999, S. 31.

Gerken, G., Abschied vom Marketing, Düsseldorf 1991.

Gerken, G., Die fraktale Marke, Düsseldorf 1994.

Ghemawat, P., Dauerhafte Wettbewerbsvorteile aufbauen, in: Simon, H., Wettbewerbsvorteile und Wettbewerbsfähigkeit, Stuttgart 1988, S. 18-29.

Ghoshal, S. S., Bartlett, Ch., Building the Entrepreneurial Organisation: New Organisational Processes, New Managerial Tasks, in: European Management Journal, Vol. 13, Heft 2, 1995, S. 139-155.

Ghoshal, S. S., Bartlett, Ch., Moran, P. A New Manifesto for Management, in: Sloan Management Review, Sonderheft Spring 1999, S. 9-20.

Gilbert, R. J., Matutes, C., Product Line Rivalry with Brand Differentiation, in: Journal of Industrial Economics, Heft 9, 1993, S. 223-240.

Gilbert, X., Strebel, P., Strategies to Outpace the Competition, in: The Journal of Business Strategy, Heft 1, 1987, S. 28-37.

Gleich, R., Performance Measurement, in: Die Betriebswirtschaft, 57. Jg., 1997, Heft 1, S. 114-117.

Gnirke, K., Controlling international tätiger Unternehmen - Grundrisse eines koordinationsorientierten Bezugsrahmens unter besonderer Berücksichtigung von Netzwerkbeziehungen, in: Controlling-Konzepte im Wandel ausgewählte Ansätze, Jöstinmeier, B. et. al (Hrsg.), Marburger Schriften zum Genossenschaftswesen 80, Göttingen 1994, S. 23-71.

Grimmeisen, M., Implementierungscontrolling: wirtschaftliche Umsetzung von Change-Programmen, Wiesbaden 1998.

Grob, H. L., Positionsbestimmung des Controlling, Arbeitsbericht Nr. 1 der Reihe Computergestützes Controlling, Grob (Hrsg.), Münster 1996.

Güldenberg, H. G., Der volkswirtschaftliche Wert der Marke, in: Markenartikel, Heft 3, 1999, S. 8.

Güldenberg H. G., Franzen, O., Operatives Markencontrolling, in: Handwörterbuch Markenartikel, Bruhn, M. (Hrsg.), Band 2, Stuttgart 1994, S. 13371351.

Gutenberg, E., Grundlagen der Betriebswirtschaftslehre, Zweiter Band, Der Absatz, 16. Aufl., Berlin, Heidelberg, New York 1979.

Gutenberg, E., Grundlagen der Betriebswirtschaftslehre, Erster Band, Die Produktion, 24. Aufl., Berlin, Heidelberg, New York 1983. 
GWA (Hrsg.), So wirkt Werbung im Marketing-Mix, Frankfurt a. M. 1997.

Haedrich, G., Tomczak, T., Strategische Markenführung: Planung und Realisierung von Marketingstrategien für eingeführte Produkte, Bern, Stuttgart 1990.

Haedrich, G., Tomczak, T., Strategische Markenführung, in: Handwörterbuch Markenartikel, Bruhn, M. (Hrsg.), Band 2, Stuttgart 1994, S. 925-948.

Haedrich, G., Tomczak, T., Produktpolitik, Stuttgart 1996.

Hahn, D., PuK. Planung und Kontrolle. Planungs- und Kontrollsysteme. Planungsund Kontrollrechnung. Controllingkonzepte, 5. Aufl., Wiesbaden 1996.

Hamman, P., Der Wert einer Marke aus betriebswirtschaftlicher und rechtlicher Sicht, in: Marke und Markenartikel als Instrumente des Wettbewerbs, Dichtl, E., Eggers, W. (Hrsg.), München 1992, S. 205-245.

Hamman, P., Schuchard-Fischer, Chr., Die Verhaltensrelevanz von Nachkaufdissonanzen im Automobilmarkt, in: Marktforschung im Automobilsektor, Dichtl, E., Raffée, H., Potucek, V., Schriftenreihe des Verbandes der Automobilindustrie e.V. (VDA), Nr. 40, Frankfurt 1983, S. 59-73

Hamprecht, M., Controlling von Konzernplanungssystemen: theoretische Ableitung und betriebliche Realität führungsstrukturabhängiger Ausprägungsmuster, Wiesbaden 1996.

Han, C., Terpstra, V., Country-of-Origin Effects for uni-national and bi-national products, in: Journal of International Business Studies, Vol. 19, Summer 1988, S. 235-255.

Hansen, G. S., Wernerfelt, B., Determinants of Firm Performance: The Relative Importance of Economic and Organizational Factors, in: Strategic Management Journal, Vol. 10, 1989, S. 399-411.

Heigl, A., Controlling - Interne Revision, Stuttgart, New York 1978.

Heinen, E., Einführung in die Betriebswirtschaftslehre, 9. Aufl., Wiesbaden 1985.

Heinen, E., Dill, H., Unternehmenskultur aus betriebswirtschaftlicher Sicht, in: Herausforderung Unternehmenskultur, Simon, H. (Hrsg.), Stuttgart 1990, S. $12-24$.

Heise, G., Internationale Marktsegmentierung im Automobilmarketing, Wiesbaden 1997.

Hermann, A., Marketing-Controlling - Erläuterung der konzeptionellen Grundlagen zur Planung, Steuerung und Kontrolle der marketingpolitischen Aktivitäten am Beispiel von Unternehmen der Automobilbranche, in: Jahrbuch der Absatz und Verbrauchsforschung, Heft 1, 1993, S. 4-22.

Herrmann, A., Huber, F., Braunstein, C., Gestaltung der Markenpersönlichkeit mittels der "means-end"-Theorie, in: Moderne Markenführung: Grundlagen - innovative Ansätze - praktische Umsetzung, Esch, F.-R. (Hrsg.), Wiesbaden 1999, S. 103-134.

Herrmann, A., Gutsche, J., Ein Modell zur Erfassung der individuellen Markenwechselneigung, in: ZfbF, 46. Jg., Heft 1, 1994, S. 63-80. 
Herzberg, F., Mausner, B., Snyderman, B., The Motivation to Work, 2. Aufl., New York 1959.

Hilker, J., Marketingimplementierung: Grundlagen und Umsetzung am Beispiel ostdeutscher Unternehmen, Wiesbaden 1993.

Hinterhuber, H., Strategische Unternehmensführung, II. Strategisches Handeln, 6. Aufl., Berlin u.a. 1997.

Hoffecker, J., Goldenberg, C., Using the Balanced Scorecard to Develop Companywide Performance Measures, in: Journal of Cost Management, 8. Jg., Heft 3, 1994, S. 5-17.

Hoffmann, F., Führungsorganisation, Band 1, Stand der Forschung und Konzeption, Tübingen 1980.

Hoffmeister, M., Multi-Franchise-Konzepte im Automobileinzelhandel: Entwicklungen und Auswirkungen auf die Absatzkanalpolitik der Automobilhersteller, Wiesbaden 1998.

Homburg, Ch. et al., Interne Kundenorientierung der Kostenrechnung - Ergebnisse der Koblenzer Studie, Band 7 der Reihe Advanced Controlling, Koblenz 1998.

Homburg, Ch., Giering, A., Messung von Markenzufriedenheit und Markenloyalität, in: Moderne Markenführung: Grundlagen - innovative Ansätze praktische Umsetzung, Esch, F.-R. (Hrsg.), Wiesbaden 1999, S. 10891100.

Hooley, G. H., Saunders, J., Competitive Positioning: The Key To Marketing Strategy, New York 1993.

Hornung, K., Mayer, J. H., Erfolgsfaktoren-basierte Balanced Scorecards zur Unterstützung einer wertorientierten Unternehmensführung, in: Controlling, Heft 8/ 9, 1999, S. 389-398.

Horváth, P., Controlling - Entwicklung und Stand einer Konzeption zur Lösung der Adaptions- und Koordinationsprobleme der Führung, in: ZfB, 48. Jg., Heft 3, 1978, S. 194-208.

Horváth, P., Controlling, in: Vahlens Großes Controllinglexikon, Horváth, P., Reichmann, Th. (Hrsg.), München 1993, S. 112-114.

Horváth, P., Controlling, 7. Aufl., München 1998.

Horváth, P. Balanced Scorecard - Wie man Strategie in konkrete Informationen umsetzt, in: Marktforschung \& Management, 42 Jg., Heft 3, S. 113-114.

Horváth, P., Kaufmann, L., Balanced Scorecard - ein Werkzeug zur Umsetzung von Strategien, in Harvard Business Manager, Heft 5, 1998, S. 39-48.

Horváth \& Partner (Hrsg.), Balanced Scorecard umsetzen, Stuttgart 2000.

Hronec, S. M., Vital Signs, New York u.a. 1993.

Hünerberg, R., Heise, G., Hoffmeister, M., Internationales Marketing für die Automobilwirtschaft - Eine Einführung, in: Internationales Automobilmarketing, Hünerberg, R., Heise, G., Hoffmeister, M., (Hrsg.), Wiesbaden 1995, S. 5-26. 
Immon, W. H., Building the Datawarehouse, New York 1996.

Jacob, H., Der Absatz, in: Jacob, H. (Hrsg.), Allgemeine Betriebswirtschaftslehre in programmierter Form, Wiesbaden 1976.

Jacob, H., Der Absatz, in: Allgemeine Betriebswirtschaftslehre: Handbuch für Studium und Prüfung, Jacob, H. (Hrsg.), 5. Aufl., Wiesbaden 1988, S. 301515.

Jenner, Th., Markenführung als Lernprozeß, in: Harvard Business Manager, No. 5, 1999, S. 20-29.

Jensen, M, Meckling, W. H., Theory of the Firm: Managerial Behaviour, Acency Costs and Ownership Structure, in: Journal of Financial Economocs, 3. Vol., 1976, S. 305-360.

Jöstingmeier, B., Zum Verständnis der Controlling-Konzepte, in: ControllingKonzepte im Wandel - ausgewählte Ansätze, Jöstinmeier, B. et. al (Hrsg.), Marburger Schriften zum Genossenschaftswesen 80, Göttingen 1994, S. 23-71.

Kaas, K.P., Langfristige Werbewirkung und Brand Equity, in: Werbeforschung und Praxis, Heft 3, 1990, S. 48-52.

Kapferer, J.-N., Die Marke - Kapital des Unternehmens, Landsberg/Lech 1992.

Kapferer, J.-N., Führung von Markenportfolios, in: Moderne Markenführung: Grundlagen - innovative Ansätze - praktische Umsetzung, Esch, F.-R. (Hrsg.), Wiesbaden 1999, S. 605-618.

Kaplan, R. S., Norton, D. P., In Search of Excellence - der Maßstab muß neu definiert werden, in: Harvard manager, Heft 4, 1992, S. 37-46.

Kaplan, R. S., Norton, D. P., Putting the Balanced Scorecard to Work, in: Harvard Business Review, Heft 5, 1993, S. 134-142.

Kaplan, R. S., Norton, D. P., Using the Balanced Scorecard as a Strategic Management System, in: Harvard Business Review, No. 1, 1996, S. 75-85.

Kaplan, R. S., Norton, D. P., Balanced Scorecard: Strategien erfolgreich umsetzen, aus dem Amerikanischen übersetzt von P. Horváth, Stuttgart 1997.

Kaplan, R. S., Norton, D. P., Strategieumsetzung mit Hilfe der Balanced Scorecard, in: Gleich, R., Seidenschwarz, W. (Hrsg.): Die Kunst des Controlling, 1997, S. 313-342.

Karnani, A., Strategic Implications of Market Share Attraction Models, in: Management Science, 1985, S. 536-547.

Kast, F., Rosenzweig, J., Organization and Management: A Contingency Approach, Tokio 1970.

Keller, K. L., Conzeptualizing, Measuring, and Managing Customer-Based Brand Equity, in: Journal of Marketing, Vol. 57., No.1, 1993, S. 1-22.

Keller, K. L., Strategic Brand Management: Building, Measuring, and Managing Brand Equity, London et al. 1998.

Kern, W., Bewertung von Warenzeichen, in: BfuP, Heft 1, 1962, S. 17-31. 
Kiener, J., Marketing-Controlling, Darmstadt 1980.

Kieser, A., Moden und Mythen des Organisierens, in: DBW, Heft 1, 1996, S. 21 39.

Kilger, W., Optimale Produktions- und Absatzplanung, Opladen 1973.

Kinast, K., Das Entstehen von Mythos und die Erstarrung einer Marke als Höhepunkt ihrer Entwicklung, in: der markt, Heft 2, 1995, S. 73-83.

Kirsch, W., Roventa, W., Trux, W., Wider den Haarschneideautomaten - Ein Plädoyer für mehr Individualität bei der Strategischen Unternehmensführung, Arbeitspapier Nr. 5 der Wissenschaftlichen Gesellschaft für Marketing und Unternehmensführung e.V., Meffert, $H$., Wagner, H. (Hrsg.), Münster 1982.

Klingebiel, N., Performance Measurement: Grundlagen - Ansätze - Fallstudien, Wiesbaden 1999.

Knoblich, H. K., Die typologische Methode in der Betriebswirtschaftslehre, in: WiSt, Heft 4, 1972, S. 141-147.

Knudsen, T. R. et al., Brand Consolidation makes al lot of Economic Sense, in The McKinsey Quarterly, No. 4, 1997, S. 189-193.

Köhler, R., Marketing-Entscheidungen als Anwendungsgebiet der quantitativen Planung, in: Entscheidungshilfen im Marketing, Köhler, R., Zimmermann, H.-J. (Hrsg.), Stuttgart 1977, S. 2-28.

Köhler, R., Beiträge zum Marketing-Management. Planung, Organisation, Controlling, 3. Aufl., Stuttgart 1993.

Köhler, R., Marke und Verbraucher - Zu den Grundlagen lebenslanger Bindungen, in: Markenartikel, Heft 7, 1993, S. 340-347.

Köhler, R., Marketing-Controlling: Konzepte und Methoden, in: MarketingControlling, Reinecke, S., Tomczak, T., Dittrich, S. (Hrsg.), St. Gallen 1998, S. 10-21.

Kolks, V., Strategieimplementierung: Ein anwendungsorientiertes Konzept, Wiesbaden 1990 .

Korte, Ch., Customer Satisfaction Measurement: Kundenzufriedenheitsmessung des Hersteller- und Handelsmarketing am Beispiel der Automobilwirtschaft, Frankfurt a. M. 1995.

Kosiol, E., Organisation der Unternehmung, 2. Aufl., Wiesbaden 1976.

Kotler, Ph., Marketing Management, 6. Aufl., Englewood Cliffs 1988.

Kotler, Ph., Bliemel, F., Marketing-Management: Analyse, Planung, Umsetzung und Steuerung, 9. Aufl., Stuttgart 1999.

Kotler, Ph., Über die Entwicklung von Wertangeboten zur Unique Selling Proposition, in: absatzwirtschaft, Heft 3, 2000, S. 46-49.

Krink, J., Kracht-Müntz, B., Operative und strategische Planung - Aufgaben, Interdependenzen und Koordinationsproblematik (I), in: Das Wirtschaftsstudium, Heft 1, 1984, S. $24-27$. 
Kroeber-Riel, W., Informationsüberlastung durch Massenmedien und Werbung in Deutschland, in: DBW, 47.Jg., Heft 3, 1987, S. 257-264.

Kroeber-Riel, W., Weinberg, P., Konsumentenverhalten, 7. Aufl., München 1999.

Krüger, B, Die Mehrmarkenstrategie des Volkswagen-Konzerns. Unveröffentlichter Vortrag zum Deutschen Marketing-Preis 1999 am 30.06.1999 in Düsseldorf.

Krulis-Randa, J. S., Theorie und Praxis des Marketing-Controlling, in: Siegwart, $\mathrm{H}$. et al, Management Controlling, Meilensteine im Management, Basel, Frankfurt 1990, S. 257-272.

Küpper, H.-U., Controlling: Konzeptionen, Aufgaben und Instrumente, Stuttgart 1995.

Küpper, H.-U., Weber, J., Zünd, A., Zum Verständnis und Selbstverständnis des Controlling, in: ZfB, Heft: 3, 1990, S. $281-294$

Laakmann, K., Value-Added Services als Profilierungsinstrument im Wettbewerb. Analyse, Generierung und Bewertung, Frankfurt a.M. u.a. 1995.

Laforet, S., Saunders, J., Managing Brand Portfolios: How The Leaders Do It, in: JoAR, Nr. 5, 1994, S. 64-76.

Laforet, S., Saunders, J., Managing Brand Portfolios: Why Leaders Do What They Do, in: JoAR, Nr. 1, 1999, S. 51-66.

Lamparter, D. H., Tenbrock, Ch., Speed, Speed, Speed, in: Die Zeit Nr. 12 vom 18.3.1999, S. 33-36.

Landwehr, R., Standardisierung der internationalen Werbeplanung - Eine Untersuchung der Prozeßstandardisierung am Beispiel der Werbebudgetierung im Automobilmarkt, Frankfurt a. M. 1988.

Langen, A., Leitbild und Unternehmenskultur: Die Rolle des Topmanagements, in: Herausforderung Unternehmenskultur, Simon, H., (Hrsg.), Stuttgart 1990, S. 41-46.

Lederer, M., Clement, R., Mit innovativen Marktleistungskonzepten zu globaler Wettbewerbsfähigkeit, in: Markendynamik, Weinhold-Strünzi, H., Reinecke, S., Schögel, M. (Hrsg.), St. Gallen 1997, S. 144-152.

Lehnert, St., Die Bedeutung von Kontingenzansätzen für das strategische Management, Frankfurt a. M., Bern, New York, 1983.

Linden, F.A., Schlacht um die Spitze, in: manager magazin, Heft 8, 1998, S. 4857.

Linden, F.A., Volles Rohr voraus, in: manager magazin, Heft 4, 1998, S. 59-75.

Loitlsberger, E., Treuhand und Revisionswesen, 2. Aufl., Stuttgart 1966.

Low, G. S., Fullerton, R. A., Brand, Brand Management, and the Brand Manager System: A Critical-Historical Evaluation, in: Journal of Marketing Research, Vol. 31, May 1994, S. 173-190.

Low, G.S., Mohr, J.J. Setting Advertising and Promotion Budgets in Multi-Brand Companies, in: JoAR, No. 1, 1999, S. 67-78. 
Luehrman, T. A., Strategy as a Portfolio of Real Options, in: Harvard Business Review, Sept.-Oct. 1998, S. 89-99.

Luhmann, N., Komplexität, in: Handwörterbuch der Organisation, Grochla, E. (Hrsg.), 2. Aufl., Stuttgart 1980, Sp. 1064-1070.

Lütke Schwienhorst, R., Strategische Kontrolle, Rahmenbedingungen, Aufgaben und Methoden, Diss, Wiesbaden 1989.

Lynch, R. L., Cross, K. F., Measure Up! Yardsticks for Continuous Improvement, Cornwall 1995.

Mag, W., Planung, in: Vahlens Kompendium der Betriebswirtschaftslehre, Bitz, M. et al. (Hrsg.), Band 2, 3. Aufl., München 1993, S. 1-56.

Malik, F., Strategie des Managements komplexer Systeme, 5. Aufl., Bern 1996.

Mansfield, E., Technological Change and the Rate of Imitation, in: Econometrica, Vol. 29, Heft 4, S. 741-766.

March, J., G., Simon, H. A., Organizations, New York, London, Sydney, 1958.

Martinsons, M., Davison, R., Tse, D., The Balanced Scorecard: a Foundation for the Strategic Management of Information Systems, in: Decision Support Systems, Vol. 25, 1999, S. 71-88.

Maslow, A. H., Motivation and Personality, 3. Aufl. New York 1989.

Mason, Ch. H., Milne, G. R., An Approach for Identifying Cannibalization within Product Line Extensions and Multi-Brand Strategies, in: JoBR, 1994, S. 163-170.

Matje, A., Unternehmensleitbilder als Führungsinstrument: Komponenten einer erfolgreichen Unternehmensidentität, Wiesbaden 1996.

Maxton, G., Wormald, J., Driving over a Cliff? Business Lessons from the World's Car Industry, Cambridge 1995.

McAlister, L., A Dynamic Attribute Satiation Model of Variety Seeking Behavior, in: Journal of Consumer Research, Vol. 9, Heft 2, 1982, S. 141-150.

Meffert, H., Die Leistungsfähigkeit der entscheidungs- und systemorientierten Marketingtheorie, in: Wissenschaftsprogramm und Ausbildungsziele der Betriebswirtschaftslehre, Tagungsberichte des Verbandes der Hochschullehrer für Betriebswirtschaft e.V., Band 1, Kortzfleisch, G. v. (Hrsg.), Berlin 1971, S. 167-187.

Meffert, H., Computergestützte Marketing-Informationssysteme und MarketingModelle, in: Computergestützte Marketing-Planung, Hansen, H. R., München 1974, S. 11-31.

Meffert, H., Größere Flexibilität als Unternehmungskonzept, in: ZfbF, 37. Jg., Heft 2, 1985, S. 121-137.

Meffert, $\boldsymbol{H}$., Kundendienstpolitik - Eine Bestandsaufnahme zu einem komplexen Marketinginstrument, in: Marketing ZFP, Heft 2, Mai 1987, S. 93-102.

Meffert, H., Strategien zur Profilierung von Marken, in: Marke und Markenartikel als Instrumente des Wettbewerbs, Dichtl, E., Eggers, W. (Hrsg.), München 1992, S. 129-156. 
Meffert, H., Marketingforschung und Käuferverhalten, 2. Aufl., Wiesbaden 1992.

Meffert, H., Entscheidungsorientierter Ansatz der Markenpolitik, in: Handwörterbuch Markenartikel, Bruhn, M. (Hrsg.), Band 1, Stuttgart 1994, S. 173197.

Meffert, H., Markenführung in der Bewährungsprobe, in: Markenartikel, Heft 10, 1994, S. 478-481.

Meffert, H., Marketing-Management: Analyse, Strategie, Implementierung, Wiesbaden 1994.

Meffert, H., Herausforderungen an die Betriebswirtschaftslehre - Die Perspektive der Wissenschaft, in: DBW, 58. Jg., Heft 6, 1998, S. 709-730.

Meffert, H., Marketing: Grundlagen marktorientierter Unternehmensführung: Konzepte - Instrumente - Praxisbeispiele, 8. Aufl., Wiesbaden 1998.

Meffert, H., Dogmen, in: Horizont Magazin, Heft 1, 1999, S. 106-107.

Meffert, H., Mehrmarkenstrategie - immer die beste Option? in: asw, Sondernummer Oktober 1999, S. 82-87.

Meffert, H., Marketingwissenschaft im Wandel - Anmerkungen zur Paradigmendiskussion, in: HHL-Arbeitspapier Nr. 30, Leipzig 1999.

Meffert, H., Bruhn, M., Beschwerdeverhalten und Zufriedenheit von Konsumenten, in: DBW, 41. Jg., Heft 4, 1981, S. 597-613.

Meffert, H., Burmann, Ch., Identitätsorientierte Markenführung - Grundlagen für das Management von Markenportfolios, Arbeitspapier Nr. 100 der Wissenschaftlichen Gesellschaft für Marketing und Unternehmensführung e.V., Meffert, H., Wagner, H., Backhaus, K. (Hrsg.), Münster 1996.

Meffert, H., Burmann, Ch., Identitätsorientierte Markenführung, in: Markenartikel, Heft 8, 1996, S. 373-380.

Meffert, H., Burmann, Ch., Towards an Identity-Oriented Approach of Branding, University of Cambridge, Research Papers in Management Studies, Nr.18, 1996.

Meffert, H., Burmann, Ch., Identitätsorientierte Markenführung - Konsequenzen für die Handelsmarke, in: Handelsmarken. Entwicklungstendenzen und Zukunftsperspektiven der Handelsmarkenpolitik, Bruhn, M. (Hrsg.), 2. Aufl., Stuttgart 1997, S. 49-69.

Meffert, H., Burmann, Ch., Abnutzbarkeit und Nutzungsdauer von Marken - Ein Beitrag zur steuerlichen Behandlung von Warenzeichen, Arbeitspapier Nr. 117 der Wissenschaftlichen Gesellschaft für Marketing und Unternehmensführung e.V., Meffert, H., Backhaus, K. (Hrsg.), Münster 1998.

Meffert, H., Burmann, Ch., Abnutzbarkeit und Nutzungsdauer von Marken, in: GfK JdAV, Heft 3, 1999, S. 244-263.

Meffert, H., Burmann, Ch., Markenbildung und Markenstrategien, in: Handbuch Produktmanagement, Albers, S., Herrmann, A., (Hrsg.), Wiesbaden 2000 (im Druck). 
Meffert, H., Koers, M., Positionierung im Wettbewerb - dargestellt am Beispiel der Automobilbranche, Fallstudie des Instituts für Marketing, Münster 2000.

Meffert, H., Perrey, J., Mehrmarkenstrategie - Ein Beitrag zum Management von Markenportfolios, Arbeitspapier Nr. 121 der Wissenschaftlichen Gesellschaft für Marketing und Unternehmensführung e.V., Meffert, H., Backhaus, K. (Hrsg.), Münster 1998.

Meffert, H., Perrey, J., Mehrmarkenstrategie - Ein Beitrag zum Management von Markenportfolios, in: Moderne Markenführung: Grundlagen - innovative Ansätze - praktische Umsetzung, Esch, F.-R. (Hrsg.), Wiesbaden 1999, S. 619-646.

Meffert, H., Wöllenstein, St., Burmann, Ch., Erfolgswirkungen des Konflikt- und Kooperationsverhaltens in vertraglichen Vertriebssystemen des Automobilhandels, in: Marketing ZFP, Heft 4, 1996, S. 279-290.

Mehrabian, A., Russel, J. A., An Approach to Environmental Psychology, MITPress, Cambridge, Mass., 1974.

Meinig, W., Dealer Satisfaction and its Significance with Regard to the Relationship between authorized Car Dealers and Manufacturers/Importers, in: der markt, Heft 1, 1998, S. 12-20.

Mellerowicz, K., Markenartikel - Die ökonomischen Gesetze ihrer Preisbildung und Preisbindung, 2. Aufl., München u.a. 1963.

Menzel, St., Mehrmarkenstrategie als Erfolgsrezept, in: Handelsblatt Nr. 147 vom 3.8.1999, S. 43.

Michael, B. M., Die Marke ist tot. Es lebe die Marke!, in: Markenartikel, Heft 1, 1994, S. 22-25.

Michael, B. M., Laudatio Deutscher Marketing-Preis 1999 an Volkswagen AG, in: Kongressdokumentation vom Deutschen Marketing Verband des 27. Deutschen Marketing-Tag am 29. Oktober 1999 in Frankfurt unter dem Thema "Chancen im 3. Jahrtausend. Information managen - Zukunft gestalten", S. 47.

Micklethwait, J., Wooldridge, A., The Witch Doctors - What the Management Gurus are Saying, Why it Matters and How to Make Sense of it, London 1996.

Miller, J. E., Shepard, R. N., Chang, J. J., An Analytical Approach to the Interpretation of Multidimensional Scaling Solution, in: American Psychologist, Vol. 19, 1964, S. 579-580.

Mooraj, St., Oyon, D., Hostettler, D., The Balanced Scorecard: a Necessary Good or an Unnecessary Evil?, in: European Management Journal, Vol. 17, Heft 5, 1999, S. 481-491.

Moorthy, K. S., Png, I. P. L., Market Segmentation, Cannibalization and the Timing of Product Introduction, in: Management Science, 38. Jg., Heft 3, 1992, S. 345-359. 
Müller, W., Strategische Marktforschung - dargestellt am Beispiel der Automobilindustrie, in: Wettbewerbsvorteile erkennen und sichern: Erfahrungsberichte aus der Marketingpraxis, Neuwied 1994, S. 35-53.

Müller-Hagedorn, L., Bausteine eines Management-Informationssystems: Balanced Scorecard - Benchmarking - Betriebsvergleich, in: Beisheim, O., Distribution im Aufbruch - Bestandsaufnahme und Perspektiven, München 1999, S. 729-754.

Müller-Hagedorn, L., Büchel, D., Zur Steuerung von Handelsunternehmen mit der Balanced Scorecard, in: Der Verbund, Heft 3, 1999, S. 17-23.

Müller, W., Riesenbeck, H.-J., Wie aus zufriedenen auch anhängliche Kunden werden, in: Harvard Manager, 13. Jg., Heft 3, 1991, S. 68-73.

Neely, A., Gregory, M., Platts, K., Performance Measurement System Design, in: International Journal of Operations \& Production Management, Heft 4, 1995, S. 80-116.

Neubauer, F.-F., Portfolio-Management: Erfolgspotentiale vor Planungsritualen, 3. Aufl., Neuwied 1989.

Newman, J. W., Werbel, R. A., Multivariate Analysis of Brand Loyalty for Major Household Appliances, in: Journal of Marketing Research, 10. Jg., November 1973, S. 404-409.

Nieschlag, R., Dichtl, E., Hörschgen, H., Marketing, 18. Aufl., Berlin 1997.

Nommensen, J. N., Die Prägnanz von Markenbildern: Prüfung der Kommunikationsstrategie bei Produktrepositionierung, Heidelberg 1990.

Norton, D. P., Use Strategy Maps to Communicate Your Strategy, in: Balanced Scorecard: Insight, Experience \& Ideas for Strategy Focused Organizations (http//www.hbsp.harvard.edu./products/bsr/index.html [5.5.2000])

o. V., Lifestyle-Typologien helfen dem Marketing kaum, in: w\&v, Heft 3, 1992, S. 12-16.

o.V., Markenwechsel ist in. Eine Sonderstudie zum Thema Markenwechsel/ Makenbewußtsein, durchgeführt von Infratest Kommunikationsforschung im Auftrag der Bayerischen Rundfunkwerbung, München 1994.

o.V., Nicht nur Junge werden untreu. Markenwechsel ist keine Frage des Alters, in: Lebensmittelzeitung, Heft 24, 1995, S. 60.

o.V., Doppeltest VW Polo Classic gegen Seat Cordoba, in: Auto, Motor und Sport, Heft 6, 1996, S. 42-44.

o.V., Marktbericht August 1997, in: Auto, Motor und Sport, Heft 21, 1997, S. 10.

o.V., autofahren in deutschland 1999, Motor-Presse Stuttgart (Hrsg.), Stuttgart 1999.

o.V., Höhere Ansprüche an die interne Revision, in: FAZ vom 29.11.1999, S. 28.

o.V., Piechs Sündenbock, in: Wirtschaftswoche vom 1.6.2000, Heft 23, S. 12.

O'Meara, J. T., Selecting Profitable Products, in: Harvard Business Review, Vol. 39, Heft 1, 1961, S. 83-89. 
Olve, N., Roy, J., Wetter, M., Performance Drivers - A Practical Guide to Using the Balanced Scorecard, Chichester u. a. 1999.

Osgood, C. E., Eine Entdeckungsreise in die Welt der Begriffe und Bedeutungen, in: Grundfragen der Kommunikationsforschung, Schramm, W. (Hrsg.), 5. Aufl., München 1973, S. 39-54.

Osgood, C. E., Suci, G. J., Tannenbaum, P. H., The Measurement of Meaning, University of Illinois Press, Chicago u.a 1957.

Otte, Th., Die Selbstähnlicheit der Marke, in: Jahrbuch Markentechnik 1995 , Brandmeyer, K., Deichsel, A., Otte, Th. (Hrsg.), Frankfurt a. M. 1995, S. 43-53.

Otte, Th., Sind die Marken milleniumfähig?, in asw, Sondernummer Oktober 1999, S. $190-193$.

Owen, S., The Landor Image Power Survey: A Global Assessment of Brand Strength, in: Brand Equity \& Advertising. Advertising's Role in Buliding Strong Brands, Aaker, D. A., Biel, A. L. (Hrsg.), Hillsdale 1993, S. 11 30.

Palloks, M., Kennzahlen, absatzwirtschaftliche, in: Handwörterbuch des Marketing, Tietz, B., Köhler, R., Zentes, J. (Hrsg.), 2. Aufl., Stuttgart 1995, Sp. 1136-1153.

Parasuram, A., Zeithaml, V., Berry, L.L., A Conceptual Model of Service Quality and its Implications for Future Research, in: Journal of Marketing, Vol. 49, 1985, S. 41-50.

Paulus, J., Fraktale Marke: Verbrauchter Mythos, in: W\&V, Heft 10, 1995, S. 8086.

Pausenberger, E., Der Konzernaufbau. Versuch einer Morphologie des Konzerns, München 1957.

Perlitz, M., Unternehmen durch „Performance Measurement" erfolgreich machen, in: Gablers Magazin, Heft 2, 1999, S. 6-10.

Perrey, J., Nutzenorientierte Marktsegmentierung - Ein integrativer Ansatz zum Zielgruppenmarketing im Verkehrsdienstleistungsbereich, Wiesbaden 1998.

Perridon, L., Steiner, M., Finanzwirtschaft der Unternehmung, 9. Aufl., München 1997.

Petersen, Th., Optimale Anreizsysteme. Betriebswirtschaftliche Implikationen der Principal-Agenten-Theorie, Wiesbaden 1989.

Pfohl, H.-Ch., Zettelmeyer, B., Strategisches Controlling: in ZfB, 57. Jg., Heft 2, 1987, S. 145-175.

Picot, A., Böhme, M., Controlling in dezentralen Unternehmensstrukturen, München 1999.

Picot, A., Dietl, H., Franck, E., Organisation: Eine ökonomische Perspektive, Stuttgart 1997. 
Porter, M. E., What is Strategy, in: Harvard Business Review, Heft 6, Nov.-Dez. 1996, S. 61-78.

Porter, M. E., Wettbewerbsvorteile: Spitzenleistungen erreichen und behaupten, 5. Aufl., Frankfurt a. M., New York 1999.

Prahalad, C. K., Hamel, G., The Core Competence of the Corporation, in: Harvard Business Review, Vol. 68, May-June 1990, S. 79-91.

Proff, H., Mikroökonomische Wurzeln eines strategischen Managements zwischen Markt- und Ressourcenorientierung, in: Strategien für die Automobilindustrie: Ansatzpunkte im strategischen Management und in der Industriepolitik, Proff, H., Proff, H. V., (Hrsg.), Wiesbaden 1998, S. 2345 .

Proff, H., Proff, H. V., Bedeutung vorwärtsgerichteter Strategien für die Automobilindustrie, in: Strategien für die Automobilindustrie: Ansatzpunkte im strategischen Management und in der Industriepolitik, Proff, H., Proff, H. V., (Hrsg.), Wiesbaden 1998, S. 1-19.

Puhlmann, M., Semlitsch, B., Wie geht das Management mit der Marke um?, in: asw, Sondernummer Oktober 1997, S. 24-32.

Quelch, J. A., Kenny, D., Extend Profits, Not Product Lines, in: Harvard Business Review, Heft 5, Sept.-Oct. 1994, S. 153-160.

Rappaport, A., Creating Shareholder Value. The New Standard for Business Performance, New York 1986.

Rasche, Ch., Wettbewerbsvorteile durch Kernkompetenzen - Ein ressourcenorientierter Ansatz, Wiesbaden 1994.

Reddy, S. K., Holak, S. L., Bhat, S., To Extend or Not to Extend: Success Determinants of Line Extensions, in: JoMR, Vol. XXXI, May 1994, S. 243262.

Reichheld, F. F., Sasser, W. E., Zero-Migration: Dienstleister im Sog der Qualitätsrevolution, in: Harvard Manager, 13. Jg., Heft 4, 1991, S. 108-116.

Reichmann, Th., Controlling mit Kennzahlen und Managementberichten: Grundlagen einer systemgestützten Controlling-Konzeption, 4. Aufl., München 1995

Reineke, S., Tomczak, T., Aufgabenorientiertes Marketingcontrolling, in: Marketing-Controlling, Reinecke, S., Tomczak, T., Dittrich, S. (Hrsg.), St. Gallen 1998, S. 90-109.

Reuter, J., Komplexität und Dynamik der Implementierung von Wettbewerbsstrategien, Wiesbaden 1998.

Riebel, P., Einzelkosten- und Deckungsbeitragsrechnung, 6. Aufl., Wiesbaden 1990.

Riedel, F., Die Markenwertmessung als Grundlage strategischer Markenführung, Heidelberg 1996.

Ries, A., Trout, J., Positioning: Die neue Werbestrategie, Hamburg 1986. 
Robertson, D., Ulrich, K., Produktplattformen: Was sie leisten, was sie erfordern in: Harvard Business Manager, No. 4, 1999, S. 75-85.

Rommel, G. et al., Einfach überlegen. Das Unternehmen, das die Schlanken schlanker macht und die Schnellen schneller macht, Stuttgart 1993.

Roters, M., Komplexität und Dynamik als Einflußgrößen der Effizienz von Organisationen. Eine empirische Untersuchung, Frankfurt a. M., 1989.

Roth, F., Ickstadt, C., Die geheime Quelle der Stärke, in: Horizont Magazin, Nr. 3, 1999, S. 88-96.

Rubinson, J.-R., Brand Equity. Zum Management komplexer Markenportfolios, in: planung\&analyse, Heft 3, 1994, S. 48-51.

Sabel, H., Produktpolitik in absatzwirtschaftlicher Sicht - Grundlagen und Entscheidungsmodelle, Wiesbaden 1971.

Sanchez, R., Strategic Product Creation: Managing New Interactions of Technology, Markets and Organizations, in: European Management Journal, Vol. 14, Heft 2, 1996, S. 121-138.

Sander, M., Die Bestimmung und Steuerung des Wertes von Marken. Eine Analyse aus Sicht des Markeninhabers, Heidelberg 1994.

Sander, M., Die Bewertung internationaler Marken auf Basis der hedonischen Theorie, in: Marketing ZFP, Heft 4, 1994, S. 234-245.

Sattler, H., Markenstrategien für neue Produkte, in: Moderne Markenführung: Grundlagen - innovative Ansätze - praktische Umsetzung, Esch, F.-R. (Hrsg.), Wiesbaden 1999, S. 337-355.

Schaaf, A., Marktorientiertes Entwicklungsmanagement in der Automobilindustrie: Ein kundennutzenorientierter Ansatz zur Steuerung des Entwicklungsprozesses, Wiesbaden 1999.

Schiele, Th. P., Markenstrategien wachstumsorientierter Unternehmen, Wiesbaden 1999.

Schinzer, H.D./Bange, C./Mertnes, H., Data Warehouse und Data Mining: Marktführende Produkte im Vergleich, 2. Aufl., München 1999.

Schmalensee, R., Do Markets Differ Much?, in: American Economic Review, Vol 75,1985, S. 341-351.

Schmidt, U., Balanced Scorecard - Von Kennzahlen zur Handlungssteuerung, in: Organisationsentwicklung, Heft 2, 1998, S. 28-40.

Schmitt, B. H., Pan, Y., Managing Corporate and Brand Identities in the Asia Pacific Region, in: California Management Review, Winter 1995, S. 15-31.

Scholl, W., Politische Prozesse in Organisationen, in: Handwörterbuch der Organisation, Frese, E. (Hrsg.), 3. Aufl., Stuttgart, Sp. 1993-2004.

Scholtys, F., Riskante Tour, in: managermagazin, Heft 10, 1999, S. 130-143.

Schöne, K., Controlling der Informationsinfrastruktur: Entwicklungsstand - Gestaltungskonzeptionen - Perspektiven, Wiesbaden 1997. 
Schott, G., Kennzahlen - Instrument der Unternehmensführung, 4. Aufl., Wiesbaden 1991.

Schulte-Zurhausen, M., Organisation, München 1995.

Schüppenhauer, A., Wo die Märkte aufreißen, in: asw, 39. Jg., Nr. 6, 1996.

Schüppenhauer, A., Multioptionales Konsumentenverhalten: Erklärungen und Empfehlungen auf Basis der Autopoiesetheorie, Wiesbaden 1998.

Schütz, K., Markenkompetenz als Ansatz der Markenführung - dargestellt an einem ausgewählten Beispiel, unveröffentlichte Diplomarbeit am Institut für Marketing der Universität Münster, Münster 2000.

Schwarzrock, K., Controlling und Führungsberichtswesen in der Praxis, in: Controlling in Theorie und Praxis, Botta, V. (Hrsg.) Aachen 1999.

Schwaiger. M., Wirkungskontrolle kommunikationspolitischer Maßnahmen, in: Marketingcontrolling, Reinecke, S., Tomczak, T., Dittrich, S. (Hrsg.), St. Gallen 1998, S. 200-209.

Schweiger, G., Friederes, G., Vom Markenmythos zum Markenwert, in: Werbeforschung und Praxis, Heft 1, 1995, S. 26-31.

Schweitzer, M., Friedl, B., Beitrag zu einer umfassenden Controlling-Konzeption, in: Controlling, Spremann, K., Zur, E. (Hrsg.), Wiesbaden 1992, S. 141167.

Schwetje, Th., Kundenzufriedenheit und Arbeitszufriedenheit bei Dienstleitungen: Operationalisierung und Erklärung der Beziehungen am Beispiel des Handels, Wiesbaden 1999.

Sheinin, D. A., Schmitt, B. H., Extending Brands with New Product Concepts: The Role of Category Attribute Congruity, Brand Affect, and Brand Breadth, in: JoBR, 1994, S. 1-10.

Shocker, A. D., Srinivasan, V., A Consumer-Based Methodology for the Identification of New Product Ideas, in: Management Science, Vol. 20, No. 6, 1974 , S. $921-937$.

Shocker, A. D., Srinivasan, V., Multiattribute Approaches for Product Concept Evaluation and Generation: A Critical Review, in: Journal of Marketing Research, Vol. 16, May 1979, S. 159-180.

Siegwart, H., Controlling-Konzepte und Controller-Funktionen in der Schweiz, in: Controlling-Konzepte im internationalen Vergleich, Mayer, E., v. Landsberg, G., Thiede, W., (Hrsg.), Freiburg i. Br., 1986, S. 105-131.

Simon, C. J., Sullivan, M. W., A Financial Approach to Estimating Firm-Level Brand Equity and Measuring the Impact of Marketing Events, MSI Working Paper Report No. 92-116, Cambridge 1992.

Simon, H., Goodwill und Marketingstrategie, Wiesbaden 1985.

Simon, H., Management strategischer Wettbewerbsvorteile, in: ZfB, 58. Jg., Heft 4, 1988, S. 461-480.

Simon, H., Homburg, C., Kundenzufriedenheit als strategischer Erfolgsfaktor Einführende Überlegungen, in: Kundenzufriedenheit: Konzepte- 
Methoden-Erfahrungen, Simon, H., Homburg, C. (Hrsg.), Wiesbaden 1995, S. 15-27.

Smith, D. C., Parc, W. C., The Effects of Brand Extensions on Market Share and Advertising Efficiency, in: Journal of Marketing Research, Vol. 29, Nr. 1, S. 296-313.

Solomon, J., Hymowitz, C., Procter and Gamble Makes Changes in the Way It Develops and Sells Its Products, in: Wall Street Journal, 11.8.1987, S. 1 und 12.

Spiegel, B., Die Struktur der Meinungsverteilung im sozialen Feld: Das psychologische Marktmodell, Bern, Stuttgart 1961.

Srinivasan, V., Shocker, A. D., Linear Programming Techniques for Multidimensional Analysis of Preferences, in: Psychometrica, Vol. 38, 1973, S. 337-354.

Srivastava, R. K., Alpert, M. J., Shocker, A. D., A Customer-oriented Approach for Determining Market Structures, in: Journal of Marketing, Vol. 48., Heft 2, S. 32-45.

Srivastava, R. K., Shocker, A. D., Brand Equity: A Perspective on Its Meaning and Measurement, MSI Technical Working Paper, Report No. 91-124, Cambridge 1991.

Stegmüller, B., Internationale Marktsegmentierung als Grundlage für internationale Marketingkonzeptionen, Bergisch-Gladbach u. a., 1995.

Sullivan, M., Measuring Image Spillovers in Umbrella-branded Products, in: Journal of Business, 63. Jg., Heft 3, 1990, S. 309-329.

Tannenbaum, R., Schmidt, W., How to choose a Leadership Pattern, in: Harvard Business Review, Vol. 36, 1958, S. 95ff.

Teece, D.J., Pisano, G., Shuen, A., Dynamic Capabilities and Strategic Management, in: Strategic Management Journal, Vol. 18, 1997, S. 509-533.

Tennagen, U., Produktrelaunch in der Konsumgüterindustrie: Diagnosekonzept zur Auswahl, Ermittlung und Bewertung von Informationen, Wiesbaden 1993.

ter Haseborg, F., Mäßen, A., Das Phänomen Variety-Seeking-Behavior: Modellierung, empirische Befunde und marketingpolitische Implikationen, in: JdAV, 43. Jg. 1997, S. 164 - 188.

Theisen, P., Preispolitik der Mehrproduktunternehmung, in: Wist, 4. Jg., Heft 6, S. 273-281.

Thompson, J. D., Organizations in Action, New York u. a. 1967.

Tolle, E., Steffenhagen, H., Kategorien des Markenerfolges und einschlägige Meßmethoden, in: Handwörterbuch Markenartikel, Bruhn, M. (Hrsg.), Band 2, Stuttgart 1994, S. 1283-1303.

Tomczak, T., Müller, F., Kommunikation als zentraler Erfolgsfaktor der strategischen Markenführung, in: Thexis, Heft 6, 1992, S. 18-22. 
Tomczak, T., Roosdorp, A., Positionierung - Neue Herausforderungen verlangen neue Ansätze, in: Positionierung: Kernentscheidung des Marketing, Tomczak, T., Rudolph, Th., Roosdorp, A., (Hrsg.), St. Gallen 1996, S. 26-42.

Traylor, M. B., Cannibalism in Multibrand Firms, in: Journal of Consumer Marketing, Vol. 3, No. 2, Spring 1986, S. 69-75.

Trommsdorff, V., Die Messung von Produktimages für das Marketing, Köln u. a 1975.

Trommsdorff, V., Die Messung von Produktimages für das Marketing. Grundlagen und Operationalisierung, Köln u. a. 1975.

Trommsdorff, V., Wettbewerbsorientierte Image-Positionierung, in: Markenartikel, Heft 10, 1992, S. 458-463.

Trommsdorff, V., Positionierung, in: Handwörterbuch des Marketing, 2. Aufl., Tietz, B, Köhler, R., Zentes, J. (Hrsg.), Stuttgart 1995, Sp. 2055-2068.

Trommsdorff, V., Konsumentenverhalten, 3. Aufl., Stuttgart-Berlin-Köln, 1998.

Trommsdorff, V., Paulssen, M., Messung und Gestaltung der Markenpositionierung, in: Moderne Markenführung: Grundlagen - innovative Ansätze praktische Umsetzung, Esch, F.-R. (Hrsg.), Wiesbaden 1999, S. 10691088.

Trommsdorff, V., Zellerhoff, C., Produkt- und Markenpositionierung, in: Handwörterbuch Markenartikel, Bruhn, M. (Hrsg.), Band 1, Stuttgart 1994, S. 349-373.

Ulrich, H., Die Unternehmung als produktives soziales System, 2. Aufl., Bern 1970.

Upshaw. L. B., Building Brand Identity: A Strategy for Success in an hostile Marketplace, New York u.a. 1995.

Vershofen, W., Handbuch der Verbrauchsforschung, Band 1, Berlin 1940

Wagner, H., Marktorientierte Unternehmensführung versus Orientierung an Mitarbeiterinteressen, Shareholder-Value und Gemeinwohlverpflichtung, in: Marktorientierte Unternehmensführung: Reflexionen-DenkanstößePerspektiven, Bruhn, M., Steffenhagen, H. (Hrsg.), Wiesbaden 1997, S. 87-102.

Waltermann, B., Internationale Markenpolitik und Produktpositionierung: markenpolitische Entscheidungen im europäischen Automobilmarkt, Wien 1989.

Weber, D., Wenn Line Extensions gefährlich werden, in: W\&V, Heft 39, 1997, S. 106-112.

Weber, J. Einführung in das Controlling, 7. Aufl., Stuttgart 1998.

Weber, J., Controlling - Entwicklungstendenzen und Zukunftsperspektiven, in: Die Unternehmung, 53. Jg., Heft 6, 1999, S. 465-480.

Weber, J. et al, Grundgedanken zur Entwicklung einer Theorie der Unternehmensführung, WHU-Forschungspapier Nr. 30, Koblenz 1995. 
Weber, J., Knorren, N., Sicherung der Rationalität durch wertorientierte Planung, WHU- Forschungsbericht Nr. 59, Koblenz 1998.

Weber, J., Schäffer, U., Balanced Scorecard - Gedanken zur Einordnung des Konzeptes in das bisherige Controlling-Instrumentarium, WHU- Forschungspapier.

Weber, J., Schäffer, U., Entwicklung von Kennzahlensystemen, WHU- Forschungspapier Nr. 62, Koblenz 1999.

Weber, J., Schäffer, U., Sicherstellung der Rationalität von Führung als Aufgabe des Controlling?, in: DBW, 59. Jg., Heft 6, 1999, S. 731-747.

Weber, J., Schäffer, U., Balanced Scorecard \& Controlling: Implementierung Nutzen für Manager und Controller - Erfahrungen in deutschen Unternehmen, 2. Aufl., Wiesbaden 2000.

Weber, M., Soziologische Grundbegriffe, in: Gesammelte Aufsätze zur Wissenschaftslehre, Wincekmann, J. (Hrsg.), Tübingen 1973.

Weißenberg, P., Hochzeiten mit Hindernissen, in: W\&V, Heft 38, 1999, S. 96-98.

Werner, H., Vom Marken- zum Preiswettbewerb? - Strategisches Marketing in der Automobilindustrie für das Jahr 2000 und darüber hinaus!, in: Das Handbuch für das Automobilmarketing - Strategien, Konzepte, Instrumente, Diez, W. (Hrsg.), 3. Aufl., Landsberg/Lech 1997, S. 21-34.

Wiedmann, K-P., Markenpolitik und Corporate Identity, in: Handwörterbuch Markenartikel, Bruhn, M. (Hrsg.), Band 2, Stuttgart 1994, S. 1033-1054.

Wiedmann, K-P., Strategisches Markencontrolling, in: Handwörterbuch Markenartikel, Bruhn, M. (Hrsg.), Band 2, Stuttgart 1994, S. 1305-1336.

Wiese, J., Implementierung der Balanced Scorecard: Grundlagen und ITFachkonzepte, Wiesbaden 2000.

Witt, F. H., Theorietradition der betriebswirtschaftlichen Forschung, Wiesbaden 1985.

Wolters, H., Hocke, R., Auf dem Weg zur Globalisierung - Chancen und Risiken, in: Die Zukunft der Automobilindustrie, Wolters, H. et al. (Hrsg.), Wiesbaden 1999.

Young, F., Day, G., Multidimensional Scaling, New York 1974.

Zahn, E., Mehrebenenansatz der Planung, in: Handwörterbuch der Planung, Szyperski, N., Winand, U. (Hrsg.), Band 9, Stuttgart 1989, Sp. 1080-1090. 


\section{SCHRIFTEN ZUM MARKETING}

Band 1 Friedrich Wehrle: Strategische Marketingplanung in Warenhäusern. Anwendung der Portfolio-Methode. 1981. 2. Auflage. 1984.

Band 2 Jürgen Althans: Die Übertragbarkeit von Werbekonzeptionen auf internationale Märkte. Analyse und Exploration auf der Grundlage einer Befragung bei europaweit tätigen Werbeagenturen. 1982.

Band 3 Günter Kimmeskamp: Die Rollenbeurteilung von Handelsvertretungen. Eine empirische Untersuchung zur Einschätzung des Dienstleistungsangebotes durch Industrie und Handel. 1982.

Band 4 Manfred Bruhn: Konsumentenzufriedenheit und Beschwerden. Erklärungsansätze und Ergebnisse einer empirischen Untersuchung in ausgewählten Konsumbereichen. 1982.

Band 5 Heribert Meffert (Hrsg.): Kundendienst-Management. Entwicklungsstand und Entscheidungsprobleme der Kundendienstpolitik. 1982.

Band 6 Ralf Becker: Die Beurteilung von Handelsvertretern und Reisenden durch Hersteller und Kunden. Eine empirische Untersuchung zum Vergleich der Funktionen und Leistungen. 1982.

Band 7 Gerd Schnetkamp: Einstellungen und Involvement als Bestimmungsfaktoren des sozialen Verhaltens. Eine empirische Analyse am Beispiel der Organspendebereitschaft in der Bundesrepublik Deutschland. 1982.

Band 8 Stephan Bentz: Kennzahlensysteme zur Erfolgskontrolle des Verkaufs und der MarketingLogistik. Entwicklung und Anwendung in der Konsumgüterindustrie. 1983.

Band 9 Jan Honsel: Das Kaufverhalten im Antiquitätenmarkt. Eine empirische Analyse der Kaufmotive, ihrer Bestimmungsfaktoren und Verhaltenswirkungen. 1984.

\section{SCHRIFTEN ZU MARKETING UND MANAGEMENT}

Band 10 Matthias Krups: Marketing innovativer Dienstleistungen am Beispiel elektronischer Wirtschaftsinformationsdienste. 1985.

Band 11 Bernd Faehsler: Emotionale Grundhaltungen als Einflußfaktoren des Käuferverhaltens. Eine empirische Analyse der Beziehungen zwischen emotionalen Grundhaltungen und ausgewählten Konsumstrukturen. 1986.

Band 12 Ernst-Otto Thiesing: Strategische Marketingplanung in filialisierten Universalbanken. Integrierte Filial- und Kundengruppenstrategien auf der Grundlage erfolgsbeeinflussender Schlüsselfaktoren. 1986.

Band 13 Rainer Landwehr: Standardisierung der internationalen Werbeplanung. Eine Untersuchung der Prozeßstandardisierung am Beispiel der Werbebudgetierung im Automobilmarkt. 1988.

Band 14 Paul-Josef Patt: Strategische Erfolgsfaktoren im Einzelhandel. Eine empirische Analyse am Beispiel des Bekleidungsfachhandels. 1988. 2. Auflage. 1990.

Band 15 Elisabeth Tolle: Der Einfluß ablenkender Tätigkeiten auf die Werbewirkung. Bestimmungsfaktoren der Art und Höhe von Ablenkungseffekten bei Rundfunkspots. 1988.

Band 16 Hanns Ostmeier: Ökologieorientierte Produktinnovationen. Eine empirische Analyse unter besonderer Berücksichtigung ihrer Erfolgseinschätzung. 1990.

Band 17 Bernd Büker: Qualitätsbeurteilung investiver Dienstleistungen. Operationalisierungsansätze an einem empirischen Beispiel zentraler EDV-Dienste. 1991.

Band 18 Kerstin Ch. Monhemius: Umweltbewußtes Kaufverhalten von Konsumenten. Ein Beitrag zur Operationalisierung, Erklärung und Typologie des Verhaltens in der Kaufsituation. 1993. 
Band 19 Uwe Schürmann: Erfolgsfaktoren der Werbung im Produktlebenszyklus. Ein Beitrag zur Werbewirkungsforschung. 1993.

Band 20 Ralf Birkelbach: Qualitätsmanagement in Dienstleistungscentem. Konzeption und typenspezifische Ausgestaltung unter besonderer Berücksichtigung von Verkehrsflughäfen. 1993.

Band 21 Simone Frömbling. Zielgruppenmarketing im Fremdenverkehr von Regionen. Ein Beitrag zur Marktsegmentierung auf der Grundlage von Werten, Motiven und Einstellungen. 1993.

Band 22 Marcus Poggenpohl: Verbundanalyse im Einzelhandel auf der Grundlage von Kundenkarteninformationen. Eine empirische Untersuchung von Verbundbeziehungen zwischen Abteilungen. 1994.

Band 23 Kai Bauche: Segmentierung von Kundendienstleistungen auf investiven Märkten. Dargestellt am Beispiel von Personal Computern. 1994.

Band 24 Ewald Werthmöller: Räumliche Identität als Aufgabenfeld des Städte- und Regionenmarketing. Ein Beitrag zur Fundierung des Placemarketing. 1995.

Band 25 Nicolaus Müller: Marketingstrategien in High-Tech-Märkten. Typologisierung, Ausgestaltungsformen und Einflußfaktoren auf der Grundlage strategischer Gruppen. 1995.

Band 26 Nicolaus Henke: Wettbewerbsvorteile durch Integration von Geschäftsaktivitäten. Ein zeitablaufbezogener wettbewerbsstrategischer Analyseansatz unter besonderer Berücksichtigung des Einsatzes von Kommunikations- und Informationssystemen (KIS). 1995.

Band 27 Kai Laakmann: Value-Added Services als Profilierungsinstrument im Wettbewerb. Analyse, Generierung und Bewertung. 1995.

Band 28 Stephan Wöllenstein: Betriebstypenprofilierung in vertraglichen Vertriebssystemen. Eine Analyse von Einflußfaktoren und Erfolgswirkungen auf der Grundlage eines Vertragshändlersystems im Automobilhandel. 1996.

Band 29 Michael Szeliga: Push und Pull in der Markenpolitik. Ein Beitrag zur modellgestützten Marketingplanung am Beispiel des Reifenmarktes. 1996.

Band 30 Hans-Ulrich Schröder: Globales Produktmanagement. Eine empirische Analyse des Instrumenteeinsatzes in ausgewählten Branchen der Konsumgüterindustrie. 1996.

Band 31 Peter Lensker: Planung und Implementierung standardisierter vs. differenzierter Sortimentsstrategien in Filialbetrieben des Einzelhandels. 1996.

Band 32 Michael H. Ceyp: Ökologieorientierte Profilierung im vertikalen Marketing. Dargestellt am Beispiel der Elektrobranche. 1996.

Band 33 Mark Unger: Die Automobil-Kaufentscheidung. Ein theoretischer Erklärungsansatz und seine empirische Überprüfung. 1998.

Band 34 Ralf Ueding: Management von Messebeteiligungen. Identifikation und Erklärung messespezifischer Grundhaltungen auf der Basis einer empirischen Untersuchung. 1998.

Band 35 Andreas Siefke: Zufriedenheit mit Dienstleistungen. Ein phasenorientierter Ansatz zur Operationalisierung und Erklärung der Kundenzufriedenheit im Verkehrsbereich auf empirischer Basis. 1998.

Band 36 Irene Giesen-Netzer: Implementierung von Rücknahme- und Recyclingsystemen bei Gebrauchsgütern. 1998.

Band 37 Frithjof Netzer: Strategische Allianzen im Luftverkehr. Nachfragerorientierte Problemfelder inrer Gestaltung. 1999.

Band 38 Silvia Danne: Messebeteiligungen von Hochschulen. Ziele und Erfolgskontrolle. 2000.

Band 39 Martin Koers: Steuerung von Markenportfolios. Ein Beitrag zum Mehrmarkencontrolling am Beispiel der Automobilwirtschaft. 2001. 


\section{Kleine und mittlere Unternehmen im Strukturwandel - Arbeitsmarkt und Strukturpolitik}

Frankfurt/M., Berlin, Bern, Bruxelles, New York, Oxford, Wien, 2000. 243 S., zahlr. Tab.

Strukturwandel und Strukturpolitik. Bd. 2

Herausgegeben von Wolfram Elsner

ISBN 3-631-36316-8 br. DM 74.- *

In der Reihe nationaler und internationaler Maßnahmen zur Ankurbelung von Wachstum und Beschäftigung besitzt die Förderung kleiner und mittlerer Unternehmen und insbesondere von beruflicher Selbständigkeit und Unternehmensgründungen einen zentralen Stellenwert. Bei aller Euphorie muß freilich auch auf ernüchternde Tatbestände hingewiesen werden. Der Band beschäftigt sich in interdisziplinärer Perspektive mit unterschiedlichen wirtschafts-, sozial- und strukturpolitischen und sozioökonomischen Aspekten der Thematik. "Arbeit in und von KMU's", "Selbständige und Unternehmensgründungen" und "Entrepreneurship und Industrie- und Strukturpolitik" sind die Kapitel, unter die sich die Beiträge von namhaften Wirtschafts- und Sozialwissenschaftlern rangieren.

Frankfurt/M - Berlin - Bern - Bruxelles - New York - Oxford - Wien

Auslieferung: Verlag Peter Lang AG

Jupiterstr. 15, CH-3000 Bern 15

Telefax (004131) 9402131

*inklusive Mehrwertsteuer

Preisänderungen vorbehalten 